\title{
How to Promote Sluggish Electrocyclization of 1, 3, 5-Hexatrienes by \\ Captodative Substitution
}

\author{
Tang-Qing Yu, Yao Fu*, Lei Liu*, Qing-Xiang Guo \\ Department of Chemistry, University of Science and Technology of China, Hefei 230026,
}

China

\section{Supporting Information}

\section{Chapter $1 \square$ Tables of Data}

Table S1. The calculated Energies, the thermal corrections to Gibbs Free Energy and Solvent correction to Gibbs Free Energy related to compounds in Table 1..........3

Table S2. The calculated Energies, the thermal corrections to Gibbs Free Energy and Solvent correction to Gibbs Free Energy related to 1-substituted hexatriene. ........ 3

Table S3. The calculated Energies, the thermal corrections to Gibbs Free Energy and Solvent correction to Gibbs Free Energy related to 2-substituted hexatriene........4

Table S4. The calculated Energies, the thermal corrections to Gibbs Free Energy and Solvent correction to Gibbs Free Energy related to 3-substituted hexatriene........4

Table S5. The calculated Energies, the thermal corrections to Gibbs Free Energy and Solvent correction to Gibbs Free Energy related to dual substituted hexatriene......4

Table S6. The calculated Energies, the thermal corrections to Gibbs Free Energy and Solvent correction to Gibbs Free Energy related to compounds in Figure 3........ 15

Table S7. The C1-C6 Distance and calculated NICS related to compounds in Table 4 and the figures of their relationship with reaction barrier......................... 15 


\section{Chapter $2 \square$ The Cartesian coordinates $(\AA)$.}

1. The Cartesian coordinates of Compounds in Table 1........................17

2. The Cartesian coordinates of 1-Substituted Hexatriene.......................24

3. The Cartesian coordinates of 2- Substituted Hexatriene........................30

4. The Cartesian coordinates of 3- Substituted Hexatriene........................36

5. The Cartesian coordinates of Dual Substituted Hexatriene

a. The Cartesian coordinates of 1, 2-Substituted Hexatriene................41

b. The Cartesian coordinates of 1, 3-Substituted Hexatriene...............88

c. The Cartesian coordinates of 1, 4-Substituted Hexatriene.................135

d. The Cartesian coordinates of 1, 5-Substituted Hexatriene................181

e. The Cartesian coordinates of 1, 6-Substituted Hexatriene...............227

f. The Cartesian coordinates of 3, 2-Substituted Hexatriene..................254

g. The Cartesian coordinates of 4, 2-Substituted Hexatriene.................299

h. The Cartesian coordinates of 5, 2-Substituted Hexatriene..................344

i. The Cartesian coordinates of 4, 3-Substituted Hexatriene..................370

6. The Cartesian coordinates of Compounds in Figure 3........................396 
Table S1. Total energies (in a.u.) calculated at

ONIOM(QCISD(T)/6-31+G(d,p):B3LYP/6-311+G(2df,2p), Thermal correction to Gibbs Free Energy (in a.u.) calculated at B3LYP/6-31+G(d) and Solvent correction to Gibbs Free Energy (in $\mathrm{kcal} / \mathrm{mol}$ ) calculated at B3LYP/6-31+g** with PCM, Solvent=benzene.

\begin{tabular}{|c|c|c|c|c|c|c|}
\hline \multirow[b]{2}{*}{ Compound } & \multicolumn{3}{|c|}{ Reactant } & \multicolumn{3}{|c|}{ Transition State } \\
\hline & TCG & $\begin{array}{c}\text { Solvent } \\
\text { effect }\end{array}$ & $\begin{array}{c}\text { Total } \\
\text { Energy }\end{array}$ & TCG & $\begin{array}{c}\text { Solvent } \\
\text { effect }\end{array}$ & $\begin{array}{c}\text { Total } \\
\text { Energy }\end{array}$ \\
\hline 1,3,5-hexatriene & 0.072163 & 3.06 & -232.733194 & 0.074821 & 1.52 & -232.683269 \\
\hline & 0.097396 & 4.56 & -272.062906 & 0.098557 & 3.35 & -272.012846 \\
\hline & 0.122495 & 5.05 & -311.387126 & 0.123505 & 3.91 & -311.339127 \\
\hline & 0.148050 & 5.62 & -350.713837 & 0.149088 & 4.28 & -350.665932 \\
\hline & 0.104496 & 4.52 & -310.155148 & 0.106553 & 2.85 & -310.109693 \\
\hline & 0.125200 & 4.93 & -349.478123 & 0.126143 & 3.56 & -349.430101 \\
\hline & 0.177769 & 7.07 & -390.034081 & 0.179351 & 5.57 & -389.985943 \\
\hline
\end{tabular}

Table S2. Total energies (in a.u.) calculated at ONIOM(QCISD(T)/6-31+G(d,p):B3LYP/6-311+G(2df,2p), Thermal correction to Gibbs Free Energy (in a.u.) calculated at B3LYP/6-31+G(d) and Solvent correction to Gibbs Free Energy (in $\mathrm{kcal} / \mathrm{mol}$ ) calculated at B3LYP/6-31+g** with PCM, Solvent=benzene.

\begin{tabular}{|c|c|c|c|c|c|c|}
\hline \multirow[t]{2}{*}{ Compound } & & & & \multicolumn{3}{|c|}{ Transition State } \\
\hline & TCG & Solvent effect & Total Energy & TCG & Solvent effect & Total Energy \\
\hline $\mathrm{R}=\mathrm{CH}_{3}$ & 0.115166 & 3.94 & -272.065643 & 0.116196 & 2.64 & -272.013266 \\
\hline $\mathrm{R}=\mathrm{NH}_{2}$ & 0.104779 & 0.02 & -288.117407 & 0.106765 & -0.61 & -288.068913 \\
\hline $\mathrm{R}=\mathrm{OH}$ & 0.092523 & -0.32 & -307.986658 & 0.094453 & -0.98 & -307.939797 \\
\hline $\mathrm{R}=\mathrm{F}$ & 0.080117 & 1.95 & -332.005035 & 0.08195 & 0.68 & -331.959686 \\
\hline $\mathrm{R}=\mathrm{CHO}$ & 0.095751 & 0.57 & -346.101119 & 0.096566 & -0.28 & -346.047826 \\
\hline $\mathrm{R}=\mathrm{CN}$ & 0.085375 & 0.85 & -325.008455 & 0.086555 & -0.28 & -324.957057 \\
\hline
\end{tabular}




$\begin{array}{lllllll}\mathrm{R}=\mathrm{NO}_{2} & 0.087969 & 0.47 & -437.312499 & 0.089505 & -0.6 & -437.264156\end{array}$

Table S3. Total energies (in a.u.) calculated at ONIOM(QCISD(T)/6-31+G(d,p):B3LYP/6-311+G(2df,2p), Thermal correction to Gibbs Free Energy (in a.u.) calculated at B3LYP/6-31+G(d) and Solvent correction to Gibbs Free Energy (in $\mathrm{kcal} / \mathrm{mol}$ ) calculated at B3LYP/6-31+g** with PCM, Solvent=benzene.

\begin{tabular}{|c|c|c|c|c|c|c|}
\hline \multirow{2}{*}{ Compound } & & & & \multicolumn{3}{|c|}{ Transition State } \\
\hline & TCG & $\begin{array}{c}\text { Solvent } \\
\text { effect }\end{array}$ & Total Energy & TCG & $\begin{array}{c}\text { Solvent } \\
\text { effect }\end{array}$ & Total Energy \\
\hline $\mathrm{R}=\mathrm{CH}_{3}$ & 0.116154 & 4.42 & -272.059397 & 0.115435 & 3.3 & -272.012686 \\
\hline $\mathrm{R}=\mathrm{NH}_{2}$ & 0.105565 & 1.92 & -288.1114851 & 0.106203 & 0.29 & -288.066386 \\
\hline $\mathrm{R}=\mathrm{OH}$ & 0.092384 & 1.34 & -307.985427 & 0.093641 & -0.33 & -307.936572 \\
\hline $\mathrm{R}=\mathrm{F}$ & 0.080166 & 3.22 & -332.006867 & 0.081148 & 1.43 & -331.957229 \\
\hline $\mathrm{R}=\mathrm{CHO}$ & 0.095875 & 2.28 & -346.092444 & 0.097284 & 0.39 & -346.050490 \\
\hline $\mathrm{R}=\mathrm{CN}$ & 0.085353 & 2.93 & -324.999440 & 0.086652 & 0.43 & -324.957010 \\
\hline $\mathrm{R}=\mathrm{NO}_{2}$ & 0.087461 & 2.24 & -437.303387 & 0.089257 & 0.18 & -437.262425 \\
\hline
\end{tabular}

Table S4. Total energies (in a.u.) calculated at ONIOM(QCISD(T)/6-31+G(d,p):B3LYP/6-311+G(2df,2p), Thermal correction to Gibbs Free Energy (in a.u.) calculated at B3LYP/6-31+G(d) and Solvent correction to Gibbs Free Energy (in $\mathrm{kcal} / \mathrm{mol}$ ) calculated at B3LYP/6-31+g** with PCM, Solvent=benzene.

\begin{tabular}{ccccccc}
\hline \multirow{2}{*}{ Compound } & \multicolumn{3}{c}{ Transition State } \\
\cline { 2 - 7 } & $\mathrm{TCG}$ & $\begin{array}{c}\text { Solvent } \\
\text { effect }\end{array}$ & Total Energy & $\mathrm{TCG}$ & $\begin{array}{c}\text { Solvent } \\
\text { effect }\end{array}$ & Total Energy \\
\cline { 2 - 7 } & 0.115837 & 4.56 & -272.062906 & 0.116114 & 3.35 & -272.012846 \\
$\mathrm{R}=\mathrm{CH}_{3}$ & 0.105154 & 1.69 & -288.1140419 & 0.10575 & 0.19 & -288.063762 \\
$\mathrm{R}=\mathrm{NH}_{2}$ & 0.092213 & 1.09 & -307.9861099 & 0.093295 & -0.31 & -307.936530 \\
$\mathrm{R}=\mathrm{OH}$ & 0.080163 & 3.14 & -332.0076231 & 0.081146 & 1.48 & -331.958161 \\
$\mathrm{R}=\mathrm{F}$ & 0.096125 & 2.25 & -346.0947405 & 0.096966 & 0.56 & -346.048467 \\
$\mathrm{R}=\mathrm{CHO}$ & 0.085329 & 2.45 & -325.0040836 & 0.08628 & 0.49 & -324.955477 \\
$\mathrm{R}=\mathrm{CN}$ & 0.087112 & 2.12 & -437.3022062 & 0.088974 & 0.36 & -437.260791 \\
$\mathrm{R}=\mathrm{NO}_{2}$ & & & & & & \\
\hline
\end{tabular}

Table S5. Total energies (in a.u.) calculated at ONIOM(QCISD(T)/6-31+G(d,p):B3LYP/6-311+G(2df,2p), Thermal correction to Gibbs Free Energy (in a.u.) calculated at B3LYP/6-31+G(d) and Solvent correction to Gibbs Free Energy (in $\mathrm{kcal} / \mathrm{mol}$ ) calculated at B3LYP/6-31+g** 


\section{with PCM, Solvent=benzene.}

\begin{tabular}{|c|c|c|c|c|c|c|c|}
\hline \multicolumn{2}{|c|}{ Compound } & \multirow[b]{2}{*}{ TCG } & \multirow{2}{*}{\multicolumn{2}{|c|}{$\begin{aligned} \mathrm{R}_{2} & \mathrm{R}_{1} \\
\begin{array}{c}\text { Solvent } \\
\text { effect }\end{array} & \text { Total Energy }\end{aligned}$}} & \multicolumn{3}{|c|}{ Transition State } \\
\hline & & & & & TCG & $\begin{array}{l}\text { Solvent } \\
\text { effect }\end{array}$ & $\begin{array}{l}\text { Total } \\
\text { Energy }\end{array}$ \\
\hline \multirow[t]{7}{*}{$\mathrm{R}_{1}=\mathrm{CH}_{3}$} & $\mathrm{R}_{2}=\mathrm{CH}_{3}$ & 0.141815 & 5.04 & -311.3885056 & 0.142785 & 4 & -311.341882 \\
\hline & $\mathrm{R}_{2}=\mathrm{NH}_{2}$ & 0.130191 & 3.09 & -327.4379279 & 0.132482 & 1.41 & -327.395273 \\
\hline & $\mathrm{R}_{2}=\mathrm{OH}$ & 0.117228 & 3 & -347.3150362 & 0.119701 & 0.86 & -347.265948 \\
\hline & $\mathrm{R}_{2}=\mathrm{F}$ & 0.106118 & 4.02 & -371.3386008 & 0.107346 & 2.54 & -371.287077 \\
\hline & $\mathrm{R}_{2}=\mathrm{CHO}$ & 0.121742 & 3.25 & -385.4212531 & 0.123782 & 1.43 & -385.379722 \\
\hline & $\mathrm{R}_{2}=\mathrm{CN}$ & 0.111432 & 3.67 & -364.3335869 & 0.112724 & 1.51 & -364.287989 \\
\hline & $\mathrm{R}_{2}=\mathrm{NO}_{2}$ & 0.111794 & 3.21 & -476.6329621 & 0.116055 & 0.99 & -476.589285 \\
\hline \multirow[t]{7}{*}{$\mathrm{R}_{1}=\mathrm{NH}_{2}$} & $\mathrm{R}_{2}=\mathrm{CH}_{3}$ & 0.131266 & 1.74 & -327.441736 & 0.132940 & 1.22 & -327.395152 \\
\hline & $\mathrm{R}_{2}=\mathrm{NH}_{2}$ & 0.121239 & -0.21 & -343.490328 & 0.122799 & -1.21 & -343.448449 \\
\hline & $\mathrm{R}_{2}=\mathrm{OH}$ & 0.108446 & 0.55 & -363.363675 & 0.110587 & -1.34 & -363.320344 \\
\hline & $\mathrm{R}_{2}=\mathrm{F}$ & 0.095923 & 0.52 & -387.388920 & 0.097496 & -0.73 & -387.339166 \\
\hline & $\mathrm{R}_{2}=\mathrm{CHO}$ & 0.112798 & 0.12 & -401.491604 & 0.114579 & -0.84 & -401.441636 \\
\hline & $\mathrm{R}_{2}=\mathrm{CN}$ & 0.100875 & -0.27 & -380.392905 & 0.102587 & -1.8 & -380.344901 \\
\hline & $\mathrm{R}_{2}=\mathrm{NO}_{2}$ & 0.104564 & -0.99 & -492.703216 & 0.106130 & -1.54 & -492.648883 \\
\hline \multirow[t]{7}{*}{$\mathrm{R}_{1}=\mathrm{OH}$} & $\mathrm{R}_{2}=\mathrm{CH}_{3}$ & 0.118691 & 2.25 & -347.311476 & 0.120329 & 1.4 & -347.266484 \\
\hline & $\mathrm{R}_{2}=\mathrm{NH}_{2}$ & 0.109403 & 0.52 & -363.362160 & 0.110526 & -1.15 & -363.320380 \\
\hline & $\mathrm{R}_{2}=\mathrm{OH}$ & 0.096131 & -0.07 & -383.235673 & 0.097537 & -2.05 & -383.189713 \\
\hline & $\mathrm{R}_{2}=\mathrm{F}$ & 0.083635 & 1.02 & -407.257405 & 0.085289 & -0.35 & -407.210657 \\
\hline & $\mathrm{R}_{2}=\mathrm{CHO}$ & 0.100683 & 1.71 & -421.361086 & 0.102471 & -0.11 & -421.315188 \\
\hline & $\mathrm{R}_{2}=\mathrm{CN}$ & 0.089069 & 1.37 & -400.258556 & 0.090543 & -1.11 & -400.213357 \\
\hline & $\mathrm{R}_{2}=\mathrm{NO}_{2}$ & 0.092475 & 1.36 & -512.571180 & 0.093957 & -0.27 & -512.521578 \\
\hline \multirow[t]{7}{*}{$\mathrm{R}_{1}=\mathrm{F}$} & $\mathrm{R}_{2}=\mathrm{CH}_{3}$ & 0.107199 & 3.53 & -371.331735 & 0.107779 & 2.53 & -371.288668 \\
\hline & $\mathrm{R}_{2}=\mathrm{NH}_{2}$ & 0.096503 & 1.19 & -387.381721 & 0.097922 & -0.14 & -387.341411 \\
\hline & $\mathrm{R}_{2}=\mathrm{OH}$ & 0.083383 & 1.13 & -407.254449 & 0.085432 & -0.11 & -407.211211 \\
\hline & $\mathrm{R}_{2}=\mathrm{F}$ & 0.071072 & 2.16 & -431.270549 & 0.072819 & 0.6 & -431.227455 \\
\hline & $\mathrm{R}_{2}=\mathrm{CHO}$ & 0.086880 & 1.72 & -445.361635 & 0.088846 & 0.06 & -445.322615 \\
\hline & $\mathrm{R}_{2}=\mathrm{CN}$ & 0.076339 & 1.94 & -424.268242 & 0.078008 & -0.2 & -424.228200 \\
\hline & $\mathrm{R}_{2}=\mathrm{NO}_{2}$ & 0.077673 & 1.2 & -536.565769 & 0.079997 & -0.62 & -536.525406 \\
\hline \multirow[t]{7}{*}{$\mathrm{R}_{1}=\mathrm{CHO}$} & $\mathrm{R}_{2}=\mathrm{CH}_{3}$ & 0.122948 & 2.51 & -385.422056 & 0.123398 & 1.51 & -385.375032 \\
\hline & $\mathrm{R}_{2}=\mathrm{NH}_{2}$ & 0.113402 & 0.42 & -401.493157 & 0.113812 & -0.59 & -401.435330 \\
\hline & $\mathrm{R}_{2}=\mathrm{OH}$ & 0.100969 & 1.42 & -421.370271 & 0.101849 & -0.08 & -421.307017 \\
\hline & $\mathrm{R}_{2}=\mathrm{F}$ & 0.086917 & 0.94 & -445.371883 & 0.087747 & -0.17 & -445.319446 \\
\hline & $\mathrm{R}_{2}=\mathrm{CHO}$ & 0.102208 & 1.43 & -459.447129 & 0.103112 & -1.06 & -459.409525 \\
\hline & $\mathrm{R}_{2}=\mathrm{CN}$ & 0.091796 & 1.44 & -438.361612 & 0.092799 & -0.7 & -438.317059 \\
\hline & $\mathrm{R}_{2}=\mathrm{NO}_{2}$ & 0.093936 & 1.52 & -550.658551 & 0.095486 & -1.33 & -550.617928 \\
\hline \multirow[t]{2}{*}{$\mathrm{R}_{1}=\mathrm{CN}$} & $\mathrm{R}_{2}=\mathrm{CH}_{3}$ & 0.122948 & 2.53 & -364.335876 & 0.112279 & 1.69 & -364.287412 \\
\hline & $\mathrm{R}_{2}=\mathrm{NH}_{2}$ & 0.113402 & -0.01 & -380.394400 & 0.102679 & -1.14 & -380.344870 \\
\hline
\end{tabular}




\begin{tabular}{cccccccc}
\hline $\mathrm{R}_{2}=\mathrm{OH}$ & 0.100969 & 0.73 & -400.265927 & 0.090068 & -0.85 & -400.212128 \\
$\mathrm{R}_{2}=\mathrm{F}$ & 0.086917 & 1.14 & -424.279187 & 0.077533 & -0.29 & -424.227506 \\
$\mathrm{R}_{2}=\mathrm{CHO}$ & 0.102208 & 1.49 & -438.361029 & 0.093434 & -0.49 & -438.318808 \\
$\mathrm{R}_{2}=\mathrm{CN}$ & 0.091796 & 1.51 & -417.267588 & 0.082542 & -0.87 & -417.223190 \\
& $\mathrm{R}_{2}=\mathrm{NO}_{2}$ & 0.093936 & 0.81 & -529.565028 & 0.084890 & -1.37 & -529.523414 \\
\hline $\mathrm{R}_{1}=\mathrm{NO}_{2}$ & $\mathrm{R}_{2}=\mathrm{CH}_{3}$ & 0.112531 & 1.75 & -476.634089 & 0.115438 & 1.23 & -476.591091 \\
& $\mathrm{R}_{2}=\mathrm{NH}_{2}$ & 0.101840 & -0.93 & -492.703920 & 0.105714 & -1.33 & -492.649163 \\
& $\mathrm{R}_{2}=\mathrm{OH}$ & 0.089069 & 0.64 & -512.579254 & 0.093320 & -0.17 & -512.519512 \\
& $\mathrm{R}_{2}=\mathrm{F}$ & 0.076424 & 0.2 & -536.575261 & 0.080272 & -0.78 & -536.529067 \\
$\mathrm{R}_{2}=\mathrm{CHO}$ & 0.091858 & 1.26 & -550.656959 & 0.095954 & -0.71 & -550.621420 \\
$\mathrm{R}_{2}=\mathrm{CN}$ & 0.081326 & 0.67 & -529.564731 & 0.085104 & -1.27 & -529.524847 \\
$\mathrm{R}_{2}=\mathrm{NO}_{2}$ & 0.082445 & 1.19 & -641.861980 & 0.087700 & -1.23 & -641.823302 \\
\hline
\end{tabular}

\begin{tabular}{|c|c|c|c|c|c|c|c|}
\hline \multirow{2}{*}{\multicolumn{2}{|c|}{ Compound }} & \multirow[b]{2}{*}{ TCG } & & \multicolumn{3}{|c|}{ Transition State } \\
\hline & & & $\begin{array}{c}\text { Solvent } \\
\text { effect }\end{array}$ & Total Energy & TCG & $\begin{array}{c}\text { Solvent } \\
\text { effect }\end{array}$ & Total Energy \\
\hline \multirow[t]{7}{*}{$\mathrm{R}_{1}=\mathrm{CH}_{3}$} & $\mathrm{R}_{3}=\mathrm{CH}_{3}$ & 0.141815 & 5.73 & -311.3949267 & 0.141769 & 4.52 & -311.3425851 \\
\hline & $\mathrm{R}_{3}=\mathrm{NH}_{2}$ & 0.131262 & 2.69 & -327.4461865 & 0.13154 & 1.32 & -327.3937904 \\
\hline & $\mathrm{R}_{3}=\mathrm{OH}$ & 0.118176 & 2.13 & -347.3187884 & 0.119073 & 0.85 & -347.2664627 \\
\hline & $\mathrm{R}_{3}=\mathrm{F}$ & 0.106064 & 4.08 & -371.340496 & 0.106823 & 2.58 & -371.2885461 \\
\hline & $\mathrm{R}_{3}=\mathrm{CHO}$ & 0.121833 & 3.52 & -385.4279225 & 0.122783 & 1.62 & -385.3788336 \\
\hline & $\mathrm{R}_{3}=\mathrm{CN}$ & 0.111275 & 3.54 & -364.3373474 & 0.112105 & 1.56 & -364.2860282 \\
\hline & $\mathrm{R}_{3}=\mathrm{NO}_{2}$ & 0.112872 & 3.41 & -476.6353885 & 0.114667 & 1.47 & -476.5914571 \\
\hline \multirow[t]{7}{*}{$\mathrm{R}_{1}=\mathrm{NH}_{2}$} & $\mathrm{R}_{3}=\mathrm{CH}_{3}$ & 0.131315 & 1.86 & -327.4461329 & 0.132331 & 1.3 & -327.3979325 \\
\hline & $\mathrm{R}_{3}=\mathrm{NH}_{2}$ & 0.120892 & -1.11 & -343.4978469 & 0.121896 & -1.82 & -343.4489085 \\
\hline & $\mathrm{R}_{3}=\mathrm{OH}$ & 0.107555 & -1.64 & -363.3715262 & 0.109469 & -2.39 & -363.3223619 \\
\hline & $\mathrm{R}_{3}=\mathrm{F}$ & 0.09558 & 0.06 & -387.3934404 & 0.097369 & -0.81 & -387.3452597 \\
\hline & $\mathrm{R}_{3}=\mathrm{CHO}$ & 0.111671 & -0.08 & -401.4828015 & 0.113042 & -1.72 & -401.4333729 \\
\hline & $\mathrm{R}_{3}=\mathrm{CN}$ & 0.100652 & -0.35 & -380.3910217 & 0.102453 & -1.85 & -380.3416296 \\
\hline & $\mathrm{R}_{3}=\mathrm{NO}_{2}$ & 0.102598 & -0.51 & -492.6902308 & 0.104917 & -1.95 & -492.6464832 \\
\hline \multirow[t]{7}{*}{$\mathrm{R}_{1}=\mathrm{OH}$} & $\mathrm{R}_{3}=\mathrm{CH}_{3}$ & 0.118436 & 1.08 & -347.3143267 & 0.120074 & 0.92 & -347.2692063 \\
\hline & $\mathrm{R}_{3}=\mathrm{NH}_{2}$ & 0.108037 & -1.90 & -363.3655655 & 0.109554 & -2.28 & -363.3204643 \\
\hline & $\mathrm{R}_{3}=\mathrm{OH}$ & 0.094935 & -2.34 & -383.2391208 & 0.097108 & -2.79 & -383.1929725 \\
\hline & $\mathrm{R}_{3}=\mathrm{F}$ & 0.082967 & -0.49 & -407.2605579 & 0.085069 & -1.03 & -407.2151424 \\
\hline & $\mathrm{R}_{3}=\mathrm{CHO}$ & 0.09927 & -0.78 & -421.3507608 & 0.100818 & -1.9 & -421.303571 \\
\hline & $\mathrm{R}_{3}=\mathrm{CN}$ & 0.088076 & -0.90 & -400.2578864 & 0.090156 & -2.03 & -400.2107522 \\
\hline & $\mathrm{R}_{3}=\mathrm{NO}_{2}$ & 0.090044 & -1.03 & -512.557711 & 0.092672 & -2.07 & -512.5156676 \\
\hline \multirow[t]{3}{*}{$\mathrm{R}_{1}=\mathrm{F}$} & $\mathrm{R}_{3}=\mathrm{CH}_{3}$ & 0.106595 & 3.67 & -371.3346881 & 0.107565 & 2.53 & -371.2894647 \\
\hline & $\mathrm{R}_{3}=\mathrm{NH}_{2}$ & 0.096011 & 0.67 & -387.3853682 & 0.09708 & -0.76 & -387.3410548 \\
\hline & $\mathrm{R}_{3}=\mathrm{OH}$ & 0.082909 & 0.24 & -407.2580239 & 0.084626 & -1.11 & -407.212833 \\
\hline
\end{tabular}




\begin{tabular}{cccccccc}
\hline & $\mathrm{R}_{3}=\mathrm{F}$ & 0.070899 & 2.29 & -431.2786295 & 0.072556 & 0.81 & -431.2339859 \\
& $\mathrm{R}_{3}=\mathrm{CHO}$ & 0.087014 & 1.6 & -445.3690827 & 0.088219 & -0.14 & -445.3223204 \\
& $\mathrm{R}_{3}=\mathrm{CN}$ & 0.075961 & 1.76 & -424.2750179 & 0.077581 & -0.07 & -424.2289612 \\
& $\mathrm{R}_{3}=\mathrm{NO}_{2}$ & 0.078376 & 1.49 & -536.575566 & 0.080138 & -0.11 & -536.5337877 \\
\hline $\mathrm{R}_{1}=\mathrm{CHO}$ & $\mathrm{R}_{3}=\mathrm{CH}_{3}$ & 0.122246 & 2.41 & -385.4311161 & 0.122024 & 1.61 & -385.3777682 \\
& $\mathrm{R}_{3}=\mathrm{NH} 2$ & 0.111618 & -0.61 & -401.4814195 & 0.111939 & -1.74 & -401.4292918 \\
& $\mathrm{R}_{3}=\mathrm{OH}$ & 0.098687 & -1.26 & -421.3527567 & 0.099501 & -2.1 & -421.300885 \\
& $\mathrm{R}_{3}=\mathrm{F}$ & 0.086479 & 0.94 & -445.3733666 & 0.087232 & -0.12 & -445.3215498 \\
& $\mathrm{R}_{3}=\mathrm{CHO}$ & 0.102535 & 0.06 & -459.4603718 & 0.102939 & -0.99 & -459.4113013 \\
& $\mathrm{R}_{3}=\mathrm{CN}$ & 0.091692 & 0.28 & -438.3682555 & 0.092154 & -1.04 & -438.3171081 \\
& $\mathrm{R}_{3}=\mathrm{NO} \mathrm{O}_{2}$ & 0.093613 & 0.11 & -550.6667415 & 0.094804 & -0.97 & -550.6222072 \\
\hline $\mathrm{R}_{1}=\mathrm{CN}$ & $\mathrm{R}_{3}=\mathrm{CH}$ & 0.111914 & 2.68 & -364.3383974 & 0.112076 & 1.62 & -364.2870361 \\
& $\mathrm{R}_{3}=\mathrm{NH}$ & 0.101269 & -0.35 & -380.3884624 & 0.101793 & -1.76 & -380.3388397 \\
& $\mathrm{R}_{3}=\mathrm{OH}$ & 0.088247 & -0.88 & -400.2605943 & 0.089323 & -2.08 & -400.2100053 \\
& $\mathrm{R}_{3}=\mathrm{F}$ & 0.076177 & 1.33 & -424.2807838 & 0.077187 & -0.09 & -424.230183 \\
& $\mathrm{R}_{3}=\mathrm{CHO}$ & 0.092394 & 0.51 & -438.3693268 & 0.092821 & -0.95 & -438.3192713 \\
& $\mathrm{R}_{3}=\mathrm{CN}$ & 0.081354 & 0.75 & -417.2759878 & 0.082231 & -0.98 & -417.2245565 \\
& $\mathrm{R}_{3}=\mathrm{NO}{ }_{2}$ & 0.082716 & 0.55 & -529.5748942 & 0.084826 & -0.93 & -529.5293902 \\
\hline $\mathrm{R}_{1}=\mathrm{NO}_{2}$ & $\mathrm{R}_{3}=\mathrm{CH}$ & 0.114405 & 2.48 & -476.6428168 & 0.115099 & 1.39 & -476.5945098 \\
& $\mathrm{R}_{3}=\mathrm{NH}$ & 0.103674 & -0.68 & -492.6925666 & 0.104741 & -2.1 & -492.6467218 \\
& $\mathrm{R}_{3}=\mathrm{OH}$ & 0.090744 & -1.23 & -512.5642286 & 0.092291 & -2.33 & -512.5174676 \\
& $\mathrm{R}_{3}=\mathrm{F}$ & 0.078627 & 1.07 & -536.58407 & 0.080034 & -0.27 & -536.5369408 \\
& $\mathrm{R}_{3}=\mathrm{CHO}$ & 0.094837 & 0.34 & -550.672991 & 0.095483 & -0.95 & -550.62537 \\
& $\mathrm{R}_{3}=\mathrm{CN}$ & 0.083751 & 0.59 & -529.5787099 & 0.08492 & -1 & -529.5302685 \\
& $\mathrm{R}_{3}=\mathrm{NO} \mathrm{N}_{2}$ & 0.086138 & 0.59 & -641.8788462 & 0.087505 & -0.91 & -641.8350438 \\
\hline & & & & & & &
\end{tabular}

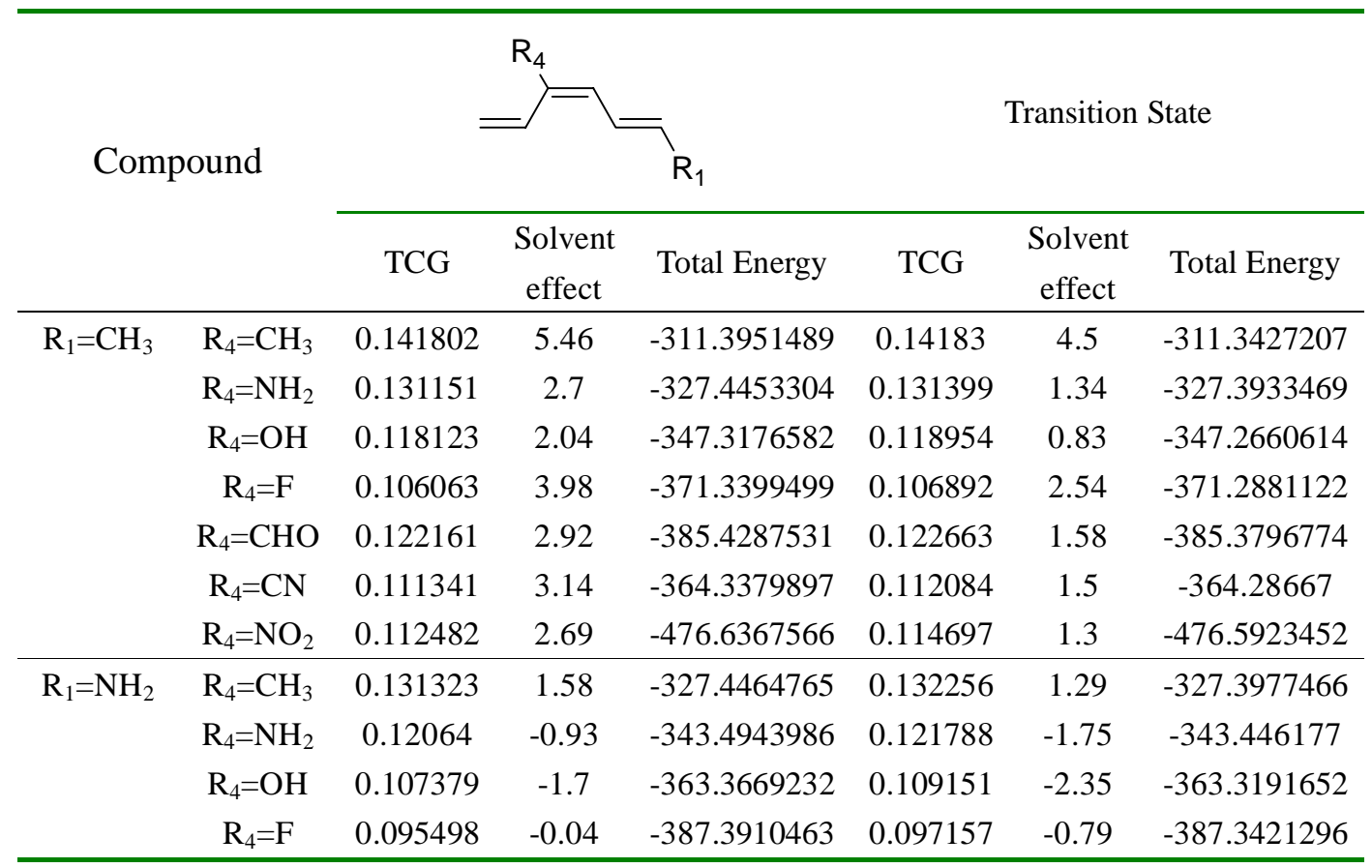




\begin{tabular}{|c|c|c|c|c|c|c|c|}
\hline & $\mathrm{R}_{4}=\mathrm{CHO}$ & 0.111832 & -2.06 & -401.484999 & 0.113458 & -2.45 & -401.4396651 \\
\hline & $\mathrm{R}_{4}=\mathrm{CN}$ & 0.100882 & -1.74 & -380.3936175 & 0.102805 & -2.46 & -380.3460618 \\
\hline & $\mathrm{R}_{4}=\mathrm{NO}_{2}$ & 0.103109 & -2.95 & -492.6950364 & 0.105604 & -3.19 & -492.6531429 \\
\hline \multirow[t]{7}{*}{$\mathrm{R}_{1}=\mathrm{OH}$} & $\mathrm{R}_{4}=\mathrm{CH}_{3}$ & 0.119027 & 1.22 & -347.3159762 & 0.119948 & 0.9 & -347.2690106 \\
\hline & $\mathrm{R}_{4}=\mathrm{NH}_{2}$ & 0.108265 & -1.44 & -363.3646875 & 0.109399 & -2.27 & -363.3180209 \\
\hline & $\mathrm{R}_{4}=\mathrm{OH}$ & 0.095053 & -2.2 & -383.2369224 & 0.096801 & -2.82 & -383.1906167 \\
\hline & $\mathrm{R}_{4}=\mathrm{F}$ & 0.083214 & -0.35 & -407.2600281 & 0.084807 & -1.12 & -407.2126877 \\
\hline & $\mathrm{R}_{4}=\mathrm{CHO}$ & 0.099761 & -1.79 & -421.3513301 & 0.101159 & -2.28 & -421.3076622 \\
\hline & $\mathrm{R}_{4}=\mathrm{CN}$ & 0.088841 & -1.57 & -400.2599469 & 0.090533 & -2.4 & -400.2140125 \\
\hline & $\mathrm{R}_{4}=\mathrm{NO}_{2}$ & 0.09095 & -2.24 & -512.5597647 & 0.093312 & -2.75 & -512.5200891 \\
\hline \multirow[t]{7}{*}{$\mathrm{R}_{1}=\mathrm{F}$} & $\mathrm{R}_{4}=\mathrm{CH}_{3}$ & 0.106618 & 3.46 & -371.3346952 & 0.107494 & 2.53 & -371.2894006 \\
\hline & $\mathrm{R}_{4}=\mathrm{NH}_{2}$ & 0.095954 & 0.58 & -387.3848768 & 0.097125 & -0.82 & -387.3398355 \\
\hline & $\mathrm{R}_{4}=\mathrm{OH}$ & 0.082899 & -0.09 & -407.2566572 & 0.08453 & -1.22 & -407.2116084 \\
\hline & $\mathrm{R}_{4}=\mathrm{F}$ & 0.070885 & 2.01 & -431.278622 & 0.072408 & 0.71 & -431.2326762 \\
\hline & $\mathrm{R}_{4}=\mathrm{CHO}$ & 0.087052 & 1.05 & -445.3669191 & 0.088472 & -0.14 & -445.3248547 \\
\hline & $\mathrm{R}_{4}=\mathrm{CN}$ & 0.076224 & 1.25 & -424.2758565 & 0.077894 & -0.22 & -424.2312123 \\
\hline & $\mathrm{R}_{4}=\mathrm{NO}_{2}$ & 0.077732 & 0.91 & -536.5744491 & 0.080549 & -0.31 & -536.53641 \\
\hline \multirow[t]{7}{*}{$\mathrm{R}_{1}=\mathrm{CHO}$} & $\mathrm{R}_{4}=\mathrm{CH}_{3}$ & 0.122246 & 2.05 & -385.4315715 & 0.122133 & 1.55 & -385.3781006 \\
\hline & $\mathrm{R}_{4}=\mathrm{NH}_{2}$ & 0.111749 & -1.55 & -401.4864854 & 0.111968 & -2.13 & -401.4317764 \\
\hline & $\mathrm{R}_{4}=\mathrm{OH}$ & 0.098952 & -1.82 & -421.356525 & 0.099521 & -2.37 & -421.3025241 \\
\hline & $\mathrm{R}_{4}=\mathrm{F}$ & 0.086666 & 0.7 & -445.3754757 & 0.087121 & -0.2 & -445.322334 \\
\hline & $\mathrm{R}_{4}=\mathrm{CHO}$ & 0.102354 & 0.25 & -459.4593695 & 0.103044 & -0.86 & -459.4108721 \\
\hline & $\mathrm{R}_{4}=\mathrm{CN}$ & 0.091657 & 0.41 & -438.3685295 & 0.092311 & -0.91 & -438.3170294 \\
\hline & $\mathrm{R}_{4}=\mathrm{NO}_{2}$ & 0.093646 & 0.25 & -550.6656936 & 0.094741 & -0.9 & -550.6216003 \\
\hline \multirow[t]{7}{*}{$\mathrm{R}_{1}=\mathrm{CN}$} & $\mathrm{R}_{4}=\mathrm{CH}_{3}$ & 0.111821 & 2.35 & -364.33886 & 0.112189 & 1.57 & -364.2874793 \\
\hline & $\mathrm{R}_{4}=\mathrm{NH}_{2}$ & 0.101331 & -1.14 & -380.39337 & 0.102083 & -2.13 & -380.3411556 \\
\hline & $\mathrm{R}_{4}=\mathrm{OH}$ & 0.088428 & -1.49 & -400.2633076 & 0.089573 & -2.34 & -400.2114685 \\
\hline & $\mathrm{R}_{4}=\mathrm{F}$ & 0.076251 & 0.99 & -424.2825378 & 0.077177 & -0.18 & -424.2308546 \\
\hline & $\mathrm{R}_{4}=\mathrm{CHO}$ & 0.091901 & 0.5 & -438.3665004 & 0.093026 & -0.82 & -438.3197017 \\
\hline & $\mathrm{R}_{4}=\mathrm{CN}$ & 0.081248 & 0.67 & -417.2756716 & 0.082403 & -0.87 & -417.2254941 \\
\hline & $\mathrm{R}_{4}=\mathrm{NO}_{2}$ & 0.083218 & 0.53 & -529.5728938 & 0.084926 & -0.83 & -529.5300065 \\
\hline \multirow[t]{7}{*}{$\mathrm{R}_{1}=\mathrm{NO}_{2}$} & $\mathrm{R}_{4}=\mathrm{CH}_{3}$ & 0.114365 & 1.99 & -476.6432618 & 0.115124 & 1.24 & -476.5951046 \\
\hline & $\mathrm{R}_{4}=\mathrm{NH}_{2}$ & 0.10396 & -2.02 & -492.6995624 & 0.105108 & -3.02 & -492.6504061 \\
\hline & $\mathrm{R}_{4}=\mathrm{OH}$ & 0.091131 & -2.04 & -512.5685784 & 0.092522 & -2.9 & -512.5194287 \\
\hline & $\mathrm{R}_{4}=\mathrm{F}$ & 0.078848 & 0.72 & -536.5867674 & 0.080035 & -0.39 & -536.5377198 \\
\hline & $\mathrm{R}_{4}=\mathrm{CHO}$ & 0.094328 & 0.37 & -550.6691176 & 0.095812 & -0.89 & -550.6255871 \\
\hline & $\mathrm{R}_{4}=\mathrm{CN}$ & 0.083712 & 0.58 & -529.5783831 & 0.085167 & -0.89 & -529.5313507 \\
\hline & $\mathrm{R}_{4}=\mathrm{NO}_{2}$ & 0.085822 & 0.51 & -641.8752503 & 0.087568 & -0.77 & -641.8353332 \\
\hline
\end{tabular}




\begin{tabular}{|c|c|c|c|c|c|c|c|}
\hline \multicolumn{2}{|c|}{ Compound } & \multirow[b]{2}{*}{ TCG } & \multirow{2}{*}{$\begin{array}{l}\mathrm{R}_{5} \\
\begin{array}{c}\text { Solvent } \\
\text { effect }\end{array}\end{array}$} & \multirow{2}{*}{$\begin{array}{l}\mathrm{R}_{1} \\
\text { Total } \\
\text { Energy }\end{array}$} & \multicolumn{3}{|c|}{ Transition State } \\
\hline & & & & & TCG & $\begin{array}{c}\text { Solvent } \\
\text { effect }\end{array}$ & $\begin{array}{c}\text { Total } \\
\text { Energy }\end{array}$ \\
\hline \multirow[t]{7}{*}{$\mathrm{R}_{1}=\mathrm{CH}_{3}$} & $\mathrm{R}_{5}=\mathrm{CH}_{3}$ & 0.142339 & 5.41 & -311.391750 & 0.140734 & 0.140734 & -311.342772 \\
\hline & $\mathrm{R}_{5}=\mathrm{NH}_{2}$ & 0.131493 & 2.91 & -327.443863 & 0.131959 & 0.131959 & -327.396710 \\
\hline & $\mathrm{R}_{5}=\mathrm{OH}$ & 0.118237 & 2.39 & -347.317973 & 0.119441 & 0.119441 & -347.267187 \\
\hline & $\mathrm{R}_{5}=\mathrm{F}$ & 0.106140 & 4.14 & -371.339783 & 0.107041 & 0.107041 & -371.287990 \\
\hline & $\mathrm{R}_{5}=\mathrm{CHO}$ & 0.121793 & 3.26 & -385.425153 & 0.122941 & 0.122941 & -385.381491 \\
\hline & $\mathrm{R}_{5}=\mathrm{CN}$ & 0.111390 & 3.99 & -364.332903 & 0.112462 & 0.112462 & -364.287853 \\
\hline & $\mathrm{R}_{5}=\mathrm{NO}_{2}$ & 0.113394 & 3.21 & -476.636549 & 0.115073 & 0.115073 & -476.593491 \\
\hline \multirow[t]{7}{*}{$\mathrm{R}_{1}=\mathrm{NH}_{2}$} & $\mathrm{R}_{5}=\mathrm{CH}_{3}$ & 0.132058 & 1.48 & -327.443450 & 0.131760 & 0.131760 & -327.399296 \\
\hline & $\mathrm{R}_{5}=\mathrm{NH}_{2}$ & 0.121097 & -1.04 & -343.495727 & 0.122205 & 0.122205 & -343.453478 \\
\hline & $\mathrm{R}_{5}=\mathrm{OH}$ & 0.108003 & -1.50 & -363.370465 & 0.109793 & 0.109793 & -363.324582 \\
\hline & $\mathrm{R}_{5}=\mathrm{F}$ & 0.095891 & 0.06 & -387.393085 & 0.097371 & 0.097371 & -387.345449 \\
\hline & $\mathrm{R}_{5}=\mathrm{CHO}$ & 0.111605 & -0.90 & -401.477917 & 0.113223 & 0.113223 & -401.437149 \\
\hline & $\mathrm{R}_{5}=\mathrm{CN}$ & 0.101110 & 0.01 & -380.387643 & 0.102806 & 0.102806 & -380.344685 \\
\hline & $\mathrm{R}_{5}=\mathrm{NO}_{2}$ & 0.102911 & -1.07 & -492.690275 & 0.105349 & 0.105349 & -492.650328 \\
\hline \multirow[t]{7}{*}{$\mathrm{R}_{1}=\mathrm{OH}$} & $\mathrm{R}_{5}=\mathrm{CH}_{3}$ & 0.119770 & 1.11 & -347.312815 & 0.119375 & 0.119375 & -347.270363 \\
\hline & $\mathrm{R}_{5}=\mathrm{NH}_{2}$ & 0.108924 & -1.35 & -363.365416 & 0.110012 & 0.110012 & -363.324263 \\
\hline & $\mathrm{R}_{5}=\mathrm{OH}$ & 0.095764 & -1.78 & -383.240063 & 0.097530 & 0.097530 & -383.194735 \\
\hline & $\mathrm{R}_{5}=\mathrm{F}$ & 0.083675 & -0.14 & -407.261966 & 0.085158 & 0.085158 & -407.214956 \\
\hline & $\mathrm{R}_{5}=\mathrm{CHO}$ & 0.099240 & -1.13 & -421.346381 & 0.100878 & 0.100878 & -421.305533 \\
\hline & $\mathrm{R}_{5}=\mathrm{CN}$ & 0.088993 & 0.01 & -400.255875 & 0.090529 & 0.090529 & -400.213405 \\
\hline & $\mathrm{R}_{5}=\mathrm{NO}_{2}$ & 0.090853 & -1.29 & -512.558003 & 0.093007 & 0.093007 & -512.518441 \\
\hline \multirow[t]{7}{*}{$\mathrm{R}_{1}=\mathrm{F}$} & $\mathrm{R}_{5}=\mathrm{CH}_{3}$ & 0.107334 & 3.39 & -371.331441 & 0.107120 & 0.107120 & -371.290365 \\
\hline & $\mathrm{R}_{5}=\mathrm{NH}_{2}$ & 0.096415 & 0.86 & -387.383563 & 0.097509 & 0.097509 & -387.343986 \\
\hline & $\mathrm{R}_{5}=\mathrm{OH}$ & 0.083249 & 0.36 & -407.257884 & 0.084981 & 0.084981 & -407.213628 \\
\hline & $\mathrm{R}_{5}=\mathrm{F}$ & 0.071151 & 2.13 & -431.278982 & 0.072630 & 0.072630 & -431.233153 \\
\hline & $\mathrm{R}_{5}=\mathrm{CHO}$ & 0.086759 & 1.21 & -445.364271 & 0.088384 & 0.088384 & -445.324701 \\
\hline & $\mathrm{R}_{5}=\mathrm{CN}$ & 0.076317 & 1.88 & -424.271311 & 0.077979 & 0.077979 & -424.231456 \\
\hline & $\mathrm{R}_{5}=\mathrm{NO}_{2}$ & 0.078397 & 1.13 & -536.575035 & 0.080494 & 0.080494 & -536.536182 \\
\hline \multirow[t]{7}{*}{$\mathrm{R}_{1}=\mathrm{CHO}$} & $\mathrm{R}_{5}=\mathrm{CH}_{3}$ & 0.122902 & 2.17 & -385.427756 & 0.121811 & 0.121811 & -385.377684 \\
\hline & $\mathrm{R}_{5}=\mathrm{NH}_{2}$ & 0.112048 & -0.51 & -401.479697 & 0.112112 & 0.112112 & -401.431292 \\
\hline & $\mathrm{R}_{5}=\mathrm{OH}$ & 0.098833 & -1.08 & -421.353719 & 0.099465 & 0.099465 & -421.300468 \\
\hline & $\mathrm{R}_{5}=\mathrm{F}$ & 0.086607 & 0.91 & -445.373894 & 0.087117 & 0.087117 & -445.319750 \\
\hline & $\mathrm{R}_{5}=\mathrm{CHO}$ & 0.102286 & 0.06 & -459.459083 & 0.103434 & 0.103434 & -459.412850 \\
\hline & $\mathrm{R}_{5}=\mathrm{CN}$ & 0.091806 & 0.81 & -438.365384 & 0.092698 & 0.092698 & -438.318147 \\
\hline & $\mathrm{R}_{5}=\mathrm{NO}_{2}$ & 0.093880 & 0.08 & -550.668666 & 0.095243 & 0.095243 & -550.622951 \\
\hline \multirow[t]{4}{*}{$\mathrm{R}_{1}=\mathrm{CN}$} & $\mathrm{R}_{5}=\mathrm{CH}_{3}$ & 0.112496 & 2.50 & -364.335259 & 0.112083 & 0.112083 & -364.287316 \\
\hline & $\mathrm{R}_{5}=\mathrm{NH}_{2}$ & 0.101681 & -0.20 & -380.387090 & 0.102136 & 0.102136 & -380.340930 \\
\hline & $\mathrm{R}_{5}=\mathrm{OH}$ & 0.088481 & -0.79 & -400.261170 & 0.089639 & 0.089639 & -400.209373 \\
\hline & $\mathrm{R}_{5}=\mathrm{F}$ & 0.076195 & 1.14 & -424.281009 & 0.077199 & 0.077199 & -424.228361 \\
\hline
\end{tabular}




\begin{tabular}{cccccccc}
\hline & $\mathrm{R}_{5}=\mathrm{CHO}$ & 0.091921 & 0.33 & -438.366525 & 0.093283 & 0.093283 & -438.321166 \\
& $\mathrm{R}_{5}=\mathrm{CN}$ & 0.081288 & 0.94 & -417.271820 & 0.082693 & 0.082693 & -417.226238 \\
& $\mathrm{R}_{5}=\mathrm{NO}_{2}$ & 0.083557 & 0.31 & -529.575866 & 0.085230 & 0.085230 & -529.530778 \\
\hline $\mathrm{R}_{1}=\mathrm{NO}_{2}$ & $\mathrm{R}_{5}=\mathrm{CH}_{3}$ & 0.115138 & 2.11 & -476.639603 & 0.115144 & 0.115144 & -476.594840 \\
& $\mathrm{R}_{5}=\mathrm{NH}_{2}$ & 0.104128 & -0.64 & -492.691007 & 0.105050 & 0.105050 & -492.648017 \\
& $\mathrm{R}_{5}=\mathrm{OH}$ & 0.090976 & -1.34 & -512.564807 & 0.092476 & 0.092476 & -512.516079 \\
& $\mathrm{R}_{5}=\mathrm{F}$ & 0.078652 & 0.74 & -536.584089 & 0.080059 & 0.080059 & -536.534524 \\
& $\mathrm{R}_{5}=\mathrm{CHO}$ & 0.094411 & 0.12 & -550.670244 & 0.095937 & 0.095937 & -550.627428 \\
& $\mathrm{R}_{5}=\mathrm{CN}$ & 0.083639 & 0.38 & -529.573996 & 0.085446 & 0.085446 & -529.532138 \\
& $\mathrm{R}_{5}=\mathrm{NO}_{2}$ & 0.086025 & 0.17 & -641.879037 & 0.087988 & 0.087988 & -641.836460 \\
\hline
\end{tabular}

\begin{tabular}{|c|c|c|c|c|c|c|c|}
\hline \multicolumn{2}{|c|}{ Compound } & \multicolumn{2}{|c|}{$\mathrm{R}_{6}^{\prime}$} & \multirow{2}{*}{$\begin{array}{c}\mathrm{R}_{1} \\
\text { Total } \\
\text { Energy }\end{array}$} & \multicolumn{3}{|c|}{ Transition State } \\
\hline & & TCG & $\begin{array}{c}\text { Solvent } \\
\text { effect }\end{array}$ & & TCG & $\begin{array}{c}\text { Solvent } \\
\text { effect }\end{array}$ & Total Energy \\
\hline \multirow[t]{7}{*}{$\mathrm{R}_{1}=\mathrm{CH}_{3}$} & $\mathrm{R}_{6}=\mathrm{CH}_{3}$ & 0.140993 & 4.86 & -311.397804 & 0.141652 & 3.51 & -311.3432379 \\
\hline & $\mathrm{R}_{6}=\mathrm{NH}_{2}$ & 0.130594 & 1.03 & -327.448887 & 0.13193 & 0.59 & -327.3985368 \\
\hline & $\mathrm{R}_{6}=\mathrm{OH}$ & 0.118348 & 0.66 & -347.318574 & 0.119981 & 0.22 & -347.2700069 \\
\hline & $\mathrm{R}_{4}=\mathrm{F}$ & 0.105969 & 2.86 & -371.337492 & 0.107467 & 1.91 & -371.2903268 \\
\hline & $\mathrm{R}_{6}=\mathrm{CHO}$ & 0.12162 & 1.36 & -385.434621 & 0.123425 & 0.81 & -385.3805798 \\
\hline & $\mathrm{R}_{6}=\mathrm{CN}$ & 0.111329 & 1.68 & -364.342004 & 0.112433 & 1 & -364.2893511 \\
\hline & $\mathrm{R}_{6}=\mathrm{NO}_{2}$ & 0.113994 & 1.34 & -476.646554 & 0.116025 & 0.47 & -476.5973712 \\
\hline \multirow[t]{6}{*}{$\mathrm{R}_{1}=\mathrm{NH}_{2}$} & $\mathrm{R}_{6}=\mathrm{NH}_{2}$ & 0.120078 & -2.5 & -343.498407 & 0.121701 & -2.12 & -343.4478611 \\
\hline & $\mathrm{R}_{6}=\mathrm{OH}$ & 0.107751 & -3.01 & -363.368943 & 0.109338 & -2.89 & -363.3209348 \\
\hline & $\mathrm{R}_{4}=\mathrm{F}$ & 0.095439 & -1.02 & -387.389154 & 0.097011 & -1.35 & -387.3430693 \\
\hline & $\mathrm{R}_{6}=\mathrm{CHO}$ & 0.111385 & -3.23 & -401.489377 & 0.113482 & -1.92 & -401.4416165 \\
\hline & $\mathrm{R}_{6}=\mathrm{CN}$ & 0.101031 & -2.8 & -380.396798 & 0.102859 & -2.46 & -380.3501259 \\
\hline & $\mathrm{R}_{6}=\mathrm{NO}_{2}$ & 0.103695 & -3.77 & -492.702846 & 0.105591 & -2.98 & -492.6592124 \\
\hline \multirow[t]{5}{*}{$\mathrm{R}_{1}=\mathrm{OH}$} & $\mathrm{R}_{6}=\mathrm{OH}$ & 0.095474 & -3.47 & -383.238822 & 0.096521 & -3.78 & -383.190733 \\
\hline & $\mathrm{R}_{4}=\mathrm{F}$ & 0.083197 & -1.4 & -407.258331 & 0.084522 & -2.07 & -407.2121873 \\
\hline & $\mathrm{R}_{6}=\mathrm{CHO}$ & 0.099257 & -3.22 & -421.356425 & 0.1012 & -2.96 & -421.3067026 \\
\hline & $\mathrm{R}_{6}=\mathrm{CN}$ & 0.088884 & -2.88 & -400.263859 & 0.090479 & -3.17 & -400.2149848 \\
\hline & $\mathrm{R}_{6}=\mathrm{NO}_{2}$ & 0.091619 & -3.51 & -512.568946 & 0.093232 & -3.63 & -512.5221064 \\
\hline \multirow[t]{4}{*}{$\mathrm{R}_{1}=\mathrm{F}$} & $\mathrm{R}_{4}=\mathrm{F}$ & 0.07081 & 0.82 & -431.276652 & 0.072264 & -0.18 & -431.2315452 \\
\hline & $\mathrm{R}_{6}=\mathrm{CHO}$ & 0.086707 & -0.64 & -445.372898 & 0.088427 & -0.89 & -445.324029 \\
\hline & $\mathrm{R}_{6}=\mathrm{CN}$ & 0.076354 & -0.36 & -424.280097 & 0.077967 & -1.07 & -424.2316012 \\
\hline & $\mathrm{R}_{6}=\mathrm{NO}_{2}$ & 0.078977 & -0.79 & -536.584189 & 0.080564 & -1.1 & -536.5374674 \\
\hline \multirow[t]{3}{*}{$\mathrm{R}_{1}=\mathrm{CHO}$} & $\mathrm{R}_{6}=\mathrm{CHO}$ & 0.101846 & -1.7 & -459.466269 & 0.102671 & -1.54 & -459.4087701 \\
\hline & $\mathrm{R}_{6}=\mathrm{CN}$ & 0.091574 & -1.49 & -438.373447 & 0.092641 & -1.7 & -438.3173548 \\
\hline & $\mathrm{R}_{6}=\mathrm{NO}_{2}$ & 0.094097 & -1.7 & -550.676561 & 0.095571 & -2.09 & -550.6222967 \\
\hline \multirow[t]{2}{*}{$\mathrm{R}_{1}=\mathrm{CN}$} & $\mathrm{R}_{6}=\mathrm{CN}$ & 0.081211 & -1.33 & -417.280432 & 0.082293 & -1.8 & -417.2248499 \\
\hline & $\mathrm{R}_{6}=\mathrm{NO}_{2}$ & 0.083663 & -1.56 & -529.583356 & 0.085228 & -1.84 & -529.5302576 \\
\hline
\end{tabular}




\begin{tabular}{llllllll}
\hline $\mathrm{R}_{1}=\mathrm{NO}_{2}$ & $\mathrm{R}_{6}=\mathrm{NO}_{2}$ & 0.085956 & -1.58 & -641.885731 & 0.087212 & -1.81 & -641.833717 \\
\hline
\end{tabular}

\begin{tabular}{|c|c|c|c|c|c|c|c|}
\hline \multicolumn{2}{|c|}{ Compound } & \multicolumn{3}{|c|}{$=\overbrace{\mathrm{R}_{2}}^{\mathrm{R}_{3}}$} & \multicolumn{3}{|c|}{ Transition State } \\
\hline & & TCG & $\begin{array}{c}\text { Solvent } \\
\text { effect }\end{array}$ & Total Energy & TCG & $\begin{array}{c}\text { Solvent } \\
\text { effect }\end{array}$ & Total Energy \\
\hline \multirow[t]{7}{*}{$\mathrm{R}_{3}=\mathrm{CH}_{3}$} & $\mathrm{R}_{2}=\mathrm{CH}_{3}$ & 0.141539 & 6.21 & -311.3904814 & 0.142425 & 4.62 & -311.340531 \\
\hline & $\mathrm{R}_{2}=\mathrm{NH}_{2}$ & 0.130774 & 3.59 & -327.4418975 & 0.13207 & 2.12 & -327.3935496 \\
\hline & $\mathrm{R}_{2}=\mathrm{OH}$ & 0.11848 & 2.9 & -347.3124269 & 0.119245 & 1.59 & -347.2644813 \\
\hline & $\mathrm{R}_{2}=\mathrm{F}$ & 0.106407 & 4.71 & -371.3352333 & 0.106764 & 3.26 & -371.2857871 \\
\hline & $\mathrm{R}_{2}=\mathrm{CHO}$ & 0.121904 & 4.15 & -385.4227771 & 0.123161 & 2.19 & -385.3792525 \\
\hline & $\mathrm{R}_{2}=\mathrm{CN}$ & 0.111215 & 4.48 & -364.3304963 & 0.112366 & 2.4 & -364.2874655 \\
\hline & $\mathrm{R}_{2}=\mathrm{NO}_{2}$ & 0.113555 & 4.3 & -476.6334074 & 0.115583 & 1.76 & -476.5893103 \\
\hline \multirow[t]{7}{*}{$\mathrm{R}_{3}=\mathrm{NH}_{2}$} & $\mathrm{R}_{2}=\mathrm{CH}_{3}$ & 0.130713 & 3.37 & -327.442323 & 0.132071 & 1.96 & -327.3922275 \\
\hline & $\mathrm{R}_{2}=\mathrm{NH}_{2}$ & 0.121125 & 0.73 & -343.4927091 & 0.121828 & -0.44 & -343.444739 \\
\hline & $\mathrm{R}_{2}=\mathrm{OH}$ & 0.108107 & 0.24 & -363.3655205 & 0.109777 & -0.38 & -363.3172959 \\
\hline & $\mathrm{R}_{2}=\mathrm{F}$ & 0.096027 & 1.86 & -387.3846876 & 0.096721 & 0.51 & -387.335611 \\
\hline & $\mathrm{R}_{2}=\mathrm{CHO}$ & 0.112186 & 1.75 & -401.4749728 & 0.113979 & 0.16 & -401.441824 \\
\hline & $\mathrm{R}_{2}=\mathrm{CN}$ & 0.101252 & 1.44 & -380.3788553 & 0.102281 & -0.41 & -380.3426508 \\
\hline & $\mathrm{R}_{2}=\mathrm{NO}_{2}$ & 0.103026 & 1.85 & -492.6835206 & 0.105539 & -0.45 & -492.6498055 \\
\hline \multirow{7}{*}{$\mathrm{R}_{3}=\mathrm{OH}$} & $\mathrm{R}_{2}=\mathrm{CH}_{3}$ & 0.118365 & 2.87 & -347.3142746 & 0.119371 & 2.11 & -347.2649476 \\
\hline & $\mathrm{R}_{2}=\mathrm{NH}_{2}$ & 0.108735 & 0.83 & -363.3659272 & 0.109669 & -0.44 & -363.3180818 \\
\hline & $\mathrm{R}_{2}=\mathrm{OH}$ & 0.095636 & 0.41 & -383.2367613 & 0.096618 & -0.96 & -383.1883389 \\
\hline & $\mathrm{R}_{2}=\mathrm{F}$ & 0.082497 & 1.36 & -407.2553266 & 0.084186 & 0.72 & -407.2078869 \\
\hline & $\mathrm{R}_{2}=\mathrm{CHO}$ & 0.099875 & 2.07 & -421.3473364 & 0.101621 & 0.77 & -421.3151928 \\
\hline & $\mathrm{R}_{2}=\mathrm{CN}$ & 0.087673 & 1.09 & -400.2498809 & 0.089656 & 0.01 & -400.2119965 \\
\hline & $\mathrm{R}_{2}=\mathrm{NO}_{2}$ & 0.090884 & 2.54 & -512.5551099 & 0.093259 & 0.64 & -512.5228339 \\
\hline \multirow[t]{7}{*}{$\mathrm{R}_{3}=\mathrm{F}$} & $\mathrm{R}_{2}=\mathrm{CH}_{3}$ & 0.107089 & 4.48 & -371.3331541 & 0.106873 & 3.22 & -371.2880629 \\
\hline & $\mathrm{R}_{2}=\mathrm{NH}_{2}$ & 0.096184 & 1.91 & -387.3835133 & 0.097029 & 0.65 & -387.3409249 \\
\hline & $\mathrm{R}_{2}=\mathrm{OH}$ & 0.08286 & 1.46 & -407.2561016 & 0.08447 & 0.7 & -407.2100194 \\
\hline & $\mathrm{R}_{2}=\mathrm{F}$ & 0.070776 & 3.63 & -431.2765343 & 0.071876 & 1.44 & -431.225638 \\
\hline & $\mathrm{R}_{2}=\mathrm{CHO}$ & 0.085435 & 2.87 & -445.3597828 & 0.08819 & 0.79 & -445.3233121 \\
\hline & $\mathrm{R}_{2}=\mathrm{CN}$ & 0.075988 & 3.44 & -424.2709201 & 0.077388 & 0.66 & -424.2281624 \\
\hline & $\mathrm{R}_{2}=\mathrm{NO}_{2}$ & 0.078122 & 2.61 & -536.5711151 & 0.07974 & -0.12 & -536.528072 \\
\hline \multirow[t]{6}{*}{$\mathrm{R}_{3}=\mathrm{CHO}$} & $\mathrm{R}_{2}=\mathrm{CH}_{3}$ & 0.122264 & 3.99 & -385.4262921 & 0.123555 & 2.18 & -385.3771641 \\
\hline & $\mathrm{R}_{2}=\mathrm{NH}_{2}$ & 0.112699 & 1.8 & -401.4791059 & 0.113695 & 0.32 & -401.4389056 \\
\hline & $\mathrm{R}_{2}=\mathrm{OH}$ & 0.10046 & 1.97 & -421.3520742 & 0.101477 & 0.8 & -421.3109316 \\
\hline & $\mathrm{R}_{2}=\mathrm{F}$ & 0.086851 & 2.64 & -445.3676744 & 0.087864 & 0.86 & -445.3201044 \\
\hline & $\mathrm{R}_{2}=\mathrm{CHO}$ & 0.102881 & 1.75 & -459.456081 & 0.103492 & -0.08 & -459.409304 \\
\hline & $\mathrm{R}_{2}=\mathrm{CN}$ & 0.094218 & 2.52 & -438.3591937 & 0.09325 & 0.34 & -438.3176796 \\
\hline
\end{tabular}




\begin{tabular}{cccccccc}
\hline & $\mathrm{R}_{2}=\mathrm{NO}_{2}$ & 0.094 & 1.82 & -550.6644905 & 0.096085 & -0.35 & -550.6197508 \\
\hline $\mathrm{R}_{3}=\mathrm{CN}$ & $\mathrm{R}_{2}=\mathrm{CH}_{3}$ & 0.11139 & 4.24 & -364.3331367 & 0.111995 & 2.33 & -364.2861126 \\
& $\mathrm{R}_{2}=\mathrm{NH}_{2}$ & 0.101383 & 1.18 & -380.3812916 & 0.102056 & -0.2 & -380.3433369 \\
& $\mathrm{R}_{2}=\mathrm{OH}$ & 0.088305 & 0.83 & -400.2542754 & 0.089535 & 0.05 & -400.2104866 \\
& $\mathrm{R}_{2}=\mathrm{F}$ & 0.076068 & 3.05 & -424.2744902 & 0.077047 & 0.59 & -424.2254584 \\
& $\mathrm{R}_{2}=\mathrm{CHO}$ & 0.091699 & 1.97 & -438.3599346 & 0.093121 & 0.22 & -438.3181373 \\
& $\mathrm{R}_{2}=\mathrm{CN}$ & 0.081245 & 2.92 & -417.2665612 & 0.082416 & -0.04 & -417.2221058 \\
& $\mathrm{R}_{2}=\mathrm{NO}_{2}$ & 0.083325 & 1.87 & -529.5687177 & 0.084937 & -0.81 & -529.5229817 \\
\hline $\mathrm{R}_{3}=\mathrm{NO}_{2}$ & $\mathrm{R}_{2}=\mathrm{CH}_{3}$ & 0.113994 & 4.02 & -476.6365853 & 0.115503 & 1.9 & -476.5885376 \\
& $\mathrm{R}_{2}=\mathrm{NH}_{2}$ & 0.103763 & 1.78 & -492.6876882 & 0.10528 & -0.2 & -492.6485108 \\
& $\mathrm{R}_{2}=\mathrm{OH}$ & 0.09147 & 2.35 & -512.5595085 & 0.09321 & 0.74 & -512.5198799 \\
& $\mathrm{R}_{2}=\mathrm{F}$ & 0.08138 & 2.79 & -536.5704373 & 0.079717 & 0.29 & -536.5257084 \\
& $\mathrm{R}_{2}=\mathrm{CHO}$ & 0.094424 & 1.78 & -550.6636667 & 0.095983 & -0.02 & -550.619568 \\
& $\mathrm{R}_{2}=\mathrm{CN}$ & 0.083565 & 1.81 & -529.5694994 & 0.085034 & -0.44 & -529.5232535 \\
& $\mathrm{R}_{2}=\mathrm{NO}$ & 0.086372 & 2.01 & -641.8738763 & 0.08778 & -0.6 & -641.8230273 \\
\hline
\end{tabular}

\begin{tabular}{|c|c|c|c|c|c|c|c|}
\hline \multicolumn{2}{|c|}{ Compound } & \multicolumn{3}{|c|}{$\mathrm{R}_{4}$} & \multicolumn{3}{|c|}{ Transition State } \\
\hline & & TCG & $\begin{array}{l}\text { Solvent } \\
\text { effect }\end{array}$ & Total Energy & TCG & $\begin{array}{c}\text { Solvent } \\
\text { effect }\end{array}$ & Total Energy \\
\hline \multirow[t]{7}{*}{$\mathrm{R}_{4}=\mathrm{CH}_{3}$} & $\mathrm{R}_{2}=\mathrm{CH}_{3}$ & 0.142892 & 5.98 & -311.3874336 & 0.141212 & 5.23 & -311.3421777 \\
\hline & $\mathrm{R}_{2}=\mathrm{NH}_{2}$ & 0.132244 & 3.49 & -327.4403471 & 0.131863 & 2.2 & -327.3965315 \\
\hline & $\mathrm{R}_{2}=\mathrm{OH}$ & 0.118867 & 2.89 & -347.3139876 & 0.119327 & 1.6 & -347.266963 \\
\hline & $\mathrm{R}_{2}=\mathrm{F}$ & 0.106557 & 4.75 & -371.3356863 & 0.106954 & 3.29 & -371.2880308 \\
\hline & $\mathrm{R}_{2}=\mathrm{CHO}$ & 0.122446 & 3.93 & -385.4225656 & 0.122986 & 2.38 & -385.3807596 \\
\hline & $\mathrm{R}_{2}=\mathrm{CN}$ & 0.11169 & 4.49 & -364.3283456 & 0.112392 & 2.36 & -364.2873604 \\
\hline & $\mathrm{R}_{2}=\mathrm{NO}_{2}$ & 0.11413 & 3.92 & -476.6338693 & 0.114996 & 2.18 & -476.5932541 \\
\hline \multirow[t]{7}{*}{$\mathrm{R}_{4}=\mathrm{NH}_{2}$} & $\mathrm{R}_{2}=\mathrm{CH}_{3}$ & 0.132284 & 3.16 & -327.4385087 & 0.130847 & 2.01 & -327.3936586 \\
\hline & $\mathrm{R}_{2}=\mathrm{NH}_{2}$ & 0.121416 & 0.75 & -343.4916687 & 0.121723 & -0.94 & -343.4492392 \\
\hline & $\mathrm{R}_{2}=\mathrm{OH}$ & 0.108134 & 0.13 & -363.3654988 & 0.109066 & -1.72 & -363.3196639 \\
\hline & $\mathrm{R}_{2}=\mathrm{F}$ & 0.095909 & 1.78 & -387.3876011 & 0.096713 & -0.1 & -387.3415031 \\
\hline & $\mathrm{R}_{2}=\mathrm{CHO}$ & 0.112239 & 1.03 & -401.4759285 & 0.112714 & -0.98 & -401.4330856 \\
\hline & $\mathrm{R}_{2}=\mathrm{CN}$ & 0.101057 & 1.48 & -380.3811649 & 0.101999 & -1.05 & -380.339566 \\
\hline & $\mathrm{R}_{2}=\mathrm{NO}_{2}$ & 0.103771 & 0.83 & -492.6876897 & 0.104626 & -1.4 & -492.6463845 \\
\hline \multirow[t]{7}{*}{$\mathrm{R}_{4}=\mathrm{OH}$} & $\mathrm{R}_{2}=\mathrm{CH}_{3}$ & 0.119224 & 2.57 & -347.3112511 & 0.118574 & 1.55 & -347.2663029 \\
\hline & $\mathrm{R}_{2}=\mathrm{NH}_{2}$ & 0.10853 & 0.05 & -363.3637503 & 0.109129 & -1.45 & -363.3221169 \\
\hline & $\mathrm{R}_{2}=\mathrm{OH}$ & 0.095122 & -0.52 & -383.2377546 & 0.096743 & -2.08 & -383.192093 \\
\hline & $\mathrm{R}_{2}=\mathrm{F}$ & 0.082905 & 1.23 & -407.2590789 & 0.084177 & -0.43 & -407.2121196 \\
\hline & $\mathrm{R}_{2}=\mathrm{CHO}$ & 0.099328 & 0.51 & -421.3466273 & 0.100364 & -1.37 & -421.3037625 \\
\hline & $\mathrm{R}_{2}=\mathrm{CN}$ & 0.087934 & 1.04 & -400.252 & 0.089446 & -1.43 & -400.2097662 \\
\hline & $\mathrm{R}_{2}=\mathrm{NO}_{2}$ & 0.090826 & 0.38 & -512.5575459 & 0.092276 & -1.64 & -512.5157966 \\
\hline
\end{tabular}




\begin{tabular}{|c|c|c|c|c|c|c|c|}
\hline \multirow[t]{7}{*}{$\mathrm{R}_{4}=\mathrm{F}$} & $\mathrm{R}_{2}=\mathrm{CH}_{3}$ & 0.107177 & 4.52 & -371.3339616 & 0.106366 & 3.29 & -371.2880244 \\
\hline & $\mathrm{R}_{2}=\mathrm{NH}_{2}$ & 0.096348 & 2 & -387.3858349 & 0.096835 & 0.17 & -387.3429239 \\
\hline & $\mathrm{R}_{2}=\mathrm{OH}$ & 0.083137 & 1.52 & -407.2599687 & 0.084412 & -0.29 & -407.2120887 \\
\hline & $\mathrm{R}_{2}=\mathrm{F}$ & 0.070907 & 3.49 & -431.2806813 & 0.071819 & 1.64 & -431.2316416 \\
\hline & $\mathrm{R}_{2}=\mathrm{CHO}$ & 0.086823 & 2.47 & -445.3664933 & 0.08791 & 0.6 & -445.3237552 \\
\hline & $\mathrm{R}_{2}=\mathrm{CN}$ & 0.076152 & 3.32 & -424.2732431 & 0.077269 & 0.74 & -424.2296228 \\
\hline & $\mathrm{R}_{2}=\mathrm{NO}_{2}$ & 0.07837 & 2.53 & -536.5766762 & 0.079887 & 0.58 & -536.5348273 \\
\hline \multirow[t]{7}{*}{$\mathrm{R}_{4}=\mathrm{CHO}$} & $\mathrm{R}_{2}=\mathrm{CH}_{3}$ & 0.122675 & 3.72 & -385.4200261 & 0.121755 & 2.49 & -385.3776282 \\
\hline & $\mathrm{R}_{2}=\mathrm{NH}_{2}$ & 0.112314 & 1.21 & -401.4727901 & 0.112569 & -0.59 & -401.4319485 \\
\hline & $\mathrm{R}_{2}=\mathrm{OH}$ & 0.099158 & 0.48 & -421.3448753 & 0.100157 & -0.99 & -421.3014728 \\
\hline & $\mathrm{R}_{2}=\mathrm{F}$ & 0.086913 & 2.63 & -445.3653614 & 0.087622 & 0.78 & -445.3202819 \\
\hline & $\mathrm{R}_{2}=\mathrm{CHO}$ & 0.102788 & 1.79 & -459.4525963 & 0.103638 & -0.21 & -459.4132876 \\
\hline & $\mathrm{R}_{2}=\mathrm{CN}$ & 0.091627 & 2.36 & -438.3565689 & 0.092966 & -0.11 & -438.3184796 \\
\hline & $\mathrm{R}_{2}=\mathrm{NO}_{2}$ & 0.094314 & 2 & -550.6619029 & 0.095467 & -0.1 & -550.6235435 \\
\hline \multirow[t]{7}{*}{$\mathrm{R}_{4}=\mathrm{CN}$} & $\mathrm{R}_{2}=\mathrm{CH}_{3}$ & 0.112384 & 3.88 & -364.3298724 & 0.111617 & 2.4 & -364.2849413 \\
\hline & $\mathrm{R}_{2}=\mathrm{NH}_{2}$ & 0.101583 & 1.33 & -380.3817986 & 0.102003 & -0.68 & -380.3396976 \\
\hline & $\mathrm{R}_{2}=\mathrm{OH}$ & 0.088499 & 0.77 & -400.2552454 & 0.089461 & -1.09 & -400.2080907 \\
\hline & $\mathrm{R}_{2}=\mathrm{F}$ & 0.076203 & 2.95 & -424.2752713 & 0.076951 & 0.88 & -424.2267382 \\
\hline & $\mathrm{R}_{2}=\mathrm{CHO}$ & 0.091789 & 1.92 & -438.3605888 & 0.093003 & -0.24 & -438.3188387 \\
\hline & $\mathrm{R}_{2}=\mathrm{CN}$ & 0.081209 & 2.76 & -417.266465 & 0.08232 & -0.07 & -417.2240498 \\
\hline & $\mathrm{R}_{2}=\mathrm{NO}_{2}$ & 0.083457 & 2.14 & -529.5696305 & 0.084826 & -0.06 & -529.5286465 \\
\hline \multirow{7}{*}{$\mathrm{R}_{4}=\mathrm{NO}_{2}$} & $\mathrm{R}_{2}=\mathrm{CH}_{3}$ & 0.114082 & 3.78 & -476.6278033 & 0.114068 & 2.34 & -476.5903397 \\
\hline & $\mathrm{R}_{2}=\mathrm{NH}_{2}$ & 0.103644 & 1.2 & -492.6802703 & 0.104513 & -0.87 & -492.6447517 \\
\hline & $\mathrm{R}_{2}=\mathrm{OH}$ & 0.09061 & 0.55 & -512.5529733 & 0.092083 & -1.19 & -512.512966 \\
\hline & $\mathrm{R}_{2}=\mathrm{F}$ & 0.078245 & 2.83 & -536.5727067 & 0.079506 & 0.82 & -536.5310583 \\
\hline & $\mathrm{R}_{2}=\mathrm{CHO}$ & 0.093928 & 1.73 & -550.6588146 & 0.095487 & -0.17 & -550.6235817 \\
\hline & $\mathrm{R}_{2}=\mathrm{CN}$ & 0.08335 & 2.72 & -529.5638642 & 0.08495 & 0 & -529.5287973 \\
\hline & $\mathrm{R}_{2}=\mathrm{NO}_{2}$ & 0.085632 & 2.04 & -641.8674797 & 0.08744 & 0.12 & -641.8331038 \\
\hline
\end{tabular}

\begin{tabular}{|c|c|c|c|c|c|c|c|}
\hline \multicolumn{2}{|c|}{ Compound } & \multicolumn{3}{|c|}{$=$} & \multicolumn{3}{|c|}{ Transition State } \\
\hline & & TCG & $\begin{array}{c}\text { Solvent } \\
\text { effect }\end{array}$ & $\begin{array}{c}\text { Total } \\
\text { Energy }\end{array}$ & TCG & $\begin{array}{c}\text { Solvent } \\
\text { effect }\end{array}$ & $\begin{array}{c}\text { Total } \\
\text { Energy }\end{array}$ \\
\hline \multirow[t]{7}{*}{$\mathrm{R}_{5}=\mathrm{CH}_{3}$} & $\mathrm{R}_{2}=\mathrm{CH}_{3}$ & 0.141513 & 5.62 & -311.384978 & 0.141009 & 5.12 & -311.341012 \\
\hline & $\mathrm{R}_{2}=\mathrm{NH}_{2}$ & 0.130981 & 3.71 & -327.438857 & 0.131737 & 2.15 & -327.395138 \\
\hline & $\mathrm{R}_{2}=\mathrm{OH}$ & 0.118341 & 2.46 & -347.306342 & 0.119005 & 1.53 & -347.265536 \\
\hline & $\mathrm{R}_{2}=\mathrm{F}$ & 0.106605 & 4.27 & -371.329281 & 0.106365 & 3.22 & -371.286765 \\
\hline & $\mathrm{R}_{2}=\mathrm{CHO}$ & 0.122029 & 3.18 & -385.416817 & 0.122711 & 2.16 & -385.381045 \\
\hline & $\mathrm{R}_{2}=\mathrm{CN}$ & 0.110786 & 4.01 & -364.325368 & 0.111393 & 2.23 & -364.287206 \\
\hline & $\mathrm{R}_{2}=\mathrm{NO}_{2}$ & 0.113846 & 3.27 & -476.628218 & 0.114408 & 1.98 & -476.593122 \\
\hline \multirow[t]{2}{*}{$\mathrm{R}_{5}=\mathrm{NH}_{2}$} & $\mathrm{R}_{2}=\mathrm{CH}_{3}$ & 0.121915 & 1.23 & -343.489055 & 0.121704 & -0.84 & -343.444896 \\
\hline & $\mathrm{R}_{2}=\mathrm{NH}_{2}$ & 0.108279 & 0.19 & -363.362231 & 0.109078 & -1.53 & -363.315678 \\
\hline
\end{tabular}




\begin{tabular}{cccccccc}
\hline \multirow{6}{*}{} & $\mathrm{R}_{2}=\mathrm{OH}$ & 0.095611 & 2.31 & -387.382859 & 0.096720 & 0.11 & -387.337780 \\
& $\mathrm{R}_{2}=\mathrm{F}$ & 0.111630 & 1.03 & -401.469513 & 0.113001 & -1.28 & -401.438725 \\
& $\mathrm{R}_{2}=\mathrm{CHO}$ & 0.100919 & 1.16 & -380.376290 & 0.102472 & -1.18 & -380.344254 \\
& $\mathrm{R}_{2}=\mathrm{CN}$ & 0.103249 & 0.93 & -492.680849 & 0.105077 & -1.88 & -492.651540 \\
\hline $\mathrm{R}_{5}=\mathrm{OH}$ & $\mathrm{R}_{2}=\mathrm{NO}_{2}$ & 0.095259 & 0.16 & -383.239256 & 0.096432 & -2.21 & -383.185843 \\
& $\mathrm{R}_{2}=\mathrm{CH}_{3}$ & 0.082530 & 1.05 & -407.252404 & 0.084099 & -0.45 & -407.207418 \\
& $\mathrm{R}_{2}=\mathrm{NH} \mathrm{H}_{2}$ & 0.098630 & 0.31 & -421.340918 & 0.100544 & -1.55 & -421.306718 \\
& $\mathrm{R}_{2}=\mathrm{OH}$ & 0.087771 & 0.31 & -400.247623 & 0.089934 & -1.55 & -400.211920 \\
& $\mathrm{R}_{2}=\mathrm{F}$ & 0.090193 & 0.16 & -512.552479 & 0.092602 & -1.95 & -512.518242 \\
\hline $\mathrm{R}_{5}=\mathrm{F}$ & $\mathrm{R}_{2}=\mathrm{CHO}$ & 0.070013 & 2.75 & -431.273395 & 0.071735 & 1.51 & -431.227667 \\
& $\mathrm{R}_{2}=\mathrm{CN}$ & 0.086512 & 2.27 & -445.362733 & 0.087966 & 0.55 & -445.324491 \\
& $\mathrm{R}_{2}=\mathrm{NO}{ }_{2}$ & 0.075617 & 2.25 & -424.268756 & 0.077405 & 0.60 & -424.229872 \\
& $\mathrm{R}_{2}=\mathrm{CH}$ & 0.077988 & 2.16 & -536.573435 & 0.080078 & 0.45 & -536.535234 \\
\hline $\mathrm{R}_{5}=\mathrm{CHO}$ & $\mathrm{R}_{2}=\mathrm{NH}$ & 0.100993 & 1.21 & -459.448376 & 0.103921 & -0.20 & -459.414395 \\
& $\mathrm{R}_{2}=\mathrm{OH}$ & 0.091628 & 1.59 & -438.356559 & 0.093224 & -0.08 & -438.320628 \\
& $\mathrm{R}_{2}=\mathrm{F}$ & 0.092889 & 1.32 & -550.659568 & 0.095710 & -0.13 & -550.625050 \\
\hline $\mathrm{R}_{5}=\mathrm{CN}$ & $\mathrm{R}_{2}=\mathrm{CHO}$ & 0.080921 & 1.64 & -417.261365 & 0.082585 & -0.02 & -417.225844 \\
& $\mathrm{R}_{2}=\mathrm{CN}$ & 0.083431 & 1.57 & -529.565860 & 0.085141 & -0.01 & -529.530247 \\
\hline $\mathrm{R}_{5}=\mathrm{NO} \mathrm{N}_{2}$ & $\mathrm{R}_{2}=\mathrm{NO}_{2}$ & 0.084751 & 1.55 & -641.869887 & 0.087556 & 0.08 & -641.834225 \\
\hline
\end{tabular}

\begin{tabular}{|c|c|c|c|c|c|c|c|}
\hline \multicolumn{2}{|c|}{ Compound } & \multicolumn{3}{|c|}{$\mathrm{R}_{4} \quad \mathrm{R}_{3}$} & \multicolumn{3}{|c|}{ Transition State } \\
\hline & & TCG & $\begin{array}{c}\text { Solvent } \\
\text { effect }\end{array}$ & $\begin{array}{c}\text { Total } \\
\text { Energy }\end{array}$ & TCG & $\begin{array}{c}\text { Solvent } \\
\text { effect }\end{array}$ & $\begin{array}{c}\text { Total } \\
\text { Energy }\end{array}$ \\
\hline \multirow[t]{7}{*}{$\mathrm{R}_{4}=\mathrm{CH}_{3}$} & $\mathrm{R}_{3}=\mathrm{CH}_{3}$ & 0.140860 & 5.48 & -311.385744 & 0.142105 & 4.60 & -311.338700 \\
\hline & $\mathrm{R}_{3}=\mathrm{NH}_{2}$ & 0.131698 & 3.41 & -327.440620 & 0.132297 & 2.15 & -327.391279 \\
\hline & $\mathrm{R}_{3}=\mathrm{OH}$ & 0.119088 & 3.38 & -347.313767 & 0.119357 & 1.54 & -347.265291 \\
\hline & $\mathrm{R}_{3}=\mathrm{F}$ & 0.106398 & 4.62 & -371.336854 & 0.107099 & 3.28 & -371.287639 \\
\hline & $\mathrm{R}_{3}=\mathrm{CHO}$ & 0.121221 & 3.75 & -385.416428 & 0.123093 & 2.19 & -385.373639 \\
\hline & $\mathrm{R}_{3}=\mathrm{CN}$ & 0.111697 & 4.01 & -364.333181 & 0.111773 & 2.38 & -364.284721 \\
\hline & $\mathrm{R}_{3}=\mathrm{NO}_{2}$ & 0.113386 & 3.99 & -476.627866 & 0.115282 & 2.30 & -476.585242 \\
\hline \multirow{6}{*}{$\mathrm{R}_{4}=\mathrm{NH}_{2}$} & $\mathrm{R}_{3}=\mathrm{CH}_{3}$ & 0.120748 & 1.34 & -343.490930 & 0.122187 & -0.44 & -343.442703 \\
\hline & $\mathrm{R}_{3}=\mathrm{NH}_{2}$ & 0.108673 & 1.68 & -363.366198 & 0.108967 & -0.28 & -363.313668 \\
\hline & $\mathrm{R}_{3}=\mathrm{OH}$ & 0.096204 & 2.18 & -387.388214 & 0.096830 & 0.52 & -387.338521 \\
\hline & $\mathrm{R}_{3}=\mathrm{F}$ & 0.112781 & 1.6 & -401.487554 & 0.113898 & 0.21 & -401.436195 \\
\hline & $\mathrm{R}_{3}=\mathrm{CHO}$ & 0.101468 & 1.43 & -380.391454 & 0.101817 & -0.12 & -380.339710 \\
\hline & $\mathrm{R}_{3}=\mathrm{CN}$ & 0.104681 & 0.33 & -492.694356 & 0.105080 & -0.47 & -492.642580 \\
\hline \multirow{5}{*}{$\mathrm{R}_{4}=\mathrm{OH}$} & $\mathrm{R}_{3}=\mathrm{NO}_{2}$ & 0.095875 & 1.21 & -383.239336 & 0.096073 & -0.98 & -383.189630 \\
\hline & $\mathrm{R}_{3}=\mathrm{CH}_{3}$ & 0.083195 & 2.38 & -407.259645 & 0.084522 & 0.90 & -407.209859 \\
\hline & $\mathrm{R}_{3}=\mathrm{NH}_{2}$ & 0.100417 & 2.68 & -421.362893 & 0.101823 & 0.69 & -421.310880 \\
\hline & $\mathrm{R}_{3}=\mathrm{OH}$ & 0.088651 & 2.38 & -400.261321 & 0.089442 & 0.60 & -400.209687 \\
\hline & $\mathrm{R}_{3}=\mathrm{F}$ & 0.091555 & 2.13 & -512.567515 & 0.093061 & 0.56 & -512.517162 \\
\hline
\end{tabular}




\begin{tabular}{cccccccc}
\hline \multirow{2}{*}{$\mathrm{R}_{4}=\mathrm{F}$} & $\mathrm{R}_{3}=\mathrm{CHO}$ & 0.070721 & 3.26 & -431.274719 & 0.071770 & 1.53 & -431.225212 \\
& $\mathrm{R}_{3}=\mathrm{CN}$ & 0.086706 & 2.61 & -445.364004 & 0.087592 & 0.83 & -445.318191 \\
& $\mathrm{R}_{3}=\mathrm{NO}_{2}$ & 0.076203 & 2.74 & -424.274938 & 0.077074 & 0.62 & -424.225507 \\
& $\mathrm{R}_{3}=\mathrm{CH}_{3}$ & 0.078182 & 2.65 & -536.567159 & 0.079465 & 0.64 & -536.522903 \\
\hline $\mathrm{R}_{4}=\mathrm{CHO}$ & $\mathrm{R}_{3}=\mathrm{NH}_{2}$ & 0.102004 & 1.74 & -459.441506 & 0.102758 & 0.01 & -459.402660 \\
& $\mathrm{R}_{3}=\mathrm{OH}$ & 0.089760 & 2.77 & -438.357033 & 0.092533 & 0.29 & -438.313616 \\
& $\mathrm{R}_{3}=\mathrm{F}$ & 0.092300 & 2.98 & -550.651359 & 0.095151 & 0.43 & -550.612524 \\
\hline $\mathrm{R}_{4}=\mathrm{CN}$ & $\mathrm{R}_{3}=\mathrm{CHO}$ & 0.080941 & 2.71 & -417.266658 & 0.081914 & -0.09 & -417.219003 \\
& $\mathrm{R}_{3}=\mathrm{CN}$ & 0.082974 & 2.68 & -529.560364 & 0.084339 & -0.26 & -529.518567 \\
\hline $\mathrm{R}_{4}=\mathrm{NO}_{2}$ & $\mathrm{R}_{3}=\mathrm{NO}_{2}$ & 0.085507 & 2.87 & -641.854790 & 0.087096 & -0.06 & -641.815496 \\
\hline
\end{tabular}

\begin{tabular}{ccccccc}
\hline & \multicolumn{3}{c}{ Reactant } & \multicolumn{3}{c}{ Transition State } \\
\cline { 2 - 7 } Compound & TCG $\begin{array}{c}\text { Solvent } \\
\text { effect }\end{array}$ & Total Energy & $\mathrm{TCG}$ & $\begin{array}{c}\text { Solvent } \\
\text { effect }\end{array}$ & Total Energy \\
\hline & & & & & & \\
\hline
\end{tabular}

Table S7. The C1-C6 Distance (a.u.) sand calculated NICS at B3LYP /6-31+g(d) level related to compounds in Table 4 and the figures of their relationship with reaction barrier

Table $\Delta \mathrm{G}^{\neq}, \mathrm{C} 1-\mathrm{C} 6$ Distance and NICS of Transition State of Hexatriene

\begin{tabular}{cccc}
\hline Hexatriene & $\Delta \mathrm{G}^{\neq}$ & $\mathrm{C} 1-\mathrm{C} 6$ Distance & NICS of Transition State \\
\hline $1-\mathrm{NO}_{2}-2-\mathrm{NH}_{2}$ & 36.4 & 2.204 & -10.56 \\
$1-\mathrm{NO}_{2}-3-\mathrm{NH}_{2}$ & 28.0 & 2.264 & -14.67 \\
$1-\mathrm{NO}_{2}-4-\mathrm{NH}_{2}$ & 30.6 & 2.234 & -12.48 \\
$1-\mathrm{NO}_{2}-5-\mathrm{NH}_{2}$ & 26.3 & 2.263 & -13.11 \\
$1-\mathrm{NO}_{2}-6-\mathrm{NH}_{2}$ & 29.4 & 2.331 & -10.72 \\
$2-\mathrm{NO}_{2}-1-\mathrm{NH}_{2}$ & 34.5 & 2.200 & -11.73 \\
$2-\mathrm{NO}_{2}-3-\mathrm{NH}_{2}$ & 20.4 & 2.324 & -13.28 \\
$2-\mathrm{NO}_{2}-4-\mathrm{NH}_{2}$ & 24.2 & 2.261 & -14.59 \\
$2-\mathrm{NO}_{2}-5-\mathrm{NH}_{2}$ & 16.7 & 2.318 & -11.46 \\
$2-\mathrm{NO}_{2}-6-\mathrm{NH}_{2}$ & 25.4 & 2.307 & -12.48 \\
$3-\mathrm{NO}_{2}-1-\mathrm{NH}_{2}$ & 27.5 & 2.280 & -13.51 \\
$3-\mathrm{NO}_{2}-2-\mathrm{NH}_{2}$ & 23.6 & 2.369 & -12.93 \\
$3-\mathrm{NO}_{2}-4-\mathrm{NH}_{2}$ & 31.9 & 2.173 & -13.86 \\
$3-\mathrm{NO}_{2}-5-\mathrm{NH}_{2}$ & 20.8 & 2.302 & -13.66 \\
$3-\mathrm{NO}_{2}-6-\mathrm{NH}_{2}$ & 27.6 & 2.282 & -11.96 \\
\hline
\end{tabular}


Relationship of Reaction Energy and C1-C6 Distance

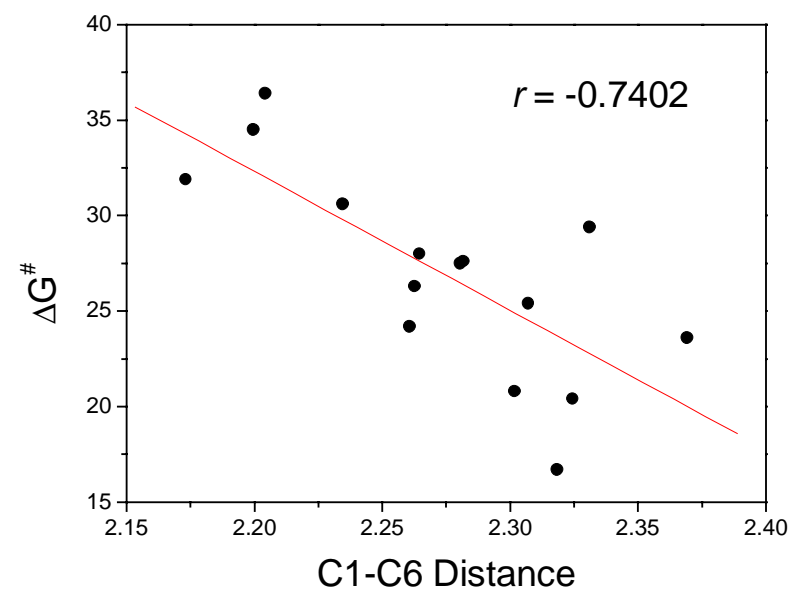

Relationship of $\Delta \mathbf{G}_{\text {transition }}$ and NICS of Transition State

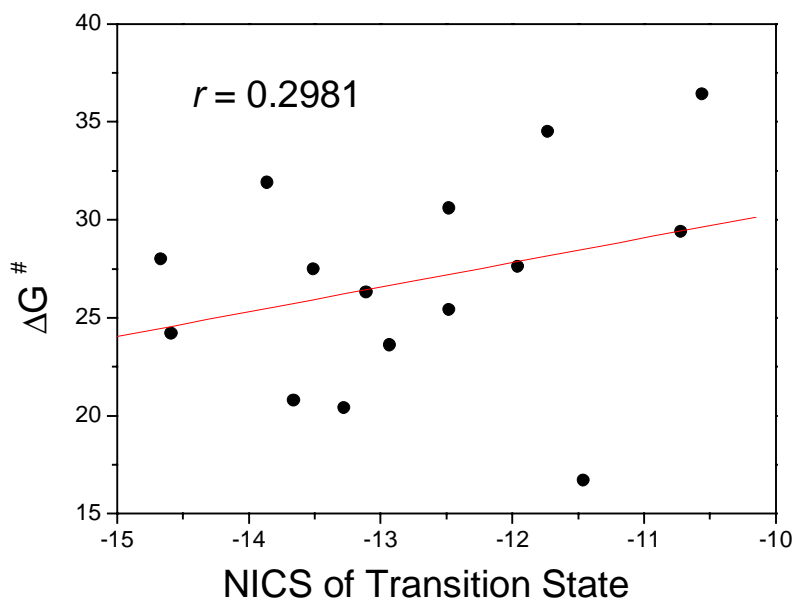

The Cartesian coordinates $(\AA)$ and calculated Energies and the thermal corrections to Gibbs Free Energy of optimized structures by B3LYP/6-31G(d) method 
(The charge and spin of all molecules are 0 and 1)

\section{TABLE S1}

1, 3, 5-hexatriene:

$\begin{array}{lrrr}\mathrm{C} & 0.000000 & 0.000000 & 0.000000 \\ \mathrm{C} & 0.000000 & 0.000000 & 1.343078 \\ \mathrm{C} & 1.209819 & 0.000000 & 2.146941 \\ \mathrm{C} & 1.292490 & -0.000019 & 3.499766 \\ \mathrm{C} & 0.189556 & -0.000043 & 4.444962 \\ \mathrm{C} & 0.353119 & -0.000061 & 5.778042 \\ \mathrm{H} & 1.342928 & -0.000058 & 6.229688 \\ \mathrm{H} & -0.492809 & -0.000047 & 6.458600 \\ \mathrm{H} & 2.287051 & -0.000016 & 3.944884 \\ \mathrm{H} & -0.822474 & -0.000030 & 4.044333 \\ \mathrm{H} & 2.142769 & 0.000000 & 1.584017 \\ \mathrm{H} & 0.927440 & 0.000000 & -0.568826 \\ \mathrm{H} & -0.922511 & -0.000032 & -0.572472 \\ \mathrm{H} & -0.955716 & -0.000017 & 1.863957\end{array}$

TS:

$\begin{array}{lrrc}\mathrm{C} & 0.000000 & 0.000000 & 0.000000 \\ \mathrm{C} & 0.000000 & 0.000000 & 1.398420 \\ \mathrm{C} & 1.139713 & 0.000000 & 2.208102 \\ \mathrm{C} & 2.345480 & -0.636120 & 1.864430 \\ \mathrm{C} & 2.542865 & -1.341525 & 0.673645 \\ \mathrm{C} & 1.956526 & -1.032193 & -0.557655 \\ \mathrm{H} & 0.511485 & 0.796506 & -0.515971 \\ \mathrm{H} & -0.903737 & -0.329184 & -0.513954 \\ \mathrm{H} & -0.947241 & -0.177042 & 1.912714 \\ \mathrm{H} & 3.019345 & -0.850078 & 2.693236 \\ \mathrm{H} & 3.067312 & -2.294977 & 0.768474 \\ \mathrm{H} & 1.911275 & -1.814283 & -1.316297 \\ \mathrm{H} & 2.117397 & -0.050716 & -0.973693 \\ \mathrm{H} & 1.003746 & 0.213300 & 3.267728\end{array}$

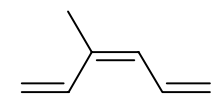

(Z)-3-methylhexa-1, 3, 5-triene :

$\begin{array}{llll}\mathrm{C} & 0.000000 & 0.000000 & 0.000000 \\ \mathrm{C} & 0.000000 & 0.000000 & 1.342966 \\ \mathrm{C} & 1.188412 & 0.000000 & 2.195920 \\ \mathrm{C} & 1.117808 & 0.000280 & 3.554461\end{array}$




$\begin{array}{crcc}\mathrm{C} & -0.067065 & 0.000635 & 4.393708 \\ \mathrm{C} & -0.021228 & 0.000501 & 5.736539 \\ \mathrm{H} & -0.930947 & -0.000016 & -0.559060 \\ \mathrm{H} & 0.913829 & 0.000000 & -0.586803 \\ \mathrm{H} & -0.963656 & -0.000034 & 1.846624 \\ \mathrm{H} & 2.060751 & 0.000185 & 4.101858 \\ \mathrm{H} & -1.042415 & 0.001080 & 3.911893 \\ \mathrm{H} & -0.924278 & 0.000791 & 6.339209 \\ \mathrm{H} & 0.924668 & 0.000096 & 6.274014 \\ \mathrm{C} & 2.538708 & -0.000281 & 1.519359 \\ \mathrm{H} & 2.662471 & 0.881018 & 0.876530 \\ \mathrm{H} & 2.661971 & -0.881454 & 0.876265 \\ \mathrm{H} & 3.350184 & -0.000613 & 2.252584\end{array}$

TS:

$\begin{array}{lrrr}\mathrm{C} & 0.000000 & 0.000000 & 0.000000 \\ \mathrm{C} & 0.000000 & 0.000000 & 1.396439 \\ \mathrm{C} & 1.136869 & 0.000000 & 2.221332 \\ \mathrm{C} & 2.316516 & -0.673426 & 1.841929 \\ \mathrm{C} & 2.484812 & -1.399518 & 0.659003 \\ \mathrm{C} & 1.901646 & -1.097880 & -0.575113 \\ \mathrm{H} & 0.526618 & 0.786712 & -0.516844 \\ \mathrm{H} & -0.910353 & -0.312038 & -0.512788 \\ \mathrm{H} & -0.950287 & -0.171687 & 1.907713 \\ \mathrm{H} & 3.001915 & -0.900268 & 2.658892 \\ \mathrm{H} & 2.985298 & -2.364766 & 0.767419 \\ \mathrm{H} & 1.828443 & -1.895298 & -1.315725 \\ \mathrm{H} & 2.085961 & -0.131353 & -1.015806 \\ \mathrm{C} & 0.985675 & 0.426887 & 3.666786 \\ \mathrm{H} & 1.188332 & 1.500655 & 3.779450 \\ \mathrm{H} & -0.037847 & 0.261248 & 4.024303 \\ \mathrm{H} & 1.672672 & -0.106633 & 4.332561\end{array}$

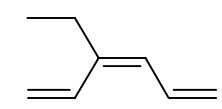

(Z)-3-ethylhexa-1, 3, 5-triene :

$\begin{array}{lrrr}\mathrm{C} & 0.000000 & 0.000000 & 0.000000 \\ \mathrm{C} & 0.000000 & 0.000000 & 1.343126 \\ \mathrm{C} & 1.176720 & 0.000000 & 2.214521 \\ \mathrm{C} & 1.075689 & 0.076235 & 3.569809 \\ \mathrm{C} & -0.124097 & 0.155893 & 4.383703 \\ \mathrm{C} & -0.103563 & 0.228910 & 5.725294 \\ \mathrm{H} & -0.932437 & 0.010530 & -0.556563\end{array}$




$\begin{array}{rrrc}\mathrm{H} & 0.910901 & -0.004690 & -0.590966 \\ \mathrm{H} & -0.967550 & 0.007945 & 1.839573 \\ \mathrm{H} & 2.007025 & 0.079704 & 4.137606 \\ \mathrm{H} & -1.090144 & 0.157126 & 3.883495 \\ \mathrm{H} & -1.017562 & 0.286487 & 6.308422 \\ \mathrm{H} & 0.831502 & 0.233383 & 6.281433 \\ \mathrm{C} & 2.552056 & -0.110710 & 1.583563 \\ \mathrm{H} & 2.620296 & 0.563090 & 0.719508 \\ \mathrm{H} & 3.302468 & 0.238525 & 2.302245 \\ \mathrm{C} & 2.911992 & -1.542346 & 1.142336 \\ \mathrm{H} & 3.906163 & -1.566889 & 0.681213 \\ \mathrm{H} & 2.191058 & -1.932815 & 0.417335 \\ \mathrm{H} & 2.919450 & -2.221083 & 2.002303\end{array}$

TS:

C $\quad 0.000000 \quad 0.000000 \quad 0.000000$

$\begin{array}{llll}\text { C } & 0.000000 & 0.000000 & 1.396477\end{array}$

$\begin{array}{llll}\text { C } & 1.138349 & 0.000000 & 2.219960\end{array}$

$\begin{array}{llll}\text { C } & 2.319618 & -0.668841 & 1.836799\end{array}$

$\begin{array}{llll}\text { C } & 2.484431 & -1.399959 & 0.656423\end{array}$

$\begin{array}{llll}\text { C } & 1.894905 & -1.107303 & -0.577060\end{array}$

$\begin{array}{llll}\mathrm{H} & 0.529989 & 0.785297 & -0.515557\end{array}$

$\begin{array}{llll}\mathrm{H} & -0.910458 & -0.309378 & -0.514108\end{array}$

$\begin{array}{llll}\mathrm{H} & -0.949899 & -0.173223 & 1.908228\end{array}$

$\begin{array}{llll}\mathrm{H} & 3.009685 & -0.890226 & 2.651475\end{array}$

$\begin{array}{llll}\mathrm{H} & 2.987644 & -2.363510 & 0.767573\end{array}$

$\mathrm{H} \quad 2.078218 \quad-0.144943 \quad-1.027199$

$\begin{array}{llll}\mathrm{H} & 1.818472 & -1.910749 & -1.310903\end{array}$

$\begin{array}{llll}\text { C } & 0.992283 & 0.436949 & 3.666423\end{array}$

$\begin{array}{llll}\mathrm{H} & 1.689528 & -0.118906 & 4.305853\end{array}$

$\begin{array}{llll}\mathrm{H} & -0.020146 & 0.196182 & 4.018660\end{array}$

$\begin{array}{llll}\text { C } & 1.233469 & 1.946288 & 3.849617\end{array}$

$\begin{array}{llll}\mathrm{H} & 0.523726 & 2.530007 & 3.252849\end{array}$

$\begin{array}{llll}\mathrm{H} & 1.117453 & 2.236944 & 4.900409\end{array}$

$\begin{array}{llll}\mathrm{H} & 2.244563 & 2.223994 & 3.530395\end{array}$

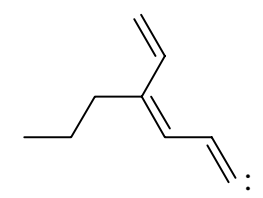

$\begin{array}{cccc}\mathrm{C} & 0.000000 & 0.000000 & 0.000000 \\ \mathrm{H} & 0.000000 & 0.000000 & 1.085869 \\ \mathrm{H} & 0.974473 & 0.000000 & -0.479289 \\ \mathrm{C} & -1.126761 & -0.005234 & -0.731077\end{array}$




$\begin{array}{llll}\mathrm{H} & -1.016148 & -0.006730 & -1.812939 \\ \mathrm{C} & -2.498383 & -0.018564 & -0.218857 \\ \mathrm{C} & -3.579370 & -0.082168 & -1.043937 \\ \mathrm{H} & -4.562924 & -0.099233 & -0.572586 \\ \mathrm{C} & -3.608154 & -0.130697 & -2.494713 \\ \mathrm{H} & -2.662652 & -0.117422 & -3.032573 \\ \mathrm{C} & -4.744408 & -0.190852 & -3.209629 \\ \mathrm{H} & -5.719922 & -0.207847 & -2.728328 \\ \mathrm{H} & -4.735608 & -0.224558 & -4.294767 \\ \mathrm{C} & -2.720286 & 0.064710 & 1.278998 \\ \mathrm{H} & -3.723352 & -0.312185 & 1.514539 \\ \mathrm{H} & -2.015927 & -0.597701 & 1.800493 \\ \mathrm{C} & -2.584894 & 1.492902 & 1.849269 \\ \mathrm{H} & -3.314550 & 2.141687 & 1.347480 \\ \mathrm{H} & -1.595750 & 1.895503 & 1.600093 \\ \mathrm{C} & -2.800633 & 1.543626 & 3.364781 \\ \mathrm{H} & -3.795093 & 1.168840 & 3.637364 \\ \mathrm{H} & -2.715060 & 2.567987 & 3.744790 \\ \mathrm{H} & -2.060795 & 0.929471 & 3.893252\end{array}$

TS:

$\begin{array}{cccc}\mathrm{H} & 0.000000 & 0.000000 & 0.000000 \\ \mathrm{C} & 0.000000 & 0.000000 & 1.078612 \\ \mathrm{H} & 1.003420 & 0.000000 & 1.505365 \\ \mathrm{C} & -0.999225 & -0.711294 & 1.746203 \\ \mathrm{H} & -0.702181 & -1.347124 & 2.583853 \\ \mathrm{C} & -2.383827 & -0.538991 & 1.580313 \\ \mathrm{C} & -2.935677 & 0.729309 & 1.303417 \\ \mathrm{H} & -4.001068 & 0.825137 & 1.514477 \\ \mathrm{C} & -2.207374 & 1.917506 & 1.190638 \\ \mathrm{H} & -2.640510 & 2.783200 & 1.697577 \\ \mathrm{C} & -0.912051 & 2.038829 & 0.678557 \\ \mathrm{H} & -0.335304 & 2.923733 & 0.951019 \\ \mathrm{H} & -0.715605 & 1.704186 & -0.327312 \\ \mathrm{C} & -3.315190 & -1.650825 & 2.025723 \\ \mathrm{H} & -4.267927 & -1.235279 & 2.380243 \\ \mathrm{H} & -2.868072 & -2.184992 & 2.876657 \\ \mathrm{C} & -3.601421 & -2.670814 & 0.905078 \\ \mathrm{H} & -4.035704 & -2.144417 & 0.044423 \\ \mathrm{H} & -2.649028 & -3.092077 & 0.557149 \\ \mathrm{C} & -4.538650 & -3.796972 & 1.351338 \\ \mathrm{H} & -5.509326 & -3.401552 & 1.675486 \\ \mathrm{H} & -4.723871 & -4.507203 & 0.537437 \\ \mathrm{H} & -4.113085 & -4.357722 & 2.192871\end{array}$




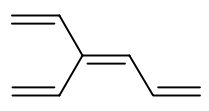

3-vinylhexa-1, 3, 5-triene :

$\begin{array}{lrrr}\mathrm{C} & 0.000000 & 0.000000 & 0.000000 \\ \mathrm{C} & 0.000000 & 0.000000 & 1.342041 \\ \mathrm{C} & 1.192205 & 0.000000 & 2.197844 \\ \mathrm{C} & 1.127785 & -0.215074 & 3.544484 \\ \mathrm{C} & -0.038171 & -0.559937 & 4.332743 \\ \mathrm{C} & -0.004957 & -0.725080 & 5.666893 \\ \mathrm{H} & -0.930748 & 0.013419 & -0.559264 \\ \mathrm{H} & 0.915441 & -0.027985 & -0.583922 \\ \mathrm{H} & -0.961796 & 0.008015 & 1.849294 \\ \mathrm{H} & 2.054124 & -0.114432 & 4.107917 \\ \mathrm{H} & -0.985406 & -0.704756 & 3.817767 \\ \mathrm{H} & -0.893351 & -0.989944 & 6.231918 \\ \mathrm{H} & 0.916075 & -0.603319 & 6.232758 \\ \mathrm{C} & 2.495208 & 0.285581 & 1.562270 \\ \mathrm{H} & 2.478384 & 1.021606 & 0.758703 \\ \mathrm{C} & 3.663145 & -0.283474 & 1.885446 \\ \mathrm{H} & 4.587029 & 0.006404 & 1.392827 \\ \mathrm{H} & 3.735680 & -1.061221 & 2.641450\end{array}$

TS:

$\begin{array}{lrrr}\text { C } & 0.000000 & 0.000000 & 0.000000 \\ \mathrm{C} & 0.000000 & 0.000000 & 1.394180 \\ \mathrm{C} & 1.152885 & 0.000000 & 2.207903 \\ \mathrm{C} & 2.312794 & -0.713968 & 1.819476 \\ \mathrm{C} & 2.445200 & -1.459142 & 0.648252 \\ \mathrm{C} & 1.840646 & -1.166786 & -0.580784 \\ \mathrm{H} & -0.912815 & -0.298230 & -0.515641 \\ \mathrm{H} & 0.544761 & 0.776786 & -0.514753 \\ \mathrm{H} & -0.939669 & -0.189764 & 1.914405 \\ \mathrm{H} & 3.008860 & -0.935854 & 2.628574 \\ \mathrm{H} & 2.937724 & -2.428184 & 0.758582 \\ \mathrm{H} & 2.050752 & -0.219925 & -1.052058 \\ \mathrm{H} & 1.726003 & -1.980068 & -1.298755 \\ \mathrm{C} & 1.056899 & 0.416453 & 3.615604 \\ \mathrm{H} & 1.822410 & 0.022812 & 4.284894 \\ \mathrm{C} & 0.130275 & 1.245845 & 4.116207 \\ \mathrm{H} & -0.625845 & 1.707874 & 3.486892 \\ \mathrm{H} & 0.107693 & 1.496092 & 5.172827\end{array}$




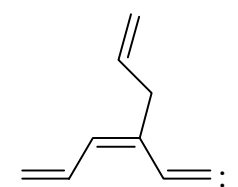

$\begin{array}{cccc}\mathrm{C} & 0.000000 & 0.000000 & 0.000000 \\ \mathrm{H} & 0.000000 & 0.000000 & 1.085801 \\ \mathrm{H} & 0.974823 & 0.000000 & -0.478489 \\ \mathrm{C} & -1.125598 & 0.006383 & -0.732582 \\ \mathrm{H} & -1.015918 & 0.015780 & -1.814272 \\ \mathrm{C} & -2.496649 & 0.018392 & -0.219606 \\ \mathrm{C} & -3.580066 & 0.090121 & -1.038372 \\ \mathrm{H} & -4.560996 & 0.120848 & -0.566749 \\ \mathrm{C} & -3.605419 & 0.143857 & -2.489556 \\ \mathrm{H} & -2.660894 & 0.118642 & -3.028626 \\ \mathrm{C} & -4.741457 & 0.222273 & -3.202624 \\ \mathrm{H} & -5.715921 & 0.251746 & -2.719958 \\ \mathrm{H} & -4.733375 & 0.260877 & -4.287668 \\ \mathrm{C} & -2.671436 & -0.018652 & 1.294383 \\ \mathrm{H} & -2.333362 & 0.937176 & 1.720574 \\ \mathrm{H} & -1.985861 & -0.780018 & 1.695891 \\ \mathrm{C} & -4.057877 & -0.329602 & 1.788741 \\ \mathrm{H} & -4.455920 & -1.303912 & 1.501993 \\ \mathrm{C} & -4.798427 & 0.475974 & 2.550801 \\ \mathrm{H} & -5.785120 & 0.184819 & 2.901220 \\ \mathrm{H} & -4.444010 & 1.457878 & 2.858433\end{array}$

TS:

$\begin{array}{cccc}\mathrm{C} & 0.000000 & 0.000000 & 0.000000 \\ \mathrm{H} & 0.000000 & 0.000000 & 1.078512 \\ \mathrm{H} & 1.001723 & 0.000000 & -0.429960 \\ \mathrm{C} & -1.005059 & 0.704744 & -0.664660 \\ \mathrm{H} & -0.718159 & 1.335018 & -1.509847 \\ \mathrm{C} & -2.385096 & 0.528522 & -0.478531 \\ \mathrm{C} & -2.935989 & -0.732310 & -0.174332 \\ \mathrm{H} & -4.007556 & -0.824446 & -0.352230 \\ \mathrm{C} & -2.208847 & -1.922464 & -0.072948 \\ \mathrm{H} & -2.660293 & -2.788893 & -0.562533 \\ \mathrm{C} & -0.902104 & -2.052073 & 0.406570 \\ \mathrm{H} & -0.337734 & -2.939532 & 0.116990 \\ \mathrm{H} & -0.674449 & -1.718226 & 1.405873 \\ \mathrm{C} & -3.334637 & 1.631853 & -0.930952 \\ \mathrm{H} & -4.217700 & 1.212276 & -1.428413 \\ \mathrm{H} & -2.816324 & 2.262312 & -1.668686 \\ \mathrm{C} & -3.780005 & 2.497353 & 0.224507\end{array}$




$\begin{array}{llll}\mathrm{H} & -2.978300 & 2.971840 & 0.791601 \\ \mathrm{C} & -5.047892 & 2.697381 & 0.585107 \\ \mathrm{H} & -5.874450 & 2.235202 & 0.048647 \\ \mathrm{H} & -5.310191 & 3.332014 & 1.427430\end{array}$

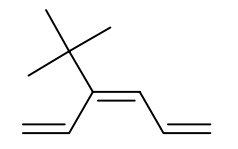

(E)-3-tert-butylhexa-1, 3, 5-triene:

$\begin{array}{cccc}\mathrm{C} & 0.000000 & 0.000000 & 0.000000 \\ \mathrm{H} & 0.000000 & 0.000000 & 1.086433 \\ \mathrm{H} & 0.970447 & 0.000000 & -0.489507 \\ \mathrm{C} & -1.135202 & 0.020842 & -0.710809 \\ \mathrm{H} & -1.048608 & 0.080997 & -1.796260 \\ \mathrm{C} & -2.508939 & 0.035725 & -0.162129 \\ \mathrm{C} & -2.902889 & -0.809571 & 0.822985 \\ \mathrm{H} & -3.914894 & -0.715462 & 1.208556 \\ \mathrm{C} & -2.142773 & -1.900243 & 1.417203 \\ \mathrm{H} & -1.180447 & -2.141523 & 0.972706 \\ \mathrm{C} & -2.583985 & -2.627701 & 2.456245 \\ \mathrm{H} & -3.536241 & -2.417515 & 2.939137 \\ \mathrm{H} & -2.006279 & -3.453740 & 2.860243 \\ \mathrm{C} & -3.472122 & 1.056764 & -0.805085 \\ \mathrm{C} & -4.828416 & 1.136232 & -0.080435 \\ \mathrm{H} & -5.376892 & 0.189130 & -0.129895 \\ \mathrm{H} & -4.710070 & 1.410384 & 0.973921 \\ \mathrm{H} & -5.452697 & 1.901906 & -0.554820 \\ \mathrm{C} & -2.827285 & 2.462913 & -0.775274 \\ \mathrm{H} & -2.645099 & 2.787659 & 0.255733 \\ \mathrm{H} & -3.489785 & 3.196186 & -1.250969 \\ \mathrm{H} & -1.868269 & 2.482550 & -1.302779 \\ \mathrm{C} & -3.744119 & 0.652629 & -2.276294 \\ \mathrm{H} & -2.832002 & 0.645101 & -2.882185 \\ \mathrm{H} & -4.436560 & 1.365530 & -2.740402 \\ \mathrm{H} & -4.195442 & -0.344409 & -2.331040\end{array}$

TS:

$\begin{array}{cccc}\mathrm{C} & 0.000000 & 0.000000 & 0.000000 \\ \mathrm{H} & 0.000000 & 0.000000 & 1.091633 \\ \mathrm{H} & 0.995313 & 0.000000 & -0.412944 \\ \mathrm{C} & -1.025840 & 0.696168 & -0.644304 \\ \mathrm{H} & -1.640461 & 1.361096 & -0.031222 \\ \mathrm{C} & -1.500381 & 0.500305 & -1.947570 \\ \mathrm{H} & -2.140744 & 1.291827 & -2.333420\end{array}$




$\begin{array}{lrrl}\mathrm{C} & -1.591909 & -0.736876 & -2.620907 \\ \mathrm{C} & -1.083202 & -1.900222 & -2.020276 \\ \mathrm{H} & -1.688107 & -2.803169 & -2.096857 \\ \mathrm{C} & 0.035758 & -1.939710 & -1.189260 \\ \mathrm{H} & 0.946674 & -1.487335 & -1.549823 \\ \mathrm{H} & 0.202138 & -2.825695 & -0.577494 \\ \mathrm{C} & -2.527072 & -0.821597 & -3.852492 \\ \mathrm{C} & -2.555783 & -2.240515 & -4.456478 \\ \mathrm{H} & -1.546165 & -2.606918 & -4.672232 \\ \mathrm{H} & -3.044004 & -2.962196 & -3.792045 \\ \mathrm{H} & -3.121392 & -2.231516 & -5.395402 \\ \mathrm{C} & -1.989861 & 0.140989 & -4.938486 \\ \mathrm{H} & -0.974687 & -0.139131 & -5.242568 \\ \mathrm{H} & -2.631843 & 0.108200 & -5.827678 \\ \mathrm{H} & -1.958275 & 1.177881 & -4.587802 \\ \mathrm{C} & -3.977768 & -0.427784 & -3.487941 \\ \mathrm{H} & -4.630000 & -0.514619 & -4.365897 \\ \mathrm{H} & -4.375201 & -1.084350 & -2.705281 \\ \mathrm{H} & -4.047041 & 0.602915 & -3.125627\end{array}$

\section{1-substituted hexatriene}

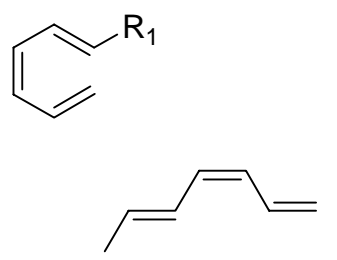

(3Z,5E)-hepta-1, 3, 5-triene :

$\begin{array}{cccc}\mathrm{C} & 0.000000 & 0.000000 & 0.000000 \\ \mathrm{H} & 0.000000 & 0.000000 & 1.087959 \\ \mathrm{H} & 0.970218 & 0.000000 & -0.487353 \\ \mathrm{C} & -1.145109 & 0.000020 & -0.702356 \\ \mathrm{H} & -1.088039 & 0.000036 & -1.789495 \\ \mathrm{C} & -2.464455 & 0.000085 & -0.095804 \\ \mathrm{H} & -2.477159 & 0.000101 & 0.993771 \\ \mathrm{C} & -3.658427 & 0.000014 & -0.738315 \\ \mathrm{H} & -4.558478 & 0.000063 & -0.123629 \\ \mathrm{C} & -3.886927 & -0.000263 & -2.171132 \\ \mathrm{H} & -3.018194 & -0.000975 & -2.828626 \\ \mathrm{C} & -5.111733 & 0.000448 & -2.728998 \\ \mathrm{H} & -5.983109 & 0.001229 & -2.071534 \\ \mathrm{C} & -5.392534 & 0.000196 & -4.200872\end{array}$




$\begin{array}{lrrr}\mathrm{H} & -4.468824 & -0.000864 & -4.789227 \\ \mathrm{H} & -5.982935 & -0.879298 & -4.493765 \\ \mathrm{H} & -5.981628 & 0.880329 & -4.494317\end{array}$

TS:

$\begin{array}{cccc}\mathrm{C} & 0.000000 & 0.000000 & 0.000000 \\ \mathrm{H} & 0.000000 & 0.000000 & 1.091103 \\ \mathrm{H} & 0.994254 & 0.000000 & -0.418436 \\ \mathrm{C} & -0.996341 & 0.732465 & -0.657630 \\ \mathrm{H} & -1.627042 & 1.397883 & -0.063643 \\ \mathrm{C} & -1.393782 & 0.532338 & -1.980425 \\ \mathrm{H} & -2.030913 & 1.287660 & -2.438749 \\ \mathrm{C} & -1.380642 & -0.724420 & -2.614602 \\ \mathrm{H} & -2.010333 & -0.811416 & -3.499487 \\ \mathrm{C} & -0.968891 & -1.911692 & -2.007831 \\ \mathrm{H} & -1.586818 & -2.789765 & -2.215708 \\ \mathrm{C} & 0.020887 & -2.037363 & -1.019271 \\ \mathrm{H} & 0.998427 & -1.657554 & -1.278063 \\ \mathrm{C} & 0.060512 & -3.255702 & -0.124490 \\ \mathrm{H} & 0.595127 & -4.087570 & -0.606883 \\ \mathrm{H} & -0.946359 & -3.603483 & 0.128888 \\ \mathrm{H} & 0.586216 & -3.040214 & 0.811998\end{array}$<smiles>C=C/C=C\C=C\N</smiles>

(1E,3Z)-hexa-1,3,5-trien-1-amine :

$\begin{array}{cccc}\mathrm{C} & 0.000000 & 0.000000 & 0.000000 \\ \mathrm{H} & 0.000000 & 0.000000 & 1.088032 \\ \mathrm{H} & 0.970298 & 0.000000 & -0.487330 \\ \mathrm{C} & -1.146117 & -0.000698 & -0.702892 \\ \mathrm{H} & -1.082887 & -0.001313 & -1.790590 \\ \mathrm{C} & -2.470290 & -0.001797 & -0.111919 \\ \mathrm{H} & -2.500667 & -0.005799 & 0.976959 \\ \mathrm{C} & -3.655957 & -0.000270 & -0.775323 \\ \mathrm{H} & -4.561630 & -0.006068 & -0.167486 \\ \mathrm{C} & -3.876232 & 0.011946 & -2.201661 \\ \mathrm{H} & -3.014729 & 0.015715 & -2.868165 \\ \mathrm{C} & -5.116302 & 0.012057 & -2.744161 \\ \mathrm{H} & -5.991669 & 0.008239 & -2.096717 \\ \mathrm{~N} & -5.421473 & -0.066126 & -4.093442 \\ \mathrm{H} & -4.663102 & 0.147734 & -4.730523 \\ \mathrm{H} & -6.291945 & 0.364777 & -4.376949\end{array}$


TS:

$\begin{array}{cccc}\mathrm{C} & 0.000000 & 0.000000 & 0.000000 \\ \mathrm{H} & 0.000000 & 0.000000 & 1.081861 \\ \mathrm{H} & 0.996685 & 0.000000 & -0.440630 \\ \mathrm{C} & -1.029673 & -0.715641 & -0.618185 \\ \mathrm{H} & -0.813073 & -1.332025 & -1.493116 \\ \mathrm{C} & -2.358759 & -0.556296 & -0.238430 \\ \mathrm{H} & -3.099694 & -1.281166 & -0.571073 \\ \mathrm{C} & -2.854328 & 0.654857 & 0.284326 \\ \mathrm{H} & -3.938281 & 0.716235 & 0.379768 \\ \mathrm{C} & -2.180832 & 1.869687 & 0.380354 \\ \mathrm{H} & -2.795066 & 2.759499 & 0.221567 \\ \mathrm{C} & -0.781029 & 2.122850 & 0.407294 \\ \mathrm{H} & -0.202415 & 1.885499 & 1.285785 \\ \mathrm{~N} & -0.331124 & 3.294862 & -0.202584 \\ \mathrm{H} & 0.660946 & 3.475598 & -0.122481 \\ \mathrm{H} & -0.661667 & 3.443561 & -1.147830\end{array}$

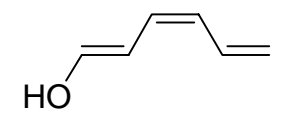

(1E,3Z)-hexa-1,3,5-trien-1-ol :

$\begin{array}{cccc}\mathrm{C} & 0.000000 & 0.000000 & 0.000000 \\ \mathrm{H} & 0.000000 & 0.000000 & 1.087915 \\ \mathrm{H} & 0.970143 & 0.000000 & -0.487443 \\ \mathrm{C} & -1.145580 & 0.000020 & -0.702370 \\ \mathrm{H} & -1.084182 & 0.000036 & -1.790005 \\ \mathrm{C} & -2.467746 & 0.000022 & -0.104472 \\ \mathrm{H} & -2.491354 & -0.000028 & 0.984460 \\ \mathrm{C} & -3.656540 & 0.000060 & -0.757909 \\ \mathrm{H} & -4.559666 & 0.000056 & -0.147849 \\ \mathrm{C} & -3.877744 & 0.000088 & -2.187792 \\ \mathrm{H} & -3.016266 & 0.000344 & -2.855470 \\ \mathrm{C} & -5.112612 & -0.000132 & -2.726754 \\ \mathrm{H} & -6.014955 & -0.000264 & -2.121456 \\ \mathrm{O} & -5.422562 & 0.000005 & -4.048645 \\ \mathrm{H} & -4.600516 & 0.000358 & -4.566996\end{array}$

TS:

$\begin{array}{cccc}\mathrm{C} & 0.000000 & 0.000000 & 0.000000 \\ \mathrm{H} & 0.000000 & 0.000000 & 1.081496 \\ \mathrm{H} & 0.993234 & 0.000000 & -0.445419 \\ \mathrm{C} & -1.046420 & -0.680592 & -0.620738 \\ \mathrm{H} & -0.851838 & -1.279854 & -1.511987\end{array}$




$\begin{array}{lrrr}\mathrm{C} & -2.372308 & -0.498914 & -0.227171 \\ \mathrm{H} & -3.128821 & -1.209261 & -0.555396 \\ \mathrm{C} & -2.838969 & 0.717024 & 0.298167 \\ \mathrm{H} & -3.920217 & 0.807082 & 0.396265 \\ \mathrm{C} & -2.127357 & 1.915605 & 0.393900 \\ \mathrm{H} & -2.712993 & 2.824130 & 0.222438 \\ \mathrm{C} & -0.730760 & 2.122744 & 0.447718 \\ \mathrm{H} & -0.139566 & 1.887549 & 1.316997 \\ \mathrm{O} & -0.208384 & 3.248820 & -0.133675 \\ \mathrm{H} & -0.766581 & 3.486870 & -0.891844\end{array}$

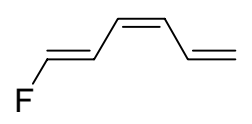

(1E,3Z)-1-fluorohexa-1, 3, 5-triene :

$\begin{array}{cccc}\text { C } & 0.000000 & 0.000000 & 0.000000 \\ \mathrm{H} & 0.000000 & 0.000000 & 1.087876 \\ \mathrm{H} & 0.970013 & 0.000000 & -0.487526 \\ \mathrm{C} & -1.144830 & 0.000000 & -0.702432 \\ \mathrm{H} & -1.088058 & 0.000000 & -1.789704 \\ \mathrm{C} & -2.463291 & 0.000000 & -0.094669 \\ \mathrm{H} & -2.477796 & 0.000000 & 0.994499 \\ \mathrm{C} & -3.656804 & 0.000000 & -0.736531 \\ \mathrm{H} & -4.556840 & 0.000000 & -0.122684 \\ \mathrm{C} & -3.873371 & 0.000000 & -2.169528 \\ \mathrm{H} & -3.025661 & 0.000000 & -2.849133 \\ \mathrm{C} & -5.099694 & 0.000000 & -2.703869 \\ \mathrm{H} & -6.033414 & 0.000000 & -2.148143 \\ \mathrm{~F} & -5.295321 & 0.000000 & -4.034762\end{array}$

TS:

$\begin{array}{cccc}\mathrm{C} & 0.000000 & 0.000000 & 0.000000 \\ \mathrm{H} & 0.000000 & 0.000000 & 1.081085 \\ \mathrm{H} & 0.993084 & 0.000000 & -0.445393 \\ \mathrm{C} & -1.046660 & -0.670784 & -0.626387 \\ \mathrm{H} & -0.851485 & -1.262298 & -1.521960 \\ \mathrm{C} & -2.377476 & -0.483412 & -0.245442 \\ \mathrm{H} & -3.128452 & -1.197182 & -0.579160 \\ \mathrm{C} & -2.857231 & 0.735387 & 0.254912 \\ \mathrm{H} & -3.939966 & 0.829602 & 0.320893 \\ \mathrm{C} & -2.138032 & 1.933603 & 0.332968 \\ \mathrm{H} & -2.687210 & 2.843849 & 0.085440 \\ \mathrm{C} & -0.756465 & 2.105655 & 0.466676 \\ \mathrm{H} & -0.197308 & 1.872024 & 1.358265\end{array}$




$$
\text { F } \quad-0.265960 \quad 3.252763 \quad-0.079603
$$

$\mathrm{OHC}$

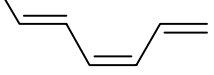

(2E,4Z)-hepta-2,4,6-trienal :

$\begin{array}{cccc}\mathrm{C} & 0.000000 & 0.000000 & 0.000000 \\ \mathrm{H} & 0.000000 & 0.000000 & 1.087706 \\ \mathrm{H} & 0.969674 & 0.000000 & -0.487950 \\ \mathrm{C} & -1.145391 & 0.000000 & -0.702501 \\ \mathrm{H} & -1.092905 & 0.000000 & -1.789259 \\ \mathrm{C} & -2.455045 & 0.000000 & -0.079847 \\ \mathrm{H} & -2.451907 & 0.000000 & 1.009628 \\ \mathrm{C} & -3.663789 & 0.000000 & -0.700844 \\ \mathrm{H} & -4.554727 & 0.000000 & -0.075356 \\ \mathrm{C} & -3.894822 & 0.000000 & -2.124923 \\ \mathrm{H} & -3.027180 & 0.000000 & -2.784574 \\ \mathrm{C} & -5.120118 & 0.000000 & -2.700981 \\ \mathrm{H} & -6.032129 & 0.000000 & -2.107288 \\ \mathrm{C} & -5.277460 & 0.000000 & -4.155725 \\ \mathrm{H} & -4.319732 & -0.000018 & -4.726261 \\ \mathrm{O} & -6.349515 & -0.000018 & -4.735964\end{array}$

TS:

$\begin{array}{llll}\text { C } & 0.000000 & 0.000000 & 0.000000\end{array}$

$\begin{array}{llll}\mathrm{H} & 0.000000 & 0.000000 & 1.089826\end{array}$

$\begin{array}{llll}\mathrm{H} & 0.992000 & 0.000000 & -0.422224\end{array}$

$\begin{array}{llll}\text { C } & -1.012928 & 0.700962 & -0.670135\end{array}$

$\begin{array}{llll}\mathrm{H} & -1.643527 & 1.373398 & -0.085251\end{array}$

$\begin{array}{llll}\text { C } & -1.421318 & 0.467992 & -1.981261\end{array}$

$\begin{array}{llll}\mathrm{H} & -2.081502 & 1.202444 & -2.440260\end{array}$

$\begin{array}{llll}\text { C } & -1.400818 & -0.801382 & -2.601168\end{array}$

$\begin{array}{llll}\mathrm{H} & -2.036604 & -0.909272 & -3.478300\end{array}$

$\begin{array}{llll}\text { C } & -0.981323 & -1.961274 & -1.961747\end{array}$

$\begin{array}{llll}\mathrm{H} & -1.569341 & -2.861188 & -2.156388\end{array}$

$\begin{array}{llll}\text { C } & 0.013310 & -2.032707 & -0.959627\end{array}$

$\begin{array}{llll}\mathrm{H} & 1.014379 & -1.707170 & -1.201435\end{array}$

$\begin{array}{llll}\text { C } & -0.015854 & -3.158528 & -0.002277\end{array}$

$\begin{array}{llll}\text { O } & 0.897439 & -3.448576 & 0.748653\end{array}$

$\begin{array}{llll}\mathrm{H} & -0.972501 & -3.731663 & 0.015568\end{array}$ 


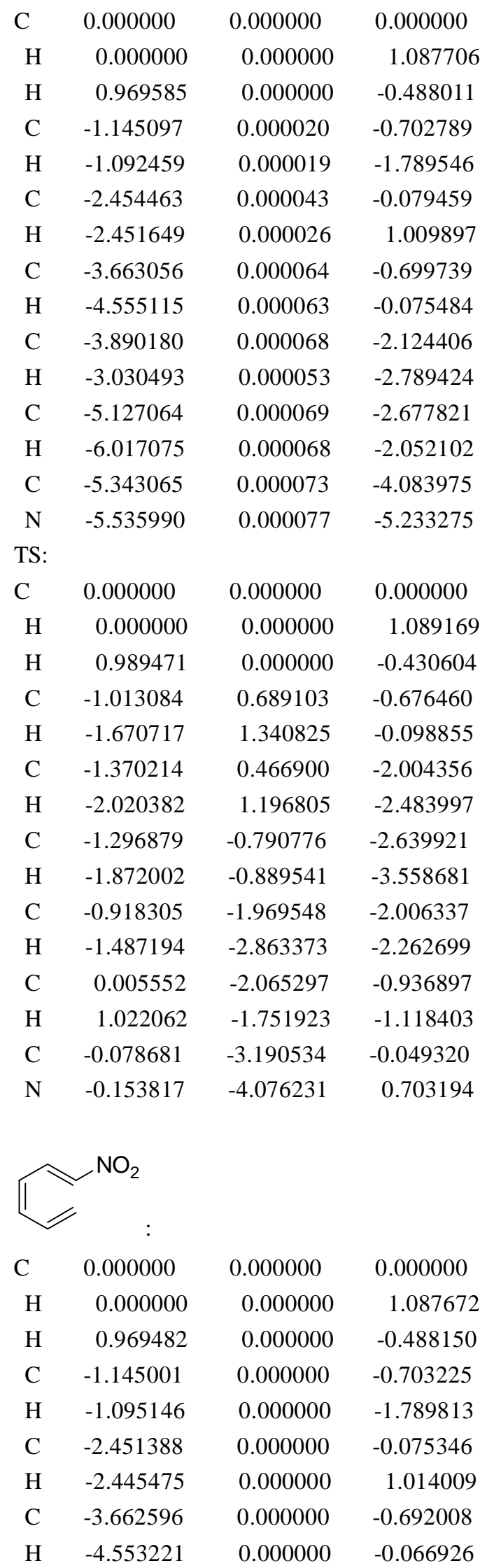




$\begin{array}{lrrr}\mathrm{C} & -3.875751 & 0.000000 & -2.117237 \\ \mathrm{H} & -3.026652 & 0.000000 & -2.793997 \\ \mathrm{C} & -5.097897 & 0.000000 & -2.679361 \\ \mathrm{H} & -6.045564 & 0.000000 & -2.157666 \\ \mathrm{~N} & -5.257296 & 0.000000 & -4.116970 \\ \mathrm{O} & -4.252169 & -0.000075 & -4.834477 \\ \mathrm{O} & -6.420651 & -0.000078 & -4.531772\end{array}$

TS:

$\begin{array}{cccc}\mathrm{C} & 0.000000 & 0.000000 & 0.000000 \\ \mathrm{H} & 0.000000 & 0.000000 & 1.088238 \\ \mathrm{H} & 0.985240 & 0.000000 & -0.440925 \\ \mathrm{C} & -1.023385 & 0.641546 & -0.695511 \\ \mathrm{H} & -1.743411 & 1.244046 & -0.140814 \\ \mathrm{C} & -1.288737 & 0.417143 & -2.046901 \\ \mathrm{H} & -1.939808 & 1.117898 & -2.566816 \\ \mathrm{C} & -1.090045 & -0.820446 & -2.687615 \\ \mathrm{H} & -1.557201 & -0.931250 & -3.663944 \\ \mathrm{C} & -0.725627 & -1.996474 & -2.033319 \\ \mathrm{H} & -1.225863 & -2.910528 & -2.349197 \\ \mathrm{C} & 0.074621 & -2.085916 & -0.882950 \\ \mathrm{H} & 1.123618 & -1.847885 & -0.875550 \\ \mathrm{~N} & -0.128920 & -3.267697 & -0.034718 \\ \mathrm{O} & 0.773743 & -3.529846 & 0.764950 \\ \mathrm{O} & -1.184571 & -3.895929 & -0.140727\end{array}$

\section{2-substituted hexatriene}

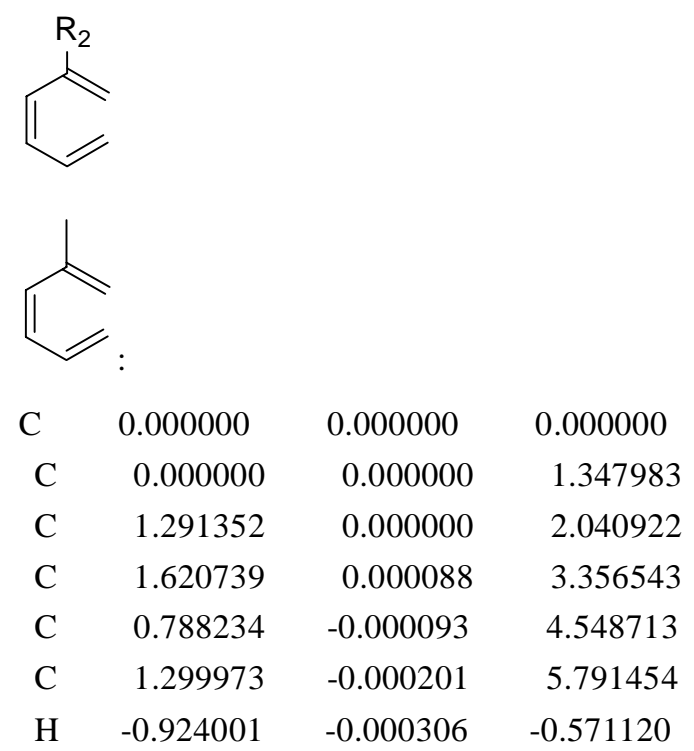




$\begin{array}{rrrr}\mathrm{H} & 0.924707 & 0.000000 & -0.571245 \\ \mathrm{H} & 2.136965 & 0.000316 & 1.353834 \\ \mathrm{H} & 2.689925 & 0.000266 & 3.571702 \\ \mathrm{H} & -0.290432 & -0.000126 & 4.435341 \\ \mathrm{H} & 2.373922 & -0.000077 & 5.965895 \\ \mathrm{H} & 0.663596 & -0.000496 & 6.671188 \\ \mathrm{C} & -1.311412 & 0.000134 & 2.099674 \\ \mathrm{H} & -1.407118 & -0.882873 & 2.742836 \\ \mathrm{H} & -1.407144 & 0.883911 & 2.741747 \\ \mathrm{H} & -2.155150 & -0.000655 & 1.403259\end{array}$

TS:

$\begin{array}{llll}\text { C } & 0.000000 & 0.000000 & 0.000000\end{array}$

$\begin{array}{llll}\mathrm{C} & 0.000000 & 0.000000 & 1.399912\end{array}$

$\begin{array}{llll}\text { C } & 1.203984 & 0.000000 & 2.121767\end{array}$

$\begin{array}{llll}\text { C } & 2.368948 & -0.667130 & 1.709550\end{array}$

$\begin{array}{llll}\text { C } & 2.468842 & -1.461869 & 0.559942\end{array}$

$\begin{array}{llll}\text { C } & 1.792713 & -1.268715 & -0.648180\end{array}$

$\begin{array}{llll}\mathrm{H} & 0.594775 & 0.750554 & -0.495931\end{array}$

$\begin{array}{llll}\mathrm{H} & -0.923269 & -0.243559 & -0.526955\end{array}$

$\begin{array}{llll}\mathrm{H} & 1.160495 & 0.255608 & 3.180341\end{array}$

$\begin{array}{llll}\mathrm{H} & 3.114719 & -0.829149 & 2.487380\end{array}$

$\begin{array}{llll}\mathrm{H} & 2.990296 & -2.411992 & 0.700503\end{array}$

$\mathrm{H} \quad 1.655023 \quad-2.137642 \quad-1.294523$

$\mathrm{H} \quad 1.932252 \quad-0.356933 \quad-1.205149$

$\begin{array}{llll}\text { C } & -1.298200 & -0.203879 & 2.160137\end{array}$

$\begin{array}{llll}\mathrm{H} & -1.636921 & -1.246217 & 2.074054\end{array}$

$\begin{array}{llll}\mathrm{H} & -1.168267 & 0.002019 & 3.227824\end{array}$

$\begin{array}{llll}\mathrm{H} & -2.107051 & 0.433059 & 1.785327\end{array}$

$\begin{array}{cccc} \\ \mathrm{C} & 0.000000 & 0.000000 & 0.000000 \\ \mathrm{H} & 0.000000 & 0.000000 & 1.086619 \\ \mathrm{H} & 0.964465 & 0.000000 & -0.494328 \\ \mathrm{C} & -1.145802 & 0.061079 & -0.713223 \\ \mathrm{C} & -1.095644 & 0.038113 & -2.180132 \\ \mathrm{H} & -0.146933 & -0.324295 & -2.569158 \\ \mathrm{C} & -2.030139 & 0.391540 & -3.094669 \\ \mathrm{H} & -1.757084 & 0.243025 & -4.139948 \\ \mathrm{C} & -3.342657 & 0.988650 & -2.893268 \\ \mathrm{H} & -3.618030 & 1.269122 & -1.880598\end{array}$




$\begin{array}{cccc}\mathrm{C} & -4.191414 & 1.239255 & -3.904050 \\ \mathrm{H} & -3.944961 & 0.986684 & -4.933504 \\ \mathrm{H} & -5.156825 & 1.707188 & -3.735858 \\ \mathrm{~N} & -2.408599 & 0.185842 & -0.101093 \\ \mathrm{H} & -3.113629 & -0.413043 & -0.519576 \\ \mathrm{H} & -2.374279 & 0.032328 & 0.901736\end{array}$

TS:

$\begin{array}{cccc}\mathrm{C} & 0.000000 & 0.000000 & 0.000000 \\ \mathrm{C} & 0.000000 & 0.000000 & 1.409360 \\ \mathrm{~N} & 1.219666 & 0.000000 & 2.097813 \\ \mathrm{C} & -1.161292 & 0.124876 & 2.208477 \\ \mathrm{C} & -2.241426 & 0.955563 & 1.889705 \\ \mathrm{C} & -2.383236 & 1.649986 & 0.681696 \\ \mathrm{C} & -1.873209 & 1.188325 & -0.532121 \\ \mathrm{H} & -1.053077 & -0.149017 & 3.257253 \\ \mathrm{H} & -2.875894 & 1.250349 & 2.725801 \\ \mathrm{H} & -2.764950 & 2.670308 & 0.743203 \\ \mathrm{H} & -1.747175 & 1.894313 & -1.353521 \\ \mathrm{H} & -2.149044 & 0.198313 & -0.860300 \\ \mathrm{H} & 0.947300 & 0.236267 & -0.492234 \\ \mathrm{H} & -0.554997 & -0.772316 & -0.509499 \\ \mathrm{H} & 2.047705 & -0.048749 & 1.519066 \\ \mathrm{H} & 1.315361 & 0.667927 & 2.853732\end{array}$

$\begin{array}{cccc}\text { C } & & \\ \mathrm{C} & 0.000000 & 0.000000 & 0.000000 \\ \mathrm{C} & 0.000000 & 0.000000 & 1.347938 \\ \mathrm{C} & 1.238164 & 0.000000 & 2.119887 \\ \mathrm{C} & 1.431365 & 0.000018 & 3.459943 \\ \mathrm{C} & 0.454261 & 0.000043 & 4.536746 \\ \mathrm{C} & 0.811578 & 0.000039 & 5.831859 \\ \mathrm{H} & -0.921686 & 0.000000 & -0.576045 \\ \mathrm{H} & 0.930162 & 0.000000 & -0.554488 \\ \mathrm{H} & 2.126055 & -0.000017 & 1.492644 \\ \mathrm{H} & 2.470598 & 0.000013 & 3.790426 \\ \mathrm{H} & -0.595125 & 0.000065 & 4.264418 \\ \mathrm{H} & 1.856050 & 0.000017 & 6.137759 \\ \mathrm{H} & 0.072440 & 0.000058 & 6.627489 \\ \mathrm{O} & -1.155515 & 0.000022 & 2.087916 \\ \mathrm{H} & -1.910113 & 0.000020 & 1.478052\end{array}$


TS:

$\begin{array}{cccc}\mathrm{C} & 0.000000 & 0.000000 & 0.000000 \\ \mathrm{C} & 0.000000 & 0.000000 & 1.400714 \\ \mathrm{C} & 1.137704 & 0.000000 & 2.230099 \\ \mathrm{C} & 2.323342 & -0.683975 & 1.927279 \\ \mathrm{C} & 2.560271 & -1.364449 & 0.728617 \\ \mathrm{C} & 2.006286 & -0.994760 & -0.498548 \\ \mathrm{H} & 0.488959 & 0.812318 & -0.511986 \\ \mathrm{H} & -0.923387 & -0.331148 & -0.475023 \\ \mathrm{H} & 0.979095 & 0.250902 & 3.279597 \\ \mathrm{H} & 2.977439 & -0.894720 & 2.772908 \\ \mathrm{H} & 3.069032 & -2.326338 & 0.808985 \\ \mathrm{H} & 2.165480 & 0.011519 & -0.851953 \\ \mathrm{H} & 1.968779 & -1.732053 & -1.300656 \\ \mathrm{O} & -1.234259 & -0.172048 & 1.992455 \\ \mathrm{H} & -1.108390 & -0.553110 & 2.875910\end{array}$<smiles>C=C/C=C\C(=C)F</smiles>

$\begin{array}{cccc}\text { C } & 0.000000 & 0.000000 & 0.000000 \\ \mathrm{C} & 0.000000 & 0.000000 & 1.338794 \\ \mathrm{C} & 1.176135 & 0.000000 & 2.188111 \\ \mathrm{C} & 1.260608 & 0.000110 & 3.539803 \\ \mathrm{C} & 0.192812 & 0.000259 & 4.526171 \\ \mathrm{C} & 0.434599 & 0.000356 & 5.847456 \\ \mathrm{H} & -0.929251 & 0.000049 & -0.557049 \\ \mathrm{H} & 0.933967 & -0.000016 & -0.548635 \\ \mathrm{H} & 2.105995 & -0.000088 & 1.624413 \\ \mathrm{H} & 2.267787 & 0.000083 & 3.956558 \\ \mathrm{H} & -0.829969 & 0.000291 & 4.166856 \\ \mathrm{H} & -0.372077 & 0.000467 & 6.574249 \\ \mathrm{H} & 1.447930 & 0.000328 & 6.244005 \\ \mathrm{~F} & -1.199549 & 0.000149 & 1.978809\end{array}$

TS:

$\begin{array}{cccc}\mathrm{C} & 0.000000 & 0.000000 & 0.000000 \\ \mathrm{C} & 0.000000 & 0.000000 & 1.395730 \\ \mathrm{C} & 1.083230 & 0.000000 & 2.275827 \\ \mathrm{C} & 2.315561 & -0.607131 & 1.988853 \\ \mathrm{C} & 2.617923 & -1.227846 & 0.775276 \\ \mathrm{C} & 2.080563 & -0.837327 & -0.453981\end{array}$




$\begin{array}{lrcc}\mathrm{H} & 0.440730 & 0.829625 & -0.526233 \\ \mathrm{H} & -0.904664 & -0.385872 & -0.470317 \\ \mathrm{H} & 0.848643 & 0.179315 & 3.322286 \\ \mathrm{H} & 2.946338 & -0.825376 & 2.849232 \\ \mathrm{H} & 3.176065 & -2.163259 & 0.834128 \\ \mathrm{H} & 2.201833 & 0.191181 & -0.755572 \\ \mathrm{H} & 2.109646 & -1.538778 & -1.287320 \\ \mathrm{~F} & -1.207737 & -0.228220 & 1.994106\end{array}$

$\begin{array}{rrrr} & & \\ \mathrm{C} & & & \\ \mathrm{C} & 0.000000 & 0.000000 & 0.000000 \\ \mathrm{C} & 0.000000 & 0.000000 & 1.348843 \\ \mathrm{C} & 2.168055 & 0.000000 & 2.236184 \\ \mathrm{C} & 2.7881390 & -0.585353 & 2.043615 \\ \mathrm{C} & 4.045695 & -1.438490 & 0.942078 \\ \mathrm{H} & 0.910544 & 0.025370 & -0.588227 \\ \mathrm{H} & -0.937722 & 0.006056 & -0.551535 \\ \mathrm{H} & 0.997901 & 0.510038 & 3.181157 \\ \mathrm{H} & 3.118068 & -0.441616 & 2.824572 \\ \mathrm{H} & 2.026658 & -1.750056 & 0.230500 \\ \mathrm{H} & 4.839877 & -1.596664 & 1.473148 \\ \mathrm{H} & 4.322185 & -2.541290 & -0.030781 \\ \mathrm{C} & -1.333306 & 0.101349 & 2.010713 \\ \mathrm{O} & -1.503559 & 0.225790 & 3.206484 \\ \mathrm{H} & -2.196795 & 0.072344 & 1.309626\end{array}$

TS:

$\begin{array}{cccc}\text { C } & 0.000000 & 0.000000 & 0.000000 \\ \text { C } & 0.000000 & 0.000000 & 1.401095 \\ \text { C } & 1.163922 & 0.000000 & 2.195843 \\ \text { C } & 2.333098 & -0.678319 & 1.846779 \\ \text { C } & 2.474570 & -1.427603 & 0.665865 \\ \text { C } & 1.908388 & -1.120657 & -0.569701 \\ \text { H } & 0.528384 & 0.783893 & -0.517669 \\ \text { H } & -0.907158 & -0.323417 & -0.513786 \\ \text { H } & 1.033536 & 0.259699 & 3.243821 \\ \mathrm{H} & 3.022739 & -0.890541 & 2.662347 \\ \mathrm{H} & 2.938898 & -2.408701 & 0.787239 \\ \mathrm{H} & 1.826364 & -1.913871 & -1.312270 \\ \mathrm{H} & 2.089497 & -0.148621 & -0.999474\end{array}$




$\begin{array}{lrrr}\mathrm{C} & -1.299513 & -0.165058 & 2.093205 \\ \mathrm{H} & -2.132257 & -0.474645 & 1.420854 \\ \mathrm{O} & -1.499486 & 0.038519 & 3.277138\end{array}$

$\begin{array}{cccc} & & \\ \end{array}$

TS:

$\begin{array}{cccc}\mathrm{C} & 0.000000 & 0.000000 & 0.000000 \\ \mathrm{C} & 0.000000 & 0.000000 & 1.402774 \\ \mathrm{C} & 1.181177 & 0.000000 & 2.180080 \\ \mathrm{C} & 2.343704 & -0.675383 & 1.801907 \\ \mathrm{C} & 2.454448 & -1.464182 & 0.644168 \\ \mathrm{C} & 1.850727 & -1.218219 & -0.586726 \\ \mathrm{H} & -0.908008 & -0.289101 & -0.524436 \\ \mathrm{H} & 0.574398 & 0.767367 & -0.492894 \\ \mathrm{H} & 1.096751 & 0.276564 & 3.228338 \\ \mathrm{H} & 3.065334 & -0.850288 & 2.598038 \\ \mathrm{H} & 2.928541 & -2.436765 & 0.792933 \\ \mathrm{H} & 1.730983 & -2.053646 & -1.276318 \\ \mathrm{H} & 2.019443 & -0.276600 & -1.083064 \\ \mathrm{C} & -1.240002 & -0.191611 & 2.099349 \\ \mathrm{~N} & -2.250013 & -0.326458 & 2.664263\end{array}$




$\begin{array}{crrr}\mathrm{C} & 0.000000 & 0.000000 & 0.000000 \\ \mathrm{C} & 0.000000 & 0.000000 & 1.338017 \\ \mathrm{C} & 1.135161 & 0.000000 & 2.258335 \\ \mathrm{C} & 2.291888 & -0.682087 & 2.103036 \\ \mathrm{C} & 2.631972 & -1.646839 & 1.070002 \\ \mathrm{C} & 3.852088 & -2.193778 & 0.950298 \\ \mathrm{H} & 0.933998 & 0.017118 & -0.548811 \\ \mathrm{H} & -0.931583 & 0.003675 & -0.552061 \\ \mathrm{H} & 1.007426 & 0.604414 & 3.149847 \\ \mathrm{H} & 3.058153 & -0.521649 & 2.860626 \\ \mathrm{H} & 1.844302 & -1.950818 & 0.384108 \\ \mathrm{H} & 4.073844 & -2.932058 & 0.185852 \\ \mathrm{H} & 4.668905 & -1.916715 & 1.613067 \\ \mathrm{~N} & -1.341271 & 0.068500 & 2.003498 \\ \mathrm{O} & -1.348008 & 0.119899 & 3.232293 \\ \mathrm{O} & -2.346555 & 0.071497 & 1.298441\end{array}$

TS:

$\begin{array}{cccc}\mathrm{C} & 0.000000 & 0.000000 & 0.000000 \\ \mathrm{C} & 0.000000 & 0.000000 & 1.391746 \\ \mathrm{C} & 1.136209 & 0.000000 & 2.216221 \\ \mathrm{C} & 2.318875 & -0.652955 & 1.861988 \\ \mathrm{C} & 2.474239 & -1.419412 & 0.694246 \\ \mathrm{C} & 1.900771 & -1.161719 & -0.548220 \\ \mathrm{H} & 0.551732 & 0.784541 & -0.491145 \\ \mathrm{H} & -0.897730 & -0.331208 & -0.511999 \\ \mathrm{H} & 1.000450 & 0.261496 & 3.259706 \\ \mathrm{H} & 3.022625 & -0.826474 & 2.673968 \\ \mathrm{H} & 2.963299 & -2.385089 & 0.839113 \\ \mathrm{H} & 2.060834 & -0.204687 & -1.017415 \\ \mathrm{H} & 1.817003 & -1.983966 & -1.258130 \\ \mathrm{~N} & -1.291491 & -0.242062 & 2.073627 \\ \mathrm{O} & -2.244453 & -0.616208 & 1.389174 \\ \mathrm{O} & -1.347665 & -0.035416 & 3.287039\end{array}$

\section{3-substituted hexatriene}

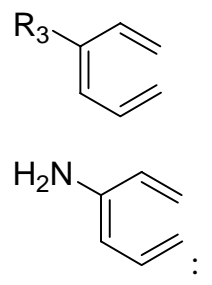




\begin{tabular}{rrrr}
$\mathrm{C}$ & \multicolumn{1}{c}{0.000000} & \multicolumn{1}{c}{0.000000} & 0.000000 \\
$\mathrm{C}$ & 0.000000 & 0.000000 & 1.341401 \\
$\mathrm{C}$ & 1.198986 & 0.000000 & 2.185408 \\
$\mathrm{C}$ & 1.177195 & -0.281490 & 3.520864 \\
$\mathrm{C}$ & 0.043232 & -0.721142 & 4.303083 \\
$\mathrm{C}$ & 0.085800 & -0.979840 & 5.622812 \\
$\mathrm{H}$ & -0.929806 & 0.027239 & -0.559940 \\
$\mathrm{H}$ & 0.916633 & -0.059469 & -0.579613 \\
$\mathrm{H}$ & -0.951266 & 0.023342 & 1.864483 \\
$\mathrm{H}$ & 2.119933 & -0.199490 & 4.064180 \\
$\mathrm{H}$ & -0.905624 & -0.856756 & 3.786853 \\
$\mathrm{H}$ & -0.794972 & -1.304423 & 6.167773 \\
$\mathrm{H}$ & 1.005318 & -0.878348 & 6.195154 \\
$\mathrm{~N}$ & 2.404163 & 0.260272 & 1.518657 \\
$\mathrm{H}$ & 2.346769 & 1.017012 & 0.845058 \\
$\mathrm{H}$ & 3.198729 & 0.383399 & 2.136132
\end{tabular}

TS:

$\begin{array}{cccc}\mathrm{C} & 0.000000 & 0.000000 & 0.000000 \\ \mathrm{C} & 0.000000 & 0.000000 & 1.396194 \\ \mathrm{C} & 1.158874 & 0.000000 & 2.196096 \\ \mathrm{C} & 2.317667 & -0.716042 & 1.814408 \\ \mathrm{C} & 2.450241 & -1.448358 & 0.634886 \\ \mathrm{C} & 1.860776 & -1.114262 & -0.591545 \\ \mathrm{H} & 0.508025 & 0.792608 & -0.527925 \\ \mathrm{H} & -0.910262 & -0.325204 & -0.504816 \\ \mathrm{H} & -0.927353 & -0.209694 & 1.930605 \\ \mathrm{H} & 2.988080 & -0.971006 & 2.637760 \\ \mathrm{H} & 2.939024 & -2.421387 & 0.729156 \\ \mathrm{H} & 2.081069 & -0.149019 & -1.019923 \\ \mathrm{H} & 1.770231 & -1.899300 & -1.343307 \\ \mathrm{~N} & 1.015854 & 0.359270 & 3.548495 \\ \mathrm{H} & 0.485988 & 1.207523 & 3.699520 \\ \mathrm{H} & 1.880558 & 0.370579 & 4.075608\end{array}$

$\begin{array}{cccc} & & & \\ \mathrm{C} & 0.000000 & 0.000000 & 0.000000 \\ \mathrm{C} & 0.000000 & 0.000000 & 1.340291 \\ \mathrm{C} & 1.205623 & 0.000000 & 2.161879 \\ \mathrm{C} & 1.237098 & -0.000984 & 3.521630 \\ \mathrm{C} & 0.104821 & -0.002316 & 4.423413 \\ \mathrm{C} & 0.205801 & -0.003245 & 5.763904\end{array}$




$\begin{array}{lrrr}\mathrm{H} & -0.931052 & -0.000081 & -0.558416 \\ \mathrm{H} & 0.923156 & 0.000000 & -0.569441 \\ \mathrm{H} & -0.943680 & 0.000017 & 1.876398 \\ \mathrm{H} & 2.218933 & -0.000742 & 3.999760 \\ \mathrm{H} & -0.891195 & -0.002629 & 3.984762 \\ \mathrm{H} & -0.673348 & -0.004216 & 6.400185 \\ \mathrm{H} & 1.171683 & -0.003046 & 6.264313 \\ \mathrm{O} & 2.356269 & 0.001022 & 1.418462 \\ \mathrm{H} & 3.117391 & 0.000174 & 2.020206\end{array}$

TS:

$\begin{array}{cccc}\mathrm{C} & 0.000000 & 0.000000 & 0.000000 \\ \mathrm{C} & 0.000000 & 0.000000 & 1.396179 \\ \mathrm{C} & 1.157073 & 0.000000 & 2.191027 \\ \mathrm{C} & 2.330300 & -0.693962 & 1.846455 \\ \mathrm{C} & 2.504142 & -1.395130 & 0.653699 \\ \mathrm{C} & 1.939581 & -1.044788 & -0.577230 \\ \mathrm{H} & 0.493906 & 0.799078 & -0.529605 \\ \mathrm{H} & -0.898648 & -0.353389 & -0.505853 \\ \mathrm{H} & -0.926602 & -0.232635 & 1.927438 \\ \mathrm{H} & 2.967008 & -0.934481 & 2.696053 \\ \mathrm{H} & 3.010487 & -2.359254 & 0.738546 \\ \mathrm{H} & 1.888663 & -1.807380 & -1.354506 \\ \mathrm{H} & 2.126591 & -0.056129 & -0.965610 \\ \mathrm{O} & 1.075085 & 0.346694 & 3.521201 \\ \mathrm{H} & 0.236403 & 0.812029 & 3.660384\end{array}$

\begin{tabular}{|c|c|c|c|}
\hline $\mathrm{C}$ & 0.000000 & 0.000000 & 0.000000 \\
\hline $\mathrm{C}$ & 0.000000 & 0.000000 & 1.341034 \\
\hline $\mathrm{C}$ & 1.204692 & 0.000000 & 2.148205 \\
\hline $\mathrm{C}$ & 1.328449 & -0.000512 & 3.492988 \\
\hline $\mathrm{C}$ & 0.244218 & -0.000953 & 4.453990 \\
\hline $\mathrm{C}$ & 0.427732 & -0.001514 & 5.784686 \\
\hline $\mathrm{H}$ & -0.928743 & 0.000016 & -0.561116 \\
\hline $\mathrm{H}$ & 0.924856 & 0.000032 & -0.566886 \\
\hline $\mathrm{H}$ & -0.939962 & -0.000085 & 1.885363 \\
\hline $\mathrm{H}$ & 2.343117 & -0.000886 & 3.884943 \\
\hline $\mathrm{H}$ & -0.774625 & -0.000584 & 4.070768 \\
\hline $\mathrm{H}$ & -0.410482 & -0.001542 & 6.474135 \\
\hline $\mathrm{H}$ & 1.423055 & -0.001786 & 6.222805 \\
\hline $\mathrm{F}$ & 2.351456 & -0.000912 & 1.421344 \\
\hline
\end{tabular}


TS:

$\begin{array}{cccc}\text { C } & 0.000000 & 0.000000 & 0.000000 \\ \mathrm{C} & 0.000000 & 0.000000 & 1.397680 \\ \mathrm{C} & 1.152949 & 0.000000 & 2.179900 \\ \mathrm{C} & 2.353648 & -0.649136 & 1.868634 \\ \mathrm{C} & 2.574522 & -1.305356 & 0.657961 \\ \mathrm{C} & 2.010627 & -0.927667 & -0.564209 \\ \mathrm{H} & 0.465324 & 0.814577 & -0.530458 \\ \mathrm{H} & -0.889434 & -0.380844 & -0.502211 \\ \mathrm{H} & -0.921224 & -0.209016 & 1.942291 \\ \mathrm{H} & 2.971445 & -0.891940 & 2.730463 \\ \mathrm{H} & 3.107384 & -2.255884 & 0.719376 \\ \mathrm{H} & 1.995094 & -1.656275 & -1.374166 \\ \mathrm{H} & 2.161402 & 0.084899 & -0.903599 \\ \mathrm{~F} & 0.983853 & 0.286522 & 3.499430\end{array}$

\begin{tabular}{|c|c|c|c|}
\hline $\mathrm{C}$ & 0.000000 & 0.000000 & 0.000000 \\
\hline $\mathrm{C}$ & 0.000000 & 0.000000 & 1.342068 \\
\hline $\mathrm{C}$ & 1.171417 & 0.000000 & 2.221209 \\
\hline $\mathrm{C}$ & 1.094829 & -0.000735 & 3.585954 \\
\hline $\mathrm{C}$ & -0.088416 & -0.002415 & 4.417892 \\
\hline $\mathrm{C}$ & -0.031089 & -0.003014 & 5.761568 \\
\hline $\mathrm{H}$ & -0.941809 & 0.000674 & -0.542045 \\
\hline $\mathrm{H}$ & 0.915707 & 0.000368 & -0.576852 \\
\hline $\mathrm{H}$ & -0.963103 & 0.000789 & 1.848566 \\
\hline $\mathrm{H}$ & -1.062771 & -0.003708 & 3.935636 \\
\hline $\mathrm{H}$ & 2.038603 & 0.000045 & 4.133416 \\
\hline $\mathrm{H}$ & 0.919373 & -0.002059 & 6.290437 \\
\hline $\mathrm{H}$ & -0.929128 & -0.004746 & 6.371462 \\
\hline $\mathrm{C}$ & 2.557427 & 0.001521 & 1.688920 \\
\hline $\mathrm{O}$ & 2.890747 & 0.001393 & 0.518718 \\
\hline $\mathrm{H}$ & 3.334611 & 0.002894 & 2.486640 \\
\hline
\end{tabular}

TS:

$\begin{array}{cccc}\mathrm{C} & 0.000000 & 0.000000 & 0.000000 \\ \mathrm{C} & 0.000000 & 0.000000 & 1.391394 \\ \mathrm{C} & 1.158196 & 0.000000 & 2.188294 \\ \mathrm{C} & 2.353100 & -0.653233 & 1.817593 \\ \mathrm{C} & 2.495207 & -1.425353 & 0.666332 \\ \mathrm{C} & 1.867032 & -1.219661 & -0.568333\end{array}$




$\begin{array}{lrrr}\text { H } & 0.594339 & 0.745182 & -0.504997 \\ \mathrm{H} & -0.914873 & -0.264636 & -0.527912 \\ \mathrm{H} & -0.947152 & -0.148443 & 1.915311 \\ \mathrm{H} & 3.051408 & -0.807355 & 2.637721 \\ \mathrm{H} & 3.029852 & -2.368295 & 0.803742 \\ \mathrm{H} & 1.756637 & -2.077567 & -1.232712 \\ \mathrm{H} & 2.025642 & -0.295478 & -1.099569 \\ \mathrm{C} & 1.005681 & 0.366889 & 3.618044 \\ \mathrm{O} & 1.910596 & 0.420953 & 4.430036 \\ \mathrm{H} & -0.036353 & 0.619870 & 3.916859\end{array}$

$\begin{array}{crrr} & & & \\ \mathrm{C} & 0.000000 & 0.000000 & 0.000000 \\ \mathrm{C} & 0.000000 & 0.000000 & 1.340427 \\ \mathrm{C} & 1.201583 & 0.000000 & 2.179755 \\ \mathrm{C} & 1.218650 & -0.000019 & 3.545405 \\ \mathrm{C} & 0.074111 & -0.000023 & 4.430388 \\ \mathrm{C} & 0.194240 & -0.000043 & 5.769543 \\ \mathrm{H} & -0.931552 & 0.000000 & -0.556794 \\ \mathrm{H} & 0.920689 & 0.000000 & -0.576831 \\ \mathrm{H} & -0.949514 & 0.000000 & 1.868009 \\ \mathrm{H} & 2.191735 & -0.000051 & 4.032117 \\ \mathrm{H} & -0.921362 & -0.000010 & 3.993364 \\ \mathrm{H} & -0.675168 & -0.000030 & 6.419328 \\ \mathrm{H} & 1.168012 & -0.000057 & 6.253584 \\ \mathrm{C} & 2.470635 & 0.000000 & 1.504505 \\ \mathrm{~N} & 3.489414 & -0.000002 & 0.941923\end{array}$

TS:

$\begin{array}{cccc}\text { C } & 0.000000 & 0.000000 & 0.000000 \\ \text { C } & 0.000000 & 0.000000 & 1.391597 \\ \text { C } & 1.171355 & 0.000000 & 2.174745 \\ \text { C } & 2.359685 & -0.669204 & 1.795379 \\ \text { C } & 2.483886 & -1.433714 & 0.635415 \\ \text { C } & 1.848006 & -1.206292 & -0.589862 \\ \text { H } & 0.577482 & 0.757641 & -0.505819 \\ \text { H } & -0.915163 & -0.276827 & -0.520642 \\ \text { H } & -0.938102 & -0.152700 & 1.924928 \\ \text { H } & 3.072147 & -0.846414 & 2.597733 \\ \text { H } & 3.010596 & -2.381820 & 0.763629 \\ \text { H } & 2.013758 & -0.276010 & -1.107882 \\ \text { H } & 1.727974 & -2.053812 & -1.265340\end{array}$




$\begin{array}{llll}\mathrm{C} & 1.053147 & 0.368074 & 3.559806 \\ \mathrm{~N} & 0.959245 & 0.689204 & 4.674667\end{array}$

$\begin{array}{lrrr} & & & \\ \mathrm{O} & & & \\ \mathrm{C} & 0.000000 & 0.000000 & 0.000000 \\ \mathrm{C} & 0.000000 & 0.000000 & 1.341928 \\ \mathrm{C} & 1.096576 & 0.000000 & 2.303735 \\ \mathrm{C} & 0.953626 & 0.007773 & 3.655563 \\ \mathrm{C} & -0.282131 & -0.003053 & 4.405577 \\ \mathrm{C} & -0.297991 & 0.000197 & 5.750410 \\ \mathrm{H} & -0.950699 & 0.028831 & -0.524731 \\ \mathrm{H} & 0.901442 & -0.029400 & -0.594595 \\ \mathrm{H} & -0.970427 & 0.026348 & 1.830940 \\ \mathrm{H} & 1.870976 & 0.022663 & 4.235519 \\ \mathrm{H} & -1.227717 & -0.014483 & 3.869919 \\ \mathrm{H} & 0.621736 & 0.011061 & 6.330150 \\ \mathrm{H} & -1.229033 & -0.006686 & 6.308470 \\ \mathrm{~N} & 2.503787 & -0.013916 & 1.833657 \\ \mathrm{O} & 2.713456 & -0.302413 & 0.655420 \\ \mathrm{O} & 3.395978 & 0.252722 & 2.640552\end{array}$

TS:

$\begin{array}{cccc}\mathrm{C} & 0.000000 & 0.000000 & 0.000000 \\ \mathrm{C} & 0.000000 & 0.000000 & 1.391905 \\ \mathrm{C} & 1.175088 & 0.000000 & 2.152162 \\ \mathrm{C} & 2.354494 & -0.683309 & 1.816000 \\ \mathrm{C} & 2.493812 & -1.428512 & 0.647741 \\ \mathrm{C} & 1.878194 & -1.167124 & -0.581811 \\ \mathrm{H} & 0.563856 & 0.764069 & -0.511091 \\ \mathrm{H} & -0.912184 & -0.293222 & -0.516582 \\ \mathrm{H} & -0.928265 & -0.151354 & 1.937015 \\ \mathrm{H} & 3.035235 & -0.860836 & 2.642185 \\ \mathrm{H} & 3.015674 & -2.380257 & 0.763386 \\ \mathrm{H} & 1.764970 & -1.993957 & -1.283167 \\ \mathrm{H} & 2.054308 & -0.221521 & -1.068147 \\ \mathrm{~N} & 1.033932 & 0.369769 & 3.580997 \\ \mathrm{O} & 2.038216 & 0.343579 & 4.292938 \\ \mathrm{O} & -0.082569 & 0.714512 & 3.971078\end{array}$

TABLE 5S

\section{1, 2-disubstituted hexatriene}


1

$\sqrt{11}$

$\begin{array}{llll}\text { C } & 0.000000 & 0.000000 & 0.000000\end{array}$

$\begin{array}{llll}\mathrm{H} & 0.000000 & 0.000000 & 1.088072\end{array}$

$\begin{array}{llll}\mathrm{H} & 0.970485 & 0.000000 & -0.486979\end{array}$

$\begin{array}{llll}\text { C } & -1.144668 & 0.000020 & -0.704783\end{array}$

$\begin{array}{llll}\mathrm{H} & -1.080667 & 0.000036 & -1.787470\end{array}$

$\begin{array}{llll}\text { C } & -2.456189 & 0.000085 & -0.076548\end{array}$

$\begin{array}{llll}\mathrm{H} & -2.413691 & 0.000151 & 1.013347\end{array}$

$\begin{array}{llll}\text { C } & -3.705651 & 0.000150 & -0.606321\end{array}$

$\begin{array}{llll}\mathrm{H} & -4.509636 & 0.000293 & 0.129595\end{array}$

$\begin{array}{llll}\text { C } & -4.203283 & -0.000029 & -1.985297\end{array}$

$\begin{array}{llll}\text { C } & -5.547967 & -0.000078 & -2.149665\end{array}$

$\begin{array}{llll}\mathrm{H} & -6.153427 & 0.000036 & -1.242263\end{array}$

$\begin{array}{llll}\text { C } & -3.237981 & -0.000126 & -3.147472\end{array}$

$\begin{array}{llll}\text { C } & -6.349115 & -0.000149 & -3.416711\end{array}$

$\begin{array}{llll}\mathrm{H} & -7.007659 & 0.878961 & -3.457520\end{array}$

H $\quad-7.007752 \quad-0.879181 \quad-3.457444$

H $\quad-5.739589 \quad-0.000196 \quad-4.323475$

$\begin{array}{llll}\mathrm{H} & -3.752449 & -0.000469 & -4.110257\end{array}$

$\mathrm{H} \quad-2.588352 \quad-0.883095 \quad-3.121311$

$\begin{array}{llll}\mathrm{H} & -2.588631 & 0.883061 & -3.121760\end{array}$

TS:

$\begin{array}{cccc}\mathrm{H} & 0.000000 & 0.000000 & 0.000000 \\ \mathrm{H} & 0.000000 & 0.000000 & 1.802165 \\ \mathrm{C} & 0.604983 & 0.000000 & 0.909712 \\ \mathrm{C} & 1.810180 & 0.712509 & 0.880009 \\ \mathrm{H} & 1.975433 & 1.393810 & 0.041257 \\ \mathrm{C} & 2.931593 & 0.478840 & 1.683200 \\ \mathrm{H} & 3.713882 & 1.237246 & 1.675393 \\ \mathrm{C} & 3.321594 & -0.786628 & 2.155656 \\ \mathrm{H} & 4.372564 & -0.877938 & 2.430187 \\ \mathrm{C} & 2.603889 & -1.971982 & 1.946089 \\ \mathrm{C} & 1.195914 & -1.964643 & 1.907455 \\ \mathrm{H} & 0.736083 & -1.446049 & 2.736574 \\ \mathrm{C} & 3.378805 & -3.227308 & 1.595536 \\ \mathrm{C} & 0.396542 & -3.139400 & 1.397160 \\ \mathrm{H} & -0.660361 & -2.875178 & 1.287714 \\ \mathrm{H} & 0.446499 & -3.991905 & 2.092286 \\ \mathrm{H} & 0.757121 & -3.490936 & 0.424722 \\ \mathrm{H} & 2.977241 & -4.122184 & 2.086861\end{array}$




$\begin{array}{cccc}\mathrm{H} & 3.356771 & -3.419804 & 0.513299 \\ \mathrm{H} & 4.431564 & -3.125144 & 1.878156 \\ & & & \\ & & & \\ & & & \\ \mathrm{C} & 0.000000 & 0.000000 & 0.000000 \\ \mathrm{H} & 0.000000 & 0.000000 & 1.087893 \\ \mathrm{H} & 0.970001 & 0.000000 & -0.487711 \\ \mathrm{C} & -1.144676 & -0.004228 & -0.703516 \\ \mathrm{H} & -1.082147 & -0.026699 & -1.789175 \\ \mathrm{C} & -2.464914 & -0.047571 & -0.091786 \\ \mathrm{H} & -2.451405 & -0.190788 & 0.988791 \\ \mathrm{C} & -3.690025 & 0.007333 & -0.670427 \\ \mathrm{H} & -4.538505 & -0.151473 & -0.007965 \\ \mathrm{C} & -4.084200 & 0.250781 & -2.063007 \\ \mathrm{C} & -5.288468 & -0.194792 & -2.499165 \\ \mathrm{H} & -5.889726 & -0.777692 & -1.807004 \\ \mathrm{C} & -5.825827 & 0.028066 & -3.881475 \\ \mathrm{~N} & -3.238688 & 0.999808 & -2.899663 \\ \mathrm{H} & -2.370946 & 1.307219 & -2.489157 \\ \mathrm{H} & -3.122369 & 0.659187 & -3.844561 \\ \mathrm{H} & -5.460963 & 0.976777 & -4.295049 \\ \mathrm{H} & -5.537625 & -0.768128 & -4.586932 \\ \mathrm{H} & -6.921338 & 0.059715 & -3.878759\end{array}$

TS:

$\begin{array}{cccc}\mathrm{H} & 0.000000 & 0.000000 & 0.000000 \\ \mathrm{H} & 0.000000 & 0.000000 & 1.808688 \\ \mathrm{C} & 0.599612 & 0.000000 & 0.911921 \\ \mathrm{C} & 1.811298 & 0.695829 & 0.909571 \\ \mathrm{H} & 2.042586 & 1.342866 & 0.061189 \\ \mathrm{C} & 2.856289 & 0.457418 & 1.808618 \\ \mathrm{H} & 3.650426 & 1.201322 & 1.873568 \\ \mathrm{C} & 3.137885 & -0.785065 & 2.393051 \\ \mathrm{H} & 4.128466 & -0.887143 & 2.838273 \\ \mathrm{C} & 2.466195 & -1.985268 & 2.070279 \\ \mathrm{C} & 1.079039 & -2.061806 & 1.806759 \\ \mathrm{H} & 0.452364 & -1.651519 & 2.584936 \\ \mathrm{C} & 0.492465 & -3.265243 & 1.107882 \\ \mathrm{~N} & 3.249727 & -3.114597 & 1.796806 \\ \mathrm{H} & 2.912770 & -3.996124 & 2.167841 \\ \mathrm{H} & 4.245794 & -2.998376 & 1.932272 \\ \mathrm{H} & 1.078957 & -3.538181 & 0.224944\end{array}$




$\begin{array}{cccc}\mathrm{H} & 0.453286 & -4.152820 & 1.762325 \\ \mathrm{H} & -0.537773 & -3.065921 & 0.793967 \\ & & & \\ & & & \\ & & & \\ \mathrm{H} & 0.000000 & 0.000000 & 0.000000 \\ \mathrm{C} & 0.000000 & 0.000000 & 1.085997 \\ \mathrm{H} & 0.973443 & 0.000000 & 1.572823 \\ \mathrm{C} & -1.143207 & -0.000279 & 1.792221 \\ \mathrm{H} & -2.096817 & -0.001058 & 1.276241 \\ \mathrm{C} & -1.162289 & 0.000364 & 3.245975 \\ \mathrm{H} & -0.176267 & 0.001954 & 3.711939 \\ \mathrm{C} & -2.216579 & -0.000659 & 4.096256 \\ \mathrm{H} & -1.989002 & -0.000678 & 5.159619 \\ \mathrm{C} & -3.648583 & -0.003424 & 3.824563 \\ \mathrm{C} & -4.551950 & 0.000009 & 4.831719 \\ \mathrm{H} & -4.155268 & 0.021566 & 5.842477 \\ \mathrm{O} & -3.990744 & -0.014131 & 2.494632 \\ \mathrm{C} & -6.047327 & -0.012889 & 4.701254 \\ \mathrm{H} & -6.399297 & -0.227731 & 3.683264 \\ \mathrm{H} & -6.493437 & 0.947208 & 4.998025 \\ \mathrm{H} & -6.490214 & -0.780500 & 5.348730 \\ \mathrm{H} & -4.956537 & 0.023380 & 2.419494\end{array}$

TS:

$\begin{array}{cccc}\mathrm{H} & 0.000000 & 0.000000 & 0.000000 \\ \mathrm{C} & 0.000000 & 0.000000 & 1.079294 \\ \mathrm{C} & 1.277101 & 0.000000 & 1.671915 \\ \mathrm{C} & 2.295732 & -0.938743 & 1.441498 \\ \mathrm{H} & 3.296964 & -0.661097 & 1.774221 \\ \mathrm{C} & 2.073311 & -2.307670 & 1.221311 \\ \mathrm{H} & 2.929106 & -2.959893 & 1.392484 \\ \mathrm{C} & 0.809509 & -2.893000 & 1.123057 \\ \mathrm{H} & 0.683831 & -3.860524 & 1.612359 \\ \mathrm{C} & -0.323389 & -2.228254 & 0.640847 \\ \mathrm{H} & -0.292193 & -1.816454 & -0.355661 \\ \mathrm{H} & -1.307009 & -2.615589 & 0.910637 \\ \mathrm{O} & 1.466306 & 0.936768 & 2.669592 \\ \mathrm{C} & -1.127324 & 0.786721 & 1.703447 \\ \mathrm{H} & 2.175799 & 0.630255 & 3.255495 \\ \mathrm{H} & -1.186492 & 0.617729 & 2.782477 \\ \mathrm{H} & -0.994530 & 1.867834 & 1.552402 \\ \mathrm{H} & -2.086232 & 0.508170 & 1.253411\end{array}$




$\begin{array}{cccc} & & & \\ & & & \\ & & & \\ \mathrm{C} & 0.000000 & 0.000000 & 0.000000 \\ \mathrm{H} & 0.000000 & 0.000000 & 1.088178 \\ \mathrm{H} & 0.970550 & 0.000000 & -0.486800 \\ \mathrm{C} & -1.143045 & -0.000020 & -0.706014 \\ \mathrm{H} & -1.102842 & -0.000036 & -1.789513 \\ \mathrm{C} & -2.451645 & 0.000000 & -0.073328 \\ \mathrm{H} & -2.432014 & 0.000067 & 1.016472 \\ \mathrm{C} & -3.679625 & -0.000053 & -0.645840 \\ \mathrm{H} & -4.541763 & 0.000009 & 0.017257 \\ \mathrm{C} & -4.046657 & -0.000124 & -2.047074 \\ \mathrm{C} & -5.301381 & 0.000132 & -2.526181 \\ \mathrm{H} & -6.103146 & 0.000450 & -1.792168 \\ \mathrm{C} & -5.673471 & 0.000056 & -3.976217 \\ \mathrm{~F} & -3.013097 & -0.000548 & -2.936609 \\ \mathrm{H} & -4.787080 & -0.000274 & -4.614515 \\ \mathrm{H} & -6.278982 & 0.881143 & -4.228348 \\ \mathrm{H} & -6.279608 & -0.880646 & -4.228127\end{array}$

TS:

$\begin{array}{cccc}\mathrm{H} & 0.000000 & 0.000000 & 0.000000 \\ \mathrm{C} & 0.000000 & 0.000000 & 1.078460 \\ \mathrm{C} & 1.259122 & 0.000000 & 1.696770 \\ \mathrm{C} & 2.291353 & -0.930790 & 1.611157 \\ \mathrm{H} & 3.240072 & -0.624843 & 2.045957 \\ \mathrm{C} & 2.094795 & -2.306908 & 1.404142 \\ \mathrm{H} & 2.929536 & -2.950735 & 1.676454 \\ \mathrm{C} & 0.843712 & -2.889791 & 1.207889 \\ \mathrm{H} & 0.668472 & -3.847637 & 1.700111 \\ \mathrm{C} & -0.232224 & -2.229422 & 0.603800 \\ \mathrm{H} & -0.083856 & -1.830155 & -0.387607 \\ \mathrm{H} & -1.240024 & -2.611986 & 0.766895 \\ \mathrm{C} & -1.128351 & 0.788159 & 1.701434 \\ \mathrm{~F} & 1.433795 & 0.945068 & 2.675232 \\ \mathrm{H} & -1.185499 & 0.627986 & 2.781788 \\ \mathrm{H} & -0.998600 & 1.867312 & 1.536565 \\ \mathrm{H} & -2.087323 & 0.502390 & 1.257413\end{array}$<smiles>C=C/C=C\C(C=O)=C/C</smiles> 


\begin{tabular}{|c|c|c|c|}
\hline $\mathrm{C}$ & 0.000000 & 0.000000 & 0.000000 \\
\hline $\mathrm{H}$ & 0.000000 & 0.000000 & 1.087885 \\
\hline $\mathrm{H}$ & 0.970567 & 0.000000 & -0.486851 \\
\hline $\mathrm{C}$ & -1.143453 & 0.002570 & -0.703510 \\
\hline $\mathrm{H}$ & -1.093385 & 0.003920 & -1.790599 \\
\hline $\mathrm{C}$ & -2.464471 & -0.038535 & -0.094120 \\
\hline $\mathrm{H}$ & -2.471488 & -0.218772 & 0.980907 \\
\hline $\mathrm{C}$ & -3.666292 & 0.065810 & -0.703308 \\
\hline $\mathrm{H}$ & -4.553490 & -0.117835 & -0.102432 \\
\hline $\mathrm{C}$ & -3.950546 & 0.350789 & -2.119731 \\
\hline $\mathrm{C}$ & -3.243885 & 1.242384 & -2.859926 \\
\hline $\mathrm{H}$ & -2.447702 & 1.780436 & -2.347412 \\
\hline $\mathrm{C}$ & -3.409500 & 1.628388 & -4.300235 \\
\hline $\mathrm{C}$ & -5.149257 & -0.333038 & -2.670861 \\
\hline $\mathrm{O}$ & -5.876522 & -1.057529 & -2.015290 \\
\hline $\mathrm{H}$ & -5.379627 & -0.139305 & -3.736594 \\
\hline $\mathrm{H}$ & -3.647438 & 2.697957 & -4.379339 \\
\hline $\mathrm{H}$ & -4.179442 & 1.069726 & -4.834318 \\
\hline $\mathrm{H}$ & -2.459672 & 1.486987 & -4.833654 \\
\hline \multicolumn{4}{|l|}{ TS: } \\
\hline $\mathrm{H}$ & 0.000000 & 0.000000 & 0.000000 \\
\hline $\mathrm{C}$ & 0.000000 & 0.000000 & 1.078120 \\
\hline $\mathrm{H}$ & 0.999841 & 0.000000 & 1.511971 \\
\hline $\mathrm{C}$ & -1.017322 & 0.662248 & 1.759850 \\
\hline $\mathrm{H}$ & -0.742532 & 1.287582 & 2.612615 \\
\hline $\mathrm{C}$ & -2.394478 & 0.450194 & 1.584906 \\
\hline $\mathrm{H}$ & -3.077275 & 1.197155 & 1.986502 \\
\hline $\mathrm{C}$ & -2.948444 & -0.801907 & 1.308492 \\
\hline $\mathrm{H}$ & -4.016454 & -0.900617 & 1.502602 \\
\hline $\mathrm{C}$ & -2.212414 & -1.999371 & 1.172669 \\
\hline $\mathrm{C}$ & -0.930844 & -2.051582 & 0.578712 \\
\hline $\mathrm{H}$ & -0.887714 & -1.594210 & -0.399578 \\
\hline $\mathrm{C}$ & 0.049896 & -3.175537 & 0.779140 \\
\hline $\mathrm{C}$ & -2.831205 & -3.183797 & 1.799076 \\
\hline $\mathrm{O}$ & -2.286587 & -4.246903 & 2.049647 \\
\hline $\mathrm{H}$ & -3.912802 & -3.045049 & 2.027941 \\
\hline $\mathrm{H}$ & -0.327359 & -4.100576 & 0.322180 \\
\hline $\mathrm{H}$ & 0.213361 & -3.398107 & 1.835205 \\
\hline $\mathrm{H}$ & 1.008288 & -2.929684 & 0.309916 \\
\hline
\end{tabular}




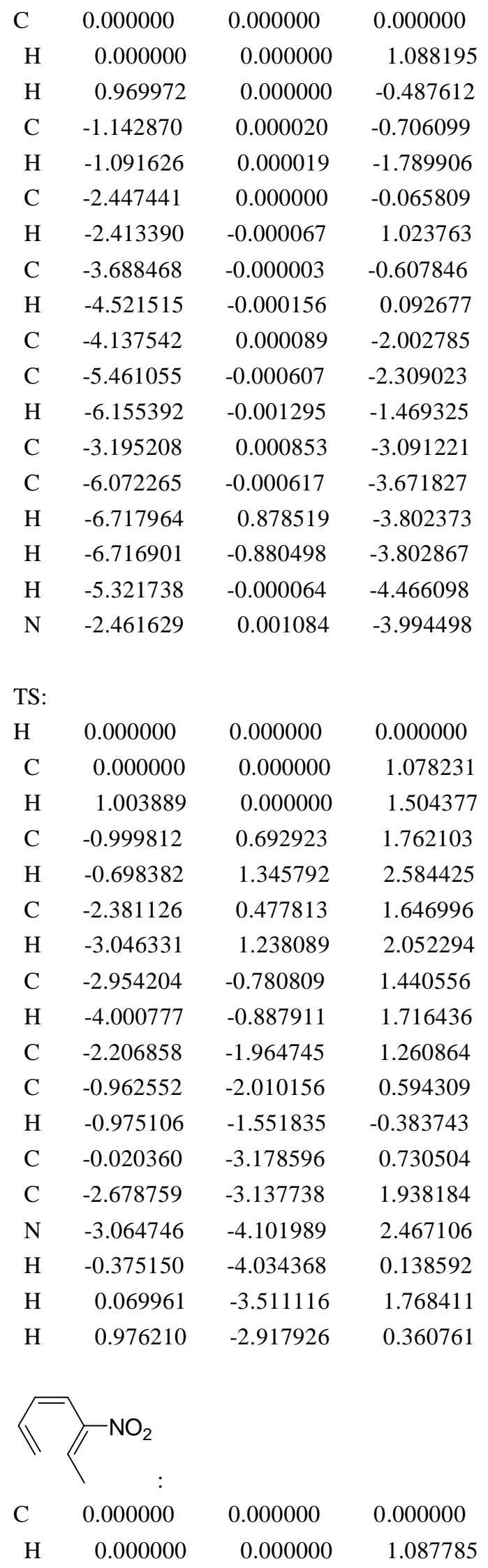




$\begin{array}{lrrr}\mathrm{H} & 0.970341 & 0.000000 & -0.486991 \\ \mathrm{C} & -1.142851 & 0.002153 & -0.704131 \\ \mathrm{H} & -1.095181 & 0.004734 & -1.791153 \\ \mathrm{C} & -2.460595 & -0.035647 & -0.088973 \\ \mathrm{H} & -2.466838 & -0.196950 & 0.988502 \\ \mathrm{C} & -3.665113 & 0.064417 & -0.693573 \\ \mathrm{H} & -4.558383 & -0.092250 & -0.098395 \\ \mathrm{C} & -3.906027 & 0.349124 & -2.111940 \\ \mathrm{C} & -3.266776 & 1.279198 & -2.851062 \\ \mathrm{H} & -2.516182 & 1.837566 & -2.296493 \\ \mathrm{~N} & -5.031210 & -0.413001 & -2.701909 \\ \mathrm{C} & -3.445634 & 1.674748 & -4.286140 \\ \mathrm{H} & -2.994456 & 2.655624 & -4.461154 \\ \mathrm{H} & -2.959123 & 0.950622 & -4.951887 \\ \mathrm{H} & -4.496940 & 1.696414 & -4.582980 \\ \mathrm{O} & -5.095015 & -0.508725 & -3.928531 \\ \mathrm{O} & -5.846091 & -0.910318 & -1.924625\end{array}$

TS:

$\begin{array}{cccc}\mathrm{H} & 0.000000 & 0.000000 & 0.000000 \\ \mathrm{H} & 0.000000 & 0.000000 & 1.808533 \\ \mathrm{C} & 0.598736 & 0.000000 & 0.911524 \\ \mathrm{C} & 1.815509 & 0.682287 & 0.892806 \\ \mathrm{H} & 2.018236 & 1.339848 & 0.044209 \\ \mathrm{C} & 2.910668 & 0.452048 & 1.739228 \\ \mathrm{H} & 3.693109 & 1.207934 & 1.769082 \\ \mathrm{C} & 3.270906 & -0.811667 & 2.216540 \\ \mathrm{H} & 4.291344 & -0.943988 & 2.558745 \\ \mathrm{C} & 2.528012 & -1.974312 & 1.959583 \\ \mathrm{C} & 1.123438 & -2.000009 & 1.854638 \\ \mathrm{H} & 0.667452 & -1.507406 & 2.701937 \\ \mathrm{C} & 0.268438 & -3.082851 & 1.249841 \\ \mathrm{~N} & 3.328930 & -3.171010 & 1.665206 \\ \mathrm{O} & 2.815935 & -4.066530 & 0.988628 \\ \mathrm{O} & 4.468720 & -3.231078 & 2.132231 \\ \mathrm{H} & 0.364679 & -4.019485 & 1.813937 \\ \mathrm{H} & 0.551151 & -3.306029 & 0.220627 \\ \mathrm{H} & -0.782546 & -2.776577 & 1.279163\end{array}$

2<smiles>C/C=C\C=C/C(C)=C/N</smiles> 


\begin{tabular}{|c|c|c|c|}
\hline $\mathrm{C}$ & 0.000000 & 0.000000 & 0.000000 \\
\hline $\mathrm{H}$ & 0.000000 & 0.000000 & 1.088124 \\
\hline $\mathrm{H}$ & 0.970398 & 0.000000 & -0.487211 \\
\hline C & -1.145831 & -0.001318 & -0.704975 \\
\hline $\mathrm{H}$ & -1.076660 & -0.006277 & -1.788448 \\
\hline $\mathrm{C}$ & -2.462644 & -0.017510 & -0.092546 \\
\hline $\mathrm{H}$ & -2.440533 & -0.067808 & 0.996470 \\
\hline $\mathrm{C}$ & -3.701977 & -0.001350 & -0.652310 \\
\hline $\mathrm{H}$ & -4.521618 & -0.069645 & 0.064476 \\
\hline $\mathrm{C}$ & -4.161947 & 0.089792 & -2.029271 \\
\hline $\mathrm{C}$ & -5.503124 & -0.027205 & -2.232111 \\
\hline $\mathrm{H}$ & -6.153995 & -0.170347 & -1.372167 \\
\hline $\mathrm{N}$ & -6.185159 & -0.074520 & -3.436453 \\
\hline $\mathrm{C}$ & -3.205824 & 0.313171 & -3.177206 \\
\hline $\mathrm{H}$ & -5.687649 & 0.244767 & -4.257891 \\
\hline $\mathrm{H}$ & -7.139437 & 0.260751 & -3.411719 \\
\hline $\mathrm{H}$ & -3.723262 & 0.574227 & -4.107404 \\
\hline $\mathrm{H}$ & -2.598602 & -0.576194 & -3.390092 \\
\hline $\mathrm{H}$ & -2.516378 & 1.136135 & -2.959114 \\
\hline \multicolumn{4}{|c|}{ TS: } \\
\hline $\mathrm{H}$ & 0.000000 & 0.000000 & 0.000000 \\
\hline $\mathrm{C}$ & 0.000000 & 0.000000 & 1.078137 \\
\hline $\mathrm{C}$ & 1.263607 & 0.000000 & 1.740505 \\
\hline $\mathrm{C}$ & 2.187886 & -1.046600 & 1.682417 \\
\hline $\mathrm{H}$ & 3.176483 & -0.816365 & 2.080708 \\
\hline $\mathrm{C}$ & 1.917231 & -2.421808 & 1.530058 \\
\hline $\mathrm{H}$ & 2.697799 & -3.100406 & 1.871399 \\
\hline $\mathrm{C}$ & 0.642646 & -2.927950 & 1.296898 \\
\hline $\mathrm{H}$ & 0.367705 & -3.859347 & 1.796985 \\
\hline $\mathrm{C}$ & -0.330060 & -2.209022 & 0.590899 \\
\hline $\mathrm{H}$ & -0.054135 & -1.852074 & -0.391246 \\
\hline $\mathrm{H}$ & -1.372058 & -2.526149 & 0.654848 \\
\hline $\mathrm{N}$ & -1.022183 & 0.817091 & 1.582210 \\
\hline $\mathrm{C}$ & 1.543760 & 1.125823 & 2.721954 \\
\hline $\mathrm{H}$ & -1.211307 & 0.697136 & 2.571108 \\
\hline $\mathrm{H}$ & -1.882541 & 0.777959 & 1.049853 \\
\hline $\mathrm{H}$ & 1.157146 & 2.086334 & 2.362921 \\
\hline $\mathrm{H}$ & 2.621523 & 1.216914 & 2.894749 \\
\hline $\mathrm{H}$ & 1.087534 & 0.944312 & 3.707560 \\
\hline
\end{tabular}




$\begin{array}{cccc}\mathrm{C} & 0.000000 & 0.000000 & 0.000000 \\ \mathrm{H} & 0.000000 & 0.000000 & 1.088157 \\ \mathrm{H} & 0.970413 & 0.000000 & -0.487176 \\ \mathrm{C} & -1.144807 & 0.004895 & -0.705950 \\ \mathrm{H} & -1.087343 & 0.027112 & -1.791785 \\ \mathrm{C} & -2.465385 & 0.046664 & -0.099474 \\ \mathrm{H} & -2.466587 & 0.186710 & 0.981470 \\ \mathrm{C} & -3.682890 & -0.018766 & -0.698686 \\ \mathrm{H} & -4.546861 & 0.141046 & -0.055198 \\ \mathrm{C} & -4.040306 & -0.272483 & -2.082230 \\ \mathrm{C} & -5.295584 & 0.010628 & -2.515146 \\ \mathrm{H} & -6.048075 & 0.374281 & -1.823005 \\ \mathrm{~N} & -5.683654 & -0.181001 & -3.853408 \\ \mathrm{~N} & -3.083184 & -0.763509 & -3.010221 \\ \mathrm{H} & -2.405116 & -1.365529 & -2.552481 \\ \mathrm{H} & -3.550367 & -1.309159 & -3.733734 \\ \mathrm{H} & -5.025461 & 0.213278 & -4.522092 \\ \mathrm{H} & -6.614608 & 0.162096 & -4.056097\end{array}$

TS:

$\begin{array}{crrr}\mathrm{H} & 0.000000 & 0.000000 & 0.000000 \\ \mathrm{C} & 0.000000 & 0.000000 & 1.078101 \\ \mathrm{C} & 1.265473 & 0.000000 & 1.734649 \\ \mathrm{C} & 2.243214 & -1.004156 & 1.640416 \\ \mathrm{H} & 3.228734 & -0.727288 & 2.018331 \\ \mathrm{C} & 2.005396 & -2.385270 & 1.520177 \\ \mathrm{H} & 2.818841 & -3.038983 & 1.833411 \\ \mathrm{C} & 0.745461 & -2.936756 & 1.301395 \\ \mathrm{H} & 0.510145 & -3.878391 & 1.800936 \\ \mathrm{C} & -0.260396 & -2.240624 & 0.620409 \\ \mathrm{H} & -0.013668 & -1.857628 & -0.359967 \\ \mathrm{H} & -1.291846 & -2.587528 & 0.698413 \\ \mathrm{~N} & -0.987249 & 0.867684 & 1.592424 \\ \mathrm{~N} & 1.419778 & 0.988354 & 2.726268 \\ \mathrm{H} & 1.159186 & 1.925679 & 2.429394 \\ \mathrm{H} & 2.310491 & 0.969114 & 3.207562 \\ \mathrm{H} & -1.090274 & 0.789871 & 2.600324 \\ \mathrm{H} & -1.885103 & 0.762881 & 1.133724\end{array}$<smiles>C=CC=C/C(O)=C\N</smiles>

$\begin{array}{llll}\text { C } & 0.000000 & 0.000000 & 0.000000\end{array}$ 


$\begin{array}{rrrr}\mathrm{H} & 0.000000 & 0.000000 & 1.088421 \\ \mathrm{H} & 0.971226 & 0.000000 & -0.485866 \\ \mathrm{C} & -1.142928 & -0.000020 & -0.706466 \\ \mathrm{H} & -1.111831 & -0.000052 & -1.790616 \\ \mathrm{C} & -2.451174 & 0.000043 & -0.072682 \\ \mathrm{H} & -2.432560 & 0.000038 & 1.017700 \\ \mathrm{C} & -3.675818 & 0.000124 & -0.650262 \\ \mathrm{H} & -4.540137 & 0.000296 & 0.010560 \\ \mathrm{C} & -4.035185 & 0.000191 & -2.058471 \\ \mathrm{C} & -5.325274 & 0.001431 & -2.476079 \\ \mathrm{H} & -6.149963 & 0.002255 & -1.766384 \\ \mathrm{~N} & -5.549043 & 0.001602 & -3.896441 \\ \mathrm{O} & -3.035855 & -0.000766 & -2.980239 \\ \mathrm{H} & -3.518285 & -0.000328 & -3.839324 \\ \mathrm{H} & -6.080120 & 0.819573 & -4.193772 \\ \mathrm{H} & -6.081385 & -0.815557 & -4.193735\end{array}$

TS:

$\begin{array}{cccc}\mathrm{H} & 0.000000 & 0.000000 & 0.000000 \\ \mathrm{C} & 0.000000 & 0.000000 & 1.081168 \\ \mathrm{H} & 0.998196 & 0.000000 & 1.518889 \\ \mathrm{C} & -1.032130 & 0.698174 & 1.710777 \\ \mathrm{H} & -0.818862 & 1.314543 & 2.585224 \\ \mathrm{C} & -2.366764 & 0.499438 & 1.359765 \\ \mathrm{H} & -3.112124 & 1.223322 & 1.686079 \\ \mathrm{C} & -2.863670 & -0.707555 & 0.843446 \\ \mathrm{H} & -3.945358 & -0.812855 & 0.792373 \\ \mathrm{C} & -2.168282 & -1.920066 & 0.775045 \\ \mathrm{C} & -0.769427 & -2.130132 & 0.621915 \\ \mathrm{H} & -0.299454 & -1.846997 & -0.305547 \\ \mathrm{~N} & -0.307101 & -3.400602 & 1.070910 \\ \mathrm{O} & -2.921484 & -3.029547 & 1.097528 \\ \mathrm{H} & -2.401098 & -3.805425 & 0.810113 \\ \mathrm{H} & -0.437661 & -3.534051 & 2.070609 \\ \mathrm{H} & 0.664767 & -3.570435 & 0.836895\end{array}$<smiles></smiles>

$\begin{array}{cccc}\mathrm{C} & 0.000000 & 0.000000 & 0.000000 \\ \mathrm{H} & 0.000000 & 0.000000 & 1.088256 \\ \mathrm{H} & 0.970649 & 0.000000 & -0.486679 \\ \mathrm{C} & -1.143993 & 0.000559 & -0.706241\end{array}$




$\begin{array}{cccc}\mathrm{H} & -1.098590 & 0.000735 & -1.790443 \\ \mathrm{C} & -2.458059 & 0.001816 & -0.089289 \\ \mathrm{H} & -2.459067 & 0.006089 & 1.000224 \\ \mathrm{C} & -3.675323 & 0.000567 & -0.691027 \\ \mathrm{H} & -4.551416 & 0.007040 & -0.044709 \\ \mathrm{C} & -4.017043 & -0.013065 & -2.085558 \\ \mathrm{C} & -5.268399 & -0.018739 & -2.596526 \\ \mathrm{H} & -6.116683 & -0.013390 & -1.920871 \\ \mathrm{~N} & -5.560256 & 0.062183 & -3.948185 \\ \mathrm{~F} & -2.995046 & -0.020243 & -3.002872 \\ \mathrm{H} & -4.773863 & -0.137990 & -4.554762 \\ \mathrm{H} & -6.405920 & -0.409535 & -4.242155\end{array}$

TS:

$\begin{array}{cccc}\mathrm{H} & 0.000000 & 0.000000 & 0.000000 \\ \mathrm{C} & 0.000000 & 0.000000 & 1.082345 \\ \mathrm{H} & 0.998706 & 0.000000 & 1.518607 \\ \mathrm{C} & -1.022765 & 0.739624 & 1.692098 \\ \mathrm{H} & -0.801822 & 1.390685 & 2.539048 \\ \mathrm{C} & -2.350503 & 0.544956 & 1.337924 \\ \mathrm{H} & -3.105150 & 1.264727 & 1.648347 \\ \mathrm{C} & -2.825824 & -0.683898 & 0.836907 \\ \mathrm{H} & -3.904741 & -0.808275 & 0.772240 \\ \mathrm{C} & -2.115836 & -1.870594 & 0.748372 \\ \mathrm{C} & -0.718701 & -2.130720 & 0.679131 \\ \mathrm{H} & -0.179409 & -1.879143 & -0.218847 \\ \mathrm{~N} & -0.265425 & -3.328253 & 1.229705 \\ \mathrm{~F} & -2.866474 & -2.994345 & 1.018436 \\ \mathrm{H} & -0.671796 & -3.583489 & 2.121147 \\ \mathrm{H} & 0.737447 & -3.454984 & 1.213633\end{array}$<smiles>N/C=C(C=O)\C=C/C=C\C=O</smiles>

$\begin{array}{cccc}\mathrm{H} & 0.000000 & 0.000000 & 0.000000 \\ \mathrm{C} & 0.000000 & 0.000000 & 1.087913 \\ \mathrm{H} & 0.970486 & 0.000000 & 1.574956 \\ \mathrm{C} & -1.144287 & 0.002194 & 1.792075 \\ \mathrm{H} & -1.085864 & 0.001514 & 2.880026 \\ \mathrm{C} & -2.471688 & 0.063145 & 1.201737 \\ \mathrm{H} & -2.498838 & 0.293509 & 0.137135 \\ \mathrm{C} & -3.662948 & -0.087188 & 1.829886 \\ \mathrm{H} & -4.552590 & 0.113613 & 1.231628\end{array}$




$\begin{array}{lrrr}\mathrm{C} & -3.945488 & -0.475470 & 3.212081 \\ \mathrm{C} & -3.148367 & -1.360578 & 3.922448 \\ \mathrm{H} & -2.299534 & -1.831458 & 3.433788 \\ \mathrm{C} & -5.174228 & 0.004173 & 3.818045 \\ \mathrm{~N} & -3.355128 & -1.716344 & 5.199512 \\ \mathrm{H} & -2.832709 & -2.470055 & 5.618060 \\ \mathrm{H} & -4.187294 & -1.336333 & 5.652033 \\ \mathrm{O} & -5.545450 & -0.211385 & 4.978254 \\ \mathrm{H} & -5.823157 & 0.608116 & 3.150307\end{array}$

TS:

$\begin{array}{cccc}\mathrm{H} & 0.000000 & 0.000000 & 0.000000 \\ \mathrm{C} & 0.000000 & 0.000000 & 1.080554 \\ \mathrm{C} & 1.300116 & 0.000000 & 1.720149 \\ \mathrm{C} & 2.176842 & -1.097249 & 1.787541 \\ \mathrm{H} & 3.180881 & -0.869119 & 2.146445 \\ \mathrm{C} & 1.818654 & -2.452669 & 1.787651 \\ \mathrm{H} & 2.529179 & -3.149206 & 2.227673 \\ \mathrm{C} & 0.503705 & -2.855047 & 1.559499 \\ \mathrm{H} & 0.111503 & -3.690485 & 2.142494 \\ \mathrm{C} & -0.349242 & -2.120900 & 0.723426 \\ \mathrm{H} & 0.032519 & -1.914369 & -0.268461 \\ \mathrm{H} & -1.419461 & -2.321513 & 0.745643 \\ \mathrm{~N} & -1.026510 & 0.733099 & 1.624449 \\ \mathrm{C} & 1.700818 & 1.143583 & 2.538002 \\ \mathrm{H} & -0.774047 & 1.273518 & 2.449541 \\ \mathrm{H} & -1.635275 & 1.218027 & 0.978504 \\ \mathrm{O} & 0.972492 & 2.013034 & 3.015528 \\ \mathrm{H} & 2.795815 & 1.191276 & 2.724545\end{array}$<smiles>C=C/C=C\C(C#N)=CN</smiles>

$\begin{array}{cccc}\mathrm{C} & 0.000000 & 0.000000 & 0.000000 \\ \mathrm{H} & 0.000000 & 0.000000 & 1.088255 \\ \mathrm{H} & 0.969849 & 0.000000 & -0.487889 \\ \mathrm{C} & -1.143858 & -0.000120 & -0.705248 \\ \mathrm{H} & -1.087223 & 0.000053 & -1.790251 \\ \mathrm{C} & -2.456352 & -0.000767 & -0.083629 \\ \mathrm{H} & -2.448575 & -0.003620 & 1.006001 \\ \mathrm{C} & -3.679255 & 0.000217 & -0.666661 \\ \mathrm{H} & -4.535188 & -0.004169 & 0.007294 \\ \mathrm{C} & -4.073776 & 0.007348 & -2.070553\end{array}$




$\begin{array}{lrrr}\mathrm{C} & -5.403239 & 0.007081 & -2.417870 \\ \mathrm{H} & -6.151337 & 0.011645 & -1.629661 \\ \mathrm{C} & -3.151428 & 0.018895 & -3.160667 \\ \mathrm{~N} & -5.903482 & -0.042323 & -3.678686 \\ \mathrm{~N} & -2.494973 & 0.034904 & -4.124983 \\ \mathrm{H} & -5.274157 & 0.062593 & -4.465037 \\ \mathrm{H} & -6.872609 & 0.181163 & -3.841657\end{array}$

TS:

$\begin{array}{cccc}\mathrm{H} & 0.000000 & 0.000000 & 0.000000 \\ \mathrm{C} & 0.000000 & 0.000000 & 1.082586 \\ \mathrm{H} & 0.991141 & 0.000000 & 1.532292 \\ \mathrm{C} & -1.047725 & 0.685508 & 1.695963 \\ \mathrm{H} & -0.861754 & 1.290605 & 2.584699 \\ \mathrm{C} & -2.366782 & 0.502454 & 1.277940 \\ \mathrm{H} & -3.131503 & 1.221995 & 1.561837 \\ \mathrm{C} & -2.813740 & -0.717165 & 0.758135 \\ \mathrm{H} & -3.885692 & -0.809204 & 0.597247 \\ \mathrm{C} & -2.101653 & -1.933789 & 0.722142 \\ \mathrm{C} & -0.670703 & -2.124578 & 0.767534 \\ \mathrm{H} & -0.083854 & -1.924898 & -0.114512 \\ \mathrm{~N} & -0.153707 & -3.144372 & 1.524099 \\ \mathrm{C} & -2.887079 & -3.121100 & 0.893025 \\ \mathrm{~N} & -3.514728 & -4.098103 & 1.012282 \\ \mathrm{H} & -0.757142 & -3.615191 & 2.185465 \\ \mathrm{H} & 0.610525 & -3.697608 & 1.162817\end{array}$<smiles>C/C=C\C(=C/N)[N+](=O)[O-]</smiles>

$\begin{array}{cccc}\mathrm{C} & 0.000000 & 0.000000 & 0.000000 \\ \mathrm{H} & 0.000000 & 0.000000 & 1.087783 \\ \mathrm{H} & 0.970368 & 0.000000 & -0.487132 \\ \mathrm{C} & -1.143580 & -0.003748 & -0.704109 \\ \mathrm{H} & -1.088675 & -0.006086 & -1.792071 \\ \mathrm{C} & -2.468499 & -0.057654 & -0.105314 \\ \mathrm{H} & -2.490998 & -0.267073 & 0.963432 \\ \mathrm{C} & -3.662984 & 0.083024 & -0.724512 \\ \mathrm{H} & -4.563713 & -0.109650 & -0.149310 \\ \mathrm{C} & -3.898485 & 0.483535 & -2.109759 \\ \mathrm{C} & -3.170078 & 1.460022 & -2.754954 \\ \mathrm{H} & -2.386454 & 1.934130 & -2.172647 \\ \mathrm{~N} & -5.035305 & -0.107653 & -2.756566\end{array}$




$\begin{array}{lrrr}\mathrm{N} & -3.299383 & 1.906165 & -4.011944 \\ \mathrm{H} & -2.764155 & 2.699337 & -4.328903 \\ \mathrm{H} & -4.058789 & 1.527008 & -4.568913 \\ \mathrm{O} & -5.307129 & 0.221979 & -3.934858 \\ \mathrm{O} & -5.712925 & -0.920656 & -2.121937\end{array}$

TS:

$\begin{array}{cccc}\mathrm{H} & 0.000000 & 0.000000 & 0.000000 \\ \mathrm{C} & 0.000000 & 0.000000 & 1.082796 \\ \mathrm{H} & 0.994435 & 0.000000 & 1.525921 \\ \mathrm{C} & -1.031851 & 0.723826 & 1.689337 \\ \mathrm{H} & -0.823821 & 1.371416 & 2.542339 \\ \mathrm{C} & -2.357646 & 0.518150 & 1.315776 \\ \mathrm{H} & -3.121341 & 1.242572 & 1.589209 \\ \mathrm{C} & -2.809609 & -0.729144 & 0.863960 \\ \mathrm{H} & -3.878703 & -0.850006 & 0.724338 \\ \mathrm{C} & -2.085638 & -1.926849 & 0.893758 \\ \mathrm{C} & -0.646456 & -2.091379 & 0.867417 \\ \mathrm{H} & -0.180138 & -1.923123 & -0.091743 \\ \mathrm{~N} & 0.003824 & -3.034141 & 1.615956 \\ \mathrm{~N} & -2.913858 & -3.105794 & 1.140290 \\ \mathrm{O} & -2.461651 & -4.032363 & 1.839834 \\ \mathrm{O} & -4.036214 & -3.145157 & 0.630226 \\ \mathrm{H} & -0.578406 & -3.595883 & 2.226858 \\ \mathrm{H} & 0.782298 & -3.517133 & 1.189078\end{array}$

3

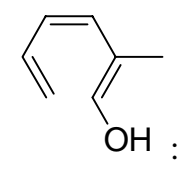

$\begin{array}{cccc}\mathrm{C} & 0.000000 & 0.000000 & 0.000000 \\ \mathrm{H} & 0.000000 & 0.000000 & 1.088032 \\ \mathrm{H} & 0.970325 & 0.000000 & -0.487174 \\ \mathrm{C} & -1.145274 & -0.000100 & -0.704550 \\ \mathrm{H} & -1.077538 & -0.000111 & -1.787823 \\ \mathrm{C} & -2.459838 & -0.000257 & -0.085262 \\ \mathrm{H} & -2.430709 & 0.000115 & 1.004584 \\ \mathrm{C} & -3.702144 & -0.000694 & -0.633910 \\ \mathrm{H} & -4.517607 & -0.000359 & 0.089812 \\ \mathrm{C} & -4.171253 & -0.001213 & -2.013960 \\ \mathrm{C} & -5.516034 & -0.000921 & -2.186708 \\ \mathrm{H} & -6.194990 & -0.000285 & -1.339233 \\ \mathrm{O} & -6.220409 & -0.001124 & -3.346296\end{array}$




$\begin{array}{cccc}\mathrm{C} & -3.218208 & -0.001672 & -3.186054 \\ \mathrm{H} & -2.570839 & 0.882786 & -3.176770 \\ \mathrm{H} & -2.571422 & -0.886519 & -3.176560 \\ \mathrm{H} & -3.731388 & -0.001559 & -4.155978 \\ \mathrm{H} & -5.609634 & -0.001629 & -4.100970\end{array}$

TS:

$\begin{array}{cccc}\mathrm{H} & 0.000000 & 0.000000 & 0.000000 \\ \mathrm{C} & 0.000000 & 0.000000 & 1.080412 \\ \mathrm{H} & 0.997904 & 0.000000 & 1.517485 \\ \mathrm{C} & -1.033845 & 0.692831 & 1.711579 \\ \mathrm{H} & -0.815092 & 1.322119 & 2.576502 \\ \mathrm{C} & -2.373009 & 0.481847 & 1.383470 \\ \mathrm{H} & -3.116819 & 1.208895 & 1.705967 \\ \mathrm{C} & -2.870444 & -0.759274 & 0.952036 \\ \mathrm{H} & -3.957355 & -0.841029 & 0.933120 \\ \mathrm{C} & -2.177083 & -1.977630 & 0.900111 \\ \mathrm{C} & -0.785170 & -2.105221 & 0.665329 \\ \mathrm{H} & -0.362516 & -1.836934 & -0.288420 \\ \mathrm{C} & -2.933189 & -3.229929 & 1.321148 \\ \mathrm{O} & -0.111030 & -3.207962 & 1.132616 \\ \mathrm{H} & -0.495182 & -3.470846 & 1.983881 \\ \mathrm{H} & -2.556850 & -4.128308 & 0.818307 \\ \mathrm{H} & -3.996185 & -3.120247 & 1.082279 \\ \mathrm{H} & -2.884272 & -3.412430 & 2.406237\end{array}$<smiles>C/C=C\C(N)=CO</smiles>

$\begin{array}{llll}\text { C } & 0.000000 & 0.000000 & 0.000000\end{array}$

$\begin{array}{llll}\mathrm{H} & 0.000000 & 0.000000 & 1.087899\end{array}$

$\begin{array}{llll}\mathrm{H} & 0.969911 & 0.000000 & -0.487793\end{array}$

$\begin{array}{llll}\text { C } & -1.145943 & -0.000200 & -0.703473\end{array}$

$\begin{array}{llll}\mathrm{H} & -1.078679 & -0.000398 & -1.788312\end{array}$

$\begin{array}{llll}\text { C } & -2.465128 & -0.000335 & -0.096018\end{array}$

$\begin{array}{llll}\mathrm{H} & -2.455714 & -0.000157 & 0.993637\end{array}$

$\begin{array}{llll}\text { C } & -3.692282 & -0.000538 & -0.680526\end{array}$

$\begin{array}{llll}\mathrm{H} & -4.539210 & -0.000416 & 0.006600\end{array}$

$\begin{array}{llll}\text { C } & -4.100899 & -0.001325 & -2.069064\end{array}$

$\begin{array}{llll}\text { C } & -5.416473 & -0.001901 & -2.412260\end{array}$

$\begin{array}{llll}\mathrm{H} & -6.227719 & -0.001624 & -1.691141\end{array}$

$\begin{array}{llll}\mathrm{N} & -3.213385 & -0.001508 & -3.213240\end{array}$

$\begin{array}{llll}\text { O } & -5.831838 & -0.001887 & -3.688498\end{array}$ 


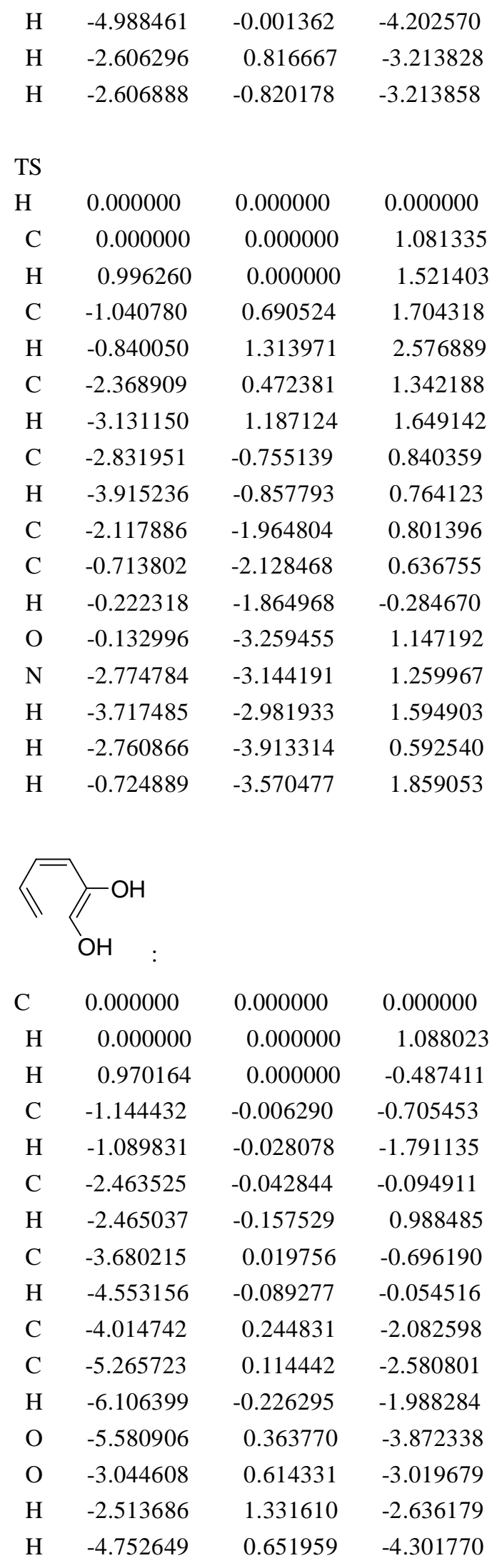


TS:

$\begin{array}{cccc}\mathrm{H} & 0.000000 & 0.000000 & 0.000000 \\ \mathrm{C} & 0.000000 & 0.000000 & 1.081469 \\ \mathrm{H} & 0.992727 & 0.000000 & 1.528097 \\ \mathrm{C} & -1.056204 & 0.656534 & 1.706594 \\ \mathrm{H} & -0.880050 & 1.246990 & 2.606283 \\ \mathrm{C} & -2.374905 & 0.446296 & 1.299871 \\ \mathrm{H} & -3.148150 & 1.146172 & 1.612065 \\ \mathrm{C} & -2.816297 & -0.752815 & 0.725464 \\ \mathrm{H} & -3.890014 & -0.877371 & 0.602907 \\ \mathrm{C} & -2.091976 & -1.949752 & 0.645568 \\ \mathrm{C} & -0.689164 & -2.145698 & 0.584595 \\ \mathrm{H} & -0.108437 & -1.881951 & -0.283024 \\ \mathrm{O} & -0.205654 & -3.345768 & 1.080342 \\ \mathrm{O} & -2.850319 & -3.083687 & 0.920369 \\ \mathrm{H} & -2.418938 & -3.837869 & 0.480603 \\ \mathrm{H} & -0.690144 & -3.542364 & 1.900377\end{array}$<smiles>C/C=C\C(F)=CO</smiles>

$\begin{array}{llll}\text { C } & 0.000000 & 0.000000 & 0.000000\end{array}$

$\begin{array}{llll}\mathrm{H} & 0.000000 & 0.000000 & 1.088118\end{array}$

$\begin{array}{llll}\text { H } & 0.970599 & 0.000000 & -0.486612\end{array}$

$\begin{array}{llll}\text { C } & -1.143370 & -0.000040 & -0.706077\end{array}$

$\begin{array}{llll}\mathrm{H} & -1.098875 & -0.000055 & -1.790295\end{array}$

$\begin{array}{llll}\text { C } & -2.455407 & -0.000086 & -0.083132\end{array}$

$\begin{array}{llll}\mathrm{H} & -2.450863 & -0.000051 & 1.006254\end{array}$

$\begin{array}{llll}\text { C } & -3.674802 & -0.000091 & -0.676633\end{array}$

$\begin{array}{llll}\mathrm{H} & -4.549584 & 0.000039 & -0.028737\end{array}$

$\begin{array}{llll}\text { C } & -4.019737 & -0.000016 & -2.071805\end{array}$

$\begin{array}{llll}\text { C } & -5.264506 & 0.000465 & -2.588449\end{array}$

$\mathrm{H} \quad-6.149547 \quad 0.000820 \quad-1.964583$

$\begin{array}{llll}\text { O } & -5.538272 & 0.000652 & -3.915201\end{array}$

$\begin{array}{llll}\text { F } & -3.004787 & 0.000289 & -3.001216\end{array}$

$\begin{array}{llll}\mathrm{H} & -4.687561 & -0.000578 & -4.388254\end{array}$

TS:

$\begin{array}{cccc}\mathrm{H} & 0.000000 & 0.000000 & 0.000000 \\ \mathrm{C} & 0.000000 & 0.000000 & 1.082307 \\ \mathrm{H} & 0.993889 & 0.000000 & 1.526021 \\ \mathrm{C} & -1.044656 & 0.700182 & 1.691583 \\ \mathrm{H} & -0.855135 & 1.326201 & 2.563841\end{array}$




$\begin{array}{lrrr}\mathrm{C} & -2.362651 & 0.489182 & 1.299809 \\ \mathrm{H} & -3.138236 & 1.190322 & 1.600333 \\ \mathrm{C} & -2.796692 & -0.736158 & 0.766076 \\ \mathrm{H} & -3.869024 & -0.884908 & 0.660712 \\ \mathrm{C} & -2.045889 & -1.901577 & 0.677557 \\ \mathrm{C} & -0.650518 & -2.132561 & 0.644395 \\ \mathrm{H} & -0.047571 & -1.894389 & -0.214862 \\ \mathrm{O} & -0.177187 & -3.281378 & 1.212738 \\ \mathrm{~F} & -2.764335 & -3.055666 & 0.948720 \\ \mathrm{H} & -0.852258 & -3.621303 & 1.823885\end{array}$

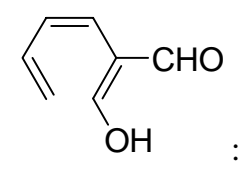

$\begin{array}{cccc}\mathrm{C} & 0.000000 & 0.000000 & 0.000000 \\ \mathrm{H} & 0.000000 & 0.000000 & 1.087851 \\ \mathrm{H} & 0.970112 & 0.000000 & -0.487406 \\ \mathrm{C} & -1.143473 & 0.002233 & -0.703743 \\ \mathrm{H} & -1.089556 & 0.004143 & -1.791352 \\ \mathrm{C} & -2.466707 & -0.045631 & -0.101788 \\ \mathrm{H} & -2.485864 & -0.240102 & 0.970021 \\ \mathrm{C} & -3.660604 & 0.083163 & -0.724506 \\ \mathrm{H} & -4.551098 & -0.081853 & -0.117546 \\ \mathrm{C} & -3.933416 & 0.411094 & -2.128425 \\ \mathrm{C} & -3.183602 & 1.302501 & -2.866942 \\ \mathrm{H} & -2.343971 & 1.844457 & -2.438356 \\ \mathrm{O} & -3.416280 & 1.616267 & -4.127609 \\ \mathrm{C} & -5.110362 & -0.151718 & -2.764754 \\ \mathrm{H} & -4.222415 & 1.083411 & -4.406937 \\ \mathrm{O} & -5.434866 & 0.047809 & -3.946034 \\ \mathrm{H} & -5.752981 & -0.796138 & -2.136344\end{array}$

TS:

$\begin{array}{cccc}\mathrm{H} & 0.000000 & 0.000000 & 0.000000 \\ \mathrm{C} & 0.000000 & 0.000000 & 1.081373 \\ \mathrm{H} & 0.999647 & 0.000000 & 1.513275 \\ \mathrm{C} & -1.009485 & 0.749054 & 1.698550 \\ \mathrm{H} & -0.756199 & 1.419096 & 2.522481 \\ \mathrm{C} & -2.366269 & 0.554952 & 1.423949 \\ \mathrm{H} & -3.086031 & 1.312342 & 1.726740 \\ \mathrm{C} & -2.876261 & -0.711762 & 1.120066 \\ \mathrm{H} & -3.960485 & -0.816204 & 1.165163 \\ \mathrm{C} & -2.144050 & -1.915852 & 1.127851\end{array}$




$\begin{array}{llll}\mathrm{C} & -0.748580 & -2.040421 & 0.801578 \\ \mathrm{H} & -0.455427 & -1.843374 & -0.219981 \\ \mathrm{O} & -0.014746 & -3.041391 & 1.316974 \\ \mathrm{C} & -2.810389 & -3.049398 & 1.748470 \\ \mathrm{H} & -3.918113 & -3.017127 & 1.734150 \\ \mathrm{O} & -2.238740 & -4.004265 & 2.286658 \\ \mathrm{H} & -0.583921 & -3.526990 & 1.968959\end{array}$<smiles>C=C/C=C\C(C#N)=CO</smiles>

$\begin{array}{cccc}\mathrm{C} & 0.000000 & 0.000000 & 0.000000 \\ \mathrm{H} & 0.000000 & 0.000000 & 1.088057 \\ \mathrm{H} & 0.969572 & 0.000000 & -0.488195 \\ \mathrm{C} & -1.143511 & -0.000040 & -0.704814 \\ \mathrm{H} & -1.088396 & -0.000071 & -1.789790 \\ \mathrm{C} & -2.453357 & -0.000086 & -0.076791 \\ \mathrm{H} & -2.439838 & -0.000051 & 1.012632 \\ \mathrm{C} & -3.678461 & -0.000128 & -0.651767 \\ \mathrm{H} & -4.535069 & -0.000057 & 0.020069 \\ \mathrm{C} & -4.067776 & -0.000239 & -2.059217 \\ \mathrm{C} & -5.385602 & 0.000207 & -2.417682 \\ \mathrm{H} & -6.174807 & 0.000410 & -1.672359 \\ \mathrm{O} & -5.878334 & -0.000020 & -3.662306 \\ \mathrm{C} & -3.148863 & -0.000122 & -3.156083 \\ \mathrm{~N} & -2.512176 & -0.000167 & -4.133019 \\ \mathrm{H} & -5.146336 & -0.000904 & -4.309787\end{array}$

$\begin{array}{cccc}\text { TS } & & & \\ \mathrm{H} & 0.000000 & 0.000000 & 0.000000 \\ \mathrm{C} & 0.000000 & 0.000000 & 1.081083 \\ \mathrm{H} & 0.993033 & 0.000000 & 1.526453 \\ \mathrm{C} & -1.044242 & 0.677465 & 1.705113 \\ \mathrm{H} & -0.845426 & 1.286507 & 2.588354 \\ \mathrm{C} & -2.379597 & 0.481887 & 1.336132 \\ \mathrm{H} & -3.129490 & 1.211714 & 1.633211 \\ \mathrm{C} & -2.857755 & -0.753368 & 0.894345 \\ \mathrm{H} & -3.938374 & -0.859621 & 0.832400 \\ \mathrm{C} & -2.128466 & -1.963744 & 0.853472 \\ \mathrm{C} & -0.716564 & -2.112595 & 0.694737 \\ \mathrm{H} & -0.247821 & -1.876724 & -0.245988 \\ \mathrm{O} & -0.068760 & -3.161920 & 1.257206 \\ \mathrm{C} & -2.836902 & -3.148390 & 1.240541\end{array}$




$\begin{array}{cccc}\mathrm{N} & -3.385557 & -4.127333 & 1.559695 \\ \mathrm{H} & -0.579944 & -3.500543 & 2.013729 \\ & & & \\ & & & \\ & & & \\ & & & \\ & & & \\ & & & \\ & & & \\ \mathrm{C} & 0.000000 & 0.000000 & 0.000000 \\ \mathrm{H} & 0.000000 & 0.000000 & 1.087711 \\ \mathrm{H} & 0.970021 & 0.000000 & -0.487446 \\ \mathrm{C} & -1.142885 & 0.000538 & -0.703960 \\ \mathrm{H} & -1.091350 & -0.000151 & -1.791535 \\ \mathrm{C} & -2.463307 & -0.042154 & -0.094657 \\ \mathrm{H} & -2.477411 & -0.216656 & 0.980370 \\ \mathrm{C} & -3.662211 & 0.075264 & -0.706862 \\ \mathrm{H} & -4.561086 & -0.085695 & -0.119000 \\ \mathrm{C} & -3.896404 & 0.414115 & -2.110145 \\ \mathrm{C} & -3.212450 & 1.380469 & -2.803250 \\ \mathrm{H} & -2.432540 & 1.938037 & -2.293790 \\ \mathrm{~N} & -4.999889 & -0.249825 & -2.753878 \\ \mathrm{O} & -3.390229 & 1.747030 & -4.056150 \\ \mathrm{H} & -4.143469 & 1.182079 & -4.396113 \\ \mathrm{O} & -5.261985 & 0.032583 & -3.955025 \\ \mathrm{O} & -5.655911 & -1.064931 & -2.114279\end{array}$

TS:

$\begin{array}{cccc}\mathrm{H} & 0.000000 & 0.000000 & 0.000000 \\ \mathrm{C} & 0.000000 & 0.000000 & 1.088840 \\ \mathrm{H} & 0.991008 & 0.000000 & 1.521245 \\ \mathrm{C} & -0.975019 & -0.729547 & 1.776112 \\ \mathrm{H} & -1.642820 & -1.394694 & 1.226018 \\ \mathrm{C} & -1.251245 & -0.514859 & 3.128452 \\ \mathrm{H} & -1.812129 & -1.260664 & 3.686967 \\ \mathrm{C} & -1.153583 & 0.752606 & 3.711536 \\ \mathrm{H} & -1.600548 & 0.886962 & 4.691157 \\ \mathrm{C} & -0.857042 & 1.937071 & 3.020527 \\ \mathrm{C} & -0.024556 & 2.064580 & 1.855546 \\ \mathrm{H} & 1.024122 & 1.851436 & 1.998983 \\ \mathrm{O} & -0.182863 & 3.035844 & 0.944928 \\ \mathrm{~N} & -1.630614 & 3.092564 & 3.441896 \\ \mathrm{O} & -1.936904 & 3.945996 & 2.575651 \\ \mathrm{O} & -1.959083 & 3.201291 & 4.619984 \\ \mathrm{H} & -1.032370 & 3.494471 & 1.153615\end{array}$




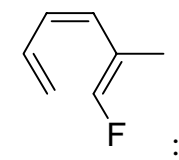

$\begin{array}{cccc}\mathrm{C} & 0.000000 & 0.000000 & 0.000000 \\ \mathrm{H} & 0.000000 & 0.000000 & 1.085713 \\ \mathrm{H} & 0.972387 & 0.000000 & -0.488103 \\ \mathrm{C} & -1.143027 & 0.000000 & -0.706885 \\ \mathrm{H} & -2.083625 & 0.000000 & -0.166192 \\ \mathrm{C} & -1.170451 & 0.000000 & -2.160298 \\ \mathrm{H} & -0.181494 & 0.000035 & -2.619231 \\ \mathrm{C} & -2.215222 & -0.000036 & -3.024761 \\ \mathrm{H} & -1.939560 & -0.000008 & -4.079498 \\ \mathrm{C} & -3.659167 & -0.000105 & -2.806050 \\ \mathrm{C} & -4.424750 & -0.000062 & -3.911239 \\ \mathrm{H} & -4.055285 & 0.000019 & -4.932152 \\ \mathrm{C} & -4.304993 & -0.000154 & -1.442445 \\ \mathrm{~F} & -5.771996 & -0.000060 & -3.862214 \\ \mathrm{H} & -5.392877 & -0.000273 & -1.534619 \\ \mathrm{H} & -4.012702 & 0.884474 & -0.865247 \\ \mathrm{H} & -4.012487 & -0.884699 & -0.865229\end{array}$

$\begin{array}{cccc}\text { TS } & & & \\ \mathrm{H} & 0.000000 & 0.000000 & 0.000000 \\ \mathrm{C} & 0.000000 & 0.000000 & 1.089101 \\ \mathrm{H} & 0.988206 & 0.000000 & 1.525055 \\ \mathrm{C} & -1.003807 & -0.672271 & 1.781155 \\ \mathrm{H} & -1.721525 & -1.282840 & 1.230918 \\ \mathrm{C} & -1.246896 & -0.459178 & 3.140942 \\ \mathrm{H} & -1.848913 & -1.185371 & 3.685120 \\ \mathrm{C} & -1.056632 & 0.776370 & 3.775282 \\ \mathrm{H} & -1.491463 & 0.862655 & 4.770467 \\ \mathrm{C} & -0.724626 & 1.992915 & 3.152687 \\ \mathrm{C} & 0.088589 & 2.092097 & 2.014607 \\ \mathrm{H} & 1.133116 & 1.825271 & 2.020129 \\ \mathrm{~F} & -0.078414 & 3.223924 & 1.269746 \\ \mathrm{C} & -1.448636 & 3.248199 & 3.605501 \\ \mathrm{H} & -0.820558 & 4.140536 & 3.519122 \\ \mathrm{H} & -2.347991 & 3.426441 & 3.001662 \\ \mathrm{H} & -1.773042 & 3.142004 & 4.645664\end{array}$




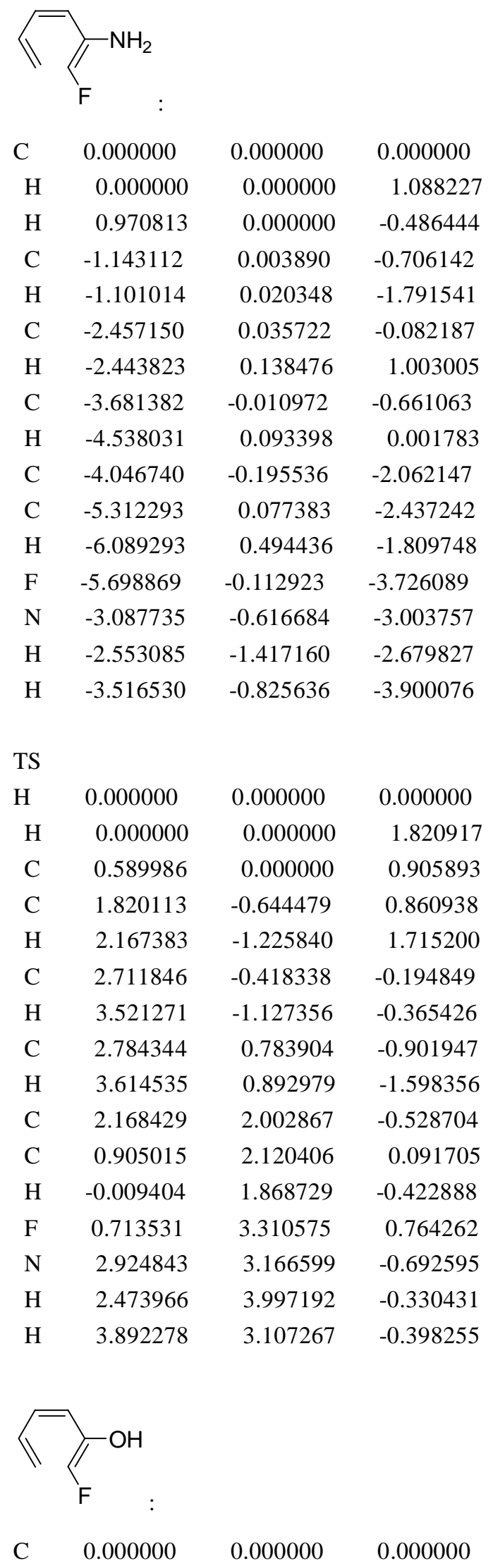




\begin{tabular}{|c|c|c|c|}
\hline $\mathrm{H}$ & 0.000000 & 0.000000 & 1.088292 \\
\hline $\mathrm{H}$ & 0.971209 & 0.000000 & -0.485667 \\
\hline $\mathrm{C}$ & -1.142618 & 0.000000 & -0.706798 \\
\hline $\mathrm{H}$ & -1.107543 & -0.000016 & -1.790956 \\
\hline $\mathrm{C}$ & -2.450463 & 0.000000 & -0.072730 \\
\hline $\mathrm{H}$ & -2.431380 & 0.000000 & 1.017254 \\
\hline $\mathrm{C}$ & -3.677464 & 0.000000 & -0.645281 \\
\hline $\mathrm{H}$ & -4.537845 & 0.000000 & 0.020730 \\
\hline $\mathrm{C}$ & -4.041960 & 0.000000 & -2.049727 \\
\hline $\mathrm{C}$ & -5.333137 & -0.000020 & -2.431455 \\
\hline $\mathrm{H}$ & -6.203298 & -0.000038 & -1.790456 \\
\hline $\mathrm{F}$ & -5.618836 & -0.000019 & -3.765259 \\
\hline $\mathrm{O}$ & -3.042252 & 0.000000 & -2.983274 \\
\hline $\mathrm{H}$ & -3.466347 & 0.000000 & -3.858488 \\
\hline \multicolumn{4}{|c|}{ TS } \\
\hline $\mathrm{C}$ & 0.000000 & 0.000000 & 0.000000 \\
\hline $\mathrm{H}$ & 0.000000 & 0.000000 & 1.081389 \\
\hline $\mathrm{H}$ & 0.993007 & 0.000000 & -0.445504 \\
\hline $\mathrm{C}$ & -1.055667 & -0.648511 & -0.630052 \\
\hline $\mathrm{H}$ & -0.880710 & -1.230691 & -1.534539 \\
\hline $\mathrm{C}$ & -2.378595 & -0.427275 & -0.233524 \\
\hline $\mathrm{H}$ & -3.149454 & -1.130576 & -0.544529 \\
\hline $\mathrm{C}$ & -2.829299 & 0.773980 & 0.320095 \\
\hline $\mathrm{H}$ & -3.904150 & 0.904023 & 0.422692 \\
\hline $\mathrm{C}$ & -2.096192 & 1.972982 & 0.375635 \\
\hline $\mathrm{C}$ & -0.699978 & 2.114328 & 0.514511 \\
\hline $\mathrm{H}$ & -0.176620 & 1.869970 & 1.424572 \\
\hline $\mathrm{F}$ & -0.256885 & 3.354872 & 0.080532 \\
\hline $\mathrm{O}$ & -2.822607 & 3.098358 & 0.090201 \\
\hline $\mathrm{H}$ & -2.231334 & 3.862200 & 0.207426 \\
\hline $\mathrm{C}$ & 0.000000 & 0.000000 & 0.000000 \\
\hline $\mathrm{H}$ & 0.000000 & 0.000000 & 1.088094 \\
\hline $\mathrm{H}$ & 0.970375 & 0.000000 & -0.486924 \\
\hline $\mathrm{C}$ & -1.142828 & 0.000000 & -0.706046 \\
\hline $\mathrm{H}$ & -1.102519 & 0.000000 & -1.789856 \\
\hline $\mathrm{C}$ & -2.450949 & 0.000000 & -0.073084 \\
\hline $\mathrm{H}$ & -2.435583 & -0.000017 & 1.016410 \\
\hline $\mathrm{C}$ & -3.675701 & 0.000018 & -0.651812 \\
\hline
\end{tabular}




$\begin{array}{lrrr}\text { H } & -4.543897 & -0.000002 & 0.003966 \\ \text { C } & -4.020207 & 0.000043 & -2.054708 \\ \text { C } & -5.282762 & 0.000001 & -2.510874 \\ \text { H } & -6.157202 & -0.000055 & -1.871303 \\ \text { F } & -3.018731 & 0.000050 & -2.964448 \\ \text { F } & -5.554990 & 0.000027 & -3.823680\end{array}$

TS

$\begin{array}{cccc}\mathrm{H} & 0.000000 & 0.000000 & 0.000000 \\ \mathrm{C} & 0.000000 & 0.000000 & 1.081675 \\ \mathrm{H} & 0.989220 & 0.000000 & 1.534221 \\ \mathrm{C} & -1.067205 & 0.634038 & 1.705055 \\ \mathrm{H} & -0.910380 & 1.208687 & 2.617575 \\ \mathrm{C} & -2.377737 & 0.417499 & 1.273389 \\ \mathrm{H} & -3.165327 & 1.102854 & 1.579869 \\ \mathrm{C} & -2.790262 & -0.780769 & 0.680885 \\ \mathrm{H} & -3.857330 & -0.924048 & 0.527971 \\ \mathrm{C} & -2.038574 & -1.955725 & 0.590405 \\ \mathrm{C} & -0.649962 & -2.154192 & 0.551734 \\ \mathrm{H} & -0.023091 & -1.885939 & -0.282200 \\ \mathrm{~F} & -0.236187 & -3.334155 & 1.078936 \\ \mathrm{~F} & -2.761554 & -3.098093 & 0.768354\end{array}$<smiles>O=CC(/C=C\C=C/F)=C/F</smiles>

$\begin{array}{cccc}\mathrm{C} & 0.000000 & 0.000000 & 0.000000 \\ \mathrm{H} & 0.000000 & 0.000000 & 1.087754 \\ \mathrm{H} & 0.970139 & 0.000000 & -0.487399 \\ \mathrm{C} & -1.143363 & 0.002690 & -0.703345 \\ \mathrm{H} & -1.092219 & 0.003784 & -1.790699 \\ \mathrm{C} & -2.463981 & -0.038994 & -0.094381 \\ \mathrm{H} & -2.474436 & -0.226026 & 0.979041 \\ \mathrm{C} & -3.664801 & 0.077141 & -0.703365 \\ \mathrm{H} & -4.558302 & -0.095476 & -0.109032 \\ \mathrm{C} & -3.948862 & 0.369311 & -2.113733 \\ \mathrm{C} & -3.220559 & 1.229242 & -2.852402 \\ \mathrm{H} & -2.376425 & 1.810966 & -2.497834 \\ \mathrm{~F} & -3.501922 & 1.481783 & -4.140311 \\ \mathrm{C} & -5.170300 & -0.237788 & -2.706510 \\ \mathrm{O} & -5.938747 & -0.939424 & -2.075844 \\ \mathrm{H} & -5.357843 & 0.000163 & -3.769961\end{array}$


TS:

$\begin{array}{cccc}\mathrm{H} & 0.000000 & 0.000000 & 0.000000 \\ \mathrm{C} & 0.000000 & 0.000000 & 1.080678 \\ \mathrm{H} & 0.989731 & 0.000000 & 1.532580 \\ \mathrm{C} & -1.060319 & 0.625323 & 1.718811 \\ \mathrm{H} & -0.880522 & 1.187880 & 2.636089 \\ \mathrm{C} & -2.394719 & 0.436442 & 1.324656 \\ \mathrm{H} & -3.145236 & 1.155371 & 1.647701 \\ \mathrm{C} & -2.870825 & -0.774317 & 0.829089 \\ \mathrm{H} & -3.948768 & -0.888350 & 0.744955 \\ \mathrm{C} & -2.156259 & -1.992025 & 0.788801 \\ \mathrm{C} & -0.767641 & -2.124726 & 0.616557 \\ \mathrm{H} & -0.246221 & -1.874144 & -0.293172 \\ \mathrm{~F} & -0.191297 & -3.217708 & 1.177914 \\ \mathrm{C} & -2.938377 & -3.229542 & 1.058025 \\ \mathrm{O} & -4.155833 & -3.269558 & 1.099447 \\ \mathrm{H} & -2.338753 & -4.150434 & 1.179379\end{array}$<smiles></smiles>

$\begin{array}{llll}\text { C } & 0.000000 & 0.000000 & 0.000000\end{array}$

$\begin{array}{llll}\mathrm{H} & 0.000000 & 0.000000 & 1.088094\end{array}$

$\begin{array}{llll}\mathrm{H} & 0.969651 & 0.000000 & -0.488023\end{array}$

$\begin{array}{llll}\text { C } & -1.142719 & 0.000000 & -0.706061\end{array}$

$\begin{array}{llll}\mathrm{H} & -1.092661 & 0.000000 & -1.790045\end{array}$

$\begin{array}{llll}\text { C } & -2.446517 & 0.000000 & -0.064993\end{array}$

$\begin{array}{llll}\mathrm{H} & -2.415898 & 0.000000 & 1.024379\end{array}$

$\begin{array}{llll}\text { C } & -3.684734 & 0.000000 & -0.611318\end{array}$

$\begin{array}{llll}\mathrm{H} & -4.523280 & 0.000000 & 0.082485\end{array}$

$\begin{array}{llll}\text { C } & -4.109087 & 0.000000 & -2.012247\end{array}$

$\begin{array}{llll}\text { C } & -5.427306 & -0.000021 & -2.307846\end{array}$

$\begin{array}{llll}\mathrm{H} & -6.222040 & -0.000038 & -1.568661\end{array}$

$\begin{array}{llll}\text { C } & -3.191929 & 0.000000 & -3.115793\end{array}$

$\begin{array}{llll}\text { F } & -5.875573 & -0.000022 & -3.562358\end{array}$

$\begin{array}{llll}\mathrm{N} & -2.462635 & -0.000002 & -4.021395\end{array}$

$\begin{array}{cccc}\text { TS } & & & \\ \mathrm{H} & 0.000000 & 0.000000 & 0.000000 \\ \mathrm{C} & 0.000000 & 0.000000 & 1.080402 \\ \mathrm{H} & 0.990517 & 0.000000 & 1.530638 \\ \mathrm{C} & -1.056294 & 0.633381 & 1.718931 \\ \mathrm{H} & -0.870347 & 1.209466 & 2.626212\end{array}$




$\begin{array}{lrrr}\mathrm{C} & -2.393150 & 0.435362 & 1.343413 \\ \mathrm{H} & -3.142503 & 1.160027 & 1.654945 \\ \mathrm{C} & -2.877154 & -0.786738 & 0.881314 \\ \mathrm{H} & -3.957679 & -0.891396 & 0.823863 \\ \mathrm{C} & -2.144600 & -1.999171 & 0.830770 \\ \mathrm{C} & -0.759065 & -2.123684 & 0.612879 \\ \mathrm{H} & -0.289082 & -1.857238 & -0.319616 \\ \mathrm{~F} & -0.169377 & -3.217885 & 1.135336 \\ \mathrm{C} & -2.849197 & -3.187035 & 1.220190 \\ \mathrm{~N} & -3.449137 & -4.141092 & 1.512944\end{array}$

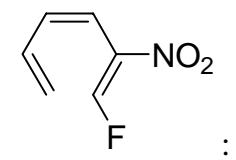

$\begin{array}{cccc}\mathrm{C} & 0.000000 & 0.000000 & 0.000000 \\ \mathrm{H} & 0.000000 & 0.000000 & 1.087667 \\ \mathrm{H} & 0.969980 & 0.000000 & -0.487467 \\ \mathrm{C} & -1.142453 & 0.001495 & -0.704271 \\ \mathrm{H} & -1.093957 & 0.003423 & -1.791479 \\ \mathrm{C} & -2.460018 & -0.036996 & -0.088569 \\ \mathrm{H} & -2.467101 & -0.197088 & 0.988874 \\ \mathrm{C} & -3.664278 & 0.063675 & -0.693072 \\ \mathrm{H} & -4.560527 & -0.087837 & -0.100388 \\ \mathrm{C} & -3.903047 & 0.356733 & -2.108211 \\ \mathrm{C} & -3.254754 & 1.332285 & -2.772467 \\ \mathrm{H} & -2.524916 & 1.960003 & -2.271056 \\ \mathrm{~F} & -3.417894 & 1.651155 & -4.046253 \\ \mathrm{~N} & -4.981907 & -0.413377 & -2.754857 \\ \mathrm{O} & -5.625799 & -1.167721 & -2.023931 \\ \mathrm{O} & -5.178439 & -0.260501 & -3.957135\end{array}$

$\begin{array}{cccc}\text { TS } & & & \\ \mathrm{H} & 0.000000 & 0.000000 & 0.000000 \\ \mathrm{C} & 0.000000 & 0.000000 & 1.080445 \\ \mathrm{H} & 0.986628 & 0.000000 & 1.538444 \\ \mathrm{C} & -1.076080 & 0.588282 & 1.721312 \\ \mathrm{H} & -0.919665 & 1.143594 & 2.646820 \\ \mathrm{C} & -2.398095 & 0.374855 & 1.303181 \\ \mathrm{H} & -3.170658 & 1.081601 & 1.598301 \\ \mathrm{C} & -2.842227 & -0.838320 & 0.784241 \\ \mathrm{H} & -3.912388 & -0.956221 & 0.656123 \\ \mathrm{C} & -2.106045 & -2.037485 & 0.732226 \\ \mathrm{C} & -0.714672 & -2.177896 & 0.591385\end{array}$




$\begin{array}{llll}\mathrm{H} & -0.185061 & -1.879070 & -0.298027 \\ \mathrm{~F} & -0.090722 & -3.198017 & 1.190366 \\ \mathrm{~N} & -2.907965 & -3.265711 & 0.978214 \\ \mathrm{O} & -2.370324 & -4.355749 & 0.794908 \\ \mathrm{O} & -4.089556 & -3.121438 & 1.299447\end{array}$

5<smiles>CC(/C=C\C=C/C=O)=C/C=O</smiles>

$\begin{array}{llll}\text { C } & 0.000000 & 0.000000 & 0.000000\end{array}$

$\begin{array}{llll}\mathrm{H} & 0.000000 & 0.000000 & 1.087880\end{array}$

$\begin{array}{llll}\mathrm{H} & 0.970045 & 0.000000 & -0.487479\end{array}$

$\begin{array}{llll}\text { C } & -1.145064 & 0.000000 & -0.705055\end{array}$

$\begin{array}{llll}\mathrm{H} & -1.088733 & -0.000017 & -1.787155\end{array}$

$\begin{array}{llll}\text { C } & -2.446243 & -0.000064 & -0.060559\end{array}$

$\begin{array}{llll}\mathrm{H} & -2.389429 & -0.000251 & 1.028603\end{array}$

$\begin{array}{llll}\text { C } & -3.706466 & -0.000023 & -0.571334\end{array}$

$\begin{array}{llll}\mathrm{H} & -4.501013 & -0.000267 & 0.173413\end{array}$

$\begin{array}{llll}\text { C } & -4.202755 & 0.000252 & -1.943739\end{array}$

$\begin{array}{llll}\text { C } & -5.561135 & -0.000239 & -2.100141\end{array}$

$\begin{array}{llll}\mathrm{H} & -6.175675 & -0.000796 & -1.200810\end{array}$

$\begin{array}{llll}\text { C } & -6.343883 & 0.000315 & -3.341202\end{array}$

$\begin{array}{llll}\text { C } & -3.260366 & 0.001239 & -3.120832\end{array}$

$\begin{array}{llll}\mathrm{H} & -2.610912 & 0.884455 & -3.090893\end{array}$

$\mathrm{H} \quad-2.611110 \quad-0.882193 \quad-3.092449$

$\mathrm{H} \quad-3.813211 \quad 0.001949 \quad-4.058066$

$\mathrm{H} \quad-7.441291 \quad 0.001125 \quad-3.158405$

$\begin{array}{llll}\text { O } & -5.931333 & -0.000009 & -4.493286\end{array}$

$\begin{array}{cccc}\text { TS } & & & \\ \mathrm{H} & 0.000000 & 0.000000 & 0.000000 \\ \mathrm{C} & 0.000000 & 0.000000 & 1.078366 \\ \mathrm{H} & 1.008753 & 0.000000 & 1.495896 \\ \mathrm{C} & -0.989414 & 0.706081 & 1.782355 \\ \mathrm{H} & -0.649042 & 1.409671 & 2.545549 \\ \mathrm{C} & -2.361443 & 0.459805 & 1.775331 \\ \mathrm{H} & -2.995509 & 1.218138 & 2.233304 \\ \mathrm{C} & -2.962864 & -0.810045 & 1.624494 \\ \mathrm{H} & -3.980586 & -0.889913 & 2.004684 \\ \mathrm{C} & -2.253260 & -1.993326 & 1.423388 \\ \mathrm{C} & -1.051165 & -1.958135 & 0.663952 \\ \mathrm{H} & -1.137971 & -1.477492 & -0.300549\end{array}$




$\begin{array}{lrrr}\mathrm{C} & -0.020444 & -3.009347 & 0.709625 \\ \mathrm{C} & -2.724896 & -3.245607 & 2.131099 \\ \mathrm{H} & -3.779464 & -3.144531 & 2.409644 \\ \mathrm{H} & -2.147591 & -3.411484 & 3.047904 \\ \mathrm{H} & -2.598367 & -4.145010 & 1.521951 \\ \mathrm{O} & 0.070618 & -3.887659 & 1.552147 \\ \mathrm{H} & 0.740863 & -2.932060 & -0.099687\end{array}$

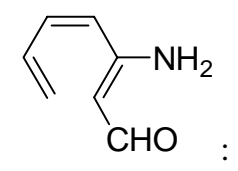

$\begin{array}{cccc}\mathrm{C} & 0.000000 & 0.000000 & 0.000000 \\ \mathrm{H} & 0.000000 & 0.000000 & 1.087953 \\ \mathrm{H} & 0.970237 & 0.000000 & -0.487194 \\ \mathrm{C} & -1.143315 & 0.000599 & -0.705023 \\ \mathrm{H} & -1.100149 & 0.012369 & -1.790891 \\ \mathrm{C} & -2.454602 & 0.035145 & -0.074638 \\ \mathrm{H} & -2.435756 & 0.176897 & 1.006019 \\ \mathrm{C} & -3.679631 & -0.029756 & -0.648660 \\ \mathrm{H} & -4.539696 & 0.117990 & -0.000762 \\ \mathrm{C} & -4.030747 & -0.249770 & -2.056373 \\ \mathrm{C} & -5.245034 & 0.216430 & -2.534135 \\ \mathrm{H} & -5.887847 & 0.777702 & -1.864761 \\ \mathrm{C} & -5.702131 & -0.020749 & -3.873750 \\ \mathrm{~N} & -3.157037 & -0.898992 & -2.873833 \\ \mathrm{H} & -2.492096 & -1.536268 & -2.460545 \\ \mathrm{H} & -3.522863 & -1.104770 & -3.806077 \\ \mathrm{O} & -5.078790 & -0.628692 & -4.758286 \\ \mathrm{H} & -6.712543 & 0.373245 & -4.106697\end{array}$

TS:

$\begin{array}{cccc}\mathrm{H} & 0.000000 & 0.000000 & 0.000000 \\ \mathrm{C} & 0.000000 & 0.000000 & 1.080478 \\ \mathrm{H} & 1.008459 & 0.000000 & 1.498010 \\ \mathrm{C} & -0.992610 & 0.747198 & 1.740772 \\ \mathrm{H} & -0.691815 & 1.438376 & 2.528322 \\ \mathrm{C} & -2.355553 & 0.493110 & 1.610252 \\ \mathrm{H} & -3.048540 & 1.244385 & 1.989097 \\ \mathrm{C} & -2.926454 & -0.760905 & 1.323429 \\ \mathrm{H} & -3.994928 & -0.857793 & 1.512284 \\ \mathrm{C} & -2.184947 & -1.957701 & 1.311491 \\ \mathrm{C} & -0.827335 & -2.016111 & 0.818778 \\ \mathrm{H} & -0.707498 & -1.753915 & -0.222463\end{array}$




$\begin{array}{lrrl}\mathrm{C} & 0.103816 & -3.068258 & 1.237450 \\ \mathrm{~N} & -2.743483 & -3.085118 & 1.863667 \\ \mathrm{H} & -3.451300 & -2.948175 & 2.573188 \\ \mathrm{H} & -2.084646 & -3.833299 & 2.060648 \\ \mathrm{O} & -0.099356 & -3.953421 & 2.066076 \\ \mathrm{H} & 1.101543 & -3.000718 & 0.752749\end{array}$<smiles></smiles>

$\begin{array}{cccc}\mathrm{C} & 0.000000 & 0.000000 & 0.000000 \\ \mathrm{H} & 0.000000 & 0.000000 & 1.088235 \\ \mathrm{H} & 0.970733 & 0.000000 & -0.486382 \\ \mathrm{C} & -1.143302 & -0.000040 & -0.707221 \\ \mathrm{H} & -1.116520 & -0.000072 & -1.790703 \\ \mathrm{C} & -2.440866 & -0.000042 & -0.057308 \\ \mathrm{H} & -2.404901 & 0.000008 & 1.032578 \\ \mathrm{C} & -3.680341 & -0.000171 & -0.609857 \\ \mathrm{H} & -4.530631 & -0.000179 & 0.066833 \\ \mathrm{C} & -4.052881 & -0.000461 & -2.014403 \\ \mathrm{C} & -5.383038 & -0.000909 & -2.402889 \\ \mathrm{H} & -6.164440 & -0.001147 & -1.652398 \\ \mathrm{C} & -5.733070 & -0.001406 & -3.789052 \\ \mathrm{O} & -3.077764 & -0.000428 & -2.921807 \\ \mathrm{H} & -3.530652 & -0.001008 & -3.823175 \\ \mathrm{O} & -4.902371 & -0.001184 & -4.721278 \\ \mathrm{H} & -6.808946 & -0.001407 & -4.039496\end{array}$

TS:

$\begin{array}{cccc}\mathrm{H} & 0.000000 & 0.000000 & 0.000000 \\ \mathrm{C} & 0.000000 & 0.000000 & 1.080230 \\ \mathrm{H} & 1.015898 & 0.000000 & 1.483848 \\ \mathrm{C} & -0.963706 & 0.764481 & 1.774717 \\ \mathrm{H} & -0.594341 & 1.505745 & 2.485308 \\ \mathrm{C} & -2.329893 & 0.505188 & 1.842131 \\ \mathrm{H} & -2.953746 & 1.280560 & 2.285112 \\ \mathrm{C} & -2.937091 & -0.765612 & 1.749185 \\ \mathrm{H} & -3.924151 & -0.876818 & 2.190554 \\ \mathrm{C} & -2.192424 & -1.930077 & 1.573448 \\ \mathrm{C} & -0.983112 & -1.932251 & 0.793752 \\ \mathrm{H} & -1.094869 & -1.544492 & -0.208264 \\ \mathrm{C} & -0.066550 & -3.067975 & 0.877565 \\ \mathrm{O} & -2.581618 & -3.025791 & 2.257209\end{array}$




$\begin{array}{rrrr}\mathrm{H} & -1.883598 & -3.713820 & 2.132746 \\ \mathrm{O} & -0.211589 & -4.040713 & 1.621474 \\ \mathrm{H} & 0.824801 & -3.018762 & 0.221686\end{array}$<smiles>C=C/C=C\C(F)=CC=O</smiles>

\begin{tabular}{cccc}
$\mathrm{C}$ & \multicolumn{1}{c}{0.000000} & 0.000000 & \multicolumn{1}{c}{0.000000} \\
$\mathrm{H}$ & 0.000000 & 0.000000 & 1.087903 \\
$\mathrm{H}$ & 0.970174 & 0.000000 & -0.487162 \\
$\mathrm{C}$ & -1.143479 & 0.000000 & -0.706475 \\
$\mathrm{H}$ & -1.105448 & 0.000000 & -1.789573 \\
$\mathrm{C}$ & -2.442694 & 0.000000 & -0.059673 \\
$\mathrm{H}$ & -2.408386 & -0.000017 & 1.029578 \\
$\mathrm{C}$ & -3.683631 & 0.000018 & -0.610591 \\
$\mathrm{H}$ & -4.536240 & -0.000002 & 0.063194 \\
$\mathrm{C}$ & -4.059292 & 0.000043 & -2.003339 \\
$\mathrm{C}$ & -5.327405 & 0.000003 & -2.477366 \\
$\mathrm{H}$ & -6.150577 & -0.000037 & -1.770201 \\
$\mathrm{~F}$ & -3.041709 & 0.000049 & -2.896804 \\
$\mathrm{C}$ & -5.669575 & 0.000030 & -3.898234 \\
$\mathrm{O}$ & -6.820054 & 0.000028 & -4.308309 \\
$\mathrm{H}$ & -4.814884 & 0.000139 & -4.602712
\end{tabular}

TS:

$\begin{array}{cccc}\mathrm{H} & 0.000000 & 0.000000 & 0.000000 \\ \mathrm{C} & 0.000000 & 0.000000 & 1.079505 \\ \mathrm{H} & 0.993845 & 0.000000 & 1.522991 \\ \mathrm{C} & -1.053391 & 0.659971 & 1.719667 \\ \mathrm{H} & -0.846288 & 1.288575 & 2.585554 \\ \mathrm{C} & -2.385722 & 0.404807 & 1.413478 \\ \mathrm{H} & -3.147536 & 1.092239 & 1.776631 \\ \mathrm{C} & -2.856006 & -0.837261 & 0.941970 \\ \mathrm{H} & -3.930273 & -1.002342 & 0.957607 \\ \mathrm{C} & -2.075256 & -1.983726 & 0.872105 \\ \mathrm{C} & -0.679292 & -2.130437 & 0.686930 \\ \mathrm{H} & -0.234104 & -1.865439 & -0.259355 \\ \mathrm{C} & 0.023962 & -3.246601 & 1.353803 \\ \mathrm{~F} & -2.739698 & -3.129246 & 1.204866 \\ \mathrm{H} & -0.512063 & -3.725573 & 2.198215 \\ \mathrm{O} & 1.151391 & -3.597693 & 1.049350\end{array}$




\begin{tabular}{|c|c|c|c|}
\hline $\mathrm{C}$ & 0.000000 & 0.000000 & 0.000000 \\
\hline $\mathrm{H}$ & 0.000000 & 0.000000 & 1.087726 \\
\hline $\mathrm{H}$ & 0.969963 & 0.000000 & -0.487607 \\
\hline $\mathrm{C}$ & -1.144564 & 0.006005 & -0.703966 \\
\hline $\mathrm{H}$ & -1.096726 & 0.004991 & -1.790060 \\
\hline $\mathrm{C}$ & -2.454736 & -0.032298 & -0.078933 \\
\hline $\mathrm{H}$ & -2.443262 & -0.216233 & 0.995409 \\
\hline $\mathrm{C}$ & -3.676962 & 0.059062 & -0.660589 \\
\hline $\mathrm{H}$ & -4.543650 & -0.148132 & -0.039046 \\
\hline $\mathrm{C}$ & -4.009928 & 0.329015 & -2.055572 \\
\hline $\mathrm{C}$ & -3.273131 & 1.136808 & -2.875025 \\
\hline $\mathrm{H}$ & -2.412417 & 1.646852 & -2.448937 \\
\hline $\mathrm{C}$ & -5.314096 & -0.271072 & -2.517868 \\
\hline $\mathrm{C}$ & -3.499700 & 1.464334 & -4.296124 \\
\hline $\mathrm{H}$ & -2.759477 & 2.191611 & -4.692737 \\
\hline $\mathrm{O}$ & -4.370956 & 1.027854 & -5.031199 \\
\hline $\mathrm{H}$ & -5.562439 & -0.129219 & -3.579175 \\
\hline $\mathrm{O}$ & -6.053206 & -0.863994 & -1.754221 \\
\hline
\end{tabular}

TS:

$\begin{array}{cccc}\mathrm{H} & 0.000000 & 0.000000 & 0.000000 \\ \mathrm{C} & 0.000000 & 0.000000 & 1.077056 \\ \mathrm{H} & 0.998250 & 0.000000 & 1.513098 \\ \mathrm{C} & -1.035957 & 0.607331 & 1.780806 \\ \mathrm{H} & -0.768321 & 1.222509 & 2.642768 \\ \mathrm{C} & -2.409230 & 0.366696 & 1.624622 \\ \mathrm{H} & -3.094669 & 1.086548 & 2.068726 \\ \mathrm{C} & -2.956989 & -0.886088 & 1.321041 \\ \mathrm{H} & -4.017055 & -1.006064 & 1.542503 \\ \mathrm{C} & -2.202120 & -2.056644 & 1.124461 \\ \mathrm{C} & -0.933418 & -2.048133 & 0.498615 \\ \mathrm{H} & -0.880885 & -1.584103 & -0.476513 \\ \mathrm{C} & 0.122931 & -3.071998 & 0.698158 \\ \mathrm{C} & -2.779752 & -3.296000 & 1.696731 \\ \mathrm{H} & -3.829474 & -3.180710 & 2.045843 \\ \mathrm{O} & -2.215052 & -4.371113 & 1.787297 \\ \mathrm{H} & 0.114211 & -3.618554 & 1.655270 \\ \mathrm{O} & 0.997315 & -3.252266 & -0.132851\end{array}$




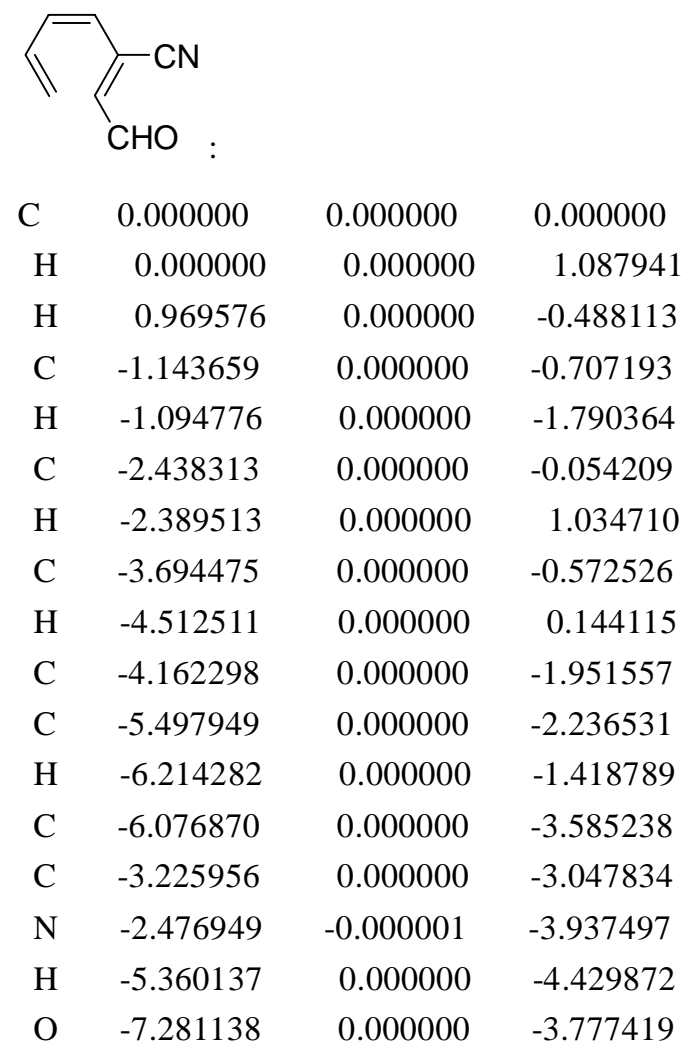

TS:

$\begin{array}{cccc}\mathrm{H} & 0.000000 & 0.000000 & 0.000000 \\ \mathrm{C} & 0.000000 & 0.000000 & 1.077612 \\ \mathrm{H} & 1.004448 & 0.000000 & 1.501494 \\ \mathrm{C} & -1.011299 & 0.652413 & 1.787819 \\ \mathrm{H} & -0.703027 & 1.317735 & 2.597410 \\ \mathrm{C} & -2.387109 & 0.396833 & 1.732784 \\ \mathrm{H} & -3.047096 & 1.135261 & 2.184098 \\ \mathrm{C} & -2.948934 & -0.875676 & 1.546963 \\ \mathrm{H} & -3.973942 & -1.009306 & 1.883635 \\ \mathrm{C} & -2.177728 & -2.026399 & 1.310332 \\ \mathrm{C} & -0.970797 & -1.983985 & 0.568264 \\ \mathrm{H} & -1.031282 & -1.518655 & -0.404770 \\ \mathrm{C} & 0.058162 & -3.044331 & 0.660200 \\ \mathrm{C} & -2.589172 & -3.236551 & 1.961079 \\ \mathrm{~N} & -2.947597 & -4.216871 & 2.478750 \\ \mathrm{H} & -0.020422 & -3.720160 & 1.536643 \\ \mathrm{O} & 0.961160 & -3.157639 & -0.147964\end{array}$<smiles>C=C/C=C\C(=C/C=O)[N+](=O)[O-]</smiles> 


$\begin{array}{cccc}\mathrm{C} & 0.000000 & 0.000000 & 0.000000 \\ \mathrm{H} & 0.000000 & 0.000000 & 1.087676 \\ \mathrm{H} & 0.969482 & 0.000000 & -0.488150 \\ \mathrm{C} & -1.143916 & 0.007466 & -0.704663 \\ \mathrm{H} & -1.097915 & 0.009153 & -1.790675 \\ \mathrm{C} & -2.450299 & -0.024665 & -0.073565 \\ \mathrm{H} & -2.440299 & -0.184802 & 1.003938 \\ \mathrm{C} & -3.673696 & 0.064383 & -0.651507 \\ \mathrm{H} & -4.551144 & -0.093118 & -0.032828 \\ \mathrm{C} & -3.962332 & 0.320924 & -2.053667 \\ \mathrm{C} & -3.287171 & 1.122680 & -2.907528 \\ \mathrm{H} & -2.440284 & 1.672204 & -2.507509 \\ \mathrm{C} & -3.579176 & 1.441803 & -4.322522 \\ \mathrm{~N} & -5.221289 & -0.335662 & -2.521747 \\ \mathrm{O} & -6.179425 & -0.298398 & -1.755113 \\ \mathrm{O} & -5.216055 & -0.866787 & -3.628636 \\ \mathrm{H} & -4.466393 & 0.981676 & -4.785065 \\ \mathrm{O} & -2.858147 & 2.203399 & -4.943469\end{array}$

$\begin{array}{cccc}\text { TS } & & & \\ \mathrm{H} & 0.000000 & 0.000000 & 0.000000 \\ \mathrm{C} & 0.000000 & 0.000000 & 1.077285 \\ \mathrm{H} & 0.997894 & 0.000000 & 1.514167 \\ \mathrm{C} & -1.035262 & 0.615501 & 1.775655 \\ \mathrm{H} & -0.769714 & 1.240101 & 2.631037 \\ \mathrm{C} & -2.406572 & 0.374101 & 1.616545 \\ \mathrm{H} & -3.096885 & 1.101667 & 2.038900 \\ \mathrm{C} & -2.959580 & -0.877597 & 1.317823 \\ \mathrm{H} & -4.013802 & -1.017693 & 1.528972 \\ \mathrm{C} & -2.191159 & -2.026913 & 1.109610 \\ \mathrm{C} & -0.915951 & -2.054769 & 0.512483 \\ \mathrm{H} & -0.850578 & -1.612692 & -0.471338 \\ \mathrm{C} & 0.157494 & -3.057431 & 0.755014 \\ \mathrm{~N} & -2.752481 & -3.280645 & 1.654490 \\ \mathrm{O} & -3.885058 & -3.252029 & 2.134841 \\ \mathrm{O} & -2.063640 & -4.301016 & 1.569402 \\ \mathrm{H} & 0.205941 & -3.504153 & 1.761510 \\ \mathrm{O} & 0.996130 & -3.297236 & -0.093775\end{array}$

6<smiles>C/C=C\C=C(C)\C=C/C</smiles> 


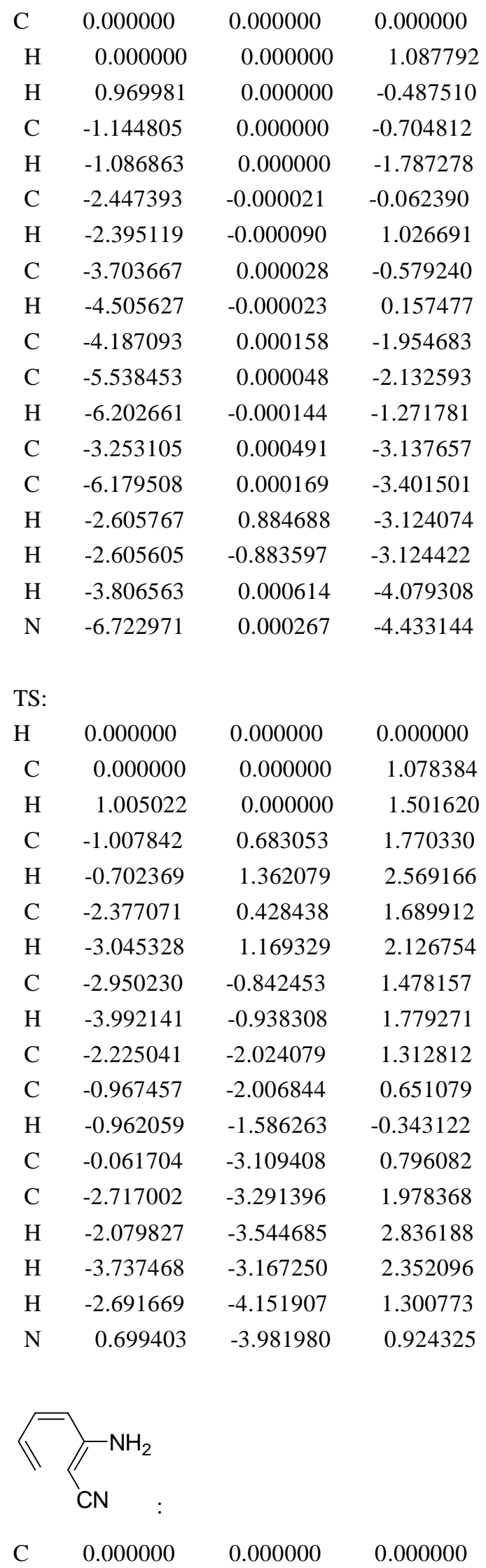




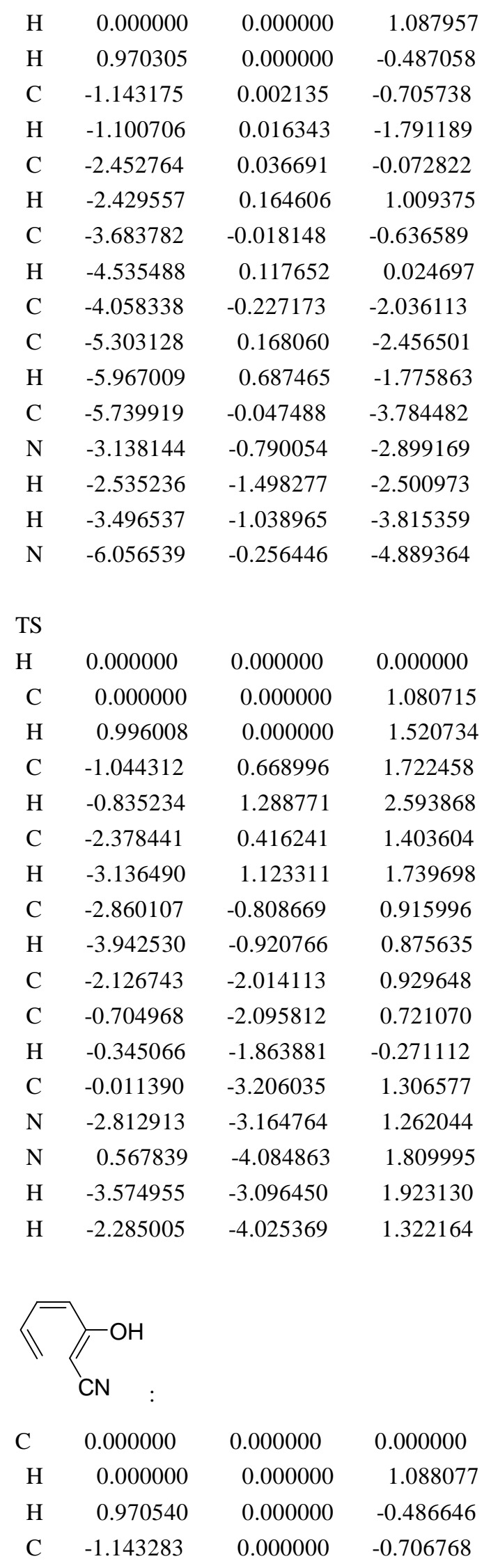




$\begin{array}{lrrr}\mathrm{H} & -1.112095 & 0.000000 & -1.790117 \\ \mathrm{C} & -2.442357 & 0.000000 & -0.058903 \\ \mathrm{H} & -2.406289 & -0.000017 & 1.030658 \\ \mathrm{C} & -3.684115 & 0.000018 & -0.604540 \\ \mathrm{H} & -4.529088 & 0.000014 & 0.078510 \\ \mathrm{C} & -4.076668 & 0.000043 & -2.001280 \\ \mathrm{C} & -5.396160 & 0.000039 & -2.365769 \\ \mathrm{H} & -6.175427 & 0.000016 & -1.614002 \\ \mathrm{C} & -5.755811 & 0.000063 & -3.733757 \\ \mathrm{O} & -3.079533 & 0.000050 & -2.914537 \\ \mathrm{H} & -3.462703 & 0.000067 & -3.811868 \\ \mathrm{~N} & -5.969698 & 0.000085 & -4.882306\end{array}$

TS:

$\begin{array}{cccc}\mathrm{H} & 0.000000 & 0.000000 & 0.000000 \\ \mathrm{C} & 0.000000 & 0.000000 & 1.080298 \\ \mathrm{H} & 0.997567 & 0.000000 & 1.517141 \\ \mathrm{C} & -1.035927 & 0.682238 & 1.726973 \\ \mathrm{H} & -0.803994 & 1.321464 & 2.578561 \\ \mathrm{C} & -2.379270 & 0.429426 & 1.462608 \\ \mathrm{H} & -3.119532 & 1.141350 & 1.824868 \\ \mathrm{C} & -2.890256 & -0.810628 & 1.038150 \\ \mathrm{H} & -3.967258 & -0.945410 & 1.096699 \\ \mathrm{C} & -2.146615 & -1.994125 & 1.005240 \\ \mathrm{C} & -0.742906 & -2.083478 & 0.727051 \\ \mathrm{H} & -0.427394 & -1.838107 & -0.276074 \\ \mathrm{C} & -0.054401 & -3.227154 & 1.251652 \\ \mathrm{O} & -2.827820 & -3.096866 & 1.428311 \\ \mathrm{H} & -2.294470 & -3.894226 & 1.268656 \\ \mathrm{~N} & 0.501954 & -4.150353 & 1.696992\end{array}$<smiles>N#C/C=C(/F)c1ccccc1</smiles>

\begin{tabular}{cccc}
$\mathrm{C}$ & 0.000000 & 0.000000 & \multicolumn{1}{c}{0.000000} \\
$\mathrm{H}$ & 0.000000 & 0.000000 & 1.087914 \\
$\mathrm{H}$ & 0.969953 & 0.000000 & -0.487433 \\
$\mathrm{C}$ & -1.143450 & 0.000000 & -0.706485 \\
$\mathrm{H}$ & -1.105715 & 0.000000 & -1.789786 \\
$\mathrm{C}$ & -2.441796 & 0.000000 & -0.058276 \\
$\mathrm{H}$ & -2.407683 & -0.000017 & 1.030863 \\
$\mathrm{C}$ & -3.682657 & 0.000018 & -0.608772 \\
$\mathrm{H}$ & -4.536158 & -0.000002 & 0.064026
\end{tabular}




$\begin{array}{lrrr}\text { C } & -4.051598 & 0.000043 & -2.002400 \\ \text { C } & -5.323787 & -0.000037 & -2.467527 \\ \text { H } & -6.144542 & -0.000110 & -1.758620 \\ \text { F } & -3.051061 & 0.000049 & -2.902182 \\ \text { C } & -5.640630 & -0.000009 & -3.849165 \\ \text { N } & -5.929307 & 0.000015 & -4.977902\end{array}$

TS:

$\begin{array}{cccc}\mathrm{H} & 0.000000 & 0.000000 & 0.000000 \\ \mathrm{C} & 0.000000 & 0.000000 & 1.080070 \\ \mathrm{H} & 0.993381 & 0.000000 & 1.524556 \\ \mathrm{C} & -1.049324 & 0.663970 & 1.721176 \\ \mathrm{H} & -0.836567 & 1.292861 & 2.585379 \\ \mathrm{C} & -2.384504 & 0.417606 & 1.419203 \\ \mathrm{H} & -3.139531 & 1.114262 & 1.778544 \\ \mathrm{C} & -2.868316 & -0.823102 & 0.960546 \\ \mathrm{H} & -3.944115 & -0.976323 & 0.980517 \\ \mathrm{C} & -2.107489 & -1.983050 & 0.894821 \\ \mathrm{C} & -0.710721 & -2.123439 & 0.691690 \\ \mathrm{H} & -0.303261 & -1.854249 & -0.270229 \\ \mathrm{C} & -0.056472 & -3.257898 & 1.275015 \\ \mathrm{~F} & -2.767757 & -3.116302 & 1.239367 \\ \mathrm{~N} & 0.530789 & -4.137490 & 1.762016\end{array}$<smiles>N#C/C=C(C=O)\C=C/C=C\C=O</smiles>

$\begin{array}{cccc}\mathrm{C} & 0.000000 & 0.000000 & 0.000000 \\ \mathrm{H} & 0.000000 & 0.000000 & 1.087661 \\ \mathrm{H} & 0.969693 & 0.000000 & -0.487959 \\ \mathrm{C} & -1.144390 & 0.006065 & -0.703803 \\ \mathrm{H} & -1.094238 & 0.002976 & -1.790089 \\ \mathrm{C} & -2.455136 & -0.031328 & -0.079290 \\ \mathrm{H} & -2.446560 & -0.220116 & 0.993985 \\ \mathrm{C} & -3.675004 & 0.074447 & -0.661027 \\ \mathrm{H} & -4.547287 & -0.120798 & -0.043135 \\ \mathrm{C} & -4.003883 & 0.359311 & -2.052053 \\ \mathrm{C} & -3.262089 & 1.134374 & -2.892535 \\ \mathrm{H} & -2.366032 & 1.632529 & -2.535156 \\ \mathrm{C} & -3.591248 & 1.395823 & -4.253757 \\ \mathrm{C} & -5.313840 & -0.182858 & -2.539588 \\ \mathrm{O} & -6.092365 & -0.779118 & -1.824388 \\ \mathrm{H} & -5.541760 & 0.007792 & -3.605836\end{array}$




$\begin{array}{cccc}\mathrm{N} & -3.813073 & 1.638383 & -5.371871 \\ \mathrm{TS}: & & & \\ \mathrm{H} & 0.000000 & 0.000000 & 0.000000 \\ \mathrm{C} & 0.000000 & 0.000000 & 1.078829 \\ \mathrm{H} & 0.996930 & 0.000000 & 1.516762 \\ \mathrm{C} & -1.031801 & 0.654523 & 1.752486 \\ \mathrm{H} & -0.768863 & 1.287397 & 2.602134 \\ \mathrm{C} & -2.399592 & 0.430997 & 1.557452 \\ \mathrm{H} & -3.093439 & 1.166194 & 1.961401 \\ \mathrm{C} & -2.951788 & -0.821944 & 1.256854 \\ \mathrm{H} & -4.018355 & -0.945092 & 1.427771 \\ \mathrm{C} & -2.213983 & -2.009870 & 1.163727 \\ \mathrm{C} & -0.886378 & -2.062123 & 0.661023 \\ \mathrm{H} & -0.733428 & -1.707658 & -0.347032 \\ \mathrm{C} & 0.001017 & -3.134067 & 1.010411 \\ \mathrm{C} & -2.847879 & -3.241493 & 1.711287 \\ \mathrm{O} & -4.032547 & -3.335911 & 1.973415 \\ \mathrm{H} & -2.163166 & -4.102672 & 1.842836 \\ \mathrm{~N} & 0.768886 & -3.961427 & 1.298266\end{array}$<smiles>N#C/C=C\C=C/C=C\C#N</smiles>

$\begin{array}{cccc}\mathrm{C} & 0.000000 & 0.000000 & 0.000000 \\ \mathrm{H} & 0.000000 & 0.000000 & 1.087918 \\ \mathrm{H} & 0.969411 & 0.000000 & -0.488327 \\ \mathrm{C} & -1.143691 & 0.000000 & -0.706992 \\ \mathrm{H} & -1.096355 & 0.000000 & -1.790276 \\ \mathrm{C} & -2.437748 & 0.000000 & -0.053317 \\ \mathrm{H} & -2.390227 & 0.000000 & 1.035535 \\ \mathrm{C} & -3.692740 & 0.000000 & -0.573639 \\ \mathrm{H} & -4.513563 & 0.000000 & 0.140118 \\ \mathrm{C} & -4.148622 & 0.000000 & -1.955334 \\ \mathrm{C} & -5.485588 & -0.000021 & -2.239296 \\ \mathrm{H} & -6.198489 & -0.000037 & -1.418879 \\ \mathrm{C} & -6.035827 & -0.000023 & -3.546664 \\ \mathrm{C} & -3.220933 & -0.000021 & -3.054351 \\ \mathrm{~N} & -2.478748 & -0.000040 & -3.949143 \\ \mathrm{~N} & -6.528465 & -0.000025 & -4.602553\end{array}$

TS

$\begin{array}{llll}\mathrm{H} & 0.000000 & 0.000000 & 0.000000\end{array}$ 


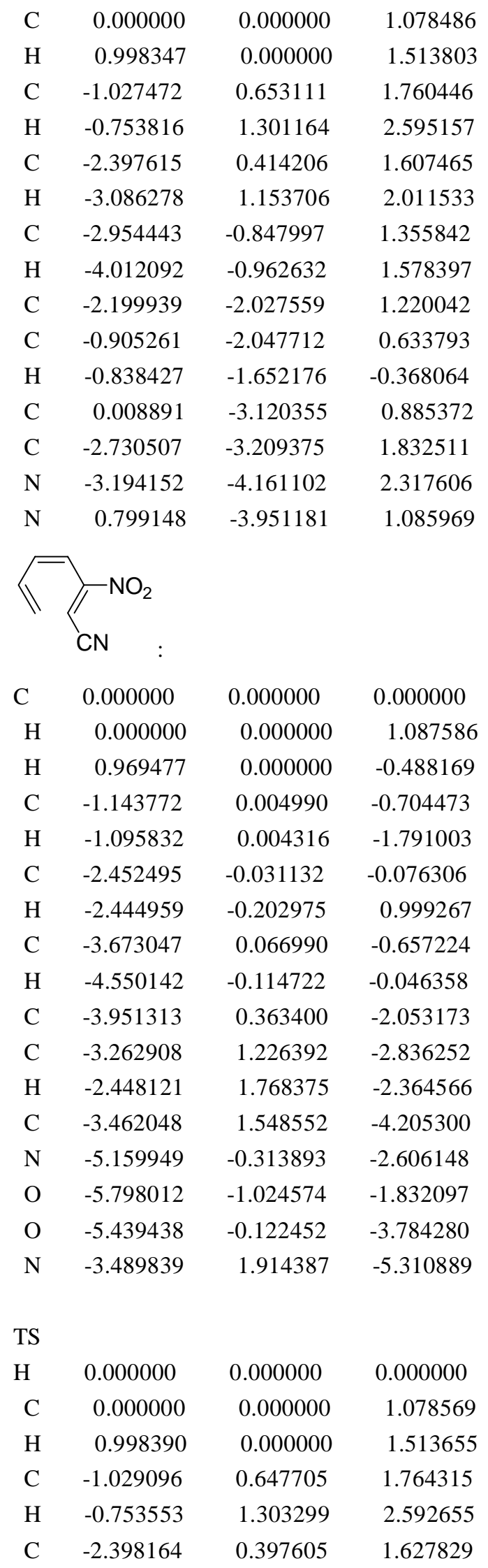




$\begin{array}{lrrr}\mathrm{H} & -3.088597 & 1.130771 & 2.039747 \\ \mathrm{C} & -2.947013 & -0.869687 & 1.377721 \\ \mathrm{H} & -3.997196 & -1.012978 & 1.606887 \\ \mathrm{C} & -2.179234 & -2.023934 & 1.218173 \\ \mathrm{C} & -0.897241 & -2.049486 & 0.625967 \\ \mathrm{H} & -0.873982 & -1.634449 & -0.371120 \\ \mathrm{C} & 0.090027 & -3.069792 & 0.808078 \\ \mathrm{~N} & -2.722730 & -3.242043 & 1.852665 \\ \mathrm{O} & -1.921858 & -4.097984 & 2.218166 \\ \mathrm{O} & -3.945683 & -3.323837 & 1.965497 \\ \mathrm{~N} & 0.996943 & -3.796888 & 0.871021\end{array}$

7<smiles>Cc1ccccc1[N+](=O)[O-]</smiles>

$\begin{array}{llll}\text { C } & 0.000000 & 0.000000 & 0.000000\end{array}$

$\begin{array}{llll}\mathrm{H} & 0.000000 & 0.000000 & 1.087772\end{array}$

$\begin{array}{llll}\mathrm{H} & 0.969889 & 0.000000 & -0.487613\end{array}$

$\begin{array}{llll}\text { C } & -1.144557 & 0.000899 & -0.705238\end{array}$

$\begin{array}{llll}\mathrm{H} & -1.088244 & -0.006433 & -1.787718\end{array}$

$\begin{array}{llll}\text { C } & -2.445360 & -0.015246 & -0.059109\end{array}$

$\begin{array}{llll}\mathrm{H} & -2.389745 & -0.081125 & 1.027779\end{array}$

$\begin{array}{llll}\text { C } & -3.704566 & 0.003187 & -0.568535\end{array}$

$\begin{array}{llll}\mathrm{H} & -4.500255 & -0.085238 & 0.168838\end{array}$

$\begin{array}{llll}\text { C } & -4.186217 & 0.097533 & -1.946435\end{array}$

$\begin{array}{llll}\text { C } & -5.517027 & -0.134915 & -2.088502\end{array}$

$\begin{array}{llll}\mathrm{H} & -6.164272 & -0.332113 & -1.244692\end{array}$

$\begin{array}{llll}\text { C } & -3.256167 & 0.467139 & -3.073326\end{array}$

N $\quad-6.286838 \quad-0.115288 \quad-3.307683$

$\begin{array}{llll}\text { O } & -5.725782 & -0.116466 & -4.410197\end{array}$

$\begin{array}{llll}\mathrm{O} & -7.514170 & -0.119063 & -3.155687\end{array}$

$\mathrm{H} \quad-2.565441 \quad 1.248374 \quad-2.745507$

$\begin{array}{llll}\mathrm{H} & -2.662078 & -0.402507 & -3.381809\end{array}$

$\mathrm{H} \quad-3.806901 \quad 0.806311 \quad-3.947225$

TS

$\begin{array}{llll}\text { C } & 0.000000 & 0.000000 & 0.000000\end{array}$

$\begin{array}{llll}\mathrm{H} & 0.000000 & 0.000000 & 1.078099\end{array}$

$\begin{array}{llll}\mathrm{H} & 1.002626 & 0.000000 & -0.428153\end{array}$

$\begin{array}{llll}\text { C } & -1.022101 & -0.649647 & -0.695169\end{array}$

$\begin{array}{llll}\mathrm{H} & -0.742861 & -1.300770 & -1.525547\end{array}$ 


\begin{tabular}{|c|c|c|c|}
\hline $\mathrm{C}$ & -2.387416 & -0.383912 & -0.564793 \\
\hline $\mathrm{H}$ & -3.079682 & -1.109706 & -0.989444 \\
\hline $\mathrm{C}$ & -2.940749 & 0.882063 & -0.295738 \\
\hline $\mathrm{H}$ & -4.000480 & 0.986044 & -0.522661 \\
\hline $\mathrm{C}$ & -2.204205 & 2.067450 & -0.168021 \\
\hline $\mathrm{C}$ & -0.934937 & 2.010348 & 0.449187 \\
\hline $\mathrm{H}$ & -0.868475 & 1.645992 & 1.459498 \\
\hline $\mathrm{N}$ & 0.037917 & 3.084768 & 0.283347 \\
\hline $\mathrm{C}$ & -2.779404 & 3.324058 & -0.788674 \\
\hline $\mathrm{H}$ & -3.872774 & 3.256613 & -0.788646 \\
\hline $\mathrm{H}$ & -2.451143 & 3.433398 & -1.827886 \\
\hline $\mathrm{H}$ & -2.478563 & 4.232930 & -0.262710 \\
\hline $\mathrm{O}$ & 0.010839 & 3.764754 & -0.746386 \\
\hline $\mathrm{O}$ & 0.877123 & 3.204948 & 1.182148 \\
\hline & $-\mathrm{NH}$ & & \\
\hline $\mathrm{C}$ & 0.000000 & 0.000000 & 0.000000 \\
\hline $\mathrm{H}$ & 0.000000 & 0.000000 & 1.087833 \\
\hline $\mathrm{H}$ & 0.970071 & 0.000000 & -0.487344 \\
\hline $\mathrm{C}$ & -1.142859 & 0.000319 & -0.705514 \\
\hline $\mathrm{H}$ & -1.099226 & 0.012352 & -1.791332 \\
\hline $\mathrm{C}$ & -2.452723 & 0.034622 & -0.072069 \\
\hline $\mathrm{H}$ & -2.432095 & 0.178635 & 1.007992 \\
\hline $\mathrm{C}$ & -3.680079 & -0.033849 & -0.639090 \\
\hline $\mathrm{H}$ & -4.538773 & 0.112691 & 0.010091 \\
\hline $\mathrm{C}$ & -4.025885 & -0.263224 & -2.048869 \\
\hline $\mathrm{C}$ & -5.229616 & 0.249209 & -2.482477 \\
\hline $\mathrm{H}$ & -5.874398 & 0.823321 & -1.835826 \\
\hline $\mathrm{N}$ & -3.160750 & -0.938791 & -2.847568 \\
\hline $\mathrm{N}$ & -5.752180 & 0.081965 & -3.781838 \\
\hline $\mathrm{O}$ & -5.091727 & -0.550158 & -4.643400 \\
\hline $\mathrm{O}$ & -6.863891 & 0.570365 & -4.016603 \\
\hline $\mathrm{H}$ & -3.513412 & -1.153365 & -3.778196 \\
\hline $\mathrm{H}$ & -2.506128 & -1.572421 & -2.413320 \\
\hline \multicolumn{4}{|l|}{$\mathrm{T}$} \\
\hline $\mathrm{H}$ & 0.000000 & 0.000000 & 0.000000 \\
\hline $\mathrm{C}$ & 0.000000 & 0.000000 & 1.080657 \\
\hline $\mathrm{H}$ & 0.998097 & 0.000000 & 1.515989 \\
\hline $\mathrm{C}$ & -1.047276 & 0.661881 & 1.724406 \\
\hline $\mathrm{H}$ & -0.843888 & 1.284539 & 2.594802 \\
\hline $\mathrm{C}$ & -2.379846 & 0.377161 & 1.419037 \\
\hline
\end{tabular}




$\begin{array}{lrrr}\mathrm{H} & -3.150091 & 1.076165 & 1.744259 \\ \mathrm{C} & -2.842217 & -0.866558 & 0.965977 \\ \mathrm{H} & -3.922647 & -0.998594 & 0.931891 \\ \mathrm{C} & -2.089197 & -2.063800 & 1.020996 \\ \mathrm{C} & -0.675418 & -2.072330 & 0.752155 \\ \mathrm{H} & -0.351955 & -1.871627 & -0.254619 \\ \mathrm{~N} & 0.184967 & -3.121547 & 1.274230 \\ \mathrm{~N} & -2.748289 & -3.212892 & 1.380776 \\ \mathrm{H} & -3.567127 & -3.103118 & 1.964830 \\ \mathrm{H} & -2.147318 & -3.981118 & 1.658468 \\ \mathrm{O} & -0.207228 & -3.871733 & 2.187438 \\ \mathrm{O} & 1.326877 & -3.162060 & 0.807415\end{array}$<smiles></smiles>

$\begin{array}{cccc}\mathrm{C} & 0.000000 & 0.000000 & 0.000000 \\ \mathrm{H} & 0.000000 & 0.000000 & 1.088072 \\ \mathrm{H} & 0.970746 & 0.000000 & -0.486240 \\ \mathrm{C} & -1.143081 & 0.000000 & -0.707581 \\ \mathrm{H} & -1.114925 & 0.000000 & -1.790735 \\ \mathrm{C} & -2.438132 & 0.000000 & -0.053687 \\ \mathrm{H} & -2.398526 & 0.000000 & 1.035769 \\ \mathrm{C} & -3.681450 & 0.000000 & -0.597371 \\ \mathrm{H} & -4.527774 & 0.000000 & 0.083821 \\ \mathrm{C} & -4.052878 & 0.000000 & -2.000688 \\ \mathrm{C} & -5.391837 & 0.000000 & -2.333957 \\ \mathrm{H} & -6.183800 & 0.000016 & -1.602054 \\ \mathrm{~N} & -5.842613 & 0.000000 & -3.665717 \\ \mathrm{O} & -3.088174 & 0.000000 & -2.913761 \\ \mathrm{H} & -3.559570 & 0.000118 & -3.801411 \\ \mathrm{O} & -4.988175 & -0.000174 & -4.602595 \\ \mathrm{O} & -7.053553 & 0.000019 & -3.880960\end{array}$

TS:

$\begin{array}{cccc}\mathrm{H} & 0.000000 & 0.000000 & 0.000000 \\ \mathrm{C} & 0.000000 & 0.000000 & 1.080248 \\ \mathrm{H} & 1.003052 & 0.000000 & 1.505895 \\ \mathrm{C} & -1.024620 & 0.699866 & 1.732416 \\ \mathrm{H} & -0.772278 & 1.365749 & 2.557347 \\ \mathrm{C} & -2.374193 & 0.414649 & 1.536331 \\ \mathrm{H} & -3.108775 & 1.132364 & 1.898983 \\ \mathrm{C} & -2.886929 & -0.853659 & 1.209379\end{array}$




$\begin{array}{rrrc}\mathrm{H} & -3.955093 & -1.005172 & 1.341090 \\ \mathrm{C} & -2.119286 & -2.025823 & 1.214621 \\ \mathrm{C} & -0.743776 & -2.031412 & 0.792392 \\ \mathrm{H} & -0.531956 & -1.820505 & -0.241509 \\ \mathrm{~N} & 0.125824 & -3.127032 & 1.189241 \\ \mathrm{O} & -2.720879 & -3.116476 & 1.723990 \\ \mathrm{H} & -2.021930 & -3.802362 & 1.847637 \\ \mathrm{O} & -0.283027 & -3.987388 & 2.001892 \\ \mathrm{O} & 1.260341 & -3.133071 & 0.719543\end{array}$<smiles></smiles>

$\begin{array}{cccc}\mathrm{C} & 0.000000 & 0.000000 & 0.000000 \\ \mathrm{H} & 0.000000 & 0.000000 & 1.087872 \\ \mathrm{H} & 0.969940 & 0.000000 & -0.487426 \\ \mathrm{C} & -1.143431 & 0.000000 & -0.706749 \\ \mathrm{H} & -1.105857 & -0.000016 & -1.789767 \\ \mathrm{C} & -2.439015 & 0.000000 & -0.053522 \\ \mathrm{H} & -2.398895 & 0.000000 & 1.035427 \\ \mathrm{C} & -3.684364 & 0.000000 & -0.594085 \\ \mathrm{H} & -4.532206 & 0.000000 & 0.085213 \\ \mathrm{C} & -4.055997 & 0.000000 & -1.989999 \\ \mathrm{C} & -5.350843 & -0.000020 & -2.388024 \\ \mathrm{H} & -6.155418 & -0.000037 & -1.668372 \\ \mathrm{~F} & -3.061667 & 0.000000 & -2.879032 \\ \mathrm{~N} & -5.813876 & -0.000001 & -3.747010 \\ \mathrm{O} & -4.998721 & -0.000001 & -4.671655 \\ \mathrm{O} & -7.043707 & -0.000026 & -3.886044\end{array}$

TS:

$\begin{array}{cccc}\mathrm{H} & 0.000000 & 0.000000 & 0.000000 \\ \mathrm{C} & 0.000000 & 0.000000 & 1.080196 \\ \mathrm{H} & 0.986768 & 0.000000 & 1.537192 \\ \mathrm{C} & -1.083481 & 0.579279 & 1.725589 \\ \mathrm{H} & -0.932994 & 1.135606 & 2.649810 \\ \mathrm{C} & -2.394817 & 0.331774 & 1.315507 \\ \mathrm{H} & -3.189416 & 0.990261 & 1.661227 \\ \mathrm{C} & -2.809333 & -0.864973 & 0.713355 \\ \mathrm{H} & -3.877522 & -1.017446 & 0.583243 \\ \mathrm{C} & -2.044166 & -2.029979 & 0.645120 \\ \mathrm{C} & -0.641126 & -2.163356 & 0.572558 \\ \mathrm{H} & -0.086157 & -1.898986 & -0.309780\end{array}$




$\begin{array}{cccc}\mathrm{N} & -0.033490 & -3.351001 & 1.170937 \\ \mathrm{~F} & -2.761443 & -3.167049 & 0.771123 \\ \mathrm{O} & -0.539581 & -3.824251 & 2.188409 \\ \mathrm{O} & 1.006286 & -3.754754 & 0.639068\end{array}$<smiles>C=C/C=C\C(C=O)=C/[N+](=O)[O-]</smiles>

$\begin{array}{cccc}\mathrm{C} & 0.000000 & 0.000000 & 0.000000 \\ \mathrm{H} & 0.000000 & 0.000000 & 1.087635 \\ \mathrm{H} & 0.969544 & 0.000000 & -0.488118 \\ \mathrm{C} & -1.144307 & 0.008164 & -0.704267 \\ \mathrm{H} & -1.095737 & 0.009426 & -1.790501 \\ \mathrm{C} & -2.452653 & -0.025406 & -0.076021 \\ \mathrm{H} & -2.439981 & -0.208487 & 0.998178 \\ \mathrm{C} & -3.677190 & 0.075165 & -0.650891 \\ \mathrm{H} & -4.543679 & -0.117621 & -0.025867 \\ \mathrm{C} & -4.005208 & 0.338112 & -2.050323 \\ \mathrm{C} & -3.240529 & 1.155488 & -2.815796 \\ \mathrm{H} & -2.414411 & 1.731547 & -2.423409 \\ \mathrm{C} & -5.322612 & -0.250688 & -2.506223 \\ \mathrm{~N} & -3.441705 & 1.455217 & -4.221050 \\ \mathrm{O} & -5.977767 & -0.958770 & -1.768687 \\ \mathrm{H} & -5.669298 & 0.019871 & -3.511489 \\ \mathrm{O} & -4.126491 & 0.704455 & -4.922899 \\ \mathrm{O} & -2.869436 & 2.469820 & -4.627355\end{array}$

TS:

$\begin{array}{cccc}\mathrm{H} & 0.000000 & 0.000000 & 0.000000 \\ \mathrm{C} & 0.000000 & 0.000000 & 1.078763 \\ \mathrm{H} & 0.989017 & 0.000000 & 1.532051 \\ \mathrm{C} & -1.072036 & 0.566728 & 1.749545 \\ \mathrm{H} & -0.882372 & 1.122649 & 2.668650 \\ \mathrm{C} & -2.415804 & 0.342107 & 1.415563 \\ \mathrm{H} & -3.159644 & 1.037835 & 1.800464 \\ \mathrm{C} & -2.917175 & -0.873119 & 0.939919 \\ \mathrm{H} & -3.997194 & -0.992533 & 0.932162 \\ \mathrm{C} & -2.186873 & -2.074045 & 0.878107 \\ \mathrm{C} & -0.813284 & -2.124818 & 0.566396 \\ \mathrm{H} & -0.431735 & -1.817898 & -0.391402 \\ \mathrm{~N} & 0.007340 & -3.222885 & 1.072436 \\ \mathrm{C} & -2.972916 & -3.324096 & 1.162876 \\ \mathrm{H} & -2.407979 & -4.268802 & 1.150901\end{array}$




$\begin{array}{lrrr}\mathrm{O} & -4.176844 & -3.313700 & 1.332592 \\ \mathrm{O} & -0.337579 & -3.776559 & 2.121458 \\ \mathrm{O} & 1.030876 & -3.484595 & 0.435836\end{array}$<smiles>C/C=C\C=C/C(C#N)=C\[N+](=O)[O-]</smiles>

$\begin{array}{cccc}\mathrm{C} & 0.000000 & 0.000000 & 0.000000 \\ \mathrm{H} & 0.000000 & 0.000000 & 1.087986 \\ \mathrm{H} & 0.969595 & 0.000000 & -0.488037 \\ \mathrm{C} & -1.143241 & 0.000000 & -0.708757 \\ \mathrm{H} & -1.097896 & 0.000000 & -1.791125 \\ \mathrm{C} & -2.432460 & 0.000000 & -0.046956 \\ \mathrm{H} & -2.372200 & -0.000017 & 1.041444 \\ \mathrm{C} & -3.699357 & 0.000000 & -0.540873 \\ \mathrm{H} & -4.498140 & 0.000000 & 0.196713 \\ \mathrm{C} & -4.198247 & 0.000000 & -1.911337 \\ \mathrm{C} & -5.544904 & 0.000063 & -2.094777 \\ \mathrm{H} & -6.243506 & 0.000080 & -1.269905 \\ \mathrm{C} & -3.278511 & 0.000000 & -3.017553 \\ \mathrm{~N} & -6.214563 & 0.000135 & -3.370073 \\ \mathrm{~N} & -2.441657 & -0.000007 & -3.824311 \\ \mathrm{O} & -5.544766 & 0.000198 & -4.404116 \\ \mathrm{O} & -7.449059 & 0.000204 & -3.317491\end{array}$

TS:

$\begin{array}{cccc}\mathrm{H} & 0.000000 & 0.000000 & 0.000000 \\ \mathrm{C} & 0.000000 & 0.000000 & 1.078218 \\ \mathrm{H} & 0.993300 & 0.000000 & 1.523479 \\ \mathrm{C} & -1.053338 & 0.596921 & 1.758780 \\ \mathrm{H} & -0.827821 & 1.190878 & 2.645706 \\ \mathrm{C} & -2.411956 & 0.359145 & 1.507326 \\ \mathrm{H} & -3.133317 & 1.076955 & 1.893381 \\ \mathrm{C} & -2.943292 & -0.881862 & 1.140113 \\ \mathrm{H} & -4.019367 & -0.996723 & 1.237787 \\ \mathrm{C} & -2.189184 & -2.072559 & 1.043412 \\ \mathrm{C} & -0.859593 & -2.082607 & 0.575270 \\ \mathrm{H} & -0.638111 & -1.741424 & -0.420109 \\ \mathrm{~N} & 0.038793 & -3.182743 & 0.921206 \\ \mathrm{C} & -2.840621 & -3.255269 & 1.529700 \\ \mathrm{~N} & -3.478691 & -4.163587 & 1.879823 \\ \mathrm{O} & -0.172955 & -3.810580 & 1.957216 \\ \mathrm{O} & 0.988910 & -3.360825 & 0.154295\end{array}$




\begin{tabular}{cccc}
\hline & \multicolumn{3}{l}{} \\
& & & \\
& & & \\
& & & \\
& & & \\
$\mathrm{NO} O_{2}$ & & \\
$\mathrm{C}$ & 0.000000 & 0.000000 & 0.000000 \\
$\mathrm{H}$ & 0.000000 & 0.000000 & 1.087563 \\
$\mathrm{H}$ & 0.969366 & 0.000000 & -0.488240 \\
$\mathrm{C}$ & -1.143486 & 0.005372 & -0.705093 \\
$\mathrm{H}$ & -1.099973 & 0.004175 & -1.791240 \\
$\mathrm{C}$ & -2.447884 & -0.023808 & -0.071116 \\
$\mathrm{H}$ & -2.438987 & -0.170894 & 1.008096 \\
$\mathrm{C}$ & -3.669248 & 0.058308 & -0.652113 \\
$\mathrm{H}$ & -4.550876 & -0.090403 & -0.033378 \\
$\mathrm{C}$ & -3.962579 & 0.295877 & -2.057060 \\
$\mathrm{C}$ & -3.305193 & 1.128468 & -2.880332 \\
$\mathrm{H}$ & -2.469443 & 1.728298 & -2.551497 \\
$\mathrm{~N}$ & -5.172153 & -0.454020 & -2.502091 \\
$\mathrm{~N}$ & -3.632562 & 1.345526 & -4.273469 \\
$\mathrm{O}$ & -4.656285 & 0.838541 & -4.731556 \\
$\mathrm{O}$ & -2.836874 & 2.049396 & -4.899238 \\
$\mathrm{O}$ & -4.980466 & -1.529785 & -3.045230 \\
$\mathrm{O}$ & -6.245587 & 0.036987 & -2.179696
\end{tabular}

TS:

$\begin{array}{cccc}\mathrm{H} & 0.000000 & 0.000000 & 0.000000 \\ \mathrm{C} & 0.000000 & 0.000000 & 1.078854 \\ \mathrm{H} & 0.983768 & 0.000000 & 1.542095 \\ \mathrm{C} & -1.092819 & 0.516037 & 1.747817 \\ \mathrm{H} & -0.940155 & 1.037723 & 2.692857 \\ \mathrm{C} & -2.419910 & 0.289831 & 1.354192 \\ \mathrm{H} & -3.190562 & 0.965222 & 1.720471 \\ \mathrm{C} & -2.883183 & -0.902817 & 0.793138 \\ \mathrm{H} & -3.958332 & -1.021431 & 0.714041 \\ \mathrm{C} & -2.131233 & -2.078594 & 0.678929 \\ \mathrm{C} & -0.753122 & -2.192110 & 0.471141 \\ \mathrm{H} & -0.262252 & -1.848953 & -0.421239 \\ \mathrm{~N} & -0.009015 & -3.314041 & 1.050122 \\ \mathrm{~N} & -2.928590 & -3.335967 & 0.857033 \\ \mathrm{O} & -2.477792 & -4.355808 & 0.345157 \\ \mathrm{O} & -4.007716 & -3.248608 & 1.437519 \\ \mathrm{O} & -0.375078 & -3.744128 & 2.141915 \\ \mathrm{O} & 0.985555 & -3.682598 & 0.423827\end{array}$




\section{1, 3-disubstituted hexatriene}

1<smiles>C/C=C\C(C)=C/C</smiles>

$\begin{array}{llll}\text { C } & 0.000000 & 0.000000 & 0.000000\end{array}$

$\begin{array}{llll}\mathrm{H} & 0.000000 & 0.000000 & 1.087979\end{array}$

$\begin{array}{llll}\mathrm{H} & 0.970199 & 0.000000 & -0.487344\end{array}$

$\begin{array}{llll}\text { C } & -1.145042 & 0.000160 & -0.703361\end{array}$

$\begin{array}{llll}\mathrm{H} & -1.081160 & 0.000218 & -1.789509\end{array}$

$\begin{array}{llll}\text { C } & -2.461554 & 0.000322 & -0.091474\end{array}$

$\begin{array}{llll}\mathrm{H} & -2.454989 & -0.000353 & 0.998814\end{array}$

$\begin{array}{llll}\text { C } & -3.675481 & 0.001236 & -0.706749\end{array}$

$\begin{array}{llll}\text { C } & -3.825452 & 0.002199 & -2.160717\end{array}$

$\begin{array}{llll}\mathrm{H} & -2.910031 & 0.001921 & -2.749378\end{array}$

$\begin{array}{llll}\text { C } & -4.990934 & 0.003393 & -2.833175\end{array}$

$\begin{array}{llll}\mathrm{H} & -5.930541 & 0.003709 & -2.282506\end{array}$

$\begin{array}{llll}\text { C } & -4.931024 & 0.001201 & 0.134081\end{array}$

$\begin{array}{llll}\text { C } & -5.109468 & 0.004359 & -4.328277\end{array}$

$\mathrm{H} \quad-5.551251 \quad 0.882944 \quad-0.073820$

$\begin{array}{llll}\mathrm{H} & -4.692849 & 0.000233 & 1.201453\end{array}$

$\begin{array}{llll}\mathrm{H} & -5.552121 & -0.879606 & -0.075221\end{array}$

$\mathrm{H} \quad-5.663967 \quad-0.874921 \quad-4.685081$

$\begin{array}{llll}\mathrm{H} & -5.662871 & 0.884704 & -4.684117\end{array}$

$\begin{array}{llll}\mathrm{H} & -4.126747 & 0.003973 & -4.811943\end{array}$

$\begin{array}{cccc}\text { TS } & & & \\ \mathrm{H} & 0.000000 & 0.000000 & 0.000000 \\ \mathrm{C} & 0.000000 & 0.000000 & 1.078674 \\ \mathrm{H} & 1.006249 & 0.000000 & 1.501042 \\ \mathrm{C} & -0.991619 & 0.727671 & 1.747352 \\ \mathrm{H} & -0.685111 & 1.394642 & 2.556932 \\ \mathrm{C} & -2.366344 & 0.529527 & 1.613073 \\ \mathrm{H} & -3.020217 & 1.296488 & 2.028397 \\ \mathrm{C} & -2.981725 & -0.718525 & 1.371424 \\ \mathrm{C} & -2.235283 & -1.896310 & 1.226781 \\ \mathrm{H} & -2.642625 & -2.774760 & 1.735991 \\ \mathrm{C} & -0.953466 & -2.016322 & 0.670356 \\ \mathrm{H} & -0.836347 & -1.638316 & -0.335199 \\ \mathrm{C} & -0.102047 & -3.231498 & 0.964003 \\ \mathrm{C} & -4.474540 & -0.834685 & 1.604768\end{array}$




$\begin{array}{lrrl}\mathrm{H} & -5.023945 & -0.761891 & 0.656180 \\ \mathrm{H} & -4.733586 & -1.805031 & 2.044834 \\ \mathrm{H} & -4.855718 & -0.046251 & 2.263026 \\ \mathrm{H} & -0.233579 & -3.575883 & 1.995084 \\ \mathrm{H} & -0.353868 & -4.066319 & 0.293211 \\ \mathrm{H} & 0.961229 & -3.013804 & 0.816325\end{array}$<smiles>C/C=C\C=C(N)/C=C\C</smiles>

$\begin{array}{lrrr}\mathrm{C} & 0.000000 & 0.000000 & 0.000000 \\ \mathrm{H} & 0.000000 & 0.000000 & 1.087924 \\ \mathrm{H} & 0.969621 & 0.000000 & -0.487859 \\ \mathrm{C} & -1.145254 & 0.005780 & -0.706486 \\ \mathrm{H} & -1.074533 & 0.020565 & -1.792927 \\ \mathrm{C} & -2.468161 & 0.003162 & -0.122894 \\ \mathrm{H} & -2.502957 & 0.036698 & 0.967236 \\ \mathrm{C} & -3.660066 & 0.001694 & -0.788309 \\ \mathrm{C} & -3.773546 & -0.120057 & -2.244213 \\ \mathrm{H} & -2.893221 & -0.488136 & -2.764859 \\ \mathrm{C} & -4.872403 & 0.166025 & -2.963157 \\ \mathrm{H} & -5.747464 & 0.570533 & -2.455669 \\ \mathrm{C} & -4.982980 & 0.002971 & -4.449740 \\ \mathrm{~N} & -4.871101 & 0.170069 & -0.099212 \\ \mathrm{H} & -4.776738 & 0.126661 & 0.909474 \\ \mathrm{H} & -5.613144 & -0.443802 & -0.419550 \\ \mathrm{H} & -4.056153 & -0.385907 & -4.884768 \\ \mathrm{H} & -5.213333 & 0.960247 & -4.937208 \\ \mathrm{H} & -5.797680 & -0.684755 & -4.716394\end{array}$

TS

$\begin{array}{cccc}\mathrm{H} & 0.000000 & 0.000000 & 0.000000 \\ \mathrm{C} & 0.000000 & 0.000000 & 1.079116 \\ \mathrm{H} & 1.005734 & 0.000000 & 1.502198 \\ \mathrm{C} & -0.987678 & 0.745810 & 1.738786 \\ \mathrm{H} & -0.682237 & 1.425475 & 2.538612 \\ \mathrm{C} & -2.358361 & 0.570090 & 1.575549 \\ \mathrm{H} & -3.018029 & 1.341228 & 1.977249 \\ \mathrm{C} & -2.983477 & -0.670725 & 1.303691 \\ \mathrm{C} & -2.254833 & -1.870637 & 1.218161 \\ \mathrm{H} & -2.674382 & -2.711823 & 1.777409 \\ \mathrm{C} & -0.963332 & -2.008913 & 0.692970\end{array}$




$\begin{array}{lrrr}\mathrm{H} & -0.813758 & -1.672996 & -0.323601 \\ \mathrm{C} & -0.122689 & -3.212527 & 1.057229 \\ \mathrm{~N} & -4.380846 & -0.732062 & 1.406972 \\ \mathrm{H} & -4.750814 & -1.668638 & 1.521510 \\ \mathrm{H} & -4.799625 & -0.081823 & 2.063553 \\ \mathrm{H} & -0.268545 & -3.504227 & 2.102649 \\ \mathrm{H} & -0.372924 & -4.078109 & 0.425918 \\ \mathrm{H} & 0.943329 & -3.008261 & 0.911481\end{array}$<smiles>C/C=C\C=C(O)/C=C\C</smiles>

$\begin{array}{lrrr}\mathrm{C} & 0.000000 & 0.000000 & 0.000000 \\ \mathrm{H} & 0.000000 & 0.000000 & 1.087845 \\ \mathrm{H} & 0.969450 & 0.000000 & -0.487879 \\ \mathrm{C} & -1.143740 & 0.000020 & -0.706940 \\ \mathrm{H} & -1.073574 & 0.000002 & -1.793159 \\ \mathrm{C} & -2.467104 & 0.000043 & -0.121500 \\ \mathrm{H} & -2.499446 & -0.000335 & 0.970127 \\ \mathrm{C} & -3.657368 & 0.000651 & -0.781111 \\ \mathrm{C} & -3.829242 & 0.001160 & -2.227857 \\ \mathrm{H} & -2.918399 & 0.001530 & -2.819953 \\ \mathrm{C} & -5.018643 & 0.001157 & -2.852042 \\ \mathrm{H} & -5.924848 & 0.000850 & -2.250189 \\ \mathrm{C} & -5.194703 & 0.001465 & -4.340005 \\ \mathrm{O} & -4.847671 & 0.000800 & -0.100190 \\ \mathrm{H} & -4.660607 & 0.000414 & 0.851698 \\ \mathrm{H} & -5.764270 & 0.881018 & -4.670785 \\ \mathrm{H} & -4.233357 & 0.001730 & -4.864822 \\ \mathrm{H} & -5.764033 & -0.878102 & -4.671159\end{array}$

TS

$\begin{array}{cccc}\mathrm{H} & 0.000000 & 0.000000 & 0.000000 \\ \mathrm{C} & 0.000000 & 0.000000 & 1.079149 \\ \mathrm{H} & 1.003650 & 0.000000 & 1.505752 \\ \mathrm{C} & -0.996019 & 0.734114 & 1.735912 \\ \mathrm{H} & -0.706093 & 1.400929 & 2.551042 \\ \mathrm{C} & -2.363034 & 0.556809 & 1.544727 \\ \mathrm{H} & -3.057109 & 1.291782 & 1.947989 \\ \mathrm{C} & -2.954533 & -0.681710 & 1.231518 \\ \mathrm{C} & -2.248023 & -1.889333 & 1.154162 \\ \mathrm{H} & -2.701628 & -2.724128 & 1.697957\end{array}$




$\begin{array}{lrrr}\mathrm{C} & -0.932081 & -2.036346 & 0.691122 \\ \mathrm{H} & -0.730626 & -1.710774 & -0.319161 \\ \mathrm{C} & -0.114124 & -3.237353 & 1.110330 \\ \mathrm{O} & -4.323695 & -0.703738 & 1.386245 \\ \mathrm{H} & -4.638366 & -1.590075 & 1.152399 \\ \mathrm{H} & -0.304994 & -3.511893 & 2.152880 \\ \mathrm{H} & -0.341276 & -4.111661 & 0.482148 \\ \mathrm{H} & 0.957837 & -3.039654 & 1.005809\end{array}$

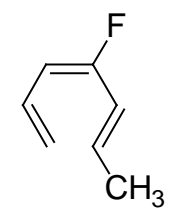

$\begin{array}{lrrr}\text { C } & 0.000000 & 0.000000 & 0.000000 \\ \text { H } & 0.000000 & 0.000000 & 1.087516 \\ \text { H } & 0.968935 & 0.000000 & -0.489126 \\ \text { C } & -1.143853 & -0.000080 & -0.704667 \\ \text { H } & -1.082550 & -0.000142 & -1.791627 \\ \text { C } & -2.462234 & -0.000172 & -0.104429 \\ \text { H } & -2.517620 & -0.000125 & 0.981781 \\ \text { C } & -3.640424 & -0.000254 & -0.765464 \\ \text { C } & -3.892412 & -0.000233 & -2.191645 \\ \text { H } & -3.015154 & -0.000222 & -2.833842 \\ \text { C } & -5.120137 & -0.000245 & -2.738623 \\ \text { H } & -5.984364 & -0.000206 & -2.077561 \\ \text { C } & -5.396670 & -0.000438 & -4.210296 \\ \text { F } & -4.769369 & -0.000121 & -0.007816 \\ \text { H } & -5.987373 & 0.879261 & -4.500741 \\ \text { H } & -5.988114 & -0.879749 & -4.500407 \\ \text { H } & -4.473899 & -0.000889 & -4.799865\end{array}$

$\begin{array}{cccc}\text { TS } & & & \\ \mathrm{H} & 0.000000 & 0.000000 & 0.000000 \\ \mathrm{C} & 0.000000 & 0.000000 & 1.079106 \\ \mathrm{H} & 1.001404 & 0.000000 & 1.509509 \\ \mathrm{C} & -1.003376 & 0.728140 & 1.729293 \\ \mathrm{H} & -0.730650 & 1.385596 & 2.556710 \\ \mathrm{C} & -2.365342 & 0.537794 & 1.513577 \\ \mathrm{H} & -3.082715 & 1.251402 & 1.912326 \\ \mathrm{C} & -2.909970 & -0.705791 & 1.163752 \\ \mathrm{C} & -2.228056 & -1.915333 & 1.088703 \\ \mathrm{H} & -2.741706 & -2.755686 & 1.559644 \\ \mathrm{C} & -0.888921 & -2.066473 & 0.693500\end{array}$




$\begin{array}{lrrc}\mathrm{H} & -0.634343 & -1.743470 & -0.304696 \\ \mathrm{C} & -0.099643 & -3.269544 & 1.157616 \\ \mathrm{~F} & -4.261881 & -0.792969 & 1.306503 \\ \mathrm{H} & -0.343472 & -3.540997 & 2.189556 \\ \mathrm{H} & -0.300676 & -4.143227 & 0.519983 \\ \mathrm{H} & 0.977046 & -3.077082 & 1.104637\end{array}$<smiles>C=C/C=C(/C=O)C=CC</smiles>

$\begin{array}{lrrr}\mathrm{C} & 0.000000 & 0.000000 & 0.000000 \\ \mathrm{H} & 0.000000 & 0.000000 & 1.087759 \\ \mathrm{H} & 0.969827 & 0.000000 & -0.487815 \\ \mathrm{C} & -1.146029 & 0.000000 & -0.704132 \\ \mathrm{H} & -1.091797 & 0.000033 & -1.790067 \\ \mathrm{C} & -2.450466 & 0.000021 & -0.079962 \\ \mathrm{H} & -2.436435 & 0.000124 & 1.010779 \\ \mathrm{C} & -3.677808 & -0.000014 & -0.684262 \\ \mathrm{C} & -3.870566 & -0.000316 & -2.133932 \\ \mathrm{H} & -2.958334 & -0.000981 & -2.729471 \\ \mathrm{C} & -5.042038 & 0.000119 & -2.795640 \\ \mathrm{H} & -5.968771 & 0.000800 & -2.230803 \\ \mathrm{C} & -4.816918 & 0.000260 & 0.268216 \\ \mathrm{C} & -5.151151 & -0.000195 & -4.290046 \\ \mathrm{O} & -6.003025 & 0.000475 & -0.006360 \\ \mathrm{H} & -4.497112 & 0.000311 & 1.334725 \\ \mathrm{H} & -5.706997 & 0.879266 & -4.643599 \\ \mathrm{H} & -5.708711 & -0.878739 & -4.643154 \\ \mathrm{H} & -4.168651 & -0.001261 & -4.774924\end{array}$

$\begin{array}{cccc}\text { TS } & & & \\ \mathrm{H} & 0.000000 & 0.000000 & 0.000000 \\ \mathrm{C} & 0.000000 & 0.000000 & 1.078314 \\ \mathrm{H} & 1.006971 & 0.000000 & 1.499064 \\ \mathrm{C} & -0.993342 & 0.713211 & 1.766435 \\ \mathrm{H} & -0.665394 & 1.409215 & 2.542486 \\ \mathrm{C} & -2.364669 & 0.486532 & 1.711847 \\ \mathrm{H} & -3.036408 & 1.224884 & 2.144382 \\ \mathrm{C} & -2.940182 & -0.791317 & 1.531113 \\ \mathrm{C} & -2.194028 & -1.957347 & 1.300825 \\ \mathrm{H} & -2.530907 & -2.858128 & 1.821793 \\ \mathrm{C} & -0.985356 & -2.014724 & 0.601974\end{array}$




$\begin{array}{cccc}\mathrm{H} & -0.992803 & -1.532861 & -0.365734 \\ \mathrm{C} & -0.079651 & -3.219566 & 0.693522 \\ \mathrm{C} & -4.348645 & -0.962417 & 1.964849 \\ \mathrm{H} & -0.094950 & -3.660335 & 1.695474 \\ \mathrm{H} & -0.381295 & -3.996123 & -0.024321 \\ \mathrm{H} & 0.956750 & -2.954658 & 0.458856 \\ \mathrm{H} & -4.672590 & -2.023469 & 2.058761 \\ \mathrm{O} & -5.130078 & -0.057192 & 2.191660\end{array}$<smiles>C=C/C=C(C#N)\C=C/C</smiles>

$\begin{array}{lrrr}\mathrm{C} & 0.000000 & 0.000000 & 0.000000 \\ \mathrm{H} & 0.000000 & 0.000000 & 1.087514 \\ \mathrm{H} & 0.969228 & 0.000000 & -0.488659 \\ \mathrm{C} & -1.145593 & -0.000020 & -0.703947 \\ \mathrm{H} & -1.092574 & -0.000036 & -1.789945 \\ \mathrm{C} & -2.449602 & 0.000000 & -0.077463 \\ \mathrm{H} & -2.451823 & 0.000135 & 1.010423 \\ \mathrm{C} & -3.662180 & -0.000132 & -0.707185 \\ \mathrm{C} & -3.876106 & -0.000322 & -2.155370 \\ \mathrm{H} & -2.981472 & -0.000427 & -2.772883 \\ \mathrm{C} & -5.077795 & -0.000450 & -2.756947 \\ \mathrm{H} & -5.976729 & -0.000380 & -2.141043 \\ \mathrm{C} & -5.284973 & -0.000579 & -4.240105 \\ \mathrm{C} & -4.832004 & -0.000103 & 0.129400 \\ \mathrm{H} & -5.861727 & 0.879286 & -4.556126 \\ \mathrm{H} & -5.862683 & -0.879850 & -4.555939 \\ \mathrm{H} & -4.335536 & -0.001035 & -4.785615 \\ \mathrm{~N} & -5.788557 & -0.000315 & 0.792541\end{array}$

TS

$\begin{array}{cccc}\mathrm{H} & 0.000000 & 0.000000 & 0.000000 \\ \mathrm{C} & 0.000000 & 0.000000 & 1.078194 \\ \mathrm{H} & 1.005938 & 0.000000 & 1.500452 \\ \mathrm{C} & -0.991637 & 0.720123 & 1.758019 \\ \mathrm{H} & -0.673379 & 1.413727 & 2.539348 \\ \mathrm{C} & -2.364278 & 0.501465 & 1.681855 \\ \mathrm{H} & -3.026920 & 1.252682 & 2.104817 \\ \mathrm{C} & -2.940092 & -0.778011 & 1.480333 \\ \mathrm{C} & -2.192556 & -1.953211 & 1.286719 \\ \mathrm{H} & -2.547456 & -2.842297 & 1.810326\end{array}$




$\begin{array}{lrrr}\mathrm{C} & -0.961734 & -2.013676 & 0.625910 \\ \mathrm{H} & -0.943219 & -1.566962 & -0.358244 \\ \mathrm{C} & -4.321805 & -0.924379 & 1.852496 \\ \mathrm{C} & -0.055095 & -3.211072 & 0.783461 \\ \mathrm{H} & -0.094782 & -3.616588 & 1.799258 \\ \mathrm{H} & -0.338476 & -4.011639 & 0.085353 \\ \mathrm{H} & 0.985542 & -2.949877 & 0.564802 \\ \mathrm{~N} & -5.445541 & -1.044770 & 2.131318\end{array}$

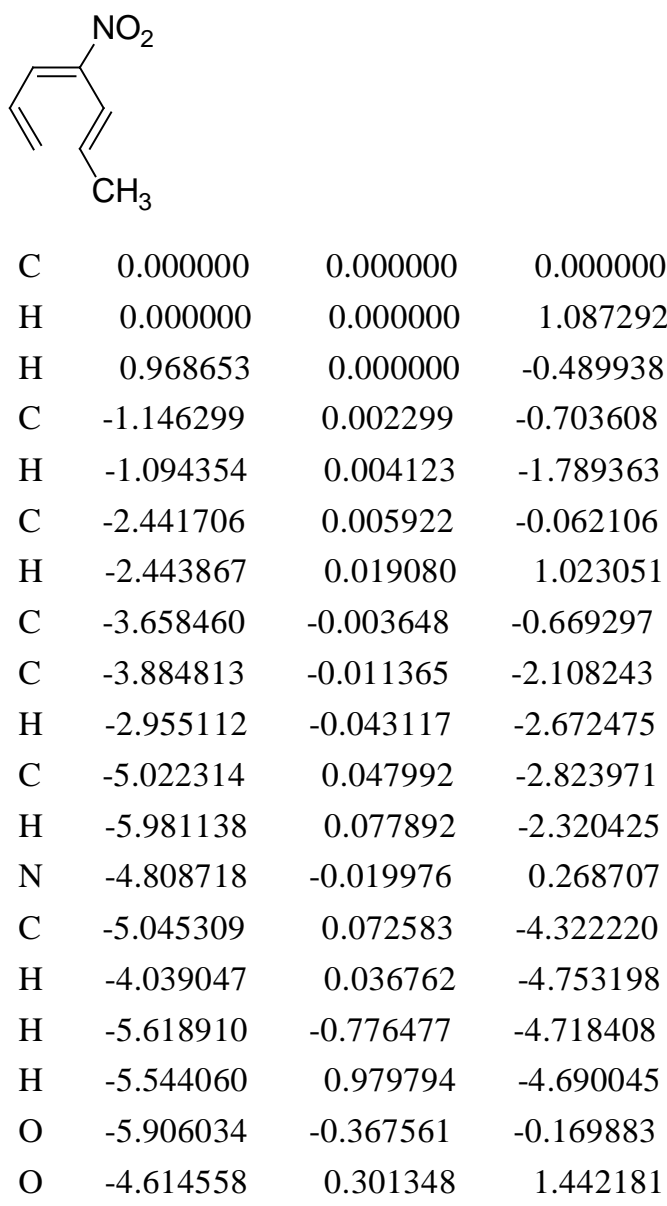

$\begin{array}{cccc}\text { TS } & & & \\ \mathrm{H} & 0.000000 & 0.000000 & 0.000000 \\ \mathrm{H} & 0.000000 & 0.000000 & 1.807604 \\ \mathrm{C} & 0.599493 & 0.000000 & 0.896454 \\ \mathrm{C} & 1.804456 & -0.716831 & 0.904480 \\ \mathrm{H} & 1.982965 & -1.410386 & 1.728380 \\ \mathrm{C} & 2.889124 & -0.495351 & 0.063779 \\ \mathrm{H} & 3.691028 & -1.224723 & 0.022482 \\ \mathrm{C} & 3.207931 & 0.776902 & -0.442281 \\ \mathrm{C} & 2.521121 & 1.960567 & -0.162338\end{array}$




$\begin{array}{lrrr}\mathrm{H} & 3.134488 & 2.832722 & 0.055318 \\ \mathrm{C} & 1.136210 & 2.033379 & 0.017361 \\ \mathrm{H} & 0.539433 & 1.609013 & -0.777464 \\ \mathrm{C} & 0.508488 & 3.225857 & 0.698852 \\ \mathrm{~N} & 4.593037 & 0.931749 & -0.950860 \\ \mathrm{O} & 5.005428 & 2.076284 & -1.146186 \\ \mathrm{O} & 5.247267 & -0.086017 & -1.180476 \\ \mathrm{H} & 1.136090 & 3.602966 & 1.512136 \\ \mathrm{H} & 0.348942 & 4.045231 & -0.016486 \\ \mathrm{H} & -0.470803 & 2.971189 & 1.117259\end{array}$

2

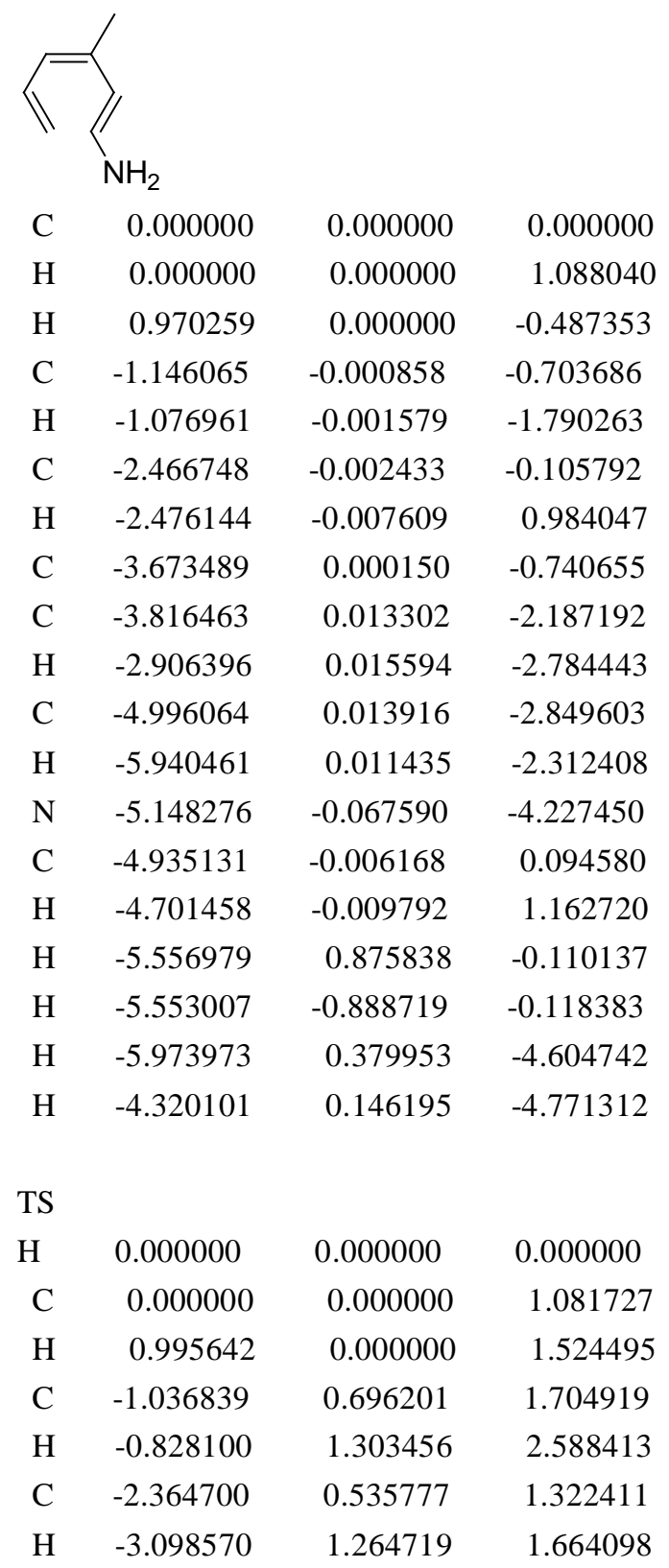




\begin{tabular}{|c|c|c|c|}
\hline $\mathrm{C}$ & -2.887196 & -0.662085 & 0.783364 \\
\hline $\mathrm{C}$ & -2.184466 & -1.869578 & 0.704891 \\
\hline $\mathrm{H}$ & -2.790055 & -2.764422 & 0.872085 \\
\hline $\mathrm{C}$ & -0.787548 & -2.122269 & 0.678918 \\
\hline $\mathrm{H}$ & -0.204125 & -1.886506 & -0.197011 \\
\hline $\mathrm{N}$ & -0.336479 & -3.291665 & 1.295309 \\
\hline $\mathrm{C}$ & -4.382743 & -0.717925 & 0.532516 \\
\hline $\mathrm{H}$ & 0.656853 & -3.467485 & 1.219638 \\
\hline $\mathrm{H}$ & -0.668023 & -3.433936 & 2.241267 \\
\hline $\mathrm{H}$ & -4.782298 & -1.725633 & 0.695038 \\
\hline $\mathrm{H}$ & -4.612072 & -0.448967 & -0.507529 \\
\hline \multirow[t]{2}{*}{$\mathrm{H}$} & -4.932893 & -0.021098 & 1.174807 \\
\hline & \multicolumn{3}{|c|}{$\mathrm{NH}_{2}$} \\
\hline $\mathrm{C}$ & 0.000000 & 0.000000 & 0.000000 \\
\hline $\mathrm{H}$ & 0.000000 & 0.000000 & 1.088017 \\
\hline $\mathrm{H}$ & 0.969876 & 0.000000 & -0.487627 \\
\hline $\mathrm{C}$ & -1.145914 & 0.006142 & -0.706782 \\
\hline $\mathrm{H}$ & -1.073105 & 0.019154 & -1.793875 \\
\hline $\mathrm{C}$ & -2.473394 & 0.009955 & -0.136488 \\
\hline $\mathrm{H}$ & -2.525924 & 0.058652 & 0.952061 \\
\hline $\mathrm{C}$ & -3.653839 & -0.009358 & -0.824194 \\
\hline $\mathrm{C}$ & -3.758251 & -0.182599 & -2.268352 \\
\hline $\mathrm{H}$ & -2.901805 & -0.614463 & -2.779923 \\
\hline $\mathrm{C}$ & -4.855037 & 0.144426 & -2.987221 \\
\hline $\mathrm{H}$ & -5.704060 & 0.618865 & -2.501871 \\
\hline $\mathrm{N}$ & -5.052315 & -0.119682 & -4.334747 \\
\hline $\mathrm{N}$ & -4.876693 & 0.191132 & -0.153811 \\
\hline $\mathrm{H}$ & -4.778429 & 0.229155 & 0.855285 \\
\hline $\mathrm{H}$ & -5.592817 & -0.479929 & -0.416530 \\
\hline $\mathrm{H}$ & -5.636084 & 0.543788 & -4.828426 \\
\hline $\mathrm{H}$ & -4.218361 & -0.355076 & -4.861049 \\
\hline \multicolumn{4}{|l|}{ TS } \\
\hline $\mathrm{H}$ & 0.000000 & 0.000000 & 0.000000 \\
\hline $\mathrm{C}$ & 0.000000 & 0.000000 & 1.081926 \\
\hline $\mathrm{H}$ & 0.995569 & 0.000000 & 1.524745 \\
\hline $\mathrm{C}$ & -1.035039 & 0.702013 & 1.705560 \\
\hline $\mathrm{H}$ & -0.824095 & 1.320632 & 2.581060 \\
\hline $\mathrm{C}$ & -2.360260 & 0.544295 & 1.325208 \\
\hline $\mathrm{H}$ & -3.099716 & 1.271546 & 1.662066 \\
\hline
\end{tabular}




$\begin{array}{lrrc}\mathrm{C} & -2.876499 & -0.654542 & 0.773723 \\ \mathrm{C} & -2.184828 & -1.875839 & 0.737265 \\ \mathrm{H} & -2.796072 & -2.754432 & 0.962456 \\ \mathrm{C} & -0.790866 & -2.122054 & 0.699850 \\ \mathrm{H} & -0.210711 & -1.910968 & -0.185610 \\ \mathrm{~N} & -0.330096 & -3.272077 & 1.348470 \\ \mathrm{~N} & -4.247565 & -0.678174 & 0.467792 \\ \mathrm{H} & -4.650918 & -1.607186 & 0.420098 \\ \mathrm{H} & -4.820527 & -0.051898 & 1.022990 \\ \mathrm{H} & -0.652294 & -3.379058 & 2.302499 \\ \mathrm{H} & 0.665407 & -3.437365 & 1.275254\end{array}$<smiles>N/C=C\C=C(O)/C=C\N</smiles>

$\begin{array}{lrrr}\mathrm{C} & 0.000000 & 0.000000 & 0.000000 \\ \mathrm{H} & 0.000000 & 0.000000 & 1.087935 \\ \mathrm{H} & 0.969554 & 0.000000 & -0.487847 \\ \mathrm{C} & -1.144771 & -0.001060 & -0.707383 \\ \mathrm{H} & -1.069447 & -0.001950 & -1.794100 \\ \mathrm{C} & -2.472284 & -0.002765 & -0.136571 \\ \mathrm{H} & -2.521853 & -0.008356 & 0.954201 \\ \mathrm{C} & -3.654246 & 0.000027 & -0.816166 \\ \mathrm{C} & -3.821230 & 0.016921 & -2.253736 \\ \mathrm{H} & -2.918920 & 0.028533 & -2.857854 \\ \mathrm{C} & -5.031072 & 0.011107 & -2.858068 \\ \mathrm{H} & -5.935133 & -0.002978 & -2.257459 \\ \mathrm{~N} & -5.251213 & -0.054421 & -4.220630 \\ \mathrm{O} & -4.851317 & -0.006031 & -0.138225 \\ \mathrm{H} & -4.663250 & -0.021083 & 0.812980 \\ \mathrm{H} & -4.465379 & 0.172984 & -4.817774 \\ \mathrm{H} & -6.121645 & 0.337333 & -4.553945\end{array}$

$\begin{array}{cccc}\text { TS } & & & \\ \mathrm{H} & 0.000000 & 0.000000 & 0.000000 \\ \mathrm{C} & 0.000000 & 0.000000 & 1.082152 \\ \mathrm{H} & 0.994972 & 0.000000 & 1.525621 \\ \mathrm{C} & -1.037777 & 0.696280 & 1.706817 \\ \mathrm{H} & -0.831764 & 1.308949 & 2.586853 \\ \mathrm{C} & -2.360987 & 0.540024 & 1.316969 \\ \mathrm{H} & -3.127315 & 1.236577 & 1.648403 \\ \mathrm{C} & -2.844900 & -0.654696 & 0.748806\end{array}$




$\begin{array}{lrrc}\text { C } & -2.172207 & -1.879752 & 0.685274 \\ \text { H } & -2.799079 & -2.752209 & 0.896415 \\ \text { C } & -0.776617 & -2.133292 & 0.689625 \\ \text { H } & -0.168989 & -1.919550 & -0.176044 \\ \text { N } & -0.343651 & -3.283763 & 1.348911 \\ \text { O } & -4.206341 & -0.650077 & 0.530057 \\ \text { H } & -4.456291 & -1.511258 & 0.161638 \\ \text { H } & -0.695767 & -3.399079 & 2.291050 \\ \text { H } & 0.649128 & -3.470130 & 1.295181\end{array}$

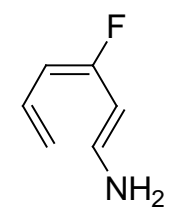

$\begin{array}{cccc}\mathrm{C} & 0.000000 & 0.000000 & 0.000000 \\ \mathrm{H} & 0.000000 & 0.000000 & 1.087598 \\ \mathrm{H} & 0.969050 & 0.000000 & -0.489057 \\ \mathrm{C} & -1.144743 & -0.001019 & -0.705215 \\ \mathrm{H} & -1.077448 & -0.001815 & -1.792712 \\ \mathrm{C} & -2.468221 & -0.002593 & -0.120580 \\ \mathrm{H} & -2.542107 & -0.006808 & 0.964072 \\ \mathrm{C} & -3.636519 & -0.000716 & -0.803410 \\ \mathrm{C} & -3.881797 & 0.013126 & -2.221870 \\ \mathrm{H} & -3.013120 & 0.022649 & -2.874459 \\ \mathrm{C} & -5.128257 & 0.007232 & -2.749817 \\ \mathrm{H} & -5.991648 & -0.005586 & -2.092008 \\ \mathrm{~N} & -5.436509 & -0.057380 & -4.092048 \\ \mathrm{~F} & -4.777357 & -0.004318 & -0.054593 \\ \mathrm{H} & -6.334318 & 0.313784 & -4.370733 \\ \mathrm{H} & -4.695230 & 0.163411 & -4.745372\end{array}$

TS

$\begin{array}{cccc}\mathrm{H} & 0.000000 & 0.000000 & 0.000000 \\ \mathrm{H} & 0.000000 & 0.000000 & 1.821535 \\ \mathrm{C} & 0.590966 & 0.000000 & 0.906816 \\ \mathrm{C} & 1.796161 & -0.707228 & 0.860065 \\ \mathrm{H} & 2.106120 & -1.320986 & 1.707452 \\ \mathrm{C} & 2.687050 & -0.547126 & -0.193066 \\ \mathrm{H} & 3.519108 & -1.230137 & -0.341288 \\ \mathrm{C} & 2.752203 & 0.654891 & -0.914518 \\ \mathrm{C} & 2.163861 & 1.878867 & -0.634360 \\ \mathrm{H} & 2.784622 & 2.741704 & -0.878985 \\ \mathrm{C} & 1.014162 & 2.133812 & 0.162969\end{array}$




$\begin{array}{cccc}\mathrm{H} & 0.025216 & 1.917787 & -0.209332 \\ \mathrm{~N} & 1.029874 & 3.284342 & 0.942962 \\ \mathrm{~F} & 3.772110 & 0.713201 & -1.818063 \\ \mathrm{H} & 1.859844 & 3.427179 & 1.503826 \\ \mathrm{H} & 0.181345 & 3.490156 & 1.452749\end{array}$

$\begin{array}{lccc} & \\ \end{array}$

$\begin{array}{cccc}\text { TS } & & & \\ \mathrm{H} & 0.000000 & 0.000000 & 0.000000 \\ \mathrm{C} & 0.000000 & 0.000000 & 1.081392 \\ \mathrm{H} & 0.997718 & 0.000000 & 1.519567 \\ \mathrm{C} & -1.025510 & 0.723632 & 1.704518 \\ \mathrm{H} & -0.786887 & 1.368982 & 2.552165 \\ \mathrm{C} & -2.360848 & 0.561570 & 1.370200 \\ \mathrm{H} & -3.103245 & 1.286468 & 1.693867 \\ \mathrm{C} & -2.883620 & -0.665742 & 0.899123 \\ \mathrm{C} & -2.196923 & -1.881164 & 0.799123 \\ \mathrm{H} & -2.792357 & -2.773626 & 1.011006 \\ \mathrm{C} & -0.803506 & -2.120318 & 0.681464 \\ \mathrm{H} & -0.292957 & -1.855726 & -0.230573 \\ \mathrm{~N} & -0.297367 & -3.290138 & 1.241446\end{array}$




$\begin{array}{lrrr}\mathrm{C} & -4.366749 & -0.770316 & 0.824016 \\ \mathrm{H} & -4.747479 & -1.805870 & 0.673233 \\ \mathrm{O} & -5.142084 & 0.163353 & 0.903458 \\ \mathrm{H} & -0.563550 & -3.468317 & 2.201579 \\ \mathrm{H} & 0.686532 & -3.463707 & 1.085058\end{array}$

C

$\begin{array}{cccc}\text { TS } & & & \\ \mathrm{H} & 0.000000 & 0.000000 & 0.000000 \\ \mathrm{C} & 0.000000 & 0.000000 & 1.081863 \\ \mathrm{H} & 0.995196 & 0.000000 & 1.524329 \\ \mathrm{C} & -1.031582 & 0.714941 & 1.699590 \\ \mathrm{H} & -0.814890 & 1.347050 & 2.562132 \\ \mathrm{C} & -2.358262 & 0.554392 & 1.327756 \\ \mathrm{H} & -3.104283 & 1.277366 & 1.646285 \\ \mathrm{C} & -2.850283 & -0.672054 & 0.810571 \\ \mathrm{C} & -2.163004 & -1.891921 & 0.735619 \\ \mathrm{H} & -2.770324 & -2.781838 & 0.904429 \\ \mathrm{C} & -0.760903 & -2.122719 & 0.704911 \\ \mathrm{H} & -0.195620 & -1.884171 & -0.181926 \\ \mathrm{~N} & -0.289845 & -3.268257 & 1.329216 \\ \mathrm{C} & -4.280492 & -0.752784 & 0.642034\end{array}$




$\begin{array}{rrrr}\mathrm{H} & -0.623623 & -3.439918 & 2.268567 \\ \mathrm{H} & 0.694410 & -3.471251 & 1.222097 \\ \mathrm{~N} & -5.433086 & -0.811335 & 0.493110\end{array}$<smiles>N/C=C\C=C(\C=C/N)[N+](=O)[O-]</smiles>

$\begin{array}{lrrr}\mathrm{C} & 0.000000 & 0.000000 & 0.000000 \\ \mathrm{H} & 0.000000 & 0.000000 & 1.087298 \\ \mathrm{H} & 0.968599 & 0.000000 & -0.490060 \\ \mathrm{C} & -1.147578 & -0.002981 & -0.703924 \\ \mathrm{H} & -1.089662 & -0.007470 & -1.790110 \\ \mathrm{C} & -2.448014 & -0.012414 & -0.078340 \\ \mathrm{H} & -2.468626 & -0.049196 & 1.005194 \\ \mathrm{C} & -3.656077 & 0.013531 & -0.711063 \\ \mathrm{C} & -3.866394 & 0.103327 & -2.138437 \\ \mathrm{H} & -2.943330 & 0.234634 & -2.699356 \\ \mathrm{C} & -5.008136 & 0.004709 & -2.865447 \\ \mathrm{H} & -5.970029 & -0.124451 & -2.388872 \\ \mathrm{~N} & -4.822421 & -0.018895 & 0.207909 \\ \mathrm{~N} & -5.046203 & -0.000596 & -4.240201 \\ \mathrm{H} & -4.223012 & 0.314262 & -4.738156 \\ \mathrm{H} & -5.915016 & 0.271564 & -4.677829 \\ \mathrm{O} & -5.934673 & 0.243493 & -0.258673 \\ \mathrm{O} & -4.638546 & -0.298740 & 1.393195\end{array}$

$\begin{array}{cccc}\text { TS } & & & \\ \mathrm{H} & 0.000000 & 0.000000 & 0.000000 \\ \mathrm{C} & 0.000000 & 0.000000 & 1.078336 \\ \mathrm{C} & 1.234384 & 0.000000 & 1.780562 \\ \mathrm{H} & 1.412769 & 0.848789 & 2.436650 \\ \mathrm{C} & 2.142118 & -1.047192 & 1.897908 \\ \mathrm{C} & 1.897324 & -2.432179 & 1.820101 \\ \mathrm{H} & 2.621279 & -3.075968 & 2.305453 \\ \mathrm{C} & 0.666789 & -2.919617 & 1.410472 \\ \mathrm{H} & 0.306373 & -3.841606 & 1.868609 \\ \mathrm{C} & -0.160225 & -2.208184 & 0.531465 \\ \mathrm{H} & 0.306467 & -1.841930 & -0.373361 \\ \mathrm{H} & -1.195128 & -2.520328 & 0.397325 \\ \mathrm{~N} & 3.438733 & -0.675143 & 2.538968 \\ \mathrm{~N} & -1.030824 & 0.767848 & 1.599762 \\ \mathrm{H} & -1.874446 & 0.828601 & 1.046632\end{array}$




$\begin{array}{rrrr}\mathrm{H} & -1.219163 & 0.659802 & 2.587710 \\ \mathrm{O} & 3.615671 & 0.501722 & 2.855336 \\ \mathrm{O} & 4.277988 & -1.561412 & 2.698505\end{array}$

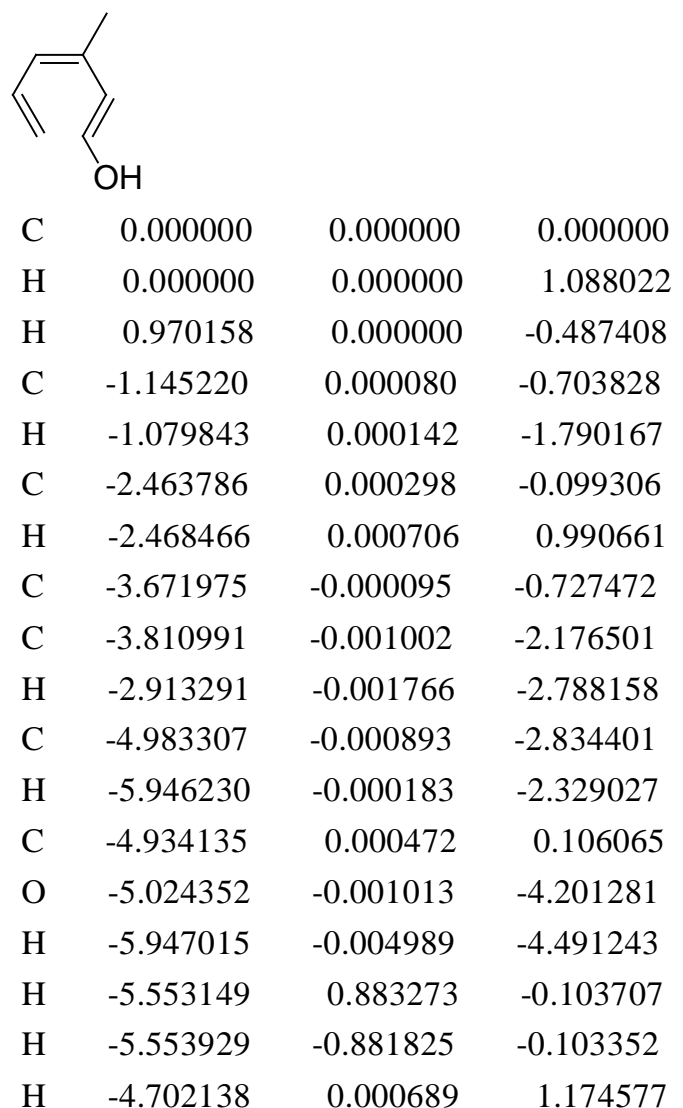

$\begin{array}{cccc}\text { TS } & & & \\ \mathrm{H} & 0.000000 & 0.000000 & 0.000000 \\ \mathrm{C} & 0.000000 & 0.000000 & 1.081485 \\ \mathrm{H} & 0.992361 & 0.000000 & 1.528698 \\ \mathrm{C} & -1.052493 & 0.663688 & 1.705795 \\ \mathrm{H} & -0.865644 & 1.254559 & 2.604520 \\ \mathrm{C} & -2.376312 & 0.481574 & 1.306954 \\ \mathrm{H} & -3.126605 & 1.196982 & 1.640790 \\ \mathrm{C} & -2.868698 & -0.721527 & 0.763979 \\ \mathrm{C} & -2.128079 & -1.912707 & 0.690548 \\ \mathrm{H} & -2.706688 & -2.825099 & 0.869745 \\ \mathrm{C} & -0.734129 & -2.120405 & 0.643838 \\ \mathrm{H} & -0.134177 & -1.892676 & -0.221613 \\ \mathrm{O} & -0.214484 & -3.241327 & 1.239686 \\ \mathrm{C} & -4.359050 & -0.821620 & 0.503268\end{array}$




\begin{tabular}{|c|c|c|c|}
\hline $\mathrm{H}$ & -0.779480 & -3.471671 & 1.995209 \\
\hline $\mathrm{H}$ & -4.580417 & -0.639052 & -0.556793 \\
\hline $\mathrm{H}$ & -4.743766 & -1.821237 & 0.738364 \\
\hline \multirow[t]{3}{*}{$\mathrm{H}$} & -4.926919 & -0.087936 & 1.085269 \\
\hline & $\mathrm{NH}_{2}$ & & \\
\hline & $\mathrm{OH}$ & & \\
\hline $\mathrm{C}$ & 0.000000 & 0.000000 & 0.000000 \\
\hline $\mathrm{H}$ & 0.000000 & 0.000000 & 1.088005 \\
\hline $\mathrm{H}$ & 0.969745 & 0.000000 & -0.487710 \\
\hline $\mathrm{C}$ & -1.145127 & -0.005520 & -0.706657 \\
\hline $\mathrm{H}$ & -1.075747 & -0.018978 & -1.793543 \\
\hline $\mathrm{C}$ & -2.470466 & -0.005377 & -0.128761 \\
\hline $\mathrm{H}$ & -2.516845 & -0.045560 & 0.960436 \\
\hline $\mathrm{C}$ & -3.653279 & 0.007134 & -0.808369 \\
\hline $\mathrm{C}$ & -3.759420 & 0.157193 & -2.256796 \\
\hline $\mathrm{H}$ & -2.917056 & 0.579922 & -2.794442 \\
\hline $\mathrm{C}$ & -4.849326 & -0.191006 & -2.960523 \\
\hline $\mathrm{H}$ & -5.711322 & -0.662481 & -2.493637 \\
\hline $\mathrm{O}$ & -4.920606 & 0.017073 & -4.308333 \\
\hline $\mathrm{N}$ & -4.877627 & -0.179187 & -0.134944 \\
\hline $\mathrm{H}$ & -4.777626 & -0.208783 & 0.874443 \\
\hline $\mathrm{H}$ & -5.585957 & 0.499791 & -0.399575 \\
\hline $\mathrm{H}$ & -5.740617 & -0.369748 & -4.645660 \\
\hline \multicolumn{4}{|c|}{ TS } \\
\hline $\mathrm{H}$ & 0.000000 & 0.000000 & 0.000000 \\
\hline $\mathrm{C}$ & 0.000000 & 0.000000 & 1.081685 \\
\hline $\mathrm{H}$ & 0.991228 & 0.000000 & 1.530909 \\
\hline $\mathrm{C}$ & -1.055907 & 0.657500 & 1.708980 \\
\hline $\mathrm{H}$ & -0.872233 & 1.252590 & 2.606084 \\
\hline $\mathrm{C}$ & -2.374937 & 0.477608 & 1.305271 \\
\hline $\mathrm{H}$ & -3.134885 & 1.183814 & 1.640791 \\
\hline $\mathrm{C}$ & -2.854149 & -0.720212 & 0.730195 \\
\hline $\mathrm{C}$ & -2.124703 & -1.926717 & 0.695439 \\
\hline $\mathrm{H}$ & -2.714611 & -2.823811 & 0.912126 \\
\hline $\mathrm{C}$ & -0.734337 & -2.132750 & 0.653934 \\
\hline $\mathrm{H}$ & -0.121076 & -1.920606 & -0.206823 \\
\hline $\mathrm{O}$ & -0.217564 & -3.238000 & 1.281857 \\
\hline $\mathrm{N}$ & -4.212259 & -0.781250 & 0.394294 \\
\hline $\mathrm{H}$ & -4.597329 & -1.716147 & 0.325977 \\
\hline $\mathrm{H}$ & -4.818405 & -0.156585 & 0.913854 \\
\hline
\end{tabular}




$\begin{array}{lccc}\mathrm{H} & -0.787179 & -3.444492 & 2.040865 \\ & & & \\ \mathrm{C} & & & \\ \mathrm{H} & 0.000000 & 0.000000 & 0.000000 \\ \mathrm{H} & 0.000000 & 0.000000 & 1.087906 \\ \mathrm{C} & -1.143897 & -0.000020 & -0.707285 \\ \mathrm{H} & -1.072644 & -0.000002 & -1.793823 \\ \mathrm{C} & -2.469242 & -0.000002 & -0.128532 \\ \mathrm{H} & -2.512604 & 0.000614 & 0.962483 \\ \mathrm{C} & -3.652961 & -0.000781 & -0.800267 \\ \mathrm{C} & -3.819114 & -0.001649 & -2.240468 \\ \mathrm{H} & -2.929830 & -0.002129 & -2.860403 \\ \mathrm{C} & -5.022368 & -0.001901 & -2.837795 \\ \mathrm{H} & -5.946711 & -0.001512 & -2.267044 \\ \mathrm{O} & -5.135027 & -0.002723 & -4.196188 \\ \mathrm{O} & -4.851244 & -0.000457 & -0.125014 \\ \mathrm{H} & -4.667601 & 0.000007 & 0.827289 \\ \mathrm{H} & -6.072178 & -0.002622 & -4.436250\end{array}$

$\begin{array}{cccc}\text { TS } & & & \\ \mathrm{H} & 0.000000 & 0.000000 & 0.000000 \\ \mathrm{C} & 0.000000 & 0.000000 & 1.081780 \\ \mathrm{H} & 0.991221 & 0.000000 & 1.530667 \\ \mathrm{C} & -1.056201 & 0.655689 & 1.710217 \\ \mathrm{H} & -0.873695 & 1.248920 & 2.608027 \\ \mathrm{C} & -2.375131 & 0.477261 & 1.303070 \\ \mathrm{H} & -3.157923 & 1.157324 & 1.630336 \\ \mathrm{C} & -2.828683 & -0.719539 & 0.726161 \\ \mathrm{C} & -2.118588 & -1.930239 & 0.666013 \\ \mathrm{H} & -2.719843 & -2.820214 & 0.884850 \\ \mathrm{C} & -0.726012 & -2.139432 & 0.644933 \\ \mathrm{H} & -0.102764 & -1.922250 & -0.207001 \\ \mathrm{O} & -0.220302 & -3.245899 & 1.274644 \\ \mathrm{O} & -4.185167 & -0.752894 & 0.495061 \\ \mathrm{H} & -4.408829 & -1.610549 & 0.102658 \\ \mathrm{H} & -0.793664 & -3.453470 & 2.030530\end{array}$




$\begin{array}{lccc} & \\ \mathrm{C} & & \\ \mathrm{H} & 0.000000 & 0.000000 & 0.000000 \\ \mathrm{H} & 0.000000 & 0.000000 & 1.087572 \\ \mathrm{C} & -1.143998 & 0.000000 & -0.705087 \\ \mathrm{H} & -1.080925 & 0.000000 & -1.792372 \\ \mathrm{C} & -2.464844 & 0.000000 & -0.112223 \\ \mathrm{H} & -2.531829 & 0.000000 & 0.973021 \\ \mathrm{C} & -3.635726 & 0.000000 & -0.786462 \\ \mathrm{C} & -3.881185 & 0.000000 & -2.207369 \\ \mathrm{H} & -3.026753 & 0.000000 & -2.875669 \\ \mathrm{C} & -5.120767 & 0.000000 & -2.728186 \\ \mathrm{H} & -6.005743 & 0.000000 & -2.097824 \\ \mathrm{~F} & -4.776098 & 0.000000 & -0.037947 \\ \mathrm{O} & -5.321312 & 0.000000 & -4.073920 \\ \mathrm{H} & -6.271745 & 0.000000 & -4.255380\end{array}$

\begin{tabular}{|c|c|c|c|}
\hline $\mathrm{H}$ & 0.000000 & 0.000000 & 0.000000 \\
\hline $\mathrm{C}$ & 0.000000 & 0.000000 & 1.081931 \\
\hline $\mathrm{H}$ & 0.991038 & 0.000000 & 1.530631 \\
\hline $\mathrm{C}$ & -1.052427 & 0.669360 & 1.704578 \\
\hline $\mathrm{H}$ & -0.871025 & 1.265912 & 2.599591 \\
\hline $\mathrm{C}$ & -2.370916 & 0.486179 & 1.296645 \\
\hline $\mathrm{H}$ & -3.163383 & 1.153325 & 1.624909 \\
\hline $\mathrm{C}$ & -2.792389 & -0.718749 & 0.725702 \\
\hline $\mathrm{C}$ & -2.108652 & -1.928934 & 0.641914 \\
\hline $\mathrm{H}$ & -2.735325 & -2.810713 & 0.792041 \\
\hline $\mathrm{C}$ & -0.712972 & -2.137716 & 0.645954 \\
\hline $\mathrm{H}$ & -0.079509 & -1.915405 & -0.196536 \\
\hline $\mathrm{O}$ & -0.220830 & -3.248511 & 1.272988 \\
\hline $\mathrm{F}$ & -4.134809 & -0.816807 & 0.524017 \\
\hline $\mathrm{H}$ & -0.815425 & -3.476517 & 2.006452 \\
\hline & 10 & & \\
\hline & & & \\
\hline & 0. & 00 & 0 \\
\hline $\mathrm{H}$ & 0.000000 & 0.000000 & 1.087785 \\
\hline & 0.969705 & 0.000000 & -0.487965 \\
\hline
\end{tabular}




$\begin{array}{lccc}\mathrm{C} & -1.146485 & 0.000000 & -0.704578 \\ \mathrm{H} & -1.090962 & 0.000000 & -1.790870 \\ \mathrm{C} & -2.452921 & 0.000000 & -0.088450 \\ \mathrm{H} & -2.451799 & 0.000000 & 1.001769 \\ \mathrm{C} & -3.674144 & 0.000000 & -0.709916 \\ \mathrm{C} & -3.856404 & 0.000000 & -2.152648 \\ \mathrm{H} & -2.964758 & 0.000000 & -2.774206 \\ \mathrm{C} & -5.038637 & 0.000000 & -2.797115 \\ \mathrm{H} & -5.987881 & 0.000000 & -2.273321 \\ \mathrm{C} & -4.824624 & 0.000000 & 0.224254 \\ \mathrm{O} & -5.072492 & 0.000000 & -4.159035 \\ \mathrm{H} & -5.995601 & 0.000000 & -4.449558 \\ \mathrm{O} & -6.007091 & 0.000000 & -0.075000 \\ \mathrm{H} & -4.527133 & 0.000000 & 1.295808\end{array}$

TS

$\begin{array}{llll}\mathrm{H} & 0.000000 & 0.000000 & 0.000000\end{array}$

$\begin{array}{llll}\text { C } & 0.000000 & 0.000000 & 1.081410\end{array}$

$\begin{array}{llll}\mathrm{H} & 0.995399 & 0.000000 & 1.522136\end{array}$

$\begin{array}{llll}\text { C } & -1.034794 & 0.711380 & 1.699170\end{array}$

$\begin{array}{llll}\mathrm{H} & -0.815611 & 1.350262 & 2.555878\end{array}$

$\begin{array}{llll}\text { C } & -2.365131 & 0.522099 & 1.348675\end{array}$

$\begin{array}{llll}\mathrm{H} & -3.111736 & 1.245210 & 1.675614\end{array}$

$\begin{array}{llll}\text { C } & -2.860144 & -0.714463 & 0.875784\end{array}$

$\begin{array}{llll}\text { C } & -2.134418 & -1.909804 & 0.794867\end{array}$

$\begin{array}{llll}\mathrm{H} & -2.718380 & -2.807949 & 1.009907\end{array}$

$\begin{array}{llll}\text { C } & -0.741655 & -2.096802 & 0.677019\end{array}$

$\begin{array}{llll}\mathrm{H} & -0.208567 & -1.869925 & -0.231751\end{array}$

$\begin{array}{llll}\text { O } & -0.169341 & -3.199187 & 1.248540\end{array}$

$\begin{array}{llll}\text { C } & -4.342827 & -0.837858 & 0.821252\end{array}$

$\begin{array}{llll}\mathrm{O} & -4.951857 & -1.868197 & 0.605503\end{array}$

$\begin{array}{llll}\mathrm{H} & -4.890316 & 0.113619 & 1.003073\end{array}$

$\begin{array}{llll}\mathrm{H} & -0.696966 & -3.456208 & 2.022712\end{array}$

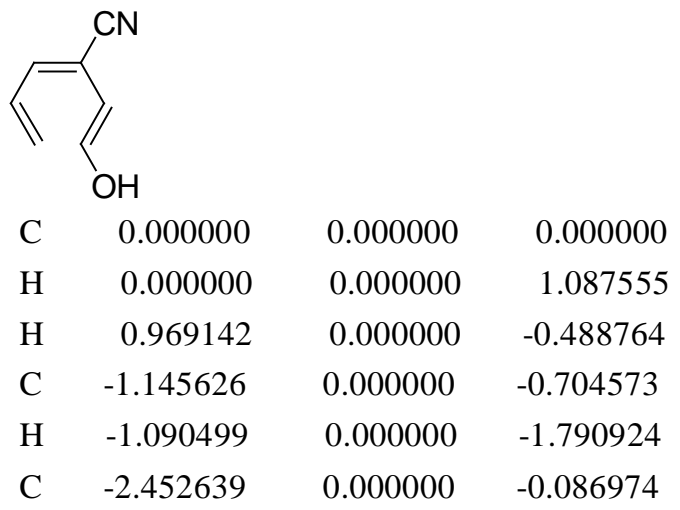




$\begin{array}{lrrr}\mathrm{H} & -2.468374 & 0.000000 & 1.000430 \\ \mathrm{C} & -3.658008 & 0.000000 & -0.732881 \\ \mathrm{C} & -3.858439 & 0.000000 & -2.175828 \\ \mathrm{H} & -2.982059 & 0.000000 & -2.815223 \\ \mathrm{C} & -5.069247 & 0.000000 & -2.761495 \\ \mathrm{H} & -5.991284 & 0.000000 & -2.183348 \\ \mathrm{O} & -5.194306 & 0.000000 & -4.114464 \\ \mathrm{C} & -4.838846 & 0.000000 & 0.089077 \\ \mathrm{~N} & -5.813381 & -0.000001 & 0.725921 \\ \mathrm{H} & -6.133159 & 0.000000 & -4.349661\end{array}$

\begin{tabular}{|c|c|c|c|}
\hline $\mathrm{H}$ & 0.000000 & 0.000000 & 0.000000 \\
\hline $\mathrm{C}$ & 0.000000 & 0.000000 & 1.081324 \\
\hline $\mathrm{H}$ & 0.992551 & 0.000000 & 1.527394 \\
\hline $\mathrm{C}$ & -1.046077 & 0.683126 & 1.702634 \\
\hline $\mathrm{H}$ & -0.847186 & 1.301732 & 2.578665 \\
\hline $\mathrm{C}$ & -2.371814 & 0.499673 & 1.325558 \\
\hline $\mathrm{H}$ & -3.129726 & 1.211385 & 1.641475 \\
\hline $\mathrm{C}$ & -2.842565 & -0.733612 & 0.817516 \\
\hline $\mathrm{C}$ & -2.117967 & -1.937998 & 0.746183 \\
\hline $\mathrm{H}$ & -2.693011 & -2.843340 & 0.951879 \\
\hline $\mathrm{C}$ & -0.722154 & -2.119687 & 0.663046 \\
\hline $\mathrm{H}$ & -0.162244 & -1.880150 & -0.225658 \\
\hline $\mathrm{O}$ & -0.161767 & -3.218403 & 1.247466 \\
\hline $\mathrm{C}$ & -4.270721 & -0.852756 & 0.664328 \\
\hline $\mathrm{N}$ & -5.422406 & -0.947085 & 0.528251 \\
\hline \multirow[t]{3}{*}{$H$} & -0.695100 & -3.470810 & 2.019221 \\
\hline & & & \\
\hline & $\mathrm{OH}$ & & \\
\hline $\mathrm{C}$ & 0.000000 & 0.000000 & 0.000000 \\
\hline $\mathrm{H}$ & 0.000000 & 0.000000 & 1.087291 \\
\hline $\mathrm{H}$ & 0.968483 & 0.000000 & -0.490214 \\
\hline $\mathrm{C}$ & -1.146921 & 0.000000 & -0.704184 \\
\hline $\mathrm{H}$ & -1.093150 & 0.000000 & -1.790018 \\
\hline $\mathrm{C}$ & -2.443011 & 0.000000 & -0.068816 \\
\hline $\mathrm{H}$ & -2.456036 & 0.000000 & 1.015801 \\
\hline $\mathrm{C}$ & -3.656283 & 0.000000 & -0.688343 \\
\hline $\mathrm{C}$ & -3.867867 & 0.000000 & -2.120666 \\
\hline $\mathrm{H}$ & -2.950506 & 0.000000 & -2.702347 \\
\hline $\mathrm{C}$ & -4.999914 & 0.000000 & -2.851060 \\
\hline
\end{tabular}




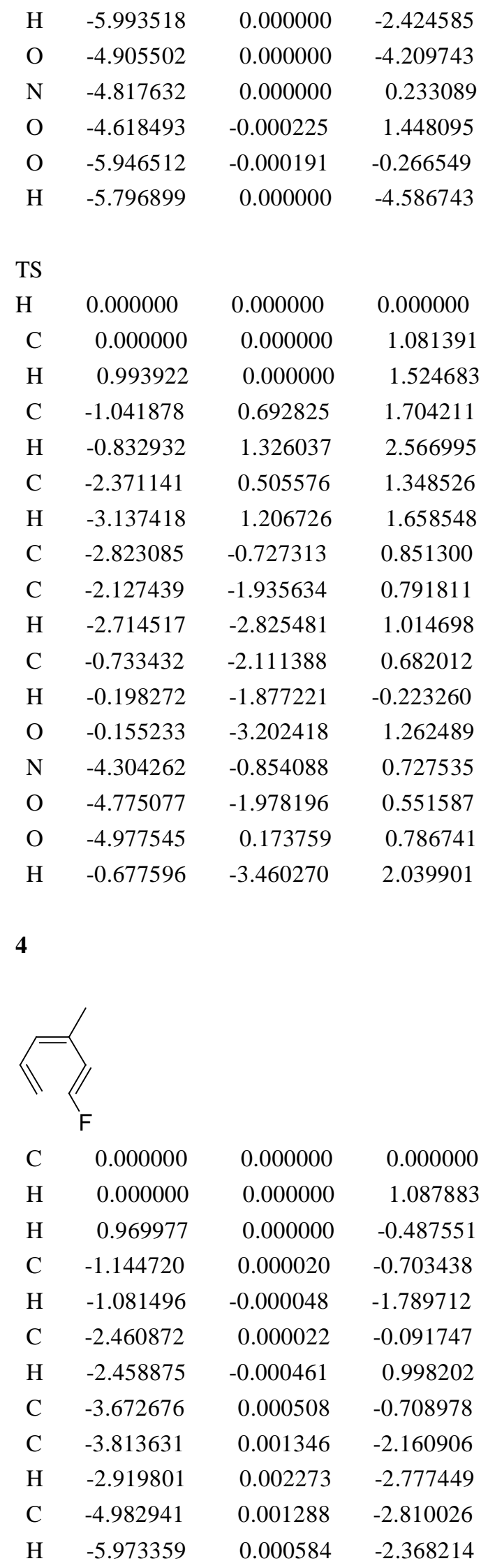




$\begin{array}{lrrr}\text { F } & -5.031680 & 0.002339 & -4.157181 \\ \text { C } & -4.935163 & 0.000364 & 0.121962 \\ \text { H } & -4.704973 & -0.000540 & 1.190789 \\ \text { H } & -5.553480 & 0.883101 & -0.089137 \\ \text { H } & -5.554161 & -0.881616 & -0.090343\end{array}$

TS

$\begin{array}{llll}\mathrm{H} & 0.000000 & 0.000000 & 0.000000\end{array}$

$\begin{array}{llll}\mathrm{C} & 0.000000 & 0.000000 & 1.081185\end{array}$

$\begin{array}{llll}\mathrm{H} & 0.992458 & 0.000000 & 1.527922\end{array}$

$\begin{array}{llll}\text { C } & -1.051392 & 0.657774 & 1.709965\end{array}$

$\begin{array}{llll}\mathrm{H} & -0.862942 & 1.242696 & 2.611520\end{array}$

$\begin{array}{llll}\text { C } & -2.379918 & 0.470636 & 1.322844\end{array}$

$\begin{array}{llll}\mathrm{H} & -3.124887 & 1.189644 & 1.661059\end{array}$

$\begin{array}{llll}\text { C } & -2.885534 & -0.735959 & 0.806319\end{array}$

$\begin{array}{llll}\text { C } & -2.137560 & -1.926560 & 0.751643\end{array}$

$\begin{array}{llll}\mathrm{H} & -2.680148 & -2.838516 & 1.010442\end{array}$

$\begin{array}{llll}\text { C } & -0.757760 & -2.100379 & 0.629708\end{array}$

$\mathrm{H} \quad-0.186718 \quad-1.880510 \quad-0.258010$

$\begin{array}{llll}\text { F } & -0.272156 & -3.241494 & 1.196291\end{array}$

$\begin{array}{llll}\text { C } & -4.380292 & -0.850285 & 0.585560\end{array}$

$\begin{array}{llll}\mathrm{H} & -4.624551 & -0.743453 & -0.479703\end{array}$

$\begin{array}{llll}\mathrm{H} & -4.757790 & -1.830767 & 0.899434\end{array}$

$\mathrm{H} \quad-4.934753 \quad-0.076939 \quad 1.127155$

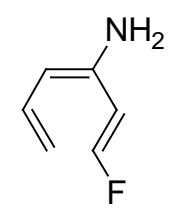

$\begin{array}{llll}\text { C } & 0.000000 & 0.000000 & 0.000000\end{array}$

$\begin{array}{llll}\mathrm{H} & 0.000000 & 0.000000 & 1.087848\end{array}$

$\begin{array}{llll}\mathrm{H} & 0.969475 & 0.000000 & -0.487977\end{array}$

$\begin{array}{llll}\text { C } & -1.144703 & 0.005738 & -0.706284\end{array}$

$\begin{array}{llll}\mathrm{H} & -1.076247 & 0.020505 & -1.793043\end{array}$

$\begin{array}{llll}\text { C } & -2.467658 & 0.002859 & -0.121404\end{array}$

$\begin{array}{llll}\mathrm{H} & -2.506634 & 0.037465 & 0.968140\end{array}$

$\begin{array}{llll}\text { C } & -3.655395 & -0.003932 & -0.789754\end{array}$

$\begin{array}{llll}\text { C } & -3.762827 & -0.131158 & -2.242956\end{array}$

$\begin{array}{llll}\mathrm{H} & -2.919252 & -0.535721 & -2.791848\end{array}$

$\begin{array}{llll}\text { C } & -4.853725 & 0.211429 & -2.934605\end{array}$

$\begin{array}{llll}\mathrm{H} & -5.752851 & 0.662588 & -2.529211\end{array}$

F $\quad-4.918795 \quad 0.039395 \quad-4.268574$

$\begin{array}{llll}\mathrm{N} & -4.877881 & 0.170809 & -0.116224\end{array}$

$\begin{array}{llll}\mathrm{H} & -4.784845 & 0.182320 & 0.893879\end{array}$ 


$\begin{array}{cccc}\mathrm{H} & -5.595717 & -0.489672 & -0.400103 \\ & & & \\ \mathrm{TS} & & & \\ \mathrm{H} & 0.000000 & 0.000000 & 0.000000 \\ \mathrm{C} & 0.000000 & 0.000000 & 1.078582 \\ \mathrm{C} & 1.131429 & 0.000000 & 1.889891 \\ \mathrm{H} & 1.185564 & 0.827123 & 2.601197 \\ \mathrm{C} & 2.076336 & -1.032293 & 2.084392 \\ \mathrm{C} & 1.744795 & -2.402850 & 2.042722 \\ \mathrm{H} & 2.397914 & -3.065156 & 2.611728 \\ \mathrm{C} & 0.518294 & -2.888146 & 1.596387 \\ \mathrm{H} & 0.092610 & -3.748553 & 2.115786 \\ \mathrm{C} & -0.223806 & -2.229826 & 0.620400 \\ \mathrm{H} & -1.258028 & -2.512339 & 0.434518 \\ \mathrm{H} & 0.310639 & -1.890278 & -0.256405 \\ \mathrm{~N} & 3.326259 & -0.671863 & 2.595134 \\ \mathrm{~F} & -1.013995 & 0.799975 & 1.517955 \\ \mathrm{H} & 3.758330 & -1.346344 & 3.216258 \\ \mathrm{H} & 3.390978 & 0.274421 & 2.951826\end{array}$

$\begin{array}{lccc} & \\ \end{array}$

$\begin{array}{cccc}\mathrm{TS} & & & \\ \mathrm{H} & 0.000000 & 0.000000 & 0.000000 \\ \mathrm{C} & 0.000000 & 0.000000 & 1.078186 \\ \mathrm{C} & 1.135761 & 0.000000 & 1.886822\end{array}$




$\begin{array}{lrrr}\mathrm{H} & 1.190683 & 0.816646 & 2.611442 \\ \mathrm{C} & 2.059781 & -1.042926 & 2.082797 \\ \mathrm{C} & 1.758581 & -2.411507 & 2.035079 \\ \mathrm{H} & 2.427936 & -3.046110 & 2.611265 \\ \mathrm{C} & 0.537486 & -2.900820 & 1.576433 \\ \mathrm{H} & 0.116213 & -3.769800 & 2.083801 \\ \mathrm{C} & -0.203554 & -2.238807 & 0.602756 \\ \mathrm{H} & 0.332730 & -1.882785 & -0.266349 \\ \mathrm{H} & -1.234103 & -2.527649 & 0.407324 \\ \mathrm{~F} & -1.013558 & 0.793496 & 1.522104 \\ \mathrm{O} & 3.259179 & -0.747204 & 2.683468 \\ \mathrm{H} & 3.360064 & 0.216473 & 2.717778 \\ & & & \\ & \mathrm{~F} & & \\ \mathrm{H} & & & \\ \mathrm{W} & \mathrm{V} & & \\ & \mathrm{F} & & \\ \mathrm{C} & 0.000000 & 0.000000 & 0.000000 \\ \mathrm{H} & 0.000000 & 0.000000 & 1.087440 \\ \mathrm{H} & 0.968740 & 0.000000 & -0.489303 \\ \mathrm{C} & -1.143485 & 0.000000 & -0.704688 \\ \mathrm{H} & -1.083082 & 0.000000 & -1.791881 \\ \mathrm{C} & -2.461483 & 0.000000 & -0.103430 \\ \mathrm{H} & -2.519929 & 0.000000 & 0.982412 \\ \mathrm{C} & -3.637212 & 0.000000 & -0.765487 \\ \mathrm{C} & -3.885777 & 0.000000 & -2.189406 \\ \mathrm{H} & -3.034933 & 0.000000 & -2.861797 \\ \mathrm{C} & -5.120291 & 0.000000 & -2.703487 \\ \mathrm{H} & -6.044056 & 0.000000 & -2.136982 \\ \mathrm{~F} & -4.775004 & 0.000000 & -0.019035 \\ \mathrm{~F} & -5.321149 & 0.000000 & -4.030968\end{array}$

$\begin{array}{cccc}\text { TS } & & & \\ \mathrm{H} & 0.000000 & 0.000000 & 0.000000 \\ \mathrm{C} & 0.000000 & 0.000000 & 1.081555 \\ \mathrm{H} & 0.990661 & 0.000000 & 1.530710 \\ \mathrm{C} & -1.054138 & 0.657139 & 1.709249 \\ \mathrm{H} & -0.875120 & 1.243295 & 2.610874 \\ \mathrm{C} & -2.375720 & 0.468160 & 1.308374 \\ \mathrm{H} & -3.165194 & 1.134961 & 1.645308 \\ \mathrm{C} & -2.808590 & -0.736998 & 0.752295 \\ \mathrm{C} & -2.118435 & -1.948113 & 0.688974 \\ \mathrm{H} & -2.713584 & -2.832907 & 0.914211 \\ \mathrm{C} & -0.734262 & -2.123524 & 0.623472\end{array}$




$\begin{array}{cccc}\text { H } & -0.122701 & -1.902480 & -0.236176 \\ \text { F } & -0.277529 & -3.256842 & 1.217959 \\ \text { F } & -4.151149 & -0.840460 & 0.573656\end{array}$

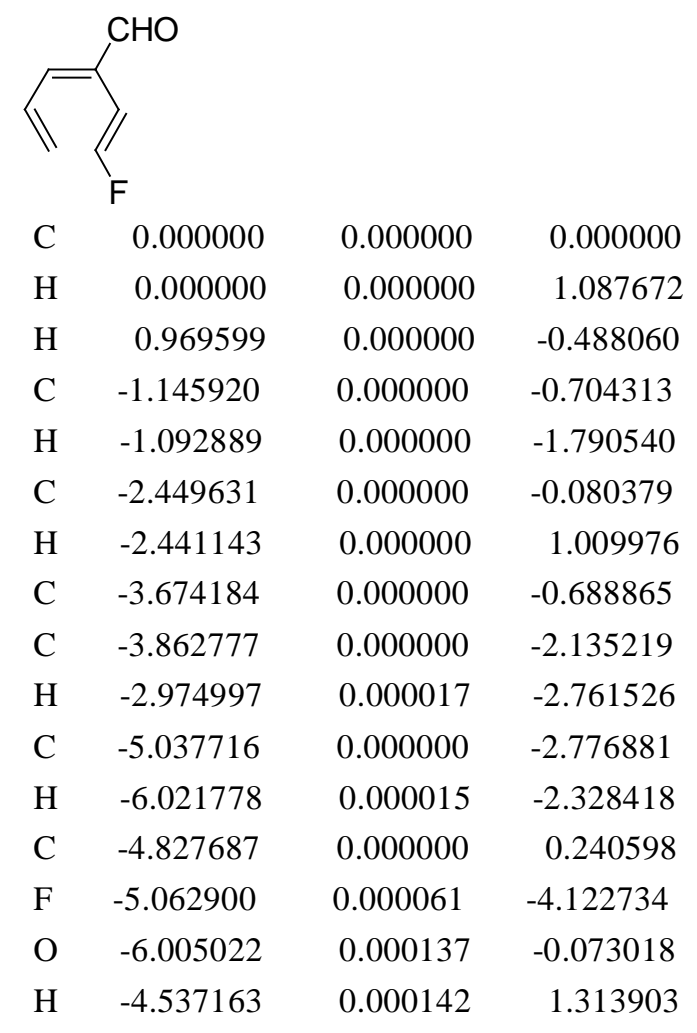

$\begin{array}{cccc}\text { TS } & & & \\ \mathrm{H} & 0.000000 & 0.000000 & 0.000000 \\ \mathrm{C} & 0.000000 & 0.000000 & 1.080855 \\ \mathrm{H} & 0.994835 & 0.000000 & 1.522578 \\ \mathrm{C} & -1.037560 & 0.693014 & 1.708228 \\ \mathrm{H} & -0.819257 & 1.319861 & 2.573467 \\ \mathrm{C} & -2.373022 & 0.495310 & 1.372346 \\ \mathrm{H} & -3.115094 & 1.219786 & 1.707214 \\ \mathrm{C} & -2.879095 & -0.742058 & 0.922470 \\ \mathrm{C} & -2.143699 & -1.936402 & 0.854358 \\ \mathrm{H} & -2.683429 & -2.836662 & 1.148759 \\ \mathrm{C} & -0.772146 & -2.086675 & 0.647885 \\ \mathrm{H} & -0.276708 & -1.851849 & -0.280887 \\ \mathrm{~F} & -0.224611 & -3.208922 & 1.181217 \\ \mathrm{C} & -4.362116 & -0.871846 & 0.904073 \\ \mathrm{O} & -4.967163 & -1.906697 & 0.704603 \\ \mathrm{H} & -4.909809 & 0.077961 & 1.093366\end{array}$




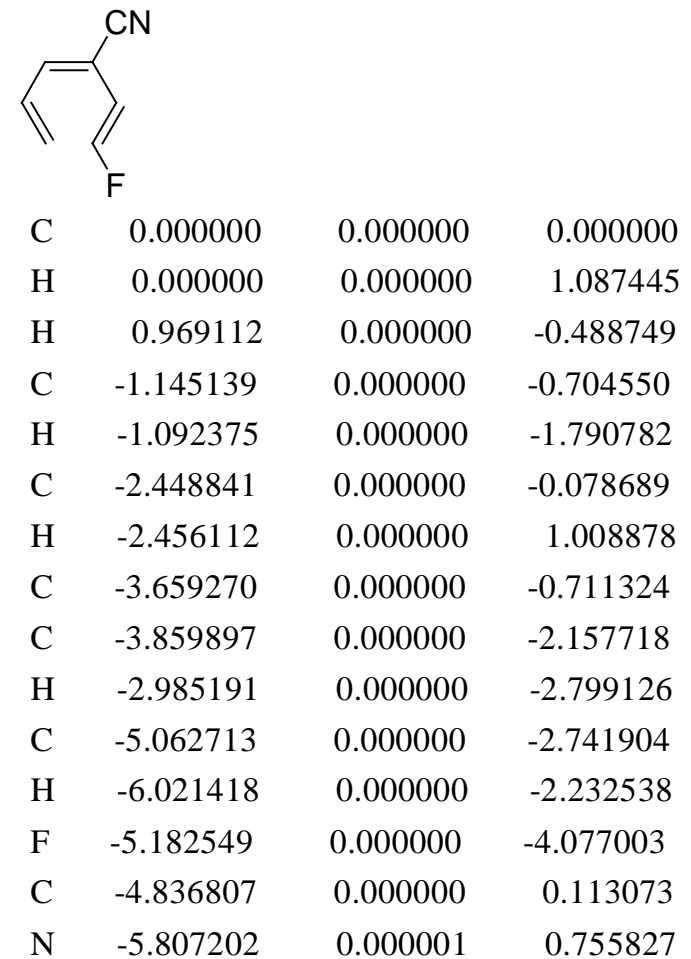

TS

$\begin{array}{llll}\mathrm{H} & 0.000000 & 0.000000 & 0.000000\end{array}$

$\begin{array}{llll}\text { C } & 0.000000 & 0.000000 & 1.080762\end{array}$

$\begin{array}{llll}\mathrm{H} & 0.992489 & 0.000000 & 1.526867\end{array}$

$\begin{array}{llll}\text { C } & -1.046184 & 0.670942 & 1.710557\end{array}$

$\begin{array}{llll}\mathrm{H} & -0.843763 & 1.283282 & 2.589698\end{array}$

$\begin{array}{llll}\text { C } & -2.378696 & 0.480578 & 1.354040\end{array}$

$\begin{array}{llll}\mathrm{H} & -3.128911 & 1.196545 & 1.679398\end{array}$

$\begin{array}{llll}\text { C } & -2.867066 & -0.756506 & 0.878794\end{array}$

$\begin{array}{llll}\text { C } & -2.133595 & -1.959250 & 0.820093\end{array}$

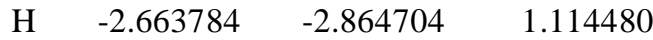

$\begin{array}{llll}\text { C } & -0.758139 & -2.104142 & 0.637405\end{array}$

$\mathrm{H} \quad-0.242162 \quad-1.860260 \quad-0.277211$

$\begin{array}{llll}\text { F } & -0.221007 & -3.223592 & 1.180955\end{array}$

$\begin{array}{llll}\text { C } & -4.298197 & -0.881273 & 0.774814\end{array}$

$\begin{array}{llll}\mathrm{N} & -5.453090 & -0.976939 & 0.672237\end{array}$

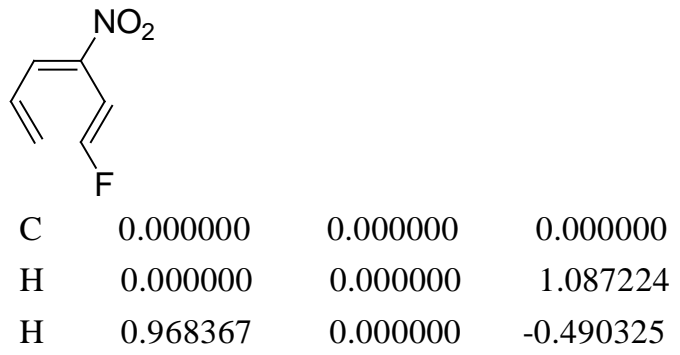




$\begin{array}{lccc}\mathrm{C} & -1.146461 & 0.000000 & -0.703902 \\ \mathrm{H} & -1.095293 & 0.000000 & -1.789736 \\ \mathrm{C} & -2.440065 & 0.000000 & -0.062005 \\ \mathrm{H} & -2.448209 & 0.000000 & 1.023164 \\ \mathrm{C} & -3.656288 & 0.000000 & -0.670248 \\ \mathrm{C} & -3.877517 & 0.000000 & -2.104593 \\ \mathrm{H} & -2.966983 & 0.000000 & -2.696172 \\ \mathrm{C} & -5.008026 & 0.000000 & -2.823642 \\ \mathrm{H} & -6.027436 & 0.000000 & -2.470122 \\ \mathrm{~F} & -4.916404 & 0.000000 & -4.166223 \\ \mathrm{~N} & -4.817052 & 0.000000 & 0.248279 \\ \mathrm{O} & -4.617419 & 0.000000 & 1.462450 \\ \mathrm{O} & -5.941275 & 0.000000 & -0.258997\end{array}$

$\begin{array}{cccc}\text { TS } & & & \\ \mathrm{H} & 0.000000 & 0.000000 & 0.000000 \\ \mathrm{C} & 0.000000 & 0.000000 & 1.080821 \\ \mathrm{H} & 0.993259 & 0.000000 & 1.525356 \\ \mathrm{C} & -1.044526 & 0.674519 & 1.713156 \\ \mathrm{H} & -0.836106 & 1.295992 & 2.584049 \\ \mathrm{C} & -2.378797 & 0.479357 & 1.372107 \\ \mathrm{H} & -3.142167 & 1.179166 & 1.693896 \\ \mathrm{C} & -2.843119 & -0.752863 & 0.892995 \\ \mathrm{C} & -2.138870 & -1.960259 & 0.843859 \\ \mathrm{H} & -2.682818 & -2.852693 & 1.143696 \\ \mathrm{C} & -0.765613 & -2.101332 & 0.646569 \\ \mathrm{H} & -0.264843 & -1.859490 & -0.277094 \\ \mathrm{~F} & -0.216597 & -3.213792 & 1.190305 \\ \mathrm{~N} & -4.324711 & -0.887354 & 0.809353 \\ \mathrm{O} & -4.786736 & -2.013517 & 0.630013 \\ \mathrm{O} & -5.002787 & 0.134985 & 0.898658\end{array}$

5

$\begin{array}{lrrr} & \\ & & & \\ \mathrm{CHO} & & \\ \mathrm{C} & 0.000000 & 0.000000 & 0.000000 \\ \mathrm{H} & 0.000000 & 0.000000 & 1.087716 \\ \mathrm{H} & 0.969708 & 0.000000 & -0.487840 \\ \mathrm{C} & -1.145110 & -0.000120 & -0.703760 \\ \mathrm{H} & -1.084557 & -0.000247 & -1.789558\end{array}$




\begin{tabular}{|c|c|c|c|}
\hline $\mathrm{C}$ & -2.453210 & -0.000193 & -0.078371 \\
\hline $\mathrm{H}$ & -2.433367 & -0.000575 & 1.011548 \\
\hline $\mathrm{C}$ & -3.679863 & 0.000189 & -0.674716 \\
\hline $\mathrm{C}$ & -3.835229 & 0.000780 & -2.118970 \\
\hline $\mathrm{H}$ & -2.924351 & 0.000857 & -2.715646 \\
\hline $\mathrm{C}$ & -5.005956 & 0.001111 & -2.798764 \\
\hline $\mathrm{H}$ & -5.973401 & 0.000975 & -2.304379 \\
\hline $\mathrm{C}$ & -5.023691 & 0.001484 & -4.262875 \\
\hline $\mathrm{C}$ & -4.929078 & 0.000023 & 0.175015 \\
\hline $\mathrm{H}$ & -5.549488 & 0.882003 & -0.028471 \\
\hline $\mathrm{H}$ & -5.550416 & -0.880897 & -0.030184 \\
\hline $\mathrm{H}$ & -4.683035 & -0.001125 & 1.240487 \\
\hline $\mathrm{H}$ & -4.016084 & 0.001555 & -4.740008 \\
\hline $\mathrm{O}$ & -6.035477 & 0.001637 & -4.942665 \\
\hline \multicolumn{4}{|l|}{ TS } \\
\hline $\mathrm{H}$ & 0.000000 & 0.000000 & 0.000000 \\
\hline $\mathrm{C}$ & 0.000000 & 0.000000 & 1.078012 \\
\hline $\mathrm{H}$ & 1.003551 & 0.000000 & 1.503519 \\
\hline $\mathrm{C}$ & -1.011282 & 0.694256 & 1.755469 \\
\hline $\mathrm{H}$ & -0.715190 & 1.365673 & 2.564334 \\
\hline $\mathrm{C}$ & -2.379774 & 0.464968 & 1.632744 \\
\hline $\mathrm{H}$ & -3.042551 & 1.210544 & 2.071598 \\
\hline $\mathrm{C}$ & -2.981836 & -0.794552 & 1.386198 \\
\hline $\mathrm{C}$ & -2.203758 & -1.944832 & 1.246120 \\
\hline $\mathrm{H}$ & -2.589287 & -2.845223 & 1.730958 \\
\hline $\mathrm{C}$ & -0.908024 & -2.012021 & 0.690182 \\
\hline $\mathrm{H}$ & -0.765648 & -1.684536 & -0.329558 \\
\hline $\mathrm{C}$ & -0.027915 & -3.137738 & 1.067721 \\
\hline $\mathrm{C}$ & -4.469720 & -0.935243 & 1.628227 \\
\hline $\mathrm{H}$ & -5.026011 & -0.812146 & 0.689341 \\
\hline $\mathrm{H}$ & -4.717300 & -1.930353 & 2.014903 \\
\hline $\mathrm{H}$ & -4.849790 & -0.186993 & 2.332164 \\
\hline $\mathrm{H}$ & -0.355453 & -3.701056 & 1.973072 \\
\hline $\mathrm{O}$ & 0.997382 & -3.438752 & 0.484355 \\
\hline & & & \\
\hline & $\mathrm{CHO}$ & & \\
\hline $\mathrm{C}$ & 0.000000 & 0.000000 & 0.000000 \\
\hline $\mathrm{H}$ & 0.000000 & 0.000000 & 1.087611 \\
\hline $\mathrm{H}$ & 0.969055 & 0.000000 & -0.488423 \\
\hline $\mathrm{C}$ & -1.145454 & 0.005023 & -0.706777 \\
\hline $\mathrm{H}$ & -1.076247 & 0.020343 & -1.792595 \\
\hline
\end{tabular}




$\begin{array}{lrrr}\mathrm{C} & -2.459307 & -0.002113 & -0.107737 \\ \mathrm{H} & -2.475484 & 0.024636 & 0.982694 \\ \mathrm{C} & -3.669504 & 0.002200 & -0.747851 \\ \mathrm{C} & -3.792830 & -0.089749 & -2.194855 \\ \mathrm{H} & -2.895122 & -0.371508 & -2.739417 \\ \mathrm{C} & -4.923092 & 0.116171 & -2.908579 \\ \mathrm{H} & -5.855297 & 0.432926 & -2.448719 \\ \mathrm{~N} & -4.865639 & 0.155813 & -0.037356 \\ \mathrm{C} & -4.934189 & -0.052111 & -4.363625 \\ \mathrm{H} & -3.956139 & -0.353671 & -4.804840 \\ \mathrm{O} & -5.913475 & 0.112922 & -5.069914 \\ \mathrm{H} & -5.628303 & -0.426521 & -0.365620 \\ \mathrm{H} & -4.764332 & 0.090238 & 0.968845\end{array}$

TS

$\begin{array}{llll}\mathrm{H} & 0.000000 & 0.000000 & 0.000000\end{array}$

$\begin{array}{llll}\text { C } & 0.000000 & 0.000000 & 1.078572\end{array}$

$\begin{array}{llll}\mathrm{H} & 1.001183 & 0.000000 & 1.508093\end{array}$

$\begin{array}{llll}\text { C } & -1.015290 & 0.704331 & 1.742775\end{array}$

$\begin{array}{llll}\mathrm{H} & -0.734635 & 1.376650 & 2.556400\end{array}$

$\begin{array}{llll}\text { C } & -2.376577 & 0.495681 & 1.562259\end{array}$

$\begin{array}{llll}\mathrm{H} & -3.056898 & 1.235564 & 1.987005\end{array}$

$\begin{array}{llll}\text { C } & -2.978064 & -0.752563 & 1.247116\end{array}$

$\begin{array}{llll}\text { C } & -2.212521 & -1.926678 & 1.194184\end{array}$

$\begin{array}{llll}\mathrm{H} & -2.631388 & -2.788870 & 1.718543\end{array}$

$\begin{array}{llll}\text { C } & -0.889544 & -2.020120 & 0.715828\end{array}$

$\begin{array}{llll}\mathrm{H} & -0.671430 & -1.752162 & -0.308393\end{array}$

$\begin{array}{llll}\mathrm{N} & -4.369726 & -0.838591 & 1.295592\end{array}$

$\begin{array}{llll}\text { C } & -0.041787 & -3.123445 & 1.215832\end{array}$

$\begin{array}{llll}\text { O } & 1.012002 & -3.469923 & 0.714557\end{array}$

$\begin{array}{llll}\mathrm{H} & -0.427417 & -3.624193 & 2.135553\end{array}$

$\begin{array}{llll}\mathrm{H} & -4.741243 & -1.776551 & 1.386622\end{array}$

$\begin{array}{llll}\mathrm{H} & -4.841544 & -0.175822 & 1.900425\end{array}$

$\begin{array}{lrrr}\text { CHO } & \\ \mathrm{C} & 0.000000 & 0.000000 & 0.000000 \\ \mathrm{H} & 0.000000 & 0.000000 & 1.087564 \\ \mathrm{H} & 0.968974 & 0.000000 & -0.488361 \\ \mathrm{C} & -1.143665 & 0.000040 & -0.707169 \\ \mathrm{H} & -1.077244 & 0.000155 & -1.793136 \\ \mathrm{C} & -2.459335 & 0.000107 & -0.107389\end{array}$




$\begin{array}{cccc}\mathrm{H} & -2.477492 & 0.000451 & 0.984128 \\ \mathrm{C} & -3.661635 & -0.000190 & -0.749762 \\ \mathrm{C} & -3.834612 & -0.001052 & -2.188851 \\ \mathrm{H} & -2.926453 & -0.002386 & -2.785876 \\ \mathrm{C} & -5.026117 & -0.000476 & -2.825110 \\ \mathrm{H} & -5.966183 & 0.000760 & -2.282604 \\ \mathrm{O} & -4.847518 & 0.000116 & -0.068069 \\ \mathrm{C} & -5.087856 & -0.001452 & -4.289593 \\ \mathrm{H} & -4.095788 & -0.002819 & -4.798046 \\ \mathrm{O} & -6.120216 & -0.000880 & -4.936264 \\ \mathrm{H} & -4.668570 & 0.001877 & 0.885462\end{array}$

TS

$\begin{array}{cccc}\mathrm{H} & 0.000000 & 0.000000 & 0.000000 \\ \mathrm{C} & 0.000000 & 0.000000 & 1.078483 \\ \mathrm{H} & 0.999780 & 0.000000 & 1.510484 \\ \mathrm{C} & -1.021612 & 0.691164 & 1.743739 \\ \mathrm{H} & -0.750715 & 1.354742 & 2.566977 \\ \mathrm{C} & -2.380916 & 0.476987 & 1.552753 \\ \mathrm{H} & -3.091167 & 1.181201 & 1.981864 \\ \mathrm{C} & -2.951956 & -0.770876 & 1.215072 \\ \mathrm{C} & -2.208232 & -1.950424 & 1.147885 \\ \mathrm{H} & -2.645770 & -2.814593 & 1.655394 \\ \mathrm{C} & -0.873776 & -2.041942 & 0.698176 \\ \mathrm{H} & -0.632225 & -1.760696 & -0.316533 \\ \mathrm{C} & -0.036292 & -3.149966 & 1.205189 \\ \mathrm{O} & -4.316917 & -0.814217 & 1.353544 \\ \mathrm{H} & -4.621214 & -1.707915 & 1.132515 \\ \mathrm{H} & -0.444193 & -3.667353 & 2.105380 \\ \mathrm{O} & 1.031545 & -3.482735 & 0.725469\end{array}$

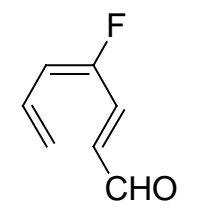

$\begin{array}{llll}\text { C } & 0.000000 & 0.000000 & 0.000000\end{array}$

$\begin{array}{llll}\mathrm{H} & 0.000000 & 0.000000 & 1.087269\end{array}$

$\begin{array}{llll}\mathrm{H} & 0.968380 & 0.000000 & -0.489801\end{array}$

$\begin{array}{llll}\text { C } & -1.144253 & 0.000000 & -0.704748\end{array}$

$\begin{array}{llll}\mathrm{H} & -1.087606 & 0.000000 & -1.791433\end{array}$

$\begin{array}{llll}\text { C } & -2.453846 & 0.000000 & -0.090063\end{array}$

$\begin{array}{llll}\mathrm{H} & -2.494787 & 0.000000 & 0.996927\end{array}$

$\begin{array}{llll}\text { C } & -3.645913 & 0.000000 & -0.732220\end{array}$

$\begin{array}{llll}\text { C } & -3.902458 & 0.000000 & -2.149237\end{array}$ 


$\begin{array}{llll}\mathrm{H} & -3.030104 & 0.000000 & -2.799066 \\ \mathrm{C} & -5.134623 & 0.000000 & -2.704261 \\ \mathrm{H} & -6.034657 & 0.000000 & -2.097272 \\ \mathrm{~F} & -4.764041 & 0.000000 & 0.033981 \\ \mathrm{C} & -5.300839 & 0.000000 & -4.160628 \\ \mathrm{H} & -4.348472 & 0.000053 & -4.739426 \\ \mathrm{O} & -6.377813 & 0.000053 & -4.728860\end{array}$

$\begin{array}{cccc}\text { TS } & & & \\ \mathrm{H} & 0.000000 & 0.000000 & 0.000000 \\ \mathrm{C} & 0.000000 & 0.000000 & 1.078620 \\ \mathrm{H} & 0.998009 & 0.000000 & 1.513665 \\ \mathrm{C} & -1.027568 & 0.688313 & 1.736404 \\ \mathrm{H} & -0.771729 & 1.344795 & 2.569118 \\ \mathrm{C} & -2.381997 & 0.461321 & 1.523471 \\ \mathrm{H} & -3.114443 & 1.146026 & 1.945051 \\ \mathrm{C} & -2.905303 & -0.792031 & 1.157177 \\ \mathrm{C} & -2.185554 & -1.974324 & 1.091098 \\ \mathrm{H} & -2.677734 & -2.842530 & 1.531527 \\ \mathrm{C} & -0.829625 & -2.067432 & 0.706490 \\ \mathrm{H} & -0.537909 & -1.786694 & -0.294377 \\ \mathrm{C} & -0.020044 & -3.180119 & 1.251758 \\ \mathrm{~F} & -4.250920 & -0.906722 & 1.293917 \\ \mathrm{H} & -0.482416 & -3.718509 & 2.111372 \\ \mathrm{O} & 1.081677 & -3.490438 & 0.839370\end{array}$

\begin{tabular}{lccc}
\multicolumn{3}{c}{ CHO } \\
CHO \\
$\mathrm{C}$ & 0.000000 & 0.000000 & 0.000000 \\
$\mathrm{H}$ & 0.000000 & 0.000000 & 1.087580 \\
$\mathrm{H}$ & 0.969380 & 0.000000 & -0.488375 \\
$\mathrm{C}$ & -1.146250 & 0.000000 & -0.704185 \\
$\mathrm{H}$ & -1.095100 & 0.000000 & -1.790010 \\
$\mathrm{C}$ & -2.443796 & 0.000000 & -0.068467 \\
$\mathrm{H}$ & -2.419292 & 0.000000 & 1.022252 \\
$\mathrm{C}$ & -3.680289 & 0.000000 & -0.655147 \\
$\mathrm{C}$ & -3.881833 & 0.000000 & -2.098166 \\
$\mathrm{H}$ & -2.973765 & 0.000000 & -2.701280 \\
$\mathrm{C}$ & -5.054263 & 0.000000 & -2.769677 \\
$\mathrm{H}$ & -6.012921 & 0.000000 & -2.265388 \\
$\mathrm{C}$ & -5.045442 & 0.000000 & -4.238865 \\
$\mathrm{C}$ & -4.821708 & 0.000000 & 0.297367
\end{tabular}




\begin{tabular}{|c|c|c|c|}
\hline $\mathrm{H}$ & -4.505801 & 0.000000 & 606 \\
\hline $\mathrm{O}$ & -6.002639 & 0.000000 & 0.010688 \\
\hline $\mathrm{H}$ & -4.029404 & -0.000088 & -4.699589 \\
\hline $\mathrm{O}$ & -6.044119 & -0.000070 & -4.934464 \\
\hline \multicolumn{4}{|l|}{ TS } \\
\hline $\mathrm{H}$ & 0.000000 & 0.000000 & 0.000000 \\
\hline $\mathrm{C}$ & 0.000000 & 0.000000 & 1.077781 \\
\hline $\mathrm{H}$ & 1.006908 & 0.000000 & 1.497205 \\
\hline $\mathrm{C}$ & -1.004754 & 0.679400 & 1.786966 \\
\hline $\mathrm{H}$ & -0.672869 & 1.388366 & 2.549301 \\
\hline $\mathrm{C}$ & -2.368882 & 0.415786 & 1.787606 \\
\hline $\mathrm{H}$ & -3.034833 & 1.137020 & 2.256632 \\
\hline $\mathrm{C}$ & -2.931089 & -0.877131 & 1.637270 \\
\hline $\mathrm{C}$ & -2.162314 & -2.010597 & 1.367600 \\
\hline $\mathrm{H}$ & -2.446126 & -2.938147 & 1.871157 \\
\hline $\mathrm{C}$ & -0.978454 & -1.992030 & 0.611402 \\
\hline $\mathrm{H}$ & -1.019837 & -1.528305 & -0.364191 \\
\hline $\mathrm{C}$ & -0.019730 & -3.112632 & 0.706253 \\
\hline $\mathrm{C}$ & -4.311556 & -1.077051 & 2.149607 \\
\hline $\mathrm{H}$ & -4.613795 & -2.142905 & 2.249701 \\
\hline $\mathrm{O}$ & -5.086373 & -0.182941 & 2.429302 \\
\hline $\mathrm{H}$ & -0.178359 & -3.791640 & 1.575668 \\
\hline $\mathrm{O}$ & 0.900112 & -3.292881 & -0.069057 \\
\hline & & & \\
\hline $\mathrm{C}$ & 0.000000 & 0.000000 & 0.000000 \\
\hline $\mathrm{H}$ & 0.000000 & 0.000000 & 1.087343 \\
\hline $\mathrm{H}$ & 0.968758 & 0.000000 & -0.489291 \\
\hline $\mathrm{C}$ & -1.146078 & 0.000000 & -0.704383 \\
\hline $\mathrm{H}$ & -1.095425 & -0.000017 & -1.790142 \\
\hline $\mathrm{C}$ & -2.442107 & 0.000000 & -0.066180 \\
\hline $\mathrm{H}$ & -2.432384 & 0.000000 & 1.021777 \\
\hline $\mathrm{C}$ & -3.666660 & 0.000000 & -0.678055 \\
\hline $\mathrm{C}$ & -3.882258 & 0.000021 & -2.118761 \\
\hline $\mathrm{H}$ & -2.988907 & 0.000003 & -2.739494 \\
\hline $\mathrm{C}$ & -5.082311 & 0.000002 & -2.736855 \\
\hline $\mathrm{H}$ & -6.019534 & 0.000033 & -2.185890 \\
\hline $\mathrm{C}$ & -5.164507 & -0.000194 & -4.203364 \\
\hline $\mathrm{C}$ & -4.831215 & 0.000000 & 0.164757 \\
\hline $\mathrm{N}$ & -5.781051 & 0.000000 & 0.836482 \\
\hline
\end{tabular}




\begin{tabular}{|c|c|c|c|}
\hline $\mathrm{H}$ & -4.180177 & 0.001835 & 25707 \\
\hline $\mathrm{O}$ & -6.206343 & 0.001847 & -4.831295 \\
\hline \multicolumn{4}{|l|}{ TS } \\
\hline $\mathrm{H}$ & 0.000000 & 0.000000 & 0.000000 \\
\hline $\mathrm{C}$ & 0.000000 & 0.000000 & 1.077702 \\
\hline $\mathrm{H}$ & 1.005904 & 0.000000 & 1.498728 \\
\hline $\mathrm{C}$ & -1.003593 & 0.684887 & 1.780183 \\
\hline $\mathrm{H}$ & -0.679549 & 1.391594 & 2.547242 \\
\hline $\mathrm{C}$ & -2.370817 & 0.431534 & 1.762423 \\
\hline $\mathrm{H}$ & -3.024689 & 1.167619 & 2.223907 \\
\hline $\mathrm{C}$ & -2.938977 & -0.860946 & 1.592357 \\
\hline $\mathrm{C}$ & -2.167052 & -2.004179 & 1.354412 \\
\hline $\mathrm{H}$ & -2.464039 & -2.921014 & 1.864240 \\
\hline $\mathrm{C}$ & -0.966360 & -1.985282 & 0.626159 \\
\hline $\mathrm{H}$ & -0.988861 & -1.547288 & -0.361707 \\
\hline $\mathrm{C}$ & -0.003312 & -3.100861 & 0.767908 \\
\hline $\mathrm{C}$ & -4.293176 & -1.035300 & 2.043470 \\
\hline $\mathrm{H}$ & -0.179801 & -3.764997 & 1.644466 \\
\hline $\mathrm{O}$ & 0.935733 & -3.285839 & 0.018502 \\
\hline \multirow[t]{3}{*}{$\mathrm{N}$} & -5.396323 & -1.176343 & 2.385695 \\
\hline & $\mathrm{O}_{2}$ & & \\
\hline & $\mathrm{CHO}$ & & \\
\hline $\mathrm{C}$ & 0.000000 & 0.000000 & 0.000000 \\
\hline $\mathrm{H}$ & 0.000000 & 0.000000 & 1.087137 \\
\hline $\mathrm{H}$ & 0.968332 & 0.000000 & -0.490434 \\
\hline $\mathrm{C}$ & -1.146839 & -0.001180 & -0.703913 \\
\hline $\mathrm{H}$ & -1.097715 & -0.001146 & -1.789473 \\
\hline $\mathrm{C}$ & -2.433728 & -0.003171 & -0.049651 \\
\hline $\mathrm{H}$ & -2.424801 & -0.007781 & 1.035924 \\
\hline $\mathrm{C}$ & -3.663017 & -0.000593 & -0.636009 \\
\hline $\mathrm{C}$ & -3.899037 & -0.007599 & -2.066408 \\
\hline $\mathrm{H}$ & -2.971295 & -0.019216 & -2.636734 \\
\hline $\mathrm{C}$ & -5.034125 & -0.023371 & -2.798715 \\
\hline $\mathrm{H}$ & -6.025488 & -0.011493 & -2.367246 \\
\hline $\mathrm{C}$ & -4.931778 & -0.057447 & -4.265536 \\
\hline $\mathrm{N}$ & -4.805285 & 0.016286 & 0.313363 \\
\hline $\mathrm{O}$ & -4.573521 & -0.174366 & 1.507569 \\
\hline $\mathrm{O}$ & -5.926904 & 0.229367 & -0.143343 \\
\hline $\mathrm{H}$ & -3.890545 & -0.060591 & -4.665203 \\
\hline $\mathrm{O}$ & -5.888207 & -0.082027 & -5.016585 \\
\hline
\end{tabular}




$\begin{array}{cccc}\text { TS } & & & \\ \mathrm{H} & 0.000000 & 0.000000 & 0.000000 \\ \mathrm{C} & 0.000000 & 0.000000 & 1.078009 \\ \mathrm{H} & 1.004095 & 0.000000 & 1.502197 \\ \mathrm{C} & -1.010416 & 0.686524 & 1.770328 \\ \mathrm{H} & -0.699657 & 1.390954 & 2.544054 \\ \mathrm{C} & -2.374423 & 0.432825 & 1.716851 \\ \mathrm{H} & -3.061762 & 1.145517 & 2.160800 \\ \mathrm{C} & -2.911665 & -0.851615 & 1.495278 \\ \mathrm{C} & -2.159861 & -2.007951 & 1.322911 \\ \mathrm{H} & -2.508494 & -2.903999 & 1.832114 \\ \mathrm{C} & -0.919365 & -2.015143 & 0.662380 \\ \mathrm{H} & -0.878857 & -1.618953 & -0.342036 \\ \mathrm{C} & 0.027403 & -3.128475 & 0.904820 \\ \mathrm{~N} & -4.327722 & -1.035158 & 1.902294 \\ \mathrm{O} & -4.747236 & -2.188936 & 1.988401 \\ \mathrm{O} & -5.008023 & -0.029363 & 2.099953 \\ \mathrm{H} & -0.214422 & -3.768113 & 1.783714 \\ \mathrm{O} & 1.017524 & -3.333795 & 0.230536\end{array}$

6

$\begin{array}{lrrr} & \\ & & & \\ \mathrm{C} & & \\ \mathrm{C} & 0.000000 & 0.000000 & 0.000000 \\ \mathrm{H} & 0.000000 & 0.000000 & 1.087716 \\ \mathrm{H} & 0.969613 & 0.000000 & -0.487940 \\ \mathrm{C} & -1.144930 & -0.000020 & -0.703842 \\ \mathrm{H} & -1.084569 & -0.000052 & -1.789601 \\ \mathrm{C} & -2.452255 & -0.000043 & -0.076635 \\ \mathrm{H} & -2.431387 & -0.000025 & 1.013164 \\ \mathrm{C} & -3.679820 & -0.000120 & -0.670327 \\ \mathrm{C} & -3.831645 & -0.000087 & -2.114845 \\ \mathrm{H} & -2.928175 & 0.000641 & -2.717066 \\ \mathrm{C} & -5.015805 & -0.000932 & -2.773426 \\ \mathrm{H} & -5.964748 & -0.001753 & -2.245251 \\ \mathrm{C} & -4.927845 & -0.000358 & 0.180986 \\ \mathrm{C} & -5.093869 & -0.000844 & -4.195074 \\ \mathrm{~N} & -5.175762 & -0.001103 & -5.357500 \\ \mathrm{H} & -5.549667 & 0.881713 & -0.019450 \\ \mathrm{H} & -5.548673 & -0.883362 & -0.018594\end{array}$




\begin{tabular}{|c|c|c|c|}
\hline $\mathrm{H}$ & -4.678764 & 0.000230 & 1.245539 \\
\hline \multicolumn{4}{|l|}{ TS } \\
\hline $\mathrm{H}$ & 0.000000 & 0.000000 & 0.000000 \\
\hline $\mathrm{H}$ & 0.000000 & 0.000000 & 1.813474 \\
\hline $\mathrm{C}$ & 0.594299 & 0.000000 & 0.912802 \\
\hline $\mathrm{C}$ & 1.815576 & 0.680771 & 0.923603 \\
\hline $\mathrm{H}$ & 2.054335 & 1.328200 & 0.077835 \\
\hline $\mathrm{C}$ & 2.841813 & 0.462380 & 1.839795 \\
\hline $\mathrm{H}$ & 3.641362 & 1.201962 & 1.873503 \\
\hline $\mathrm{C}$ & 3.159922 & -0.784087 & 2.431103 \\
\hline $\mathrm{C}$ & 2.480342 & -1.954927 & 2.082848 \\
\hline $\mathrm{H}$ & 3.095636 & -2.847434 & 1.963996 \\
\hline $\mathrm{C}$ & 1.121336 & -2.052380 & 1.704093 \\
\hline $\mathrm{H}$ & 0.371788 & -1.747068 & 2.418137 \\
\hline $\mathrm{C}$ & 4.474678 & -0.907662 & 3.172454 \\
\hline $\mathrm{C}$ & 0.705462 & -3.174310 & 0.909947 \\
\hline $\mathrm{H}$ & 5.202713 & -0.154626 & 2.852757 \\
\hline $\mathrm{H}$ & 4.320718 & -0.776092 & 4.251722 \\
\hline $\mathrm{H}$ & 4.919278 & -1.899489 & 3.033962 \\
\hline $\mathrm{N}$ & 0.356020 & -4.058037 & 0.236681 \\
\hline & & & \\
\hline & & & \\
\hline $\mathrm{C}$ & 0.000000 & 0.000000 & 0.000000 \\
\hline $\mathrm{H}$ & 0.000000 & 0.000000 & 1.087638 \\
\hline $\mathrm{H}$ & 0.969029 & 0.000000 & -0.488452 \\
\hline $\mathrm{C}$ & -1.144996 & 0.004902 & -0.706550 \\
\hline $\mathrm{H}$ & -1.077822 & 0.021277 & -1.792487 \\
\hline $\mathrm{C}$ & -2.458894 & -0.003989 & -0.105147 \\
\hline $\mathrm{H}$ & -2.475246 & 0.022362 & 0.985166 \\
\hline $\mathrm{C}$ & -3.666857 & -0.001355 & -0.745581 \\
\hline $\mathrm{C}$ & -3.788130 & -0.100352 & -2.192928 \\
\hline $\mathrm{H}$ & -2.910941 & -0.424745 & -2.741996 \\
\hline $\mathrm{C}$ & -4.923683 & 0.159550 & -2.881927 \\
\hline $\mathrm{H}$ & -5.813341 & 0.529400 & -2.379932 \\
\hline $\mathrm{C}$ & -5.011408 & 0.004956 & -4.294416 \\
\hline $\mathrm{N}$ & -4.870079 & 0.161372 & -0.042124 \\
\hline $\mathrm{H}$ & -4.759133 & 0.157718 & 0.965737 \\
\hline $\mathrm{H}$ & -5.603215 & -0.482794 & -0.322401 \\
\hline $\mathrm{N}$ & -5.102537 & -0.119066 & -5.449369 \\
\hline
\end{tabular}




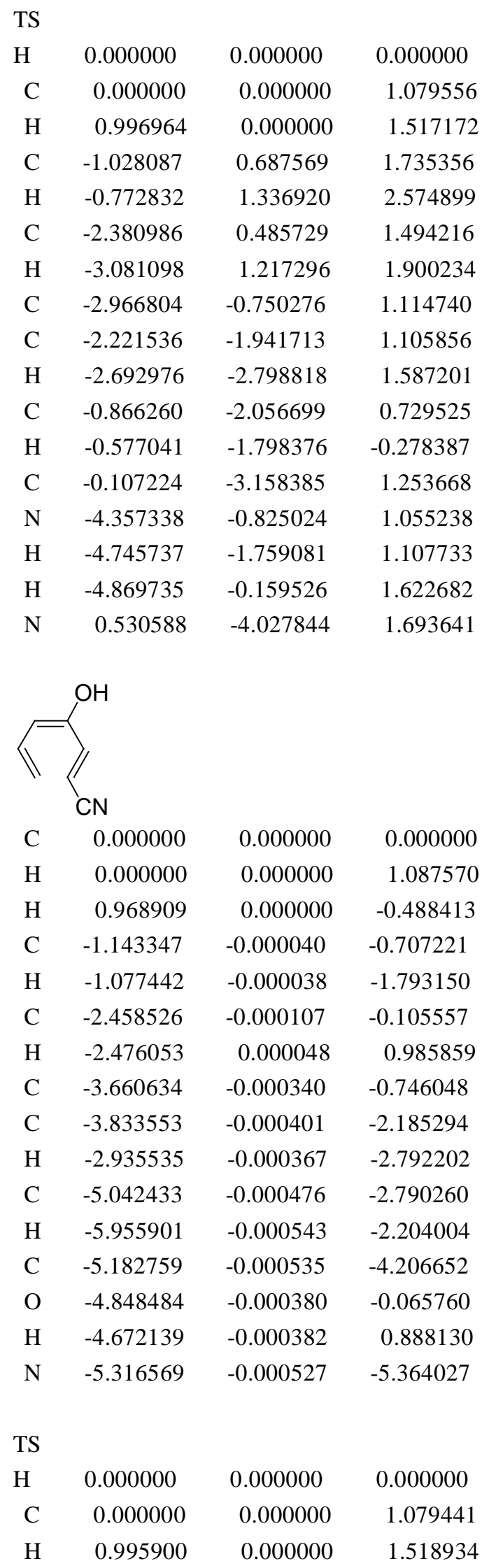




$\begin{array}{lccc}\mathrm{C} & -1.033107 & 0.676201 & 1.736732 \\ \mathrm{H} & -0.785022 & 1.318985 & 2.582714 \\ \mathrm{C} & -2.384452 & 0.469949 & 1.489681 \\ \mathrm{H} & -3.112890 & 1.167727 & 1.897880 \\ \mathrm{C} & -2.941200 & -0.765602 & 1.094370 \\ \mathrm{C} & -2.217714 & -1.961865 & 1.062207 \\ \mathrm{H} & -2.705289 & -2.822646 & 1.522910 \\ \mathrm{C} & -0.854385 & -2.075478 & 0.708994 \\ \mathrm{H} & -0.547050 & -1.801062 & -0.288641 \\ \mathrm{O} & -4.311456 & -0.796614 & 1.133762 \\ \mathrm{C} & -0.106174 & -3.183512 & 1.232179 \\ \mathrm{~N} & 0.522070 & -4.060136 & 1.671670 \\ \mathrm{H} & -4.611280 & -1.687857 & 0.896503\end{array}$

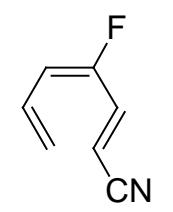

$\begin{array}{lccc}\mathrm{C} & 0.000000 & 0.000000 & 0.000000 \\ \mathrm{H} & 0.000000 & 0.000000 & 1.087253 \\ \mathrm{H} & 0.968355 & 0.000000 & -0.489788 \\ \mathrm{C} & -1.143768 & 0.000000 & -0.705303 \\ \mathrm{H} & -1.087217 & 0.000000 & -1.791970 \\ \mathrm{C} & -2.452716 & 0.000000 & -0.088995 \\ \mathrm{H} & -2.493162 & 0.000000 & 0.997968 \\ \mathrm{C} & -3.644413 & 0.000000 & -0.729880 \\ \mathrm{C} & -3.898914 & 0.000000 & -2.147601 \\ \mathrm{H} & -3.035949 & 0.000000 & -2.804885 \\ \mathrm{C} & -5.144862 & 0.000000 & -2.674525 \\ \mathrm{H} & -6.017924 & 0.000000 & -2.029412 \\ \mathrm{C} & -5.379514 & 0.000000 & -4.077204 \\ \mathrm{~F} & -4.765219 & 0.000000 & 0.033966 \\ \mathrm{~N} & -5.585974 & 0.000000 & -5.223970\end{array}$

$\begin{array}{cccc}\text { TS } & & & \\ \mathrm{H} & 0.000000 & 0.000000 & 0.000000 \\ \mathrm{C} & 0.000000 & 0.000000 & 1.079566 \\ \mathrm{H} & 0.994521 & 0.000000 & 1.521420 \\ \mathrm{C} & -1.037001 & 0.675909 & 1.730572 \\ \mathrm{H} & -0.800805 & 1.314063 & 2.582529 \\ \mathrm{C} & -2.383667 & 0.457776 & 1.466542 \\ \mathrm{H} & -3.131824 & 1.137667 & 1.867533 \\ \mathrm{C} & -2.893179 & -0.783699 & 1.049948 \\ \mathrm{C} & -2.194155 & -1.981814 & 1.009496\end{array}$




$\begin{array}{lccc}\mathrm{H} & -2.728055 & -2.846342 & 1.400715 \\ \mathrm{C} & -0.815813 & -2.093608 & 0.711110 \\ \mathrm{H} & -0.471050 & -1.816475 & -0.273005 \\ \mathrm{C} & -0.088450 & -3.202699 & 1.260078 \\ \mathrm{~F} & -4.244394 & -0.887616 & 1.096317 \\ \mathrm{~N} & 0.527853 & -4.074826 & 1.724308\end{array}$

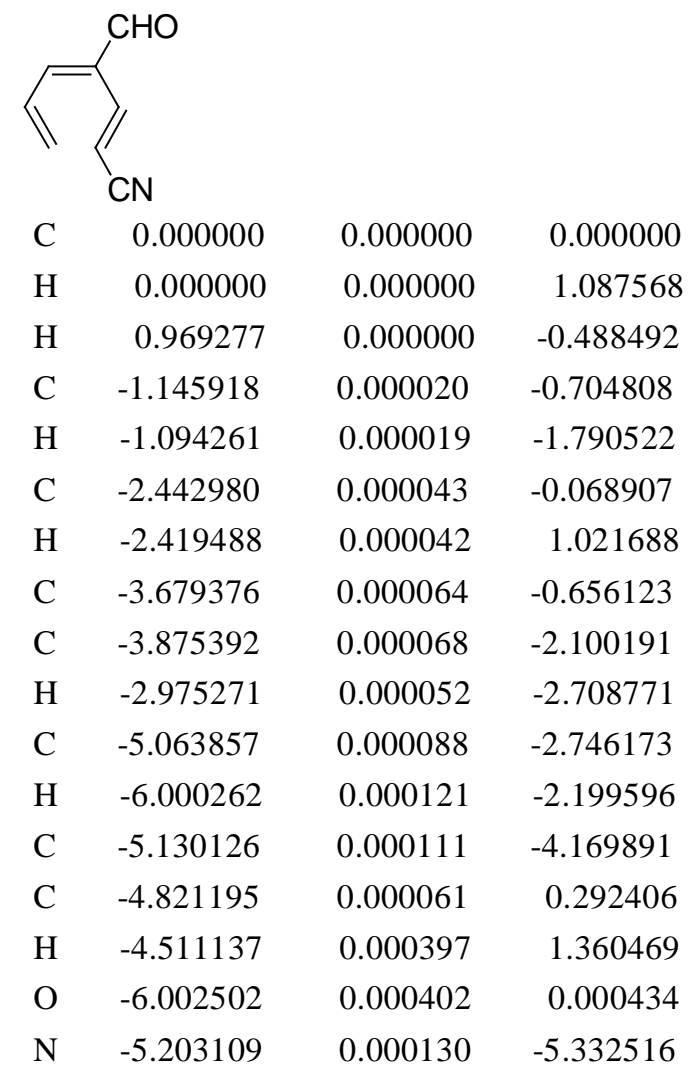

$\begin{array}{cccc}\text { TS } & & & \\ \mathrm{H} & 0.000000 & 0.000000 & 0.000000 \\ \mathrm{C} & 0.000000 & 0.000000 & 1.078594 \\ \mathrm{H} & 1.001078 & 0.000000 & 1.508919 \\ \mathrm{C} & -1.019899 & 0.678210 & 1.761576 \\ \mathrm{H} & -0.720066 & 1.364473 & 2.556284 \\ \mathrm{C} & -2.383725 & 0.436008 & 1.660837 \\ \mathrm{H} & -3.077129 & 1.158451 & 2.086069 \\ \mathrm{C} & -2.949473 & -0.842602 & 1.435422 \\ \mathrm{C} & -2.195643 & -2.005645 & 1.260100 \\ \mathrm{H} & -2.571229 & -2.915361 & 1.730346 \\ \mathrm{C} & -0.923016 & -2.046426 & 0.656106 \\ \mathrm{H} & -0.841091 & -1.642969 & -0.341954 \\ \mathrm{C} & -0.062023 & -3.169132 & 0.890693 \\ \mathrm{C} & -4.386216 & -1.011489 & 1.788851\end{array}$




$\begin{array}{crrr}\mathrm{H} & -4.724514 & -2.068622 & 1.852985 \\ \mathrm{O} & -5.162528 & -0.096921 & 1.980893 \\ \mathrm{~N} & 0.663730 & -4.055881 & 1.096627\end{array}$

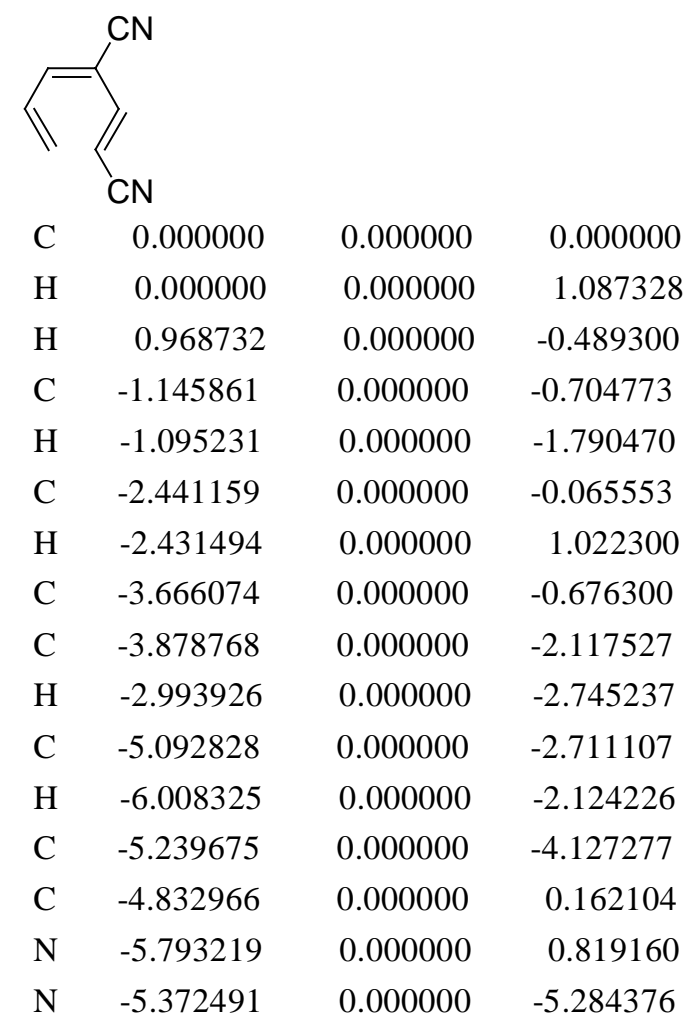

$\begin{array}{cccc}\text { TS } & & & \\ \mathrm{H} & 0.000000 & 0.000000 & 0.000000 \\ \mathrm{C} & 0.000000 & 0.000000 & 1.078647 \\ \mathrm{H} & 0.999762 & 0.000000 & 1.511179 \\ \mathrm{C} & -1.020076 & 0.681284 & 1.754964 \\ \mathrm{H} & -0.731135 & 1.363725 & 2.556253 \\ \mathrm{C} & -2.384265 & 0.446506 & 1.630775 \\ \mathrm{H} & -3.068861 & 1.180254 & 2.049255 \\ \mathrm{C} & -2.949067 & -0.832686 & 1.379716 \\ \mathrm{C} & -2.193177 & -2.004717 & 1.240908 \\ \mathrm{H} & -2.584564 & -2.903982 & 1.712895 \\ \mathrm{C} & -0.903541 & -2.044318 & 0.675141 \\ \mathrm{H} & -0.795295 & -1.669446 & -0.331463 \\ \mathrm{C} & -0.041916 & -3.154086 & 0.965866 \\ \mathrm{C} & -4.353102 & -0.979157 & 1.658636 \\ \mathrm{~N} & -5.493390 & -1.091834 & 1.859342 \\ \mathrm{~N} & 0.686791 & -4.026653 & 1.215509\end{array}$




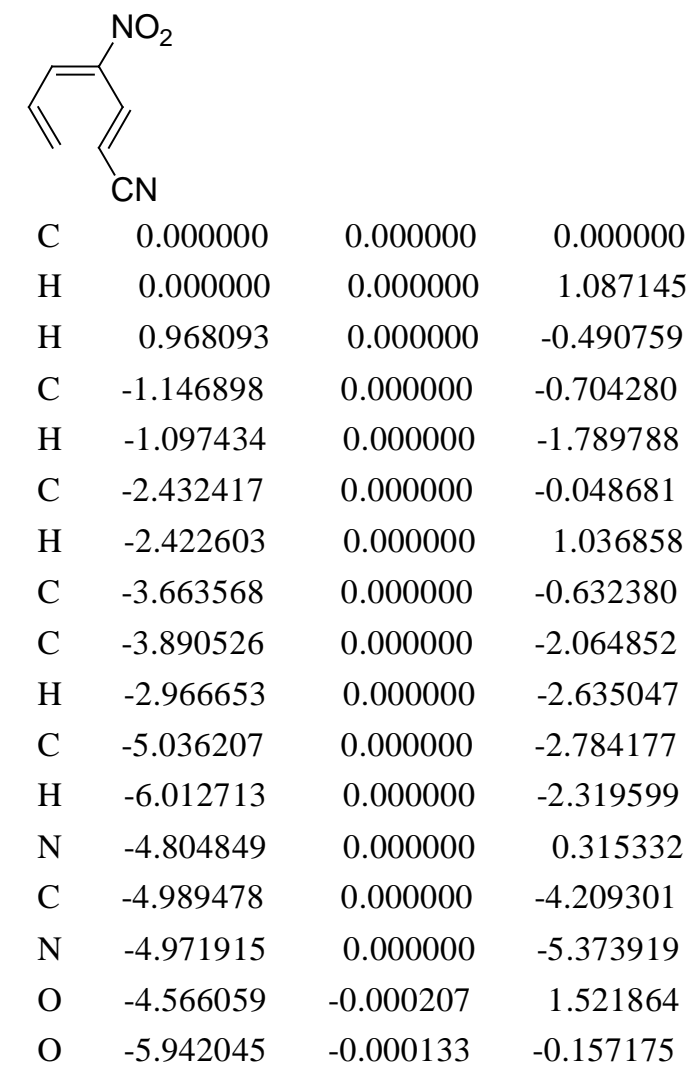

$\begin{array}{cccc}\text { TS } & & & \\ \mathrm{H} & 0.000000 & 0.000000 & 0.000000 \\ \mathrm{C} & 0.000000 & 0.000000 & 1.079018 \\ \mathrm{H} & 0.999114 & 0.000000 & 1.512595 \\ \mathrm{C} & -1.023799 & 0.680468 & 1.752101 \\ \mathrm{H} & -0.742340 & 1.362014 & 2.556076 \\ \mathrm{C} & -2.383976 & 0.441806 & 1.611473 \\ \mathrm{H} & -3.095917 & 1.151792 & 2.019569 \\ \mathrm{C} & -2.914484 & -0.829751 & 1.320241 \\ \mathrm{C} & -2.183735 & -2.009627 & 1.220117 \\ \mathrm{H} & -2.610636 & -2.894202 & 1.684064 \\ \mathrm{C} & -0.876132 & -2.058893 & 0.696693 \\ \mathrm{H} & -0.732809 & -1.705181 & -0.313049 \\ \mathrm{C} & -0.024518 & -3.161483 & 1.039670 \\ \mathrm{~N} & -4.371802 & -0.988900 & 1.575334 \\ \mathrm{O} & -4.822258 & -2.133069 & 1.593140 \\ \mathrm{O} & -5.044915 & 0.029655 & 1.721480 \\ \mathrm{~N} & 0.698619 & -4.025199 & 1.332361\end{array}$




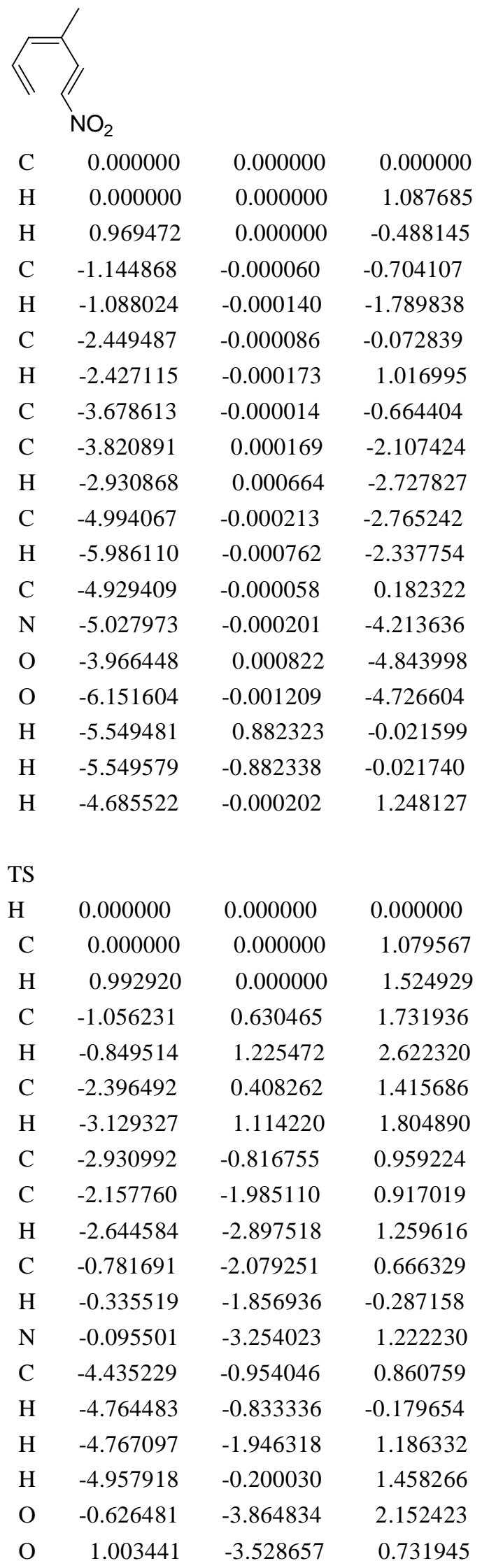




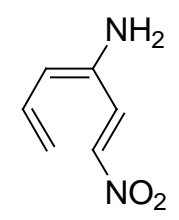

$\begin{array}{lrrr}\mathrm{C} & 0.000000 & 0.000000 & 0.000000 \\ \mathrm{H} & 0.000000 & 0.000000 & 1.087587 \\ \mathrm{H} & 0.968781 & 0.000000 & -0.488772 \\ \mathrm{C} & -1.145244 & 0.004203 & -0.706650 \\ \mathrm{H} & -1.080933 & 0.018980 & -1.792474 \\ \mathrm{C} & -2.455429 & -0.007260 & -0.099786 \\ \mathrm{H} & -2.467004 & 0.011085 & 0.990768 \\ \mathrm{C} & -3.668446 & 0.000379 & -0.733467 \\ \mathrm{C} & -3.784267 & -0.070604 & -2.179163 \\ \mathrm{H} & -2.911533 & -0.350458 & -2.757450 \\ \mathrm{C} & -4.918191 & 0.166999 & -2.860326 \\ \mathrm{H} & -5.863071 & 0.496371 & -2.452950 \\ \mathrm{~N} & -4.955011 & 0.035784 & -4.302161 \\ \mathrm{~N} & -4.867705 & 0.146451 & -0.022673 \\ \mathrm{H} & -4.758590 & 0.103604 & 0.984301 \\ \mathrm{H} & -5.612915 & -0.470866 & -0.328836 \\ \mathrm{O} & -3.936686 & -0.320684 & -4.901984 \\ \mathrm{O} & -6.035945 & 0.296657 & -4.839724\end{array}$

$\begin{array}{cccc}\text { TS } & & & \\ \mathrm{H} & 0.000000 & 0.000000 & 0.000000 \\ \mathrm{C} & 0.000000 & 0.000000 & 1.080081 \\ \mathrm{H} & 0.990790 & 0.000000 & 1.529044 \\ \mathrm{C} & -1.063006 & 0.623188 & 1.730632 \\ \mathrm{H} & -0.869519 & 1.213940 & 2.626920 \\ \mathrm{C} & -2.393076 & 0.407590 & 1.385288 \\ \mathrm{H} & -3.142944 & 1.091872 & 1.784043 \\ \mathrm{C} & -2.907214 & -0.803623 & 0.860359 \\ \mathrm{C} & -2.146942 & -1.989829 & 0.877755 \\ \mathrm{H} & -2.653725 & -2.877854 & 1.253346 \\ \mathrm{C} & -0.767210 & -2.092187 & 0.677662 \\ \mathrm{H} & -0.268525 & -1.899021 & -0.256524 \\ \mathrm{~N} & -0.107886 & -3.247675 & 1.305121 \\ \mathrm{~N} & -4.273205 & -0.893946 & 0.617624 \\ \mathrm{H} & -4.656902 & -1.831204 & 0.598865 \\ \mathrm{H} & -4.866270 & -0.235539 & 1.109008 \\ \mathrm{O} & -0.680857 & -3.830437 & 2.228816 \\ \mathrm{O} & 1.011226 & -3.539361 & 0.873988\end{array}$




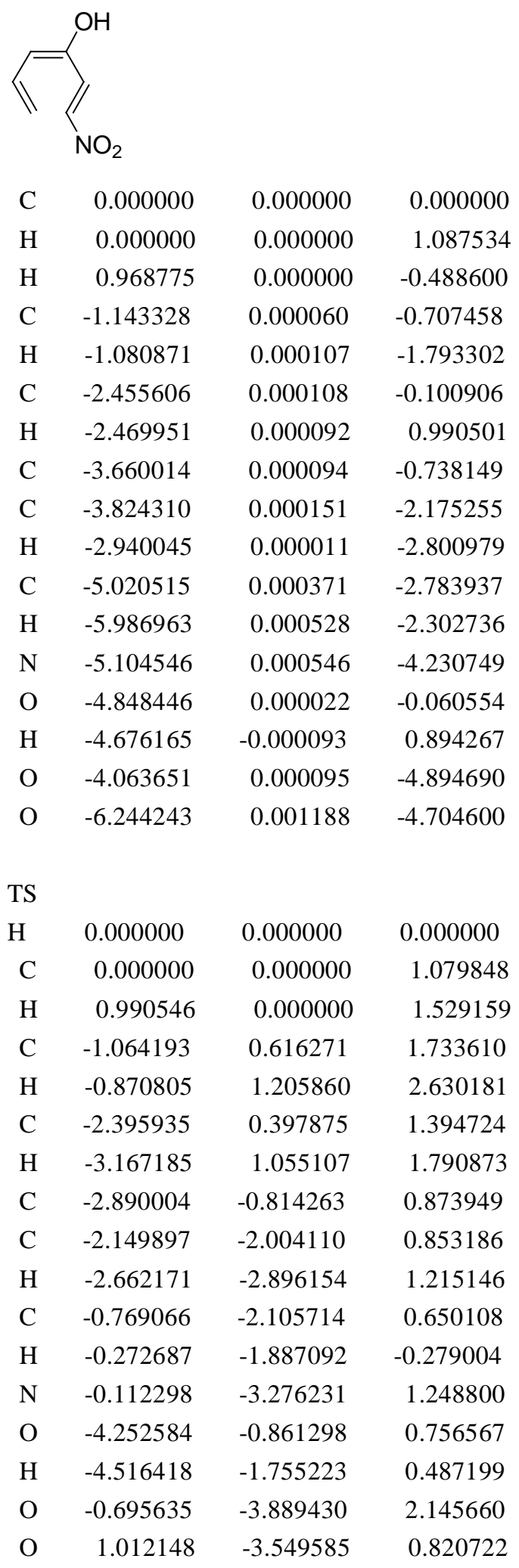




$\begin{array}{lccc}\text { Z } & & \\ \mathrm{C} & & & \\ \mathrm{H} & 0.000000 & 0.000000 & 0.000000 \\ \mathrm{H} & 0.000000 & 0.000000 & 1.087247 \\ \mathrm{C} & -1.143947 & 0.000000 & -0.705276 \\ \mathrm{H} & -1.090861 & 0.000000 & -1.791877 \\ \mathrm{C} & -2.450324 & 0.000000 & -0.084963 \\ \mathrm{H} & -2.488552 & 0.000000 & 1.002137 \\ \mathrm{C} & -3.643758 & 0.000000 & -0.723831 \\ \mathrm{C} & -3.889915 & 0.000000 & -2.139790 \\ \mathrm{H} & -3.041885 & 0.000000 & -2.815480 \\ \mathrm{C} & -5.123169 & 0.000000 & -2.671066 \\ \mathrm{H} & -6.056037 & 0.000000 & -2.127185 \\ \mathrm{~F} & -4.765310 & 0.000000 & 0.037660 \\ \mathrm{~N} & -5.301210 & 0.000000 & -4.108447 \\ \mathrm{O} & -4.304505 & -0.000132 & -4.836750 \\ \mathrm{O} & -6.469140 & -0.000136 & -4.506202\end{array}$

$\begin{array}{cccc}\text { TS } & & & \\ \mathrm{H} & 0.000000 & 0.000000 & 0.000000 \\ \mathrm{C} & 0.000000 & 0.000000 & 1.079869 \\ \mathrm{H} & 0.990233 & 0.000000 & 1.529517 \\ \mathrm{C} & -1.063041 & 0.623458 & 1.729244 \\ \mathrm{H} & -0.873314 & 1.214956 & 2.624672 \\ \mathrm{C} & -2.393621 & 0.396065 & 1.387640 \\ \mathrm{H} & -3.176315 & 1.043635 & 1.775852 \\ \mathrm{C} & -2.850735 & -0.824258 & 0.871545 \\ \mathrm{C} & -2.134678 & -2.016095 & 0.833144 \\ \mathrm{H} & -2.677657 & -2.906544 & 1.142861 \\ \mathrm{C} & -0.749138 & -2.111016 & 0.649358 \\ \mathrm{H} & -0.246198 & -1.883433 & -0.273722 \\ \mathrm{~N} & -0.097044 & -3.285781 & 1.246484 \\ \mathrm{~F} & -4.196804 & -0.944816 & 0.789514 \\ \mathrm{O} & -0.690864 & -3.903230 & 2.132024 \\ \mathrm{O} & 1.033328 & -3.550868 & 0.830222\end{array}$<smiles>C=C/C=C(/C=O)C=C[N+](=O)[O-]</smiles> 


$\begin{array}{lrrr}\mathrm{C} & 0.000000 & 0.000000 & 0.000000 \\ \mathrm{H} & 0.000000 & 0.000000 & 1.087553 \\ \mathrm{H} & 0.969242 & 0.000000 & -0.488581 \\ \mathrm{C} & -1.145928 & 0.000000 & -0.704952 \\ \mathrm{H} & -1.097463 & 0.000000 & -1.790642 \\ \mathrm{C} & -2.440614 & 0.000000 & -0.065051 \\ \mathrm{H} & -2.415731 & 0.000000 & 1.025575 \\ \mathrm{C} & -3.677781 & 0.000000 & -0.649936 \\ \mathrm{C} & -3.867044 & 0.000000 & -2.092779 \\ \mathrm{H} & -2.981628 & 0.000000 & -2.721105 \\ \mathrm{C} & -5.040211 & 0.000000 & -2.743505 \\ \mathrm{H} & -6.027872 & 0.000000 & -2.311020 \\ \mathrm{C} & -4.828397 & 0.000000 & 0.287113 \\ \mathrm{~N} & -5.047407 & 0.000000 & -4.200406 \\ \mathrm{O} & -3.975831 & 0.000000 & -4.813159 \\ \mathrm{O} & -6.160986 & 0.000000 & -4.729006 \\ \mathrm{O} & -6.003391 & 0.000052 & -0.025858 \\ \mathrm{H} & -4.532818 & 0.000053 & 1.359108\end{array}$

$\begin{array}{cccc}\text { TS } & & & \\ \mathrm{H} & 0.000000 & 0.000000 & 0.000000 \\ \mathrm{C} & 0.000000 & 0.000000 & 1.078749 \\ \mathrm{H} & 0.995757 & 0.000000 & 1.518906 \\ \mathrm{C} & -1.044969 & 0.642995 & 1.746926 \\ \mathrm{H} & -0.796841 & 1.280532 & 2.596865 \\ \mathrm{C} & -2.397792 & 0.408489 & 1.525609 \\ \mathrm{H} & -3.129489 & 1.109965 & 1.920704 \\ \mathrm{C} & -2.934811 & -0.850352 & 1.171492 \\ \mathrm{C} & -2.172873 & -2.018422 & 1.053014 \\ \mathrm{H} & -2.602665 & -2.939138 & 1.447448 \\ \mathrm{C} & -0.841044 & -2.074933 & 0.631326 \\ \mathrm{H} & -0.534529 & -1.777201 & -0.355857 \\ \mathrm{~N} & -0.065150 & -3.255932 & 1.029035 \\ \mathrm{C} & -4.407800 & -1.020076 & 1.331985 \\ \mathrm{H} & -4.756566 & -2.075060 & 1.313100 \\ \mathrm{O} & -5.194749 & -0.104250 & 1.459861 \\ \mathrm{O} & -0.476068 & -3.940398 & 1.967761 \\ \mathrm{O} & 0.977682 & -3.458544 & 0.403353\end{array}$<smiles>C=C/C=C(/C#N)C=C[N+](=O)[O-]</smiles> 


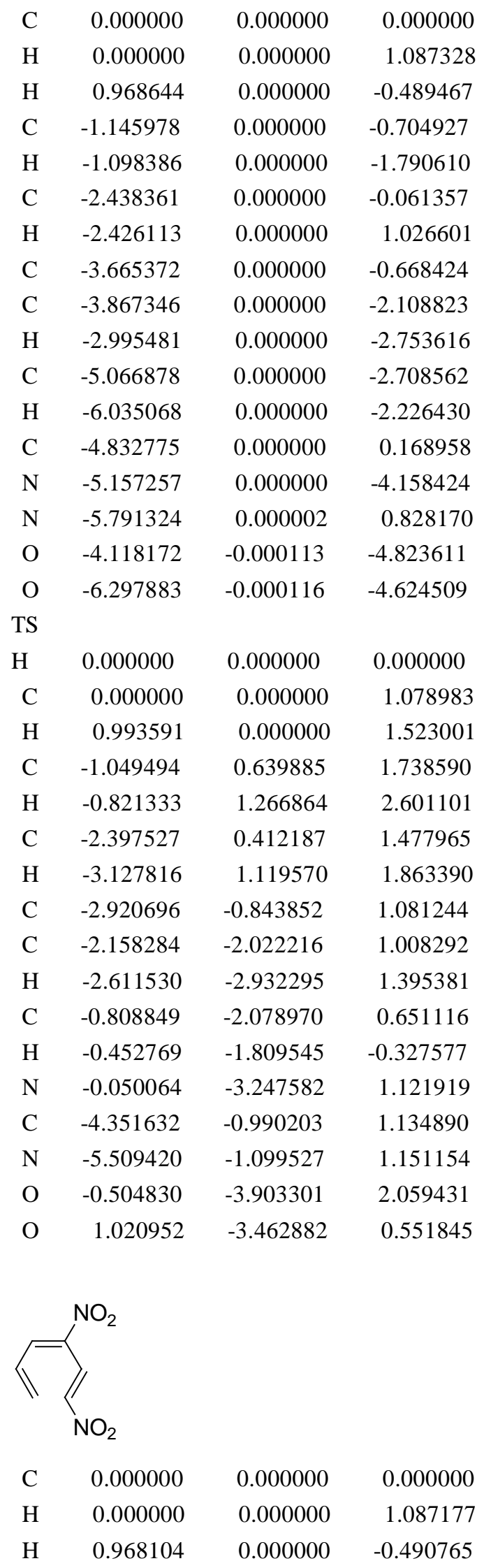




$\begin{array}{cccc}\mathrm{C} & -1.146663 & 0.000000 & -0.704853 \\ \mathrm{H} & -1.100823 & 0.000000 & -1.790407 \\ \mathrm{C} & -2.430354 & 0.000000 & -0.046937 \\ \mathrm{H} & -2.422962 & 0.000000 & 1.038911 \\ \mathrm{C} & -3.659559 & 0.000000 & -0.633738 \\ \mathrm{C} & -3.880753 & -0.000021 & -2.062895 \\ \mathrm{H} & -2.973591 & -0.000037 & -2.659470 \\ \mathrm{C} & -5.012879 & -0.000024 & -2.782446 \\ \mathrm{H} & -6.031724 & -0.000040 & -2.436297 \\ \mathrm{~N} & -4.810938 & 0.000000 & 0.299162 \\ \mathrm{~N} & -4.909160 & -0.000003 & -4.239013 \\ \mathrm{O} & -5.939134 & -0.000095 & -0.191953 \\ \mathrm{O} & -4.585259 & -0.000076 & 1.508336 \\ \mathrm{O} & -3.795918 & -0.001593 & -4.771411 \\ \mathrm{O} & -5.981298 & -0.001637 & -4.845428 \\ & & & \\ \mathrm{TS} & & & \\ \mathrm{H} & 0.000000 & 0.000000 & 0.000000 \\ \mathrm{C} & 0.000000 & 0.000000 & 1.079271 \\ \mathrm{H} & 0.993692 & 0.000000 & 1.522980 \\ \mathrm{C} & -1.050799 & 0.639750 & 1.739328 \\ \mathrm{H} & -0.823956 & 1.269650 & 2.599540 \\ \mathrm{C} & -2.396488 & 0.406694 & 1.479316 \\ \mathrm{H} & -3.148020 & 1.091214 & 1.859357 \\ \mathrm{C} & -2.885865 & -0.841370 & 1.058443 \\ \mathrm{C} & -2.152620 & -2.025742 & 1.002895 \\ \mathrm{H} & -2.629055 & -2.924902 & 1.382761 \\ \mathrm{C} & -0.799196 & -2.082997 & 0.660383 \\ \mathrm{H} & -0.434111 & -1.820693 & -0.317037 \\ \mathrm{~N} & -0.044766 & -3.245913 & 1.150912 \\ \mathrm{~N} & -4.365609 & -1.003329 & 1.113877 \\ \mathrm{O} & -4.813390 & -2.141206 & 0.991852 \\ \mathrm{O} & -5.049216 & 0.009722 & 1.245607 \\ \mathrm{O} & -0.510038 & -3.891353 & 2.089976 \\ \mathrm{O} & 1.033221 & -3.464756 & 0.595613\end{array}$

\section{1-4 substituted hexatriene}

1<smiles>C/C=C\C=C(C)\C=C/C</smiles> 


\begin{tabular}{|c|c|c|c|}
\hline $\mathrm{C}$ & 0.000000 & 0.000000 & 0.000000 \\
\hline $\mathrm{H}$ & 0.000000 & 0.000000 & 1.085954 \\
\hline $\mathrm{H}$ & 0.973836 & 0.000000 & -0.480623 \\
\hline $\mathrm{C}$ & -1.129746 & -0.000020 & -0.726649 \\
\hline $\mathrm{H}$ & -1.030592 & -0.000069 & -1.809672 \\
\hline $\mathrm{C}$ & -2.491024 & -0.000086 & -0.193006 \\
\hline $\mathrm{C}$ & -3.591961 & 0.000057 & -0.992825 \\
\hline $\mathrm{H}$ & -4.563613 & -0.000075 & -0.497616 \\
\hline $\mathrm{C}$ & -3.654325 & 0.000369 & -2.442089 \\
\hline $\mathrm{H}$ & -2.722157 & 0.000782 & -3.004652 \\
\hline $\mathrm{C}$ & -4.810196 & 0.000231 & -3.132466 \\
\hline $\mathrm{H}$ & -5.749439 & -0.000132 & -2.576424 \\
\hline $\mathrm{C}$ & -4.923651 & 0.000540 & -4.626713 \\
\hline $\mathrm{C}$ & -2.658519 & -0.000369 & 1.308053 \\
\hline $\mathrm{H}$ & -2.187347 & -0.881641 & 1.762993 \\
\hline $\mathrm{H}$ & -3.715604 & -0.000323 & 1.589361 \\
\hline $\mathrm{H}$ & -2.187214 & 0.880640 & 1.763358 \\
\hline $\mathrm{H}$ & -5.476334 & 0.880425 & -4.984922 \\
\hline $\mathrm{H}$ & -5.476135 & -0.879322 & -4.985282 \\
\hline $\mathrm{H}$ & -3.939168 & 0.000738 & -5.106729 \\
\hline \multicolumn{4}{|l|}{ is } \\
\hline $\mathrm{H}$ & 0.000000 & 0.000000 & 0.000000 \\
\hline $\mathrm{H}$ & 0.000000 & 0.000000 & 1.808388 \\
\hline $\mathrm{C}$ & 0.599767 & 0.000000 & 0.911290 \\
\hline $\mathrm{C}$ & 1.792661 & 0.730052 & 0.911516 \\
\hline $\mathrm{H}$ & 1.996194 & 1.384087 & 0.059990 \\
\hline $\mathrm{C}$ & 2.854756 & 0.554794 & 1.809718 \\
\hline $\mathrm{C}$ & 3.161994 & -0.720860 & 2.331683 \\
\hline $\mathrm{H}$ & 4.168877 & -0.821123 & 2.738056 \\
\hline $\mathrm{C}$ & 2.493327 & -1.908085 & 2.030232 \\
\hline $\mathrm{H}$ & 3.134641 & -2.777995 & 1.859909 \\
\hline $\mathrm{C}$ & 1.128583 & -2.047244 & 1.732931 \\
\hline $\mathrm{H}$ & 0.437058 & -1.690178 & 2.481965 \\
\hline $\mathrm{C}$ & 0.629958 & -3.259638 & 0.978574 \\
\hline $\mathrm{C}$ & 3.882092 & 1.658028 & 1.954538 \\
\hline $\mathrm{H}$ & 3.921209 & 2.283633 & 1.054579 \\
\hline $\mathrm{H}$ & 3.627051 & 2.324269 & 2.790045 \\
\hline $\mathrm{H}$ & 4.888002 & 1.267793 & 2.145156 \\
\hline $\mathrm{H}$ & 1.347872 & -3.586533 & 0.219177 \\
\hline $\mathrm{H}$ & 0.446810 & -4.105359 & 1.658234 \\
\hline $\mathrm{H}$ & -0.318410 & -3.047153 & 0.473095 \\
\hline
\end{tabular}




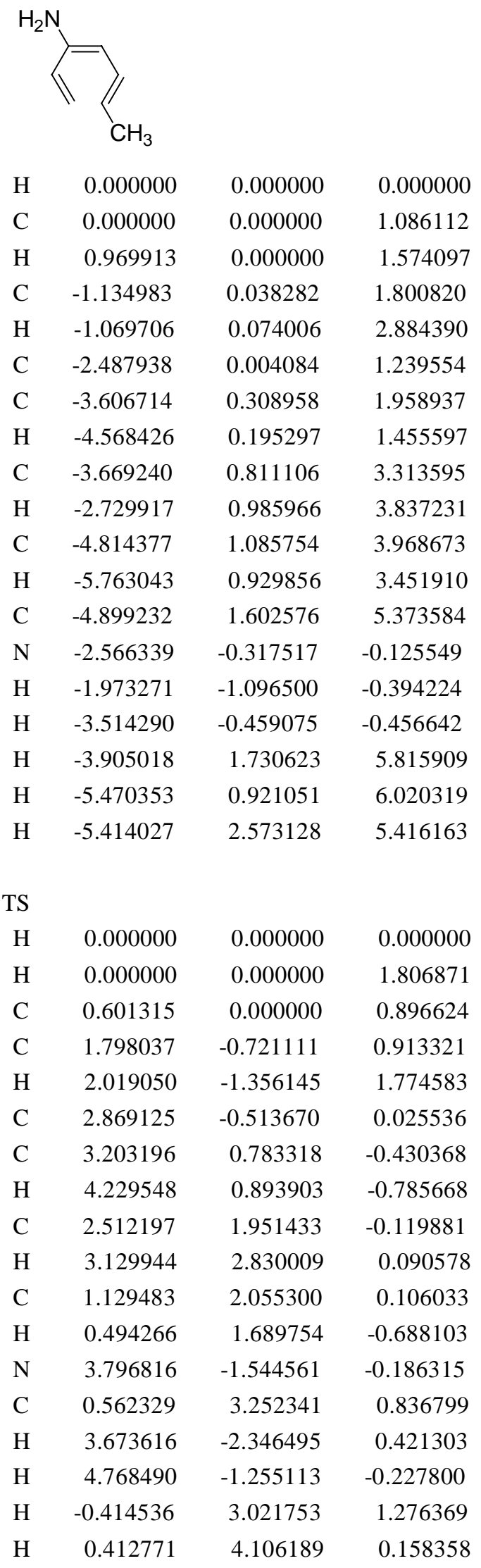




$\begin{array}{lrrr}\mathrm{H} & 1.221774 & 3.580294 & 1.647472 \\ & & & \\ \mathrm{H} & & & \\ \mathrm{H} & & & \\ \mathrm{C} & 0.000000 & 0.000000 & 0.000000 \\ \mathrm{H} & 0.900000 & 0.000000 & 1.084683 \\ \mathrm{C} & -1.140950 & -0.000020 & 1.788550 \\ \mathrm{H} & -1.101492 & 0.000047 & 2.873416 \\ \mathrm{C} & -2.473466 & -0.000002 & 1.196975 \\ \mathrm{C} & -3.645495 & 0.000056 & 1.886994 \\ \mathrm{H} & -4.568043 & 0.000385 & 1.302250 \\ \mathrm{C} & -3.819654 & -0.000191 & 3.323857 \\ \mathrm{H} & -2.925587 & -0.001143 & 3.946060 \\ \mathrm{C} & -5.016781 & 0.000604 & 3.940365 \\ \mathrm{H} & -5.920715 & 0.001495 & 3.328678 \\ \mathrm{C} & -5.219115 & 0.000303 & 5.425918 \\ \mathrm{O} & -2.447944 & 0.000260 & -0.174671 \\ \mathrm{H} & -3.360663 & 0.000306 & -0.502802 \\ \mathrm{H} & -4.263956 & -0.001066 & 5.962499 \\ \mathrm{H} & -5.791330 & -0.879175 & 5.753758 \\ \mathrm{H} & -5.789179 & 0.880939 & 5.754430 \\ & & & \end{array}$

$\begin{array}{crrr}\text { TS } & & & \\ \mathrm{H} & 0.000000 & 0.000000 & 0.000000 \\ \mathrm{C} & 0.000000 & 0.000000 & 1.079032 \\ \mathrm{H} & 1.006162 & 0.000000 & 1.500228 \\ \mathrm{C} & -0.984602 & 0.720936 & 1.762950 \\ \mathrm{H} & -0.691730 & 1.359349 & 2.600973 \\ \mathrm{C} & -2.360594 & 0.484042 & 1.646364 \\ \mathrm{C} & -2.906940 & -0.801499 & 1.474885 \\ \mathrm{H} & -3.942549 & -0.887251 & 1.799498 \\ \mathrm{C} & -2.158725 & -1.964120 & 1.313511 \\ \mathrm{H} & -2.532895 & -2.849263 & 1.835492 \\ \mathrm{C} & -0.904577 & -2.050824 & 0.688787 \\ \mathrm{H} & -0.852946 & -1.655723 & -0.315808 \\ \mathrm{C} & -0.010939 & -3.248143 & 0.921865 \\ \mathrm{O} & -3.263863 & 1.428899 & 2.083374 \\ \mathrm{H} & -2.794954 & 2.271767 & 2.177714 \\ \mathrm{H} & -0.073446 & -3.603108 & 1.955941 \\ \mathrm{H} & -0.280823 & -4.085534 & 0.260847\end{array}$




$\begin{array}{lrrr}\mathrm{H} & 1.037446 & -3.005960 & 0.715638 \\ & & & \\ & & & \\ & & & \\ & & & \\ & & & \\ \mathrm{H} & & & \\ \mathrm{C} & & & \\ \mathrm{C} & 0.000000 & 0.000000 & 0.000000 \\ \mathrm{H} & 0.000000 & 0.000000 & 1.084815 \\ \mathrm{C} & -1.142940 & 0.000020 & 1.786802 \\ \mathrm{H} & -1.113950 & 0.000020 & 2.872880 \\ \mathrm{C} & -2.462192 & 0.000022 & 1.187308 \\ \mathrm{C} & -3.671209 & -0.000123 & 1.789627 \\ \mathrm{H} & -4.536021 & -0.000241 & 1.129576 \\ \mathrm{C} & -3.924224 & -0.000280 & 3.215238 \\ \mathrm{H} & -3.067347 & -0.000233 & 3.888033 \\ \mathrm{C} & -5.157960 & -0.000401 & 3.753372 \\ \mathrm{H} & -6.019166 & -0.000499 & 3.083472 \\ \mathrm{C} & -5.456292 & -0.000350 & 5.221818 \\ \mathrm{~F} & -2.447743 & -0.000280 & -0.172025 \\ \mathrm{H} & -6.048544 & -0.880519 & 5.508564 \\ \mathrm{H} & -4.539224 & 0.000084 & 5.820719 \\ \mathrm{H} & -6.049279 & 0.879387 & 5.508392\end{array}$

$\begin{array}{crrr}\text { TS } & & & \\ \mathrm{H} & 0.000000 & 0.000000 & 0.000000 \\ \mathrm{C} & 0.000000 & 0.000000 & 1.078358 \\ \mathrm{H} & 1.006200 & 0.000000 & 1.499384 \\ \mathrm{C} & -0.981106 & 0.728661 & 1.762260 \\ \mathrm{H} & -0.696970 & 1.407953 & 2.566737 \\ \mathrm{C} & -2.343977 & 0.469175 & 1.667307 \\ \mathrm{C} & -2.922328 & -0.797439 & 1.506744 \\ \mathrm{H} & -3.947576 & -0.871959 & 1.862952 \\ \mathrm{C} & -2.186508 & -1.961225 & 1.303244 \\ \mathrm{H} & -2.548312 & -2.854431 & 1.818281 \\ \mathrm{C} & -0.947065 & -2.029916 & 0.647927 \\ \mathrm{H} & -0.922462 & -1.603055 & -0.344662 \\ \mathrm{C} & -0.046697 & -3.231026 & 0.823261 \\ \mathrm{~F} & -3.179407 & 1.419937 & 2.169924 \\ \mathrm{H} & -0.077808 & -3.612442 & 1.849095 \\ \mathrm{H} & -0.338899 & -4.049557 & 0.148831 \\ \mathrm{H} & 0.994185 & -2.982509 & 0.590009\end{array}$




\begin{tabular}{|c|c|c|c|}
\hline $\mathrm{H}$ & 0.000000 & 0.000000 & 0.000000 \\
\hline $\mathrm{C}$ & 0.000000 & 0.000000 & 1.082253 \\
\hline $\mathrm{H}$ & 0.960271 & 0.000000 & 1.590969 \\
\hline $\mathrm{C}$ & -1.136507 & -0.000244 & 1.796039 \\
\hline $\mathrm{H}$ & -1.053088 & -0.000427 & 2.881154 \\
\hline $\mathrm{C}$ & -2.504793 & -0.000435 & 1.272994 \\
\hline $\mathrm{C}$ & -3.618718 & 0.000474 & 2.067520 \\
\hline $\mathrm{H}$ & -4.583587 & 0.000029 & 1.557812 \\
\hline $\mathrm{C}$ & -3.694320 & 0.002040 & 3.508632 \\
\hline $\mathrm{H}$ & -2.769329 & 0.002840 & 4.081658 \\
\hline $\mathrm{C}$ & -4.866340 & 0.002654 & 4.176137 \\
\hline $\mathrm{H}$ & -5.792834 & 0.001934 & 3.599424 \\
\hline $\mathrm{C}$ & -5.012289 & 0.004159 & 5.664904 \\
\hline $\mathrm{C}$ & -2.792572 & -0.001690 & -0.180664 \\
\hline $\mathrm{O}$ & -1.981243 & -0.002220 & -1.088692 \\
\hline $\mathrm{H}$ & -3.882210 & -0.002176 & -0.412969 \\
\hline $\mathrm{H}$ & -5.575955 & -0.875174 & 6.005966 \\
\hline $\mathrm{H}$ & -4.040851 & 0.005027 & 6.169331 \\
\hline $\mathrm{H}$ & -5.576677 & 0.883695 & 6.004203 \\
\hline \multicolumn{4}{|l|}{ TS } \\
\hline $\mathrm{H}$ & 0.000000 & 0.000000 & 0.000000 \\
\hline $\mathrm{H}$ & 0.000000 & 0.000000 & 1.816452 \\
\hline $\mathrm{C}$ & 0.592294 & 0.000000 & 0.902270 \\
\hline $\mathrm{C}$ & 1.797687 & -0.697238 & 0.877247 \\
\hline $\mathrm{H}$ & 2.067249 & -1.320309 & 1.733227 \\
\hline $\mathrm{C}$ & 2.782111 & -0.520384 & -0.107532 \\
\hline $\mathrm{C}$ & 3.021494 & 0.722825 & -0.737590 \\
\hline $\mathrm{H}$ & 3.970750 & 0.777604 & -1.266771 \\
\hline $\mathrm{C}$ & 2.384387 & 1.913048 & -0.407301 \\
\hline $\mathrm{H}$ & 3.035405 & 2.791669 & -0.375732 \\
\hline $\mathrm{C}$ & 1.073161 & 2.089110 & 0.075728 \\
\hline $\mathrm{H}$ & 0.266980 & 1.754808 & -0.559840 \\
\hline $\mathrm{C}$ & 0.725050 & 3.303125 & 0.903791 \\
\hline $\mathrm{C}$ & 3.838153 & -1.552179 & -0.222572 \\
\hline $\mathrm{O}$ & 4.752094 & -1.539159 & -1.027385 \\
\hline $\mathrm{H}$ & 3.733436 & -2.395127 & 0.497375 \\
\hline $\mathrm{H}$ & 1.554937 & 3.607513 & 1.549069 \\
\hline $\mathrm{H}$ & 0.462238 & 4.155656 & 0.259960 \\
\hline
\end{tabular}




\begin{tabular}{|c|c|c|c|}
\hline & -0.144672 & 3.106163 & 1.539509 \\
\hline & $\begin{array}{l}\rangle_{\mathrm{CH}_{3}} \\
\end{array}$ & & \\
\hline $\mathrm{H}$ & 0.000000 & 0.000000 & 0.000000 \\
\hline $\mathrm{C}$ & 0.000000 & 0.000000 & 1.086490 \\
\hline $\mathrm{H}$ & 0.966508 & 0.000000 & 1.580205 \\
\hline $\mathrm{C}$ & -1.136263 & -0.000020 & 1.797692 \\
\hline $\mathrm{H}$ & -1.078837 & -0.000036 & 2.882585 \\
\hline $\mathrm{C}$ & -2.486542 & -0.000002 & 1.227951 \\
\hline $\mathrm{C}$ & -3.650928 & -0.000100 & 1.943838 \\
\hline $\mathrm{H}$ & -4.580142 & -0.000088 & 1.377511 \\
\hline $\mathrm{C}$ & -3.792595 & -0.000339 & 3.380668 \\
\hline $\mathrm{H}$ & -2.895394 & -0.000593 & 3.996214 \\
\hline $\mathrm{C}$ & -4.994803 & -0.000240 & 3.991137 \\
\hline $\mathrm{H}$ & -5.892528 & 0.000032 & 3.371028 \\
\hline $\mathrm{C}$ & -5.210369 & -0.000516 & 5.471502 \\
\hline $\mathrm{C}$ & -2.592890 & 0.000040 & -0.204832 \\
\hline $\mathrm{H}$ & -5.789582 & -0.880073 & 5.784515 \\
\hline $\mathrm{H}$ & -5.789537 & 0.878912 & 5.784898 \\
\hline $\mathrm{H}$ & -4.263715 & -0.000727 & 6.021186 \\
\hline & -2.660204 & 0.000092 & -1.366980 \\
\hline
\end{tabular}

TS

$\begin{array}{lrrr}\mathrm{H} & 0.000000 & 0.000000 & 0.000000 \\ \mathrm{C} & 0.000000 & 0.000000 & 1.079223 \\ \mathrm{H} & 0.996365 & 0.000000 & 1.518755 \\ \mathrm{C} & -1.012515 & 0.709998 & 1.720813 \\ \mathrm{H} & -0.769740 & 1.342759 & 2.574290 \\ \mathrm{C} & -2.377550 & 0.518385 & 1.442525 \\ \mathrm{C} & -2.919448 & -0.741282 & 1.081627 \\ \mathrm{H} & -4.000158 & -0.825031 & 1.171438 \\ \mathrm{C} & -2.190243 & -1.924759 & 1.018893 \\ \mathrm{H} & -2.703798 & -2.800268 & 1.425581 \\ \mathrm{C} & -0.833036 & -2.086504 & 0.685272 \\ \mathrm{H} & -0.530520 & -1.760782 & -0.298522 \\ \mathrm{C} & -0.066442 & -3.287307 & 1.187040 \\ \mathrm{C} & -3.307633 & 1.521324 & 1.882607 \\ \mathrm{~N} & -4.057081 & 2.345939 & 2.219921 \\ \mathrm{H} & -0.204033 & -4.148562 & 0.516797 \\ \mathrm{H} & 1.008189 & -3.079329 & 1.219246\end{array}$




\begin{tabular}{|c|c|c|c|}
\hline $\mathrm{H}$ & -0.384592 & -3.582999 & 2.191516 \\
\hline $\mathrm{O}_{2} \mathrm{~N}$ & $\begin{array}{l}\left\langle\mathrm{CH}_{3}\right. \\
\end{array}$ & & \\
\hline $\mathrm{H}$ & 0.000000 & 0.000000 & 0.000000 \\
\hline $\mathrm{C}$ & 0.000000 & 0.000000 & 1.079753 \\
\hline $\mathrm{H}$ & 0.961350 & 0.000000 & 1.585972 \\
\hline $\mathrm{C}$ & -1.119430 & 0.000041 & 1.820266 \\
\hline $\mathrm{H}$ & -0.986256 & 0.000121 & 2.899274 \\
\hline $\mathrm{C}$ & -2.531018 & -0.000627 & 1.451785 \\
\hline $\mathrm{C}$ & -3.564555 & 0.000421 & 2.338290 \\
\hline $\mathrm{H}$ & -4.560018 & -0.000860 & 1.905575 \\
\hline $\mathrm{C}$ & -3.489302 & 0.002902 & 3.777583 \\
\hline $\mathrm{H}$ & -2.516799 & 0.004279 & 4.264639 \\
\hline $\mathrm{C}$ & -4.597878 & 0.003659 & 4.547403 \\
\hline $\mathrm{H}$ & -5.571163 & 0.002272 & 4.054995 \\
\hline $\mathrm{C}$ & -4.608550 & 0.006405 & 6.042193 \\
\hline $\mathrm{N}$ & -2.938078 & -0.002616 & 0.028638 \\
\hline $\mathrm{O}$ & -2.050047 & 0.002803 & -0.824820 \\
\hline $\mathrm{O}$ & -4.141116 & -0.009303 & -0.245238 \\
\hline $\mathrm{H}$ & -3.596362 & 0.007970 & 6.458517 \\
\hline $\mathrm{H}$ & -5.140668 & -0.872464 & 6.431190 \\
\hline $\mathrm{H}$ & -5.142017 & 0.885975 & 6.427795 \\
\hline \\
\hline $\mathrm{H}$ & 0.000000 & 0.000000 & 0.000000 \\
\hline $\mathrm{C}$ & 0.000000 & 0.000000 & 1.078995 \\
\hline $\mathrm{H}$ & 0.996662 & 0.000000 & 1.517662 \\
\hline $\mathrm{C}$ & -1.008570 & 0.709466 & 1.727957 \\
\hline $\mathrm{H}$ & -0.773410 & 1.354078 & 2.571130 \\
\hline $\mathrm{C}$ & -2.362737 & 0.492644 & 1.460391 \\
\hline $\mathrm{C}$ & -2.927984 & -0.760208 & 1.160483 \\
\hline $\mathrm{H}$ & -4.002112 & -0.823270 & 1.301527 \\
\hline $\mathrm{C}$ & -2.193936 & -1.935820 & 1.072380 \\
\hline $\mathrm{H}$ & -2.679488 & -2.817992 & 1.496964 \\
\hline $\mathrm{C}$ & -0.850170 & -2.077156 & 0.675517 \\
\hline $\mathrm{H}$ & -0.601925 & -1.737552 & -0.319104 \\
\hline $\mathrm{C}$ & -0.043168 & -3.269392 & 1.129264 \\
\hline $\mathrm{N}$ & -3.305321 & 1.528147 & 1.933939 \\
\hline $\mathrm{O}$ & -4.511540 & 1.346420 & 1.759617 \\
\hline $\mathrm{O}$ & -2.826925 & 2.538023 & 2.454571 \\
\hline
\end{tabular}




$\begin{array}{lrrr}\mathrm{H} & -0.297833 & -3.568883 & 2.150481 \\ \mathrm{H} & -0.214367 & -4.130769 & 0.467031 \\ \mathrm{H} & 1.029397 & -3.051848 & 1.095840\end{array}$

2

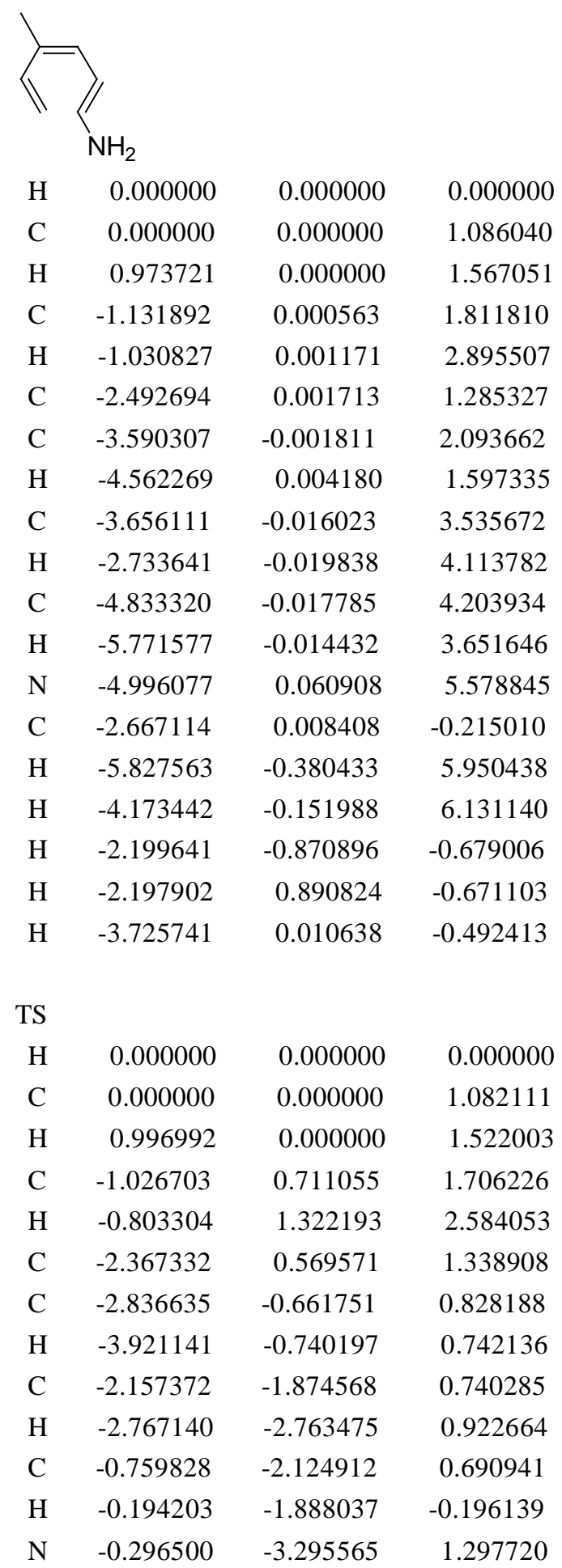




$\begin{array}{lrrr}\mathrm{C} & -3.374283 & 1.632694 & 1.722596 \\ \mathrm{H} & -3.497829 & 2.377259 & 0.923480 \\ \mathrm{H} & -3.044990 & 2.181746 & 2.613571 \\ \mathrm{H} & -4.365301 & 1.213055 & 1.930119 \\ \mathrm{H} & -0.610289 & -3.437794 & 2.249799 \\ \mathrm{H} & 0.696754 & -3.465679 & 1.207804\end{array}$<smiles>C=C/C(N)=C\C=C/N</smiles>

$\begin{array}{lrrr}\mathrm{H} & 0.000000 & 0.000000 & 0.000000 \\ \mathrm{C} & 0.000000 & 0.000000 & 1.085970 \\ \mathrm{H} & 0.969138 & 0.000000 & 1.575666 \\ \mathrm{C} & -1.138194 & -0.037620 & 1.798392 \\ \mathrm{H} & -1.073426 & -0.071232 & 2.882895 \\ \mathrm{C} & -2.487972 & 0.005087 & 1.240634 \\ \mathrm{C} & -3.609298 & -0.267949 & 1.968807 \\ \mathrm{H} & -4.568927 & -0.135998 & 1.464451 \\ \mathrm{C} & -3.687620 & -0.759091 & 3.323669 \\ \mathrm{H} & -2.761862 & -0.965023 & 3.859525 \\ \mathrm{C} & -4.858456 & -0.981004 & 3.963298 \\ \mathrm{H} & -5.802995 & -0.801986 & 3.452149 \\ \mathrm{~N} & -5.007929 & -1.351235 & 5.299890 \\ \mathrm{~N} & -2.564923 & 0.303993 & -0.138222 \\ \mathrm{H} & -1.996404 & 1.106297 & -0.396984 \\ \mathrm{H} & -3.517850 & 0.441167 & -0.459306 \\ \mathrm{H} & -4.166563 & -1.719083 & 5.731226 \\ \mathrm{H} & -5.802090 & -1.952757 & 5.486711\end{array}$

$\begin{array}{rrrr}\text { TS } & & & \\ \text { H } & 0.000000 & 0.000000 & 0.000000 \\ \text { C } & 0.000000 & 0.000000 & 1.081896 \\ \text { H } & 0.999228 & 0.000000 & 1.516725 \\ \text { C } & -1.016784 & 0.709820 & 1.724396 \\ \text { H } & -0.790902 & 1.318050 & 2.603529 \\ \text { C } & -2.364028 & 0.534183 & 1.389758 \\ \text { C } & -2.847181 & -0.722033 & 0.956467 \\ \text { H } & -3.935308 & -0.807698 & 0.932198 \\ \text { C } & -2.150387 & -1.924058 & 0.884883 \\ \text { H } & -2.725548 & -2.820355 & 1.134517 \\ \text { C } & -0.757338 & -2.135767 & 0.724864 \\ \text { H } & -0.283397 & -1.883204 & -0.210303\end{array}$




$\begin{array}{lrrr}\mathrm{N} & -0.218506 & -3.309959 & 1.279876 \\ \mathrm{~N} & -3.311015 & 1.540389 & 1.679352 \\ \mathrm{H} & -4.168144 & 1.202464 & 2.106547 \\ \mathrm{H} & -2.925577 & 2.307859 & 2.218805 \\ \mathrm{H} & -0.428700 & -3.437827 & 2.262766 \\ \mathrm{H} & 0.772758 & -3.431936 & 1.113632\end{array}$

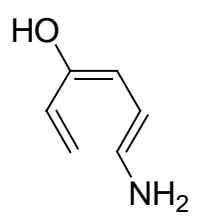

$\begin{array}{lrrr}\mathrm{H} & 0.000000 & 0.000000 & 0.000000 \\ \mathrm{C} & 0.000000 & 0.000000 & 1.084659 \\ \mathrm{H} & 0.964026 & 0.000000 & 1.584192 \\ \mathrm{C} & -1.142597 & 0.000683 & 1.788139 \\ \mathrm{H} & -1.100386 & 0.001219 & 2.873601 \\ \mathrm{C} & -2.475499 & 0.001312 & 1.205310 \\ \mathrm{C} & -3.643306 & 0.000132 & 1.903313 \\ \mathrm{H} & -4.566604 & 0.004795 & 1.318645 \\ \mathrm{C} & -3.821472 & -0.008826 & 3.336663 \\ \mathrm{H} & -2.937657 & -0.004063 & 3.972994 \\ \mathrm{C} & -5.036440 & -0.012037 & 3.929407 \\ \mathrm{H} & -5.940594 & -0.017321 & 3.322848 \\ \mathrm{O} & -2.459387 & 0.003354 & -0.172443 \\ \mathrm{~N} & -5.283557 & 0.085009 & 5.297836 \\ \mathrm{H} & -4.483438 & -0.114801 & 5.888384 \\ \mathrm{H} & -6.105641 & -0.413265 & 5.618355 \\ \mathrm{H} & -3.374850 & 0.009859 & -0.491490\end{array}$

TS

$\begin{array}{lrrr}\mathrm{H} & 0.000000 & 0.000000 & 0.000000 \\ \mathrm{C} & 0.000000 & 0.000000 & 1.081497 \\ \mathrm{H} & 0.999412 & 0.000000 & 1.515494 \\ \mathrm{C} & -1.013153 & 0.719584 & 1.723694 \\ \mathrm{H} & -0.788676 & 1.337281 & 2.597076 \\ \mathrm{C} & -2.355863 & 0.516384 & 1.408656 \\ \mathrm{C} & -2.861838 & -0.735680 & 1.009864 \\ \mathrm{H} & -3.948173 & -0.802098 & 1.045055 \\ \mathrm{C} & -2.161960 & -1.932753 & 0.918101 \\ \mathrm{H} & -2.725134 & -2.830771 & 1.184855 \\ \mathrm{C} & -0.772303 & -2.135326 & 0.717613 \\ \mathrm{H} & -0.327934 & -1.872576 & -0.229186 \\ \mathrm{~N} & -0.209345 & -3.305273 & 1.249532\end{array}$




$\begin{array}{lrrr}\mathrm{O} & -3.324201 & 1.435758 & 1.769211 \\ \mathrm{H} & -2.880483 & 2.275264 & 1.959932 \\ \mathrm{H} & -0.400918 & -3.451912 & 2.233438 \\ \mathrm{H} & 0.777450 & -3.422605 & 1.057206\end{array}$

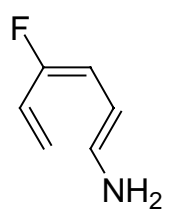

$\begin{array}{lrcc}\mathrm{H} & 0.000000 & 0.000000 & 0.000000 \\ \mathrm{C} & 0.000000 & 0.000000 & 1.084851 \\ \mathrm{H} & 0.963880 & 0.000000 & 1.583327 \\ \mathrm{C} & -1.144566 & 0.000822 & 1.786682 \\ \mathrm{H} & -1.111934 & 0.001589 & 2.873390 \\ \mathrm{C} & -2.465425 & 0.002125 & 1.198928 \\ \mathrm{C} & -3.671207 & 0.000787 & 1.811924 \\ \mathrm{H} & -4.535473 & 0.007611 & 1.150110 \\ \mathrm{C} & -3.931429 & -0.011591 & 3.230022 \\ \mathrm{H} & -3.089146 & -0.015702 & 3.920539 \\ \mathrm{C} & -5.186020 & -0.009408 & 3.737831 \\ \mathrm{H} & -6.043095 & -0.004804 & 3.067031 \\ \mathrm{~N} & -5.526109 & 0.072466 & 5.079444 \\ \mathrm{~F} & -2.465447 & 0.005515 & -0.165190 \\ \mathrm{H} & -4.784109 & -0.140765 & 5.735867 \\ \mathrm{H} & -6.400799 & -0.364714 & 5.339936\end{array}$

TS

$\begin{array}{lrrr}\mathrm{H} & 0.000000 & 0.000000 & 0.000000 \\ \mathrm{C} & 0.000000 & 0.000000 & 1.081197 \\ \mathrm{H} & 0.998399 & 0.000000 & 1.516806 \\ \mathrm{C} & -1.012114 & 0.731761 & 1.714409 \\ \mathrm{H} & -0.804064 & 1.380678 & 2.565080 \\ \mathrm{C} & -2.339327 & 0.508324 & 1.391062 \\ \mathrm{C} & -2.869550 & -0.725830 & 0.985325 \\ \mathrm{H} & -3.956073 & -0.777683 & 1.016176 \\ \mathrm{C} & -2.178163 & -1.925317 & 0.876943 \\ \mathrm{H} & -2.749986 & -2.823498 & 1.120061 \\ \mathrm{C} & -0.782393 & -2.130542 & 0.703704 \\ \mathrm{H} & -0.319758 & -1.871551 & -0.235265 \\ \mathrm{~N} & -0.233233 & -3.294094 & 1.249828 \\ \mathrm{~F} & -3.259443 & 1.430762 & 1.794835 \\ \mathrm{H} & -0.449319 & -3.451788 & 2.226474 \\ \mathrm{H} & 0.751973 & -3.432489 & 1.065978\end{array}$




\begin{tabular}{|c|c|c|c|}
\hline $\mathrm{H}$ & 0.000000 & 0.000000 & 0.000000 \\
\hline $\mathrm{C}$ & 0.000000 & 0.000000 & 1.082377 \\
\hline $\mathrm{H}$ & 0.959799 & 0.000000 & 1.592410 \\
\hline $\mathrm{C}$ & -1.138747 & 0.000730 & 1.793309 \\
\hline $\mathrm{H}$ & -1.054535 & 0.001586 & 2.879307 \\
\hline $\mathrm{C}$ & -2.507760 & 0.001608 & 1.273707 \\
\hline $\mathrm{C}$ & -3.618782 & -0.003261 & 2.082411 \\
\hline $\mathrm{H}$ & -4.583766 & 0.001235 & 1.571197 \\
\hline $\mathrm{C}$ & -3.692720 & -0.017176 & 3.509274 \\
\hline $\mathrm{H}$ & -2.777769 & -0.019997 & 4.097708 \\
\hline $\mathrm{C}$ & -4.889027 & -0.023959 & 4.158883 \\
\hline $\mathrm{H}$ & -5.814797 & -0.022340 & 3.586678 \\
\hline $\mathrm{N}$ & -5.072558 & 0.026170 & 5.513788 \\
\hline $\mathrm{C}$ & -2.799549 & 0.008098 & -0.168962 \\
\hline $\mathrm{O}$ & -1.994391 & 0.010709 & -1.086649 \\
\hline $\mathrm{H}$ & -3.891746 & 0.010296 & -0.396946 \\
\hline $\mathrm{H}$ & -4.277144 & -0.145595 & 6.114362 \\
\hline $\mathrm{H}$ & -5.959232 & -0.272009 & 5.892610 \\
\hline \multicolumn{4}{|l|}{$\mathrm{TS}$} \\
\hline $\mathrm{H}$ & 0.000000 & 0.000000 & 0.000000 \\
\hline $\mathrm{C}$ & 0.000000 & 0.000000 & 1.082750 \\
\hline $\mathrm{H}$ & 0.989473 & 0.000000 & 1.536330 \\
\hline $\mathrm{C}$ & -1.051944 & 0.668846 & 1.697058 \\
\hline $\mathrm{H}$ & -0.877497 & 1.240649 & 2.611279 \\
\hline $\mathrm{C}$ & -2.369905 & 0.537324 & 1.239687 \\
\hline $\mathrm{C}$ & -2.821808 & -0.648277 & 0.602524 \\
\hline $\mathrm{H}$ & -3.892489 & -0.666007 & 0.408514 \\
\hline $\mathrm{C}$ & -2.151699 & -1.851128 & 0.499605 \\
\hline $\mathrm{H}$ & -2.781448 & -2.743419 & 0.497538 \\
\hline $\mathrm{C}$ & -0.751787 & -2.134195 & 0.631287 \\
\hline $\mathrm{H}$ & -0.066017 & -1.914060 & -0.172244 \\
\hline $\mathrm{N}$ & -0.396028 & -3.276474 & 1.312561 \\
\hline $\mathrm{C}$ & -3.379337 & 1.513663 & 1.670877 \\
\hline $\mathrm{O}$ & -4.554348 & 1.522859 & 1.337063 \\
\hline $\mathrm{H}$ & -2.985182 & 2.301308 & 2.354098 \\
\hline $\mathrm{H}$ & -0.876572 & -3.471938 & 2.180448 \\
\hline $\mathrm{H}$ & 67 & 545623 & 1.31 \\
\hline
\end{tabular}




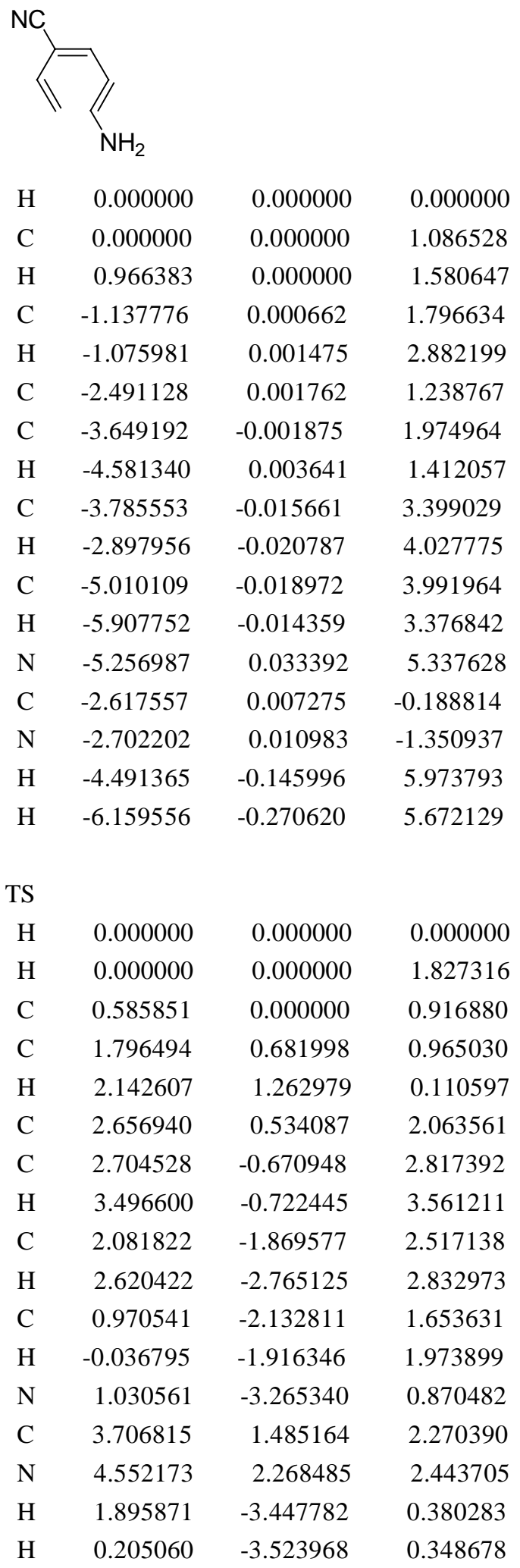




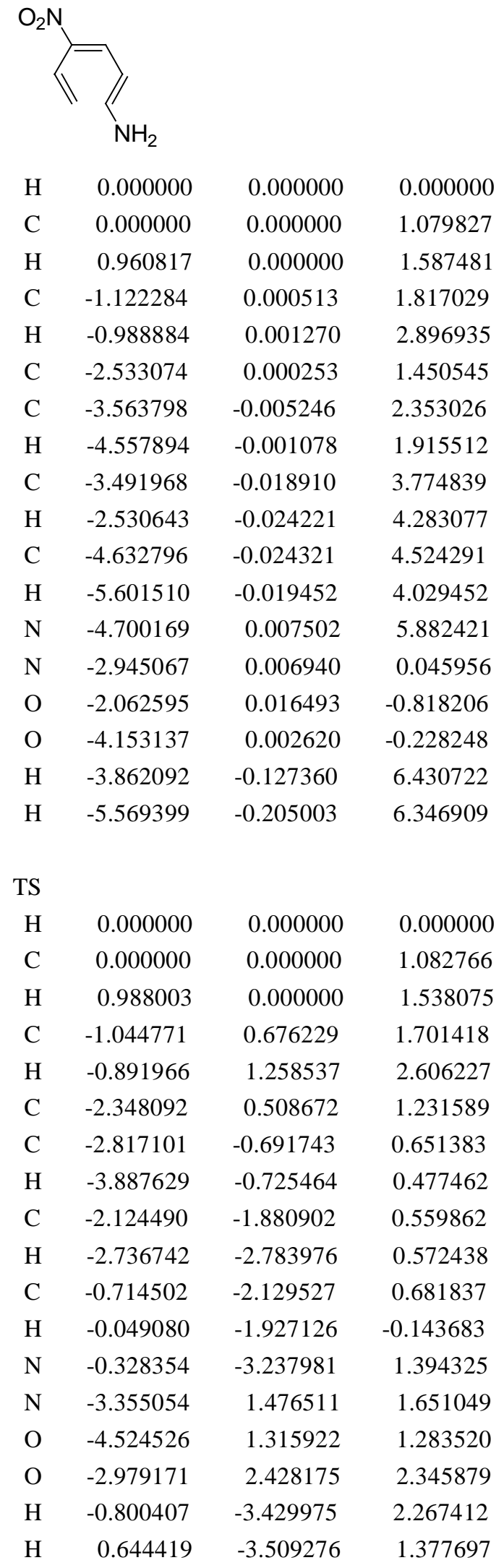




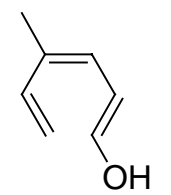

$\begin{array}{lrrr}\mathrm{H} & 0.000000 & 0.000000 & 0.000000 \\ \mathrm{C} & 0.000000 & 0.000000 & 1.085935 \\ \mathrm{H} & 0.973624 & 0.000000 & 1.566940 \\ \mathrm{C} & -1.131057 & -0.000040 & 1.811475 \\ \mathrm{H} & -1.030155 & -0.000188 & 2.895050 \\ \mathrm{C} & -2.492035 & -0.000253 & 1.281317 \\ \mathrm{C} & -3.590762 & 0.000460 & 2.084875 \\ \mathrm{H} & -4.562465 & -0.000048 & 1.589875 \\ \mathrm{C} & -3.652546 & 0.001922 & 3.530643 \\ \mathrm{H} & -2.728345 & 0.003454 & 4.107250 \\ \mathrm{C} & -4.823162 & 0.001674 & 4.198135 \\ \mathrm{H} & -5.785278 & 0.000385 & 3.693421 \\ \mathrm{O} & -4.989760 & 0.003140 & 5.546398 \\ \mathrm{C} & -2.663486 & -0.001498 & -0.219292 \\ \mathrm{H} & -4.116698 & 0.004335 & 5.973180 \\ \mathrm{H} & -2.194336 & -0.883183 & -0.676064 \\ \mathrm{H} & -2.193575 & 0.878970 & -0.677631 \\ \mathrm{H} & -3.721279 & -0.001196 & -0.498853\end{array}$

TS

$\begin{array}{lrrr}\mathrm{H} & 0.000000 & 0.000000 & 0.000000 \\ \mathrm{C} & 0.000000 & 0.000000 & 1.081875 \\ \mathrm{H} & 0.993589 & 0.000000 & 1.526495 \\ \mathrm{C} & -1.043055 & 0.678041 & 1.707249 \\ \mathrm{H} & -0.842166 & 1.270759 & 2.602295 \\ \mathrm{C} & -2.380184 & 0.516722 & 1.321979 \\ \mathrm{C} & -2.818590 & -0.718135 & 0.805122 \\ \mathrm{H} & -3.899400 & -0.824303 & 0.709306 \\ \mathrm{C} & -2.102096 & -1.914976 & 0.719675 \\ \mathrm{H} & -2.685336 & -2.822270 & 0.908048 \\ \mathrm{C} & -0.707154 & -2.121618 & 0.655505 \\ \mathrm{H} & -0.121088 & -1.893591 & -0.219274 \\ \mathrm{O} & -0.179546 & -3.243574 & 1.243209 \\ \mathrm{C} & -3.407536 & 1.562086 & 1.699195 \\ \mathrm{H} & -0.735193 & -3.477029 & 2.004577 \\ \mathrm{H} & -4.405564 & 1.133028 & 1.840904 \\ \mathrm{H} & -3.123195 & 2.074871 & 2.626462\end{array}$




\begin{tabular}{|c|c|c|c|}
\hline $\mathrm{H}$ & -3.491734 & 2.337335 & 0.925123 \\
\hline \multicolumn{4}{|c|}{$\mathrm{H}_{2} \mathrm{~N}$} \\
\hline $\mathrm{H}$ & 0.000000 & 0.000000 & 0.000000 \\
\hline $\mathrm{C}$ & 0.000000 & 0.000000 & 1.085960 \\
\hline $\mathrm{H}$ & 0.969100 & 0.000000 & 1.575572 \\
\hline $\mathrm{C}$ & -1.137432 & -0.037116 & 1.798053 \\
\hline $\mathrm{H}$ & -1.072907 & -0.070039 & 2.882503 \\
\hline $\mathrm{C}$ & -2.487967 & 0.003466 & 1.237092 \\
\hline $\mathrm{C}$ & -3.608085 & -0.287304 & 1.958383 \\
\hline $\mathrm{H}$ & -4.568422 & -0.162234 & 1.455468 \\
\hline $\mathrm{C}$ & -3.677294 & -0.792008 & 3.311340 \\
\hline $\mathrm{H}$ & -2.747946 & -1.003302 & 3.840199 \\
\hline $\mathrm{C}$ & -4.839615 & -1.030085 & 3.949722 \\
\hline $\mathrm{H}$ & -5.809222 & -0.862039 & 3.489684 \\
\hline $\mathrm{O}$ & -4.986957 & -1.505097 & 5.219433 \\
\hline $\mathrm{N}$ & -2.563218 & 0.317627 & -0.135042 \\
\hline $\mathrm{H}$ & -1.990235 & 1.116678 & -0.391535 \\
\hline $\mathrm{H}$ & -3.513697 & 0.449796 & -0.464190 \\
\hline $\mathrm{H}$ & -4.106588 & -1.649403 & 5.603876 \\
\hline \multicolumn{4}{|l|}{ TS } \\
\hline $\mathrm{H}$ & 0.000000 & 0.000000 & 0.000000 \\
\hline $\mathrm{C}$ & 0.000000 & 0.000000 & 1.081675 \\
\hline $\mathrm{H}$ & 0.995725 & 0.000000 & 1.521424 \\
\hline $\mathrm{C}$ & -1.033612 & 0.677820 & 1.723637 \\
\hline $\mathrm{H}$ & -0.832033 & 1.265043 & 2.621991 \\
\hline $\mathrm{C}$ & -2.375964 & 0.483774 & 1.364980 \\
\hline $\mathrm{C}$ & -2.824866 & -0.776458 & 0.916818 \\
\hline $\mathrm{H}$ & -3.909422 & -0.890848 & 0.875496 \\
\hline $\mathrm{C}$ & -2.091961 & -1.961374 & 0.846391 \\
\hline $\mathrm{H}$ & -2.644023 & -2.875963 & 1.089209 \\
\hline $\mathrm{C}$ & -0.699970 & -2.131641 & 0.691559 \\
\hline $\mathrm{H}$ & -0.191360 & -1.895893 & -0.228574 \\
\hline $\mathrm{O}$ & -0.108513 & -3.249025 & 1.238629 \\
\hline $\mathrm{N}$ & -3.339456 & 1.470874 & 1.646543 \\
\hline $\mathrm{H}$ & -4.210033 & 1.122794 & 2.035138 \\
\hline $\mathrm{H}$ & -2.981362 & 2.242548 & 2.197740 \\
\hline $\mathrm{H}$ & -0.598667 & -3.486367 & 2.041887 \\
\hline
\end{tabular}




\begin{tabular}{|c|c|c|c|}
\hline $\mathrm{H}$ & 0.000000 & 0.000000 & 0.000000 \\
\hline $\mathrm{C}$ & 0.000000 & 0.000000 & 1.084639 \\
\hline $\mathrm{H}$ & 0.963850 & 0.000000 & 1.584358 \\
\hline $\mathrm{C}$ & -1.141876 & 0.000000 & 1.787922 \\
\hline $\mathrm{H}$ & -1.099819 & 0.000000 & 2.873312 \\
\hline $\mathrm{C}$ & -2.474785 & 0.000105 & 1.201040 \\
\hline $\mathrm{C}$ & -3.643287 & -0.000048 & 1.895729 \\
\hline $\mathrm{H}$ & -4.566741 & 0.000298 & 1.312825 \\
\hline $\mathrm{C}$ & -3.816559 & -0.000760 & 3.331985 \\
\hline $\mathrm{H}$ & -2.931076 & -0.001645 & 3.967005 \\
\hline $\mathrm{C}$ & -5.025469 & -0.000262 & 3.925298 \\
\hline $\mathrm{H}$ & -5.955925 & 0.000393 & 3.364475 \\
\hline $\mathrm{O}$ & -5.273821 & -0.000866 & 5.264896 \\
\hline $\mathrm{O}$ & -2.453979 & 0.000427 & -0.173807 \\
\hline $\mathrm{H}$ & -3.367219 & 0.000144 & -0.499572 \\
\hline $\mathrm{H}$ & -4.427826 & -0.000481 & 5.742349 \\
\hline \multicolumn{4}{|l|}{ TS } \\
\hline $\mathrm{H}$ & 0.000000 & 0.000000 & 0.000000 \\
\hline $\mathrm{C}$ & 0.000000 & 0.000000 & 1.081171 \\
\hline $\mathrm{H}$ & 0.995710 & 0.000000 & 1.520560 \\
\hline $\mathrm{C}$ & -1.031693 & 0.684703 & 1.723062 \\
\hline $\mathrm{H}$ & -0.833623 & 1.279120 & 2.617952 \\
\hline $\mathrm{C}$ & -2.368269 & 0.462371 & 1.380204 \\
\hline $\mathrm{C}$ & -2.838379 & -0.793200 & 0.961178 \\
\hline $\mathrm{H}$ & -3.922756 & -0.888160 & 0.974416 \\
\hline $\mathrm{C}$ & -2.101758 & -1.973169 & 0.873410 \\
\hline $\mathrm{H}$ & -2.642185 & -2.888853 & 1.133848 \\
\hline $\mathrm{C}$ & -0.713126 & -2.133776 & 0.682297 \\
\hline $\mathrm{H}$ & -0.230911 & -1.886590 & -0.248958 \\
\hline $\mathrm{O}$ & -0.097833 & -3.245169 & 1.208110 \\
\hline $\mathrm{O}$ & -3.356992 & 1.358712 & 1.732493 \\
\hline $\mathrm{H}$ & -2.935099 & 2.207114 & 1.933560 \\
\hline $\mathrm{H}$ & -0.563866 & -3.493463 & 2.022588 \\
\hline & $\mathrm{OH}$ & & \\
\hline $\mathrm{H}$ & 0.000000 & 0.000000 & 0.000000 \\
\hline $\mathrm{C}$ & 0.000000 & 0.000000 & 1.084809 \\
\hline
\end{tabular}




\begin{tabular}{|c|c|c|c|}
\hline $\mathrm{H}$ & 0.963845 & 0.000000 & 3245 \\
\hline $\mathrm{C}$ & -1.143694 & 0.000000 & 1.786490 \\
\hline $\mathrm{H}$ & -1.111920 & 0.000000 & 2.873055 \\
\hline $\mathrm{C}$ & -2.463951 & 0.000042 & 1.193004 \\
\hline $\mathrm{C}$ & -3.670733 & 0.000027 & 1.800322 \\
\hline $\mathrm{H}$ & -4.534863 & 0.000170 & 1.139382 \\
\hline $\mathrm{C}$ & -3.927350 & -0.000196 & 3.222269 \\
\hline $\mathrm{H}$ & -3.083290 & -0.000692 & 3.911625 \\
\hline $\mathrm{C}$ & -5.175210 & 0.000167 & 3.730495 \\
\hline $\mathrm{H}$ & -6.062357 & 0.000505 & 3.103567 \\
\hline $\mathrm{O}$ & -5.515914 & -0.000095 & 5.045060 \\
\hline $\mathrm{F}$ & -2.458011 & 0.000299 & -0.168357 \\
\hline $\mathrm{H}$ & -4.707779 & -0.000741 & 5.584773 \\
\hline \multicolumn{4}{|l|}{ TS } \\
\hline $\mathrm{H}$ & 0.000000 & 0.000000 & 0.000000 \\
\hline $\mathrm{C}$ & 0.000000 & 0.000000 & 1.080782 \\
\hline $\mathrm{H}$ & 0.994705 & 0.000000 & 1.521865 \\
\hline $\mathrm{C}$ & -1.031184 & 0.694940 & 1.715195 \\
\hline $\mathrm{H}$ & -0.848681 & 1.321805 & 2.587478 \\
\hline $\mathrm{C}$ & -2.352588 & 0.451684 & 1.367333 \\
\hline $\mathrm{C}$ & -2.848478 & -0.786323 & 0.944011 \\
\hline $\mathrm{H}$ & -3.933210 & -0.867381 & 0.959540 \\
\hline $\mathrm{C}$ & -2.119295 & -1.968607 & 0.836666 \\
\hline $\mathrm{H}$ & -2.665680 & -2.884524 & 1.079244 \\
\hline $\mathrm{C}$ & -0.727071 & -2.130165 & 0.661031 \\
\hline $\mathrm{H}$ & -0.234214 & -1.879489 & -0.263591 \\
\hline $\mathrm{O}$ & -0.119328 & -3.239433 & 1.190341 \\
\hline $\mathrm{F}$ & -3.293002 & 1.351727 & 1.765497 \\
\hline $\mathrm{H}$ & -0.591525 & -3.491595 & 2.000332 \\
\hline \multicolumn{4}{|c|}{$\mathrm{OH}$} \\
\hline $\mathrm{H}$ & 0.000000 & 0.000000 & 0.000000 \\
\hline $\mathrm{C}$ & 0.000000 & 0.000000 & 1.082297 \\
\hline $\mathrm{H}$ & 0.959938 & 0.000000 & 1.591695 \\
\hline $\mathrm{C}$ & -1.137676 & 0.000122 & 1.794273 \\
\hline $\mathrm{H}$ & -1.053083 & 0.000231 & 2.880025 \\
\hline $\mathrm{C}$ & -2.506171 & 0.000085 & 1.272653 \\
\hline $\mathrm{C}$ & -3.618417 & 0.000058 & 2.072796 \\
\hline $\mathrm{H}$ & -4.583109 & -0.000122 & 1.562772 \\
\hline $\mathrm{C}$ & -3.689947 & 0.000275 & 3.506614 \\
\hline
\end{tabular}




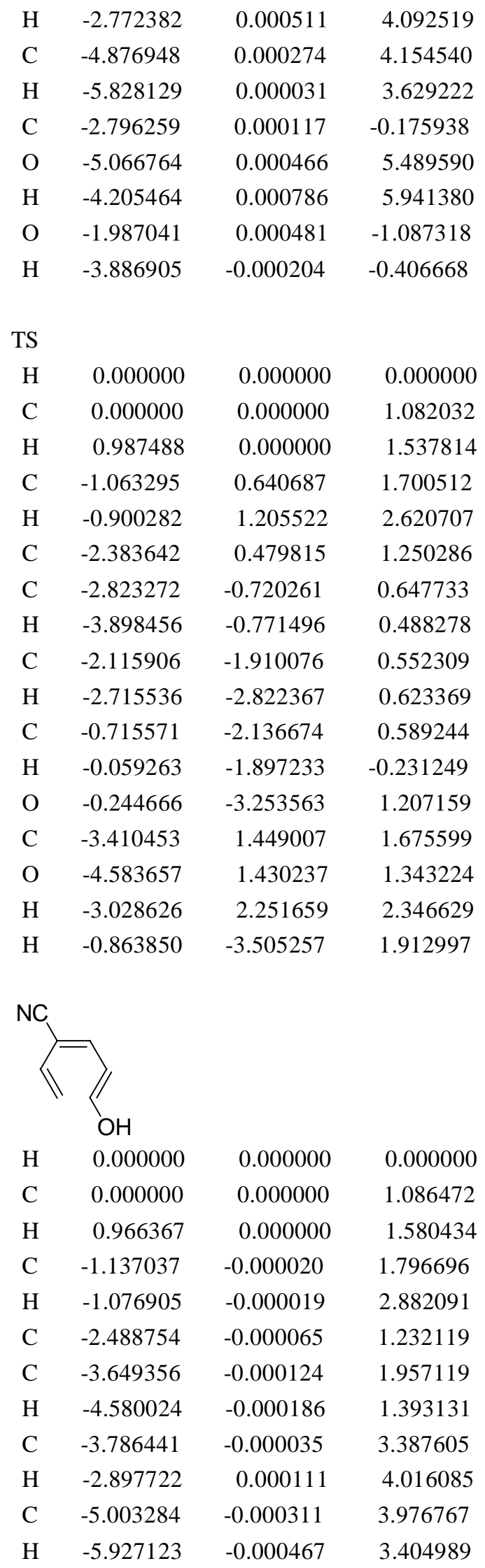




\begin{tabular}{|c|c|c|c|}
\hline $\mathrm{O}$ & -5.257896 & -0.000565 & 5.301189 \\
\hline $\mathrm{C}$ & -2.604879 & -0.000089 & -0.198073 \\
\hline $\mathrm{H}$ & -4.420440 & -0.000986 & 5.795314 \\
\hline $\mathrm{N}$ & -2.679174 & -0.000092 & -1.360170 \\
\hline \multicolumn{4}{|l|}{ TS } \\
\hline $\mathrm{H}$ & 0.000000 & 0.000000 & 0.000000 \\
\hline $\mathrm{C}$ & 0.000000 & 0.000000 & 1.081970 \\
\hline $\mathrm{H}$ & 0.986953 & 0.000000 & 1.538152 \\
\hline $\mathrm{C}$ & -1.055573 & 0.654636 & 1.700360 \\
\hline $\mathrm{H}$ & -0.896157 & 1.230096 & 2.611474 \\
\hline $\mathrm{C}$ & -2.376087 & 0.478492 & 1.249047 \\
\hline $\mathrm{C}$ & -2.811305 & -0.740559 & 0.674663 \\
\hline $\mathrm{H}$ & -3.883406 & -0.825085 & 0.512987 \\
\hline $\mathrm{C}$ & -2.088659 & -1.925379 & 0.598772 \\
\hline $\mathrm{H}$ & -2.681122 & -2.839371 & 0.695158 \\
\hline $\mathrm{C}$ & -0.686884 & -2.132904 & 0.631320 \\
\hline $\mathrm{H}$ & -0.042550 & -1.901299 & -0.200827 \\
\hline $\mathrm{C}$ & -3.383292 & 1.421757 & 1.636847 \\
\hline $\mathrm{O}$ & -0.197978 & -3.234733 & 1.262926 \\
\hline $\mathrm{H}$ & -0.801364 & -3.477908 & 1.985035 \\
\hline $\mathrm{N}$ & -4.195636 & 2.198814 & 1.941625 \\
\hline \multicolumn{4}{|c|}{$\mathrm{O}_{2} \mathrm{~N}$} \\
\hline $\mathrm{H}$ & 0.000000 & 0.000000 & 0.000000 \\
\hline $\mathrm{C}$ & 0.000000 & 0.000000 & 1.079829 \\
\hline $\mathrm{H}$ & 0.960942 & 0.000000 & 1.586798 \\
\hline $\mathrm{C}$ & -1.121004 & 0.000021 & 1.818153 \\
\hline $\mathrm{H}$ & -0.987076 & 0.000035 & 2.897694 \\
\hline $\mathrm{C}$ & -2.532034 & 0.000027 & 1.449502 \\
\hline $\mathrm{C}$ & -3.564583 & 0.000187 & 2.341656 \\
\hline $\mathrm{H}$ & -4.558755 & 0.000083 & 1.905348 \\
\hline $\mathrm{C}$ & -3.489736 & 0.000492 & 3.771727 \\
\hline $\mathrm{H}$ & -2.525251 & 0.000790 & 4.275777 \\
\hline $\mathrm{C}$ & -4.618972 & 0.000455 & 4.519634 \\
\hline $\mathrm{H}$ & -5.610484 & 0.000213 & 4.075694 \\
\hline $\mathrm{O}$ & -4.691752 & 0.000699 & 5.862170 \\
\hline $\mathrm{N}$ & -2.941929 & -0.000210 & 0.035229 \\
\hline $\mathrm{O}$ & -2.056167 & -0.000224 & -0.822505 \\
\hline $\mathrm{O}$ & -4.147083 & 0.000049 & -0.237580 \\
\hline $\mathrm{H}$ & -3.796044 & 0.001313 & 6.241822 \\
\hline
\end{tabular}




$\begin{array}{crrr}\text { TS } & & & \\ \mathrm{H} & 0.000000 & 0.000000 & 0.000000 \\ \mathrm{C} & 0.000000 & 0.000000 & 1.081899 \\ \mathrm{H} & 0.986542 & 0.000000 & 1.538623 \\ \mathrm{C} & -1.055124 & 0.651047 & 1.704731 \\ \mathrm{H} & -0.910726 & 1.231179 & 2.612113 \\ \mathrm{C} & -2.361193 & 0.451575 & 1.247600 \\ \mathrm{C} & -2.821813 & -0.763700 & 0.711131 \\ \mathrm{H} & -3.897298 & -0.829023 & 0.582061 \\ \mathrm{C} & -2.094747 & -1.940938 & 0.627564 \\ \mathrm{H} & -2.675963 & -2.861142 & 0.726813 \\ \mathrm{C} & -0.687501 & -2.133626 & 0.636357 \\ \mathrm{H} & -0.062730 & -1.904867 & -0.211763 \\ \mathrm{O} & -0.176238 & -3.220322 & 1.270171 \\ \mathrm{~N} & -3.384459 & 1.416851 & 1.661486 \\ \mathrm{H} & -0.766489 & -3.467085 & 2.002273 \\ \mathrm{O} & -3.014965 & 2.377218 & 2.343179 \\ \mathrm{O} & -4.549423 & 1.236688 & 1.296465\end{array}$

4

\begin{tabular}{lrrr}
\hline & & & \\
& & & \\
& & & \\
& & & \\
$\mathrm{H}$ & 0.000000 & 0.000000 & 0.000000 \\
$\mathrm{C}$ & 0.000000 & 0.000000 & 1.085908 \\
$\mathrm{H}$ & 0.973556 & 0.000000 & 1.566837 \\
$\mathrm{C}$ & -1.129619 & -0.000020 & 1.812224 \\
$\mathrm{H}$ & -1.030892 & -0.000170 & 2.895376 \\
$\mathrm{C}$ & -2.490150 & -0.000127 & 1.276900 \\
$\mathrm{C}$ & -3.590747 & 0.000319 & 2.076003 \\
$\mathrm{H}$ & -4.562532 & 0.000042 & 1.582071 \\
$\mathrm{C}$ & -3.640028 & 0.001109 & 3.524177 \\
$\mathrm{H}$ & -2.725389 & 0.002055 & 4.109675 \\
$\mathrm{C}$ & -4.798008 & 0.001050 & 4.194435 \\
$\mathrm{H}$ & -5.789586 & 0.000492 & 3.750216 \\
$\mathrm{~F}$ & -4.839092 & 0.002385 & 5.539888 \\
$\mathrm{C}$ & -2.658577 & -0.001063 & -0.223715 \\
$\mathrm{H}$ & -2.188257 & -0.883087 & -0.677658 \\
$\mathrm{H}$ & -2.186541 & 0.879353 & -0.678984 \\
$\mathrm{H}$ & -3.715459 & -0.000171 & -0.505681
\end{tabular}




$\begin{array}{cccc}\text { TS } & & & \\ \mathrm{H} & 0.000000 & 0.000000 & 0.000000 \\ \mathrm{C} & 0.000000 & 0.000000 & 1.081539 \\ \mathrm{H} & 0.993422 & 0.000000 & 1.526189 \\ \mathrm{C} & -1.043302 & 0.668631 & 1.711921 \\ \mathrm{H} & -0.842385 & 1.251463 & 2.612567 \\ \mathrm{C} & -2.384850 & 0.503542 & 1.335507 \\ \mathrm{C} & -2.833325 & -0.734076 & 0.839564 \\ \mathrm{H} & -3.915066 & -0.844599 & 0.770143 \\ \mathrm{C} & -2.109363 & -1.930168 & 0.772666 \\ \mathrm{H} & -2.657825 & -2.838763 & 1.029224 \\ \mathrm{C} & -0.728731 & -2.103719 & 0.637851 \\ \mathrm{H} & -0.168678 & -1.880453 & -0.256035 \\ \mathrm{~F} & -0.239117 & -3.247835 & 1.194813 \\ \mathrm{C} & -3.405565 & 1.554105 & 1.716053 \\ \mathrm{H} & -3.474119 & 2.334786 & 0.946337 \\ \mathrm{H} & -3.122320 & 2.056641 & 2.648897 \\ \mathrm{H} & -4.407842 & 1.132838 & 1.846661\end{array}$

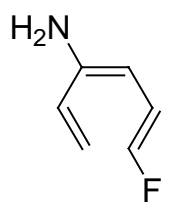

$\begin{array}{lrrr}\mathrm{H} & 0.000000 & 0.000000 & 0.000000 \\ \mathrm{C} & 0.000000 & 0.000000 & 1.086075 \\ \mathrm{H} & 0.969555 & 0.000000 & 1.574559 \\ \mathrm{C} & -1.134932 & 0.037618 & 1.800294 \\ \mathrm{H} & -1.070203 & 0.072999 & 2.884040 \\ \mathrm{C} & -2.487321 & 0.003685 & 1.237007 \\ \mathrm{C} & -3.604660 & 0.318148 & 1.953449 \\ \mathrm{H} & -4.567104 & 0.205311 & 1.452541 \\ \mathrm{C} & -3.651648 & 0.832703 & 3.302997 \\ \mathrm{H} & -2.728080 & 1.018998 & 3.844344 \\ \mathrm{C} & -4.795544 & 1.111794 & 3.940296 \\ \mathrm{H} & -5.797053 & 0.997369 & 3.535566 \\ \mathrm{~F} & -4.803016 & 1.588339 & 5.203891 \\ \mathrm{~N} & -2.566231 & -0.326983 & -0.124611 \\ \mathrm{H} & -1.973649 & -1.107338 & -0.389654 \\ \mathrm{H} & -3.513111 & -0.464736 & -0.459991\end{array}$

TS

$\begin{array}{llll}\mathrm{H} & 0.000000 & 0.000000 & 0.000000\end{array}$

$\begin{array}{llll}\text { C } & 0.000000 & 0.000000 & 1.081393\end{array}$

$\begin{array}{llll}\mathrm{H} & 0.994819 & 0.000000 & 1.522569\end{array}$ 


\begin{tabular}{|c|c|c|c|}
\hline $\mathrm{C}$ & -1.036057 & 0.661771 & 1.728690 \\
\hline $\mathrm{H}$ & -0.839637 & 1.228461 & 2.640316 \\
\hline $\mathrm{C}$ & -2.382014 & 0.465298 & 1.370207 \\
\hline $\mathrm{C}$ & -2.834179 & -0.796334 & 0.930931 \\
\hline $\mathrm{H}$ & -3.918120 & -0.917478 & 0.910765 \\
\hline $\mathrm{C}$ & -2.091634 & -1.976822 & 0.869772 \\
\hline $\mathrm{H}$ & -2.610793 & -2.895146 & 1.153228 \\
\hline $\mathrm{C}$ & -0.714448 & -2.116594 & 0.664223 \\
\hline $\mathrm{H}$ & -0.219986 & -1.885529 & -0.266111 \\
\hline $\mathrm{F}$ & -0.176184 & -3.259602 & 1.187525 \\
\hline $\mathrm{N}$ & -3.337483 & 1.449797 & 1.657283 \\
\hline $\mathrm{H}$ & -4.226725 & 1.110170 & 2.007114 \\
\hline $\mathrm{H}$ & -2.988476 & 2.219874 & 2.215408 \\
\hline \multicolumn{4}{|l|}{$\mathrm{HO}$} \\
\hline $\mathrm{H}$ & 0.000000 & 0.000000 & 0.000000 \\
\hline $\mathrm{C}$ & 0.000000 & 0.000000 & 1.084666 \\
\hline $\mathrm{H}$ & 0.963785 & 0.000000 & 1.584330 \\
\hline $\mathrm{C}$ & -1.140552 & 0.000000 & 1.788701 \\
\hline $\mathrm{H}$ & -1.100732 & 0.000000 & 2.873633 \\
\hline $\mathrm{C}$ & -2.472756 & 0.000000 & 1.196011 \\
\hline $\mathrm{C}$ & -3.643641 & 0.000000 & 1.887092 \\
\hline $\mathrm{H}$ & -4.567347 & 0.000000 & 1.305381 \\
\hline $\mathrm{C}$ & -3.802423 & 0.000000 & 3.324632 \\
\hline $\mathrm{H}$ & -2.926140 & 0.000000 & 3.966749 \\
\hline $\mathrm{C}$ & -4.997881 & 0.000000 & 3.925832 \\
\hline $\mathrm{H}$ & -5.963269 & 0.000000 & 3.427729 \\
\hline $\mathrm{F}$ & -5.112362 & 0.000000 & 5.270020 \\
\hline $\mathrm{O}$ & -2.447689 & 0.000000 & -0.174279 \\
\hline $\mathrm{H}$ & -3.359020 & 0.000000 & -0.506246 \\
\hline \multicolumn{4}{|l|}{ TS } \\
\hline $\mathrm{H}$ & 0.000000 & 0.000000 & 0.000000 \\
\hline $\mathrm{C}$ & 0.000000 & 0.000000 & 1.080792 \\
\hline $\mathrm{H}$ & 0.994713 & 0.000000 & 1.521879 \\
\hline $\mathrm{C}$ & -1.034599 & 0.668109 & 1.728149 \\
\hline $\mathrm{H}$ & -0.840931 & 1.242333 & 2.636335 \\
\hline $\mathrm{C}$ & -2.374847 & 0.443268 & 1.386849 \\
\hline $\mathrm{C}$ & -2.848853 & -0.812722 & 0.977486 \\
\hline $\mathrm{H}$ & -3.931944 & -0.912123 & 1.011537 \\
\hline $\mathrm{C}$ & -2.102445 & -1.989012 & 0.900503 \\
\hline
\end{tabular}




\begin{tabular}{|c|c|c|c|}
\hline $\mathrm{H}$ & -2.606719 & -2.907477 & 1.206762 \\
\hline $\mathrm{C}$ & -0.731726 & -2.117922 & 0.653673 \\
\hline $\mathrm{H}$ & -0.267573 & -1.872949 & -0.288592 \\
\hline $\mathrm{F}$ & -0.165172 & -3.253115 & 1.155532 \\
\hline $\mathrm{O}$ & -3.357328 & 1.339225 & 1.740715 \\
\hline $\mathrm{H}$ & -2.934162 & 2.181961 & 1.963267 \\
\hline & $\mathrm{F}$ & & \\
\hline $\mathrm{H}$ & 0.000000 & 0.000000 & 0.000000 \\
\hline $\mathrm{C}$ & 0.000000 & 0.000000 & 1.084810 \\
\hline $\mathrm{H}$ & 0.963815 & 0.000000 & 1.583188 \\
\hline $\mathrm{C}$ & -1.142437 & 0.000000 & 1.787202 \\
\hline $\mathrm{H}$ & -1.113218 & 0.000000 & 2.873318 \\
\hline $\mathrm{C}$ & -2.461286 & 0.000000 & 1.186696 \\
\hline $\mathrm{C}$ & -3.670045 & 0.000000 & 1.788545 \\
\hline $\mathrm{H}$ & -4.534595 & 0.000000 & 1.128741 \\
\hline $\mathrm{C}$ & -3.912792 & 0.000000 & 3.214066 \\
\hline $\mathrm{H}$ & -3.078231 & 0.000000 & 3.909790 \\
\hline $\mathrm{C}$ & -5.147533 & 0.000000 & 3.729481 \\
\hline $\mathrm{H}$ & -6.073756 & 0.000000 & 3.161957 \\
\hline $\mathrm{F}$ & -2.449625 & 0.000000 & -0.171247 \\
\hline & -5.357049 & 0.000000 & 5.058466 \\
\hline
\end{tabular}

TS

$\begin{array}{lrrr}\mathrm{H} & 0.000000 & 0.000000 & 0.000000 \\ \mathrm{C} & 0.000000 & 0.000000 & 1.080338 \\ \mathrm{H} & 0.993931 & 0.000000 & 1.522761 \\ \mathrm{C} & -1.033253 & 0.679753 & 1.721161 \\ \mathrm{H} & -0.852491 & 1.291305 & 2.604026 \\ \mathrm{C} & -2.359300 & 0.433515 & 1.381171 \\ \mathrm{C} & -2.862734 & -0.804825 & 0.973480 \\ \mathrm{H} & -3.945981 & -0.889688 & 1.017225 \\ \mathrm{C} & -2.124484 & -1.984657 & 0.876246 \\ \mathrm{H} & -2.631610 & -2.902653 & 1.176668 \\ \mathrm{C} & -0.752678 & -2.113474 & 0.632620 \\ \mathrm{H} & -0.285093 & -1.861669 & -0.305865 \\ \mathrm{~F} & -0.187101 & -3.245711 & 1.132603 \\ \mathrm{~F} & -3.290895 & 1.335135 & 1.785609\end{array}$




\begin{tabular}{|c|c|c|c|}
\hline $\mathrm{H}$ & 0.000000 & 0.000000 & 0.000000 \\
\hline $\mathrm{C}$ & 0.000000 & 0.000000 & 1.082252 \\
\hline $\mathrm{H}$ & 0.960113 & 0.000000 & 1.590970 \\
\hline $\mathrm{C}$ & -1.136212 & 0.000223 & 1.796379 \\
\hline $\mathrm{H}$ & -1.052481 & 0.000409 & 2.881594 \\
\hline $\mathrm{C}$ & -2.503683 & 0.000104 & 1.272770 \\
\hline $\mathrm{C}$ & -3.616970 & -0.000128 & 2.066723 \\
\hline $\mathrm{H}$ & -4.581961 & -0.000453 & 1.557984 \\
\hline $\mathrm{C}$ & -3.679014 & 0.000121 & 3.506208 \\
\hline $\mathrm{H}$ & -2.771665 & 0.000072 & 4.102270 \\
\hline $\mathrm{C}$ & -4.854324 & 0.000400 & 4.151753 \\
\hline $\mathrm{H}$ & -5.834407 & 0.000433 & 3.682176 \\
\hline $\mathrm{F}$ & -4.925459 & 0.000594 & 5.488113 \\
\hline $\mathrm{C}$ & -2.793541 & 0.000139 & -0.181263 \\
\hline $\mathrm{O}$ & -1.982179 & 0.000961 & -1.088340 \\
\hline $\mathrm{H}$ & -3.883229 & -0.000572 & -0.413241 \\
\hline \multicolumn{4}{|l|}{ TS } \\
\hline $\mathrm{H}$ & 0.000000 & 0.000000 & 0.000000 \\
\hline $\mathrm{C}$ & 0.000000 & 0.000000 & 1.081503 \\
\hline $\mathrm{H}$ & 0.988187 & 0.000000 & 1.535747 \\
\hline $\mathrm{C}$ & -1.060649 & 0.639042 & 1.704566 \\
\hline $\mathrm{H}$ & -0.891644 & 1.205766 & 2.621970 \\
\hline $\mathrm{C}$ & -2.387813 & 0.466558 & 1.275995 \\
\hline $\mathrm{C}$ & -2.846777 & -0.743336 & 0.718819 \\
\hline $\mathrm{H}$ & -3.926710 & -0.803046 & 0.602139 \\
\hline $\mathrm{C}$ & -2.131162 & -1.935669 & 0.650342 \\
\hline $\mathrm{H}$ & -2.696607 & -2.850586 & 0.836401 \\
\hline $\mathrm{C}$ & -0.743296 & -2.120001 & 0.578976 \\
\hline $\mathrm{H}$ & -0.134950 & -1.877555 & -0.277508 \\
\hline $\mathrm{F}$ & -0.283422 & -3.259150 & 1.149281 \\
\hline $\mathrm{C}$ & -3.407485 & 1.453558 & 1.698820 \\
\hline $\mathrm{O}$ & -4.580709 & 1.434030 & 1.374240 \\
\hline $\mathrm{H}$ & -3.015156 & 2.264645 & 2.352052 \\
\hline & $r$ & & \\
\hline $\mathrm{H}$ & 0.000000 & 0.000000 & 0.000000 \\
\hline
\end{tabular}




$\begin{array}{crrr}\mathrm{C} & 0.000000 & 0.000000 & 1.086475 \\ \mathrm{H} & 0.966377 & 0.000000 & 1.580250 \\ \mathrm{C} & -1.136061 & 0.000000 & 1.797772 \\ \mathrm{H} & -1.078951 & 0.000000 & 2.882797 \\ \mathrm{C} & -2.485363 & 0.000000 & 1.226583 \\ \mathrm{C} & -3.649189 & 0.000000 & 1.941734 \\ \mathrm{H} & -4.578375 & 0.000000 & 1.375913 \\ \mathrm{C} & -3.779688 & 0.000000 & 3.377343 \\ \mathrm{H} & -2.902662 & 0.000000 & 4.016976 \\ \mathrm{C} & -4.985053 & 0.000000 & 3.964484 \\ \mathrm{H} & -5.940911 & 0.000000 & 3.447739 \\ \mathrm{~F} & -5.118859 & 0.000000 & 5.295883 \\ \mathrm{C} & -2.593224 & 0.000000 & -0.205906 \\ \mathrm{~N} & -2.662008 & 0.000000 & -1.367787 \\ & & & \\ \mathrm{TS} & & & \\ \mathrm{H} & 0.000000 & 0.000000 & 0.000000 \\ \mathrm{C} & 0.000000 & 0.000000 & 1.081486 \\ \mathrm{H} & 0.987528 & 0.000000 & 1.536450 \\ \mathrm{C} & -1.053413 & 0.651109 & 1.704632 \\ \mathrm{H} & -0.889343 & 1.225956 & 2.614796 \\ \mathrm{C} & -2.380818 & 0.465174 & 1.272966 \\ \mathrm{C} & -2.834324 & -0.761982 & 0.740090 \\ \mathrm{H} & -3.911078 & -0.853972 & 0.621892 \\ \mathrm{C} & -2.103677 & -1.948036 & 0.687306 \\ \mathrm{H} & -2.662102 & -2.863276 & 0.889907 \\ \mathrm{C} & -0.714886 & -2.117230 & 0.614941 \\ \mathrm{H} & -0.114717 & -1.883317 & -0.249658 \\ \mathrm{~F} & -0.242808 & -3.242751 & 1.200890 \\ \mathrm{C} & -3.378421 & 1.423129 & 1.656953 \\ \mathrm{~N} & -4.179796 & 2.213501 & 1.953983\end{array}$

$\begin{array}{lrll}\mathrm{O}_{2} \mathrm{~N} & \\ & = & & \\ & & & \\ \mathrm{F} & & & \\ \mathrm{H} & 0.000000 & 0.000000 & 0.000000 \\ \mathrm{C} & 0.000000 & 0.000000 & 1.079819 \\ \mathrm{H} & 0.961155 & 0.000000 & 1.586064 \\ \mathrm{C} & -1.119469 & 0.000000 & 1.820133 \\ \mathrm{H} & -0.986672 & 0.000000 & 2.899340 \\ \mathrm{C} & -2.529866 & 0.000000 & 1.449175 \\ \mathrm{C} & -3.564486 & 0.000000 & 2.333042 \\ \mathrm{H} & -4.558730 & 0.000000 & 1.897407\end{array}$




$\begin{array}{llll}\mathrm{C} & -3.480693 & 0.000000 & 3.768831 \\ \mathrm{H} & -2.525784 & 0.000000 & 4.284611 \\ \mathrm{C} & -4.598605 & 0.000000 & 4.511626 \\ \mathrm{H} & -5.614116 & 0.000000 & 4.125018 \\ \mathrm{~F} & -4.553876 & 0.000000 & 5.845904 \\ \mathrm{~N} & -2.938852 & 0.000000 & 0.026059 \\ \mathrm{O} & -2.051725 & 0.000000 & -0.827405 \\ \mathrm{O} & -4.142327 & 0.000019 & -0.244449\end{array}$

TS

$\begin{array}{lrrr}\mathrm{H} & 0.000000 & 0.000000 & 0.000000 \\ \mathrm{C} & 0.000000 & 0.000000 & 1.081267 \\ \mathrm{H} & 0.987467 & 0.000000 & 1.536140 \\ \mathrm{C} & -1.051234 & 0.651615 & 1.709062 \\ \mathrm{H} & -0.897801 & 1.236979 & 2.611210 \\ \mathrm{C} & -2.365075 & 0.439539 & 1.278785 \\ \mathrm{C} & -2.847234 & -0.783269 & 0.792661 \\ \mathrm{H} & -3.927083 & -0.854387 & 0.713875 \\ \mathrm{C} & -2.113247 & -1.963524 & 0.730935 \\ \mathrm{H} & -2.657775 & -2.882393 & 0.951705 \\ \mathrm{C} & -0.723815 & -2.117923 & 0.619160 \\ \mathrm{H} & -0.153815 & -1.882224 & -0.265434 \\ \mathrm{~F} & -0.220960 & -3.230617 & 1.198937 \\ \mathrm{~N} & -3.382218 & 1.425161 & 1.690864 \\ \mathrm{O} & -4.547812 & 1.249736 & 1.333201 \\ \mathrm{O} & -2.996512 & 2.388639 & 2.355144\end{array}$

5

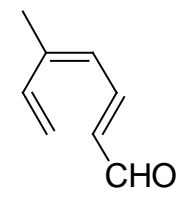

$\begin{array}{lrrl}\mathrm{H} & 0.000000 & 0.000000 & 0.000000 \\ \mathrm{C} & 0.000000 & 0.000000 & 1.085733 \\ \mathrm{H} & 0.973412 & 0.000000 & 1.566654 \\ \mathrm{C} & -1.128932 & -0.000060 & 1.813197 \\ \mathrm{H} & -1.030517 & -0.000173 & 2.895649 \\ \mathrm{C} & -2.486186 & -0.000154 & 1.270664 \\ \mathrm{C} & -3.597594 & 0.000509 & 2.062692 \\ \mathrm{H} & -4.565841 & 0.000258 & 1.563884 \\ \mathrm{C} & -3.653383 & 0.001599 & 3.503125 \\ \mathrm{H} & -2.718683 & 0.002150 & 4.062355\end{array}$




$\begin{array}{crrc}\mathrm{C} & -4.804372 & 0.002109 & 4.218021 \\ \mathrm{H} & -5.778957 & 0.001682 & 3.733872 \\ \mathrm{C} & -4.792276 & 0.003217 & 5.680096 \\ \mathrm{C} & -2.649372 & -0.001093 & -0.229541 \\ \mathrm{H} & -3.774842 & 0.003609 & 6.135877 \\ \mathrm{O} & -5.789532 & 0.003686 & 6.381994 \\ \mathrm{H} & -2.175448 & -0.882792 & -0.679274 \\ \mathrm{H} & -2.175459 & 0.880035 & -0.680414 \\ \mathrm{H} & -3.704824 & -0.001219 & -0.514363 \\ & & & \\ \mathrm{TS} & & & \\ \mathrm{H} & 0.000000 & 0.000000 & 0.000000 \\ \mathrm{C} & 0.000000 & 0.000000 & 1.078552 \\ \mathrm{H} & 1.001706 & 0.000000 & 1.507160 \\ \mathrm{C} & -1.013472 & 0.699409 & 1.744302 \\ \mathrm{H} & -0.724007 & 1.355189 & 2.567939 \\ \mathrm{C} & -2.388325 & 0.497946 & 1.580417 \\ \mathrm{C} & -2.914981 & -0.787814 & 1.295195 \\ \mathrm{H} & -3.978279 & -0.907346 & 1.500291 \\ \mathrm{C} & -2.162673 & -1.950497 & 1.190617 \\ \mathrm{H} & -2.599303 & -2.843357 & 1.645072 \\ \mathrm{C} & -0.833788 & -2.046407 & 0.716577 \\ \mathrm{H} & -0.617635 & -1.748761 & -0.298832 \\ \mathrm{C} & 0.005288 & -3.170417 & 1.179835 \\ \mathrm{C} & -3.348960 & 1.570257 & 2.047012 \\ \mathrm{H} & -3.669819 & 2.190338 & 1.198912 \\ \mathrm{H} & -2.874551 & 2.242925 & 2.770077 \\ \mathrm{H} & -4.252234 & 1.152070 & 2.503978 \\ \mathrm{H} & -0.397399 & -3.720503 & 2.062884 \\ \mathrm{O} & 1.074098 & -3.483388 & 0.686936\end{array}$

$\begin{array}{lrrr}{ }^{\mathrm{H}_{2} \mathrm{~N}} & & \\ & & & \\ \mathrm{CHO} & & \\ \mathrm{H} & 0.000000 & 0.000000 & 0.000000 \\ \mathrm{C} & 0.000000 & 0.000000 & 1.086362 \\ \mathrm{H} & 0.970853 & 0.000000 & 1.571851 \\ \mathrm{C} & -1.131047 & -0.039475 & 1.805201 \\ \mathrm{H} & -1.064172 & -0.079394 & 2.887792 \\ \mathrm{C} & -2.486360 & -0.013375 & 1.241632 \\ \mathrm{C} & -3.601954 & -0.400135 & 1.942439 \\ \mathrm{H} & -4.567082 & -0.287475 & 1.448557 \\ \mathrm{C} & -3.624428 & -0.977006 & 3.250630\end{array}$




$\begin{array}{lrrr}\mathrm{H} & -2.672075 & -1.138519 & 3.755807 \\ \mathrm{C} & -4.746423 & -1.350551 & 3.921905 \\ \mathrm{H} & -5.737265 & -1.238146 & 3.486348 \\ \mathrm{C} & -4.670694 & -1.923443 & 5.257730 \\ \mathrm{~N} & -2.582760 & 0.385950 & -0.081655 \\ \mathrm{H} & -1.943191 & 1.114580 & -0.373259 \\ \mathrm{H} & -3.521117 & 0.520079 & -0.437136 \\ \mathrm{H} & -3.633381 & -2.021264 & 5.658602 \\ \mathrm{O} & -5.629246 & -2.282003 & 5.925217\end{array}$

TS

$\begin{array}{llll}\mathrm{H} & 0.000000 & 0.000000 & 0.000000\end{array}$

$\begin{array}{llll}\mathrm{C} & 0.000000 & 0.000000 & 1.079117\end{array}$

$\begin{array}{llll}\mathrm{H} & 1.003776 & 0.000000 & 1.502633\end{array}$

$\begin{array}{llll}\text { C } & -1.001268 & 0.701851 & 1.757600\end{array}$

$\begin{array}{llll}\mathrm{H} & -0.721679 & 1.333176 & 2.602532\end{array}$

$\begin{array}{llll}\text { C } & -2.378308 & 0.472748 & 1.595620\end{array}$

$\begin{array}{llll}\text { C } & -2.887708 & -0.841809 & 1.381238\end{array}$

$\begin{array}{llll}\mathrm{H} & -3.942476 & -0.971762 & 1.627039\end{array}$

$\begin{array}{llll}\text { C } & -2.112938 & -1.981143 & 1.285344\end{array}$

$\begin{array}{llll}\mathrm{H} & -2.526627 & -2.885713 & 1.738176\end{array}$

$\begin{array}{llll}\text { C } & -0.788428 & -2.045508 & 0.772066\end{array}$

$\begin{array}{llll}\mathrm{H} & -0.627944 & -1.772614 & -0.261410\end{array}$

$\begin{array}{llll}\text { C } & 0.091963 & -3.133878 & 1.225387\end{array}$

$\begin{array}{llll}\mathrm{N} & -3.285510 & 1.465348 & 1.941092\end{array}$

$\mathrm{H} \quad-4.158597 \quad 1.169941 \quad 2.361376$

$\begin{array}{llll}\mathrm{H} & -2.894914 & 2.292641 & 2.374271\end{array}$

$\begin{array}{llll}\mathrm{H} & -0.264373 & -3.671602 & 2.136938\end{array}$

$\begin{array}{llll}\text { O } & 1.152373 & -3.436394 & 0.703700\end{array}$

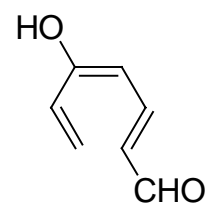

$\begin{array}{lrll}\mathrm{H} & 0.000000 & 0.000000 & 0.000000 \\ \mathrm{C} & 0.000000 & 0.000000 & 1.084660 \\ \mathrm{H} & 0.963801 & 0.000000 & 1.583969 \\ \mathrm{C} & -1.139591 & 0.000000 & 1.790218 \\ \mathrm{H} & -1.100742 & 0.000000 & 2.874481 \\ \mathrm{C} & -2.468884 & 0.000000 & 1.189712 \\ \mathrm{C} & -3.651752 & 0.000000 & 1.874293 \\ \mathrm{H} & -4.570853 & 0.000000 & 1.286504 \\ \mathrm{C} & -3.814801 & 0.000000 & 3.298287 \\ \mathrm{H} & -2.918246 & 0.000000 & 3.917657\end{array}$




$\begin{array}{lrrc}\mathrm{C} & -5.009616 & 0.000000 & 3.942221 \\ \mathrm{H} & -5.953014 & 0.000000 & 3.399896 \\ \mathrm{C} & -5.083790 & -0.000022 & 5.398384 \\ \mathrm{O} & -2.440462 & 0.000000 & -0.170517 \\ \mathrm{H} & -3.349452 & 0.000000 & -0.511514 \\ \mathrm{H} & -4.093141 & 0.000210 & 5.911843 \\ \mathrm{O} & -6.118850 & 0.000221 & 6.045287\end{array}$

$\begin{array}{rrrr}\text { TS } & & & \\ \mathrm{H} & 0.000000 & 0.000000 & 0.000000 \\ \mathrm{H} & 0.000000 & 0.000000 & 1.806289 \\ \mathrm{C} & 0.599395 & 0.000000 & 0.909894 \\ \mathrm{C} & 1.812474 & 0.699037 & 0.894996 \\ \mathrm{H} & 2.041762 & 1.339305 & 0.039971 \\ \mathrm{C} & 2.886319 & 0.440287 & 1.754304 \\ \mathrm{C} & 3.228394 & -0.860748 & 2.197636 \\ \mathrm{H} & 4.265765 & -0.962694 & 2.509276 \\ \mathrm{C} & 2.502990 & -1.995965 & 1.887007 \\ \mathrm{H} & 3.079404 & -2.903869 & 1.696014 \\ \mathrm{C} & 1.103228 & -2.038117 & 1.667401 \\ \mathrm{H} & 0.452908 & -1.716732 & 2.468520 \\ \mathrm{C} & 0.538263 & -3.135599 & 0.862326 \\ \mathrm{O} & 3.896887 & 1.353057 & 1.894327 \\ \mathrm{H} & 3.597353 & 2.204026 & 1.537980 \\ \mathrm{H} & 1.286637 & -3.709358 & 0.265765 \\ \mathrm{O} & -0.648132 & -3.406708 & 0.796237\end{array}$

$\begin{array}{llll} & & \\ & & & \\ & & & \\ \mathrm{CHO} & & \\ \mathrm{H} & 0.000000 & 0.000000 & 0.000000 \\ \mathrm{C} & 0.000000 & 0.000000 & 1.084733 \\ \mathrm{H} & 0.963729 & 0.000000 & 1.583088 \\ \mathrm{C} & -1.142203 & 0.000000 & 1.787916 \\ \mathrm{H} & -1.115125 & 0.000000 & 2.873403 \\ \mathrm{C} & -2.456132 & 0.000000 & 1.176616 \\ \mathrm{C} & -3.675717 & 0.000000 & 1.767789 \\ \mathrm{H} & -4.536371 & 0.000000 & 1.103871 \\ \mathrm{C} & -3.920052 & 0.000000 & 3.184399 \\ \mathrm{H} & -3.060303 & 0.000000 & 3.854347 \\ \mathrm{C} & -5.151323 & 0.000000 & 3.749367 \\ \mathrm{H} & -6.058006 & 0.000000 & 3.148177 \\ \mathrm{C} & -5.317553 & 0.000000 & 5.202041\end{array}$




\begin{tabular}{|c|c|c|c|}
\hline $\mathrm{F}$ & -2.433636 & 0.000000 & -0.174356 \\
\hline $\mathrm{H}$ & -4.362868 & -0.000195 & 5.778308 \\
\hline $\mathrm{O}$ & -6.392684 & -0.000193 & 5.776566 \\
\hline \multicolumn{4}{|l|}{ TS } \\
\hline $\mathrm{H}$ & 0.000000 & 0.000000 & 0.000000 \\
\hline $\mathrm{C}$ & 0.000000 & 0.000000 & 1.077629 \\
\hline $\mathrm{H}$ & 1.005665 & 0.000000 & 1.498122 \\
\hline $\mathrm{C}$ & -0.992934 & 0.701399 & 1.776761 \\
\hline $\mathrm{H}$ & -0.705648 & 1.391894 & 2.570056 \\
\hline $\mathrm{C}$ & -2.350620 & 0.413093 & 1.723608 \\
\hline $\mathrm{C}$ & -2.915741 & -0.870323 & 1.587712 \\
\hline $\mathrm{H}$ & -3.923692 & -0.964501 & 1.984481 \\
\hline $\mathrm{C}$ & -2.152952 & -2.002795 & 1.362737 \\
\hline $\mathrm{H}$ & -2.474186 & -2.921441 & 1.856815 \\
\hline $\mathrm{C}$ & -0.923133 & -2.005206 & 0.665603 \\
\hline $\mathrm{H}$ & -0.917617 & -1.615708 & -0.342580 \\
\hline $\mathrm{C}$ & 0.028120 & -3.114407 & 0.876061 \\
\hline $\mathrm{F}$ & -3.183406 & 1.338849 & 2.259098 \\
\hline $\mathrm{H}$ & -0.175809 & -3.739993 & 1.776384 \\
\hline $\mathrm{O}$ & 0.989496 & -3.340569 & 0.164308 \\
\hline \multicolumn{4}{|c|}{$\mathrm{OH}$} \\
\hline $\mathrm{H}$ & 0.000000 & 0.000000 & 0.000 \\
\hline $\mathrm{C}$ & 0.000000 & 0.000000 & 1.082127 \\
\hline $\mathrm{H}$ & 0.960144 & 0.000000 & 1.590454 \\
\hline $\mathrm{C}$ & -1.136211 & -0.000041 & 1.798302 \\
\hline $\mathrm{H}$ & -1.051200 & -0.000071 & 2.882832 \\
\hline $\mathrm{C}$ & -2.499759 & 0.000054 & 1.271770 \\
\hline $\mathrm{C}$ & -3.621547 & -0.000490 & 2.056875 \\
\hline $\mathrm{H}$ & -4.584440 & -0.000115 & 1.545780 \\
\hline $\mathrm{C}$ & -3.691848 & -0.001610 & 3.494795 \\
\hline $\mathrm{H}$ & -2.763047 & -0.002146 & 4.062984 \\
\hline $\mathrm{C}$ & -4.856253 & -0.002098 & 4.186278 \\
\hline $\mathrm{H}$ & -5.821592 & -0.001630 & 3.683947 \\
\hline $\mathrm{C}$ & -4.876227 & -0.003287 & 5.653423 \\
\hline $\mathrm{C}$ & -2.784417 & 0.000874 & -0.191031 \\
\hline $\mathrm{O}$ & -1.965810 & 0.000889 & -1.089093 \\
\hline $\mathrm{H}$ & -3.871333 & 0.001466 & -0.428216 \\
\hline $\mathrm{H}$ & -3.872744 & -0.003777 & 6.135654 \\
\hline $\mathrm{O}$ & -5.894144 & -0.003748 & 6.321662 \\
\hline
\end{tabular}




\begin{tabular}{|c|c|c|c|}
\hline $\mathrm{H}$ & 0.000000 & 0.000000 & 0.000000 \\
\hline $\mathrm{C}$ & 0.000000 & 0.000000 & 1.079002 \\
\hline $\mathrm{H}$ & 0.994440 & 0.000000 & 1.521048 \\
\hline $\mathrm{C}$ & -1.041625 & 0.664637 & 1.726198 \\
\hline $\mathrm{H}$ & -0.807562 & 1.295859 & 2.586219 \\
\hline $\mathrm{C}$ & -2.399045 & 0.449792 & 1.456243 \\
\hline $\mathrm{C}$ & -2.925998 & -0.803583 & 1.060176 \\
\hline $\mathrm{H}$ & -4.007360 & -0.882431 & 1.146708 \\
\hline $\mathrm{C}$ & -2.176594 & -1.968722 & 0.994852 \\
\hline $\mathrm{H}$ & -2.673206 & -2.867478 & 1.369403 \\
\hline $\mathrm{C}$ & -0.803061 & -2.086622 & 0.677523 \\
\hline $\mathrm{H}$ & -0.451463 & -1.793982 & -0.300370 \\
\hline $\mathrm{C}$ & -0.033262 & -3.214129 & 1.252040 \\
\hline $\mathrm{C}$ & -3.369142 & 1.457853 & 1.961338 \\
\hline $\mathrm{O}$ & -4.573034 & 1.405100 & 1.801415 \\
\hline $\mathrm{H}$ & -2.904577 & 2.312514 & 2.500509 \\
\hline $\mathrm{H}$ & -0.549234 & -3.769259 & 2.068714 \\
\hline $\mathrm{O}$ & 1.093038 & -3.516014 & 0.904931 \\
\hline${ }^{\mathrm{NC}}$ & UतO & & \\
\hline $\mathrm{H}$ & 0.000000 & 0.000000 & 0.000000 \\
\hline $\mathrm{C}$ & 0.000000 & 0.000000 & 1.086397 \\
\hline $\mathrm{H}$ & 0.966376 & 0.000000 & 1.580023 \\
\hline $\mathrm{C}$ & -1.136210 & 0.000000 & 1.799057 \\
\hline $\mathrm{H}$ & -1.079604 & 0.000000 & 2.883502 \\
\hline $\mathrm{C}$ & -2.480348 & 0.000000 & 1.220124 \\
\hline $\mathrm{C}$ & -3.655382 & 0.000000 & 1.921642 \\
\hline $\mathrm{H}$ & -4.580246 & 0.000000 & 1.350033 \\
\hline $\mathrm{C}$ & -3.794831 & 0.000000 & 3.354411 \\
\hline $\mathrm{H}$ & -2.894991 & 0.000000 & 3.967344 \\
\hline $\mathrm{C}$ & -4.991639 & 0.000000 & 3.987527 \\
\hline $\mathrm{H}$ & -5.931134 & 0.000000 & 3.438795 \\
\hline $\mathrm{C}$ & -5.080665 & 0.000000 & 5.452316 \\
\hline $\mathrm{C}$ & -2.573407 & 0.000000 & -0.214642 \\
\hline $\mathrm{N}$ & -2.630080 & -0.000001 & -1.376728 \\
\hline $\mathrm{H}$ & -4.100610 & 0.000053 & 5.980905 \\
\hline $\mathrm{O}$ & -6.128381 & 0.000053 & 6.072181 \\
\hline
\end{tabular}

TS

$\begin{array}{llll}\mathrm{H} & 0.000000 & 0.000000 & 0.000000\end{array}$ 


$\begin{array}{rrrr}\mathrm{C} & 0.000000 & 0.000000 & 1.078909 \\ \mathrm{H} & 0.993933 & 0.000000 & 1.521561 \\ \mathrm{C} & -1.032928 & 0.678378 & 1.726055 \\ \mathrm{H} & -0.801554 & 1.316337 & 2.578349 \\ \mathrm{C} & -2.391965 & 0.455084 & 1.453734 \\ \mathrm{C} & -2.915408 & -0.814747 & 1.087434 \\ \mathrm{H} & -3.993550 & -0.919698 & 1.178991 \\ \mathrm{C} & -2.154012 & -1.973801 & 1.034539 \\ \mathrm{H} & -2.643670 & -2.869946 & 1.422915 \\ \mathrm{C} & -0.782639 & -2.081207 & 0.709561 \\ \mathrm{H} & -0.443882 & -1.799078 & -0.275867 \\ \mathrm{C} & -0.000775 & -3.200360 & 1.287671 \\ \mathrm{C} & -3.342000 & 1.431006 & 1.914013 \\ \mathrm{H} & -0.507415 & -3.754676 & 2.110486 \\ \mathrm{O} & 1.125022 & -3.494077 & 0.934260 \\ \mathrm{~N} & -4.108817 & 2.233017 & 2.264673\end{array}$

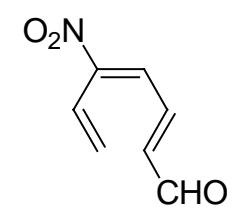

$\begin{array}{lrrr}\mathrm{H} & 0.000000 & 0.000000 & 0.000000 \\ \mathrm{C} & 0.000000 & 0.000000 & 1.081092 \\ \mathrm{H} & 0.962317 & 0.000000 & 1.584243 \\ \mathrm{C} & -1.122174 & 0.004628 & 1.818217 \\ \mathrm{H} & -1.005327 & 0.010847 & 2.898653 \\ \mathrm{C} & -2.516980 & -0.042295 & 1.405815 \\ \mathrm{C} & -3.595204 & -0.060516 & 2.235076 \\ \mathrm{H} & -4.570240 & -0.126405 & 1.763311 \\ \mathrm{C} & -3.572907 & 0.000136 & 3.673689 \\ \mathrm{H} & -2.615405 & 0.064045 & 4.187554 \\ \mathrm{C} & -4.697017 & -0.017669 & 4.427320 \\ \mathrm{H} & -5.686784 & -0.079524 & 3.980051 \\ \mathrm{C} & -4.635559 & 0.044776 & 5.893874 \\ \mathrm{~N} & -2.858710 & -0.075142 & -0.041762 \\ \mathrm{O} & -2.038880 & 0.388758 & -0.832390 \\ \mathrm{O} & -3.946076 & -0.547873 & -0.368620 \\ \mathrm{H} & -3.608431 & 0.106627 & 6.318530 \\ \mathrm{O} & -5.615046 & 0.032343 & 6.615714\end{array}$

TS

$\begin{array}{llll}\mathrm{H} & 0.000000 & 0.000000 & 0.000000 \\ \mathrm{C} & 0.000000 & 0.000000 & 1.078451 \\ \mathrm{H} & 0.995796 & 0.000000 & 1.517400\end{array}$




$\begin{array}{lrrr}\mathrm{C} & -1.023711 & 0.681569 & 1.739271 \\ \mathrm{H} & -0.788609 & 1.342179 & 2.570113 \\ \mathrm{C} & -2.375455 & 0.429838 & 1.508497 \\ \mathrm{C} & -2.931950 & -0.833493 & 1.227906 \\ \mathrm{H} & -3.999136 & -0.917364 & 1.403813 \\ \mathrm{C} & -2.166099 & -1.984628 & 1.128231 \\ \mathrm{H} & -2.608219 & -2.885435 & 1.558959 \\ \mathrm{C} & -0.826784 & -2.064562 & 0.686036 \\ \mathrm{H} & -0.585399 & -1.752297 & -0.319033 \\ \mathrm{C} & 0.023360 & -3.180641 & 1.167246 \\ \mathrm{~N} & -3.331263 & 1.450277 & 2.014136 \\ \mathrm{O} & -4.533278 & 1.247653 & 1.849358 \\ \mathrm{O} & -2.859522 & 2.457809 & 2.539789 \\ \mathrm{H} & -0.392951 & -3.755060 & 2.025707 \\ \mathrm{O} & 1.112681 & -3.450822 & 0.700451\end{array}$

6

\begin{tabular}{lrrr}
\hline & & & \\
& & & \\
& & & \\
$\mathrm{H}$ & 0.000000 & 0.000000 & 0.000000 \\
$\mathrm{C}$ & 0.000000 & 0.000000 & 1.085767 \\
$\mathrm{H}$ & 0.972988 & 0.000000 & 1.567366 \\
$\mathrm{C}$ & -1.129394 & 0.000040 & 1.812301 \\
$\mathrm{H}$ & -1.032283 & 0.000087 & 2.894866 \\
$\mathrm{C}$ & -2.485493 & 0.000068 & 1.266328 \\
$\mathrm{C}$ & -3.598753 & 0.000489 & 2.054736 \\
$\mathrm{H}$ & -4.566731 & 0.000439 & 1.554954 \\
$\mathrm{C}$ & -3.653009 & 0.001001 & 3.495239 \\
$\mathrm{H}$ & -2.725984 & 0.001162 & 4.061520 \\
$\mathrm{C}$ & -4.818040 & 0.001257 & 4.188855 \\
$\mathrm{H}$ & -5.774923 & 0.001080 & 3.671168 \\
$\mathrm{C}$ & -4.868943 & 0.001700 & 5.610269 \\
$\mathrm{C}$ & -2.644500 & -0.000299 & -0.234433 \\
$\mathrm{H}$ & -2.168361 & -0.881661 & -0.682216 \\
$\mathrm{H}$ & -2.168829 & 0.881185 & -0.682534 \\
$\mathrm{H}$ & -3.698799 & -0.000563 & -0.523430 \\
$\mathrm{~N}$ & -4.927952 & 0.002040 & 6.774308
\end{tabular}

TS

$\begin{array}{llll}\mathrm{H} & 0.000000 & 0.000000 & 0.000000\end{array}$ 


\begin{tabular}{|c|c|c|c|}
\hline $\mathrm{C}$ & 0.000000 & 0.000000 & 1.079575 \\
\hline $\mathrm{H}$ & 0.997965 & 0.000000 & 1.515368 \\
\hline $\mathrm{C}$ & -1.023857 & 0.686086 & 1.737490 \\
\hline $\mathrm{H}$ & -0.757291 & 1.320334 & 2.584776 \\
\hline $\mathrm{C}$ & -2.391936 & 0.494425 & 1.514162 \\
\hline $\mathrm{C}$ & -2.907847 & -0.779631 & 1.171726 \\
\hline $\mathrm{H}$ & -3.983644 & -0.891658 & 1.300296 \\
\hline $\mathrm{C}$ & -2.176153 & -1.959948 & 1.102677 \\
\hline $\mathrm{H}$ & -2.659209 & -2.848411 & 1.510639 \\
\hline $\mathrm{C}$ & -0.816288 & -2.075119 & 0.722655 \\
\hline $\mathrm{H}$ & -0.539118 & -1.784645 & -0.278926 \\
\hline $\mathrm{C}$ & -0.061029 & -3.197978 & 1.201438 \\
\hline $\mathrm{C}$ & -3.368678 & 1.561550 & 1.958089 \\
\hline $\mathrm{H}$ & -3.632718 & 2.212711 & 1.113744 \\
\hline $\mathrm{H}$ & -2.930124 & 2.204500 & 2.729182 \\
\hline $\mathrm{H}$ & -4.300537 & 1.139538 & 2.348690 \\
\hline $\mathrm{N}$ & 0.579078 & -4.081208 & 1.610143 \\
\hline \multicolumn{4}{|c|}{$\mathrm{H}_{2} \mathrm{~N}$} \\
\hline $\mathrm{H}$ & 0.000000 & 0.000000 & 0.000000 \\
\hline $\mathrm{C}$ & 0.000000 & 0.000000 & 1.086304 \\
\hline $\mathrm{H}$ & 0.970681 & 0.000000 & 1.572046 \\
\hline $\mathrm{C}$ & -1.130810 & 0.039863 & 1.805262 \\
\hline $\mathrm{H}$ & -1.063458 & 0.079865 & 2.887835 \\
\hline $\mathrm{C}$ & -2.486139 & 0.014355 & 1.241278 \\
\hline $\mathrm{C}$ & -3.600172 & 0.407024 & 1.939883 \\
\hline $\mathrm{H}$ & -4.566800 & 0.295414 & 1.448445 \\
\hline $\mathrm{C}$ & -3.615130 & 0.990944 & 3.245878 \\
\hline $\mathrm{H}$ & -2.667368 & 1.153852 & 3.752755 \\
\hline $\mathrm{C}$ & -4.747150 & 1.366090 & 3.900164 \\
\hline $\mathrm{H}$ & -5.724673 & 1.242931 & 3.439306 \\
\hline $\mathrm{C}$ & -4.726816 & 1.935139 & 5.200868 \\
\hline $\mathrm{N}$ & -2.583162 & -0.390845 & -0.080535 \\
\hline $\mathrm{H}$ & -1.945405 & -1.123165 & -0.366947 \\
\hline $\mathrm{H}$ & -3.521521 & -0.525349 & -0.435875 \\
\hline $\mathrm{N}$ & -4.725661 & 2.403001 & 6.269471 \\
\hline \multicolumn{4}{|c|}{$\mathrm{TS}$} \\
\hline $\mathrm{H}$ & 0.000000 & 0.000000 & 0.000000 \\
\hline $\mathrm{C}$ & 0.000000 & 0.000000 & 1.080043 \\
\hline $\mathrm{H}$ & 0.999081 & 0.000000 & 1.512632 \\
\hline $\mathrm{C}$ & -1.015655 & 0.682543 & 1.749550 \\
\hline
\end{tabular}




\begin{tabular}{|c|c|c|c|}
\hline $\mathrm{H}$ & -0.765870 & 1.286042 & 2.622987 \\
\hline $\mathrm{C}$ & -2.383769 & 0.463677 & 1.513708 \\
\hline $\mathrm{C}$ & -2.882298 & -0.836737 & 1.217471 \\
\hline $\mathrm{H}$ & -3.955516 & -0.961136 & 1.365798 \\
\hline $\mathrm{C}$ & -2.128370 & -1.995034 & 1.161597 \\
\hline $\mathrm{H}$ & -2.598810 & -2.897929 & 1.553211 \\
\hline $\mathrm{C}$ & -0.762504 & -2.081639 & 0.767920 \\
\hline $\mathrm{H}$ & -0.517533 & -1.817083 & -0.250095 \\
\hline $\mathrm{C}$ & 0.015498 & -3.182676 & 1.254874 \\
\hline $\mathrm{N}$ & -3.306738 & 1.447463 & 1.840771 \\
\hline $\mathrm{N}$ & 0.674902 & -4.047394 & 1.674700 \\
\hline $\mathrm{H}$ & -4.199788 & 1.142722 & 2.209030 \\
\hline $\mathrm{H}$ & -2.938056 & 2.261692 & 2.315614 \\
\hline \multicolumn{4}{|l|}{$\mathrm{HO}$} \\
\hline $\mathrm{H}$ & 0.000000 & 0.000000 & 0.000000 \\
\hline $\mathrm{C}$ & 0.000000 & 0.000000 & 1.084630 \\
\hline $\mathrm{H}$ & 0.963671 & 0.000000 & 1.584064 \\
\hline $\mathrm{C}$ & -1.139615 & 0.000000 & 1.790037 \\
\hline $\mathrm{H}$ & -1.100599 & 0.000000 & 2.874214 \\
\hline $\mathrm{C}$ & -2.468245 & 0.000000 & 1.188266 \\
\hline $\mathrm{C}$ & -3.651676 & 0.000000 & 1.871300 \\
\hline $\mathrm{H}$ & -4.571290 & 0.000000 & 1.284177 \\
\hline $\mathrm{C}$ & -3.810604 & 0.000000 & 3.296257 \\
\hline $\mathrm{H}$ & -2.920014 & 0.000000 & 3.918974 \\
\hline $\mathrm{C}$ & -5.015600 & 0.000000 & 3.922469 \\
\hline $\mathrm{H}$ & -5.941803 & 0.000000 & 3.352107 \\
\hline $\mathrm{C}$ & -5.143187 & 0.000000 & 5.337557 \\
\hline $\mathrm{O}$ & -2.438610 & 0.000000 & -0.172095 \\
\hline $\mathrm{H}$ & -3.346734 & 0.000000 & -0.514758 \\
\hline $\mathrm{N}$ & -5.263389 & -0.000001 & 6.497357 \\
\hline \multicolumn{4}{|c|}{ TS } \\
\hline $\mathrm{H}$ & 0.000000 & 0.000000 & 0.000000 \\
\hline $\mathrm{C}$ & 0.000000 & 0.000000 & 1.079286 \\
\hline $\mathrm{H}$ & 0.999156 & 0.000000 & 1.511763 \\
\hline $\mathrm{C}$ & -1.014684 & 0.684078 & 1.753018 \\
\hline $\mathrm{H}$ & -0.761014 & 1.299320 & 2.618644 \\
\hline $\mathrm{C}$ & -2.377618 & 0.435639 & 1.555237 \\
\hline $\mathrm{C}$ & -2.899732 & -0.853946 & 1.298889 \\
\hline $\mathrm{H}$ & -3.960828 & -0.950723 & 1.518096 \\
\hline
\end{tabular}




\begin{tabular}{|c|c|c|c|}
\hline $\mathrm{C}$ & -2.141875 & -2.008823 & 1.207878 \\
\hline $\mathrm{H}$ & -2.578778 & -2.911860 & 1.634525 \\
\hline $\mathrm{C}$ & -0.803497 & -2.078077 & 0.738061 \\
\hline $\mathrm{H}$ & -0.616810 & -1.776658 & -0.281853 \\
\hline $\mathrm{C}$ & 0.013977 & -3.181484 & 1.150608 \\
\hline $\mathrm{O}$ & -3.318774 & 1.339325 & 1.966764 \\
\hline $\mathrm{N}$ & 0.706235 & -4.049082 & 1.505041 \\
\hline $\mathrm{H}$ & -2.880570 & 2.186489 & 2.142912 \\
\hline$\rangle$ & $\mathrm{CN}$ & & \\
\hline $\mathrm{H}$ & 0.000000 & 0.000000 & 0.000000 \\
\hline $\mathrm{C}$ & 0.000000 & 0.000000 & 1.084739 \\
\hline $\mathrm{H}$ & 0.963576 & 0.000000 & 1.583314 \\
\hline $\mathrm{C}$ & -1.142246 & 0.000000 & 1.787728 \\
\hline $\mathrm{H}$ & -1.115320 & 0.000000 & 2.873188 \\
\hline $\mathrm{C}$ & -2.455303 & 0.000000 & 1.174491 \\
\hline $\mathrm{C}$ & -3.675687 & 0.000000 & 1.763528 \\
\hline $\mathrm{H}$ & -4.536022 & 0.000000 & 1.099017 \\
\hline $\mathrm{C}$ & -3.917867 & 0.000000 & 3.180339 \\
\hline $\mathrm{H}$ & -3.066321 & 0.000000 & 3.855896 \\
\hline $\mathrm{C}$ & -5.160197 & 0.000000 & 3.723332 \\
\hline $\mathrm{H}$ & -6.045426 & 0.000000 & 3.091414 \\
\hline $\mathrm{C}$ & -5.382858 & 0.000000 & 5.127732 \\
\hline $\mathrm{F}$ & -2.432388 & 0.000000 & -0.176187 \\
\hline $\mathrm{N}$ & -5.579711 & -0.000002 & 6.276473 \\
\hline \multicolumn{4}{|l|}{ TS } \\
\hline $\mathrm{H}$ & 0.000000 & 0.000000 & 0.000000 \\
\hline $\mathrm{C}$ & 0.000000 & 0.000000 & 1.078614 \\
\hline $\mathrm{H}$ & 0.999391 & 0.000000 & 1.510799 \\
\hline $\mathrm{C}$ & -1.011326 & 0.691433 & 1.754596 \\
\hline $\mathrm{H}$ & -0.762023 & 1.354955 & 2.582089 \\
\hline $\mathrm{C}$ & -2.362832 & 0.416787 & 1.593831 \\
\hline $\mathrm{C}$ & -2.920907 & -0.854706 & 1.365051 \\
\hline $\mathrm{H}$ & -3.971034 & -0.939383 & 1.633443 \\
\hline $\mathrm{C}$ & -2.171207 & -2.012000 & 1.227797 \\
\hline $\mathrm{H}$ & -2.582804 & -2.917924 & 1.671344 \\
\hline $\mathrm{C}$ & -0.857044 & -2.063212 & 0.699352 \\
\hline $\mathrm{H}$ & -0.717922 & -1.724592 & -0.316288 \\
\hline $\mathrm{C}$ & -0.015676 & -3.173700 & 1.040595 \\
\hline $\mathrm{F}$ & -3.233061 & 1.333364 & 2.079854 \\
\hline
\end{tabular}




\begin{tabular}{|c|c|c|c|}
\hline $\mathrm{N}$ & 0.694470 & -4.048738 & 1.334401 \\
\hline \multicolumn{4}{|c|}{$\mathrm{OHC}$} \\
\hline $\mathrm{H}$ & 0.000000 & 0.000000 & 0.000000 \\
\hline $\mathrm{C}$ & 0.000000 & 0.000000 & 1.082093 \\
\hline $\mathrm{H}$ & 0.960062 & 0.000000 & 1.590441 \\
\hline $\mathrm{C}$ & -1.136000 & 0.000102 & 1.798468 \\
\hline $\mathrm{H}$ & -1.050894 & 0.000245 & 2.882994 \\
\hline $\mathrm{C}$ & -2.499144 & -0.000002 & 1.271162 \\
\hline $\mathrm{C}$ & -3.621461 & -0.000999 & 2.054945 \\
\hline $\mathrm{H}$ & -4.584512 & -0.000951 & 1.543744 \\
\hline $\mathrm{C}$ & -3.688617 & -0.002081 & 3.492323 \\
\hline $\mathrm{H}$ & -2.767615 & -0.002455 & 4.067717 \\
\hline $\mathrm{C}$ & -4.867266 & -0.002716 & 4.162759 \\
\hline $\mathrm{H}$ & -5.813879 & -0.002456 & 3.626319 \\
\hline $\mathrm{C}$ & -4.947685 & -0.003786 & 5.582935 \\
\hline $\mathrm{C}$ & -2.784449 & 0.001078 & -0.191829 \\
\hline $\mathrm{O}$ & -1.966217 & 0.002333 & -1.089856 \\
\hline $\mathrm{H}$ & -3.871631 & 0.000704 & -0.428714 \\
\hline $\mathrm{N}$ & -5.032321 & -0.004640 & 6.744897 \\
\hline \multicolumn{4}{|l|}{$\mathrm{TS}$} \\
\hline $\mathrm{H}$ & 0.000000 & 0.000000 & 0.000000 \\
\hline $\mathrm{C}$ & 0.000000 & 0.000000 & 1.079619 \\
\hline $\mathrm{H}$ & 0.992125 & 0.000000 & 1.526310 \\
\hline $\mathrm{C}$ & -1.045722 & 0.655574 & 1.725597 \\
\hline $\mathrm{H}$ & -0.820949 & 1.273508 & 2.597300 \\
\hline $\mathrm{C}$ & -2.399296 & 0.449630 & 1.429526 \\
\hline $\mathrm{C}$ & -2.924055 & -0.794897 & 1.008335 \\
\hline $\mathrm{H}$ & -4.008349 & -0.863511 & 1.059294 \\
\hline $\mathrm{C}$ & -2.196867 & -1.975236 & 0.954499 \\
\hline $\mathrm{H}$ & -2.713542 & -2.870933 & 1.300942 \\
\hline $\mathrm{C}$ & -0.813546 & -2.100864 & 0.670846 \\
\hline $\mathrm{H}$ & -0.454117 & -1.797974 & -0.300302 \\
\hline $\mathrm{C}$ & -0.097176 & -3.223617 & 1.203622 \\
\hline $\mathrm{C}$ & -3.376688 & 1.459509 & 1.918568 \\
\hline $\mathrm{H}$ & -2.922543 & 2.304057 & 2.481412 \\
\hline $\mathrm{O}$ & -4.575147 & 1.416172 & 1.721647 \\
\hline $\mathrm{N}$ & 0.510861 & -4.106414 & 1.658819 \\
\hline
\end{tabular}




\begin{tabular}{|c|c|c|c|}
\hline $\mathrm{H}$ & 0.000000 & 0.000000 & 0.000000 \\
\hline $\mathrm{C}$ & 0.000000 & 0.000000 & 1.086389 \\
\hline $\mathrm{H}$ & 0.966199 & 0.000000 & 1.580265 \\
\hline $\mathrm{C}$ & -1.136138 & 0.000000 & 1.799059 \\
\hline $\mathrm{H}$ & -1.079833 & 0.000000 & 2.883555 \\
\hline $\mathrm{C}$ & -2.479618 & 0.000000 & 1.218685 \\
\hline $\mathrm{C}$ & -3.655347 & 0.000000 & 1.918504 \\
\hline $\mathrm{H}$ & -4.580087 & 0.000000 & 1.346391 \\
\hline $\mathrm{C}$ & -3.793000 & 0.000000 & 3.350713 \\
\hline $\mathrm{H}$ & -2.901943 & 0.000000 & 3.971352 \\
\hline $\mathrm{C}$ & -5.003589 & 0.000000 & 3.961006 \\
\hline $\mathrm{H}$ & -5.922109 & 0.000000 & 3.378050 \\
\hline $\mathrm{C}$ & -5.152072 & 0.000000 & 5.375625 \\
\hline $\mathrm{C}$ & -2.573145 & 0.000000 & -0.215937 \\
\hline $\mathrm{N}$ & -2.632148 & 0.000001 & -1.377863 \\
\hline $\mathrm{N}$ & -5.289606 & 0.000000 & 6.532452 \\
\hline \multicolumn{4}{|c|}{ TS } \\
\hline $\mathrm{H}$ & 0.000000 & 0.000000 & 0.000000 \\
\hline $\mathrm{C}$ & 0.000000 & 0.000000 & 1.079638 \\
\hline $\mathrm{H}$ & 0.991617 & 0.000000 & 1.526891 \\
\hline $\mathrm{C}$ & -1.037169 & 0.668999 & 1.725939 \\
\hline $\mathrm{H}$ & -0.814958 & 1.294345 & 2.589683 \\
\hline $\mathrm{C}$ & -2.392232 & 0.453779 & 1.427804 \\
\hline $\mathrm{C}$ & -2.912425 & -0.807730 & 1.034441 \\
\hline $\mathrm{H}$ & -3.993656 & -0.904487 & 1.089488 \\
\hline $\mathrm{C}$ & -2.172792 & -1.981797 & 0.992222 \\
\hline $\mathrm{H}$ & -2.683767 & -2.875883 & 1.349890 \\
\hline $\mathrm{C}$ & -0.790764 & -2.097437 & 0.703397 \\
\hline $\mathrm{H}$ & -0.442395 & -1.806614 & -0.275446 \\
\hline $\mathrm{C}$ & -0.064068 & -3.210302 & 1.244001 \\
\hline $\mathrm{C}$ & -3.347772 & 1.430106 & 1.874986 \\
\hline $\mathrm{N}$ & -4.117866 & 2.233463 & 2.214955 \\
\hline $\mathrm{N}$ & 0.552420 & -4.084380 & 1.704066 \\
\hline $\mathrm{O}_{2} \mathrm{I}$ & CIN & & \\
\hline $\mathrm{H}$ & 0.000000 & 0.000000 & 0.000000 \\
\hline
\end{tabular}




$\begin{array}{rrrr}\mathrm{C} & 0.000000 & 0.000000 & 1.080939 \\ \mathrm{H} & 0.962102 & 0.000000 & 1.584427 \\ \mathrm{C} & -1.121821 & -0.003913 & 1.818619 \\ \mathrm{H} & -1.003372 & -0.009580 & 2.898892 \\ \mathrm{C} & -2.517029 & 0.041485 & 1.407603 \\ \mathrm{C} & -3.593118 & 0.070028 & 2.239284 \\ \mathrm{H} & -4.569344 & 0.129830 & 1.768918 \\ \mathrm{C} & -3.563773 & 0.029925 & 3.677346 \\ \mathrm{H} & -2.612440 & -0.024097 & 4.197816 \\ \mathrm{C} & -4.701440 & 0.058079 & 4.413769 \\ \mathrm{H} & -5.675870 & 0.112095 & 3.933300 \\ \mathrm{C} & -4.697600 & 0.021992 & 5.835963 \\ \mathrm{~N} & -2.864653 & 0.059492 & -0.039020 \\ \mathrm{O} & -2.037489 & -0.387795 & -0.831003 \\ \mathrm{O} & -3.964164 & 0.505494 & -0.364020 \\ \mathrm{~N} & -4.710403 & -0.006959 & 7.000362\end{array}$

TS

$\begin{array}{llll}\mathrm{H} & 0.000000 & 0.000000 & 0.000000\end{array}$

$\begin{array}{llll}\text { C } & 0.000000 & 0.000000 & 1.079314\end{array}$

$\begin{array}{llll}\mathrm{H} & 0.992346 & 0.000000 & 1.524980\end{array}$

$\begin{array}{llll}\text { C } & -1.032078 & 0.671725 & 1.733356\end{array}$

$\begin{array}{llll}\mathrm{H} & -0.814646 & 1.316334 & 2.581071\end{array}$

$\begin{array}{llll}\text { C } & -2.376293 & 0.428157 & 1.455938\end{array}$

$\begin{array}{llll}\text { C } & -2.925116 & -0.826195 & 1.131409\end{array}$

$\begin{array}{llll}\mathrm{H} & -4.001249 & -0.901427 & 1.246550\end{array}$

$\begin{array}{llll}\text { C } & -2.180001 & -1.993822 & 1.062085\end{array}$

$\begin{array}{llll}\mathrm{H} & -2.658788 & -2.891120 & 1.453145\end{array}$

$\begin{array}{llll}\text { C } & -0.815450 & -2.088721 & 0.694290\end{array}$

$\begin{array}{llll}\mathrm{H} & -0.531172 & -1.781419 & -0.300373\end{array}$

$\begin{array}{llll}\text { C } & -0.041427 & -3.194822 & 1.180023\end{array}$

$\begin{array}{llll}\mathrm{N} & -3.347200 & 1.445689 & 1.937363\end{array}$

$\begin{array}{llll}\mathrm{O} & -4.542168 & 1.252000 & 1.720772\end{array}$

$\begin{array}{llll}\text { O } & -2.890363 & 2.441365 & 2.496938\end{array}$

$\begin{array}{llll}\mathrm{N} & 0.613171 & -4.064143 & 1.593714\end{array}$

7<smiles>Cc1ccccc1[N+](=O)[O-]</smiles>

$\begin{array}{llll}\mathrm{H} & 0.000000 & 0.000000 & 0.000000\end{array}$ 


$\begin{array}{lrrr}\mathrm{C} & 0.000000 & 0.000000 & 1.085774 \\ \mathrm{H} & 0.972860 & 0.000000 & 1.567522 \\ \mathrm{C} & -1.129009 & -0.000020 & 1.812785 \\ \mathrm{H} & -1.034080 & -0.000002 & 2.895214 \\ \mathrm{C} & -2.483636 & -0.000044 & 1.263939 \\ \mathrm{C} & -3.598677 & -0.000104 & 2.051652 \\ \mathrm{H} & -4.566120 & -0.000101 & 1.552317 \\ \mathrm{C} & -3.637188 & -0.000152 & 3.490671 \\ \mathrm{H} & -2.718936 & -0.000224 & 4.069261 \\ \mathrm{C} & -4.786842 & -0.000131 & 4.191129 \\ \mathrm{H} & -5.788180 & -0.000078 & 3.782042 \\ \mathrm{~N} & -4.779956 & -0.000181 & 5.636233 \\ \mathrm{C} & -2.642853 & -0.000047 & -0.236306 \\ \mathrm{H} & -2.166182 & -0.881370 & -0.683186 \\ \mathrm{H} & -2.166669 & 0.881597 & -0.683086 \\ \mathrm{H} & -3.696911 & -0.000350 & -0.525574 \\ \mathrm{O} & -3.699327 & -0.000281 & 6.234817 \\ \mathrm{O} & -5.888042 & -0.000118 & 6.182752\end{array}$

$\begin{array}{crrr}\text { TS } & & & \\ \mathrm{H} & 0.000000 & 0.000000 & 0.000000 \\ \mathrm{C} & 0.000000 & 0.000000 & 1.079954 \\ \mathrm{H} & 0.992746 & 0.000000 & 1.525321 \\ \mathrm{C} & -1.051872 & 0.636986 & 1.730557 \\ \mathrm{H} & -0.839838 & 1.221220 & 2.626783 \\ \mathrm{C} & -2.401338 & 0.442488 & 1.403844 \\ \mathrm{C} & -2.864465 & -0.809490 & 0.938642 \\ \mathrm{H} & -3.946047 & -0.933805 & 0.933987 \\ \mathrm{C} & -2.118855 & -1.985703 & 0.891461 \\ \mathrm{H} & -2.629306 & -2.897815 & 1.197555 \\ \mathrm{C} & -0.734892 & -2.090316 & 0.671148 \\ \mathrm{H} & -0.265906 & -1.868880 & -0.271389 \\ \mathrm{~N} & -0.074645 & -3.271778 & 1.237090 \\ \mathrm{C} & -3.423556 & 1.471446 & 1.832288 \\ \mathrm{H} & -3.591626 & 2.199497 & 1.027122 \\ \mathrm{H} & -3.073892 & 2.037113 & 2.702984 \\ \mathrm{H} & -4.391303 & 1.022782 & 2.077252 \\ \mathrm{O} & -0.635119 & -3.880480 & 2.152198 \\ \mathrm{O} & 1.034119 & -3.556132 & 0.774096\end{array}$<smiles>C=C/C(N)=C\C=C\[N+](=O)[O-]</smiles> 


$\begin{array}{lrrr}\mathrm{H} & 0.000000 & 0.000000 & 0.000000 \\ \mathrm{C} & 0.000000 & 0.000000 & 1.086455 \\ \mathrm{H} & 0.971279 & 0.000000 & 1.570823 \\ \mathrm{C} & -1.128709 & 0.041019 & 1.807876 \\ \mathrm{H} & -1.060612 & 0.085037 & 2.889853 \\ \mathrm{C} & -2.485354 & 0.022049 & 1.244618 \\ \mathrm{C} & -3.596244 & 0.435171 & 1.942480 \\ \mathrm{H} & -4.563952 & 0.334204 & 1.452170 \\ \mathrm{C} & -3.585640 & 1.020327 & 3.242471 \\ \mathrm{H} & -2.645204 & 1.167180 & 3.765113 \\ \mathrm{C} & -4.694951 & 1.426394 & 3.901245 \\ \mathrm{H} & -5.711611 & 1.375192 & 3.536550 \\ \mathrm{~N} & -4.604765 & 1.997371 & 5.215222 \\ \mathrm{~N} & -2.592791 & -0.396856 & -0.065072 \\ \mathrm{H} & -1.938121 & -1.103358 & -0.373833 \\ \mathrm{H} & -3.528170 & -0.522289 & -0.429876 \\ \mathrm{O} & -3.495310 & 2.113743 & 5.753883 \\ \mathrm{O} & -5.673048 & 2.344076 & 5.736557\end{array}$

TS

$\begin{array}{llll}\mathrm{H} & 0.000000 & 0.000000 & 0.000000\end{array}$

$\begin{array}{llll}\text { C } & 0.000000 & 0.000000 & 1.080214\end{array}$

$\begin{array}{llll}\mathrm{H} & 0.993874 & 0.000000 & 1.522237\end{array}$

$\begin{array}{llll}\text { C } & -1.043763 & 0.631254 & 1.743424\end{array}$

$\begin{array}{llll}\mathrm{H} & -0.848332 & 1.180265 & 2.664363\end{array}$

$\begin{array}{llll}\text { C } & -2.393421 & 0.408352 & 1.406811\end{array}$

$\begin{array}{llll}\text { C } & -2.842854 & -0.873146 & 0.979473\end{array}$

$\begin{array}{llll}\mathrm{H} & -3.923973 & -1.011940 & 0.995640\end{array}$

$\begin{array}{llll}\text { C } & -2.076017 & -2.023837 & 0.939741\end{array}$

$\begin{array}{llll}\mathrm{H} & -2.572418 & -2.954594 & 1.212033\end{array}$

$\begin{array}{llll}\text { C } & -0.680282 & -2.095922 & 0.709993\end{array}$

$\begin{array}{llll}\mathrm{H} & -0.245239 & -1.893946 & -0.254220\end{array}$

$\begin{array}{llll}\mathrm{N} & -0.003021 & -3.247507 & 1.286462\end{array}$

$\begin{array}{llll}\mathrm{N} & -3.350015 & 1.350772 & 1.731742\end{array}$

$\begin{array}{llll}\mathrm{H} & -4.275352 & 1.024235 & 1.979676\end{array}$

$\begin{array}{llll}\mathrm{H} & -3.040279 & 2.149850 & 2.268783\end{array}$

$\begin{array}{llll}\text { O } & -0.544942 & -3.839395 & 2.228496\end{array}$

$\begin{array}{llll}\text { O } & 1.104801 & -3.537970 & 0.818638\end{array}$<smiles>C=CC(O)=CC=C[N+](=O)[O-]</smiles> 


\begin{tabular}{|c|c|c|c|}
\hline $\mathrm{H}$ & 0.000000 & 0.000000 & 0.000000 \\
\hline $\mathrm{C}$ & 0.000000 & 0.000000 & 1.084669 \\
\hline $\mathrm{H}$ & 0.963628 & 0.000000 & 1.584060 \\
\hline $\mathrm{C}$ & -1.139128 & 0.000000 & 1.790738 \\
\hline $\mathrm{H}$ & -1.102319 & 0.000000 & 2.874659 \\
\hline $\mathrm{C}$ & -2.466408 & 0.000000 & 1.186002 \\
\hline $\mathrm{C}$ & -3.652366 & 0.000000 & 1.868895 \\
\hline $\mathrm{H}$ & -4.571654 & 0.000000 & 1.282273 \\
\hline $\mathrm{C}$ & -3.794180 & 0.000000 & 3.291704 \\
\hline $\mathrm{H}$ & -2.913565 & 0.000000 & 3.926800 \\
\hline $\mathrm{C}$ & -4.983643 & 0.000000 & 3.929087 \\
\hline $\mathrm{H}$ & -5.960990 & 0.000000 & 3.466305 \\
\hline $\mathrm{N}$ & -5.050232 & 0.000000 & 5.367227 \\
\hline $\mathrm{O}$ & -2.437630 & 0.000000 & -0.170851 \\
\hline $\mathrm{H}$ & -3.345176 & 0.000000 & -0.515958 \\
\hline $\mathrm{O}$ & -4.001001 & 0.000056 & 6.022706 \\
\hline $\mathrm{O}$ & -6.184546 & 0.000058 & 5.859899 \\
\hline \multicolumn{4}{|l|}{ TS } \\
\hline $\mathrm{H}$ & 0.000000 & 0.000000 & 0.000000 \\
\hline $\mathrm{C}$ & 0.000000 & 0.000000 & 1.079377 \\
\hline $\mathrm{H}$ & 0.993951 & 0.000000 & 1.521372 \\
\hline $\mathrm{C}$ & -1.041998 & 0.637312 & 1.744528 \\
\hline $\mathrm{H}$ & -0.841461 & 1.201114 & 2.656875 \\
\hline $\mathrm{C}$ & -2.388288 & 0.387684 & 1.441333 \\
\hline $\mathrm{C}$ & -2.869263 & -0.885355 & 1.059813 \\
\hline $\mathrm{H}$ & -3.947356 & -0.996879 & 1.148996 \\
\hline $\mathrm{C}$ & -2.099201 & -2.035879 & 0.997952 \\
\hline $\mathrm{H}$ & -2.568652 & -2.962324 & 1.325120 \\
\hline $\mathrm{C}$ & -0.724162 & -2.095542 & 0.688736 \\
\hline $\mathrm{H}$ & -0.342866 & -1.865976 & -0.291410 \\
\hline $\mathrm{N}$ & 0.003879 & -3.249416 & 1.208142 \\
\hline $\mathrm{O}$ & -3.366541 & 1.256225 & 1.828362 \\
\hline $\mathrm{H}$ & -2.956517 & 2.095490 & 2.090482 \\
\hline $\mathrm{O}$ & -0.476805 & -3.868492 & 2.162906 \\
\hline $\mathrm{O}$ & 1.087862 & -3.507072 & 0.673733 \\
\hline & $\mathrm{NO}_{2}$ & & \\
\hline $\mathrm{H}$ & 0.000000 & 0.000000 & 0.000000 \\
\hline $\mathrm{C}$ & 0.000000 & 0.000000 & 1.084787 \\
\hline
\end{tabular}




$\begin{array}{cccc}\mathrm{H} & 0.963543 & 0.000000 & 1.583366 \\ \mathrm{C} & -1.141835 & 0.000000 & 1.788458 \\ \mathrm{H} & -1.116905 & 0.000000 & 2.873672 \\ \mathrm{C} & -2.453111 & 0.000000 & 1.171698 \\ \mathrm{C} & -3.675492 & 0.000000 & 1.759360 \\ \mathrm{H} & -4.535987 & 0.000000 & 1.095900 \\ \mathrm{C} & -3.900419 & 0.000000 & 3.176609 \\ \mathrm{H} & -3.058701 & 0.000000 & 3.862804 \\ \mathrm{C} & -5.127033 & 0.000000 & 3.731503 \\ \mathrm{H} & -6.071969 & 0.000000 & 3.205537 \\ \mathrm{~N} & -5.288556 & 0.000000 & 5.166988 \\ \mathrm{~F} & -2.429460 & 0.000000 & -0.176525 \\ \mathrm{O} & -4.283965 & -0.000151 & 5.886496 \\ \mathrm{O} & -6.452331 & -0.000136 & 5.580566 \\ \mathrm{TS} & & & \\ \mathrm{H} & 0.000000 & 0.000000 & 0.000000 \\ \mathrm{C} & 0.000000 & 0.000000 & 1.078776 \\ \mathrm{H} & 0.993992 & 0.000000 & 1.520893 \\ \mathrm{C} & -1.039216 & 0.647736 & 1.743654 \\ \mathrm{H} & -0.843877 & 1.260934 & 2.621882 \\ \mathrm{C} & -2.374716 & 0.372890 & 1.468237 \\ \mathrm{C} & -2.894009 & -0.882532 & 1.109391 \\ \mathrm{H} & -3.968733 & -0.980792 & 1.238778 \\ \mathrm{C} & -2.130319 & -2.037860 & 1.014329 \\ \mathrm{H} & -2.583060 & -2.962407 & 1.367643 \\ \mathrm{C} & -0.769969 & -2.087556 & 0.659758 \\ \mathrm{H} & -0.420054 & -1.834029 & -0.325754 \\ \mathrm{~N} & -0.015745 & -3.249939 & 1.134917 \\ \mathrm{~F} & -3.290570 & 1.253990 & 1.926217 \\ \mathrm{O} & -0.465710 & -3.894312 & 2.085673 \\ \mathrm{O} & 1.053030 & -3.482031 & 0.563274 \\ & & & \\ & & \end{array}$

$\begin{array}{lrll} & \mathrm{OHC} \\ & & & \\ & & & \\ & & & \\ \mathrm{NO} & & & \\ \mathrm{H} & 0.000000 & 0.000000 & 0.000000 \\ \mathrm{C} & 0.000000 & 0.000000 & 1.082089 \\ \mathrm{H} & 0.960076 & 0.000000 & 1.590252 \\ \mathrm{C} & -1.135744 & 0.000325 & 1.799494 \\ \mathrm{H} & -1.051870 & 0.000536 & 2.883837 \\ \mathrm{C} & -2.497254 & 0.000449 & 1.270961 \\ \mathrm{C} & -3.620596 & 0.000389 & 2.052926\end{array}$




$\begin{array}{lrrr}\mathrm{H} & -4.583396 & 0.000342 & 1.542547 \\ \mathrm{C} & -3.673838 & 0.000399 & 3.491190 \\ \mathrm{H} & -2.761591 & 0.000379 & 4.079116 \\ \mathrm{C} & -4.837171 & 0.000434 & 4.165958 \\ \mathrm{H} & -5.830220 & 0.000428 & 3.736309 \\ \mathrm{~N} & -4.861171 & 0.000444 & 5.616380 \\ \mathrm{C} & -2.783088 & 0.000656 & -0.194748 \\ \mathrm{O} & -1.963700 & 0.001101 & -1.090584 \\ \mathrm{H} & -3.869781 & 0.000392 & -0.431983 \\ \mathrm{O} & -3.792820 & 0.000401 & 6.232223 \\ \mathrm{O} & -5.981109 & 0.000497 & 6.133864\end{array}$

TS

$\begin{array}{lrrr}\mathrm{H} & 0.000000 & 0.000000 & 0.000000 \\ \mathrm{C} & 0.000000 & 0.000000 & 1.079818 \\ \mathrm{H} & 0.988823 & 0.000000 & 1.532599 \\ \mathrm{C} & -1.066563 & 0.612236 & 1.724762 \\ \mathrm{H} & -0.879398 & 1.193458 & 2.629367 \\ \mathrm{C} & -2.406393 & 0.398536 & 1.366706 \\ \mathrm{C} & -2.890559 & -0.830043 & 0.870388 \\ \mathrm{H} & -3.974676 & -0.910758 & 0.844268 \\ \mathrm{C} & -2.146385 & -2.004703 & 0.820511 \\ \mathrm{H} & -2.658342 & -2.920093 & 1.114363 \\ \mathrm{C} & -0.762118 & -2.106667 & 0.615303 \\ \mathrm{H} & -0.275454 & -1.856051 & -0.310565 \\ \mathrm{~N} & -0.096513 & -3.291861 & 1.176641 \\ \mathrm{C} & -3.421219 & 1.381320 & 1.841092 \\ \mathrm{O} & -4.605922 & 1.330647 & 1.578423 \\ \mathrm{H} & -3.008022 & 2.209457 & 2.456558 \\ \mathrm{O} & -0.672302 & -3.925786 & 2.062114 \\ \mathrm{O} & 1.026407 & -3.545091 & 0.734174\end{array}$

$\begin{array}{lrrr} & \\ & & & \\ & & & \\ & & & \\ & & & \\ \mathrm{NO} & & & \\ \mathrm{H} & 0.000000 & 0.000000 & 0.000000 \\ \mathrm{C} & 0.000000 & 0.000000 & 1.086412 \\ \mathrm{H} & 0.966154 & 0.000000 & 1.580329 \\ \mathrm{C} & -1.135844 & 0.000000 & 1.799948 \\ \mathrm{H} & -1.081292 & 0.000000 & 2.884318 \\ \mathrm{C} & -2.477384 & 0.000000 & 1.216769 \\ \mathrm{C} & -3.654662 & 0.000000 & 1.913869\end{array}$




$\begin{array}{lrrr}\mathrm{H} & -4.578856 & 0.000000 & 1.341894 \\ \mathrm{C} & -3.778124 & 0.000000 & 3.347239 \\ \mathrm{H} & -2.896695 & 0.000000 & 3.980237 \\ \mathrm{C} & -4.972442 & 0.000000 & 3.965336 \\ \mathrm{H} & -5.944292 & 0.000000 & 3.489884 \\ \mathrm{~N} & -5.060916 & 0.000000 & 5.413112 \\ \mathrm{C} & -2.568517 & 0.000000 & -0.218546 \\ \mathrm{~N} & -2.627974 & 0.000000 & -1.380219 \\ \mathrm{O} & -4.020460 & 0.000056 & 6.075649 \\ \mathrm{O} & -6.202180 & 0.000058 & 5.880574\end{array}$

$\begin{array}{crrr}\text { TS } & & & \\ \mathrm{H} & 0.000000 & 0.000000 & 0.000000 \\ \mathrm{C} & 0.000000 & 0.000000 & 1.079863 \\ \mathrm{H} & 0.988178 & 0.000000 & 1.533434 \\ \mathrm{C} & -1.059083 & 0.625193 & 1.724398 \\ \mathrm{H} & -0.877371 & 1.212193 & 2.623237 \\ \mathrm{C} & -2.399230 & 0.404095 & 1.357791 \\ \mathrm{C} & -2.877938 & -0.841147 & 0.882510 \\ \mathrm{H} & -3.958749 & -0.949574 & 0.852137 \\ \mathrm{C} & -2.122522 & -2.009877 & 0.846625 \\ \mathrm{H} & -2.631840 & -2.924927 & 1.145062 \\ \mathrm{C} & -0.737275 & -2.102799 & 0.648361 \\ \mathrm{H} & -0.250650 & -1.863421 & -0.280540 \\ \mathrm{~N} & -0.068281 & -3.279335 & 1.226649 \\ \mathrm{C} & -3.388723 & 1.353053 & 1.789360 \\ \mathrm{~N} & -4.183672 & 2.136459 & 2.117911 \\ \mathrm{O} & -0.651809 & -3.913090 & 2.106698 \\ \mathrm{O} & 1.062623 & -3.522913 & 0.801163\end{array}$

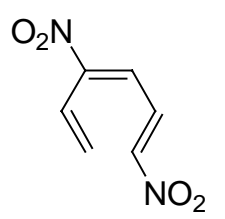

$\begin{array}{lrrr}\mathrm{H} & 0.000000 & 0.000000 & 0.000000 \\ \mathrm{C} & 0.000000 & 0.000000 & 1.081424 \\ \mathrm{H} & 0.962519 & 0.000000 & 1.583783 \\ \mathrm{C} & -1.122447 & -0.005280 & 1.818335 \\ \mathrm{H} & -1.011019 & -0.011591 & 2.899095 \\ \mathrm{C} & -2.511773 & 0.043776 & 1.392117 \\ \mathrm{C} & -3.601175 & 0.074041 & 2.204727 \\ \mathrm{H} & -4.570543 & 0.143513 & 1.722329 \\ \mathrm{C} & -3.577145 & 0.022939 & 3.643157\end{array}$




$\begin{array}{rrrr}\mathrm{H} & -2.640508 & -0.042141 & 4.187251 \\ \mathrm{C} & -4.706473 & 0.053440 & 4.371126 \\ \mathrm{H} & -5.717374 & 0.116691 & 3.990730 \\ \mathrm{~N} & -4.657931 & 0.002660 & 5.821967 \\ \mathrm{~N} & -2.836622 & 0.068207 & -0.061713 \\ \mathrm{O} & -2.037187 & -0.458047 & -0.832340 \\ \mathrm{O} & -3.892245 & 0.596234 & -0.404644 \\ \mathrm{O} & -3.560747 & -0.069881 & 6.379397 \\ \mathrm{O} & -5.748669 & 0.036977 & 6.395221\end{array}$

TS

$\begin{array}{llll}\mathrm{H} & 0.000000 & 0.000000 & 0.000000\end{array}$

$\begin{array}{llll}\mathrm{C} & 0.000000 & 0.000000 & 1.079567\end{array}$

$\begin{array}{llll}\mathrm{H} & 0.988501 & 0.000000 & 1.532389\end{array}$

$\begin{array}{llll}\text { C } & -1.056005 & 0.627085 & 1.730175\end{array}$

$\begin{array}{llll}\mathrm{H} & -0.881964 & 1.230457 & 2.616771\end{array}$

$\begin{array}{llll}\text { C } & -2.382832 & 0.376883 & 1.374917\end{array}$

$\begin{array}{llll}\text { C } & -2.890532 & -0.861095 & 0.953650\end{array}$

$\begin{array}{llll}\mathrm{H} & -3.971660 & -0.948835 & 0.969439\end{array}$

$\begin{array}{llll}\text { C } & -2.130132 & -2.025151 & 0.900687\end{array}$

$\begin{array}{llll}\mathrm{H} & -2.617484 & -2.941247 & 1.229750\end{array}$

$\begin{array}{llll}\text { C } & -0.754208 & -2.102240 & 0.644657\end{array}$

$\begin{array}{llll}\mathrm{H} & -0.310702 & -1.852404 & -0.302969\end{array}$

$\begin{array}{llll}\mathrm{N} & -0.044413 & -3.270740 & 1.192950\end{array}$

$\begin{array}{llll}\mathrm{N} & -3.396141 & 1.362329 & 1.840370\end{array}$

$\begin{array}{llll}\text { O } & -4.569629 & 1.174398 & 1.527300\end{array}$

$\begin{array}{llll}\mathrm{O} & -2.987070 & 2.325427 & 2.485722\end{array}$

$\begin{array}{llll}\text { O } & -0.584292 & -3.916167 & 2.091375\end{array}$

$\begin{array}{llll}\text { O } & 1.072578 & -3.493743 & 0.723144\end{array}$

\section{1, 5-substituted hexatriene}

1

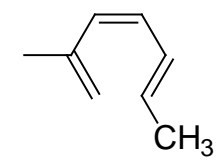

$\begin{array}{lrrr}\mathrm{H} & 0.000000 & 0.000000 & 0.000000 \\ \mathrm{C} & 0.000000 & 0.000000 & 1.091590 \\ \mathrm{C} & 1.182998 & 0.000000 & 1.735029 \\ \mathrm{H} & 1.176253 & 0.000134 & 2.820646 \\ \mathrm{C} & 2.455852 & -0.000598 & 1.034722 \\ \mathrm{H} & 2.355144 & -0.000430 & -0.051502 \\ \mathrm{C} & 3.731345 & -0.000786 & 1.497149\end{array}$




\begin{tabular}{|c|c|c|c|}
\hline $\mathrm{H}$ & 4.501495 & -0.000616 & 0.726435 \\
\hline $\mathrm{C}$ & 4.287413 & -0.000972 & 2.852646 \\
\hline $\mathrm{C}$ & 5.627987 & -0.000518 & 2.995652 \\
\hline $\mathrm{H}$ & 6.293692 & 0.000024 & 2.136378 \\
\hline $\mathrm{H}$ & 6.098415 & -0.000692 & 3.974790 \\
\hline $\mathrm{C}$ & 3.402113 & -0.001788 & 4.077889 \\
\hline $\mathrm{C}$ & -1.344202 & -0.000150 & 1.753865 \\
\hline $\mathrm{H}$ & -1.934250 & -0.879341 & 1.459596 \\
\hline $\mathrm{H}$ & -1.933045 & 0.880267 & 1.460527 \\
\hline $\mathrm{H}$ & -1.257698 & -0.000439 & 2.845689 \\
\hline $\mathrm{H}$ & 2.752146 & 0.881070 & 4.104878 \\
\hline $\mathrm{H}$ & 2.753251 & -0.885461 & 4.104520 \\
\hline $\mathrm{H}$ & 4.005559 & -0.001576 & 4.990461 \\
\hline \multicolumn{4}{|l|}{ TS } \\
\hline $\mathrm{H}$ & 0.000000 & 0.000000 & 0.000000 \\
\hline $\mathrm{H}$ & 0.000000 & 0.000000 & 1.813603 \\
\hline $\mathrm{C}$ & 0.596147 & 0.000000 & 0.914453 \\
\hline $\mathrm{C}$ & 1.792371 & 0.732040 & 0.933905 \\
\hline $\mathrm{C}$ & 2.753895 & 0.518361 & 1.930652 \\
\hline $\mathrm{H}$ & 3.543416 & 1.260744 & 2.043819 \\
\hline $\mathrm{C}$ & 3.013012 & -0.733719 & 2.515445 \\
\hline $\mathrm{H}$ & 3.966068 & -0.814723 & 3.037809 \\
\hline $\mathrm{C}$ & 2.386280 & -1.933912 & 2.166579 \\
\hline $\mathrm{H}$ & 3.053131 & -2.797382 & 2.084661 \\
\hline $\mathrm{C}$ & 1.071176 & -2.100934 & 1.706784 \\
\hline $\mathrm{H}$ & 0.275246 & -1.765207 & 2.353856 \\
\hline $\mathrm{C}$ & 0.701341 & -3.312982 & 0.881186 \\
\hline $\mathrm{C}$ & 2.135674 & 1.638269 & -0.234578 \\
\hline $\mathrm{H}$ & 2.149085 & 1.061605 & -1.169782 \\
\hline $\mathrm{H}$ & 1.399880 & 2.441322 & -0.366640 \\
\hline $\mathrm{H}$ & 3.123033 & 2.096126 & -0.119094 \\
\hline $\mathrm{H}$ & 1.530303 & -3.638690 & 0.244316 \\
\hline $\mathrm{H}$ & 0.411502 & -4.159204 & 1.521989 \\
\hline $\mathrm{H}$ & -0.155442 & -3.098829 & 0.232908 \\
\hline $\mathrm{H}$ & 0.000000 & 0.000000 & 0.000000 \\
\hline $\mathrm{C}$ & 0.000000 & 0.000000 & 1.086669 \\
\hline $\mathrm{H}$ & 0.964411 & 0.000000 & 1.581160 \\
\hline $\mathrm{C}$ & -1.145941 & -0.059898 & 1.800016 \\
\hline
\end{tabular}




$\begin{array}{lrrl}\mathrm{C} & -1.100064 & -0.032361 & 3.266634 \\ \mathrm{H} & -0.150668 & 0.325877 & 3.657756 \\ \mathrm{C} & -2.040024 & -0.378185 & 4.179251 \\ \mathrm{H} & -1.766462 & -0.225702 & 5.224054 \\ \mathrm{C} & -3.354657 & -0.967631 & 3.981421 \\ \mathrm{H} & -3.636812 & -1.253415 & 2.971138 \\ \mathrm{C} & -4.204849 & -1.210802 & 4.996602 \\ \mathrm{H} & -3.904710 & -0.933905 & 6.009187 \\ \mathrm{C} & -5.555427 & -1.845092 & 4.856313 \\ \mathrm{~N} & -2.408082 & -0.184518 & 1.185074 \\ \mathrm{H} & -3.113708 & 0.412418 & 1.605263 \\ \mathrm{H} & -2.371499 & -0.025485 & 0.183155 \\ \mathrm{H} & -5.778145 & -2.093032 & 3.813170 \\ \mathrm{H} & -6.349336 & -1.181028 & 5.226336 \\ \mathrm{H} & -5.624822 & -2.769151 & 5.447283\end{array}$

TS

$\begin{array}{llll}\mathrm{H} & 0.000000 & 0.000000 & 0.000000\end{array}$

$\begin{array}{llll}\text { C } & 0.000000 & 0.000000 & 1.081131\end{array}$

$\begin{array}{llll}\mathrm{C} & 1.263642 & 0.000000 & 1.686404\end{array}$

$\begin{array}{llll}\mathrm{H} & 1.432761 & 0.660212 & 2.539901\end{array}$

$\begin{array}{llll}\text { C } & 2.264972 & -0.930923 & 1.398666\end{array}$

$\begin{array}{llll}\mathrm{H} & 3.277409 & -0.704179 & 1.733751\end{array}$

$\begin{array}{llll}\text { C } & 2.045791 & -2.248817 & 0.975839\end{array}$

$\begin{array}{llll}\mathrm{H} & 2.902251 & -2.920756 & 1.015070\end{array}$

$\begin{array}{llll}\text { C } & 0.772281 & -2.859286 & 0.950650\end{array}$

$\begin{array}{llll}\text { C } & -0.424355 & -2.187543 & 0.616505\end{array}$

$\begin{array}{llll}\mathrm{H} & -0.517066 & -1.781266 & -0.379378\end{array}$

$\begin{array}{llll}\mathrm{H} & -1.353876 & -2.642298 & 0.972201\end{array}$

$\begin{array}{llll}\mathrm{N} & 0.711729 & -4.179955 & 1.411387\end{array}$

$\begin{array}{llll}\text { C } & -1.158819 & 0.752410 & 1.692395\end{array}$

$\begin{array}{llll}\mathrm{H} & -2.117967 & 0.349442 & 1.350313\end{array}$

$\begin{array}{llll}\mathrm{H} & -1.136100 & 1.813999 & 1.404660\end{array}$

$\begin{array}{llll}\mathrm{H} & -1.141741 & 0.699916 & 2.786077\end{array}$

$\begin{array}{llll}\mathrm{H} & -0.210805 & -4.593534 & 1.434542\end{array}$

$\begin{array}{llll}\mathrm{H} & 1.258092 & -4.401967 & 2.235271\end{array}$<smiles>C=C(O)/C=C\C=C/C</smiles>
$\begin{array}{llll}\mathrm{H} & 0.000000 & 0.000000 & 0.000000\end{array}$
$\begin{array}{llll}\text { C } & 0.000000 & 0.000000 & 1.086991\end{array}$
$\begin{array}{llll}\mathrm{H} & 0.963204 & 0.000000 & 1.581819\end{array}$ 


$\begin{array}{lrrr}\mathrm{C} & -1.143544 & -0.000080 & 1.801252 \\ \mathrm{C} & -1.146497 & -0.000256 & 3.259573 \\ \mathrm{H} & -0.145480 & 0.000772 & 3.683469 \\ \mathrm{C} & -2.183869 & -0.001204 & 4.130648 \\ \mathrm{H} & -1.912353 & -0.000765 & 5.187061 \\ \mathrm{C} & -3.613000 & -0.002436 & 3.874948 \\ \mathrm{H} & -3.945717 & -0.002789 & 2.842002 \\ \mathrm{C} & -4.521384 & -0.003736 & 4.868745 \\ \mathrm{H} & -4.167822 & -0.003815 & 5.901882 \\ \mathrm{C} & -6.007755 & -0.005478 & 4.678019 \\ \mathrm{O} & -2.382491 & -0.000023 & 1.210463 \\ \mathrm{H} & -2.261759 & -0.000067 & 0.247741 \\ \mathrm{H} & -6.278613 & -0.005258 & 3.617103 \\ \mathrm{H} & -6.468515 & -0.886223 & 5.147076 \\ \mathrm{H} & -6.471127 & 0.873364 & 5.148096\end{array}$

TS

$\begin{array}{llll}\mathrm{H} & 0.000000 & 0.000000 & 0.000000\end{array}$

$\begin{array}{llll}\mathrm{H} & 0.000000 & 0.000000 & 1.815861\end{array}$

$\begin{array}{llll}\text { C } & 0.592917 & 0.000000 & 0.915318\end{array}$

$\begin{array}{llll}\text { C } & 1.752680 & 0.789388 & 0.886225\end{array}$

$\begin{array}{llll}\text { C } & 2.863531 & 0.651234 & 1.735511\end{array}$

$\begin{array}{llll}\mathrm{H} & 3.581324 & 1.472246 & 1.747839\end{array}$

$\begin{array}{llll}\text { C } & 3.322735 & -0.586427 & 2.213470\end{array}$

$\begin{array}{llll}\mathrm{H} & 4.357748 & -0.605806 & 2.554003\end{array}$

$\begin{array}{llll}\text { C } & 2.685799 & -1.810559 & 2.008942\end{array}$

$\begin{array}{llll}\mathrm{H} & 3.331764 & -2.668647 & 1.810440\end{array}$

$\begin{array}{llll}\text { C } & 1.302019 & -1.969609 & 1.842769\end{array}$

$\begin{array}{llll}\mathrm{H} & 0.684816 & -1.555665 & 2.627167\end{array}$

$\begin{array}{llll}\text { C } & 0.733191 & -3.217678 & 1.210587\end{array}$

$\begin{array}{llll}\mathrm{O} & 1.848857 & 1.660828 & -0.178817\end{array}$

$\begin{array}{llll}\mathrm{H} & 2.785848 & 1.856024 & -0.339416\end{array}$

$\begin{array}{llll}\mathrm{H} & -0.266570 & -3.033973 & 0.803773\end{array}$

$\begin{array}{llll}\mathrm{H} & 0.635022 & -4.024349 & 1.952119\end{array}$

$\begin{array}{llll}\mathrm{H} & 1.367490 & -3.582922 & 0.396399\end{array}$<smiles>C=C(F)/C=C\C=C/C</smiles>

$\begin{array}{cccc}\mathrm{H} & 0.000000 & 0.000000 & 0.000000 \\ \mathrm{C} & 0.000000 & 0.000000 & 1.083422 \\ \mathrm{H} & 0.950624 & 0.000000 & 1.602688 \\ \mathrm{C} & -1.148906 & 0.000000 & 1.771382\end{array}$




$\begin{array}{lrrr}\mathrm{C} & -1.279150 & 0.000020 & 3.215370 \\ \mathrm{H} & -0.320494 & 0.000124 & 3.728364 \\ \mathrm{C} & -2.399855 & 0.000095 & 3.977315 \\ \mathrm{H} & -2.239816 & 0.000266 & 5.055752 \\ \mathrm{C} & -3.793110 & 0.000117 & 3.570716 \\ \mathrm{H} & -4.019488 & 0.000002 & 2.509520 \\ \mathrm{C} & -4.801995 & 0.000096 & 4.462455 \\ \mathrm{H} & -4.559646 & 0.000177 & 5.527007 \\ \mathrm{C} & -6.259041 & -0.000097 & 4.115257 \\ \mathrm{~F} & -2.313162 & 0.000086 & 1.068753 \\ \mathrm{H} & -6.416330 & 0.000506 & 3.031784 \\ \mathrm{H} & -6.766771 & -0.880346 & 4.533752 \\ \mathrm{H} & -6.767295 & 0.879254 & 4.534935\end{array}$

TS

$\begin{array}{llll}\mathrm{H} & 0.000000 & 0.000000 & 0.000000\end{array}$

$\begin{array}{llll}\mathrm{H} & 0.000000 & 0.000000 & 1.812631\end{array}$

$\begin{array}{llll}\text { C } & 0.595008 & 0.000000 & 0.914204\end{array}$

$\begin{array}{llll}\text { C } & 1.758222 & 0.774647 & 0.871313\end{array}$

$\begin{array}{llll}\text { C } & 2.918176 & 0.649005 & 1.631749\end{array}$

$\begin{array}{llll}\mathrm{H} & 3.639807 & 1.457847 & 1.548509\end{array}$

$\begin{array}{llll}\text { C } & 3.381738 & -0.581331 & 2.128916\end{array}$

$\begin{array}{llll}\mathrm{H} & 4.433578 & -0.609217 & 2.409762\end{array}$

$\begin{array}{llll}\text { C } & 2.714658 & -1.795597 & 1.986762\end{array}$

$\begin{array}{llll}\mathrm{H} & 3.335030 & -2.671851 & 1.787137\end{array}$

$\begin{array}{llll}\text { C } & 1.320739 & -1.933096 & 1.891425\end{array}$

$\begin{array}{llll}\mathrm{H} & 0.756161 & -1.481418 & 2.694893\end{array}$

$\begin{array}{llll}\text { C } & 0.699363 & -3.191313 & 1.334291\end{array}$

$\begin{array}{llll}\text { F } & 1.863069 & 1.641716 & -0.180875\end{array}$

$\begin{array}{llll}\mathrm{H} & -0.323151 & -3.008228 & 0.988568\end{array}$

$\begin{array}{llll}\mathrm{H} & 0.643088 & -3.974712 & 2.104342\end{array}$

$\begin{array}{llll}\mathrm{H} & 1.275845 & -3.586660 & 0.491872\end{array}$<smiles>C=C(C=O)/C=C\C=C/C</smiles>

$\begin{array}{lrrr}\mathrm{H} & 0.000000 & 0.000000 & 0.000000 \\ \mathrm{C} & 0.000000 & 0.000000 & 1.091445 \\ \mathrm{C} & 1.182669 & 0.000000 & 1.733903 \\ \mathrm{H} & 1.187551 & -0.000533 & 2.822371 \\ \mathrm{C} & 2.461327 & -0.042428 & 1.047201 \\ \mathrm{H} & 2.400265 & -0.200655 & -0.029814 \\ \mathrm{C} & 3.704179 & 0.036267 & 1.575815\end{array}$




$\begin{array}{lrrr}\mathrm{H} & 4.547640 & -0.134195 & 0.911486 \\ \mathrm{C} & 4.094081 & 0.266582 & 2.970012 \\ \mathrm{C} & 3.444910 & 1.005106 & 3.894114 \\ \mathrm{H} & 2.533740 & 1.551264 & 3.677682 \\ \mathrm{H} & 3.843806 & 1.101520 & 4.901650 \\ \mathrm{C} & 5.403280 & -0.310744 & 3.393352 \\ \mathrm{C} & -1.343440 & 0.003759 & 1.754244 \\ \mathrm{H} & -1.930500 & 0.884351 & 1.458597 \\ \mathrm{H} & -1.933040 & -0.875110 & 1.458766 \\ \mathrm{H} & -1.256670 & 0.003276 & 2.845869 \\ \mathrm{H} & 5.654177 & -0.140199 & 4.464045 \\ \mathrm{O} & 6.168044 & -0.904834 & 2.660679\end{array}$

TS

$\begin{array}{llll}\mathrm{H} & 0.000000 & 0.000000 & 0.000000\end{array}$

$\begin{array}{llll}\mathrm{H} & 0.000000 & 0.000000 & 1.825526\end{array}$

$\begin{array}{llll}\text { C } & 0.583806 & 0.000000 & 0.919364\end{array}$

$\begin{array}{llll}\text { C } & 1.794133 & 0.703885 & 0.903863\end{array}$

$\begin{array}{llll}\text { C } & 2.848658 & 0.479694 & 1.811981\end{array}$

$\begin{array}{llll}\mathrm{H} & 3.627847 & 1.238893 & 1.877797\end{array}$

$\begin{array}{llll}\text { C } & 3.169646 & -0.779519 & 2.326765\end{array}$

$\begin{array}{llll}\mathrm{H} & 4.173476 & -0.884769 & 2.735637\end{array}$

$\begin{array}{llll}\text { C } & 2.480558 & -1.959585 & 2.007913\end{array}$

$\begin{array}{llll}\mathrm{H} & 3.113143 & -2.826884 & 1.799568\end{array}$

$\begin{array}{llll}\text { C } & 1.112731 & -2.089013 & 1.753629\end{array}$

$\begin{array}{llll}\mathrm{H} & 0.444778 & -1.683342 & 2.498746\end{array}$

$\begin{array}{llll}\text { C } & 0.566948 & -3.290695 & 1.020137\end{array}$

$\begin{array}{llll}\text { C } & 2.098762 & 1.579335 & -0.252339\end{array}$

$\begin{array}{llll}\mathrm{O} & 1.426750 & 1.665194 & -1.264898\end{array}$

$\begin{array}{llll}\mathrm{H} & 3.025457 & 2.185409 & -0.135237\end{array}$

$\begin{array}{llll}\mathrm{H} & -0.360196 & -3.041198 & 0.493513\end{array}$

$\mathrm{H} \quad 0.327905 \quad-4.105547 \quad 1.718914$

$\begin{array}{llll}\mathrm{H} & 1.279348 & -3.672570 & 0.282072\end{array}$<smiles>C=C(C#N)/C=C\C=C/C</smiles>

$\begin{array}{lrll}\mathrm{H} & 0.000000 & 0.000000 & 0.000000 \\ \mathrm{C} & 0.000000 & 0.000000 & 1.083966 \\ \mathrm{H} & 0.962535 & 0.000000 & 1.586718 \\ \mathrm{C} & -1.149483 & 0.000040 & 1.797398 \\ \mathrm{C} & -1.147874 & 0.000107 & 3.261187 \\ \mathrm{H} & -0.140589 & 0.001032 & 3.672622\end{array}$




$\begin{array}{lrrr}\mathrm{C} & -2.168827 & -0.000952 & 4.152827 \\ \mathrm{H} & -1.868637 & -0.000686 & 5.201026 \\ \mathrm{C} & -3.603377 & -0.002311 & 3.941502 \\ \mathrm{H} & -3.988413 & -0.002775 & 2.926447 \\ \mathrm{C} & -4.475702 & -0.003060 & 4.967896 \\ \mathrm{H} & -4.084357 & -0.002590 & 5.987119 \\ \mathrm{C} & -5.966464 & -0.004253 & 4.829209 \\ \mathrm{C} & -2.379348 & 0.000250 & 1.044533 \\ \mathrm{~N} & -3.353905 & 0.000705 & 0.409811 \\ \mathrm{H} & -6.274992 & -0.005128 & 3.779093 \\ \mathrm{H} & -6.408018 & -0.883828 & 5.317805 \\ \mathrm{H} & -6.408854 & 0.875721 & 5.316454\end{array}$

TS

$\begin{array}{llll}\mathrm{H} & 0.000000 & 0.000000 & 0.000000\end{array}$

$\begin{array}{llll}\mathrm{H} & 0.000000 & 0.000000 & 1.819751\end{array}$

$\begin{array}{llll}\text { C } & 0.587876 & 0.000000 & 0.915676\end{array}$

$\begin{array}{llll}\text { C } & 1.793781 & 0.720789 & 0.943107\end{array}$

$\begin{array}{llll}\text { C } & 2.794354 & 0.503936 & 1.913665\end{array}$

$\begin{array}{llll}\mathrm{H} & 3.565339 & 1.261649 & 2.030002\end{array}$

$\begin{array}{llll}\text { C } & 3.064586 & -0.757784 & 2.453638\end{array}$

$\begin{array}{llll}\mathrm{H} & 4.026285 & -0.855355 & 2.954737\end{array}$

$\begin{array}{llll}\text { C } & 2.423689 & -1.944313 & 2.070151\end{array}$

$\begin{array}{llll}\mathrm{H} & 3.089748 & -2.798133 & 1.918218\end{array}$

$\begin{array}{llll}\text { C } & 1.089967 & -2.100028 & 1.674707\end{array}$

$\begin{array}{llll}\mathrm{H} & 0.334144 & -1.759036 & 2.366368\end{array}$

$\begin{array}{llll}\text { C } & 0.666638 & -3.288053 & 0.843068\end{array}$

$\begin{array}{llll}\text { C } & 2.116313 & 1.587314 & -0.154197\end{array}$

$\begin{array}{llll}\mathrm{N} & 2.366274 & 2.304810 & -1.037591\end{array}$

$\mathrm{H} \quad 1.456336 \quad-3.603707 \quad 0.154276$

$\begin{array}{llll}\mathrm{H} & 0.406751 & -4.143937 & 1.482642\end{array}$

$\begin{array}{llll}\mathrm{H} & -0.223522 & -3.053291 & 0.250003\end{array}$<smiles>C=C(/C=C\C=C/C)[N+](=O)[O-]</smiles>

$\begin{array}{lrrr}\mathrm{H} & 0.000000 & 0.000000 & 0.000000 \\ \mathrm{C} & 0.000000 & 0.000000 & 1.091359 \\ \mathrm{C} & 1.182616 & 0.000000 & 1.733975 \\ \mathrm{H} & 1.191281 & 0.002693 & 2.822538 \\ \mathrm{C} & 2.458142 & -0.041447 & 1.043040 \\ \mathrm{H} & 2.397752 & -0.188360 & -0.035029 \\ \mathrm{C} & 3.702412 & 0.034325 & 1.569014\end{array}$




$\begin{array}{rrrr}\mathrm{H} & 4.550599 & -0.123627 & 0.912084 \\ \mathrm{C} & 4.037341 & 0.293452 & 2.966399 \\ \mathrm{C} & 3.444712 & 1.116943 & 3.839414 \\ \mathrm{H} & 2.601149 & 1.723999 & 3.533703 \\ \mathrm{H} & 3.811631 & 1.203005 & 4.854438 \\ \mathrm{~N} & 5.253725 & -0.431713 & 3.461181 \\ \mathrm{C} & -1.342937 & 0.002434 & 1.753854 \\ \mathrm{H} & -1.930600 & -0.876638 & 1.455792 \\ \mathrm{H} & -1.929805 & 0.882702 & 1.457540 \\ \mathrm{H} & -1.257423 & 0.000428 & 2.845361 \\ \mathrm{O} & 5.595699 & -0.274210 & 4.630220 \\ \mathrm{O} & 5.841704 & -1.147010 & 2.651725 \\ & & & \\ \mathrm{TS} & & & \\ \mathrm{H} & 0.000000 & 0.000000 & 0.000000 \\ \mathrm{H} & 0.000000 & 0.000000 & 1.827894 \\ \mathrm{C} & 0.578943 & 0.000000 & 0.918350 \\ \mathrm{C} & 1.778177 & 0.710816 & 0.936529 \\ \mathrm{C} & 2.831275 & 0.509336 & 1.838020 \\ \mathrm{H} & 3.592851 & 1.277724 & 1.908149 \\ \mathrm{C} & 3.131372 & -0.753194 & 2.359806 \\ \mathrm{H} & 4.114450 & -0.850189 & 2.817140 \\ \mathrm{C} & 2.470938 & -1.938514 & 2.007159 \\ \mathrm{H} & 3.126995 & -2.794215 & 1.826004 \\ \mathrm{C} & 1.118964 & -2.091001 & 1.679155 \\ \mathrm{H} & 0.402545 & -1.737203 & 2.405628 \\ \mathrm{C} & 0.646728 & -3.280917 & 0.878221 \\ \mathrm{~N} & 2.068737 & 1.595481 & -0.213055 \\ \mathrm{O} & 1.335215 & 1.519257 & -1.199945 \\ \mathrm{O} & 3.017584 & 2.375654 & -0.113870 \\ \mathrm{H} & -0.270352 & -3.043601 & 0.329103 \\ \mathrm{H} & 0.413809 & -4.130174 & 1.536626 \\ & 1.398794 & -3.606596 & 0.153058\end{array}$

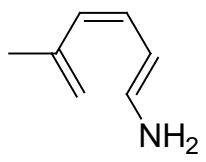

$\begin{array}{llll}\mathrm{H} & 0.000000 & 0.000000 & 0.000000 \\ \mathrm{C} & 0.000000 & 0.000000 & 1.086308 \\ \mathrm{H} & 0.972040 & 0.000000 & 1.573027\end{array}$




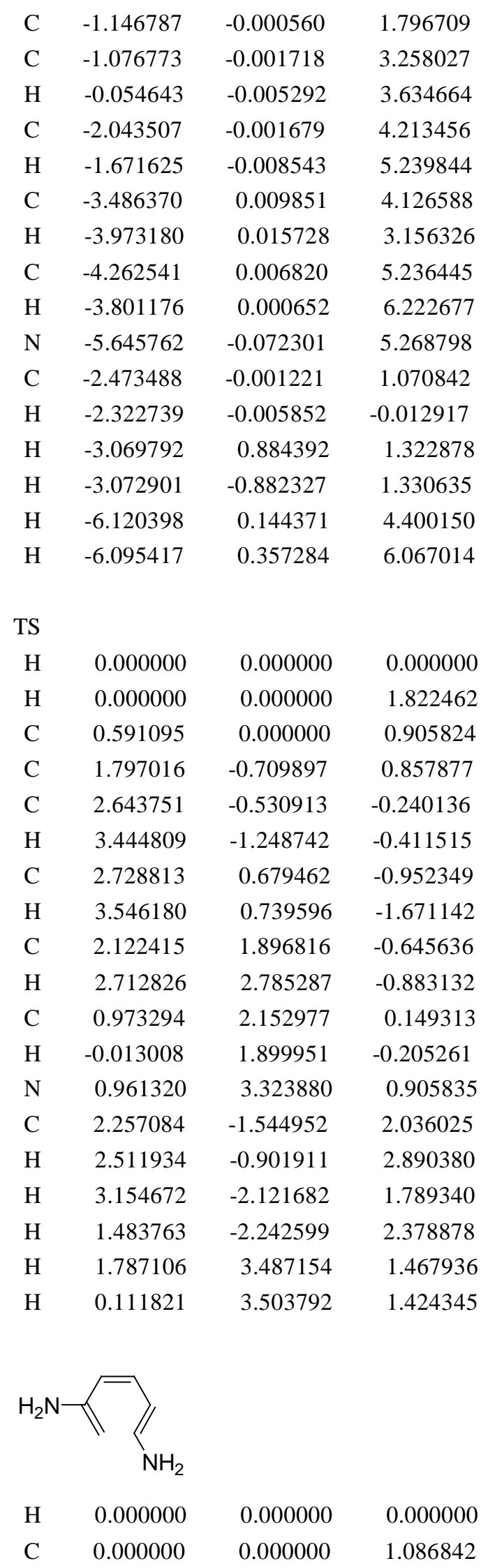




$\begin{array}{cccc}\mathrm{H} & 0.964400 & 0.000000 & 1.581626 \\ \mathrm{C} & -1.146345 & -0.057087 & 1.800361 \\ \mathrm{C} & -1.119526 & -0.015798 & 3.265201 \\ \mathrm{H} & -0.178934 & 0.348899 & 3.670443 \\ \mathrm{C} & -2.075330 & -0.365539 & 4.163838 \\ \mathrm{H} & -1.814594 & -0.202891 & 5.211167 \\ \mathrm{C} & -3.378636 & -0.957709 & 3.958243 \\ \mathrm{H} & -3.663892 & -1.254812 & 2.952220 \\ \mathrm{C} & -4.218672 & -1.206097 & 4.990782 \\ \mathrm{H} & -3.935852 & -0.926919 & 6.004723 \\ \mathrm{~N} & -5.426552 & -1.880687 & 4.917251 \\ \mathrm{~N} & -2.407709 & -0.183427 & 1.176433 \\ \mathrm{H} & -2.359914 & -0.022298 & 0.174997 \\ \mathrm{H} & -3.107429 & 0.426123 & 1.589269 \\ \mathrm{H} & -5.821607 & -1.979737 & 3.989588 \\ \mathrm{H} & -6.121673 & -1.628645 & 5.607741\end{array}$

TS

$\begin{array}{llll}\mathrm{H} & 0.000000 & 0.000000 & 0.000000\end{array}$

$\begin{array}{llll}\mathrm{H} & 0.000000 & 0.000000 & 1.827631\end{array}$

$\begin{array}{llll}\text { C } & 0.587150 & 0.000000 & 0.919621\end{array}$

$\begin{array}{llll}\text { C } & 1.771310 & 0.754917 & 0.954926\end{array}$

$\begin{array}{llll}\text { C } & 2.696480 & 0.594011 & 2.001252\end{array}$

$\begin{array}{llll}\mathrm{H} & 3.451164 & 1.365806 & 2.151393\end{array}$

$\begin{array}{llll}\text { C } & 2.924327 & -0.643385 & 2.622467\end{array}$

$\begin{array}{llll}\mathrm{H} & 3.805087 & -0.683788 & 3.264275\end{array}$

$\begin{array}{llll}\text { C } & 2.340090 & -1.877106 & 2.341703\end{array}$

$\begin{array}{llll}\mathrm{H} & 2.978062 & -2.750253 & 2.493503\end{array}$

$\begin{array}{llll}\text { C } & 1.115121 & -2.143807 & 1.676070\end{array}$

$\begin{array}{llll}\mathrm{H} & 0.172046 & -1.899629 & 2.138595\end{array}$

$\begin{array}{llll}\mathrm{N} & 2.114885 & 1.542406 & -0.151615\end{array}$

$\begin{array}{llll}\mathrm{N} & 1.028990 & -3.301121 & 0.909001\end{array}$

$\begin{array}{llll}\mathrm{H} & 1.797835 & -3.455366 & 0.268835\end{array}$

$\begin{array}{llll}\mathrm{H} & 0.133257 & -3.476519 & 0.473530\end{array}$

$\begin{array}{llll}\mathrm{H} & 1.380478 & 2.138129 & -0.513864\end{array}$

$\begin{array}{llll}\mathrm{H} & 2.999559 & 2.029292 & -0.078385\end{array}$<smiles>C=C(O)/C=C\C=C/N</smiles>
$\begin{array}{llll}\mathrm{H} & 0.000000 & 0.000000 & 0.000000\end{array}$
$\begin{array}{llll}\text { C } & 0.000000 & 0.000000 & 1.087121\end{array}$
$\begin{array}{llll}\mathrm{H} & 0.963177 & 0.000000 & 1.582191\end{array}$ 


$\begin{array}{cccc}\mathrm{C} & -1.144558 & -0.001338 & 1.801849 \\ \mathrm{C} & -1.163814 & -0.000899 & 3.257262 \\ \mathrm{H} & -0.170169 & 0.005486 & 3.697157 \\ \mathrm{C} & -2.218741 & -0.009701 & 4.112291 \\ \mathrm{H} & -1.957328 & -0.010611 & 5.172109 \\ \mathrm{C} & -3.636178 & -0.014406 & 3.846304 \\ \mathrm{H} & -3.973937 & -0.018989 & 2.815086 \\ \mathrm{C} & -4.541863 & -0.019480 & 4.853235 \\ \mathrm{H} & -4.208003 & -0.014349 & 5.889888 \\ \mathrm{~N} & -5.916371 & -0.112702 & 4.712148 \\ \mathrm{O} & -2.381370 & -0.002573 & 1.199096 \\ \mathrm{H} & -2.246918 & -0.010412 & 0.238386 \\ \mathrm{H} & -6.277896 & 0.089151 & 3.787380 \\ \mathrm{H} & -6.469547 & 0.314856 & 5.443488\end{array}$

TS

$\begin{array}{lrrr}\mathrm{H} & 0.000000 & 0.000000 & 0.000000 \\ \mathrm{H} & 0.000000 & 0.000000 & 1.829606 \\ \mathrm{C} & 0.583242 & 0.000000 & 0.910067 \\ \mathrm{C} & 1.753491 & -0.763922 & 0.881714 \\ \mathrm{C} & 2.697167 & -0.653619 & -0.144535 \\ \mathrm{H} & 3.439641 & -1.442090 & -0.265117 \\ \mathrm{C} & 2.929416 & 0.561050 & -0.813331 \\ \mathrm{H} & 3.803049 & 0.571865 & -1.465221 \\ \mathrm{C} & 2.358098 & 1.805036 & -0.566823 \\ \mathrm{H} & 2.996206 & 2.667616 & -0.768160 \\ \mathrm{C} & 1.151850 & 2.109329 & 0.126942 \\ \mathrm{H} & 0.192917 & 1.895196 & -0.317475 \\ \mathrm{~N} & 1.118648 & 3.273827 & 0.881157 \\ \mathrm{O} & 2.006693 & -1.562606 & 1.974452 \\ \mathrm{H} & 2.954931 & -1.768730 & 1.988228 \\ \mathrm{H} & 1.907955 & 3.425283 & 1.496045 \\ \mathrm{H} & 0.237280 & 3.497774 & 1.323112\end{array}$

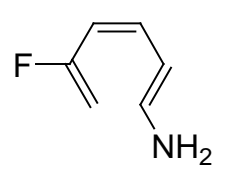

$\begin{array}{cccc}\mathrm{H} & 0.000000 & 0.000000 & 0.000000 \\ \mathrm{C} & 0.000000 & 0.000000 & 1.083346 \\ \mathrm{H} & 0.950698 & 0.000000 & 1.602609 \\ \mathrm{C} & -1.149567 & -0.001344 & 1.772493 \\ \mathrm{C} & -1.296092 & -0.001762 & 3.212004 \\ \mathrm{H} & -0.346619 & 0.001430 & 3.740911\end{array}$




$\begin{array}{llll}\mathrm{C} & -2.433620 & -0.007252 & 3.955625 \\ \mathrm{H} & -2.284256 & -0.008705 & 5.036414 \\ \mathrm{C} & -3.812941 & -0.007896 & 3.539225 \\ \mathrm{H} & -4.044254 & -0.012735 & 2.479283 \\ \mathrm{C} & -4.821777 & -0.009055 & 4.444452 \\ \mathrm{H} & -4.598858 & -0.003088 & 5.510315 \\ \mathrm{~N} & -6.169978 & -0.094419 & 4.158764 \\ \mathrm{~F} & -2.312609 & -0.002362 & 1.061315 \\ \mathrm{H} & -6.439811 & 0.099967 & 3.202091 \\ \mathrm{H} & -6.804172 & 0.307724 & 4.835867\end{array}$

$\begin{array}{cccc}\text { TS } & & & \\ \mathrm{H} & 0.000000 & 0.000000 & 0.000000 \\ \mathrm{C} & 0.000000 & 0.000000 & 1.088351 \\ \mathrm{H} & 0.980171 & 0.000000 & 1.543768 \\ \mathrm{C} & -0.972963 & -0.753858 & 1.740280 \\ \mathrm{C} & -1.273025 & -0.664285 & 3.089916 \\ \mathrm{H} & -1.884689 & -1.440331 & 3.541255 \\ \mathrm{C} & -1.079897 & 0.534467 & 3.803915 \\ \mathrm{H} & -1.477672 & 0.540367 & 4.818175 \\ \mathrm{C} & -0.702659 & 1.775351 & 3.307255 \\ \mathrm{H} & -1.115515 & 2.637520 & 3.834331 \\ \mathrm{C} & -0.057089 & 2.094530 & 2.074950 \\ \mathrm{H} & 0.992157 & 1.887644 & 1.937119 \\ \mathrm{~N} & -0.437495 & 3.265469 & 1.439471 \\ \mathrm{~F} & -1.780397 & -1.547029 & 0.979587 \\ \mathrm{H} & 0.074302 & 3.516595 & 0.604675 \\ \mathrm{H} & -1.432986 & 3.422212 & 1.349067\end{array}$

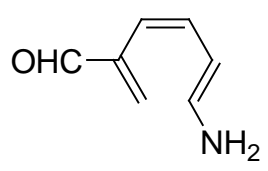

$\begin{array}{lrrr}\mathrm{H} & 0.000000 & 0.000000 & 0.000000 \\ \mathrm{C} & 0.000000 & 0.000000 & 1.088709 \\ \mathrm{C} & 1.176294 & 0.000000 & 1.762624 \\ \mathrm{H} & 1.159119 & -0.001678 & 2.849926 \\ \mathrm{C} & 2.443991 & -0.042445 & 1.077509 \\ \mathrm{H} & 2.371220 & -0.158415 & -0.005183 \\ \mathrm{C} & 3.702998 & -0.011202 & 1.583697 \\ \mathrm{H} & 4.524611 & -0.173323 & 0.891463 \\ \mathrm{C} & 4.136145 & 0.155638 & 2.968886 \\ \mathrm{C} & 3.491449 & 0.771653 & 3.985259 \\ \mathrm{H} & 3.943281 & 0.818695 & 4.973621\end{array}$




$\begin{array}{rrrr}\mathrm{H} & 2.536281 & 1.269458 & 3.865923 \\ \mathrm{C} & 5.500252 & -0.355576 & 3.294930 \\ \mathrm{~N} & -1.260124 & 0.082910 & 1.645037 \\ \mathrm{H} & -1.334529 & -0.125269 & 2.633314 \\ \mathrm{H} & -2.022532 & -0.300784 & 1.103176 \\ \mathrm{H} & 5.789378 & -0.234717 & 4.363233 \\ \mathrm{O} & 6.270699 & -0.850357 & 2.496625\end{array}$

$\begin{array}{rrrr}\text { TS } & & & \\ \mathrm{H} & 0.000000 & 0.000000 & 0.000000 \\ \mathrm{H} & 0.000000 & 0.000000 & 1.833243 \\ \mathrm{C} & 0.579401 & 0.000000 & 0.921072 \\ \mathrm{C} & 1.788362 & 0.704313 & 0.939487 \\ \mathrm{C} & 2.737210 & 0.520773 & 1.957784 \\ \mathrm{H} & 3.520480 & 1.266861 & 2.085577 \\ \mathrm{C} & 2.927073 & -0.709743 & 2.601336 \\ \mathrm{H} & 3.824278 & -0.788885 & 3.213913 \\ \mathrm{C} & 2.302619 & -1.920234 & 2.292146 \\ \mathrm{H} & 2.932297 & -2.807556 & 2.390020 \\ \mathrm{C} & 1.051330 & -2.151787 & 1.670044 \\ \mathrm{H} & 0.140766 & -1.866156 & 2.171161 \\ \mathrm{C} & 2.179646 & 1.538515 & -0.218171 \\ \mathrm{~N} & 0.895420 & -3.303093 & 0.909132 \\ \mathrm{H} & -0.025700 & -3.455444 & 0.520937 \\ \mathrm{H} & 1.626081 & -3.488005 & 0.233524 \\ \mathrm{O} & 1.587291 & 1.585682 & -1.282305 \\ \mathrm{H} & 3.098085 & 2.147171 & -0.054864\end{array}$

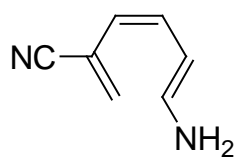

$\begin{array}{cccc}\mathrm{H} & 0.000000 & 0.000000 & 0.000000 \\ \mathrm{C} & 0.000000 & 0.000000 & 1.083806 \\ \mathrm{H} & 0.962267 & 0.000000 & 1.587081 \\ \mathrm{C} & -1.150849 & -0.000060 & 1.798781 \\ \mathrm{C} & -1.169550 & 0.003393 & 3.258325 \\ \mathrm{H} & -0.171075 & 0.019709 & 3.689445 \\ \mathrm{C} & -2.212827 & -0.011742 & 4.131818 \\ \mathrm{H} & -1.925099 & -0.003487 & 5.184342 \\ \mathrm{C} & -3.632211 & -0.035491 & 3.904850 \\ \mathrm{H} & -4.019252 & -0.056446 & 2.891160 \\ \mathrm{C} & -4.507957 & -0.038750 & 4.942177 \\ \mathrm{H} & -4.140861 & -0.014823 & 5.967031\end{array}$




$\begin{array}{rrrr}\mathrm{N} & -5.875522 & -0.140511 & 4.842528 \\ \mathrm{C} & -2.377484 & 0.002912 & 1.038840 \\ \mathrm{~N} & -3.353323 & 0.008074 & 0.405536 \\ \mathrm{H} & -6.285493 & 0.019723 & 3.930822 \\ \mathrm{H} & -6.428171 & 0.221991 & 5.606561\end{array}$

$\begin{array}{crrr}\text { TS } & & & \\ \mathrm{H} & 0.000000 & 0.000000 & 0.000000 \\ \mathrm{H} & 0.000000 & 0.000000 & 1.827902 \\ \mathrm{C} & 0.583397 & 0.000000 & 0.917621 \\ \mathrm{C} & 1.791332 & 0.714179 & 0.972184 \\ \mathrm{C} & 2.690011 & 0.534549 & 2.035605 \\ \mathrm{H} & 3.470531 & 1.272484 & 2.199416 \\ \mathrm{C} & 2.812505 & -0.693202 & 2.703538 \\ \mathrm{H} & 3.655730 & -0.766724 & 3.388759 \\ \mathrm{C} & 2.210136 & -1.905224 & 2.366484 \\ \mathrm{H} & 2.825723 & -2.791329 & 2.535857 \\ \mathrm{C} & 1.021207 & -2.150468 & 1.629340 \\ \mathrm{H} & 0.060080 & -1.901372 & 2.050113 \\ \mathrm{~N} & 0.966823 & -3.286654 & 0.839337 \\ \mathrm{C} & 2.186501 & 1.540914 & -0.133054 \\ \mathrm{~N} & 2.496439 & 2.217268 & -1.029489 \\ \mathrm{H} & 0.086682 & -3.481457 & 0.382328 \\ \mathrm{H} & 1.763888 & -3.459581 & 0.240731\end{array}$<smiles>C=C(/C=C\C=C/N)[N+](=O)[O-]</smiles>

$\begin{array}{lrrr}\mathrm{H} & 0.000000 & 0.000000 & 0.000000 \\ \mathrm{C} & 0.000000 & 0.000000 & 1.088606 \\ \mathrm{C} & 1.177133 & 0.000000 & 1.763302 \\ \mathrm{H} & 1.163315 & 0.002837 & 2.850908 \\ \mathrm{C} & 2.441540 & -0.045530 & 1.077034 \\ \mathrm{H} & 2.372846 & -0.148132 & -0.006608 \\ \mathrm{C} & 3.701020 & -0.025598 & 1.584937 \\ \mathrm{H} & 4.529064 & -0.182850 & 0.904212 \\ \mathrm{C} & 4.070518 & 0.159188 & 2.979824 \\ \mathrm{C} & 3.478805 & 0.890098 & 3.935691 \\ \mathrm{H} & 2.606590 & 1.489318 & 3.704691 \\ \mathrm{H} & 3.877101 & 0.912673 & 4.941673 \\ \mathrm{~N} & 5.333770 & -0.545918 & 3.389367 \\ \mathrm{~N} & -1.256139 & 0.077416 & 1.644683 \\ \mathrm{H} & -1.338010 & -0.124812 & 2.633185\end{array}$




$\begin{array}{crrr}\mathrm{H} & -2.028179 & -0.275155 & 1.096456 \\ \mathrm{O} & 5.706921 & -0.452189 & 4.556711 \\ \mathrm{O} & 5.929450 & -1.181140 & 2.520766 \\ & & & \\ \mathrm{TS} & & & \\ \mathrm{H} & 0.000000 & 0.000000 & 0.000000 \\ \mathrm{H} & 0.000000 & 0.000000 & 1.835636 \\ \mathrm{C} & 0.574617 & 0.000000 & 0.920045 \\ \mathrm{C} & 1.773974 & 0.707420 & 0.967612 \\ \mathrm{C} & 2.735542 & 0.542913 & 1.962587 \\ \mathrm{H} & 3.504923 & 1.296621 & 2.081924 \\ \mathrm{C} & 2.907744 & -0.692511 & 2.606571 \\ \mathrm{H} & 3.786327 & -0.766512 & 3.245401 \\ \mathrm{C} & 2.292874 & -1.902580 & 2.289914 \\ \mathrm{H} & 2.917476 & -2.788718 & 2.419984 \\ \mathrm{C} & 1.063453 & -2.143484 & 1.619415 \\ \mathrm{H} & 0.130793 & -1.893024 & 2.099189 \\ \mathrm{~N} & 0.960097 & -3.273519 & 0.828367 \\ \mathrm{~N} & 2.132961 & 1.561800 & -0.183945 \\ \mathrm{O} & 1.460712 & 1.454978 & -1.211507 \\ \mathrm{O} & 3.077806 & 2.341765 & -0.052124 \\ \mathrm{H} & 0.054863 & -3.462733 & 0.420638 \\ \mathrm{H} & 1.721945 & -3.448599 & 0.186037 \\ & & & \end{array}$

3

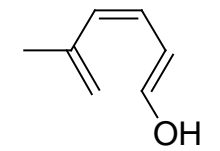

$\begin{array}{lrrr}\mathrm{H} & 0.000000 & 0.000000 & 0.000000 \\ \mathrm{C} & 0.000000 & 0.000000 & 1.086246 \\ \mathrm{H} & 0.972058 & 0.000000 & 1.572698 \\ \mathrm{C} & -1.146323 & 0.000080 & 1.796110 \\ \mathrm{C} & -1.069802 & 0.000124 & 3.258177 \\ \mathrm{H} & -0.045932 & -0.000125 & 3.629642 \\ \mathrm{C} & -2.029087 & 0.000449 & 4.218233 \\ \mathrm{H} & -1.655835 & 0.000300 & 5.243233 \\ \mathrm{C} & -3.475273 & 0.001059 & 4.129806 \\ \mathrm{H} & -3.961851 & 0.001535 & 3.158511 \\ \mathrm{C} & -4.248143 & 0.001015 & 5.234399 \\ \mathrm{H} & -3.834810 & 0.000621 & 6.239261 \\ \mathrm{O} & -5.605564 & 0.001547 & 5.275208 \\ \mathrm{C} & -2.474093 & 0.000212 & 1.072608 \\ \mathrm{H} & -2.324748 & 0.000048 & -0.011167\end{array}$




\begin{tabular}{|c|c|c|c|}
\hline $\mathrm{H}$ & -3.071251 & -0.883828 & 1.328145 \\
\hline $\mathrm{H}$ & -3.070987 & 0.884504 & 1.327937 \\
\hline $\mathrm{H}$ & -5.949641 & 0.001305 & 4.366361 \\
\hline \multicolumn{4}{|l|}{ TS } \\
\hline $\mathrm{H}$ & 0.000000 & 0.000000 & 0.000000 \\
\hline $\mathrm{C}$ & 0.000000 & 0.000000 & 1.077254 \\
\hline $\mathrm{C}$ & 1.177834 & 0.000000 & 1.854896 \\
\hline $\mathrm{H}$ & 1.282620 & 0.863885 & 2.518689 \\
\hline $\mathrm{C}$ & 2.095519 & -1.034459 & 2.069107 \\
\hline $\mathrm{H}$ & 2.998788 & -0.749454 & 2.607854 \\
\hline $\mathrm{C}$ & 1.862156 & -2.412716 & 1.962977 \\
\hline $\mathrm{H}$ & 2.554534 & -3.063649 & 2.495004 \\
\hline $\mathrm{C}$ & 0.650124 & -2.942583 & 1.497517 \\
\hline $\mathrm{C}$ & -0.067494 & -2.228553 & 0.536201 \\
\hline $\mathrm{H}$ & 0.505373 & -1.824694 & -0.287323 \\
\hline $\mathrm{H}$ & -1.082122 & -2.526150 & 0.274555 \\
\hline $\mathrm{C}$ & 0.073416 & -4.176738 & 2.160105 \\
\hline $\mathrm{O}$ & -1.072086 & 0.740129 & 1.503211 \\
\hline $\mathrm{H}$ & -1.048884 & 0.777197 & 2.473465 \\
\hline $\mathrm{H}$ & -0.478012 & -4.808392 & 1.455680 \\
\hline $\mathrm{H}$ & -0.620192 & -3.900846 & 2.967011 \\
\hline $\mathrm{H}$ & 0.865258 & -4.781238 & 2.616241 \\
\hline $\mathrm{H}$ & 0.000000 & 0.000000 & 0.000000 \\
\hline $\mathrm{C}$ & 0.000000 & 0.000000 & 1.083911 \\
\hline $\mathrm{H}$ & 0.967134 & 0.000000 & 1.579585 \\
\hline $\mathrm{C}$ & -1.157238 & 0.056639 & 1.777910 \\
\hline $\mathrm{C}$ & -2.447497 & 0.020456 & 1.080830 \\
\hline $\mathrm{H}$ & -2.381038 & -0.360174 & 0.064711 \\
\hline $\mathrm{C}$ & -3.677423 & 0.392617 & 1.512517 \\
\hline $\mathrm{H}$ & -4.494856 & 0.233502 & 0.808220 \\
\hline $\mathrm{C}$ & -4.068672 & 1.016817 & 2.760956 \\
\hline $\mathrm{H}$ & -3.289536 & 1.304803 & 3.463680 \\
\hline $\mathrm{C}$ & -5.356949 & 1.292042 & 3.043514 \\
\hline $\mathrm{H}$ & -6.174934 & 1.038906 & 2.374242 \\
\hline $\mathrm{O}$ & -5.825915 & 1.904781 & 4.161436 \\
\hline $\mathrm{N}$ & -1.182909 & 0.181965 & 3.185107 \\
\hline $\mathrm{H}$ & -0.268028 & 0.036997 & 3.601287 \\
\hline $\mathrm{H}$ & -1.857707 & -0.442464 & 3.617535 \\
\hline $\mathrm{H}$ & -5.073111 & 2.134818 & 4.731618 \\
\hline
\end{tabular}




\begin{tabular}{|c|c|c|c|}
\hline \multicolumn{4}{|l|}{ TS } \\
\hline \multicolumn{4}{|l|}{$\mathrm{H}$} \\
\hline $\mathrm{C}$ & 0.000000 & 0.000000 & 1.077453 \\
\hline $\mathrm{C}$ & 1.177659 & 0.000000 & 1.854419 \\
\hline \multirow{2}{*}{$\begin{array}{l}\mathrm{H} \\
\mathrm{C}\end{array}$} & 1.286076 & 0.858167 & 2.523553 \\
\hline & 2.102950 & -1.035069 & 2.034413 \\
\hline $\mathrm{C}$ & 3.018047 & -0.754077 & 2.555788 \\
\hline $\mathrm{C}$ & 1.907622 & -2.407786 & 1.862572 \\
\hline $\mathrm{H}$ & 2.631161 & -3.078774 & 2.321559 \\
\hline $\mathrm{C}$ & 0.675046 & -2.941925 & 1.435291 \\
\hline C & -0.127689 & -2.234110 & 0.528782 \\
\hline $\mathrm{H}$ & 0.372093 & -1.866195 & -0.356768 \\
\hline $\mathrm{H}$ & -1.152839 & -2.565301 & 0.356297 \\
\hline $\mathrm{N}$ & 0.246759 & -4.147130 & 1.994074 \\
\hline $\mathrm{O}$ & -1.073807 & 0.735156 & 1.503505 \\
\hline $\mathrm{H}$ & -1.036861 & 0.785480 & 2.473137 \\
\hline $\mathrm{H}$ & 0.410240 & -4.271454 & 2.985626 \\
\hline $\mathrm{H}$ & -0.672649 & -4.458680 & 1.709307 \\
\hline $\mathrm{H}$ & & & \\
\hline $\mathrm{C}$ & 0.000000 & 0.000000 & 1.086976 \\
\hline $\mathrm{H}$ & 0.963011 & 0.000000 & 1.582258 \\
\hline $\mathrm{C}$ & -1.143521 & 0.000000 & 1.801444 \\
\hline $\mathrm{C}$ & -1.157428 & 0.000021 & 3.258158 \\
\hline $\mathrm{H}$ & -0.162228 & 0.000107 & 3.694399 \\
\hline $\mathrm{C}$ & -2.206914 & -0.000012 & 4.116296 \\
\hline $\mathrm{H}$ & -1.946576 & 0.000067 & 5.175485 \\
\hline $\mathrm{C}$ & -3.627019 & -0.000195 & 3.844009 \\
\hline $\mathrm{H}$ & -3.960036 & -0.000398 & 2.810255 \\
\hline $\mathrm{C}$ & -4.532475 & 0.000082 & 4.842342 \\
\hline $\mathrm{H}$ & -4.253003 & 0.000214 & 5.892600 \\
\hline $\mathrm{O}$ & -5.883137 & 0.000121 & 4.705742 \\
\hline $\mathrm{O}$ & -2.382285 & -0.000020 & 1.203375 \\
\hline $\mathrm{H}$ & -2.253348 & -0.000114 & 0.241900 \\
\hline & -6.101158 & 0.000846 & 3.758600 \\
\hline
\end{tabular}

TS

$\begin{array}{llll}\mathrm{H} & 0.000000 & 0.000000 & 0.000000\end{array}$

$\begin{array}{llll}\text { C } & 0.000000 & 0.000000 & 1.087796\end{array}$

$\begin{array}{llll}\mathrm{H} & 0.976546 & 0.000000 & 1.549866\end{array}$ 


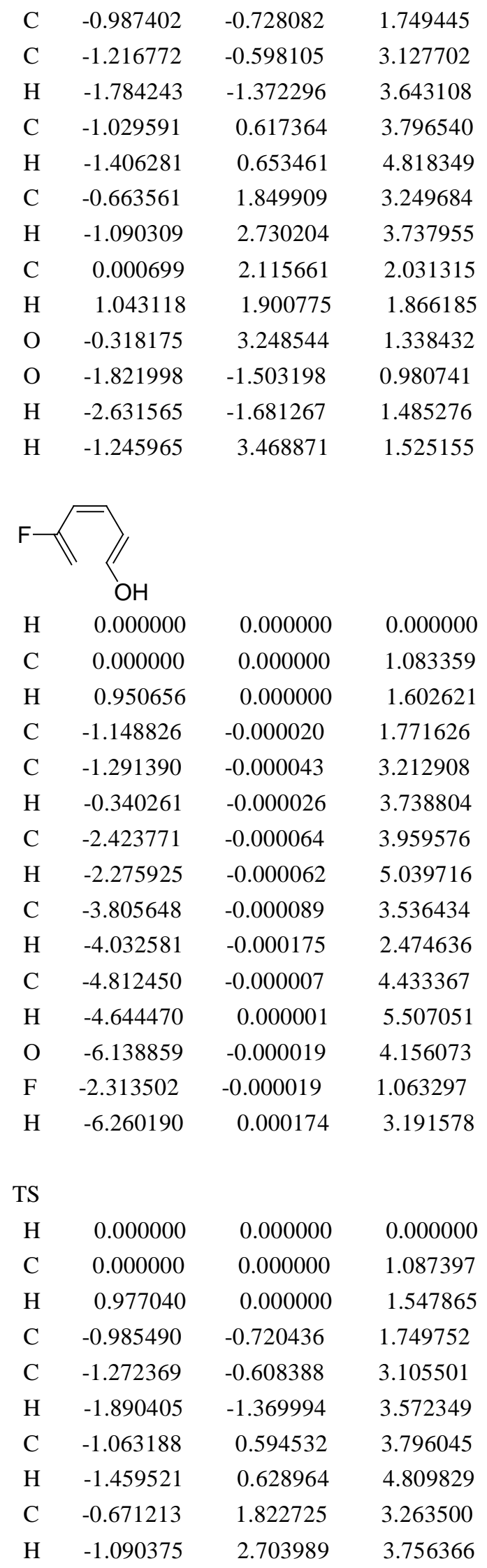




$\begin{array}{cccc}\mathrm{C} & 0.003306 & 2.096220 & 2.049464 \\ \mathrm{H} & 1.049578 & 1.888283 & 1.899964 \\ \mathrm{O} & -0.309760 & 3.234452 & 1.365400 \\ \mathrm{~F} & -1.827703 & -1.488846 & 1.007435 \\ \mathrm{H} & -1.241109 & 3.451267 & 1.538417\end{array}$<smiles>C=C(C=O)/C=C\C=C/O</smiles>

$\begin{array}{llll}\mathrm{H} & 0.000000 & 0.000000 & 0.000000\end{array}$

$\begin{array}{llll}\text { C } & 0.000000 & 0.000000 & 1.086567\end{array}$

$\begin{array}{llll}\text { C } & 1.136568 & 0.000000 & 1.812036\end{array}$

$\begin{array}{llll}\mathrm{H} & 1.069271 & 0.002526 & 2.898654\end{array}$

$\begin{array}{llll}\text { C } & 2.441072 & -0.052167 & 1.189350\end{array}$

$\begin{array}{llll}\mathrm{H} & 2.427952 & -0.192492 & 0.108190\end{array}$

$\begin{array}{llll}\text { C } & 3.663589 & -0.002722 & 1.770104\end{array}$

$\begin{array}{llll}\mathrm{H} & 4.528196 & -0.176405 & 1.135284\end{array}$

$\begin{array}{llll}\text { C } & 4.002859 & 0.191852 & 3.180337\end{array}$

$\begin{array}{llll}\text { C } & 3.313469 & 0.881866 & 4.114394\end{array}$

$\begin{array}{llll}\mathrm{H} & 2.399633 & 1.422268 & 3.894920\end{array}$

$\begin{array}{llll}\mathrm{H} & 3.684651 & 0.948994 & 5.134660\end{array}$

$\begin{array}{llll}\text { C } & 5.310859 & -0.373456 & 3.625898\end{array}$

$\begin{array}{llll}\text { O } & -1.267507 & 0.007558 & 1.565071\end{array}$

$\begin{array}{llll}\mathrm{H} & -1.242605 & -0.003172 & 2.536739\end{array}$

$\begin{array}{llll}\mathrm{H} & 5.525571 & -0.231656 & 4.708675\end{array}$

$\begin{array}{llll}\text { O } & 6.110359 & -0.929504 & 2.900538\end{array}$

TS

$\begin{array}{lrrr}\mathrm{H} & 0.000000 & 0.000000 & 0.000000 \\ \mathrm{C} & 0.000000 & 0.000000 & 1.077412 \\ \mathrm{C} & 1.192612 & 0.000000 & 1.827481 \\ \mathrm{H} & 1.289954 & 0.831388 & 2.531867 \\ \mathrm{C} & 2.157754 & -1.007992 & 1.952850 \\ \mathrm{H} & 3.077979 & -0.717415 & 2.457211 \\ \mathrm{C} & 1.943247 & -2.382233 & 1.816741 \\ \mathrm{H} & 2.670003 & -3.055757 & 2.262906 \\ \mathrm{C} & 0.697539 & -2.910846 & 1.444550 \\ \mathrm{C} & -0.160137 & -2.225327 & 0.576372 \\ \mathrm{H} & 0.267211 & -1.858172 & -0.345617 \\ \mathrm{H} & -1.201085 & -2.537937 & 0.491969 \\ \mathrm{C} & 0.232991 & -4.168150 & 2.074725 \\ \mathrm{O} & -1.070741 & 0.728590 & 1.514490 \\ \mathrm{H} & -1.047251 & 0.766048 & 2.484736 \\ \mathrm{H} & -0.850766 & -4.377929 & 1.923888\end{array}$




$\begin{array}{lccc}\mathrm{O} & 0.938485 & -4.950121 & 2.685541 \\ & & & \\ & & & \\ \mathrm{H} & 0.000000 & 0.000000 & 0.000000 \\ \mathrm{C} & 0.000000 & 0.000000 & 1.083867 \\ \mathrm{H} & 0.962292 & 0.000000 & 1.586984 \\ \mathrm{C} & -1.149920 & -0.000020 & 1.797876 \\ \mathrm{C} & -1.162090 & -0.000021 & 3.259632 \\ \mathrm{H} & -0.161544 & -0.000407 & 3.686133 \\ \mathrm{C} & -2.198552 & 0.000554 & 4.136317 \\ \mathrm{H} & -1.911571 & 0.000578 & 5.188228 \\ \mathrm{C} & -3.622905 & 0.001102 & 3.904729 \\ \mathrm{H} & -4.007372 & 0.001008 & 2.888931 \\ \mathrm{C} & -4.495469 & 0.001756 & 4.934496 \\ \mathrm{H} & -4.177943 & 0.001758 & 5.973985 \\ \mathrm{C} & -2.379670 & -0.000192 & 1.044018 \\ \mathrm{O} & -5.843918 & 0.002120 & 4.844601 \\ \mathrm{H} & -6.102519 & 0.002077 & 3.907098 \\ \mathrm{~N} & -3.361655 & -0.000435 & 0.420448\end{array}$

$\begin{array}{cccc}\text { TS } & & & \\ \mathrm{H} & 0.000000 & 0.000000 & 0.000000 \\ \mathrm{C} & 0.000000 & 0.000000 & 1.086689 \\ \mathrm{H} & 0.978439 & 0.000000 & 1.545336 \\ \mathrm{C} & -1.001512 & -0.682458 & 1.789009 \\ \mathrm{C} & -1.187756 & -0.478486 & 3.169486 \\ \mathrm{H} & -1.762161 & -1.204558 & 3.738154 \\ \mathrm{C} & -0.936629 & 0.757586 & 3.773094 \\ \mathrm{H} & -1.293374 & 0.861086 & 4.796381 \\ \mathrm{C} & -0.595696 & 1.952535 & 3.128276 \\ \mathrm{H} & -1.046813 & 2.854719 & 3.550977 \\ \mathrm{C} & 0.064542 & 2.145734 & 1.898560 \\ \mathrm{H} & 1.102720 & 1.897061 & 1.752884 \\ \mathrm{O} & -0.234318 & 3.235547 & 1.136396 \\ \mathrm{C} & -1.953197 & -1.492274 & 1.082433 \\ \mathrm{~N} & -2.717116 & -2.159295 & 0.509767 \\ \mathrm{H} & -1.161269 & 3.483143 & 1.288971 \\ & & & \\ \mathrm{O} & \mathrm{N}-\mathrm{W} & & \\ & =\mathrm{OH} & & \\ \mathrm{H} & 0.000000 & 0.000000 & 0.000000\end{array}$




$\begin{array}{rrrr}\mathrm{C} & 0.000000 & 0.000000 & 1.086612 \\ \mathrm{C} & 1.137236 & 0.000000 & 1.812116 \\ \mathrm{H} & 1.073925 & 0.006818 & 2.899112 \\ \mathrm{C} & 2.438530 & -0.053220 & 1.186520 \\ \mathrm{H} & 2.428049 & -0.179627 & 0.104221 \\ \mathrm{C} & 3.661624 & -0.011974 & 1.767624 \\ \mathrm{H} & 4.532021 & -0.175895 & 1.143004 \\ \mathrm{C} & 3.939968 & 0.204979 & 3.182010 \\ \mathrm{C} & 3.313354 & 0.998117 & 4.060763 \\ \mathrm{H} & 2.487076 & 1.622385 & 3.742341 \\ \mathrm{H} & 3.638431 & 1.046906 & 5.092152 \\ \mathrm{~N} & 5.136632 & -0.537468 & 3.704105 \\ \mathrm{O} & -1.264865 & 0.007337 & 1.564801 \\ \mathrm{H} & -1.242941 & -0.008159 & 2.536678 \\ \mathrm{O} & 5.425973 & -0.421825 & 4.892368 \\ \mathrm{O} & 5.763261 & -1.222474 & 2.897767\end{array}$

TS

$\begin{array}{llll}\mathrm{H} & 0.000000 & 0.000000 & 0.000000\end{array}$

$\begin{array}{llll}\mathrm{H} & 0.000000 & 0.000000 & 1.836116\end{array}$

$\begin{array}{llll}\text { C } & 0.573383 & 0.000000 & 0.920569\end{array}$

$\begin{array}{llll}\text { C } & 1.784927 & 0.679968 & 0.969801\end{array}$

$\begin{array}{llll}\text { C } & 2.747382 & 0.493210 & 1.964682\end{array}$

$\begin{array}{llll}\mathrm{H} & 3.527676 & 1.235144 & 2.087185\end{array}$

$\begin{array}{llll}\text { C } & 2.910010 & -0.749181 & 2.587729\end{array}$

$\begin{array}{llll}\mathrm{H} & 3.796449 & -0.850664 & 3.211098\end{array}$

$\begin{array}{llll}\text { C } & 2.263889 & -1.943451 & 2.252097\end{array}$

$\begin{array}{llll}\mathrm{H} & 2.878797 & -2.844318 & 2.328641\end{array}$

$\begin{array}{llll}\text { C } & 1.008603 & -2.135591 & 1.639937\end{array}$

$\begin{array}{llll}\mathrm{H} & 0.084675 & -1.885440 & 2.134601\end{array}$

$\begin{array}{llll}\mathrm{O} & 0.801888 & -3.222966 & 0.847044\end{array}$

$\begin{array}{llll}\mathrm{N} & 2.168258 & 1.520215 & -0.185957\end{array}$

$\begin{array}{llll}\mathrm{O} & 1.500756 & 1.419953 & -1.215483\end{array}$

$\begin{array}{llll}\mathrm{O} & 3.125782 & 2.282637 & -0.047633\end{array}$

$\begin{array}{llll}\mathrm{H} & 1.639576 & -3.470672 & 0.421819\end{array}$

4

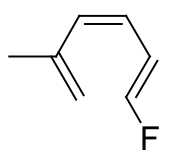

$\begin{array}{llll}\mathrm{H} & 0.000000 & 0.000000 & 0.000000\end{array}$

$\begin{array}{llll}\text { C } & 0.000000 & 0.000000 & 1.086191\end{array}$ 


$\begin{array}{lrrr}\mathrm{H} & 0.972162 & 0.000000 & 1.572377 \\ \mathrm{C} & -1.146385 & 0.000000 & 1.795236 \\ \mathrm{C} & -1.059460 & 0.000000 & 3.257480 \\ \mathrm{H} & -0.032858 & -0.000070 & 3.621422 \\ \mathrm{C} & -2.010058 & 0.000105 & 4.224276 \\ \mathrm{H} & -1.634064 & 0.000055 & 5.247850 \\ \mathrm{C} & -3.457677 & 0.000275 & 4.127693 \\ \mathrm{H} & -3.956400 & 0.000356 & 3.166217 \\ \mathrm{C} & -4.227505 & 0.000339 & 5.222521 \\ \mathrm{H} & -3.872103 & 0.000274 & 6.249382 \\ \mathrm{~F} & -5.570890 & 0.000496 & 5.145179 \\ \mathrm{C} & -2.476183 & 0.000135 & 1.076517 \\ \mathrm{H} & -2.329072 & 0.000257 & -0.007403 \\ \mathrm{H} & -3.072748 & -0.883728 & 1.332914 \\ \mathrm{H} & -3.072683 & 0.883983 & 1.333123\end{array}$

TS

$\begin{array}{llll}\mathrm{H} & 0.000000 & 0.000000 & 0.000000\end{array}$

$\begin{array}{llll}\mathrm{H} & 0.000000 & 0.000000 & 1.823854\end{array}$

$\begin{array}{llll}\text { C } & 0.588069 & 0.000000 & 0.916539\end{array}$

$\begin{array}{llll}\text { C } & 1.810428 & 0.668352 & 0.968832\end{array}$

$\begin{array}{llll}\text { C } & 2.637895 & 0.472322 & 2.086274\end{array}$

$\begin{array}{llll}\mathrm{H} & 3.443233 & 1.181092 & 2.274781\end{array}$

$\begin{array}{llll}\text { C } & 2.709077 & -0.742721 & 2.777672\end{array}$

$\begin{array}{llll}\mathrm{H} & 3.523169 & -0.832813 & 3.495466\end{array}$

$\begin{array}{llll}\text { C } & 2.086057 & -1.945540 & 2.416593\end{array}$

$\begin{array}{llll}\mathrm{H} & 2.673244 & -2.854129 & 2.562023\end{array}$

$\begin{array}{llll}\text { C } & 0.909686 & -2.124182 & 1.683073\end{array}$

$\begin{array}{llll}\mathrm{H} & -0.082877 & -1.897169 & 2.037357\end{array}$

$\begin{array}{llll}\text { F } & 0.862981 & -3.269635 & 0.945783\end{array}$

$\begin{array}{llll}\text { C } & 2.338904 & 1.453167 & -0.213736\end{array}$

$\begin{array}{llll}\mathrm{H} & 1.536939 & 1.920104 & -0.794005\end{array}$

$\begin{array}{llll}\mathrm{H} & 3.023352 & 2.241431 & 0.120077\end{array}$

$\begin{array}{llll}\mathrm{H} & 2.909181 & 0.805170 & -0.893717\end{array}$<smiles>C=C(N)/C=C\C=C/F</smiles>

$\begin{array}{lrrr}\mathrm{H} & 0.000000 & 0.000000 & 0.000000 \\ \mathrm{H} & 0.000000 & 0.000000 & 1.851922 \\ \mathrm{C} & 0.565931 & 0.000000 & 0.927581 \\ \mathrm{C} & 1.915226 & 0.060623 & 0.939448 \\ \mathrm{C} & 2.640123 & 0.036338 & 2.215121 \\ \mathrm{H} & 2.041063 & -0.330217 & 3.045018\end{array}$




\begin{tabular}{|c|c|c|c|}
\hline $\mathrm{C}$ & 3.912893 & 0.399996 & 2.501135 \\
\hline $\mathrm{H}$ & 4.229835 & 0.254158 & 3.534075 \\
\hline $\mathrm{C}$ & 4.909504 & 1.001698 & 1.633200 \\
\hline $\mathrm{H}$ & 4.639734 & 1.283981 & 0.620929 \\
\hline $\mathrm{C}$ & 6.147720 & 1.260758 & 2.068111 \\
\hline $\mathrm{H}$ & 6.532498 & 1.042224 & 3.060902 \\
\hline $\mathrm{F}$ & 7.070674 & 1.842838 & 1.280377 \\
\hline $\mathrm{N}$ & 2.675069 & 0.189192 & -0.241168 \\
\hline $\mathrm{H}$ & 2.118952 & 0.054882 & -1.079764 \\
\hline $\mathrm{H}$ & 3.482874 & -0.426076 & -0.261291 \\
\hline \multicolumn{4}{|l|}{$\mathrm{TS}$} \\
\hline $\mathrm{H}$ & 0.000000 & 0.000000 & 0.000000 \\
\hline $\mathrm{C}$ & 0.000000 & 0.000000 & 1.078160 \\
\hline $\mathrm{C}$ & 1.142807 & 0.000000 & 1.880207 \\
\hline $\mathrm{H}$ & 1.217645 & 0.835212 & 2.577537 \\
\hline $\mathrm{C}$ & 2.070522 & -1.040073 & 2.041143 \\
\hline $\mathrm{H}$ & 2.993456 & -0.771650 & 2.553994 \\
\hline $\mathrm{C}$ & 1.846994 & -2.407217 & 1.876248 \\
\hline $\mathrm{H}$ & 2.564852 & -3.087754 & 2.330161 \\
\hline $\mathrm{C}$ & 0.595645 & -2.927267 & 1.480482 \\
\hline $\mathrm{C}$ & -0.208798 & -2.226112 & 0.572154 \\
\hline $\mathrm{H}$ & 0.279892 & -1.883379 & -0.329301 \\
\hline $\mathrm{H}$ & -1.242712 & -2.541339 & 0.425536 \\
\hline $\mathrm{N}$ & 0.155825 & -4.107776 & 2.079063 \\
\hline $\mathrm{F}$ & -1.016445 & 0.793344 & 1.518407 \\
\hline $\mathrm{H}$ & 0.325481 & -4.202982 & 3.072902 \\
\hline $\mathrm{H}$ & -0.771813 & -4.412547 & 1.814266 \\
\hline $\mathrm{H}$ & 0.000000 & 0.000000 & 0.000000 \\
\hline $\mathrm{H}$ & 0.000000 & 0.000000 & 1.852097 \\
\hline $\mathrm{C}$ & 0.565118 & 0.000000 & 0.928353 \\
\hline $\mathrm{C}$ & 1.912863 & 0.000000 & 0.944208 \\
\hline $\mathrm{C}$ & 2.673384 & 0.000000 & 2.188432 \\
\hline $\mathrm{H}$ & 2.042723 & 0.000017 & 3.073371 \\
\hline $\mathrm{C}$ & 4.012450 & -0.000036 & 2.387601 \\
\hline $\mathrm{H}$ & 4.335560 & -0.000044 & 3.428956 \\
\hline $\mathrm{C}$ & 5.085274 & -0.000066 & 1.412638 \\
\hline $\mathrm{H}$ & 4.852061 & -0.000061 & 0.355005 \\
\hline $\mathrm{C}$ & 6.365664 & -0.000081 & 1.800552 \\
\hline $\mathrm{H}$ & 6.719137 & -0.000072 & 2.828452 \\
\hline
\end{tabular}




$\begin{array}{cccc}\text { F } & 7.377546 & -0.000109 & 0.914092 \\ \mathrm{O} & 2.667684 & -0.000043 & -0.201974 \\ \mathrm{H} & 2.068406 & -0.000039 & -0.964861 \\ & & & \\ \mathrm{TS} & & & \\ \mathrm{H} & 0.000000 & 0.000000 & 0.000000 \\ \mathrm{C} & 0.000000 & 0.000000 & 1.077696 \\ \mathrm{C} & 1.144130 & 0.000000 & 1.880760 \\ \mathrm{H} & 1.216906 & 0.838013 & 2.574942 \\ \mathrm{C} & 2.069830 & -1.036223 & 2.055130 \\ \mathrm{H} & 2.986236 & -0.767957 & 2.578783 \\ \mathrm{C} & 1.841090 & -2.405974 & 1.896859 \\ \mathrm{H} & 2.549039 & -3.086688 & 2.368537 \\ \mathrm{C} & 0.604940 & -2.913942 & 1.463227 \\ \mathrm{C} & -0.199801 & -2.235328 & 0.550808 \\ \mathrm{H} & 0.293146 & -1.868747 & -0.337538 \\ \mathrm{H} & -1.220510 & -2.582470 & 0.406616 \\ \mathrm{O} & 0.093250 & -4.055645 & 2.027710 \\ \mathrm{~F} & -1.014909 & 0.787489 & 1.521324 \\ \mathrm{H} & 0.555733 & -4.227884 & 2.863102\end{array}$

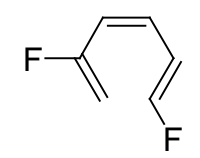

$\begin{array}{lrll}\mathrm{H} & 0.000000 & 0.000000 & 0.000000 \\ \mathrm{C} & 0.000000 & 0.000000 & 1.083399 \\ \mathrm{H} & 0.950725 & 0.000000 & 1.602526 \\ \mathrm{C} & -1.148646 & 0.000060 & 1.770849 \\ \mathrm{C} & -1.280415 & 0.000150 & 3.214563 \\ \mathrm{H} & -0.324769 & 0.000061 & 3.732465 \\ \mathrm{C} & -2.403906 & 0.000342 & 3.970798 \\ \mathrm{H} & -2.250151 & 0.000375 & 5.049782 \\ \mathrm{C} & -3.789335 & 0.000512 & 3.545987 \\ \mathrm{H} & -4.036472 & 0.000510 & 2.491568 \\ \mathrm{C} & -4.781166 & 0.000693 & 4.444505 \\ \mathrm{H} & -4.659174 & 0.000730 & 5.524601 \\ \mathrm{~F} & -6.072537 & 0.000872 & 4.076453 \\ \mathrm{~F} & -2.314373 & 0.000186 & 1.070189\end{array}$

TS

$\begin{array}{llll}\mathrm{H} & 0.000000 & 0.000000 & 0.000000 \\ \mathrm{C} & 0.000000 & 0.000000 & 1.077904 \\ \mathrm{C} & 1.152506 & 0.000000 & 1.873232 \\ \mathrm{H} & 1.237372 & 0.849663 & 2.551745\end{array}$




$\begin{array}{lrrr}\mathrm{C} & 2.068802 & -1.038267 & 2.065269 \\ \mathrm{H} & 2.984349 & -0.771300 & 2.589905 \\ \mathrm{C} & 1.816287 & -2.410297 & 1.944713 \\ \mathrm{H} & 2.482621 & -3.099572 & 2.455835 \\ \mathrm{C} & 0.591411 & -2.890757 & 1.488789 \\ \mathrm{C} & -0.233356 & -2.230986 & 0.588369 \\ \mathrm{H} & 0.236433 & -1.873571 & -0.315590 \\ \mathrm{H} & -1.255549 & -2.582734 & 0.470737 \\ \mathrm{~F} & 0.118313 & -4.014128 & 2.087898 \\ \mathrm{~F} & -1.002662 & 0.799585 & 1.522852\end{array}$

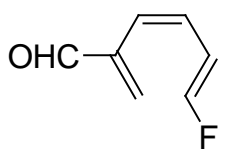

$\begin{array}{lrrr}\mathrm{H} & 0.000000 & 0.000000 & 0.000000 \\ \mathrm{C} & 0.000000 & 0.000000 & 1.087801 \\ \mathrm{H} & 0.970005 & 0.000000 & 1.572316 \\ \mathrm{C} & -1.162925 & 0.040244 & 1.770521 \\ \mathrm{C} & -1.344516 & 0.052210 & 3.225006 \\ \mathrm{H} & -2.245326 & 0.567499 & 3.547879 \\ \mathrm{C} & -0.582406 & -0.532757 & 4.176374 \\ \mathrm{H} & -0.892804 & -0.381566 & 5.210076 \\ \mathrm{C} & 0.574186 & -1.387712 & 3.992635 \\ \mathrm{H} & 0.856554 & -1.711610 & 2.995514 \\ \mathrm{C} & 1.296978 & -1.829971 & 5.027867 \\ \mathrm{H} & 1.125998 & -1.583563 & 6.072313 \\ \mathrm{~F} & 2.347980 & -2.649612 & 4.860266 \\ \mathrm{C} & -2.403759 & 0.173939 & 0.951910 \\ \mathrm{H} & -2.234972 & 0.132359 & -0.146881 \\ \mathrm{O} & -3.517725 & 0.335910 & 1.407031\end{array}$

$\begin{array}{cccc}\text { TS } & & & \\ \mathrm{H} & 0.000000 & 0.000000 & 0.000000 \\ \mathrm{C} & 0.000000 & 0.000000 & 1.078179 \\ \mathrm{C} & 1.156357 & 0.000000 & 1.857418 \\ \mathrm{H} & 1.213975 & 0.804578 & 2.592335 \\ \mathrm{C} & 2.132588 & -1.004650 & 1.955983 \\ \mathrm{H} & 3.057121 & -0.721961 & 2.455860 \\ \mathrm{C} & 1.905550 & -2.373650 & 1.814258 \\ \mathrm{H} & 2.635592 & -3.053114 & 2.246261 \\ \mathrm{C} & 0.647172 & -2.899978 & 1.472878 \\ \mathrm{C} & -0.228928 & -2.228907 & 0.616533 \\ \mathrm{H} & 0.170346 & -1.870864 & -0.320964 \\ \mathrm{H} & -1.272931 & -2.538799 & 0.567861\end{array}$




$\begin{array}{rrrr}\text { F } & -1.014853 & 0.775355 & 1.536731 \\ \mathrm{C} & 0.191474 & -4.144178 & 2.137670 \\ \mathrm{O} & 0.913041 & -4.923320 & 2.731655 \\ \mathrm{H} & -0.898436 & -4.344498 & 2.028729\end{array}$

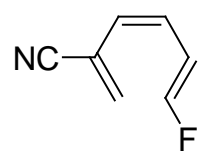

$\begin{array}{lrrr}\mathrm{H} & 0.000000 & 0.000000 & 0.000000 \\ \mathrm{C} & 0.000000 & 0.000000 & 1.083936 \\ \mathrm{H} & 0.962481 & 0.000000 & 1.586745 \\ \mathrm{C} & -1.149460 & 0.000040 & 1.796687 \\ \mathrm{C} & -1.148949 & 0.000107 & 3.260160 \\ \mathrm{H} & -0.143897 & 0.000084 & 3.676219 \\ \mathrm{C} & -2.172128 & 0.000207 & 4.147998 \\ \mathrm{H} & -1.875301 & 0.000237 & 5.196820 \\ \mathrm{C} & -3.602669 & 0.000282 & 3.923648 \\ \mathrm{H} & -4.014721 & 0.000261 & 2.921056 \\ \mathrm{C} & -4.449811 & 0.000359 & 4.960609 \\ \mathrm{H} & -4.166187 & 0.000357 & 6.010091 \\ \mathrm{~F} & -5.778877 & 0.000429 & 4.791035 \\ \mathrm{C} & -2.381946 & 0.000101 & 1.048737 \\ \mathrm{~N} & -3.362281 & 0.000151 & 0.423112\end{array}$

TS

$\begin{array}{lrrr}\mathrm{H} & 0.000000 & 0.000000 & 0.000000 \\ \mathrm{C} & 0.000000 & 0.000000 & 1.078121 \\ \mathrm{C} & 1.149373 & 0.000000 & 1.867354 \\ \mathrm{H} & 1.202543 & 0.808840 & 2.597723 \\ \mathrm{C} & 2.122353 & -1.004587 & 1.985492 \\ \mathrm{H} & 3.044028 & -0.716872 & 2.487265 \\ \mathrm{C} & 1.904462 & -2.377111 & 1.859955 \\ \mathrm{H} & 2.637107 & -3.039349 & 2.313286 \\ \mathrm{C} & 0.656115 & -2.907887 & 1.472564 \\ \mathrm{C} & -0.173537 & -2.229636 & 0.574203 \\ \mathrm{H} & 0.301951 & -1.854923 & -0.320411 \\ \mathrm{H} & -1.209403 & -2.534925 & 0.453155 \\ \mathrm{C} & 0.184221 & -4.089423 & 2.136606 \\ \mathrm{~F} & -1.022917 & 0.760398 & 1.535385 \\ \mathrm{~N} & -0.190188 & -5.059132 & 2.661401\end{array}$<smiles>C=C(/C=C\C=C\F)[N+](=O)[O-]</smiles> 


$\begin{array}{rrrr}\mathrm{H} & 0.000000 & 0.000000 & 0.000000 \\ \mathrm{C} & 0.000000 & 0.000000 & 1.082780 \\ \mathrm{H} & 0.949197 & 0.000000 & 1.605210 \\ \mathrm{C} & -1.150967 & 0.027606 & 1.764985 \\ \mathrm{C} & -1.367331 & 0.033017 & 3.209195 \\ \mathrm{H} & -2.198768 & 0.638866 & 3.551328 \\ \mathrm{C} & -0.652813 & -0.652490 & 4.129988 \\ \mathrm{H} & -0.924631 & -0.489661 & 5.172151 \\ \mathrm{C} & 0.405328 & -1.615224 & 3.900232 \\ \mathrm{H} & 0.642309 & -1.930985 & 2.888602 \\ \mathrm{C} & 1.089589 & -2.160947 & 4.912414 \\ \mathrm{H} & 0.954312 & -1.931217 & 5.965935 \\ \mathrm{~F} & 2.048561 & -3.075242 & 4.706212 \\ \mathrm{~N} & -2.404930 & 0.121316 & 0.947870 \\ \mathrm{O} & -2.308778 & 0.109893 & -0.276029 \\ \mathrm{O} & -3.464694 & 0.207474 & 1.566023\end{array}$

$\begin{array}{cccc}\text { TS } & & & \\ \mathrm{H} & 0.000000 & 0.000000 & 0.000000 \\ \mathrm{C} & 0.000000 & 0.000000 & 1.079824 \\ \mathrm{H} & 0.974371 & 0.000000 & 1.556552 \\ \mathrm{C} & -1.052762 & 0.669341 & 1.687853 \\ \mathrm{C} & -2.400497 & 0.483198 & 1.359430 \\ \mathrm{H} & -3.121455 & 1.227235 & 1.677854 \\ \mathrm{C} & -2.880818 & -0.754197 & 0.926109 \\ \mathrm{H} & -3.963413 & -0.854878 & 0.887859 \\ \mathrm{C} & -2.149330 & -1.949900 & 0.880159 \\ \mathrm{H} & -2.674746 & -2.848309 & 1.207335 \\ \mathrm{C} & -0.780913 & -2.116480 & 0.667217 \\ \mathrm{H} & -0.276442 & -1.867049 & -0.252385 \\ \mathrm{~F} & -0.236781 & -3.232370 & 1.203757 \\ \mathrm{~N} & -0.762903 & 1.495579 & 2.880940 \\ \mathrm{O} & -1.652429 & 2.244934 & 3.285009 \\ \mathrm{O} & 0.353957 & 1.399436 & 3.387522\end{array}$

5

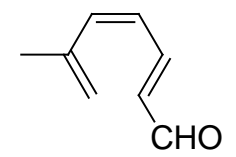

$\begin{array}{llll}\mathrm{H} & 0.000000 & 0.000000 & 0.000000 \\ \mathrm{C} & 0.000000 & 0.000000 & 1.086104 \\ \mathrm{H} & 0.971966 & 0.000000 & 1.572150\end{array}$




$\begin{array}{rrrr}\text { C } & -1.147621 & 0.000000 & 1.794972 \\ \text { C } & -1.044418 & 0.000000 & 3.254372 \\ \text { H } & -0.012648 & -0.000035 & 3.603707 \\ \text { C } & -1.980810 & 0.000053 & 4.240536 \\ \text { H } & -1.592944 & 0.000036 & 5.258654 \\ \text { C } & -3.422434 & 0.000147 & 4.156756 \\ \text { H } & -3.893796 & 0.000202 & 3.178344 \\ \text { C } & -4.234340 & 0.000190 & 5.241579 \\ \text { H } & -3.837368 & 0.000156 & 6.254896 \\ \text { C } & -5.690266 & 0.000286 & 5.102159 \\ \text { C } & -2.478338 & 0.000067 & 1.078785 \\ \text { H } & -3.073766 & 0.884402 & 1.335392 \\ \text { H } & -3.073857 & -0.884205 & 1.335396 \\ \text { H } & -2.332106 & 0.000056 & -0.005050 \\ \text { H } & -6.056031 & 0.000301 & 4.048926 \\ \text { O } & -6.475791 & 0.000312 & 6.034734\end{array}$

$\begin{array}{cccc}\mathrm{TS} & & & \\ \mathrm{H} & 0.000000 & 0.000000 & 0.000000 \\ \mathrm{C} & 0.000000 & 0.000000 & 1.089863 \\ \mathrm{H} & 0.987021 & 0.000000 & 1.525068 \\ \mathrm{C} & -1.007931 & -0.690761 & 1.779978 \\ \mathrm{C} & -1.234645 & -0.448161 & 3.138545 \\ \mathrm{H} & -1.856828 & -1.156641 & 3.684242 \\ \mathrm{C} & -1.070716 & 0.807896 & 3.759196 \\ \mathrm{H} & -1.541010 & 0.911333 & 4.735892 \\ \mathrm{C} & -0.749154 & 1.984038 & 3.085706 \\ \mathrm{H} & -1.297823 & 2.872183 & 3.409586 \\ \mathrm{C} & 0.036921 & 2.107719 & 1.917993 \\ \mathrm{H} & 1.080898 & 1.834079 & 1.931423 \\ \mathrm{C} & -0.246991 & 3.226947 & 0.994932 \\ \mathrm{C} & -1.968770 & -1.580700 & 1.013599 \\ \mathrm{H} & -2.505103 & -0.993535 & 0.255953 \\ \mathrm{H} & -1.450007 & -2.388776 & 0.484563 \\ \mathrm{H} & -2.718742 & -2.028693 & 1.672329 \\ \mathrm{H} & -1.203272 & 3.766176 & 1.192390 \\ \mathrm{O} & 0.463416 & 3.544117 & 0.057970 \\ & & & \\ \mathrm{H} \mathrm{N}- & - & & \\ & - & & \\ \mathrm{H} & 0.000000 & 0.000000 & 0.000000 \\ \mathrm{C} & 0.000000 & 0.000000 & 1.086392 \\ \mathrm{H} & 0.964587 & 0.000000 & 1.580294\end{array}$




$\begin{array}{lrrr}\mathrm{C} & -1.146469 & -0.064187 & 1.799761 \\ \mathrm{C} & -1.081903 & -0.055368 & 3.263639 \\ \mathrm{H} & -0.128916 & 0.300154 & 3.647992 \\ \mathrm{C} & -2.009409 & -0.414672 & 4.188111 \\ \mathrm{H} & -1.728874 & -0.275692 & 5.231570 \\ \mathrm{C} & -3.310259 & -1.010875 & 3.978015 \\ \mathrm{H} & -3.590108 & -1.268409 & 2.958735 \\ \mathrm{C} & -4.171303 & -1.303117 & 4.981808 \\ \mathrm{H} & -3.939542 & -1.080715 & 6.021863 \\ \mathrm{C} & -5.463671 & -1.934715 & 4.714306 \\ \mathrm{~N} & -2.410793 & -0.189582 & 1.193100 \\ \mathrm{H} & -2.378190 & -0.058486 & 0.187013 \\ \mathrm{H} & -3.110484 & 0.424986 & 1.598458 \\ \mathrm{H} & -5.659637 & -2.153753 & 3.639362 \\ \mathrm{O} & -6.288005 & -2.213841 & 5.568640\end{array}$

TS

$\begin{array}{llll}\mathrm{H} & 0.000000 & 0.000000 & 0.000000\end{array}$

$\begin{array}{llll}\mathrm{H} & 0.000000 & 0.000000 & 1.808514\end{array}$

$\begin{array}{llll}\text { C } & 0.600675 & 0.000000 & 0.912566\end{array}$

$\begin{array}{llll}\text { C } & 1.786681 & 0.771806 & 0.901328\end{array}$

$\begin{array}{llll}\text { C } & 2.882967 & 0.553820 & 1.763436\end{array}$

$\begin{array}{llll}\mathrm{H} & 3.623397 & 1.351347 & 1.807276\end{array}$

$\begin{array}{llll}\text { C } & 3.316456 & -0.716737 & 2.176881\end{array}$

$\begin{array}{llll}\mathrm{H} & 4.351349 & -0.779015 & 2.511490\end{array}$

$\begin{array}{llll}\text { C } & 2.641673 & -1.905089 & 1.908536\end{array}$

$\begin{array}{llll}\mathrm{H} & 3.245968 & -2.782747 & 1.673344\end{array}$

$\begin{array}{llll}\text { C } & 1.244411 & -1.985348 & 1.746711\end{array}$

$\begin{array}{llll}\mathrm{H} & 0.612840 & -1.635954 & 2.551038\end{array}$

$\begin{array}{llll}\text { C } & 0.669021 & -3.122578 & 0.997166\end{array}$

$\begin{array}{llll}\mathrm{N} & 1.958599 & 1.737934 & -0.090112\end{array}$

$\begin{array}{llll}\mathrm{H} & 1.181016 & 1.878287 & -0.720234\end{array}$

$\begin{array}{llll}\mathrm{H} & 2.857890 & 1.779174 & -0.553386\end{array}$

$\begin{array}{llll}\mathrm{O} & -0.512110 & -3.417034 & 0.985820\end{array}$

$\begin{array}{llll}\mathrm{H} & 1.406797 & -3.702267 & 0.393996\end{array}$<smiles>C=C(O)/C=C\C=C/C</smiles>

$\begin{array}{rrrr}\mathrm{H} & 0.000000 & 0.000000 & 0.000000 \\ \mathrm{C} & 0.000000 & 0.000000 & 1.086699 \\ \mathrm{H} & 0.963451 & 0.000000 & 1.580889 \\ \mathrm{C} & -1.142652 & 0.000000 & 1.802150 \\ \mathrm{C} & -1.127465 & 0.000000 & 3.259685\end{array}$




$\begin{array}{lrrr}\mathrm{H} & -0.121814 & -0.000017 & 3.672112 \\ \mathrm{C} & -2.154332 & 0.000018 & 4.146178 \\ \mathrm{H} & -1.877880 & 0.000013 & 5.199948 \\ \mathrm{C} & -3.576225 & 0.000043 & 3.884262 \\ \mathrm{H} & -3.900505 & 0.000065 & 2.847901 \\ \mathrm{C} & -4.510042 & 0.000059 & 4.864763 \\ \mathrm{H} & -4.239465 & 0.000055 & 5.919124 \\ \mathrm{C} & -5.939530 & 0.000106 & 4.550522 \\ \mathrm{O} & -2.384907 & 0.000022 & 1.224117 \\ \mathrm{H} & -2.281205 & 0.000036 & 0.259485 \\ \mathrm{H} & -6.175466 & 0.000020 & 3.461851 \\ \mathrm{O} & -6.830923 & 0.000018 & 5.382807\end{array}$

TS

$\begin{array}{llll}\mathrm{H} & 0.000000 & 0.000000 & 0.000000\end{array}$

$\begin{array}{llll}\mathrm{H} & 0.000000 & 0.000000 & 1.809058\end{array}$

$\begin{array}{llll}\text { C } & 0.599602 & 0.000000 & 0.913849\end{array}$

$\begin{array}{llll}\text { C } & 1.789367 & 0.757284 & 0.888065\end{array}$

$\begin{array}{llll}\text { C } & 2.922168 & 0.546229 & 1.686090\end{array}$

$\begin{array}{llll}\mathrm{H} & 3.674724 & 1.330254 & 1.647981\end{array}$

$\begin{array}{llll}\text { C } & 3.352533 & -0.719443 & 2.123679\end{array}$

$\begin{array}{llll}\mathrm{H} & 4.400218 & -0.788076 & 2.412109\end{array}$

$\begin{array}{llll}\text { C } & 2.651297 & -1.901002 & 1.910160\end{array}$

$\begin{array}{llll}\mathrm{H} & 3.234715 & -2.792679 & 1.675616\end{array}$

$\begin{array}{llll}\text { C } & 1.248070 & -1.964315 & 1.800259\end{array}$

$\mathrm{H} \quad 0.655514 \quad-1.581141 \quad 2.618881$

$\begin{array}{llll}\text { C } & 0.622557 & -3.111369 & 1.105958\end{array}$

$\begin{array}{llll}\text { O } & 1.989991 & 1.670115 & -0.124152\end{array}$

$\mathrm{H} \quad 1.127300 \quad 1.953290 \quad-0.462526$

$\begin{array}{llll}\text { O } & -0.561738 & -3.385589 & 1.162057\end{array}$

$\begin{array}{llll}\mathrm{H} & 1.320680 & -3.715891 & 0.481793\end{array}$

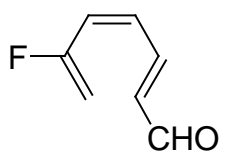

$\begin{array}{lrrr}\mathrm{H} & 0.000000 & 0.000000 & 0.000000 \\ \mathrm{C} & 0.000000 & 0.000000 & 1.083475 \\ \mathrm{H} & 0.950859 & 0.000000 & 1.602201 \\ \mathrm{C} & -1.148911 & 0.000020 & 1.771275 \\ \mathrm{C} & -1.263710 & 0.000064 & 3.215743 \\ \mathrm{H} & -0.299591 & 0.000010 & 3.718252 \\ \mathrm{C} & -2.375347 & 0.000160 & 3.993686 \\ \mathrm{H} & -2.210013 & 0.000175 & 5.070003 \\ \mathrm{C} & -3.761532 & 0.000245 & 3.581895\end{array}$




$\begin{array}{llll}\mathrm{H} & -3.979940 & 0.000234 & 2.518365 \\ \mathrm{C} & -4.793005 & 0.000338 & 4.457825 \\ \mathrm{H} & -4.634791 & 0.000353 & 5.534706 \\ \mathrm{C} & -6.183509 & 0.000422 & 3.995939 \\ \mathrm{~F} & -2.315349 & 0.000083 & 1.076352 \\ \mathrm{H} & -6.306885 & 0.000386 & 2.889217 \\ \mathrm{O} & -7.153210 & 0.000490 & 4.734306\end{array}$

$\begin{array}{crrr}\text { TS } & & & \\ \mathrm{H} & 0.000000 & 0.000000 & 0.000000 \\ \mathrm{H} & 0.000000 & 0.000000 & 1.812073 \\ \mathrm{C} & 0.593940 & 0.000000 & 0.914099 \\ \mathrm{C} & 1.779380 & 0.745892 & 0.866597 \\ \mathrm{C} & 2.946425 & 0.585623 & 1.607489 \\ \mathrm{H} & 3.688257 & 1.373377 & 1.502295 \\ \mathrm{C} & 3.397678 & -0.655631 & 2.098654 \\ \mathrm{H} & 4.452384 & -0.705179 & 2.362136 \\ \mathrm{C} & 2.698379 & -1.844988 & 1.942583 \\ \mathrm{H} & 3.283161 & -2.742987 & 1.737322 \\ \mathrm{C} & 1.292501 & -1.921419 & 1.859694 \\ \mathrm{H} & 0.717835 & -1.515121 & 2.679865 \\ \mathrm{C} & 0.660799 & -3.101030 & 1.224952 \\ \mathrm{~F} & 1.887008 & 1.619011 & -0.175150 \\ \mathrm{H} & 1.345251 & -3.717213 & 0.597723 \\ \mathrm{O} & -0.516046 & -3.387335 & 1.334242\end{array}$

$\begin{array}{lrrr}\mathrm{OHC} & = & & \\ & & & \\ \mathrm{CHO} & & \\ \mathrm{H} & 0.000000 & 0.000000 & 0.000000 \\ \mathrm{C} & 0.000000 & 0.000000 & 1.087848 \\ \mathrm{H} & 0.969392 & 0.000000 & 1.574257 \\ \mathrm{C} & -1.161303 & 0.044471 & 1.771535 \\ \mathrm{C} & -1.320981 & 0.064934 & 3.229029 \\ \mathrm{H} & -2.179367 & 0.639541 & 3.568727 \\ \mathrm{C} & -0.576282 & -0.569269 & 4.165521 \\ \mathrm{H} & -0.838555 & -0.396930 & 5.208302 \\ \mathrm{C} & 0.503294 & -1.504789 & 3.938071 \\ \mathrm{H} & 0.675906 & -1.838547 & 2.915372 \\ \mathrm{C} & 1.282789 & -2.019074 & 4.916522 \\ \mathrm{H} & 1.165650 & -1.726163 & 5.957984 \\ \mathrm{C} & -2.411706 & 0.186962 & 0.968014 \\ \mathrm{C} & 2.333835 & -2.995808 & 4.618327 \\ \mathrm{H} & 2.419708 & -3.272850 & 3.542324\end{array}$




\begin{tabular}{|c|c|c|c|}
\hline $\mathrm{O}$ & 3.074366 & -3.487275 & 5.451377 \\
\hline $\mathrm{H}$ & -2.259192 & 0.138741 & -0.132265 \\
\hline $\mathrm{O}$ & -3.514816 & 0.361189 & 1.442716 \\
\hline \multicolumn{4}{|l|}{ TS } \\
\hline $\mathrm{H}$ & 0.000000 & 0.000000 & 0.000000 \\
\hline $\mathrm{C}$ & 0.000000 & 0.000000 & 1.091231 \\
\hline $\mathrm{H}$ & 0.990628 & 0.000000 & 1.516245 \\
\hline $\mathrm{C}$ & -1.018629 & -0.699909 & 1.759040 \\
\hline $\mathrm{C}$ & -1.411504 & -0.455637 & 3.084581 \\
\hline $\mathrm{H}$ & -2.042277 & -1.211968 & 3.546150 \\
\hline $\mathrm{C}$ & -1.410362 & 0.817786 & 3.673412 \\
\hline $\mathrm{H}$ & -2.038484 & 0.936623 & 4.554188 \\
\hline $\mathrm{C}$ & -1.017581 & 1.973091 & 2.995126 \\
\hline $\mathrm{H}$ & -1.643119 & 2.855291 & 3.150733 \\
\hline $\mathrm{C}$ & -0.015160 & 2.050842 & 2.008925 \\
\hline $\mathrm{H}$ & 0.985425 & 1.730654 & 2.260010 \\
\hline $\mathrm{C}$ & -0.047380 & 3.164052 & 1.033764 \\
\hline $\mathrm{C}$ & -1.840627 & -1.652954 & 0.969503 \\
\hline $\mathrm{O}$ & -2.583508 & -2.492173 & 1.442136 \\
\hline $\mathrm{H}$ & -1.705411 & -1.563456 & -0.131543 \\
\hline $\mathrm{O}$ & 0.869926 & 3.442405 & 0.285253 \\
\hline $\mathrm{H}$ & -1.004866 & 3.733818 & 1.003348 \\
\hline & טח ט & & \\
\hline $\mathrm{H}$ & 0.000000 & 0.000000 & 0.000000 \\
\hline $\mathrm{C}$ & 0.000000 & 0.000000 & 1.084082 \\
\hline $\mathrm{H}$ & 0.962767 & 0.000000 & 1.586207 \\
\hline $\mathrm{C}$ & -1.149736 & 0.000000 & 1.796282 \\
\hline $\mathrm{C}$ & -1.134944 & 0.000000 & 3.259994 \\
\hline $\mathrm{H}$ & -0.124020 & 0.000000 & 3.662290 \\
\hline $\mathrm{C}$ & -2.144225 & 0.000000 & 4.166048 \\
\hline $\mathrm{H}$ & -1.837409 & 0.000000 & 5.211079 \\
\hline $\mathrm{C}$ & -3.575520 & 0.000000 & 3.955101 \\
\hline $\mathrm{H}$ & -3.960194 & 0.000000 & 2.938860 \\
\hline $\mathrm{C}$ & -4.462787 & 0.000000 & 4.976252 \\
\hline $\mathrm{H}$ & -4.142431 & 0.000000 & 6.016550 \\
\hline $\mathrm{C}$ & -5.910215 & 0.000000 & 4.733620 \\
\hline $\mathrm{C}$ & -2.384629 & 0.000000 & 1.053638 \\
\hline $\mathrm{N}$ & -3.367899 & 0.000002 & 0.432952 \\
\hline $\mathrm{H}$ & -6.206219 & 0.000018 & 3.661252 \\
\hline $\mathrm{O}$ & -6.748477 & 0.000035 & 5.617674 \\
\hline
\end{tabular}




$\begin{array}{crrr}\text { TS } & & & \\ \mathrm{H} & 0.000000 & 0.000000 & 0.000000 \\ \mathrm{H} & 0.000000 & 0.000000 & 1.820070 \\ \mathrm{C} & 0.586687 & 0.000000 & 0.915721 \\ \mathrm{C} & 1.809823 & 0.694712 & 0.945546 \\ \mathrm{C} & 2.810345 & 0.455933 & 1.908425 \\ \mathrm{H} & 3.599984 & 1.196375 & 2.010520 \\ \mathrm{C} & 3.070843 & -0.811204 & 2.450887 \\ \mathrm{H} & 4.033136 & -0.921729 & 2.946520 \\ \mathrm{C} & 2.412565 & -1.975994 & 2.049652 \\ \mathrm{H} & 3.051200 & -2.850739 & 1.905647 \\ \mathrm{C} & 1.070606 & -2.081496 & 1.639219 \\ \mathrm{H} & 0.283771 & -1.790732 & 2.319049 \\ \mathrm{C} & 0.679474 & -3.196638 & 0.743298 \\ \mathrm{C} & 2.143075 & 1.564713 & -0.145997 \\ \mathrm{~N} & 2.404519 & 2.285641 & -1.022594 \\ \mathrm{O} & -0.472754 & -3.496052 & 0.498332 \\ \mathrm{H} & 1.528096 & -3.743170 & 0.272112\end{array}$

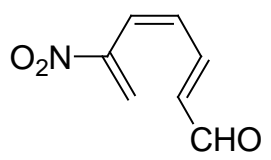

$\begin{array}{lrrr}\mathrm{H} & 0.000000 & 0.000000 & 0.000000 \\ \mathrm{C} & 0.000000 & 0.000000 & 1.088176 \\ \mathrm{C} & 1.147618 & 0.000000 & 1.802422 \\ \mathrm{H} & 1.079381 & 0.005140 & 2.889842 \\ \mathrm{C} & 2.467325 & -0.044149 & 1.210345 \\ \mathrm{H} & 2.499099 & -0.218761 & 0.136413 \\ \mathrm{C} & 3.656152 & 0.062822 & 1.848828 \\ \mathrm{H} & 4.564181 & -0.105127 & 1.279554 \\ \mathrm{C} & 3.854891 & 0.378451 & 3.261630 \\ \mathrm{C} & 3.195712 & 1.257891 & 4.024426 \\ \mathrm{H} & 3.465752 & 1.395347 & 5.064327 \\ \mathrm{H} & 2.400516 & 1.863479 & 3.606024 \\ \mathrm{~N} & 4.999888 & -0.343012 & 3.903896 \\ \mathrm{C} & -1.310619 & 0.006998 & 1.748283 \\ \mathrm{H} & -1.273062 & 0.000942 & 2.861483 \\ \mathrm{O} & -2.374657 & 0.019160 & 1.156939 \\ \mathrm{O} & 5.224923 & -0.140158 & 5.092593 \\ \mathrm{O} & 5.646246 & -1.101539 & 3.183463\end{array}$

TS

$\begin{array}{llll}\mathrm{H} & 0.000000 & 0.000000 & 0.000000\end{array}$ 


$\begin{array}{rrrr}\mathrm{C} & 0.000000 & 0.000000 & 1.079899 \\ \mathrm{C} & 1.230501 & 0.000000 & 1.761949 \\ \mathrm{H} & 1.364094 & 0.733704 & 2.560300 \\ \mathrm{C} & 2.239238 & -0.958798 & 1.649567 \\ \mathrm{H} & 3.217599 & -0.715702 & 2.058343 \\ \mathrm{C} & 1.991157 & -2.323137 & 1.434750 \\ \mathrm{H} & 2.770874 & -3.021338 & 1.718946 \\ \mathrm{C} & 0.704675 & -2.837760 & 1.247358 \\ \mathrm{C} & -0.364435 & -2.182177 & 0.633300 \\ \mathrm{H} & -1.371975 & -2.515667 & 0.861539 \\ \mathrm{H} & -0.213071 & -1.831592 & -0.374375 \\ \mathrm{~N} & 0.406735 & -4.102407 & 1.964046 \\ \mathrm{C} & -1.149289 & 0.735130 & 1.662202 \\ \mathrm{O} & -2.169906 & 0.985427 & 1.052251 \\ \mathrm{H} & -1.028937 & 1.030491 & 2.729225 \\ \mathrm{O} & -0.773644 & -4.376138 & 2.174574 \\ \mathrm{O} & 1.358242 & -4.812294 & 2.288973\end{array}$

6

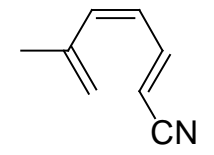

$\begin{array}{lrrr}\mathrm{H} & 0.000000 & 0.000000 & 0.000000 \\ \mathrm{C} & 0.000000 & 0.000000 & 1.086039 \\ \mathrm{H} & 0.971996 & 0.000000 & 1.572100 \\ \mathrm{C} & -1.147745 & -0.000020 & 1.794513 \\ \mathrm{C} & -1.044060 & 0.000003 & 3.253841 \\ \mathrm{H} & -0.012710 & 0.000421 & 3.604138 \\ \mathrm{C} & -1.980846 & -0.000411 & 4.238870 \\ \mathrm{H} & -1.596001 & -0.000175 & 5.258408 \\ \mathrm{C} & -3.422006 & -0.001198 & 4.148427 \\ \mathrm{H} & -3.897907 & -0.001661 & 3.175273 \\ \mathrm{C} & -4.217641 & -0.001465 & 5.246464 \\ \mathrm{H} & -3.788633 & -0.001056 & 6.246354 \\ \mathrm{C} & -5.638024 & -0.002239 & 5.168047 \\ \mathrm{C} & -2.478977 & -0.000065 & 1.079366 \\ \mathrm{H} & -2.333567 & 0.000281 & -0.004508 \\ \mathrm{H} & -3.074409 & -0.884665 & 1.335113 \\ \mathrm{H} & -3.074771 & 0.884126 & 1.335646 \\ \mathrm{~N} & -6.802466 & -0.002872 & 5.119202\end{array}$

TS 


\begin{tabular}{|c|c|c|c|}
\hline $\mathrm{H}$ & 0.000000 & 0.000000 & 0.000000 \\
\hline $\mathrm{C}$ & 0.000000 & 0.000000 & 1.078578 \\
\hline $\mathrm{C}$ & 1.233753 & 0.000000 & 1.772571 \\
\hline $\mathrm{H}$ & 1.387320 & 0.785202 & 2.513569 \\
\hline $\mathrm{C}$ & 2.191342 & -1.012886 & 1.755540 \\
\hline $\mathrm{H}$ & 3.155606 & -0.772814 & 2.200297 \\
\hline $\mathrm{C}$ & 1.915245 & -2.387290 & 1.614810 \\
\hline $\mathrm{H}$ & 2.671665 & -3.056781 & 2.022397 \\
\hline $\mathrm{C}$ & 0.643692 & -2.912401 & 1.359700 \\
\hline $\mathrm{C}$ & -0.264130 & -2.205188 & 0.560473 \\
\hline $\mathrm{H}$ & 0.094994 & -1.840633 & -0.389953 \\
\hline $\mathrm{H}$ & -1.314898 & -2.493025 & 0.557518 \\
\hline $\mathrm{C}$ & 0.197012 & -4.162867 & 2.093038 \\
\hline $\mathrm{C}$ & -1.093784 & 0.750782 & 1.627829 \\
\hline $\mathrm{N}$ & -2.005021 & 1.320140 & 2.077740 \\
\hline $\mathrm{H}$ & -0.536731 & -3.906412 & 2.869338 \\
\hline $\mathrm{H}$ & -0.278113 & -4.888579 & 1.423936 \\
\hline $\mathrm{H}$ & 1.037937 & -4.654393 & 2.591833 \\
\hline $\mathrm{H}$ & 0.000000 & 0.000000 & 0.000000 \\
\hline $\mathrm{C}$ & 0.000000 & 0.000000 & 1.086324 \\
\hline $\mathrm{H}$ & 0.964527 & 0.000000 & 1.580407 \\
\hline $\mathrm{C}$ & -1.146647 & -0.064858 & 1.799115 \\
\hline $\mathrm{C}$ & -1.082190 & -0.057571 & 3.263019 \\
\hline $\mathrm{H}$ & -0.130342 & 0.298376 & 3.649535 \\
\hline $\mathrm{C}$ & -2.009417 & -0.420972 & 4.185507 \\
\hline $\mathrm{H}$ & -1.730825 & -0.286910 & 5.230388 \\
\hline $\mathrm{C}$ & -3.309998 & -1.014606 & 3.967708 \\
\hline $\mathrm{H}$ & -3.597518 & -1.265808 & 2.951708 \\
\hline $\mathrm{C}$ & -4.155801 & -1.303188 & 4.986588 \\
\hline $\mathrm{H}$ & -3.888559 & -1.076830 & 6.016938 \\
\hline $\mathrm{C}$ & -5.427983 & -1.907875 & 4.784695 \\
\hline $\mathrm{N}$ & -2.411020 & -0.193920 & 1.193996 \\
\hline $\mathrm{H}$ & -2.378997 & -0.078936 & 0.185971 \\
\hline $\mathrm{H}$ & -3.110421 & 0.427476 & 1.589594 \\
\hline $\mathrm{N}$ & -6.474050 & -2.400205 & 4.637738 \\
\hline \multicolumn{4}{|c|}{ TS } \\
\hline $\mathrm{H}$ & 0.000000 & 0.000000 & 0.000000 \\
\hline $\mathrm{H}$ & 0.000000 & 0.000000 & 1.814088 \\
\hline $\mathrm{C}$ & 0.596468 & 0.000000 & 0.914178 \\
\hline
\end{tabular}




$\begin{array}{lrrr}\mathrm{C} & 1.784015 & 0.764171 & 0.921154 \\ \mathrm{C} & 2.825298 & 0.561541 & 1.851408 \\ \mathrm{H} & 3.564906 & 1.356259 & 1.934763 \\ \mathrm{C} & 3.217056 & -0.701473 & 2.320452 \\ \mathrm{H} & 4.214345 & -0.754739 & 2.754917 \\ \mathrm{C} & 2.598374 & -1.909906 & 2.001880 \\ \mathrm{H} & 3.242877 & -2.772458 & 1.839320 \\ \mathrm{C} & 1.225726 & -2.030491 & 1.691782 \\ \mathrm{H} & 0.507768 & -1.727969 & 2.439110 \\ \mathrm{C} & 0.781712 & -3.153670 & 0.914003 \\ \mathrm{~N} & 2.010129 & 1.698895 & -0.086779 \\ \mathrm{H} & 1.268404 & 1.817391 & -0.763224 \\ \mathrm{H} & 2.932586 & 1.720483 & -0.503264 \\ \mathrm{~N} & 0.408902 & -4.037349 & 0.253467\end{array}$

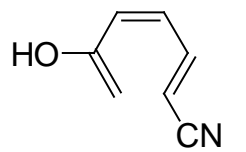

$\begin{array}{lrrr}\mathrm{H} & 0.000000 & 0.000000 & 0.000000 \\ \mathrm{C} & 0.000000 & 0.000000 & 1.086583 \\ \mathrm{H} & 0.963047 & 0.000000 & 1.581586 \\ \mathrm{C} & -1.142801 & 0.000000 & 1.801655 \\ \mathrm{C} & -1.127996 & 0.000000 & 3.259056 \\ \mathrm{H} & -0.123507 & -0.000017 & 3.673795 \\ \mathrm{C} & -2.156478 & 0.000018 & 4.143128 \\ \mathrm{H} & -1.883900 & -0.000004 & 5.198095 \\ \mathrm{C} & -3.576762 & 0.000043 & 3.873787 \\ \mathrm{H} & -3.906134 & 0.000065 & 2.841790 \\ \mathrm{C} & -4.495228 & 0.000039 & 4.869921 \\ \mathrm{H} & -4.189461 & 0.000017 & 5.914410 \\ \mathrm{C} & -5.897373 & 0.000063 & 4.626140 \\ \mathrm{O} & -2.385894 & 0.000022 & 1.227003 \\ \mathrm{H} & -2.286678 & 0.000036 & 0.261804 \\ \mathrm{~N} & -7.048945 & 0.000084 & 4.447394\end{array}$

$\begin{array}{rrrr}\text { TS } & & & \\ \mathrm{H} & 0.000000 & 0.000000 & 0.000000 \\ \mathrm{H} & 0.000000 & 0.000000 & 1.823026 \\ \mathrm{C} & 0.585606 & 0.000000 & 0.917697 \\ \mathrm{C} & 1.771028 & 0.751187 & 0.910471 \\ \mathrm{C} & 2.820714 & 0.595740 & 1.827739 \\ \mathrm{H} & 3.566885 & 1.389259 & 1.870091 \\ \mathrm{C} & 3.203542 & -0.642553 & 2.372029 \\ \mathrm{H} & 4.193781 & -0.672418 & 2.823322\end{array}$




$\begin{array}{lrrr}\mathrm{C} & 2.597186 & -1.864874 & 2.093729 \\ \mathrm{H} & 3.255270 & -2.726447 & 1.989419 \\ \mathrm{C} & 1.235820 & -2.022666 & 1.747198 \\ \mathrm{H} & 0.488338 & -1.713995 & 2.461474 \\ \mathrm{C} & 0.841279 & -3.179092 & 0.992511 \\ \mathrm{O} & 1.933577 & 1.595224 & -0.160054 \\ \mathrm{H} & 2.878837 & 1.773147 & -0.288705 \\ \mathrm{~N} & 0.510102 & -4.093979 & 0.352908\end{array}$

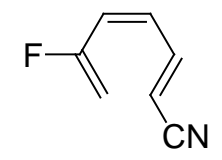

$\begin{array}{lrrr}\mathrm{H} & 0.000000 & 0.000000 & 0.000000 \\ \mathrm{C} & 0.000000 & 0.000000 & 1.083426 \\ \mathrm{H} & 0.950673 & 0.000000 & 1.602547 \\ \mathrm{C} & -1.148813 & -0.000040 & 1.771112 \\ \mathrm{C} & -1.262862 & -0.000086 & 3.215734 \\ \mathrm{H} & -0.299573 & -0.000085 & 3.719522 \\ \mathrm{C} & -2.375049 & -0.000128 & 3.992242 \\ \mathrm{H} & -2.212883 & -0.000158 & 5.069219 \\ \mathrm{C} & -3.758893 & -0.000139 & 3.573855 \\ \mathrm{H} & -3.982566 & -0.000110 & 2.514100 \\ \mathrm{C} & -4.776809 & -0.000183 & 4.467330 \\ \mathrm{H} & -4.581987 & -0.000212 & 5.537987 \\ \mathrm{C} & -6.145939 & -0.000194 & 4.078717 \\ \mathrm{~F} & -2.316163 & -0.000038 & 1.078459 \\ \mathrm{~N} & -7.272755 & -0.000202 & 3.782367\end{array}$

$\begin{array}{cccc}\text { TS } & & & \\ \mathrm{H} & 0.000000 & 0.000000 & 0.000000 \\ \mathrm{H} & 0.000000 & 0.000000 & 1.819665 \\ \mathrm{C} & 0.587809 & 0.000000 & 0.916521 \\ \mathrm{C} & 1.773213 & 0.741241 & 0.899153 \\ \mathrm{C} & 2.874644 & 0.599511 & 1.735574 \\ \mathrm{H} & 3.626872 & 1.382719 & 1.687349 \\ \mathrm{C} & 3.266014 & -0.630222 & 2.298511 \\ \mathrm{H} & 4.277497 & -0.663948 & 2.697934 \\ \mathrm{C} & 2.632631 & -1.845848 & 2.067059 \\ \mathrm{H} & 3.274125 & -2.718812 & 1.954785 \\ \mathrm{C} & 1.254422 & -1.992451 & 1.780200 \\ \mathrm{H} & 0.549326 & -1.665239 & 2.528943 \\ \mathrm{C} & 0.812002 & -3.159903 & 1.070521 \\ \mathrm{~F} & 1.947570 & 1.581108 & -0.155824 \\ \mathrm{~N} & 0.439176 & -4.083776 & 0.468218\end{array}$




\begin{tabular}{|c|c|c|c|}
\hline $\mathrm{H}$ & 0.000000 & 0.000000 & 0.000000 \\
\hline $\mathrm{C}$ & 0.000000 & 0.000000 & 1.086534 \\
\hline $\mathrm{C}$ & 1.187615 & 0.000000 & 1.736330 \\
\hline $\mathrm{H}$ & 1.229438 & 0.002249 & 2.823497 \\
\hline $\mathrm{C}$ & -1.272672 & -0.040382 & 1.771983 \\
\hline $\mathrm{H}$ & -1.225873 & -0.212342 & 2.846485 \\
\hline $\mathrm{C}$ & -2.502148 & 0.058678 & 1.214088 \\
\hline $\mathrm{H}$ & -3.362952 & -0.117467 & 1.854597 \\
\hline $\mathrm{C}$ & -2.849943 & 0.324089 & -0.185548 \\
\hline $\mathrm{C}$ & -2.192395 & 1.131323 & -1.042250 \\
\hline $\mathrm{H}$ & -2.549705 & 1.261224 & -2.061418 \\
\hline $\mathrm{H}$ & -1.317882 & 1.706364 & -0.757686 \\
\hline $\mathrm{C}$ & -4.119867 & -0.289798 & -0.676005 \\
\hline $\mathrm{C}$ & 2.437826 & 0.005077 & 1.055803 \\
\hline $\mathrm{N}$ & 3.468109 & 0.011101 & 0.511895 \\
\hline $\mathrm{O}$ & -4.879375 & -0.935756 & 0.015674 \\
\hline $\mathrm{H}$ & -4.340889 & -0.095226 & -1.747933 \\
\hline \multicolumn{4}{|l|}{ TS } \\
\hline $\mathrm{H}$ & 0.000000 & 0.000000 & 0.000000 \\
\hline $\mathrm{H}$ & 0.000000 & 0.000000 & 1.815336 \\
\hline $\mathrm{C}$ & 0.593971 & 0.000000 & 0.914914 \\
\hline $\mathrm{C}$ & 1.815076 & 0.689795 & 0.921334 \\
\hline $\mathrm{C}$ & 2.846654 & 0.454490 & 1.843287 \\
\hline $\mathrm{H}$ & 3.628220 & 1.208754 & 1.897725 \\
\hline $\mathrm{C}$ & 3.147613 & -0.811083 & 2.366487 \\
\hline $\mathrm{H}$ & 4.137637 & -0.922229 & 2.803998 \\
\hline $\mathrm{C}$ & 2.476990 & -1.983005 & 2.009528 \\
\hline $\mathrm{H}$ & 3.096898 & -2.864715 & 1.846793 \\
\hline $\mathrm{C}$ & 1.109010 & -2.073556 & 1.673545 \\
\hline $\mathrm{H}$ & 0.387283 & -1.751020 & 2.408433 \\
\hline $\mathrm{C}$ & 0.654102 & -3.183175 & 0.885208 \\
\hline $\mathrm{C}$ & 2.095927 & 1.626871 & -0.198640 \\
\hline $\mathrm{H}$ & 1.394965 & 1.531286 & -1.057295 \\
\hline $\mathrm{O}$ & 2.982440 & 2.458737 & -0.202506 \\
\hline $\mathrm{N}$ & 0.271123 & -4.056061 & 0.216772 \\
\hline
\end{tabular}




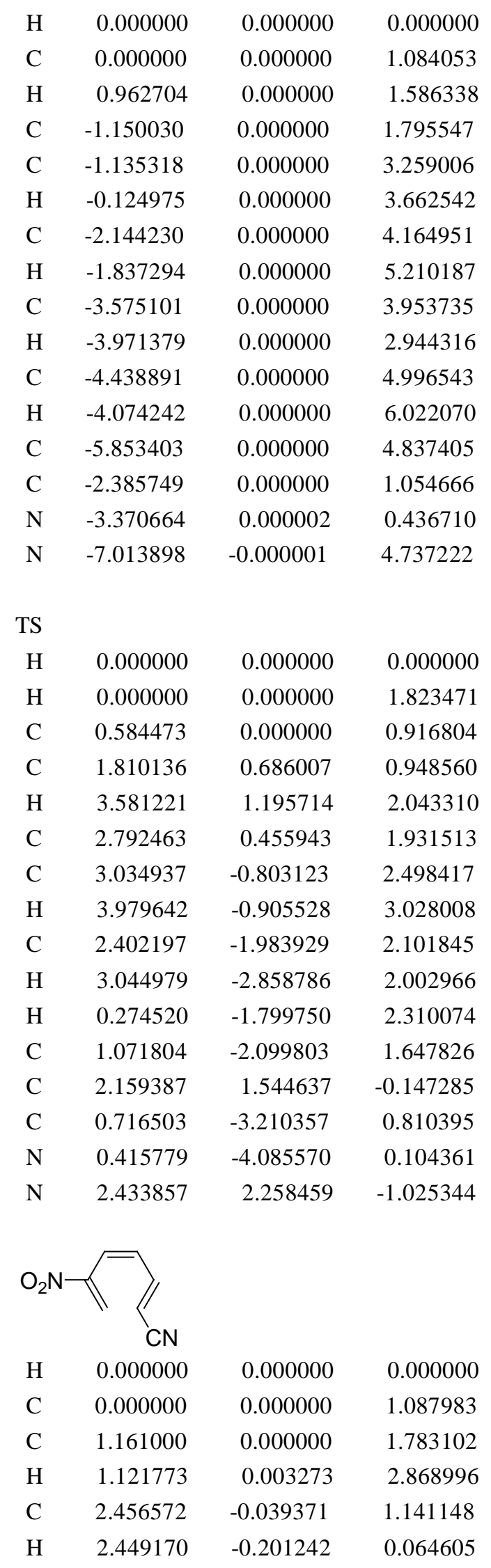




$\begin{array}{lrrr}\mathrm{C} & 3.666801 & 0.059159 & 1.738703 \\ \mathrm{H} & 4.553091 & -0.102121 & 1.134457 \\ \mathrm{C} & 3.917812 & 0.354104 & 3.147783 \\ \mathrm{C} & 3.287324 & 1.220340 & 3.948931 \\ \mathrm{H} & 2.475300 & 1.831520 & 3.572949 \\ \mathrm{H} & 3.597227 & 1.341372 & 4.979735 \\ \mathrm{~N} & 5.087406 & -0.375870 & 3.733647 \\ \mathrm{C} & -1.276158 & 0.007709 & 1.718989 \\ \mathrm{~N} & -2.327442 & 0.016916 & 2.220493 \\ \mathrm{O} & 5.361682 & -0.187127 & 4.913983 \\ \mathrm{O} & 5.702203 & -1.126299 & 2.977796\end{array}$

TS

$\begin{array}{lrrr}\mathrm{H} & 0.000000 & 0.000000 & 0.000000 \\ \mathrm{H} & 0.000000 & 0.000000 & 1.831146 \\ \mathrm{C} & 0.576345 & 0.000000 & 0.919700 \\ \mathrm{C} & 1.792206 & 0.681703 & 0.940919 \\ \mathrm{C} & 2.832364 & 0.473613 & 1.850291 \\ \mathrm{H} & 3.611510 & 1.226154 & 1.902730 \\ \mathrm{C} & 3.112125 & -0.782632 & 2.408048 \\ \mathrm{H} & 4.082727 & -0.878751 & 2.889332 \\ \mathrm{C} & 2.461514 & -1.965539 & 2.051405 \\ \mathrm{H} & 3.098672 & -2.841570 & 1.929588 \\ \mathrm{C} & 1.109984 & -2.084700 & 1.664912 \\ \mathrm{H} & 0.350157 & -1.772857 & 2.364785 \\ \mathrm{C} & 0.710573 & -3.205954 & 0.863070 \\ \mathrm{~N} & 2.102157 & 1.563168 & -0.212920 \\ \mathrm{O} & 1.387456 & 1.471706 & -1.208695 \\ \mathrm{O} & 3.043934 & 2.345734 & -0.093879 \\ \mathrm{~N} & 0.374135 & -4.092508 & 0.188113\end{array}$

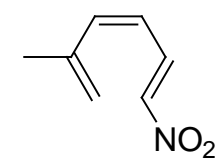

$\begin{array}{cccc}\mathrm{H} & 0.000000 & 0.000000 & 0.000000 \\ \mathrm{C} & 0.000000 & 0.000000 & 1.086022 \\ \mathrm{H} & 0.972028 & 0.000000 & 1.571971 \\ \mathrm{C} & -1.148409 & 0.000000 & 1.794131 \\ \mathrm{C} & -1.038178 & -0.000023 & 3.252326 \\ \mathrm{H} & -0.005709 & -0.000107 & 3.599209\end{array}$




$\begin{array}{lrrr}\mathrm{C} & -1.971953 & 0.000006 & 4.241216 \\ \mathrm{H} & -1.586413 & -0.000040 & 5.259743 \\ \mathrm{C} & -3.409905 & 0.000110 & 4.134762 \\ \mathrm{H} & -3.897360 & 0.000195 & 3.168340 \\ \mathrm{C} & -4.213239 & 0.000126 & 5.215033 \\ \mathrm{H} & -3.899468 & 0.000052 & 6.250308 \\ \mathrm{~N} & -5.651700 & 0.000253 & 5.072115 \\ \mathrm{C} & -2.480442 & 0.000067 & 1.081069 \\ \mathrm{H} & -2.335328 & -0.000014 & -0.002756 \\ \mathrm{H} & -3.076437 & -0.883806 & 1.337094 \\ \mathrm{H} & -3.076267 & 0.884090 & 1.336980 \\ \mathrm{O} & -6.144859 & 0.000366 & 3.939387 \\ \mathrm{O} & -6.300642 & 0.000278 & 6.123253\end{array}$

$\begin{array}{crrr}\text { TS } & & & \\ \mathrm{H} & 0.000000 & 0.000000 & 0.000000 \\ \mathrm{C} & 0.000000 & 0.000000 & 1.088535 \\ \mathrm{H} & 0.982899 & 0.000000 & 1.535701 \\ \mathrm{C} & -1.021295 & -0.635863 & 1.795956 \\ \mathrm{C} & -1.143626 & -0.406750 & 3.174076 \\ \mathrm{H} & -1.753833 & -1.093953 & 3.758992 \\ \mathrm{C} & -0.849621 & 0.819622 & 3.794503 \\ \mathrm{H} & -1.191217 & 0.924064 & 4.822449 \\ \mathrm{C} & -0.543926 & 2.004992 & 3.121202 \\ \mathrm{H} & -0.996214 & 2.914866 & 3.512797 \\ \mathrm{C} & 0.109469 & 2.117048 & 1.883775 \\ \mathrm{H} & 1.150585 & 1.897121 & 1.726184 \\ \mathrm{C} & -2.100657 & -1.421949 & 1.079203 \\ \mathrm{~N} & -0.222436 & 3.301871 & 1.079559 \\ \mathrm{O} & 0.565796 & 3.587480 & 0.173579 \\ \mathrm{O} & -1.268015 & 3.908840 & 1.323577 \\ \mathrm{H} & -2.904128 & -0.756337 & 0.735444 \\ \mathrm{H} & -1.713835 & -1.955771 & 0.206127 \\ \mathrm{H} & -2.559115 & -2.153723 & 1.753147\end{array}$<smiles>C=C(N)/C=C\C=C\[N+](=O)[O-]</smiles>

$\begin{array}{rrrr}\mathrm{H} & 0.000000 & 0.000000 & 0.000000 \\ \mathrm{C} & 0.000000 & 0.000000 & 1.086244 \\ \mathrm{H} & 0.964543 & 0.000000 & 1.580144 \\ \mathrm{C} & -1.147642 & -0.066686 & 1.799118 \\ \mathrm{C} & -1.072431 & -0.066956 & 3.261473\end{array}$




$\begin{array}{rrrr}\mathrm{H} & -0.110155 & 0.267462 & 3.641240 \\ \mathrm{C} & -1.997891 & -0.413997 & 4.193128 \\ \mathrm{H} & -1.704843 & -0.292468 & 5.234864 \\ \mathrm{C} & -3.312427 & -0.970751 & 3.973945 \\ \mathrm{H} & -3.634107 & -1.207717 & 2.965567 \\ \mathrm{C} & -4.152222 & -1.250717 & 4.987287 \\ \mathrm{H} & -3.963971 & -1.092370 & 6.040944 \\ \mathrm{~N} & -5.457063 & -1.823991 & 4.740870 \\ \mathrm{~N} & -2.410066 & -0.198563 & 1.196950 \\ \mathrm{H} & -2.383400 & -0.093702 & 0.187989 \\ \mathrm{H} & -3.117631 & 0.413684 & 1.591185 \\ \mathrm{O} & -5.811695 & -2.038853 & 3.577979 \\ \mathrm{O} & -6.142283 & -2.058683 & 5.742034\end{array}$

$\begin{array}{rrrr}\text { TS } & & & \\ \mathrm{H} & 0.000000 & 0.000000 & 0.000000 \\ \mathrm{C} & 0.000000 & 0.000000 & 1.076001 \\ \mathrm{C} & 1.163245 & 0.000000 & 1.856196 \\ \mathrm{H} & 1.284315 & 0.818309 & 2.562872 \\ \mathrm{C} & 2.071112 & -1.060510 & 1.944694 \\ \mathrm{H} & 3.030324 & -0.842079 & 2.410829 \\ \mathrm{C} & 1.758266 & -2.417452 & 1.803625 \\ \mathrm{H} & 2.463279 & -3.126344 & 2.234202 \\ \mathrm{C} & 0.457983 & -2.889270 & 1.519505 \\ \mathrm{C} & -0.398687 & -2.187242 & 0.655308 \\ \mathrm{H} & 0.001695 & -1.906899 & -0.307431 \\ \mathrm{H} & -1.458468 & -2.442685 & 0.639089 \\ \mathrm{~N} & 0.020276 & -4.026069 & 2.192463 \\ \mathrm{~N} & -1.091824 & 0.879686 & 1.523863 \\ \mathrm{O} & -1.951538 & 1.157480 & 0.682855 \\ \mathrm{O} & -1.110236 & 1.248749 & 2.700190 \\ \mathrm{H} & -0.925539 & -4.324613 & 1.995522 \\ \mathrm{H} & 0.260481 & -4.104643 & 3.172741\end{array}$<smiles>C=C(O)/C=C\C=C\[N+](=O)[O-]</smiles>

$\begin{array}{cccc}\mathrm{H} & 0.000000 & 0.000000 & 0.000000 \\ \mathrm{C} & 0.000000 & 0.000000 & 1.086511 \\ \mathrm{H} & 0.962992 & 0.000000 & 1.581592 \\ \mathrm{C} & -1.143068 & 0.000000 & 1.801584 \\ \mathrm{C} & -1.121982 & 0.000000 & 3.258836 \\ \mathrm{H} & -0.116261 & -0.000017 & 3.670534\end{array}$




$\begin{array}{llll}\mathrm{C} & -2.147721 & 0.000018 & 4.146514 \\ \mathrm{H} & -1.873673 & 0.000014 & 5.200412 \\ \mathrm{C} & -3.564675 & 0.000043 & 3.865204 \\ \mathrm{H} & -3.907590 & 0.000065 & 2.838194 \\ \mathrm{C} & -4.482760 & 0.000039 & 4.847975 \\ \mathrm{H} & -4.286852 & 0.000018 & 5.912152 \\ \mathrm{~N} & -5.899004 & 0.000064 & 4.550404 \\ \mathrm{O} & -2.386784 & 0.000022 & 1.233190 \\ \mathrm{H} & -2.296548 & 0.000036 & 0.267080 \\ \mathrm{O} & -6.268385 & 0.000183 & 3.373076 \\ \mathrm{O} & -6.655718 & 0.000155 & 5.527502\end{array}$

TS

$\begin{array}{lrrr}\mathrm{H} & 0.000000 & 0.000000 & 0.000000 \\ \mathrm{C} & 0.000000 & 0.000000 & 1.075557 \\ \mathrm{C} & 1.162759 & 0.000000 & 1.857399 \\ \mathrm{H} & 1.281200 & 0.822329 & 2.560127 \\ \mathrm{C} & 2.070139 & -1.056662 & 1.964435 \\ \mathrm{H} & 3.021563 & -0.835803 & 2.444235 \\ \mathrm{C} & 1.753031 & -2.416446 & 1.836191 \\ \mathrm{H} & 2.445398 & -3.121156 & 2.296097 \\ \mathrm{C} & 0.470012 & -2.880522 & 1.503487 \\ \mathrm{C} & -0.382578 & -2.201391 & 0.629926 \\ \mathrm{H} & 0.030435 & -1.891164 & -0.316707 \\ \mathrm{H} & -1.429815 & -2.494070 & 0.606938 \\ \mathrm{O} & -0.046205 & -3.981798 & 2.134705 \\ \mathrm{~N} & -1.090165 & 0.883171 & 1.524506 \\ \mathrm{O} & -1.102872 & 1.254133 & 2.699699 \\ \mathrm{O} & -1.947332 & 1.163315 & 0.683095 \\ \mathrm{H} & 0.446605 & -4.145973 & 2.954263\end{array}$

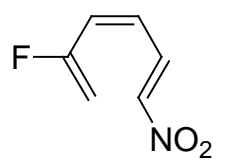

$\begin{array}{lrrr}\mathrm{H} & 0.000000 & 0.000000 & 0.000000 \\ \mathrm{H} & 0.000000 & 0.000000 & 1.863334 \\ \mathrm{C} & 0.552795 & 0.000000 & 0.931840 \\ \mathrm{C} & 1.891865 & -0.000020 & 0.936444 \\ \mathrm{C} & 2.722101 & -0.000064 & 2.124084 \\ \mathrm{H} & 2.147250 & -0.000010 & 3.046760 \\ \mathrm{C} & 4.074404 & -0.000178 & 2.230441 \\ \mathrm{H} & 4.481409 & -0.000207 & 3.239994 \\ \mathrm{C} & 5.043370 & -0.000267 & 1.158404\end{array}$




$\begin{array}{llll}\mathrm{H} & 4.711383 & -0.000246 & 0.127847 \\ \mathrm{C} & 6.365627 & -0.000378 & 1.399295 \\ \mathrm{H} & 6.843396 & -0.000413 & 2.370164 \\ \mathrm{~N} & 7.325186 & -0.000467 & 0.313642 \\ \mathrm{~F} & 2.544069 & -0.000105 & -0.251875 \\ \mathrm{O} & 6.916200 & -0.000345 & -0.849550 \\ \mathrm{O} & 8.514297 & -0.000469 & 0.647215\end{array}$

$\begin{array}{cccc}\text { TS } & & & \\ \mathrm{H} & 0.000000 & 0.000000 & 0.000000 \\ \mathrm{H} & 0.000000 & 0.000000 & 1.827126 \\ \mathrm{C} & 0.581074 & 0.000000 & 0.919381 \\ \mathrm{C} & 1.785318 & 0.697293 & 0.931770 \\ \mathrm{C} & 2.799805 & 0.552525 & 1.874408 \\ \mathrm{H} & 3.585206 & 1.303822 & 1.882518 \\ \mathrm{C} & 3.061086 & -0.660596 & 2.532182 \\ \mathrm{H} & 3.997214 & -0.707383 & 3.083986 \\ \mathrm{C} & 2.440820 & -1.875763 & 2.242932 \\ \mathrm{H} & 3.073697 & -2.761073 & 2.245056 \\ \mathrm{C} & 1.136801 & -2.034253 & 1.749572 \\ \mathrm{H} & 0.255378 & -1.824931 & 2.329664 \\ \mathrm{~N} & 0.878634 & -3.253743 & 0.963198 \\ \mathrm{~F} & 2.074150 & 1.470525 & -0.143635 \\ \mathrm{O} & -0.304431 & -3.573826 & 0.828909 \\ \mathrm{O} & 1.836707 & -3.847581 & 0.466744\end{array}$

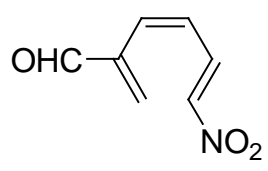

$\begin{array}{lrrl}\mathrm{H} & 0.000000 & 0.000000 & 0.000000 \\ \mathrm{C} & 0.000000 & 0.000000 & 1.081826 \\ \mathrm{C} & 1.078692 & 0.000000 & 1.882583 \\ \mathrm{H} & 0.904018 & 0.001498 & 2.954066 \\ \mathrm{C} & 2.433266 & -0.038819 & 1.380771 \\ \mathrm{H} & 2.548114 & -0.221730 & 0.313927 \\ \mathrm{C} & 3.563569 & 0.079956 & 2.116755 \\ \mathrm{H} & 4.512931 & -0.092613 & 1.615366 \\ \mathrm{C} & 3.692532 & 0.364749 & 3.549282 \\ \mathrm{C} & 2.903323 & 1.174241 & 4.283759 \\ \mathrm{H} & 3.098869 & 1.319805 & 5.343818 \\ \mathrm{H} & 2.076871 & 1.736026 & 3.862232 \\ \mathrm{C} & 4.880266 & -0.229555 & 4.232371 \\ \mathrm{~N} & -1.339690 & 0.004252 & 1.635480\end{array}$




$\begin{array}{lrrr}\mathrm{O} & -2.261840 & -0.005478 & 0.815470 \\ \mathrm{O} & -1.482703 & 0.020687 & 2.861096 \\ \mathrm{H} & 4.935043 & -0.023820 & 5.323157 \\ \mathrm{O} & 5.741402 & -0.871804 & 3.668239\end{array}$

$\begin{array}{crrr}\text { TS } & & & \\ \mathrm{H} & 0.000000 & 0.000000 & 0.000000 \\ \mathrm{H} & 0.000000 & 0.000000 & 1.819527 \\ \mathrm{C} & 0.590193 & 0.000000 & 0.916608 \\ \mathrm{C} & 1.828034 & 0.647606 & 0.932464 \\ \mathrm{C} & 2.810084 & 0.413386 & 1.910555 \\ \mathrm{H} & 3.610370 & 1.146002 & 1.984446 \\ \mathrm{C} & 3.036310 & -0.837046 & 2.496629 \\ \mathrm{H} & 3.983789 & -0.958477 & 3.016889 \\ \mathrm{C} & 2.366183 & -2.007840 & 2.123374 \\ \mathrm{H} & 2.967291 & -2.910007 & 2.020727 \\ \mathrm{C} & 1.039630 & -2.085600 & 1.686696 \\ \mathrm{H} & 0.201547 & -1.830723 & 2.311285 \\ \mathrm{~N} & 0.675286 & -3.250224 & 0.868493 \\ \mathrm{C} & 2.182331 & 1.531525 & -0.210177 \\ \mathrm{H} & 1.503922 & 1.436917 & -1.086389 \\ \mathrm{O} & 3.104273 & 2.323035 & -0.211233 \\ \mathrm{O} & 1.574816 & -3.889320 & 0.320686 \\ \mathrm{O} & -0.530774 & -3.482708 & 0.760706\end{array}$

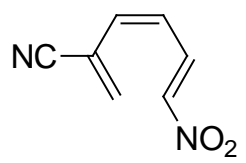

$\begin{array}{lrrl}\mathrm{H} & 0.000000 & 0.000000 & 0.000000 \\ \mathrm{C} & 0.000000 & 0.000000 & 1.084087 \\ \mathrm{H} & 0.962710 & 0.000000 & 1.586374 \\ \mathrm{C} & -1.150424 & 0.000000 & 1.794880 \\ \mathrm{C} & -1.129276 & 0.000000 & 3.258158 \\ \mathrm{H} & -0.117294 & 0.000000 & 3.657298 \\ \mathrm{C} & -2.132204 & 0.000000 & 4.170144 \\ \mathrm{H} & -1.819894 & 0.000000 & 5.213044 \\ \mathrm{C} & -3.562822 & 0.000000 & 3.956439 \\ \mathrm{H} & -3.982869 & 0.000000 & 2.956307 \\ \mathrm{C} & -4.415405 & 0.000000 & 4.994759 \\ \mathrm{H} & -4.148268 & -0.000015 & 6.043466 \\ \mathrm{~N} & -5.852283 & 0.000000 & 4.793842 \\ \mathrm{C} & -2.386913 & 0.000000 & 1.055970 \\ \mathrm{~N} & -3.368286 & 0.000001 & 0.432619\end{array}$




$\begin{array}{llll}\mathrm{O} & -6.296169 & 0.000244 & 3.645274 \\ \mathrm{O} & -6.535398 & 0.000253 & 5.822520\end{array}$

TS

$\begin{array}{lrrr}\mathrm{H} & 0.000000 & 0.000000 & 0.000000 \\ \mathrm{H} & 0.000000 & 0.000000 & 1.827413 \\ \mathrm{C} & 0.581003 & 0.000000 & 0.918453 \\ \mathrm{C} & 1.823359 & 0.643160 & 0.960286 \\ \mathrm{C} & 2.755564 & 0.411656 & 1.994388 \\ \mathrm{H} & 3.560154 & 1.130443 & 2.127620 \\ \mathrm{C} & 2.918904 & -0.831193 & 2.614859 \\ \mathrm{H} & 3.816115 & -0.944702 & 3.218850 \\ \mathrm{C} & 2.280023 & -2.009144 & 2.207312 \\ \mathrm{H} & 2.893787 & -2.907575 & 2.164079 \\ \mathrm{C} & 0.995706 & -2.105477 & 1.666235 \\ \mathrm{H} & 0.099584 & -1.862978 & 2.209626 \\ \mathrm{C} & 2.243511 & 1.448780 & -0.150544 \\ \mathrm{~N} & 0.719220 & -3.278283 & 0.819142 \\ \mathrm{O} & -0.469346 & -3.516521 & 0.598574 \\ \mathrm{O} & 1.671108 & -3.914320 & 0.366613 \\ \mathrm{~N} & 2.577722 & 2.121615 & -1.039795\end{array}$<smiles>C=C(/C=C\C=C/[N+](=O)[O-])[N+](=O)[O-]</smiles>

$\begin{array}{llll}\mathrm{H} & 0.000000 & 0.000000 & 0.000000\end{array}$

$\begin{array}{llll}\text { C } & 0.000000 & 0.000000 & 1.081923\end{array}$

$\begin{array}{llll}\mathrm{C} & 1.077157 & 0.000000 & 1.883087\end{array}$

$\begin{array}{llll}\mathrm{H} & 0.904442 & 0.000891 & 2.955033\end{array}$

$\begin{array}{llll}\text { C } & 2.430500 & -0.033099 & 1.374983\end{array}$

$\begin{array}{llll}\mathrm{H} & 2.543448 & -0.199215 & 0.305671\end{array}$

$\begin{array}{llll}\text { C } & 3.564578 & 0.081466 & 2.104376\end{array}$

$\begin{array}{llll}\mathrm{H} & 4.514630 & -0.071959 & 1.603695\end{array}$

$\begin{array}{llll}\text { C } & 3.653475 & 0.384958 & 3.531267\end{array}$

$\begin{array}{llll}\text { C } & 2.925013 & 1.244338 & 4.252596\end{array}$

$\begin{array}{llll}\mathrm{H} & 3.118597 & 1.373948 & 5.310516\end{array}$

$\begin{array}{llll}\mathrm{H} & 2.149005 & 1.841368 & 3.788385\end{array}$

$\begin{array}{llll}\mathrm{N} & 4.761255 & -0.326525 & 4.244466\end{array}$

$\begin{array}{llll}\mathrm{N} & -1.341725 & 0.002391 & 1.636256\end{array}$

$\begin{array}{llll}\text { O } & -1.482430 & 0.021852 & 2.861257\end{array}$

$\begin{array}{llll}\text { O } & -2.261769 & -0.011733 & 0.815115\end{array}$

$\begin{array}{llll}\text { O } & 5.461541 & -1.074016 & 3.563590\end{array}$

$\begin{array}{llll}\text { O } & 4.904463 & -0.127179 & 5.445560\end{array}$ 


$\begin{array}{rrrr}\text { TS } & & & \\ \mathrm{H} & 0.000000 & 0.000000 & 0.000000 \\ \mathrm{C} & 0.000000 & 0.000000 & 1.075712 \\ \mathrm{C} & 1.166894 & 0.000000 & 1.844056 \\ \mathrm{H} & 1.261979 & 0.793678 & 2.583544 \\ \mathrm{C} & 2.124256 & -1.020137 & 1.891478 \\ \mathrm{H} & 3.077310 & -0.779041 & 2.356009 \\ \mathrm{C} & 1.829293 & -2.383157 & 1.772983 \\ \mathrm{H} & 2.534568 & -3.086716 & 2.201750 \\ \mathrm{C} & 0.549114 & -2.852783 & 1.456617 \\ \mathrm{C} & -0.365167 & -2.201687 & 0.638400 \\ \mathrm{H} & -0.010480 & -1.873824 & -0.325685 \\ \mathrm{H} & -1.412384 & -2.481395 & 0.690407 \\ \mathrm{~N} & 0.077249 & -4.038978 & 2.212797 \\ \mathrm{~N} & -1.101728 & 0.859025 & 1.541705 \\ \mathrm{O} & -1.985915 & 1.096957 & 0.717924 \\ \mathrm{O} & -1.086192 & 1.251238 & 2.708365 \\ \mathrm{O} & -1.133120 & -4.245445 & 2.250776 \\ \mathrm{O} & 0.931342 & -4.752279 & 2.736580\end{array}$

\section{1, 6-subtituted hexatriene}

1<smiles>C/C=C\C=C/C</smiles>

$\begin{array}{lrrr}\mathrm{H} & 0.000000 & 0.000000 & 0.000000 \\ \mathrm{C} & 0.000000 & 0.000000 & 1.091562 \\ \mathrm{C} & 1.183066 & 0.000000 & 1.733513 \\ \mathrm{H} & 1.180080 & -0.000017 & 2.823142 \\ \mathrm{C} & 2.466436 & -0.000105 & 1.056955 \\ \mathrm{H} & 2.419601 & 0.000518 & -0.031882 \\ \mathrm{C} & 3.696123 & -0.001017 & 1.629087 \\ \mathrm{H} & 4.559189 & -0.000931 & 0.963597 \\ \mathrm{C} & 4.005208 & -0.001992 & 3.046563 \\ \mathrm{H} & 3.173626 & -0.002719 & 3.750673 \\ \mathrm{C} & 5.258273 & -0.001929 & 3.538063 \\ \mathrm{H} & 6.093256 & -0.001184 & 2.834993 \\ \mathrm{C} & -1.343951 & -0.000470 & 1.754646 \\ \mathrm{C} & 5.616685 & -0.002339 & 4.993201 \\ \mathrm{H} & 6.219974 & 0.877989 & 5.256460 \\ \mathrm{H} & 4.724886 & -0.003325 & 5.629034\end{array}$




$\begin{array}{rrrr}\mathrm{H} & 6.221744 & -0.881548 & 5.255873 \\ \mathrm{H} & -1.255922 & -0.000200 & 2.846361 \\ \mathrm{H} & -1.934876 & 0.878751 & 1.461596 \\ \mathrm{H} & -1.933626 & -0.880785 & 1.462127\end{array}$

$\begin{array}{crrr}\text { TS } & & & \\ \mathrm{H} & 0.000000 & 0.000000 & 0.000000 \\ \mathrm{C} & 0.000000 & 0.000000 & 1.080841 \\ \mathrm{C} & 1.270983 & 0.000000 & 1.688376 \\ \mathrm{H} & 1.452542 & -0.731625 & 2.480649 \\ \mathrm{C} & 2.256676 & 0.966102 & 1.511808 \\ \mathrm{H} & 3.252110 & 0.743309 & 1.894171 \\ \mathrm{C} & 1.992151 & 2.326446 & 1.249012 \\ \mathrm{H} & 2.810240 & 3.015597 & 1.455234 \\ \mathrm{C} & 0.713981 & 2.864371 & 1.134904 \\ \mathrm{H} & 0.562645 & 3.844158 & 1.596477 \\ \mathrm{C} & -0.425038 & 2.186187 & 0.658267 \\ \mathrm{H} & -0.349285 & 1.797632 & -0.347471 \\ \mathrm{C} & -1.807592 & 2.687406 & 1.007815 \\ \mathrm{C} & -1.128335 & -0.805588 & 1.683108 \\ \mathrm{H} & -1.052643 & -0.853072 & 2.774678 \\ \mathrm{H} & -1.137911 & -1.835633 & 1.297201 \\ \mathrm{H} & -2.104067 & -0.370108 & 1.436526 \\ \mathrm{H} & -2.540052 & 1.871345 & 1.001901 \\ \mathrm{H} & -1.831145 & 3.147013 & 2.001653 \\ \mathrm{H} & -2.161595 & 3.432070 & 0.279690\end{array}$<smiles>C/C=C\C=C/C=C\N</smiles>

$\begin{array}{lrrr}\mathrm{H} & 0.000000 & 0.000000 & 0.000000 \\ \mathrm{C} & 0.000000 & 0.000000 & 1.091585 \\ \mathrm{C} & 1.183198 & 0.000000 & 1.734517 \\ \mathrm{H} & 1.173759 & -0.000622 & 2.824913 \\ \mathrm{C} & 2.473042 & -0.000773 & 1.073022 \\ \mathrm{H} & 2.444049 & -0.005102 & -0.016067 \\ \mathrm{C} & 3.694803 & 0.001102 & 1.666960 \\ \mathrm{H} & 4.564405 & -0.004861 & 1.008733 \\ \mathrm{C} & 3.994923 & 0.013991 & 3.079519 \\ \mathrm{H} & 3.170820 & 0.017186 & 3.792013 \\ \mathrm{C} & 5.261934 & 0.015207 & 3.554266 \\ \mathrm{H} & 6.101416 & 0.011679 & 2.860878 \\ \mathrm{~N} & 5.640198 & -0.064724 & 4.887218\end{array}$




$\begin{array}{rrrr}\mathrm{C} & -1.344300 & -0.000877 & 1.755833 \\ \mathrm{H} & 4.913736 & 0.150799 & 5.560368 \\ \mathrm{H} & 6.516913 & 0.383525 & 5.121806 \\ \mathrm{H} & -1.936052 & -0.881254 & 1.466677 \\ \mathrm{H} & -1.253590 & -0.001052 & 2.847721 \\ \mathrm{H} & -1.937521 & 0.878563 & 1.466767 \\ & & & \\ \mathrm{TS} & & & \\ \mathrm{H} & 0.000000 & 0.000000 & 0.000000 \\ \mathrm{C} & 0.000000 & 0.000000 & 1.083666 \\ \mathrm{C} & 1.280937 & 0.000000 & 1.656576 \\ \mathrm{H} & 1.475258 & 0.641032 & 2.520767 \\ \mathrm{C} & 2.279027 & -0.883734 & 1.262848 \\ \mathrm{H} & 3.304404 & -0.693962 & 1.575826 \\ \mathrm{C} & 2.004465 & -2.175249 & 0.766333 \\ \mathrm{H} & 2.865415 & -2.836799 & 0.673058 \\ \mathrm{C} & 0.760500 & -2.793162 & 0.701042 \\ \mathrm{H} & 0.753465 & -3.870849 & 0.881680 \\ \mathrm{C} & -0.524052 & -2.181459 & 0.671544 \\ \mathrm{H} & -0.855356 & -1.681704 & -0.225597 \\ \mathrm{~N} & -1.577025 & -2.855192 & 1.298284 \\ \mathrm{C} & -1.151513 & 0.744149 & 1.715469 \\ \mathrm{H} & -2.486905 & -2.421976 & 1.206533 \\ \mathrm{H} & -1.396254 & -3.138802 & 2.253495 \\ \mathrm{H} & -2.102056 & 0.210022 & 1.577957 \\ \mathrm{H} & -1.287922 & 1.744214 & 1.279256 \\ \mathrm{H} & -1.003286 & 0.865593 & 2.794490\end{array}$<smiles>C/C=C\C=C/C=C\CO</smiles>

$\begin{array}{lrrr}\mathrm{H} & 0.000000 & 0.000000 & 0.000000 \\ \mathrm{C} & 0.000000 & 0.000000 & 1.091492 \\ \mathrm{C} & 1.183195 & 0.000000 & 1.733487 \\ \mathrm{H} & 1.175717 & -0.000017 & 2.823753 \\ \mathrm{C} & 2.470416 & 0.000168 & 1.065486 \\ \mathrm{H} & 2.434963 & 0.000196 & -0.023303 \\ \mathrm{C} & 3.694842 & 0.000178 & 1.649612 \\ \mathrm{H} & 4.561965 & 0.000232 & 0.989495 \\ \mathrm{C} & 3.995712 & 0.000004 & 3.065458 \\ \mathrm{H} & 3.171422 & -0.000373 & 3.778776 \\ \mathrm{C} & 5.257242 & 0.000290 & 3.537853 \\ \mathrm{H} & 6.126595 & 0.000588 & 2.886168\end{array}$




$\begin{array}{rrrr}\mathrm{O} & 5.636936 & 0.000198 & 4.842983 \\ \mathrm{C} & -1.343849 & 0.000043 & 1.755547 \\ \mathrm{H} & 4.842468 & -0.000097 & 5.402547 \\ \mathrm{H} & -1.254467 & -0.000512 & 2.847386 \\ \mathrm{H} & -1.935419 & -0.879473 & 1.464231 \\ \mathrm{H} & -1.935049 & 0.880120 & 1.465121 \\ & & & \\ \mathrm{TS} & & & \\ \mathrm{H} & 0.000000 & 0.000000 & 0.000000 \\ \mathrm{C} & 0.000000 & 0.000000 & 1.077864 \\ \mathrm{C} & 1.199420 & 0.000000 & 1.823885 \\ \mathrm{H} & 1.341680 & -0.864907 & 2.479083 \\ \mathrm{C} & 2.098238 & 1.053379 & 1.995562 \\ \mathrm{H} & 3.032026 & 0.804375 & 2.498203 \\ \mathrm{C} & 1.799165 & 2.423855 & 1.899735 \\ \mathrm{H} & 2.491769 & 3.104728 & 2.391473 \\ \mathrm{C} & 0.546267 & 2.899427 & 1.516698 \\ \mathrm{H} & 0.161081 & 3.786557 & 2.025811 \\ \mathrm{C} & -0.292143 & 2.209587 & 0.633213 \\ \mathrm{H} & 0.196716 & 1.870360 & -0.271950 \\ \mathrm{C} & -1.754600 & 2.547887 & 0.481120 \\ \mathrm{O} & -1.057177 & -0.767116 & 1.504682 \\ \mathrm{H} & -1.029242 & -0.808171 & 2.474563 \\ \mathrm{H} & -2.128659 & 3.091913 & 1.355717 \\ \mathrm{H} & -2.359746 & 1.638654 & 0.377740 \\ \mathrm{H} & -1.942400 & 3.164524 & -0.408946\end{array}$

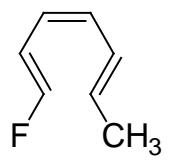

$\begin{array}{lrrr}\mathrm{H} & 0.000000 & 0.000000 & 0.000000 \\ \mathrm{C} & 0.000000 & 0.000000 & 1.091469 \\ \mathrm{C} & 1.183011 & 0.000000 & 1.733337 \\ \mathrm{H} & 1.180348 & 0.000033 & 2.822982 \\ \mathrm{C} & 2.465397 & -0.000021 & 1.055867 \\ \mathrm{H} & 2.420763 & -0.000072 & -0.032685 \\ \mathrm{C} & 3.694376 & -0.000041 & 1.627905 \\ \mathrm{H} & 4.557968 & -0.000089 & 0.963882 \\ \mathrm{C} & 3.990272 & -0.000067 & 3.046628 \\ \mathrm{H} & 3.179802 & -0.000054 & 3.770534 \\ \mathrm{C} & 5.242319 & -0.000086 & 3.517391 \\ \mathrm{H} & 6.146933 & -0.000069 & 2.915479 \\ \mathrm{~F} & 5.505591 & 0.000011 & 4.837933\end{array}$




$\begin{array}{lrrr}\mathrm{C} & -1.343270 & -0.000085 & 1.755384 \\ \mathrm{H} & -1.933072 & 0.879817 & 1.462533 \\ \mathrm{H} & -1.933142 & -0.879824 & 1.462135 \\ \mathrm{H} & -1.254580 & -0.000380 & 2.846976\end{array}$

$\begin{array}{cccc}\text { TS } & & & \\ \mathrm{H} & 0.000000 & 0.000000 & 0.000000 \\ \mathrm{C} & 0.000000 & 0.000000 & 1.078771 \\ \mathrm{C} & 1.164629 & 0.000000 & 1.853486 \\ \mathrm{H} & 1.270437 & -0.844958 & 2.536344 \\ \mathrm{C} & 2.071574 & 1.052588 & 2.007747 \\ \mathrm{H} & 3.005011 & 0.810972 & 2.513336 \\ \mathrm{C} & 1.760302 & 2.417691 & 1.909097 \\ \mathrm{H} & 2.450175 & 3.103101 & 2.398534 \\ \mathrm{C} & 0.501674 & 2.891172 & 1.535800 \\ \mathrm{H} & 0.114902 & 3.766080 & 2.063266 \\ \mathrm{C} & -0.338242 & 2.210042 & 0.650805 \\ \mathrm{H} & 0.146005 & 1.873029 & -0.257609 \\ \mathrm{C} & -1.799485 & 2.553842 & 0.500268 \\ \mathrm{~F} & -0.998092 & -0.817369 & 1.523463 \\ \mathrm{H} & -2.390077 & 1.666440 & 0.245125 \\ \mathrm{H} & -2.209223 & 2.967086 & 1.427660 \\ \mathrm{H} & -1.959194 & 3.289120 & -0.301172\end{array}$<smiles>C/C=C\C=C/C=C\C=O</smiles>

$\begin{array}{lrrr}\mathrm{H} & 0.000000 & 0.000000 & 0.000000 \\ \mathrm{C} & 0.000000 & 0.000000 & 1.091339 \\ \mathrm{C} & 1.185132 & 0.000000 & 1.732111 \\ \mathrm{H} & 1.188230 & 0.000033 & 2.821102 \\ \mathrm{C} & 2.455567 & -0.000042 & 1.038756 \\ \mathrm{H} & 2.391356 & 0.000355 & -0.049102 \\ \mathrm{C} & 3.700607 & -0.000516 & 1.586457 \\ \mathrm{H} & 4.552364 & -0.000402 & 0.908672 \\ \mathrm{C} & 4.014667 & -0.001204 & 2.993093 \\ \mathrm{H} & 3.186226 & -0.001336 & 3.701549 \\ \mathrm{C} & 5.271171 & -0.001759 & 3.499938 \\ \mathrm{H} & 6.147893 & -0.001686 & 2.855309 \\ \mathrm{C} & 5.509683 & -0.002442 & 4.942081 \\ \mathrm{C} & -1.341952 & 0.000043 & 1.754136 \\ \mathrm{H} & 4.584718 & -0.002567 & 5.564757 \\ \mathrm{O} & 6.611969 & -0.002892 & 5.464141\end{array}$




$\begin{array}{rrrr}\mathrm{H} & -1.929409 & 0.879241 & 1.455603 \\ \mathrm{H} & -1.928961 & -0.879864 & 1.456855 \\ \mathrm{H} & -1.256726 & 0.000855 & 2.845558 \\ & & & \\ \mathrm{TS} & & & \\ \mathrm{H} & 0.000000 & 0.000000 & 0.000000 \\ \mathrm{C} & 0.000000 & 0.000000 & 1.081254 \\ \mathrm{C} & 1.279667 & 0.000000 & 1.675717 \\ \mathrm{H} & 1.460151 & 0.705517 & 2.489699 \\ \mathrm{C} & 2.277281 & -0.929511 & 1.422809 \\ \mathrm{H} & 3.275023 & -0.710348 & 1.800595 \\ \mathrm{C} & 2.046378 & -2.283325 & 1.071457 \\ \mathrm{H} & 2.898786 & -2.951934 & 1.177680 \\ \mathrm{C} & 0.785359 & -2.853742 & 1.019254 \\ \mathrm{H} & 0.703036 & -3.877307 & 1.393307 \\ \mathrm{C} & -0.422685 & -2.171679 & 0.713181 \\ \mathrm{H} & -0.559981 & -1.763492 & -0.277045 \\ \mathrm{C} & -1.163128 & 0.731874 & 1.695142 \\ \mathrm{C} & -1.674135 & -2.635248 & 1.330558 \\ \mathrm{H} & -1.553947 & -3.421444 & 2.112450 \\ \mathrm{O} & -2.778290 & -2.177573 & 1.078301 \\ \mathrm{H} & -1.061149 & 0.816107 & 2.782047 \\ \mathrm{H} & -2.100056 & 0.205424 & 1.478154 \\ \mathrm{H} & -1.254698 & 1.743771 & 1.275807\end{array}$

\begin{tabular}{lrrr} 
& $=$ & & \\
$\mathrm{NC}$ & \multicolumn{3}{l}{$\mathrm{CH}_{3}$} \\
$\mathrm{H}$ & & \\
$\mathrm{H}$ & 0.000000 & 0.000000 & 0.000000 \\
$\mathrm{C}$ & 0.000000 & 0.000000 & 1.091338 \\
$\mathrm{C}$ & 1.184953 & 0.000000 & 1.732307 \\
$\mathrm{H}$ & 1.187633 & 0.000017 & 2.821219 \\
$\mathrm{C}$ & 2.455210 & 0.000042 & 1.038590 \\
$\mathrm{H}$ & 2.391555 & -0.000235 & -0.049198 \\
$\mathrm{C}$ & 3.699931 & 0.000291 & 1.585839 \\
$\mathrm{H}$ & 4.552972 & 0.000183 & 0.909339 \\
$\mathrm{C}$ & 4.009378 & 0.000648 & 2.993519 \\
$\mathrm{H}$ & 3.188454 & 0.000783 & 3.706131 \\
$\mathrm{C}$ & 5.275341 & 0.000864 & 3.478843 \\
$\mathrm{H}$ & 6.129533 & 0.000757 & 2.805114 \\
$\mathrm{C}$ & 5.567620 & 0.001258 & 4.870744 \\
$\mathrm{C}$ & -1.341605 & -0.000043 & 1.754750 \\
$\mathrm{H}$ & -1.928506 & -0.879922 & 1.457301
\end{tabular}




$\begin{array}{rrrr}\mathrm{H} & -1.928932 & 0.879297 & 1.456584 \\ \mathrm{H} & -1.255678 & 0.000369 & 2.846080 \\ \mathrm{~N} & 5.822942 & 0.001540 & 6.008045 \\ & & & \\ \mathrm{TS} & & & \\ \mathrm{H} & 0.000000 & 0.000000 & 0.000000 \\ \mathrm{C} & 0.000000 & 0.000000 & 1.080592 \\ \mathrm{C} & 1.262112 & 0.000000 & 1.707906 \\ \mathrm{H} & 1.418405 & 0.740037 & 2.496889 \\ \mathrm{C} & 2.264746 & -0.954315 & 1.574519 \\ \mathrm{H} & 3.239659 & -0.704083 & 1.990346 \\ \mathrm{C} & 2.047555 & -2.331310 & 1.335153 \\ \mathrm{H} & 2.873276 & -2.993012 & 1.589315 \\ \mathrm{C} & 0.791284 & -2.897105 & 1.186001 \\ \mathrm{H} & 0.620738 & -3.877686 & 1.629402 \\ \mathrm{C} & -0.313413 & -2.199627 & 0.635828 \\ \mathrm{H} & -0.198217 & -1.833995 & -0.374294 \\ \mathrm{C} & -1.650113 & -2.620466 & 0.930352 \\ \mathrm{C} & -1.168652 & 0.728554 & 1.694461 \\ \mathrm{~N} & -2.754092 & -2.887891 & 1.190874 \\ \mathrm{H} & -2.098054 & 0.167845 & 1.539515 \\ \mathrm{H} & -1.308378 & 1.715375 & 1.231614 \\ \mathrm{H} & -1.038177 & 0.864531 & 2.772693\end{array}$<smiles>C/C=C\C=C/C=C\[N+](=O)[O-]</smiles>

$\begin{array}{lrrr}\mathrm{H} & 0.000000 & 0.000000 & 0.000000 \\ \mathrm{C} & 0.000000 & 0.000000 & 1.081654 \\ \mathrm{C} & 1.084221 & 0.000000 & 1.879843 \\ \mathrm{H} & 0.902215 & -0.000017 & 2.950466 \\ \mathrm{C} & 2.434127 & 0.000021 & 1.380792 \\ \mathrm{H} & 2.554559 & 0.000122 & 0.299449 \\ \mathrm{C} & 3.558645 & -0.000017 & 2.147081 \\ \mathrm{H} & 4.514494 & 0.000108 & 1.623898 \\ \mathrm{C} & 3.643216 & -0.000105 & 3.590569 \\ \mathrm{H} & 2.719295 & -0.000431 & 4.166174 \\ \mathrm{C} & 4.817709 & 0.000181 & 4.251579 \\ \mathrm{H} & 5.740953 & 0.000474 & 3.669709 \\ \mathrm{C} & 4.971150 & 0.000110 & 5.739704 \\ \mathrm{~N} & -1.334360 & -0.000065 & 1.634828 \\ \mathrm{O} & -1.479692 & -0.000027 & 2.861821 \\ \mathrm{O} & -2.260361 & -0.000078 & 0.816435\end{array}$




$\begin{array}{rrrr}\mathrm{H} & 4.002390 & -0.000084 & 6.249092 \\ \mathrm{H} & 5.536817 & 0.879669 & 6.076369 \\ \mathrm{H} & 5.537083 & -0.879340 & 6.076223 \\ & & & \\ \mathrm{TS} & & & \\ \mathrm{H} & 0.000000 & 0.000000 & 0.000000 \\ \mathrm{C} & 0.000000 & 0.000000 & 1.075799 \\ \mathrm{C} & 1.182950 & 0.000000 & 1.840440 \\ \mathrm{H} & 1.336066 & -0.844457 & 2.510622 \\ \mathrm{C} & 2.062173 & 1.070630 & 1.950571 \\ \mathrm{H} & 3.014351 & 0.877359 & 2.439971 \\ \mathrm{C} & 1.673076 & 2.425649 & 1.868840 \\ \mathrm{H} & 2.336426 & 3.132302 & 2.365118 \\ \mathrm{C} & 0.394201 & 2.861420 & 1.538279 \\ \mathrm{H} & 0.005190 & 3.722368 & 2.084863 \\ \mathrm{C} & -0.472855 & 2.180061 & 0.672165 \\ \mathrm{H} & -0.035055 & 1.876113 & -0.269404 \\ \mathrm{C} & -1.951240 & 2.450895 & 0.622129 \\ \mathrm{~N} & -1.088869 & -0.858051 & 1.533677 \\ \mathrm{O} & -1.047781 & -1.321899 & 2.675459 \\ \mathrm{O} & -2.025568 & -1.022161 & 0.740158 \\ \mathrm{H} & -2.319137 & 2.859608 & 1.568346 \\ \mathrm{H} & -2.191472 & 3.160791 & -0.181536 \\ \mathrm{H} & -2.501199 & 1.526579 & 0.410608\end{array}$<smiles>N/C=C\C=C/C=C\N</smiles>

$\begin{array}{lrrr}\mathrm{H} & 0.000000 & 0.000000 & 0.000000 \\ \mathrm{C} & 0.000000 & 0.000000 & 1.088904 \\ \mathrm{C} & 1.170694 & 0.000000 & 1.764641 \\ \mathrm{H} & 1.139494 & 0.000100 & 2.854351 \\ \mathrm{C} & 2.460290 & 0.005558 & 1.110880 \\ \mathrm{H} & 2.431588 & 0.002089 & 0.021069 \\ \mathrm{C} & 3.686205 & 0.008240 & 1.697226 \\ \mathrm{H} & 4.552616 & -0.000542 & 1.035558 \\ \mathrm{C} & 3.986802 & 0.028264 & 3.111311 \\ \mathrm{H} & 3.158159 & 0.041018 & 3.819576 \\ \mathrm{C} & 5.247703 & 0.026766 & 3.598478 \\ \mathrm{H} & 6.095271 & 0.013999 & 2.914996\end{array}$




$\begin{array}{rrrr}\mathrm{N} & 5.613178 & -0.051801 & 4.941905 \\ \mathrm{~N} & -1.274749 & 0.092057 & 1.646783 \\ \mathrm{H} & -1.998541 & -0.400562 & 1.136549 \\ \mathrm{H} & -1.318030 & -0.122638 & 2.637233 \\ \mathrm{H} & 4.870781 & 0.174867 & 5.594893 \\ \mathrm{H} & 6.467737 & 0.438372 & 5.179204\end{array}$

TS

$\begin{array}{lrrr}\mathrm{H} & 0.000000 & 0.000000 & 0.000000 \\ \mathrm{C} & 0.000000 & 0.000000 & 1.080444 \\ \mathrm{C} & 1.262644 & 0.000000 & 1.722767 \\ \mathrm{H} & 1.456331 & 0.809024 & 2.431631 \\ \mathrm{C} & 2.184630 & -1.035527 & 1.668041 \\ \mathrm{H} & 3.181112 & -0.834332 & 2.059808 \\ \mathrm{C} & 1.867431 & -2.396652 & 1.465457 \\ \mathrm{H} & 2.647896 & -3.110770 & 1.724885 \\ \mathrm{C} & 0.585964 & -2.898245 & 1.275235 \\ \mathrm{H} & 0.362489 & -3.876456 & 1.713547 \\ \mathrm{C} & -0.498251 & -2.178414 & 0.720921 \\ \mathrm{H} & -0.411330 & -1.852673 & -0.305345 \\ \mathrm{~N} & -1.833579 & -2.462858 & 1.082635 \\ \mathrm{~N} & -1.044822 & 0.775714 & 1.609641 \\ \mathrm{H} & -1.172218 & 0.626207 & 2.605571 \\ \mathrm{H} & -1.928755 & 0.604269 & 1.141923 \\ \mathrm{H} & -1.907517 & -3.085043 & 1.882178 \\ \mathrm{H} & -2.420980 & -2.795660 & 0.322770\end{array}$

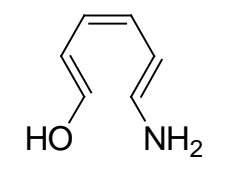

$\begin{array}{lrrr}\mathrm{H} & 0.000000 & 0.000000 & 0.000000 \\ \mathrm{C} & 0.000000 & 0.000000 & 1.086393 \\ \mathrm{C} & 1.132779 & 0.000000 & 1.814294 \\ \mathrm{H} & 1.048595 & -0.000218 & 2.901905 \\ \mathrm{C} & 2.454423 & -0.002455 & 1.222182 \\ \mathrm{H} & 2.480999 & -0.009314 & 0.133148 \\ \mathrm{C} & 3.645519 & 0.000089 & 1.874009 \\ \mathrm{H} & 4.545243 & -0.008744 & 1.258547 \\ \mathrm{C} & 3.873396 & 0.017091 & 3.301178 \\ \mathrm{H} & 3.012080 & 0.025575 & 3.969139 \\ \mathrm{C} & 5.110855 & 0.015406 & 3.846594 \\ \mathrm{H} & 5.988232 & 0.006010 & 3.201938 \\ \mathrm{~N} & 5.413911 & -0.064205 & 5.201676\end{array}$




$\begin{array}{rrrc}\mathrm{O} & -1.276447 & -0.000095 & 1.565847 \\ \mathrm{H} & -1.244103 & -0.003490 & 2.536801 \\ \mathrm{H} & 4.648360 & 0.159187 & 5.827738 \\ \mathrm{H} & 6.269428 & 0.399793 & 5.481501\end{array}$

$\begin{array}{rrrr}\text { TS } & & & \\ \mathrm{H} & 0.000000 & 0.000000 & 0.000000 \\ \mathrm{C} & 0.000000 & 0.000000 & 1.079270 \\ \mathrm{C} & 1.225004 & 0.000000 & 1.778170 \\ \mathrm{H} & 1.401799 & -0.840221 & 2.457641 \\ \mathrm{C} & 2.136144 & 1.050082 & 1.810407 \\ \mathrm{H} & 3.115033 & 0.840877 & 2.238968 \\ \mathrm{C} & 1.814516 & 2.412021 & 1.637582 \\ \mathrm{H} & 2.571208 & 3.122885 & 1.966531 \\ \mathrm{C} & 0.544458 & 2.910913 & 1.382845 \\ \mathrm{H} & 0.280880 & 3.874481 & 1.825057 \\ \mathrm{C} & -0.489650 & 2.200933 & 0.730551 \\ \mathrm{H} & -0.296639 & 1.883183 & -0.283801 \\ \mathrm{~N} & -1.824657 & 2.593911 & 0.918745 \\ \mathrm{O} & -1.046465 & -0.788226 & 1.519030 \\ \mathrm{H} & -0.974407 & -0.866228 & 2.483847 \\ \mathrm{H} & -2.080604 & 2.702776 & 1.893958 \\ \mathrm{H} & -2.493009 & 1.987842 & 0.457181\end{array}$<smiles>N/C=C\C=C/C=C\F</smiles>

$\begin{array}{cccc}\mathrm{H} & 0.000000 & 0.000000 & 0.000000 \\ \mathrm{C} & 0.000000 & 0.000000 & 1.086386 \\ \mathrm{C} & 1.084117 & 0.000000 & 1.870349 \\ \mathrm{H} & 0.921130 & -0.000675 & 2.945383 \\ \mathrm{C} & 2.436252 & -0.000212 & 1.350825 \\ \mathrm{H} & 2.531938 & -0.004063 & 0.266106 \\ \mathrm{C} & 3.580471 & 0.002178 & 2.082131 \\ \mathrm{H} & 4.519054 & -0.002725 & 1.527381 \\ \mathrm{C} & 3.713771 & 0.014071 & 3.519165 \\ \mathrm{H} & 2.812709 & 0.016055 & 4.131405 \\ \mathrm{C} & 4.917801 & 0.015527 & 4.137502 \\ \mathrm{H} & 5.831475 & 0.013564 & 3.545452 \\ \mathrm{~N} & 5.138972 & -0.063807 & 5.503119 \\ \mathrm{~F} & -1.249315 & 0.000040 & 1.599048 \\ \mathrm{H} & 4.342019 & 0.147551 & 6.092164 \\ \mathrm{H} & 5.988737 & 0.369828 & 5.840281\end{array}$




$\begin{array}{cccc}\text { TS } & & & \\ \mathrm{H} & 0.000000 & 0.000000 & 0.000000 \\ \mathrm{C} & 0.000000 & 0.000000 & 1.079505 \\ \mathrm{C} & 1.250391 & 0.000000 & 1.743706 \\ \mathrm{H} & 1.405067 & 0.799095 & 2.471904 \\ \mathrm{C} & 2.214102 & -0.999855 & 1.696959 \\ \mathrm{H} & 3.185587 & -0.755851 & 2.124814 \\ \mathrm{C} & 1.984661 & -2.368522 & 1.455329 \\ \mathrm{H} & 2.791649 & -3.046290 & 1.726625 \\ \mathrm{C} & 0.733834 & -2.925120 & 1.208255 \\ \mathrm{H} & 0.508511 & -3.913141 & 1.613370 \\ \mathrm{C} & -0.315778 & -2.230158 & 0.601859 \\ \mathrm{H} & -0.225024 & -1.873834 & -0.414844 \\ \mathrm{~F} & -1.575622 & -2.719644 & 0.815209 \\ \mathrm{~N} & -1.046438 & 0.759738 & 1.607017 \\ \mathrm{H} & -1.216354 & 0.610763 & 2.594656 \\ \mathrm{H} & -1.911473 & 0.710068 & 1.083610\end{array}$

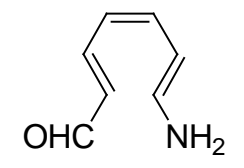

$\begin{array}{lrrr}\mathrm{H} & 0.000000 & 0.000000 & 0.000000 \\ \mathrm{C} & 0.000000 & 0.000000 & 1.088415 \\ \mathrm{C} & 1.179596 & 0.000000 & 1.762692 \\ \mathrm{H} & 1.160939 & -0.000650 & 2.851136 \\ \mathrm{C} & 2.440630 & 0.005173 & 1.078639 \\ \mathrm{H} & 2.376840 & 0.007496 & -0.010149 \\ \mathrm{C} & 3.696368 & 0.004040 & 1.616453 \\ \mathrm{H} & 4.539780 & 0.002898 & 0.928942 \\ \mathrm{C} & 4.016214 & 0.005345 & 3.015401 \\ \mathrm{H} & 3.187319 & 0.010590 & 3.724783 \\ \mathrm{C} & 5.272032 & 0.001130 & 3.533217 \\ \mathrm{H} & 6.152522 & -0.004064 & 2.893729 \\ \mathrm{C} & 5.497249 & 0.003096 & 4.972337 \\ \mathrm{~N} & -1.253138 & 0.066939 & 1.644749 \\ \mathrm{H} & -2.032129 & -0.266297 & 1.095161 \\ \mathrm{H} & -1.339707 & -0.117792 & 2.635849 \\ \mathrm{H} & 4.563624 & 0.008903 & 5.584201 \\ \mathrm{O} & 6.592532 & -0.001288 & 5.513497\end{array}$

TS

$\begin{array}{llll}\mathrm{H} & 0.000000 & 0.000000 & 0.000000\end{array}$ 


$\begin{array}{lrrr}\mathrm{C} & 0.000000 & 0.000000 & 1.081229 \\ \mathrm{C} & 1.265465 & 0.000000 & 1.729302 \\ \mathrm{H} & 1.457982 & -0.727095 & 2.521899 \\ \mathrm{C} & 2.210171 & 0.971899 & 1.487104 \\ \mathrm{H} & 3.216962 & 0.840339 & 1.878627 \\ \mathrm{C} & 1.874156 & 2.294658 & 1.071251 \\ \mathrm{H} & 2.687304 & 3.016203 & 1.143454 \\ \mathrm{C} & 0.615645 & 2.837643 & 0.908402 \\ \mathrm{H} & 0.513059 & 3.903037 & 1.122869 \\ \mathrm{C} & -0.613549 & 2.145173 & 0.655847 \\ \mathrm{H} & -0.762294 & 1.684508 & -0.307558 \\ \mathrm{~N} & -1.795969 & 2.604737 & 1.181256 \\ \mathrm{C} & -1.182806 & -0.547518 & 1.721718 \\ \mathrm{H} & -1.018637 & -0.975028 & 2.739173 \\ \mathrm{O} & -2.321005 & -0.477276 & 1.262581 \\ \mathrm{H} & -1.762326 & 2.925002 & 2.142056 \\ \mathrm{H} & -2.591821 & 1.999813 & 1.003559\end{array}$

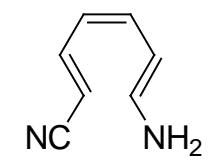

$\begin{array}{lrrr}\mathrm{H} & 0.000000 & 0.000000 & 0.000000 \\ \mathrm{C} & 0.000000 & 0.000000 & 1.088431 \\ \mathrm{C} & 1.179471 & 0.000000 & 1.762964 \\ \mathrm{H} & 1.159885 & -0.001133 & 2.851306 \\ \mathrm{C} & 2.440817 & 0.006364 & 1.079430 \\ \mathrm{H} & 2.378357 & 0.011014 & -0.009326 \\ \mathrm{C} & 3.695687 & 0.004185 & 1.617769 \\ \mathrm{H} & 4.541344 & 0.005145 & 0.932713 \\ \mathrm{C} & 4.009104 & 0.001112 & 3.018702 \\ \mathrm{H} & 3.186016 & 0.002808 & 3.730098 \\ \mathrm{C} & 5.272938 & -0.003902 & 3.518177 \\ \mathrm{H} & 6.133817 & -0.005370 & 2.853177 \\ \mathrm{C} & 5.548087 & -0.008086 & 4.911936 \\ \mathrm{~N} & -1.252515 & 0.066052 & 1.644994 \\ \mathrm{H} & -2.032716 & -0.264537 & 1.095645 \\ \mathrm{H} & -1.339391 & -0.116070 & 2.636544 \\ \mathrm{~N} & 5.787448 & -0.012254 & 6.053565\end{array}$

TS

$\begin{array}{llll}\mathrm{H} & 0.000000 & 0.000000 & 0.000000\end{array}$

$\begin{array}{llll}\text { C } & 0.000000 & 0.000000 & 1.082918\end{array}$

$\begin{array}{llll}\text { C } & 1.277842 & 0.000000 & 1.692226\end{array}$ 


$\begin{array}{lrrr}\mathrm{H} & 1.481482 & -0.679506 & 2.519376 \\ \mathrm{C} & 2.235959 & 0.922485 & 1.320989 \\ \mathrm{H} & 3.263630 & 0.775680 & 1.645796 \\ \mathrm{C} & 1.915117 & 2.210077 & 0.818722 \\ \mathrm{H} & 2.757846 & 2.895560 & 0.737453 \\ \mathrm{C} & 0.666549 & 2.803016 & 0.729967 \\ \mathrm{H} & 0.644971 & 3.885166 & 0.874202 \\ \mathrm{C} & -0.624471 & 2.188884 & 0.698099 \\ \mathrm{H} & -0.956782 & 1.669118 & -0.186912 \\ \mathrm{~N} & -1.661851 & 2.796109 & 1.364180 \\ \mathrm{C} & -1.120508 & -0.654444 & 1.672675 \\ \mathrm{~N} & -2.082535 & -1.107180 & 2.153383 \\ \mathrm{H} & -1.465152 & 3.150170 & 2.291591 \\ \mathrm{H} & -2.570993 & 2.358339 & 1.292426\end{array}$<smiles>N/C=C\C=C/C=[N+]([O-])[O-]</smiles>

$\begin{array}{lrrr}\mathrm{H} & 0.000000 & 0.000000 & 0.000000 \\ \mathrm{C} & 0.000000 & 0.000000 & 1.081448 \\ \mathrm{C} & 1.090066 & 0.000000 & 1.879548 \\ \mathrm{H} & 0.900497 & -0.000596 & 2.949404 \\ \mathrm{C} & 2.438573 & 0.001405 & 1.399897 \\ \mathrm{H} & 2.580765 & 0.000898 & 0.321598 \\ \mathrm{C} & 3.555368 & 0.003638 & 2.190516 \\ \mathrm{H} & 4.515909 & 0.003544 & 1.674034 \\ \mathrm{C} & 3.629596 & 0.008966 & 3.619326 \\ \mathrm{H} & 2.712389 & 0.004677 & 4.204761 \\ \mathrm{C} & 4.824589 & 0.015744 & 4.270764 \\ \mathrm{H} & 5.751088 & 0.021648 & 3.699812 \\ \mathrm{~N} & 5.005368 & -0.040972 & 5.624239 \\ \mathrm{~N} & -1.326179 & 0.000258 & 1.634977 \\ \mathrm{O} & -2.259452 & -0.000559 & 0.821262 \\ \mathrm{O} & -1.472924 & 0.001745 & 2.864886 \\ \mathrm{H} & 4.208832 & 0.114448 & 6.227698 \\ \mathrm{H} & 5.892610 & 0.246608 & 6.009326\end{array}$

$\begin{array}{crrr}\text { TS } & & & \\ \mathrm{H} & 0.000000 & 0.000000 & 0.000000 \\ \mathrm{C} & 0.000000 & 0.000000 & 1.076723 \\ \mathrm{C} & 1.210033 & 0.000000 & 1.794274 \\ \mathrm{H} & 1.367597 & -0.754658 & 2.562936 \\ \mathrm{C} & 2.132377 & 1.014359 & 1.623242\end{array}$




$\begin{array}{lrrr}\mathrm{H} & 3.130684 & 0.882083 & 2.034122 \\ \mathrm{C} & 1.779678 & 2.346307 & 1.273224 \\ \mathrm{H} & 2.569856 & 3.079134 & 1.432435 \\ \mathrm{C} & 0.516214 & 2.878847 & 1.077756 \\ \mathrm{H} & 0.378910 & 3.917576 & 1.382921 \\ \mathrm{C} & -0.672991 & 2.197813 & 0.688506 \\ \mathrm{H} & -0.727077 & 1.727680 & -0.279457 \\ \mathrm{~N} & -1.898205 & 2.599516 & 1.137942 \\ \mathrm{~N} & -1.155102 & -0.673577 & 1.591378 \\ \mathrm{H} & -2.686644 & 2.037371 & 0.840519 \\ \mathrm{H} & -1.962373 & 2.909770 & 2.099605 \\ \mathrm{O} & -2.166685 & -0.670397 & 0.863490 \\ \mathrm{O} & -1.126325 & -1.144988 & 2.734298\end{array}$

\begin{tabular}{crrr} 
& \multicolumn{3}{l}{} \\
$\mathrm{HO}$ & \multicolumn{3}{l}{} \\
$\mathrm{H}$ & 0.000000 & 0.000000 & 0.000000 \\
$\mathrm{C}$ & 0.000000 & 0.000000 & 1.086437 \\
$\mathrm{C}$ & 1.134428 & 0.000000 & 1.812325 \\
$\mathrm{H}$ & 1.053921 & -0.000151 & 2.900043 \\
$\mathrm{C}$ & 2.451619 & 0.000212 & 1.210824 \\
$\mathrm{H}$ & 2.468976 & 0.000117 & 0.121664 \\
$\mathrm{C}$ & 3.648369 & 0.000476 & 1.849185 \\
$\mathrm{H}$ & 4.543269 & 0.000641 & 1.228117 \\
$\mathrm{C}$ & 3.882451 & 0.000625 & 3.278172 \\
$\mathrm{H}$ & 3.023901 & 0.000721 & 3.950853 \\
$\mathrm{C}$ & 5.117172 & 0.000630 & 3.816043 \\
$\mathrm{H}$ & 6.019488 & 0.000683 & 3.210923 \\
$\mathrm{O}$ & 5.428698 & 0.000841 & 5.140914 \\
$\mathrm{O}$ & -1.273855 & -0.000266 & 1.565628 \\
$\mathrm{H}$ & -1.244490 & -0.000013 & 2.536754 \\
$\mathrm{H}$ & 4.605794 & 0.000541 & 5.657421
\end{tabular}

$\begin{array}{crrr}\text { TS } & & & \\ \mathrm{H} & 0.000000 & 0.000000 & 0.000000 \\ \mathrm{C} & 0.000000 & 0.000000 & 1.079261 \\ \mathrm{C} & 1.230752 & 0.000000 & 1.770577 \\ \mathrm{H} & 1.400495 & 0.827581 & 2.465656 \\ \mathrm{C} & 2.161377 & -1.030965 & 1.761440 \\ \mathrm{H} & 3.141417 & -0.818020 & 2.184781\end{array}$




$\begin{array}{lccc}\mathrm{C} & 1.863645 & -2.389706 & 1.541098 \\ \mathrm{H} & 2.641135 & -3.100818 & 1.814720 \\ \mathrm{C} & 0.595079 & -2.901015 & 1.299668 \\ \mathrm{H} & 0.365730 & -3.893253 & 1.699283 \\ \mathrm{C} & -0.479298 & -2.189946 & 0.722882 \\ \mathrm{H} & -0.406468 & -1.863473 & -0.303242 \\ \mathrm{O} & -1.773720 & -2.579488 & 0.973831 \\ \mathrm{O} & -1.036306 & 0.787073 & 1.522961 \\ \mathrm{H} & -0.982228 & 0.843400 & 2.490235 \\ \mathrm{H} & -1.812621 & -2.942820 & 1.872821\end{array}$<smiles>O/C=C\C=C/C=C\F</smiles>

$\begin{array}{lrrr}\mathrm{H} & 0.000000 & 0.000000 & 0.000000 \\ \mathrm{C} & 0.000000 & 0.000000 & 1.086415 \\ \mathrm{C} & 1.085105 & 0.000000 & 1.868816 \\ \mathrm{H} & 0.925562 & 0.000000 & 2.944174 \\ \mathrm{C} & 2.434030 & -0.000064 & 1.339608 \\ \mathrm{H} & 2.520559 & -0.000181 & 0.254196 \\ \mathrm{C} & 3.583966 & -0.000030 & 2.058223 \\ \mathrm{H} & 4.518733 & -0.000123 & 1.498710 \\ \mathrm{C} & 3.721544 & 0.000075 & 3.498361 \\ \mathrm{H} & 2.821945 & 0.000653 & 4.113983 \\ \mathrm{C} & 4.922018 & -0.000397 & 4.110138 \\ \mathrm{H} & 5.858774 & -0.000868 & 3.559601 \\ \mathrm{O} & 5.152852 & -0.000193 & 5.448059 \\ \mathrm{~F} & -1.246573 & 0.000020 & 1.599630 \\ \mathrm{H} & 4.301803 & 0.000904 & 5.917360\end{array}$

TS

$\begin{array}{lrrr}\mathrm{H} & 0.000000 & 0.000000 & 0.000000 \\ \mathrm{C} & 0.000000 & 0.000000 & 1.080662 \\ \mathrm{C} & 1.200443 & 0.000000 & 1.796099 \\ \mathrm{H} & 1.336421 & -0.795242 & 2.530410 \\ \mathrm{C} & 2.139970 & 1.025257 & 1.741619 \\ \mathrm{H} & 3.124592 & 0.826616 & 2.159769 \\ \mathrm{C} & 1.830947 & 2.374046 & 1.492871 \\ \mathrm{H} & 2.612597 & 3.092428 & 1.734039 \\ \mathrm{C} & 0.557467 & 2.889813 & 1.272588 \\ \mathrm{H} & 0.348485 & 3.885380 & 1.673423 \\ \mathrm{C} & -0.544896 & 2.194491 & 0.735213 \\ \mathrm{H} & -0.516134 & 1.840865 & -0.283603 \\ \mathrm{O} & -1.820467 & 2.595627 & 1.029356\end{array}$




$\begin{array}{llrc}\mathrm{F} & -0.984119 & -0.813512 & 1.552936 \\ \mathrm{H} & -1.831160 & 2.947876 & 1.933891\end{array}$<smiles>O=C/C=C\C=C/CO</smiles>

$\begin{array}{lrrr}\mathrm{H} & 0.000000 & 0.000000 & 0.000000 \\ \mathrm{C} & 0.000000 & 0.000000 & 1.086607 \\ \mathrm{C} & 1.140240 & 0.000000 & 1.810253 \\ \mathrm{H} & 1.072332 & -0.000183 & 2.897500 \\ \mathrm{C} & 2.436705 & 0.000000 & 1.181675 \\ \mathrm{H} & 2.424894 & -0.000086 & 0.091996 \\ \mathrm{C} & 3.658554 & 0.000113 & 1.783747 \\ \mathrm{H} & 4.537010 & 0.000143 & 1.141732 \\ \mathrm{C} & 3.908211 & 0.000379 & 3.200347 \\ \mathrm{H} & 3.047002 & 0.000135 & 3.869791 \\ \mathrm{C} & 5.137946 & 0.001029 & 3.772110 \\ \mathrm{H} & 6.046320 & 0.001323 & 3.173011 \\ \mathrm{C} & 5.300641 & 0.001867 & 5.222800 \\ \mathrm{O} & -1.262957 & -0.000359 & 1.565754 \\ \mathrm{H} & -1.242220 & -0.001187 & 2.537881 \\ \mathrm{H} & 4.342947 & 0.001261 & 5.795197 \\ \mathrm{O} & 6.372764 & 0.003353 & 5.805424\end{array}$

TS

$\begin{array}{lrrr}\mathrm{H} & 0.000000 & 0.000000 & 0.000000 \\ \mathrm{C} & 0.000000 & 0.000000 & 1.084289 \\ \mathrm{C} & 1.268952 & 0.000000 & 1.696878 \\ \mathrm{H} & 1.448553 & -0.623019 & 2.574617 \\ \mathrm{C} & 2.262516 & 0.853654 & 1.253968 \\ \mathrm{H} & 3.290171 & 0.706686 & 1.578411 \\ \mathrm{C} & 1.962747 & 2.090783 & 0.637297 \\ \mathrm{H} & 2.813696 & 2.746192 & 0.457540 \\ \mathrm{C} & 0.717125 & 2.698408 & 0.533502 \\ \mathrm{H} & 0.725817 & 3.791810 & 0.567532 \\ \mathrm{C} & -0.586485 & 2.134174 & 0.641942 \\ \mathrm{H} & -1.073883 & 1.582105 & -0.145495 \\ \mathrm{C} & -1.157785 & -0.659874 & 1.696770 \\ \mathrm{O} & -1.533500 & 2.834313 & 1.321501 \\ \mathrm{H} & -1.098206 & 3.372557 & 2.003938 \\ \mathrm{H} & -1.032085 & -0.926105 & 2.772622 \\ \mathrm{O} & -2.207575 & -0.885865 & 1.118260\end{array}$




\begin{tabular}{lrrr} 
& $=$ & & \\
$\mathrm{NC}$ & \multicolumn{3}{l}{} \\
$\mathrm{H}$ & 0.000000 & 0.000000 & 0.000000 \\
$\mathrm{C}$ & 0.000000 & 0.000000 & 1.086593 \\
$\mathrm{C}$ & 1.140452 & 0.000000 & 1.809955 \\
$\mathrm{H}$ & 1.072817 & 0.000017 & 2.897084 \\
$\mathrm{C}$ & 2.436335 & -0.000193 & 1.180009 \\
$\mathrm{H}$ & 2.423916 & -0.000113 & 0.090422 \\
$\mathrm{C}$ & 3.658545 & -0.000385 & 1.780246 \\
$\mathrm{H}$ & 4.537636 & -0.000412 & 1.138778 \\
$\mathrm{C}$ & 3.904163 & -0.000556 & 3.197799 \\
$\mathrm{H}$ & 3.049796 & -0.000551 & 3.870966 \\
$\mathrm{C}$ & 5.143781 & -0.000745 & 3.749687 \\
$\mathrm{H}$ & 6.032324 & -0.000759 & 3.122099 \\
$\mathrm{C}$ & 5.359506 & -0.000912 & 5.154860 \\
$\mathrm{O}$ & -1.262451 & -0.000057 & 1.565674 \\
$\mathrm{H}$ & -1.242122 & -0.000611 & 2.537806 \\
$\mathrm{~N}$ & 5.549423 & -0.001037 & 6.305172
\end{tabular}

TS

$\begin{array}{llll}\mathrm{H} & 0.000000 & 0.000000 & 0.000000\end{array}$

$\begin{array}{llll}\text { C } & 0.000000 & 0.000000 & 1.082332\end{array}$

$\begin{array}{llll}\text { C } & 1.275109 & 0.000000 & 1.691730\end{array}$

$\begin{array}{llll}\mathrm{H} & 1.470747 & -0.679400 & 2.520413\end{array}$

$\begin{array}{llll}\text { C } & 2.243291 & 0.922659 & 1.336461\end{array}$

$\begin{array}{llll}\mathrm{H} & 3.266886 & 0.763313 & 1.667442\end{array}$

$\begin{array}{llll}\text { C } & 1.933053 & 2.215367 & 0.858391\end{array}$

$\begin{array}{llll}\mathrm{H} & 2.770584 & 2.909505 & 0.810452\end{array}$

$\begin{array}{llll}\text { C } & 0.673955 & 2.793524 & 0.759423\end{array}$

$\begin{array}{llll}\mathrm{H} & 0.628000 & 3.870385 & 0.943175\end{array}$

$\begin{array}{llll}\text { C } & -0.594266 & 2.165024 & 0.652373\end{array}$

$\begin{array}{llll}\mathrm{H} & -0.918177 & 1.672983 & -0.250655\end{array}$

$\begin{array}{llll}\text { O } & -1.672786 & 2.737975 & 1.244658\end{array}$

$\begin{array}{llll}\text { C } & -1.087630 & -0.719711 & 1.668789\end{array}$

$\begin{array}{llll}\mathrm{N} & -1.992118 & -1.269360 & 2.155770\end{array}$

$\mathrm{H} \quad-1.384168 \quad 3.211297 \quad 2.042941$<smiles>O=[N+]([O-])C/C=C\C=C/O</smiles>

$\begin{array}{llll}\mathrm{H} & 0.000000 & 0.000000 & 0.000000\end{array}$

$\begin{array}{llll}\text { C } & 0.000000 & 0.000000 & 1.086662\end{array}$ 


$\begin{array}{rrrr}\mathrm{C} & 1.141650 & 0.000000 & 1.810281 \\ \mathrm{H} & 1.077033 & -0.000150 & 2.897269 \\ \mathrm{C} & 2.432983 & 0.000000 & 1.176797 \\ \mathrm{H} & 2.418720 & -0.000206 & 0.087223 \\ \mathrm{C} & 3.658125 & 0.000244 & 1.775054 \\ \mathrm{H} & 4.536090 & 0.000218 & 1.133210 \\ \mathrm{C} & 3.888302 & 0.000597 & 3.192133 \\ \mathrm{H} & 3.044487 & 0.000801 & 3.876392 \\ \mathrm{C} & 5.113459 & 0.000755 & 3.754373 \\ \mathrm{H} & 6.059572 & 0.000595 & 3.230366 \\ \mathrm{~N} & 5.273382 & 0.001153 & 5.187111 \\ \mathrm{O} & -1.259586 & -0.000226 & 1.565914 \\ \mathrm{H} & -1.240930 & -0.000843 & 2.538387 \\ \mathrm{O} & 4.268526 & 0.001409 & 5.908534 \\ \mathrm{O} & 6.437077 & 0.001212 & 5.604805\end{array}$

$\begin{array}{crrr}\text { TS } & & & \\ \mathrm{H} & 0.000000 & 0.000000 & 0.000000 \\ \mathrm{C} & 0.000000 & 0.000000 & 1.078467 \\ \mathrm{C} & 1.219464 & 0.000000 & 1.768210 \\ \mathrm{H} & 1.370541 & -0.716889 & 2.572249 \\ \mathrm{C} & 2.172407 & 0.967425 & 1.499867 \\ \mathrm{H} & 3.184496 & 0.827323 & 1.871274 \\ \mathrm{C} & 1.840008 & 2.266032 & 1.054097 \\ \mathrm{H} & 2.655036 & 2.987739 & 1.073632 \\ \mathrm{C} & 0.569772 & 2.811759 & 0.908509 \\ \mathrm{H} & 0.486722 & 3.879012 & 1.130238 \\ \mathrm{C} & -0.676208 & 2.162263 & 0.716278 \\ \mathrm{H} & -0.951166 & 1.665601 & -0.199800 \\ \mathrm{O} & -1.790580 & 2.679991 & 1.282626 \\ \mathrm{~N} & -1.100854 & -0.797394 & 1.582437 \\ \mathrm{O} & -2.021459 & -1.031387 & 0.791634 \\ \mathrm{O} & -1.074307 & -1.158551 & 2.763254 \\ \mathrm{H} & -1.547695 & 3.156104 & 2.095017\end{array}$

4<smiles>F/C=C\C=C/F</smiles>

$\begin{array}{llll}\mathrm{H} & 0.000000 & 0.000000 & 0.000000\end{array}$

$\begin{array}{llll}\text { C } & 0.000000 & 0.000000 & 1.086568\end{array}$

$\begin{array}{llll}\text { C } & 1.086233 & 0.000000 & 1.867393\end{array}$ 


$\begin{array}{lrll}\mathrm{H} & 0.934147 & 0.000000 & 2.943409 \\ \mathrm{C} & 2.429396 & 0.000000 & 1.324393 \\ \mathrm{H} & 2.503408 & 0.000000 & 0.237909 \\ \mathrm{C} & 3.587709 & 0.000000 & 2.027302 \\ \mathrm{H} & 4.517297 & 0.000000 & 1.460035 \\ \mathrm{C} & 3.726164 & 0.000000 & 3.469436 \\ \mathrm{H} & 2.841896 & 0.000000 & 4.101111 \\ \mathrm{C} & 4.920265 & 0.000000 & 4.072490 \\ \mathrm{H} & 5.884123 & 0.000000 & 3.570886 \\ \mathrm{~F} & 5.039542 & 0.000000 & 5.412395 \\ \mathrm{~F} & -1.243631 & 0.000000 & 1.599334\end{array}$

TS

$\begin{array}{lrrr}\mathrm{H} & 0.000000 & 0.000000 & 0.000000 \\ \mathrm{C} & 0.000000 & 0.000000 & 1.080295 \\ \mathrm{C} & 1.192262 & 0.000000 & 1.808055 \\ \mathrm{H} & 1.309197 & -0.793878 & 2.546465 \\ \mathrm{C} & 2.144439 & 1.016756 & 1.778802 \\ \mathrm{H} & 3.116140 & 0.796503 & 2.216074 \\ \mathrm{C} & 1.867059 & 2.373003 & 1.545207 \\ \mathrm{H} & 2.650094 & 3.075372 & 1.823512 \\ \mathrm{C} & 0.599174 & 2.900095 & 1.308679 \\ \mathrm{H} & 0.355573 & 3.870100 & 1.743325 \\ \mathrm{C} & -0.452413 & 2.211194 & 0.699777 \\ \mathrm{H} & -0.381728 & 1.864012 & -0.320761 \\ \mathrm{~F} & -1.701345 & 2.680273 & 0.949206 \\ \mathrm{~F} & -0.988917 & -0.803049 & 1.548491\end{array}$<smiles>O=C/C=C\C=C/C=C/F</smiles>

$\begin{array}{lccc}\mathrm{H} & 0.000000 & 0.000000 & 0.000000 \\ \mathrm{C} & 0.000000 & 0.000000 & 1.088178 \\ \mathrm{C} & 1.152138 & 0.000000 & 1.799947 \\ \mathrm{H} & 1.073070 & 0.000017 & 2.887299 \\ \mathrm{C} & 2.470542 & -0.000041 & 1.217059 \\ \mathrm{H} & 2.512163 & 0.000250 & 0.129677 \\ \mathrm{C} & 3.649939 & -0.000559 & 1.892752 \\ \mathrm{H} & 4.563183 & -0.000647 & 1.298914 \\ \mathrm{C} & 3.834992 & -0.001052 & 3.326204 \\ \mathrm{H} & 2.977861 & -0.001068 & 3.993390 \\ \mathrm{C} & 5.056512 & -0.001482 & 3.875868 \\ \mathrm{H} & 5.996032 & -0.001505 & 3.329801 \\ \mathrm{~F} & 5.234034 & -0.001861 & 5.204036\end{array}$




$\begin{array}{rrrr}\text { C } & -1.304759 & -0.000305 & 1.749690 \\ \mathrm{O} & -2.375828 & -0.000970 & 1.167445 \\ \mathrm{H} & -1.260916 & 0.000088 & 2.863595 \\ & & & \\ \mathrm{TS} & & & \\ \mathrm{H} & 0.000000 & 0.000000 & 0.000000 \\ \mathrm{C} & 0.000000 & 0.000000 & 1.083781 \\ \mathrm{C} & 1.262700 & 0.000000 & 1.700241 \\ \mathrm{H} & 1.437360 & -0.621454 & 2.579576 \\ \mathrm{C} & 2.258569 & 0.868159 & 1.278890 \\ \mathrm{H} & 3.284474 & 0.708373 & 1.603241 \\ \mathrm{C} & 1.966230 & 2.129052 & 0.719163 \\ \mathrm{H} & 2.814953 & 2.799032 & 0.594065 \\ \mathrm{C} & 0.710713 & 2.732148 & 0.652666 \\ \mathrm{H} & 0.688671 & 3.810313 & 0.821360 \\ \mathrm{C} & -0.560197 & 2.129172 & 0.643877 \\ \mathrm{H} & -1.004154 & 1.609609 & -0.190459 \\ \mathrm{~F} & -1.519674 & 2.834896 & 1.288926 \\ \mathrm{C} & -1.161181 & -0.670441 & 1.692053 \\ \mathrm{H} & -1.041716 & -0.935968 & 2.767446 \\ \mathrm{O} & -2.200864 & -0.902648 & 1.101504\end{array}$

$\begin{array}{cccc} & & & \\ \mathrm{NC} & \mathrm{F} & & \\ \mathrm{H} & 0.000000 & 0.000000 & 0.000000 \\ \mathrm{C} & 0.000000 & 0.000000 & 1.087988 \\ \mathrm{C} & 1.165071 & 0.000000 & 1.780389 \\ \mathrm{H} & 1.115825 & 0.000000 & 2.866541 \\ \mathrm{C} & 2.460521 & 0.000000 & 1.148277 \\ \mathrm{H} & 2.463161 & 0.000000 & 0.059909 \\ \mathrm{C} & 3.662039 & 0.000000 & 1.782865 \\ \mathrm{H} & 4.553658 & 0.000000 & 1.157177 \\ \mathrm{C} & 3.897455 & 0.000000 & 3.208915 \\ \mathrm{H} & 3.065281 & 0.000000 & 3.906944 \\ \mathrm{C} & 5.138193 & 0.000000 & 3.713836 \\ \mathrm{H} & 6.057304 & 0.000000 & 3.134100 \\ \mathrm{~F} & 5.363376 & 0.000000 & 5.034407 \\ \mathrm{C} & -1.274139 & 0.000000 & 1.720282 \\ \mathrm{~N} & -2.324920 & 0.000000 & 2.224273 \\ & & & \\ \mathrm{TS} & & & \\ \mathrm{H} & 0.000000 & 0.000000 & 0.000000 \\ \mathrm{C} & 0.000000 & 0.000000 & 1.079000\end{array}$




$\begin{array}{lrrr}\mathrm{C} & 1.163429 & 0.000000 & 1.864689 \\ \mathrm{H} & 1.258443 & 0.857809 & 2.532213 \\ \mathrm{C} & 2.078936 & -1.034341 & 2.045257 \\ \mathrm{H} & 2.993378 & -0.769482 & 2.572579 \\ \mathrm{C} & 1.806099 & -2.413820 & 1.954175 \\ \mathrm{H} & 2.503637 & -3.076055 & 2.461810 \\ \mathrm{C} & 0.575271 & -2.919815 & 1.563104 \\ \mathrm{H} & 0.205402 & -3.827324 & 2.037494 \\ \mathrm{C} & -0.258920 & -2.218258 & 0.669016 \\ \mathrm{H} & 0.210588 & -1.888175 & -0.248405 \\ \mathrm{C} & -1.636414 & -2.579331 & 0.518431 \\ \mathrm{~F} & -1.000638 & 0.786115 & 1.532364 \\ \mathrm{~N} & -2.765035 & -2.841392 & 0.403333\end{array}$

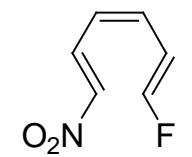

$\begin{array}{lrrr}\mathrm{H} & 0.000000 & 0.000000 & 0.000000 \\ \mathrm{C} & 0.000000 & 0.000000 & 1.081681 \\ \mathrm{C} & 1.083332 & 0.000000 & 1.879916 \\ \mathrm{H} & 0.901402 & 0.000000 & 2.950779 \\ \mathrm{C} & 2.433080 & 0.000000 & 1.379209 \\ \mathrm{H} & 2.554155 & 0.000000 & 0.298266 \\ \mathrm{C} & 3.558174 & 0.000000 & 2.142789 \\ \mathrm{H} & 4.512676 & 0.000000 & 1.617896 \\ \mathrm{C} & 3.635557 & 0.000000 & 3.584667 \\ \mathrm{H} & 2.732560 & 0.000000 & 4.187962 \\ \mathrm{C} & 4.817023 & 0.000000 & 4.217129 \\ \mathrm{H} & 5.791268 & 0.000000 & 3.735458 \\ \mathrm{~F} & 4.903484 & 0.000000 & 5.551624 \\ \mathrm{~N} & -1.335406 & 0.000000 & 1.635616 \\ \mathrm{O} & -2.260482 & 0.000000 & 0.817005 \\ \mathrm{O} & -1.478964 & -0.000019 & 2.862292\end{array}$

TS

$\begin{array}{lrrr}\mathrm{H} & 0.000000 & 0.000000 & 0.000000 \\ \mathrm{C} & 0.000000 & 0.000000 & 1.078158 \\ \mathrm{C} & 1.213885 & 0.000000 & 1.770995 \\ \mathrm{H} & 1.357437 & -0.713292 & 2.579220 \\ \mathrm{C} & 2.169633 & 0.974376 & 1.520019 \\ \mathrm{H} & 3.179024 & 0.823652 & 1.894809 \\ \mathrm{C} & 1.843667 & 2.282125 & 1.110250 \\ \mathrm{H} & 2.653069 & 3.007310 & 1.168052\end{array}$




$\begin{array}{lccc}\mathrm{C} & 0.562239 & 2.817052 & 0.986471 \\ \mathrm{H} & 0.436539 & 3.853474 & 1.302667 \\ \mathrm{C} & -0.638692 & 2.147525 & 0.710469 \\ \mathrm{H} & -0.886395 & 1.686600 & -0.232463 \\ \mathrm{~F} & -1.742823 & 2.702165 & 1.249557 \\ \mathrm{~N} & -1.099097 & -0.820112 & 1.573493 \\ \mathrm{O} & -2.018898 & -1.037236 & 0.779349 \\ \mathrm{O} & -1.060101 & -1.212655 & 2.740808\end{array}$

5

$\begin{array}{lrrr} & = & & \\ & & & \\ \mathrm{OHC} & \mathrm{CHO} & & \\ \mathrm{H} & 0.000000 & 0.000000 & 0.000000 \\ \mathrm{C} & 0.000000 & 0.000000 & 1.088176 \\ \mathrm{C} & 1.150321 & 0.000000 & 1.800905 \\ \mathrm{H} & 1.074372 & 0.000000 & 2.887844 \\ \mathrm{C} & 2.464784 & 0.000000 & 1.205993 \\ \mathrm{H} & 2.491264 & 0.000000 & 0.117813 \\ \mathrm{C} & 3.656080 & 0.000000 & 1.864166 \\ \mathrm{H} & 4.563199 & 0.000000 & 1.262527 \\ \mathrm{C} & 3.852118 & 0.000000 & 3.293608 \\ \mathrm{H} & 2.972379 & 0.000000 & 3.936466 \\ \mathrm{C} & 5.067788 & 0.000000 & 3.888055 \\ \mathrm{H} & 5.989000 & 0.000000 & 3.308834 \\ \mathrm{C} & 5.205590 & 0.000000 & 5.348294 \\ \mathrm{C} & -1.309538 & 0.000000 & 1.748782 \\ \mathrm{O} & -2.374116 & -0.000018 & 1.157802 \\ \mathrm{H} & -1.271442 & -0.000018 & 2.861993 \\ \mathrm{H} & 4.242907 & 0.000035 & 5.908589 \\ \mathrm{O} & 6.272551 & 0.000018 & 5.934958\end{array}$

$\begin{array}{cccc}\text { TS } & & & \\ \mathrm{H} & 0.000000 & 0.000000 & 0.000000 \\ \mathrm{C} & 0.000000 & 0.000000 & 1.080946 \\ \mathrm{C} & 1.255899 & 0.000000 & 1.735757 \\ \mathrm{H} & 1.426660 & 0.742607 & 2.517198 \\ \mathrm{C} & 2.227416 & -0.967936 & 1.556976 \\ \mathrm{H} & 3.218087 & -0.778912 & 1.966354 \\ \mathrm{C} & 1.941460 & -2.322732 & 1.242168 \\ \mathrm{H} & 2.754567 & -3.025274 & 1.415577 \\ \mathrm{C} & 0.662076 & -2.843896 & 1.157284\end{array}$




$\begin{array}{rrrr}\mathrm{H} & 0.508800 & -3.838096 & 1.578323 \\ \mathrm{C} & -0.511129 & -2.142502 & 0.774633 \\ \mathrm{H} & -0.587540 & -1.774794 & -0.238147 \\ \mathrm{C} & -1.809365 & -2.584343 & 1.350787 \\ \mathrm{C} & -1.126943 & 0.763909 & 1.666343 \\ \mathrm{O} & -2.161883 & 1.007425 & 1.075069 \\ \mathrm{H} & -0.979185 & 1.087103 & 2.721754 \\ \mathrm{H} & -2.709445 & -2.110425 & 0.909111 \\ \mathrm{O} & -1.906123 & -3.385776 & 2.262836\end{array}$

$\begin{array}{cccc} & \\ \mathrm{NC} & \mathrm{CHO} & & \\ \mathrm{H} & 0.000000 & 0.000000 & 0.000000 \\ \mathrm{C} & 0.000000 & 0.000000 & 1.088161 \\ \mathrm{C} & 1.150246 & 0.000000 & 1.800677 \\ \mathrm{H} & 1.075358 & 0.000000 & 2.887709 \\ \mathrm{C} & 2.464028 & 0.000000 & 1.203529 \\ \mathrm{H} & 2.488968 & 0.000000 & 0.115405 \\ \mathrm{C} & 3.656692 & 0.000000 & 1.858280 \\ \mathrm{H} & 4.563190 & 0.000000 & 1.255320 \\ \mathrm{C} & 3.852526 & 0.000000 & 3.287350 \\ \mathrm{H} & 2.982191 & 0.000000 & 3.937923 \\ \mathrm{C} & 5.081556 & 0.000000 & 3.857325 \\ \mathrm{H} & 5.980177 & 0.000000 & 3.244120 \\ \mathrm{C} & 5.278078 & 0.000000 & 5.266539 \\ \mathrm{C} & -1.310342 & -0.000022 & 1.748312 \\ \mathrm{O} & -2.373769 & 0.000188 & 1.155176 \\ \mathrm{H} & -1.273931 & 0.000174 & 2.861154 \\ \mathrm{~N} & 5.455341 & 0.000000 & 6.417968\end{array}$

$\begin{array}{crrr}\text { TS } & & & \\ \text { H } & 0.000000 & 0.000000 & 0.000000 \\ \text { C } & 0.000000 & 0.000000 & 1.082146 \\ \text { C } & 1.260349 & 0.000000 & 1.718021 \\ \text { H } & 1.432610 & 0.690467 & 2.544803 \\ \text { C } & 2.243842 & -0.926983 & 1.424575 \\ \text { H } & 3.251704 & -0.755389 & 1.797302 \\ \text { C } & 1.960942 & -2.249008 & 0.998462 \\ \text { H } & 2.798949 & -2.942524 & 1.027663 \\ \text { C } & 0.694911 & -2.815120 & 0.966936 \\ \text { H } & 0.634790 & -3.856798 & 1.283841 \\ \text { C } & -0.543359 & -2.136598 & 0.773282 \\ \text { H } & -0.771686 & -1.717168 & -0.194872\end{array}$




$\begin{array}{rrrr}\mathrm{C} & -1.704751 & -2.675692 & 1.424798 \\ \mathrm{C} & -1.141038 & 0.736672 & 1.675151 \\ \mathrm{O} & -2.153122 & 1.013853 & 1.062513 \\ \mathrm{H} & -1.023069 & 1.006729 & 2.748335 \\ \mathrm{~N} & -2.639644 & -3.084708 & 1.985377\end{array}$<smiles>O=C/C=C\C=C/C=C\[N+](=O)[O-]</smiles>

$\begin{array}{lrrr}\mathrm{H} & 0.000000 & 0.000000 & 0.000000 \\ \mathrm{C} & 0.000000 & 0.000000 & 1.081882 \\ \mathrm{C} & 1.080473 & 0.000000 & 1.881186 \\ \mathrm{H} & 0.900309 & 0.000000 & 2.951833 \\ \mathrm{C} & 2.428633 & 0.000021 & 1.369391 \\ \mathrm{H} & 2.535774 & 0.000020 & 0.286606 \\ \mathrm{C} & 3.564484 & 0.000022 & 2.118063 \\ \mathrm{H} & 4.514914 & 0.000040 & 1.587581 \\ \mathrm{C} & 3.650153 & 0.000003 & 3.559190 \\ \mathrm{H} & 2.724746 & 0.000002 & 4.133952 \\ \mathrm{C} & 4.820150 & 0.000006 & 4.237687 \\ \mathrm{H} & 5.780185 & -0.000009 & 3.725270 \\ \mathrm{C} & 4.857240 & 0.000073 & 5.705996 \\ \mathrm{~N} & -1.339680 & -0.000022 & 1.637289 \\ \mathrm{O} & -2.261134 & -0.000795 & 0.817111 \\ \mathrm{O} & -1.479509 & -0.000793 & 2.862843 \\ \mathrm{H} & 3.860490 & -0.000760 & 6.201156 \\ \mathrm{O} & 5.884334 & -0.000768 & 6.359168\end{array}$

TS

$\begin{array}{lrrr}\mathrm{H} & 0.000000 & 0.000000 & 0.000000 \\ \mathrm{C} & 0.000000 & 0.000000 & 1.082608 \\ \mathrm{C} & 1.254771 & 0.000000 & 1.716933 \\ \mathrm{H} & 1.413659 & 0.644922 & 2.581780 \\ \mathrm{C} & 2.259105 & -0.887073 & 1.360874 \\ \mathrm{H} & 3.270266 & -0.708697 & 1.720936 \\ \mathrm{C} & 1.999667 & -2.183947 & 0.862861 \\ \mathrm{H} & 2.854486 & -2.855026 & 0.815895 \\ \mathrm{C} & 0.743801 & -2.780814 & 0.836283 \\ \mathrm{H} & 0.705977 & -3.836588 & 1.100415 \\ \mathrm{C} & -0.502914 & -2.130724 & 0.719007 \\ \mathrm{H} & -0.876224 & -1.701198 & -0.195128 \\ \mathrm{~N} & -1.630996 & -2.796016 & 1.398786 \\ \mathrm{C} & -1.152876 & 0.718584 & 1.680242\end{array}$




$\begin{array}{rrrr}\mathrm{O} & -2.139236 & 1.041368 & 1.050996 \\ \mathrm{H} & -1.060993 & 0.936304 & 2.767452 \\ \mathrm{O} & -2.759918 & -2.458025 & 1.042020 \\ \mathrm{O} & -1.377255 & -3.605010 & 2.294308 \\ 6 & & & \end{array}$

\begin{tabular}{lrll} 
& \\
$\mathrm{NC}$ & \multicolumn{3}{l}{} \\
$\mathrm{H}$ & 0.000000 & 0.000000 & 0.000000 \\
$\mathrm{C}$ & 0.000000 & 0.000000 & 1.088069 \\
$\mathrm{C}$ & 1.164454 & 0.000000 & 1.780296 \\
$\mathrm{H}$ & 1.120083 & 0.000000 & 2.866124 \\
$\mathrm{C}$ & 2.452653 & 0.000000 & 1.131526 \\
$\mathrm{H}$ & 2.435839 & 0.000000 & 0.043057 \\
$\mathrm{C}$ & 3.668753 & 0.000000 & 1.740928 \\
$\mathrm{H}$ & 4.550762 & 0.000000 & 1.102883 \\
$\mathrm{C}$ & 3.920134 & 0.000000 & 3.161214 \\
$\mathrm{H}$ & 3.076885 & 0.000000 & 3.846723 \\
$\mathrm{C}$ & 5.171686 & 0.000000 & 3.679598 \\
$\mathrm{H}$ & 6.043255 & 0.000000 & 3.028221 \\
$\mathrm{C}$ & 5.429920 & 0.000000 & 5.078763 \\
$\mathrm{C}$ & -1.275383 & 0.000000 & 1.718735 \\
$\mathrm{~N}$ & -2.327660 & 0.000000 & 2.218460 \\
$\mathrm{~N}$ & 5.658837 & 0.000000 & 6.220956
\end{tabular}

TS

$\begin{array}{lrrr}\mathrm{H} & 0.000000 & 0.000000 & 0.000000 \\ \mathrm{C} & 0.000000 & 0.000000 & 1.080438 \\ \mathrm{C} & 1.264346 & 0.000000 & 1.727938 \\ \mathrm{H} & 1.452698 & 0.776594 & 2.468395 \\ \mathrm{C} & 2.212956 & -1.000489 & 1.606043 \\ \mathrm{H} & 3.202663 & -0.797590 & 2.009771 \\ \mathrm{C} & 1.913283 & -2.366974 & 1.381215 \\ \mathrm{H} & 2.704522 & -3.068828 & 1.636216 \\ \mathrm{C} & 0.633189 & -2.877787 & 1.254251 \\ \mathrm{H} & 0.437881 & -3.849217 & 1.707039 \\ \mathrm{C} & -0.474158 & -2.163602 & 0.723721 \\ \mathrm{H} & -0.400676 & -1.832455 & -0.302093 \\ \mathrm{C} & -1.800249 & -2.573625 & 1.086784 \\ \mathrm{C} & -1.064333 & 0.781996 & 1.640924 \\ \mathrm{~N} & -1.942690 & 1.390608 & 2.102228 \\ \mathrm{~N} & -2.879576 & -2.880036 & 1.396433\end{array}$


$\left.{ }_{\mathrm{O}_{2} \mathrm{~N}}\right\rangle_{\mathrm{CN}}$

$\begin{array}{lrrr}\mathrm{H} & 0.000000 & 0.000000 & 0.000000 \\ \mathrm{C} & 0.000000 & 0.000000 & 1.081922 \\ \mathrm{C} & 1.080334 & 0.000000 & 1.881152 \\ \mathrm{H} & 0.901006 & 0.000000 & 2.952085 \\ \mathrm{C} & 2.427352 & 0.000000 & 1.365779 \\ \mathrm{H} & 2.531328 & 0.000000 & 0.282779 \\ \mathrm{C} & 3.566356 & 0.000000 & 2.108909 \\ \mathrm{H} & 4.514797 & 0.000000 & 1.574490 \\ \mathrm{C} & 3.655727 & 0.000000 & 3.549067 \\ \mathrm{H} & 2.741188 & 0.000000 & 4.135785 \\ \mathrm{C} & 4.843441 & 0.000000 & 4.199780 \\ \mathrm{H} & 5.780735 & 0.000000 & 3.647143 \\ \mathrm{C} & 4.949512 & 0.000000 & 5.618752 \\ \mathrm{~N} & -1.340193 & 0.000000 & 1.636911 \\ \mathrm{O} & -1.479915 & -0.000075 & 2.862098 \\ \mathrm{O} & -2.260726 & -0.000058 & 0.815736 \\ \mathrm{~N} & 5.055988 & 0.000000 & 6.778636\end{array}$

$\begin{array}{rrrr}\text { TS } & & & \\ \mathrm{H} & 0.000000 & 0.000000 & 0.000000 \\ \mathrm{C} & 0.000000 & 0.000000 & 1.080762 \\ \mathrm{C} & 1.262631 & 0.000000 & 1.714695 \\ \mathrm{H} & 1.434729 & 0.702036 & 2.528683 \\ \mathrm{C} & 2.242361 & -0.945607 & 1.452051 \\ \mathrm{H} & 3.244677 & -0.751622 & 1.827851 \\ \mathrm{C} & 1.976820 & -2.281933 & 1.076973 \\ \mathrm{H} & 2.813242 & -2.971858 & 1.163095 \\ \mathrm{C} & 0.707752 & -2.841952 & 1.009592 \\ \mathrm{H} & 0.591804 & -3.861347 & 1.373487 \\ \mathrm{C} & -0.475664 & -2.151149 & 0.688955 \\ \mathrm{H} & -0.675027 & -1.769342 & -0.297984 \\ \mathrm{~N} & -1.728245 & -2.708090 & 1.227588 \\ \mathrm{C} & -1.086726 & 0.738243 & 1.655309 \\ \mathrm{~N} & -1.976511 & 1.320166 & 2.127994 \\ \mathrm{O} & -2.767245 & -2.310992 & 0.699239 \\ \mathrm{O} & -1.659131 & -3.492189 & 2.174646\end{array}$


$\underset{\mathrm{O}_{2} \mathrm{~N}}{\overline{\mathrm{NO}_{2}}}$

$\begin{array}{lrll}\mathrm{H} & 0.000000 & 0.000000 & 0.000000 \\ \mathrm{C} & 0.000000 & 0.000000 & 1.082026 \\ \mathrm{C} & 1.079737 & 0.000000 & 1.880756 \\ \mathrm{H} & 0.905548 & 0.000000 & 2.952558 \\ \mathrm{C} & 2.424995 & 0.000000 & 1.357371 \\ \mathrm{H} & 2.522339 & 0.000000 & 0.273708 \\ \mathrm{C} & 3.568420 & 0.000000 & 2.092339 \\ \mathrm{H} & 4.513805 & 0.000000 & 1.553778 \\ \mathrm{C} & 3.650935 & 0.000000 & 3.533465 \\ \mathrm{H} & 2.748261 & 0.000000 & 4.137023 \\ \mathrm{C} & 4.825918 & 0.000000 & 4.184019 \\ \mathrm{H} & 5.810237 & 0.000000 & 3.734691 \\ \mathrm{~N} & 4.878555 & 0.000000 & 5.635415 \\ \mathrm{~N} & -1.342196 & 0.000000 & 1.636857 \\ \mathrm{O} & -1.481160 & 0.000000 & 2.861280 \\ \mathrm{O} & -2.260760 & 0.000019 & 0.814151 \\ \mathrm{O} & 3.822402 & 0.000038 & 6.270292 \\ \mathrm{O} & 6.008418 & 0.000019 & 6.129393\end{array}$

TS

$\begin{array}{llll}\mathrm{H} & 0.000000 & 0.000000 & 0.000000\end{array}$

$\begin{array}{llll}\mathrm{C} & 0.000000 & 0.000000 & 1.077077\end{array}$

$\begin{array}{llll}\text { C } & 1.191197 & 0.000000 & 1.816975\end{array}$

$\begin{array}{llll}\mathrm{H} & 1.319948 & 0.793555 & 2.550118\end{array}$

$\begin{array}{llll}\text { C } & 2.125278 & -1.028367 & 1.793137\end{array}$

$\begin{array}{llll}\mathrm{H} & 3.096251 & -0.829967 & 2.241157\end{array}$

$\begin{array}{llll}\text { C } & 1.802477 & -2.388186 & 1.601353\end{array}$

$\begin{array}{llll}\mathrm{H} & 2.556326 & -3.104233 & 1.920512\end{array}$

$\begin{array}{llll}\text { C } & 0.511105 & -2.865015 & 1.412703\end{array}$

$\begin{array}{llll}\mathrm{H} & 0.230583 & -3.795069 & 1.902713\end{array}$

$\begin{array}{llll}\text { C } & -0.509205 & -2.145511 & 0.774223\end{array}$

$\begin{array}{llll}\mathrm{H} & -0.441969 & -1.862903 & -0.262937\end{array}$

$\begin{array}{llll}\mathrm{N} & -1.077309 & 0.888906 & 1.550332\end{array}$

$\begin{array}{llll}\mathrm{N} & -1.895332 & -2.557277 & 1.063708\end{array}$

$\begin{array}{llll}\text { O } & -2.107557 & -3.187790 & 2.099293\end{array}$

$\begin{array}{llll}\text { O } & -2.745242 & -2.208689 & 0.245681\end{array}$

$\begin{array}{llll}\text { O } & -1.053661 & 1.251732 & 2.726268\end{array}$

$\begin{array}{llll}\text { O } & -1.941024 & 1.179473 & 0.724134\end{array}$

\section{2, 3-subsituted hexatriene}




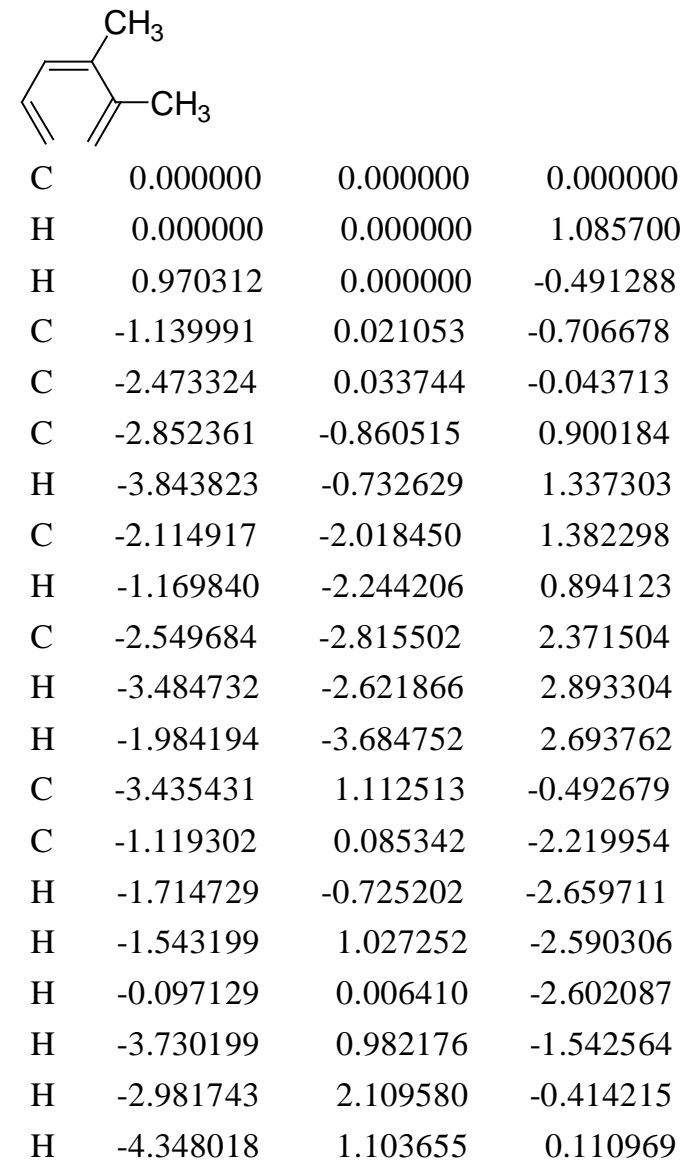

TS

$\begin{array}{lrrr}\mathrm{C} & 0.000000 & 0.000000 & 0.000000 \\ \mathrm{H} & 0.000000 & 0.000000 & 1.089762 \\ \mathrm{H} & 0.988423 & 0.000000 & -0.434299 \\ \mathrm{C} & -0.992053 & -0.715645 & -0.679093 \\ \mathrm{C} & -1.163131 & -0.541736 & -2.068531 \\ \mathrm{C} & -0.954296 & 0.715193 & -2.672535 \\ \mathrm{H} & -1.348416 & 0.807187 & -3.685357 \\ \mathrm{C} & -0.696439 & 1.919515 & -2.003631 \\ \mathrm{H} & -1.276415 & 2.771490 & -2.369186 \\ \mathrm{C} & 0.021361 & 2.097953 & -0.815352 \\ \mathrm{H} & 1.069774 & 1.853030 & -0.762641 \\ \mathrm{H} & -0.224169 & 2.977653 & -0.215319 \\ \mathrm{C} & -1.915132 & -1.586409 & -2.870660 \\ \mathrm{C} & -1.966388 & -1.582224 & 0.094732 \\ \mathrm{H} & -1.865644 & -1.408408 & 1.170815 \\ \mathrm{H} & -3.012508 & -1.402607 & -0.184786 \\ \mathrm{H} & -1.770796 & -2.650191 & -0.076640\end{array}$




$\begin{array}{llll}\mathrm{H} & -2.968638 & -1.656477 & -2.564924 \\ \mathrm{H} & -1.481869 & -2.585710 & -2.741476 \\ \mathrm{H} & -1.900622 & -1.350445 & -3.939180\end{array}$<smiles>Cc1ccccc1N</smiles>

$\begin{array}{lrrr}\mathrm{H} & 0.000000 & 0.000000 & 0.000000 \\ \mathrm{C} & 0.000000 & 0.000000 & 1.088209 \\ \mathrm{H} & 0.971165 & 0.000000 & 1.574130 \\ \mathrm{C} & -1.141765 & 0.005201 & 1.794597 \\ \mathrm{H} & -1.099473 & 0.019015 & 2.880854 \\ \mathrm{C} & -2.461893 & -0.017035 & 1.182906 \\ \mathrm{H} & -2.465327 & -0.115428 & 0.096373 \\ \mathrm{C} & -3.665427 & 0.036952 & 1.799684 \\ \mathrm{C} & -3.823009 & 0.217924 & 3.269828 \\ \mathrm{C} & -3.258131 & 1.238747 & 3.942357 \\ \mathrm{H} & -2.688270 & 1.999678 & 3.424083 \\ \mathrm{H} & -3.401703 & 1.362919 & 5.013484 \\ \mathrm{~N} & -4.723558 & -0.684215 & 3.870245 \\ \mathrm{C} & -4.946866 & -0.089565 & 1.005709 \\ \mathrm{H} & -4.740061 & -0.166514 & -0.066238 \\ \mathrm{H} & -5.528226 & -0.969596 & 1.306715 \\ \mathrm{H} & -5.597333 & 0.778126 & 1.174664 \\ \mathrm{H} & -4.596144 & -1.647179 & 3.574947 \\ \mathrm{H} & -4.735175 & -0.624848 & 4.883221\end{array}$

TS

$\begin{array}{rrrl}\mathrm{H} & 0.000000 & 0.000000 & 0.000000 \\ \mathrm{C} & 0.000000 & 0.000000 & 1.091696 \\ \mathrm{H} & 0.994716 & 0.000000 & 1.507366 \\ \mathrm{C} & -1.022536 & -0.686382 & 1.748038 \\ \mathrm{H} & -1.669938 & -1.337133 & 1.156104 \\ \mathrm{C} & -1.435340 & -0.463360 & 3.068952 \\ \mathrm{H} & -2.059934 & -1.235512 & 3.521535 \\ \mathrm{C} & -1.426791 & 0.763256 & 3.753247 \\ \mathrm{C} & -0.987091 & 1.954045 & 3.117880 \\ \mathrm{C} & 0.071488 & 1.994741 & 2.193142 \\ \mathrm{H} & 0.160472 & 2.890314 & 1.573463 \\ \mathrm{H} & 1.025189 & 1.604821 & 2.513758 \\ \mathrm{~N} & -1.716048 & 3.122238 & 3.384392 \\ \mathrm{C} & -2.275229 & 0.879543 & 5.006076 \\ \mathrm{H} & -1.881928 & 1.643697 & 5.684118 \\ \mathrm{H} & -3.319233 & 1.155383 & 4.786859\end{array}$




$\begin{array}{lrrr}\mathrm{H} & -2.309009 & -0.075551 & 5.540228 \\ \mathrm{H} & -2.726983 & 3.030612 & 3.398004 \\ \mathrm{H} & -1.417916 & 3.935683 & 2.861261\end{array}$

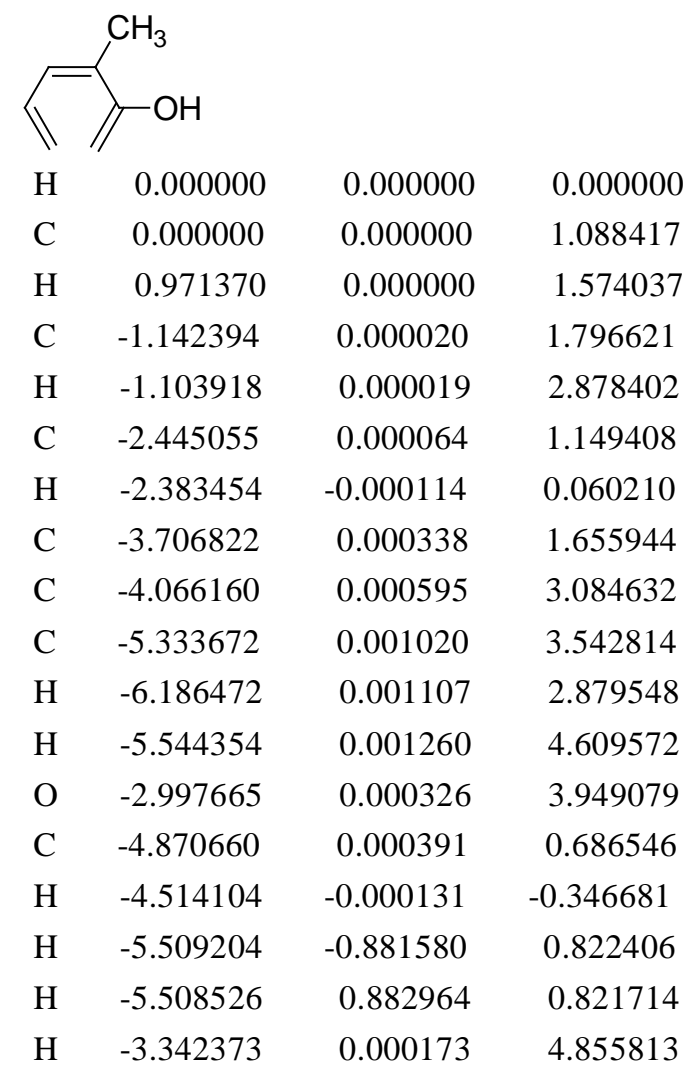

TS

$\begin{array}{lrrr}\mathrm{H} & 0.000000 & 0.000000 & 0.000000 \\ \mathrm{C} & 0.000000 & 0.000000 & 1.078191 \\ \mathrm{H} & 1.006734 & 0.000000 & 1.499482 \\ \mathrm{C} & -0.998613 & 0.696357 & 1.763115 \\ \mathrm{H} & -0.699860 & 1.353072 & 2.582768 \\ \mathrm{C} & -2.375155 & 0.475688 & 1.641484 \\ \mathrm{H} & -3.031744 & 1.247603 & 2.044655 \\ \mathrm{C} & -3.004609 & -0.755742 & 1.384478 \\ \mathrm{C} & -2.246383 & -1.931255 & 1.208571 \\ \mathrm{C} & -0.975227 & -2.000625 & 0.620128 \\ \mathrm{H} & -0.869506 & -1.635513 & -0.388661 \\ \mathrm{H} & -0.387508 & -2.901306 & 0.815791 \\ \mathrm{O} & -2.777621 & -3.043464 & 1.835054 \\ \mathrm{C} & -4.479383 & -0.893065 & 1.707993 \\ \mathrm{H} & -4.966580 & 0.086963 & 1.724986 \\ \mathrm{H} & -4.634270 & -1.365254 & 2.686354 \\ \mathrm{H} & -4.994921 & -1.519492 & 0.971835\end{array}$


$\mathrm{H}$

$-2.335355$

$-3.829870$

1.482783

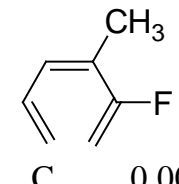

C $\quad 0.000000$

$\mathrm{H} \quad 0.000000$

$\mathrm{H} \quad 0.970657$

C $\quad-1.142497$

H $\quad-1.097595$

C -2.445222

$\mathrm{H} \quad-2.390921$

C $\quad-3.701831$

C $\quad-4.031573$

C $\quad-5.250350$

$\mathrm{H} \quad-6.147058$

H $\quad-5.356337$

F $\quad-2.969405$

C $\quad-4.889687$

$\mathrm{H} \quad-4.559933$

$\mathrm{H} \quad-5.523645$

$\mathrm{H} \quad-5.523275$

TS

$\begin{array}{llll}\mathrm{H} & 0.000000 & 0.000000 & 0.000000\end{array}$

$\begin{array}{llll}\text { C } & 0.000000 & 0.000000 & 1.078111\end{array}$

$\begin{array}{llll}\mathrm{H} & 1.004668 & 0.000000 & 1.502479\end{array}$

C -1.000962

$0.705316 \quad 1.752677$

$\mathrm{H} \quad-0.706491$

1.366923

2.569665

C $\quad-2.375264$

0.492001

1.617436

H $\quad-3.037593$

1.260003

2.016795

C $\quad-2.994053$

$-0.746647$

1.357829

C $\quad-2.223291$

$-1.901018$

1.171616

C $\quad-0.944029$

$-2.020529$

0.632769

$\mathrm{H} \quad-0.777716 \quad-1.676083$

$-0.374235$

H $\quad-0.408160$

F $\quad-2.734416$

$-2.936540$

0.879987

$-3.028018$

1.760949

C $\quad-4.467594$

$-0.913004$

0.058937

1.669974

$\mathrm{H} \quad-4.950760$

H $\quad-4.615554$

$-1.498602$

1.806466

H $\quad-4.990410$

$-1.443087$

2.585112

0.865878 


\begin{tabular}{lrrr}
\multicolumn{3}{l}{$\mathrm{CH}_{3}$} \\
$\mathrm{C}$
\end{tabular}

$\begin{array}{crrr}\text { TS } & & & \\ \mathrm{H} & 0.000000 & 0.000000 & 0.000000 \\ \mathrm{C} & 0.000000 & 0.000000 & 1.078136 \\ \mathrm{H} & 1.003459 & 0.000000 & 1.504948 \\ \mathrm{C} & -1.004340 & 0.667787 & 1.772982 \\ \mathrm{H} & -0.704640 & 1.298897 & 2.613609 \\ \mathrm{C} & -2.393416 & 0.478159 & 1.642599 \\ \mathrm{H} & -3.029566 & 1.270615 & 2.036702 \\ \mathrm{C} & -3.036944 & -0.752234 & 1.446779 \\ \mathrm{C} & -2.255988 & -1.937421 & 1.296097 \\ \mathrm{C} & -1.047302 & -1.937942 & 0.589811 \\ \mathrm{H} & -1.046577 & -1.523685 & -0.406511 \\ \mathrm{H} & -0.366354 & -2.779628 & 0.719086 \\ \mathrm{C} & -2.569841 & -3.173158 & 2.039955 \\ \mathrm{C} & -4.522541 & -0.830516 & 1.708054 \\ \mathrm{O} & -3.642083 & -3.503712 & 2.519738 \\ \mathrm{H} & -1.690793 & -3.853360 & 2.125672 \\ \mathrm{H} & -4.735878 & -1.466679 & 2.572457 \\ \mathrm{H} & -5.042586 & -1.291116 & 0.859786 \\ \mathrm{H} & -4.945491 & 0.165170 & 1.873314\end{array}$




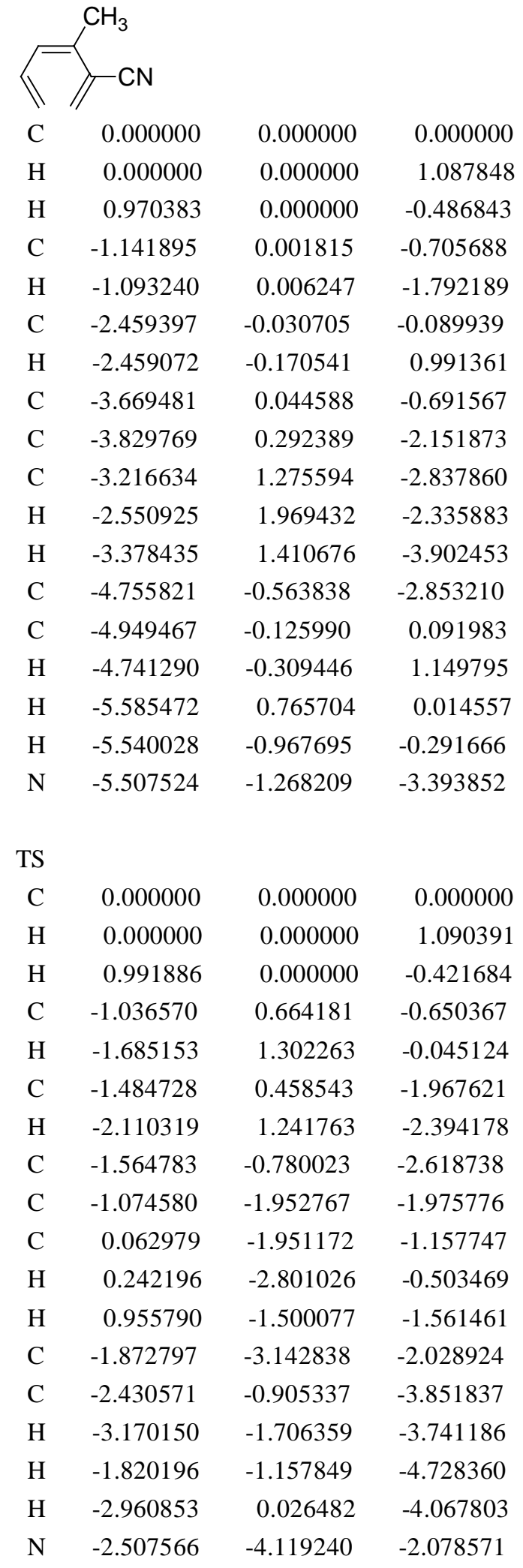




\begin{tabular}{lrrr}
\multicolumn{3}{l}{$\mathrm{CH}_{3}$} \\
$\mathrm{C}$
\end{tabular}

$\begin{array}{crrr}\text { TS } & & & \\ \mathrm{H} & 0.000000 & 0.000000 & 0.000000 \\ \mathrm{C} & 0.000000 & 0.000000 & 1.077926 \\ \mathrm{H} & 1.004043 & 0.000000 & 1.503352 \\ \mathrm{C} & -1.005928 & 0.647556 & 1.787580 \\ \mathrm{H} & -0.700458 & 1.291881 & 2.616118 \\ \mathrm{C} & -2.395584 & 0.432899 & 1.704148 \\ \mathrm{H} & -3.029388 & 1.227317 & 2.097793 \\ \mathrm{C} & -3.035125 & -0.807714 & 1.558980 \\ \mathrm{C} & -2.228185 & -1.949352 & 1.299641 \\ \mathrm{C} & -1.081225 & -1.901028 & 0.516458 \\ \mathrm{H} & -1.179831 & -1.412602 & -0.440779 \\ \mathrm{H} & -0.382992 & -2.728998 & 0.562561 \\ \mathrm{~N} & -2.448136 & -3.184172 & 2.067865 \\ \mathrm{C} & -4.504430 & -0.874301 & 1.917969 \\ \mathrm{O} & -1.517595 & -3.990094 & 2.142946 \\ \mathrm{O} & -3.552645 & -3.364176 & 2.588427 \\ \mathrm{H} & -5.037106 & -1.598916 & 1.297938 \\ \mathrm{H} & -4.652603 & -1.186598 & 2.956538 \\ \mathrm{H} & -4.960971 & 0.112062 & 1.784061\end{array}$




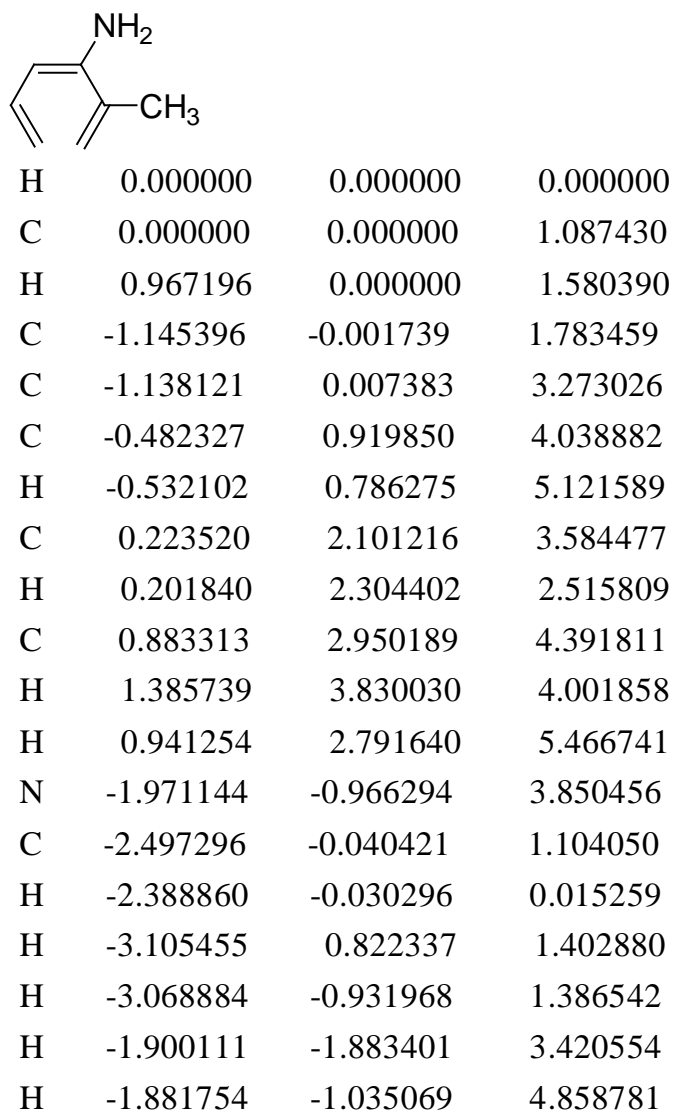

$\begin{array}{rrrr}\text { TS } & & & \\ \mathrm{H} & 0.000000 & 0.000000 & 0.000000 \\ \mathrm{C} & 0.000000 & 0.000000 & 1.091934 \\ \mathrm{H} & 0.997664 & 0.000000 & 1.500801 \\ \mathrm{C} & -1.012713 & -0.726243 & 1.732451 \\ \mathrm{H} & -1.610525 & -1.413281 & 1.127005 \\ \mathrm{C} & -1.472067 & -0.529427 & 3.036891 \\ \mathrm{H} & -2.118594 & -1.308089 & 3.447819 \\ \mathrm{C} & -1.563427 & 0.716204 & 3.700352 \\ \mathrm{C} & -1.135822 & 1.915238 & 3.085501 \\ \mathrm{C} & -0.001372 & 1.919352 & 2.266609 \\ \mathrm{H} & 0.164310 & 2.784500 & 1.623919 \\ \mathrm{H} & 0.912363 & 1.497411 & 2.656595 \\ \mathrm{C} & -2.013839 & 3.145146 & 3.192755 \\ \mathrm{~N} & -2.453637 & 0.811837 & 4.788396 \\ \mathrm{H} & -2.174448 & 1.478394 & 5.498081 \\ \mathrm{H} & -2.694705 & -0.078650 & 5.207220 \\ \mathrm{H} & -3.075997 & 2.888417 & 3.269677 \\ \mathrm{H} & -1.863314 & 3.790056 & 2.319124\end{array}$




\begin{tabular}{|c|c|c|c|}
\hline $\mathrm{H}$ & -1.765023 & 3.756295 & 4.074044 \\
\hline & \multicolumn{3}{|l|}{$\mathrm{NH}_{2}$} \\
\hline & \multicolumn{3}{|l|}{$\mathrm{NH}_{2}$} \\
\hline $\mathrm{C}$ & 0.000000 & 0.000000 & 0.000000 \\
\hline $\mathrm{H}$ & 0.000000 & 0.000000 & 1.082134 \\
\hline $\mathrm{H}$ & 0.962092 & 0.000000 & -0.505453 \\
\hline $\mathrm{C}$ & -1.154125 & -0.099349 & -0.692049 \\
\hline $\mathrm{C}$ & -2.474133 & -0.028649 & -0.009253 \\
\hline $\mathrm{C}$ & -3.623150 & -0.640507 & -0.416530 \\
\hline $\mathrm{H}$ & -4.523425 & -0.398236 & 0.152075 \\
\hline $\mathrm{C}$ & -3.799230 & -1.650069 & -1.442348 \\
\hline $\mathrm{H}$ & -2.908897 & -1.984904 & -1.967447 \\
\hline $\mathrm{C}$ & -4.989064 & -2.191585 & -1.760442 \\
\hline $\mathrm{H}$ & -5.908870 & -1.891117 & -1.262190 \\
\hline $\mathrm{H}$ & -5.077866 & -2.957486 & -2.524814 \\
\hline $\mathrm{N}$ & -2.443930 & 0.722667 & 1.170635 \\
\hline $\mathrm{N}$ & -1.194849 & -0.283281 & -2.080919 \\
\hline $\mathrm{H}$ & -1.951759 & 0.204635 & -2.546390 \\
\hline $\mathrm{H}$ & -0.305876 & -0.127787 & -2.542464 \\
\hline $\mathrm{H}$ & -1.872345 & 1.558396 & 1.128263 \\
\hline $\mathrm{H}$ & -3.350122 & 0.890712 & 1.591352 \\
\hline TS & & & \\
\hline $\mathrm{C}$ & 0.000000 & 0.000000 & 0.000000 \\
\hline $\mathrm{H}$ & 0.000000 & 0.000000 & 1.079080 \\
\hline $\mathrm{H}$ & 1.002125 & 0.000000 & -0.429470 \\
\hline $\mathrm{C}$ & -1.013094 & -0.696853 & -0.660449 \\
\hline $\mathrm{H}$ & -0.756271 & -1.332816 & -1.509936 \\
\hline $\mathrm{C}$ & -2.374682 & -0.488976 & -0.426836 \\
\hline $\mathrm{H}$ & -3.071901 & -1.231853 & -0.820129 \\
\hline $\mathrm{C}$ & -2.966304 & 0.730795 & -0.049947 \\
\hline $\mathrm{C}$ & -2.230880 & 1.945380 & 0.010832 \\
\hline $\mathrm{C}$ & -0.885118 & 2.047430 & 0.410967 \\
\hline $\mathrm{H}$ & -0.617709 & 1.767865 & 1.417823 \\
\hline $\mathrm{H}$ & -0.357045 & 2.945096 & 0.075384 \\
\hline $\mathrm{N}$ & -2.913149 & 3.079886 & -0.462143 \\
\hline $\mathrm{N}$ & -4.368515 & 0.869662 & -0.220479 \\
\hline $\mathrm{H}$ & -4.866767 & -0.010061 & -0.146002 \\
\hline $\mathrm{H}$ & -4.770593 & 1.559388 & 0.407004 \\
\hline $\mathrm{H}$ & -3.494227 & 2.915265 & -1.281467 \\
\hline $\mathrm{H}$ & -2.333587 & 3.905619 & -0.551848 \\
\hline
\end{tabular}




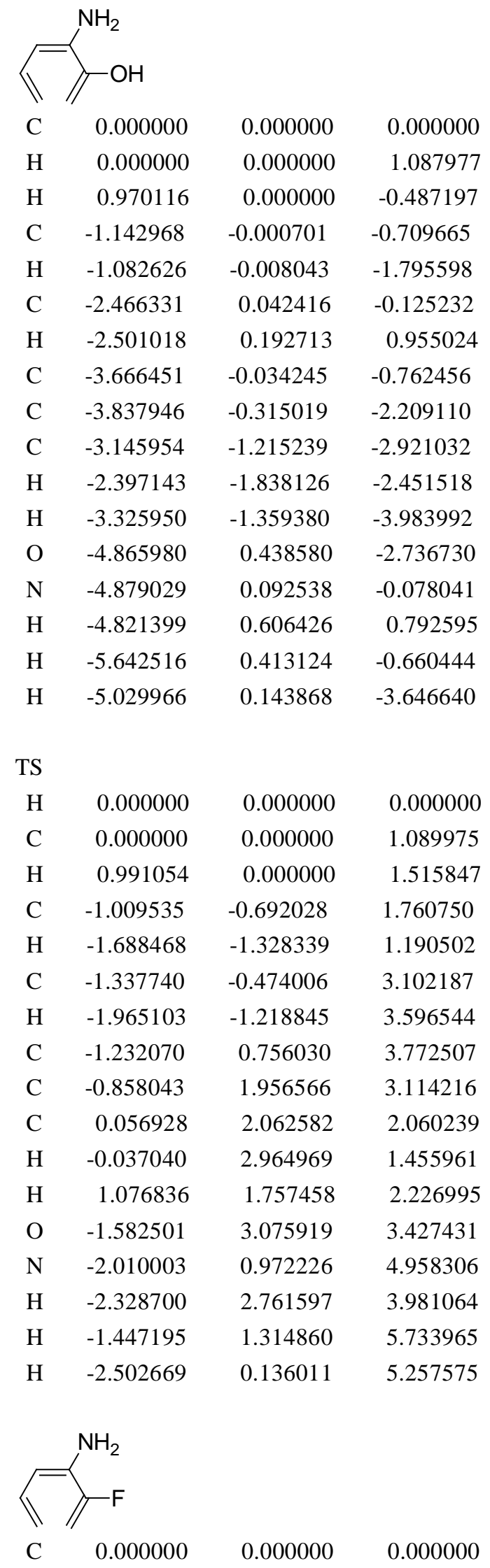




$\begin{array}{crrc}\mathrm{H} & 0.000000 & 0.000000 & 1.088137 \\ \mathrm{H} & 0.969977 & 0.000000 & -0.487275 \\ \mathrm{C} & -1.142783 & 0.000340 & -0.708832 \\ \mathrm{H} & -1.089731 & -0.000421 & -1.792020 \\ \mathrm{C} & -2.455255 & -0.004459 & -0.094758 \\ \mathrm{H} & -2.453143 & -0.033081 & 0.996362 \\ \mathrm{C} & -3.680424 & -0.032529 & -0.687903 \\ \mathrm{C} & -3.931735 & 0.061695 & -2.134561 \\ \mathrm{C} & -5.027806 & -0.344625 & -2.780765 \\ \mathrm{H} & -5.813307 & -0.866271 & -2.250185 \\ \mathrm{H} & -5.124238 & -0.191750 & -3.849279 \\ \mathrm{~F} & -2.942246 & 0.659074 & -2.844069 \\ \mathrm{~N} & -4.854196 & -0.246707 & 0.057215 \\ \mathrm{H} & -4.698223 & -0.257359 & 1.059173 \\ \mathrm{H} & -5.613413 & 0.383097 & -0.184088 \\ & & & \\ \mathrm{TS} & & & \\ \mathrm{H} & 0.000000 & 0.000000 & 0.000000 \\ \mathrm{C} & 0.000000 & 0.000000 & 1.078847 \\ \mathrm{H} & 1.001424 & 0.000000 & 1.508865 \\ \mathrm{C} & -2.499884 & -0.022677 & -0.121384 \\ \mathrm{H} & -2.850293 & -0.867216 & 0.890221 \\ \mathrm{H} & -3.875535 & -0.782178 & 1.254376 \\ \mathrm{H} & -0.733551 & 1.378047 & 2.558990 \\ \mathrm{C} & -2.371581 & 0.521797 & 1.531164 \\ \mathrm{H} & -3.054612 & 1.280553 & 1.915886 \\ \mathrm{C} & -2.979545 & -0.710516 & 1.205112 \\ \mathrm{C} & -2.219204 & -1.887004 & 1.091554 \\ \mathrm{C} & -0.899368 & -2.039764 & 0.678784 \\ \mathrm{H} & -0.620354 & -1.770606 & -0.326552 \\ \mathrm{H} & -0.403299 & -2.941072 & 1.040291 \\ \mathrm{~F} & -2.813867 & -2.987467 & 1.676418 \\ \mathrm{~N} & -4.375482 & -0.827332 & 1.284723 \\ \mathrm{H} & -4.812719 & -0.186688 & 1.938361 \\ \mathrm{H} & -4.679793 & -1.778931 & 1.453707 \\ & & & \\ & & & \\ \mathrm{NH}\end{array}$




$\begin{array}{lrrr}\mathrm{C} & -2.055628 & -1.906110 & 1.509198 \\ \mathrm{H} & -1.082311 & -2.119149 & 1.070606 \\ \mathrm{C} & -2.454455 & -2.645391 & 2.560294 \\ \mathrm{H} & -3.412903 & -2.478882 & 3.046999 \\ \mathrm{H} & -1.833232 & -3.438335 & 2.964650 \\ \mathrm{~N} & -3.393082 & 0.915632 & -0.643343 \\ \mathrm{C} & -0.968830 & 0.152377 & -2.191499 \\ \mathrm{O} & -1.851309 & 0.397081 & -2.995133 \\ \mathrm{H} & 0.080064 & 0.027601 & -2.535386 \\ \mathrm{H} & -3.260136 & 1.084005 & -1.636086 \\ \mathrm{H} & -4.363803 & 0.723710 & -0.422332\end{array}$

TS

$\begin{array}{lrrr}\mathrm{C} & 0.000000 & 0.000000 & 0.000000 \\ \mathrm{H} & 0.000000 & 0.000000 & 1.090137 \\ \mathrm{H} & 0.983818 & 0.000000 & -0.440330 \\ \mathrm{C} & -1.050777 & -0.633299 & -0.661143 \\ \mathrm{C} & -1.371578 & -0.380184 & -2.043722 \\ \mathrm{C} & -1.352110 & 0.937386 & -2.540696 \\ \mathrm{H} & -1.975107 & 1.100309 & -3.421391 \\ \mathrm{C} & -0.958844 & 2.079988 & -1.826393 \\ \mathrm{C} & 0.064724 & 2.170453 & -0.891588 \\ \mathrm{H} & 0.089471 & 3.034472 & -0.229215 \\ \mathrm{H} & 1.037575 & 1.766512 & -1.119629 \\ \mathrm{H} & -1.606133 & 2.951762 & -1.955505 \\ \mathrm{~N} & -1.965753 & -1.381481 & -2.774361 \\ \mathrm{C} & -1.955248 & -1.452665 & 0.142480 \\ \mathrm{H} & -1.862540 & -1.278967 & 1.236329 \\ \mathrm{O} & -2.744227 & -2.299402 & -0.272883 \\ \mathrm{H} & -2.377183 & -2.119289 & -2.204089 \\ \mathrm{H} & -2.537798 & -1.088850 & -3.557726\end{array}$

\begin{tabular}{rrrr} 
\} $&{\multicolumn{3}{l}{}} \\
{\mathrm{C}} &{0.000000} &{0.000000} &{0.000000} \\
{\mathrm{H}} &{0.000000} &{0.000000} &{1.088019} \\
{\mathrm{H}} &{0.969184} &{0.000000} &{-0.488276} \\
{\mathrm{C}} &{-1.143004} &{-0.000240} &{-0.708306} \\
{\mathrm{H}} &{-1.079717} &{-0.005325} &{-1.792175} \\
{\mathrm{C}} &{-2.453798} &{-0.000410} &{-0.093141} \\
{\mathrm{H}} &{-2.449226} &{-0.046773} &{0.997109} \\
{\mathrm{C}} &{-3.680720} &{-0.019059} &{-0.683190} \\
{\mathrm{C}} &{-3.954095} &{0.109248} &{-2.135824}$
\end{tabular}




$\begin{array}{lrrr}\mathrm{C} & -5.058441 & -0.403162 & -2.720395 \\ \mathrm{H} & -5.761245 & -0.996127 & -2.146295 \\ \mathrm{H} & -5.255129 & -0.254994 & -3.776377 \\ \mathrm{C} & -3.063064 & 0.881269 & -2.962335 \\ \mathrm{~N} & -4.842983 & -0.263931 & 0.068710 \\ \mathrm{H} & -4.675162 & -0.310676 & 1.067851 \\ \mathrm{H} & -5.611318 & 0.367821 & -0.139232 \\ \mathrm{~N} & -2.376718 & 1.511141 & -3.658685\end{array}$

TS

$\begin{array}{lrrr}\mathrm{H} & 0.000000 & 0.000000 & 0.000000 \\ \mathrm{C} & 0.000000 & 0.000000 & 1.077762 \\ \mathrm{H} & 0.998687 & 0.000000 & 1.513061 \\ \mathrm{C} & -1.018176 & 0.652679 & 1.767541 \\ \mathrm{H} & -0.738436 & 1.277376 & 2.619707 \\ \mathrm{C} & -2.397296 & 0.478523 & 1.584919 \\ \mathrm{H} & -3.050900 & 1.248197 & 1.997563 \\ \mathrm{C} & -3.034765 & -0.741021 & 1.295254 \\ \mathrm{C} & -2.269272 & -1.949051 & 1.181488 \\ \mathrm{C} & -1.024802 & -1.999361 & 0.548187 \\ \mathrm{H} & -0.938082 & -1.586439 & -0.444032 \\ \mathrm{H} & -0.377697 & -2.851608 & 0.740701 \\ \mathrm{C} & -2.738536 & -3.075752 & 1.925188 \\ \mathrm{~N} & -4.413154 & -0.824175 & 1.378245 \\ \mathrm{H} & -4.796785 & -1.753622 & 1.502606 \\ \mathrm{H} & -4.882109 & -0.122323 & 1.937674 \\ \mathrm{~N} & -3.156960 & -3.992046 & 2.514663\end{array}$

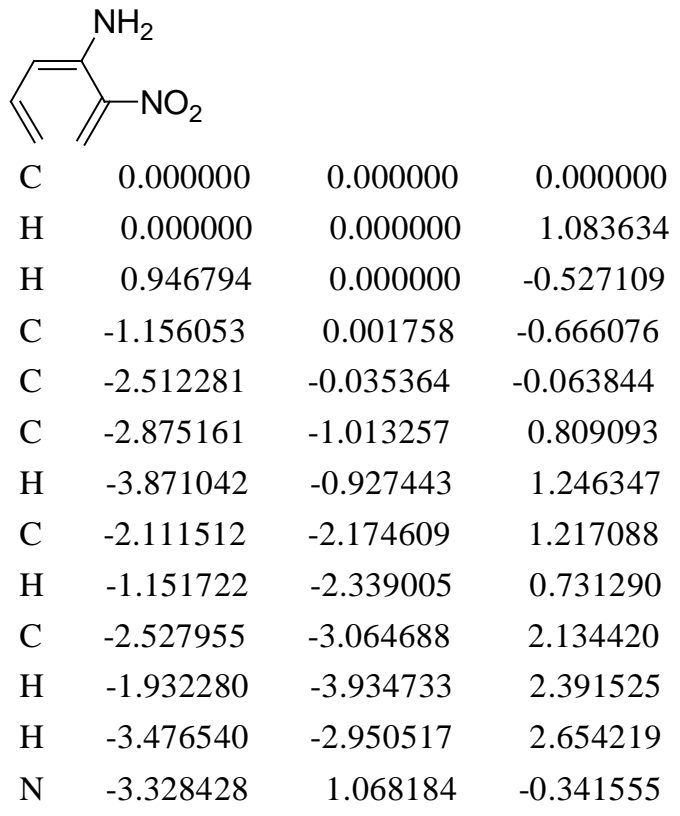




$\begin{array}{rrrr}\mathrm{N} & -1.069531 & 0.059574 & -2.156521 \\ \mathrm{O} & -2.127293 & 0.199383 & -2.776473 \\ \mathrm{O} & 0.033370 & -0.031061 & -2.683795 \\ \mathrm{H} & -3.279969 & 1.355840 & -1.313636 \\ \mathrm{H} & -4.294129 & 0.925617 & -0.064001\end{array}$

$\begin{array}{rrrr}\text { TS } & & & \\ \mathrm{H} & 0.000000 & 0.000000 & 0.000000 \\ \mathrm{C} & 0.000000 & 0.000000 & 1.077619 \\ \mathrm{H} & 0.996812 & 0.000000 & 1.516351 \\ \mathrm{C} & -1.030352 & 0.608160 & 1.782858 \\ \mathrm{H} & -0.757464 & 1.215232 & 2.650420 \\ \mathrm{C} & -2.412760 & 0.430972 & 1.603423 \\ \mathrm{H} & -3.058936 & 1.203298 & 2.022466 \\ \mathrm{C} & -3.070813 & -0.778416 & 1.307608 \\ \mathrm{C} & -2.280307 & -1.970795 & 1.135408 \\ \mathrm{C} & -1.067400 & -1.972692 & 0.467854 \\ \mathrm{H} & -1.037519 & -1.507326 & -0.504353 \\ \mathrm{H} & -0.387200 & -2.801471 & 0.627302 \\ \mathrm{~N} & -2.608024 & -3.144390 & 1.932614 \\ \mathrm{~N} & -4.441257 & -0.839604 & 1.380793 \\ \mathrm{H} & -4.803500 & -1.759286 & 1.611464 \\ \mathrm{H} & -4.901867 & -0.074007 & 1.857350 \\ \mathrm{O} & -3.787913 & -3.311017 & 2.295358 \\ \mathrm{O} & -1.709117 & -3.943880 & 2.197779\end{array}$

\begin{tabular}{lrrr} 
I & \multicolumn{3}{l}{} \\
$\mathrm{C}$ & 0.000000 & 0.000000 & 0.000000 \\
$\mathrm{H}$ & 0.000000 & 0.000000 & 1.087988 \\
$\mathrm{H}$ & 0.970191 & 0.000000 & -0.486831 \\
$\mathrm{C}$ & -1.141107 & -0.001660 & -0.709447 \\
$\mathrm{H}$ & -1.085403 & -0.009886 & -1.795693 \\
$\mathrm{C}$ & -2.467501 & 0.033503 & -0.123873 \\
$\mathrm{H}$ & -2.502034 & 0.175960 & 0.959069 \\
$\mathrm{C}$ & -3.656031 & -0.040075 & -0.772144 \\
$\mathrm{C}$ & -3.877549 & -0.268157 & -2.218994 \\
$\mathrm{C}$ & -3.209777 & -1.222073 & -2.883859 \\
$\mathrm{H}$ & -2.506728 & -1.883764 & -2.388813 \\
$\mathrm{H}$ & -3.366140 & -1.377060 & -3.948375
\end{tabular}




\begin{tabular}{|c|c|c|c|}
\hline $\mathrm{C}$ & -4.920906 & 0.599962 & -2.884981 \\
\hline $\mathrm{O}$ & -4.848386 & 0.135821 & -0.111580 \\
\hline $\mathrm{H}$ & -4.660185 & 0.361743 & 0.813876 \\
\hline $\mathrm{H}$ & -4.682730 & 1.664688 & -2.768636 \\
\hline $\mathrm{H}$ & -5.904553 & 0.443807 & -2.429306 \\
\hline $\mathrm{H}$ & -4.990771 & 0.374745 & -3.953169 \\
\hline \multicolumn{4}{|l|}{ TS } \\
\hline $\mathrm{H}$ & 0.000000 & 0.000000 & 0.000000 \\
\hline $\mathrm{C}$ & 0.000000 & 0.000000 & 1.091109 \\
\hline $\mathrm{H}$ & 0.994510 & 0.000000 & 1.506999 \\
\hline $\mathrm{C}$ & -1.016276 & -0.709922 & 1.739435 \\
\hline $\mathrm{H}$ & -1.639404 & -1.378730 & 1.140657 \\
\hline $\mathrm{C}$ & -1.442210 & -0.515295 & 3.054739 \\
\hline $\mathrm{H}$ & -2.097946 & -1.260599 & 3.502306 \\
\hline $\mathrm{C}$ & -1.462980 & 0.723615 & 3.718437 \\
\hline $\mathrm{C}$ & -1.069139 & 1.934734 & 3.116223 \\
\hline $\mathrm{C}$ & 0.016389 & 1.966115 & 2.233274 \\
\hline $\mathrm{H}$ & 0.962297 & 1.567483 & 2.564360 \\
\hline $\mathrm{H}$ & 0.118375 & 2.832331 & 1.578796 \\
\hline $\mathrm{C}$ & -1.961539 & 3.150053 & 3.292100 \\
\hline $\mathrm{O}$ & -2.268253 & 0.737849 & 4.839841 \\
\hline $\mathrm{H}$ & -2.177745 & 1.600050 & 5.271570 \\
\hline $\mathrm{H}$ & -3.016843 & 2.882868 & 3.424926 \\
\hline $\mathrm{H}$ & -1.664079 & 3.767672 & 4.153865 \\
\hline $\mathrm{H}$ & -1.880955 & 3.799401 & 2.413314 \\
\hline & \multicolumn{3}{|l|}{$\mathrm{OH}$} \\
\hline & \multicolumn{3}{|l|}{$\mathrm{NH}_{2}$} \\
\hline $\mathrm{C}$ & 0.000000 & 0.000000 & 0.000000 \\
\hline $\mathrm{H}$ & 0.000000 & 0.000000 & 1.087750 \\
\hline $\mathrm{H}$ & 0.969716 & 0.000000 & -0.488162 \\
\hline $\mathrm{C}$ & -1.143086 & -0.002234 & -0.707751 \\
\hline $\mathrm{H}$ & -1.087387 & -0.011247 & -1.794195 \\
\hline $\mathrm{C}$ & -2.464443 & 0.034090 & -0.115438 \\
\hline $\mathrm{H}$ & -2.520509 & 0.187535 & 0.960911 \\
\hline $\mathrm{C}$ & -3.653837 & -0.047812 & -0.758242 \\
\hline $\mathrm{C}$ & -3.884328 & -0.307597 & -2.199687 \\
\hline $\mathrm{C}$ & -3.192644 & -1.197332 & -2.931907 \\
\hline $\mathrm{H}$ & -2.440356 & -1.830513 & -2.479600 \\
\hline $\mathrm{H}$ & -3.398486 & -1.340180 & -3.989708 \\
\hline $\mathrm{N}$ & -5.040254 & 0.361950 & -2.681316 \\
\hline $\mathrm{O}$ & -4.800727 & 0.157283 & -0.033187 \\
\hline
\end{tabular}




\begin{tabular}{|c|c|c|c|}
\hline $\mathrm{H}$ & -5.547818 & 0.023063 & -0.645144 \\
\hline $\mathrm{H}$ & -5.008445 & 1.369380 & -2.538775 \\
\hline $\mathrm{H}$ & -5.238421 & 0.157307 & -3.655761 \\
\hline \multicolumn{4}{|l|}{ S } \\
\hline $\mathrm{H}$ & 0.000000 & 0.000000 & 0.000000 \\
\hline $\mathrm{C}$ & 0.000000 & 0.000000 & 1.079154 \\
\hline $\mathrm{H}$ & 1.000749 & 0.000000 & 1.510849 \\
\hline $\mathrm{C}$ & -1.014267 & 0.705564 & 1.732290 \\
\hline $\mathrm{H}$ & -0.757802 & 1.347304 & 2.577010 \\
\hline $\mathrm{C}$ & -2.372999 & 0.509384 & 1.486517 \\
\hline $\mathrm{H}$ & -3.094122 & 1.233717 & 1.861538 \\
\hline $\mathrm{C}$ & -2.936528 & -0.714834 & 1.094293 \\
\hline $\mathrm{C}$ & -2.217299 & -1.931420 & 1.051385 \\
\hline $\mathrm{C}$ & -0.867740 & -2.055242 & 0.680373 \\
\hline $\mathrm{H}$ & -0.566536 & -1.793037 & -0.321763 \\
\hline $\mathrm{H}$ & -0.346065 & -2.939749 & 1.056477 \\
\hline $\mathrm{N}$ & -2.949985 & -3.046789 & 1.545323 \\
\hline $\mathrm{O}$ & -4.309440 & -0.784550 & 1.178045 \\
\hline $\mathrm{H}$ & -4.547086 & -1.701198 & 0.945784 \\
\hline $\mathrm{H}$ & -3.284954 & -2.939165 & 2.502389 \\
\hline $\mathrm{H}$ & -2.459311 & -3.927155 & 1.441245 \\
\hline & & & \\
\hline & $-\Omega H$ & & \\
\hline $\mathrm{C}$ & 0.000000 & 0.000000 & 0.000000 \\
\hline $\mathrm{H}$ & 0.000000 & 0.000000 & 1.087641 \\
\hline $\mathrm{H}$ & 0.969533 & 0.000000 & -0.488409 \\
\hline $\mathrm{C}$ & -1.143138 & 0.000300 & -0.707671 \\
\hline $\mathrm{H}$ & -1.085572 & -0.003561 & -1.793856 \\
\hline $\mathrm{C}$ & -2.462795 & 0.048282 & -0.113145 \\
\hline $\mathrm{H}$ & -2.515032 & 0.238753 & 0.957266 \\
\hline $\mathrm{C}$ & -3.658483 & -0.062920 & -0.739089 \\
\hline $\mathrm{C}$ & -3.898156 & -0.393929 & -2.159927 \\
\hline $\mathrm{C}$ & -3.164074 & -1.204578 & -2.935150 \\
\hline $\mathrm{H}$ & -2.307227 & -1.731126 & -2.538933 \\
\hline $\mathrm{H}$ & -3.419041 & -1.374268 & -3.978390 \\
\hline $\mathrm{O}$ & -5.068500 & 0.206460 & -2.586914 \\
\hline $\mathrm{O}$ & -4.797168 & 0.131034 & -0.002310 \\
\hline $\mathrm{H}$ & -5.545606 & 0.155134 & -0.622446 \\
\hline $\mathrm{H}$ & -5.308509 & -0.150293 & -3.456546 \\
\hline
\end{tabular}

TS 


\begin{tabular}{|c|c|c|c|}
\hline $\mathrm{H}$ & 0.000000 & 0.000000 & 0.000000 \\
\hline $\mathrm{C}$ & 0.000000 & 0.000000 & 1.079447 \\
\hline $\mathrm{H}$ & 0.997210 & 0.000000 & 1.517379 \\
\hline $\mathrm{C}$ & -1.028855 & 0.683984 & 1.728604 \\
\hline $\mathrm{H}$ & -0.795522 & 1.308349 & 2.592072 \\
\hline $\mathrm{C}$ & -2.378484 & 0.480342 & 1.451688 \\
\hline $\mathrm{H}$ & -3.116596 & 1.182424 & 1.834320 \\
\hline $\mathrm{C}$ & -2.923920 & -0.733970 & 1.004443 \\
\hline $\mathrm{C}$ & -2.191811 & -1.936043 & 0.928061 \\
\hline $\mathrm{C}$ & -0.822859 & -2.089191 & 0.669898 \\
\hline $\mathrm{H}$ & -0.421255 & -1.835389 & -0.297441 \\
\hline $\mathrm{H}$ & -0.365559 & -2.987769 & 1.095085 \\
\hline $\mathrm{O}$ & -2.941280 & -3.021148 & 1.396113 \\
\hline $\mathrm{O}$ & -4.294612 & -0.809281 & 1.030797 \\
\hline $\mathrm{H}$ & -4.520884 & -1.755314 & 1.005832 \\
\hline $\mathrm{H}$ & -2.580180 & -3.828842 & 1.003764 \\
\hline & \multicolumn{3}{|l|}{ 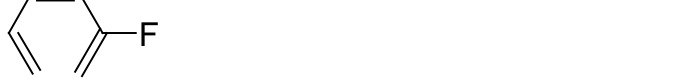 } \\
\hline $\mathrm{C}$ & 0.000000 & 0.000000 & 0.000000 \\
\hline $\mathrm{H}$ & 0.000000 & 0.000000 & 1.088094 \\
\hline $\mathrm{H}$ & 0.969907 & 0.000000 & -0.487134 \\
\hline $\mathrm{C}$ & -1.141178 & 0.000000 & -0.710293 \\
\hline $\mathrm{H}$ & -1.089773 & 0.000033 & -1.792315 \\
\hline $\mathrm{C}$ & -2.451755 & -0.000021 & -0.089274 \\
\hline $\mathrm{H}$ & -2.435696 & -0.000003 & 1.003456 \\
\hline $\mathrm{C}$ & -3.686917 & 0.000030 & -0.652534 \\
\hline $\mathrm{C}$ & -4.034384 & -0.000379 & -2.076173 \\
\hline $\mathrm{C}$ & -5.266105 & -0.000124 & -2.588663 \\
\hline $\mathrm{H}$ & -6.128617 & 0.000598 & -1.937036 \\
\hline $\mathrm{H}$ & -5.410152 & -0.000623 & -3.662666 \\
\hline $\mathrm{F}$ & -2.969981 & -0.001233 & -2.915341 \\
\hline $\mathrm{O}$ & -4.826838 & 0.000202 & 0.113115 \\
\hline $\mathrm{H}$ & -4.570805 & 0.001971 & 1.048631 \\
\hline \multicolumn{4}{|l|}{ TS } \\
\hline $\mathrm{H}$ & 0.000000 & 0.000000 & 0.000000 \\
\hline $\mathrm{C}$ & 0.000000 & 0.000000 & 1.079383 \\
\hline $\mathrm{H}$ & 0.996478 & 0.000000 & 1.518218 \\
\hline $\mathrm{C}$ & -1.028418 & 0.690834 & 1.724620 \\
\hline $\mathrm{H}$ & -0.793660 & 1.323300 & 2.581548 \\
\hline $\mathrm{C}$ & -2.377570 & 0.489748 & 1.448751 \\
\hline $\mathrm{H}$ & -3.116878 & 1.191261 & 1.829318 \\
\hline
\end{tabular}




$\begin{array}{llll}\text { C } & -2.917930 & -0.732474 & 1.014589 \\ \text { C } & -2.165907 & -1.912071 & 0.916146 \\ \text { C } & -0.808164 & -2.094945 & 0.669022 \\ \text { H } & -0.386113 & -1.844553 & -0.289821 \\ \text { H } & -0.385375 & -3.003571 & 1.101129 \\ \text { F } & -2.870612 & -3.013880 & 1.373610 \\ \text { O } & -4.285549 & -0.824210 & 1.053761 \\ \text { H } & -4.514812 & -1.767348 & 1.012217\end{array}$

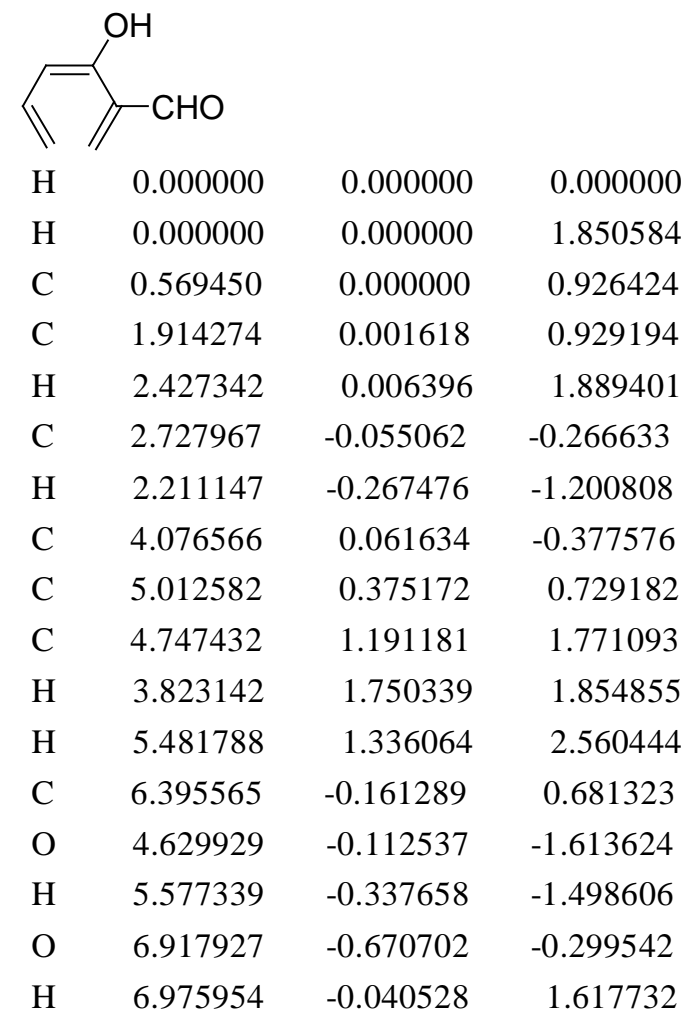

TS

$\begin{array}{lrrr}\mathrm{H} & 0.000000 & 0.000000 & 0.000000 \\ \mathrm{C} & 0.000000 & 0.000000 & 1.077534 \\ \mathrm{H} & 0.993786 & 0.000000 & 1.521993 \\ \mathrm{C} & -1.039295 & 0.601804 & 1.771355 \\ \mathrm{H} & -0.784954 & 1.174109 & 2.666972 \\ \mathrm{C} & -2.417194 & 0.449181 & 1.542937 \\ \mathrm{H} & -3.096120 & 1.172736 & 1.989235 \\ \mathrm{C} & -3.044607 & -0.743130 & 1.163645 \\ \mathrm{C} & -2.314128 & -1.971716 & 1.021505 \\ \mathrm{C} & -1.031169 & -2.053631 & 0.479429 \\ \mathrm{H} & -0.827983 & -1.616166 & -0.483618 \\ \mathrm{H} & -0.430954 & -2.935949 & 0.702789 \\ \mathrm{C} & -2.900671 & -3.178447 & 1.588527\end{array}$




$\begin{array}{llll}\mathrm{O} & -4.388478 & -0.769760 & 1.273746 \\ \mathrm{H} & -4.657037 & -1.718122 & 1.371167 \\ \mathrm{O} & -4.103751 & -3.338762 & 1.812383 \\ \mathrm{H} & -2.191616 & -4.008041 & 1.781490\end{array}$

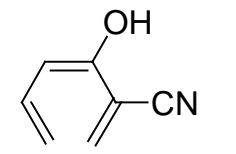

$\begin{array}{lrrr}\mathrm{C} & 0.000000 & 0.000000 & 0.000000 \\ \mathrm{H} & 0.000000 & 0.000000 & 1.088079 \\ \mathrm{H} & 0.969223 & 0.000000 & -0.488062 \\ \mathrm{C} & -1.141068 & 0.000000 & -0.710777 \\ \mathrm{H} & -1.079764 & -0.000017 & -1.793041 \\ \mathrm{C} & -2.447341 & 0.000021 & -0.083626 \\ \mathrm{H} & -2.417056 & 0.000055 & 1.008770 \\ \mathrm{C} & -3.694041 & 0.000079 & -0.623018 \\ \mathrm{C} & -4.102390 & -0.000041 & -2.040002 \\ \mathrm{C} & -5.401081 & 0.000279 & -2.412274 \\ \mathrm{H} & -6.190102 & 0.000638 & -1.671437 \\ \mathrm{H} & -5.677912 & 0.000167 & -3.460405 \\ \mathrm{C} & -3.120096 & -0.000575 & -3.089961 \\ \mathrm{O} & -4.805219 & 0.000294 & 0.183145 \\ \mathrm{H} & -4.519012 & 0.000432 & 1.109733 \\ \mathrm{~N} & -2.372196 & -0.001273 & -3.980547\end{array}$

$\begin{array}{crrr}\text { TS } & & & \\ \mathrm{H} & 0.000000 & 0.000000 & 0.000000 \\ \mathrm{C} & 0.000000 & 0.000000 & 1.077536 \\ \mathrm{H} & 0.997604 & 0.000000 & 1.514644 \\ \mathrm{C} & -1.022215 & 0.640390 & 1.769617 \\ \mathrm{H} & -0.748430 & 1.255457 & 2.629888 \\ \mathrm{C} & -2.401417 & 0.461855 & 1.588271 \\ \mathrm{H} & -3.079315 & 1.198603 & 2.014251 \\ \mathrm{C} & -3.015278 & -0.757520 & 1.287220 \\ \mathrm{C} & -2.274471 & -1.968240 & 1.140519 \\ \mathrm{C} & -1.017586 & -2.019070 & 0.528520 \\ \mathrm{H} & -0.908755 & -1.588547 & -0.453162 \\ \mathrm{H} & -0.383475 & -2.881880 & 0.717171 \\ \mathrm{C} & -2.799357 & -3.113103 & 1.818534 \\ \mathrm{O} & -4.363941 & -0.788305 & 1.472868 \\ \mathrm{H} & -4.682659 & -1.703779 & 1.396102 \\ \mathrm{~N} & -3.283884 & -4.036884 & 2.341086\end{array}$




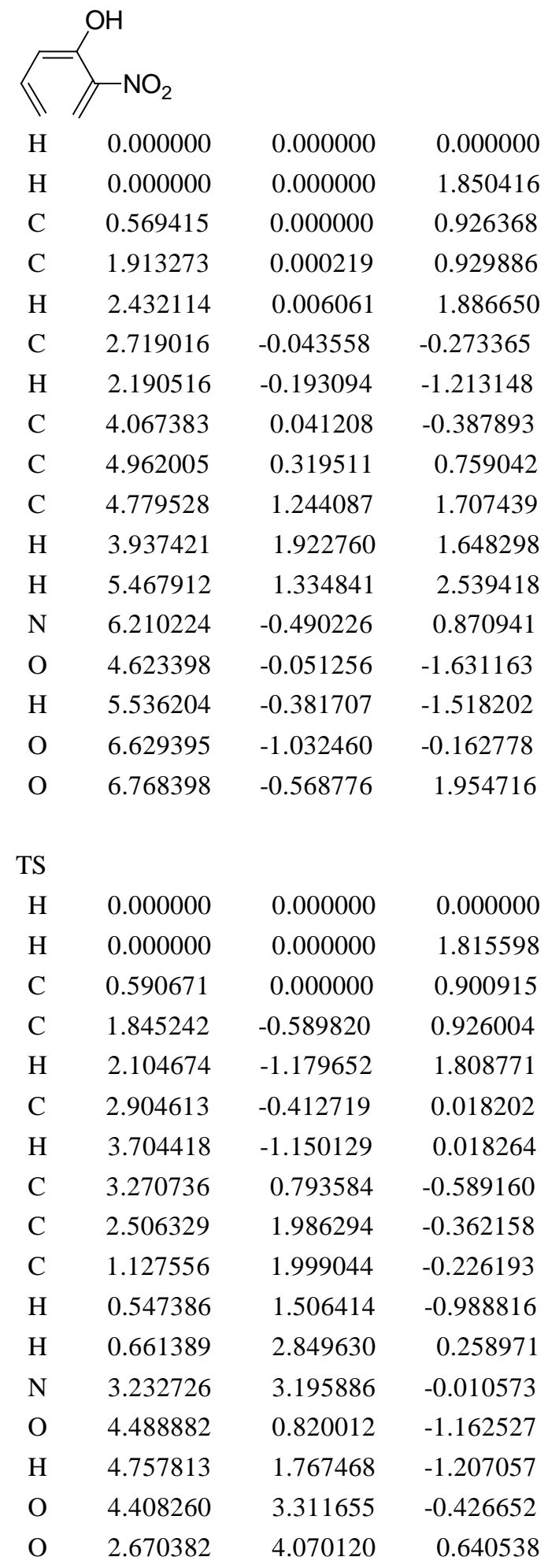




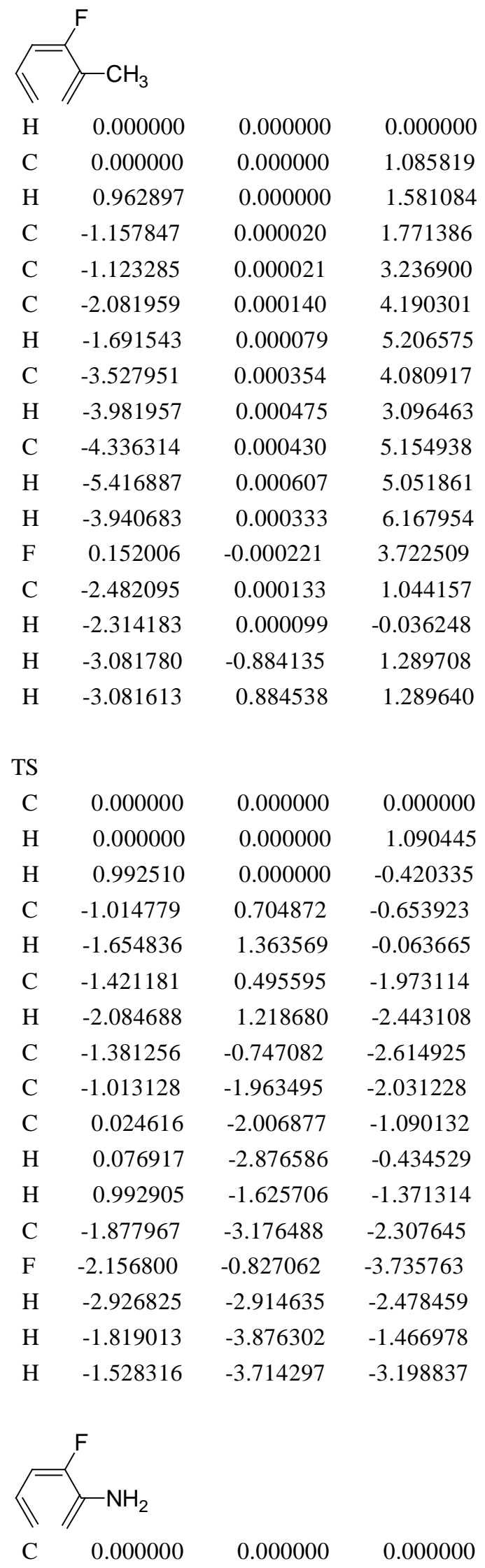




\begin{tabular}{|c|c|c|c|}
\hline $\mathrm{H}$ & 0.000000 & 0.000000 & 7906 \\
\hline $\mathrm{H}$ & 0.969968 & 0.000000 & -0.487674 \\
\hline $\mathrm{C}$ & -1.141329 & 0.004761 & -0.708681 \\
\hline $\mathrm{H}$ & -1.098472 & 0.015039 & -1.793761 \\
\hline $\mathrm{C}$ & -2.454129 & 0.034609 & -0.088103 \\
\hline $\mathrm{H}$ & -2.476465 & 0.143248 & 0.994892 \\
\hline $\mathrm{C}$ & -3.668380 & -0.004533 & -0.675521 \\
\hline $\mathrm{C}$ & -4.073919 & -0.199400 & -2.075570 \\
\hline $\mathrm{C}$ & -5.306772 & 0.155871 & -2.486576 \\
\hline $\mathrm{H}$ & -6.001281 & 0.643384 & -1.816422 \\
\hline $\mathrm{H}$ & -5.625151 & -0.007759 & -3.512072 \\
\hline $\mathrm{N}$ & -3.086035 & -0.715255 & -2.937572 \\
\hline $\mathrm{F}$ & -4.737719 & 0.152943 & 0.148920 \\
\hline $\mathrm{H}$ & -2.610054 & -1.529406 & -2.559978 \\
\hline $\mathrm{H}$ & -3.452714 & -0.924427 & -3.860477 \\
\hline \multicolumn{4}{|l|}{ TS } \\
\hline $\mathrm{H}$ & 0.000000 & 0.000000 & 0.000000 \\
\hline $\mathrm{C}$ & 0.000000 & 0.000000 & 1.078879 \\
\hline $\mathrm{H}$ & 0.996427 & 0.000000 & 1.518501 \\
\hline $\mathrm{C}$ & -1.034904 & 0.657759 & 1.737791 \\
\hline $\mathrm{H}$ & -0.815824 & 1.262333 & 2.618232 \\
\hline $\mathrm{C}$ & -2.384065 & 0.431772 & 1.450269 \\
\hline $\mathrm{H}$ & -3.140803 & 1.121565 & 1.819301 \\
\hline $\mathrm{C}$ & -2.881798 & -0.777225 & 0.971290 \\
\hline $\mathrm{C}$ & -2.188316 & -2.008644 & 0.928483 \\
\hline $\mathrm{C}$ & -0.821823 & -2.108346 & 0.609725 \\
\hline $\mathrm{H}$ & -0.488420 & -1.800756 & -0.368114 \\
\hline $\mathrm{H}$ & -0.314373 & -2.997684 & 0.987810 \\
\hline $\mathrm{N}$ & -2.842726 & -3.119493 & 1.469794 \\
\hline $\mathrm{F}$ & -4.253012 & -0.892229 & 1.025658 \\
\hline $\mathrm{H}$ & -3.806202 & -2.950087 & 1.729039 \\
\hline $\mathrm{H}$ & -2.736130 & -3.981766 & 0.951747 \\
\hline & \\
\hline & \multicolumn{3}{|l|}{${ }_{5} \mathrm{OH}$} \\
\hline . & 0.000000 & 0.000000 & 0.000000 \\
\hline $\mathrm{H}$ & 0.000000 & 0.000000 & 1.850216 \\
\hline $\mathrm{C}$ & 0.570291 & 0.000000 & 0.926415 \\
\hline $\mathrm{C}$ & 1.913827 & 0.000000 & 0.931058 \\
\hline $\mathrm{H}$ & 2.450044 & 0.000000 & 1.872980 \\
\hline $\mathrm{C}$ & 2.693831 & 0.000000 & -0.292407 \\
\hline $\mathrm{H}$ & 2.132098 & -0.000017 & -1.225074 \\
\hline
\end{tabular}




$\begin{array}{cccc}\text { C } & 4.032662 & 0.000018 & -0.450300 \\ \text { C } & 5.133166 & 0.000024 & 0.515836 \\ \text { C } & 6.423660 & 0.000004 & 0.141695 \\ \text { H } & 6.697862 & -0.000018 & -0.903204 \\ \text { H } & 7.223413 & 0.000009 & 0.877131 \\ \text { O } & 4.712943 & 0.000009 & 1.820297 \\ \text { F } & 4.493822 & 0.000013 & -1.729961 \\ \text { H } & 5.496817 & 0.000014 & 2.391594\end{array}$

TS

$\begin{array}{lrrc}\mathrm{H} & 0.000000 & 0.000000 & 0.000000 \\ \mathrm{C} & 0.000000 & 0.000000 & 1.079030 \\ \mathrm{H} & 0.996001 & 0.000000 & 1.518880 \\ \mathrm{C} & -1.034277 & 0.669411 & 1.731151 \\ \mathrm{H} & -0.816073 & 1.283717 & 2.604592 \\ \mathrm{C} & -2.380419 & 0.439886 & 1.443247 \\ \mathrm{H} & -3.142995 & 1.120652 & 1.815908 \\ \mathrm{C} & -2.865266 & -0.773582 & 0.960169 \\ \mathrm{C} & -2.162488 & -1.991338 & 0.897336 \\ \mathrm{C} & -0.794012 & -2.117472 & 0.626572 \\ \mathrm{H} & -0.415183 & -1.820699 & -0.336758 \\ \mathrm{H} & -0.333319 & -3.024755 & 1.018314 \\ \mathrm{O} & -2.822742 & -3.111924 & 1.328036 \\ \mathrm{~F} & -4.232526 & -0.931481 & 1.050910 \\ \mathrm{H} & -3.675669 & -2.832472 & 1.703916\end{array}$

$\begin{array}{lrrr}\text { N } & & & \\ \mathrm{C} & & & \\ \mathrm{H} & & & \\ \mathrm{H} & 0.000000 & 0.000000 & 0.000000 \\ \mathrm{H} & 0.9600000 & 0.000000 & 1.087688 \\ \mathrm{C} & -1.141499 & 0.000000 & -0.488466 \\ \mathrm{H} & -1.098639 & 0.000000 & -0.707980 \\ \mathrm{C} & -2.447403 & 0.0000000 & -1.791124 \\ \mathrm{H} & -2.462829 & 0.000000 & -0.075781 \\ \mathrm{C} & -3.665372 & 0.000018 & -0.653647 \\ \mathrm{C} & -4.067325 & 0.000024 & -2.053947 \\ \mathrm{C} & -5.321181 & -0.000051 & -2.511180 \\ \mathrm{H} & -6.154018 & -0.000125 & -1.821684 \\ \mathrm{H} & -5.515793 & -0.000039 & -3.576879 \\ \mathrm{~F} & -3.031638 & 0.000009 & -2.927746 \\ \mathrm{~F} & -4.747776 & 0.000014 & 0.167704\end{array}$




\begin{tabular}{|c|c|c|c|}
\hline $\mathrm{C}$ & 0.000000 & 0.000000 & 0.000000 \\
\hline $\mathrm{H}$ & 0.000000 & 0.000000 & 1.079024 \\
\hline $\mathrm{H}$ & 0.998454 & 0.000000 & -0.435073 \\
\hline $\mathrm{C}$ & -1.020410 & -0.700863 & -0.649096 \\
\hline $\mathrm{H}$ & -0.776383 & -1.346467 & -1.492896 \\
\hline $\mathrm{C}$ & -2.373724 & -0.482544 & -0.398742 \\
\hline $\mathrm{H}$ & -3.116664 & -1.189367 & -0.761990 \\
\hline $\mathrm{C}$ & -2.896023 & 0.752665 & -0.004086 \\
\hline $\mathrm{C}$ & -2.166360 & 1.942838 & 0.071613 \\
\hline $\mathrm{C}$ & -0.818914 & 2.078043 & 0.404580 \\
\hline $\mathrm{H}$ & -0.484407 & 1.817026 & 1.394754 \\
\hline $\mathrm{H}$ & -0.331989 & 2.964825 & -0.001886 \\
\hline $\mathrm{F}$ & -2.793441 & 3.018584 & -0.476303 \\
\hline $\mathrm{F}$ & -4.241720 & 0.886202 & -0.102681 \\
\hline & $F$ & & \\
\hline & & & \\
\hline $\mathrm{C}$ & 0.000000 & 0.000000 & 0.000000 \\
\hline $\mathrm{H}$ & 0.000000 & 0.000000 & 1.088139 \\
\hline $\mathrm{H}$ & 0.970124 & 0.000000 & -0.487413 \\
\hline $\mathrm{C}$ & -1.140273 & -0.000501 & -0.711443 \\
\hline $\mathrm{H}$ & -1.108635 & -0.012361 & -1.793201 \\
\hline $\mathrm{C}$ & -2.435573 & 0.001909 & -0.062129 \\
\hline $\mathrm{H}$ & -2.411954 & -0.019280 & 1.026685 \\
\hline $\mathrm{C}$ & -3.692626 & 0.048630 & -0.552879 \\
\hline $\mathrm{C}$ & -4.307200 & 0.119373 & -1.886087 \\
\hline $\mathrm{C}$ & -5.634291 & 0.355399 & -2.003685 \\
\hline $\mathrm{H}$ & -6.275283 & 0.501166 & -1.144196 \\
\hline $\mathrm{H}$ & -6.100124 & 0.401901 & -2.983661 \\
\hline $\mathrm{C}$ & -3.605629 & -0.094629 & -3.182618 \\
\hline $\mathrm{F}$ & -4.665611 & 0.075746 & 0.412131 \\
\hline $\mathrm{O}$ & -2.443594 & -0.389996 & -3.368904 \\
\hline $\mathrm{H}$ & -4.289536 & 0.029282 & -4.049163 \\
\hline TS & & & \\
\hline $\mathrm{C}$ & 0.000000 & 0.000000 & 0.000000 \\
\hline $\mathrm{H}$ & 0.000000 & 0.000000 & 1.088779 \\
\hline $\mathrm{H}$ & 0.986105 & 0.000000 & -0.434862 \\
\hline $\mathrm{C}$ & -1.035492 & 0.648878 & -0.664074 \\
\hline $\mathrm{H}$ & -1.724694 & 1.260909 & -0.079026 \\
\hline $\mathrm{C}$ & -1.392033 & 0.456651 & -2.008041 \\
\hline $\mathrm{H}$ & -2.043251 & 1.178713 & -2.495575 \\
\hline
\end{tabular}




$\begin{array}{lrll}\mathrm{C} & -1.310581 & -0.776531 & -2.646301 \\ \mathrm{C} & -0.929965 & -1.991891 & -2.036658 \\ \mathrm{C} & 0.087220 & -2.044115 & -1.080212 \\ \mathrm{H} & 1.062280 & -1.659518 & -1.329747 \\ \mathrm{H} & 0.083905 & -2.901041 & -0.409665 \\ \mathrm{C} & -1.829662 & -3.160119 & -2.206652 \\ \mathrm{~F} & -1.993525 & -0.867782 & -3.816262 \\ \mathrm{H} & -2.610823 & -3.048089 & -2.982504 \\ \mathrm{O} & -1.743243 & -4.181986 & -1.548061\end{array}$

\begin{tabular}{lrrr} 
& & & \\
\hline & & & \\
$\mathrm{C}$ & & & \\
$\mathrm{H}$ & 0.000000 & 0.000000 & 0.000000 \\
$\mathrm{H}$ & 0.000000 & 0.000000 & 1.087693 \\
$\mathrm{C}$ & -1.141515 & 0.000000 & -0.708486 \\
$\mathrm{H}$ & -1.089533 & 0.000000 & -1.791859 \\
$\mathrm{C}$ & -2.442531 & 0.000000 & -0.068504 \\
$\mathrm{H}$ & -2.442037 & 0.000000 & 1.019988 \\
$\mathrm{C}$ & -3.675375 & 0.000000 & -0.616087 \\
$\mathrm{C}$ & -4.148387 & 0.000000 & -2.003489 \\
$\mathrm{C}$ & -5.463760 & 0.000000 & -2.311135 \\
$\mathrm{H}$ & -6.215732 & 0.000000 & -1.532200 \\
$\mathrm{H}$ & -5.794196 & 0.000000 & -3.343233 \\
$\mathrm{C}$ & -3.200959 & 0.000000 & -3.085423 \\
$\mathrm{~F}$ & -4.719884 & 0.000000 & 0.254474 \\
$\mathrm{~N}$ & -2.464265 & -0.000001 & -3.985006 \\
& & &
\end{tabular}

TS

$\begin{array}{lrrr}\mathrm{C} & 0.000000 & 0.000000 & 0.000000 \\ \mathrm{H} & 0.000000 & 0.000000 & 1.077728 \\ \mathrm{H} & 0.998255 & 0.000000 & -0.435546 \\ \mathrm{C} & -1.017039 & -0.660347 & -0.683745 \\ \mathrm{H} & -0.745590 & -1.288440 & -1.534203 \\ \mathrm{C} & -2.393610 & -0.461737 & -0.504761 \\ \mathrm{H} & -3.091412 & -1.189548 & -0.912579 \\ \mathrm{C} & -2.962165 & 0.774234 & -0.213241 \\ \mathrm{C} & -2.243832 & 1.983501 & -0.085770 \\ \mathrm{C} & -0.973379 & 2.024198 & 0.506733 \\ \mathrm{H} & -0.866779 & 1.622713 & 1.501018 \\ \mathrm{H} & -0.333663 & 2.876973 & 0.293071 \\ \mathrm{C} & -2.752077 & 3.129033 & -0.778226 \\ \mathrm{~F} & -4.290456 & 0.876157 & -0.429126\end{array}$




$\begin{array}{lrrr}\mathrm{N} & -3.148881 & 4.079660 & -1.321365 \\ \mathrm{C} & & & \\ \mathrm{C} & & & \\ \mathrm{H} & 0.000000 & 0.000000 & 0.000000 \\ \mathrm{H} & 0.000000 & 0.000000 & 1.087348 \\ \mathrm{C} & -1.969089 & 0.000000 & -0.488716 \\ \mathrm{H} & -1.093919 & -0.001032 & -1.793149 \\ \mathrm{C} & -2.457759 & -0.023936 & -0.094595 \\ \mathrm{H} & -2.507234 & -0.118641 & 0.988236 \\ \mathrm{C} & -3.637251 & 0.031811 & -0.734629 \\ \mathrm{C} & -3.878893 & 0.231264 & -2.168624 \\ \mathrm{C} & -3.378648 & 1.200613 & -2.935812 \\ \mathrm{H} & -2.737686 & 1.963243 & -2.508394 \\ \mathrm{H} & -3.609674 & 1.240344 & -3.993973 \\ \mathrm{~N} & -4.770442 & -0.777877 & -2.811709 \\ \mathrm{~F} & -4.779184 & -0.055442 & -0.015745 \\ \mathrm{O} & -4.978259 & -1.806068 & -2.174561 \\ \mathrm{O} & -5.216433 & -0.527242 & -3.927676\end{array}$

$\begin{array}{crrc}\text { TS } & & & \\ \mathrm{H} & 0.000000 & 0.000000 & 0.000000 \\ \mathrm{C} & 0.000000 & 0.000000 & 1.078250 \\ \mathrm{H} & 0.999708 & 0.000000 & 1.510966 \\ \mathrm{C} & -1.012563 & 0.667510 & 1.762904 \\ \mathrm{H} & -0.735357 & 1.313427 & 2.598224 \\ \mathrm{C} & -2.391226 & 0.460458 & 1.596882 \\ \mathrm{H} & -3.087973 & 1.211343 & 1.963014 \\ \mathrm{C} & -2.967301 & -0.785446 & 1.363236 \\ \mathrm{C} & -2.210249 & -1.972623 & 1.228747 \\ \mathrm{C} & -0.981801 & -1.970803 & 0.575888 \\ \mathrm{H} & -0.959300 & -1.590070 & -0.433051 \\ \mathrm{H} & -0.295647 & -2.783109 & 0.791542 \\ \mathrm{~N} & -2.535601 & -3.121857 & 2.086518 \\ \mathrm{~F} & -4.294570 & -0.851238 & 1.538432 \\ \mathrm{O} & -3.598057 & -3.108609 & 2.706023 \\ \mathrm{O} & -1.714192 & -4.041757 & 2.130145\end{array}$




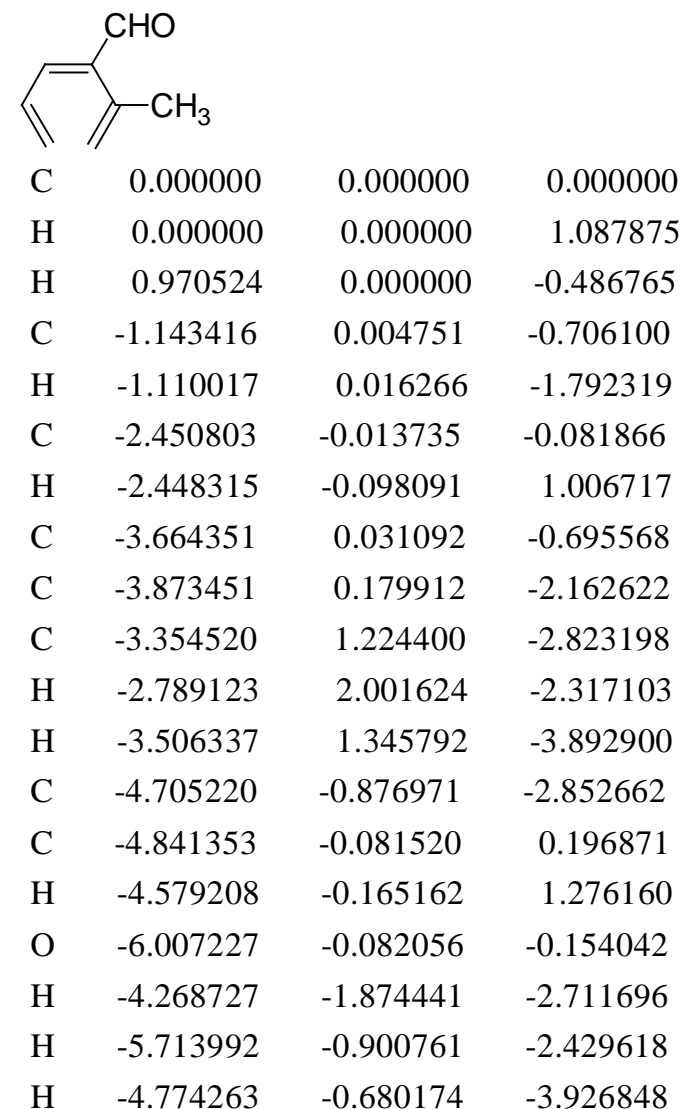

$\begin{array}{crrr}\text { TS } & & & \\ \mathrm{H} & 0.000000 & 0.000000 & 0.000000 \\ \mathrm{C} & 0.000000 & 0.000000 & 1.077990 \\ \mathrm{H} & 1.011637 & 0.000000 & 1.490234 \\ \mathrm{C} & -0.985864 & 0.681703 & 1.803660 \\ \mathrm{H} & -0.641078 & 1.384117 & 2.566823 \\ \mathrm{C} & -2.355246 & 0.409506 & 1.841233 \\ \mathrm{H} & -2.992487 & 1.171253 & 2.292789 \\ \mathrm{C} & -2.941726 & -0.873087 & 1.741342 \\ \mathrm{C} & -2.172605 & -2.025564 & 1.433700 \\ \mathrm{C} & -1.113633 & -1.891824 & 0.536134 \\ \mathrm{H} & -1.304827 & -1.319189 & -0.360088 \\ \mathrm{H} & -0.415131 & -2.716697 & 0.404537 \\ \mathrm{C} & -2.399435 & -3.340260 & 2.146815 \\ \mathrm{C} & -4.315569 & -0.974173 & 2.278416 \\ \mathrm{H} & -4.780215 & 0.011711 & 2.510722 \\ \mathrm{O} & -4.947830 & -2.001470 & 2.451846 \\ \mathrm{H} & -2.635716 & -3.213053 & 3.207236 \\ \mathrm{H} & -3.247207 & -3.874393 & 1.703585 \\ \mathrm{H} & -1.511134 & -3.973971 & 2.050062\end{array}$




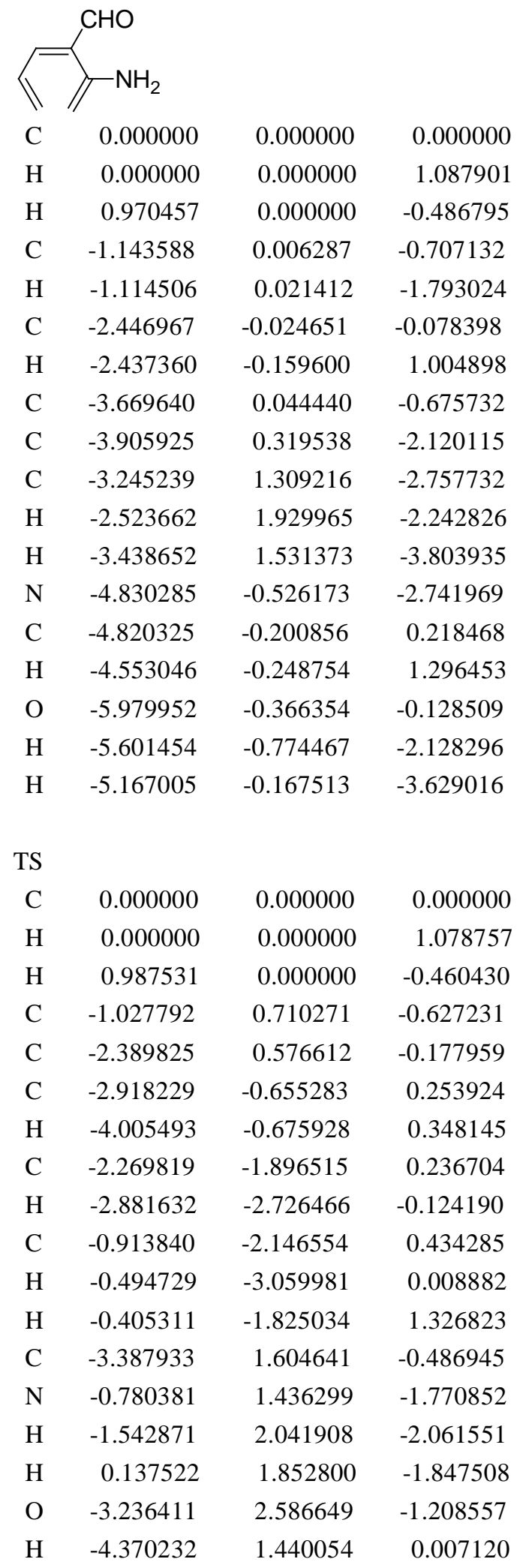




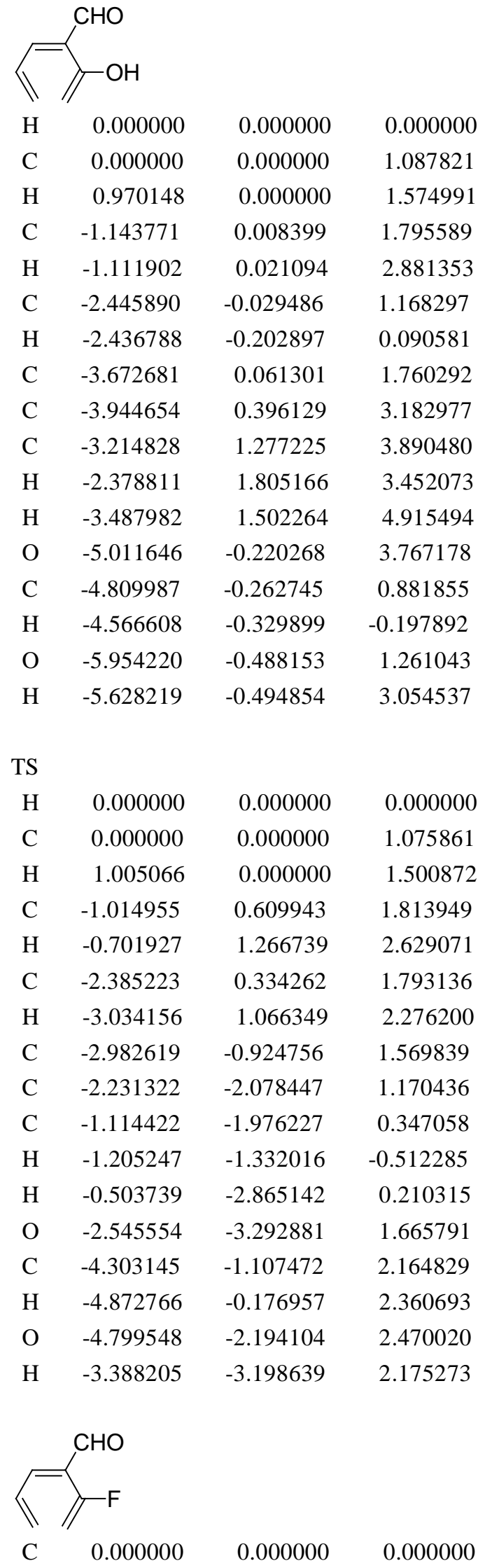




$\begin{array}{cccc}\mathrm{H} & 0.000000 & 0.000000 & 1.087966 \\ \mathrm{H} & 0.970218 & 0.000000 & -0.487163 \\ \mathrm{C} & -1.143784 & 0.000000 & -0.708707 \\ \mathrm{H} & -1.109062 & 0.000000 & -1.790147 \\ \mathrm{C} & -2.434311 & 0.000000 & -0.052568 \\ \mathrm{H} & -2.373671 & 0.000000 & 1.037173 \\ \mathrm{C} & -3.703579 & 0.000000 & -0.561113 \\ \mathrm{C} & -4.066909 & 0.000000 & -1.980197 \\ \mathrm{C} & -5.284608 & 0.000146 & -2.527350 \\ \mathrm{H} & -6.164260 & 0.000303 & -1.900989 \\ \mathrm{H} & -5.383366 & 0.000221 & -3.607088 \\ \mathrm{~F} & -2.995673 & 0.000218 & -2.826785 \\ \mathrm{C} & -4.769473 & 0.000000 & 0.475894 \\ \mathrm{H} & -4.362596 & 0.000072 & 1.510718 \\ \mathrm{O} & -5.972534 & 0.000000 & 0.298563\end{array}$

TS

$\begin{array}{lrrr}\mathrm{H} & 0.000000 & 0.000000 & 0.000000 \\ \mathrm{C} & 0.000000 & 0.000000 & 1.077864 \\ \mathrm{H} & 1.003735 & 0.000000 & 1.504152 \\ \mathrm{C} & -1.010204 & 0.682931 & 1.767030 \\ \mathrm{H} & -0.707203 & 1.362177 & 2.566266 \\ \mathrm{C} & -2.377639 & 0.442302 & 1.673476 \\ \mathrm{H} & -3.073768 & 1.175820 & 2.075637 \\ \mathrm{C} & -2.956312 & -0.823488 & 1.441894 \\ \mathrm{C} & -2.170486 & -1.968918 & 1.220869 \\ \mathrm{C} & -0.943279 & -2.035076 & 0.576478 \\ \mathrm{H} & -0.874147 & -1.620717 & -0.415904 \\ \mathrm{H} & -0.348251 & -2.931353 & 0.740309 \\ \mathrm{~F} & -2.581504 & -3.093927 & 1.871533 \\ \mathrm{C} & -4.392478 & -0.989227 & 1.777966 \\ \mathrm{O} & -5.144235 & -0.064694 & 2.032710 \\ \mathrm{H} & -4.764878 & -2.031121 & 1.757992\end{array}$

\begin{tabular}{lrrr} 
Z & \multicolumn{3}{l}{} \\
$\mathrm{H}$ & 0.000000 & 0.000000 & 0.000000 \\
$\mathrm{C}$ & 0.000000 & 0.000000 & 1.082685 \\
$\mathrm{H}$ & 0.969386 & 0.000000 & 1.576996 \\
$\mathrm{C}$ & -1.133795 & 0.009295 & 1.809708 \\
$\mathrm{C}$ & -2.480974 & 0.087266 & 1.200723 \\
$\mathrm{C}$ & -3.575200 & -0.635388 & 1.563957 \\
$\mathrm{H}$ & -4.498924 & -0.412088 & 1.026289
\end{tabular}




$\begin{array}{lrrr}\mathrm{C} & -3.680057 & -1.707908 & 2.530031 \\ \mathrm{H} & -2.794224 & -1.987716 & 3.089192 \\ \mathrm{C} & -4.835014 & -2.360444 & 2.744994 \\ \mathrm{H} & -4.906969 & -3.169129 & 3.465611 \\ \mathrm{H} & -5.746056 & -2.100010 & 2.210653 \\ \mathrm{C} & -2.688154 & 1.034480 & 0.077784 \\ \mathrm{C} & -0.965846 & 0.067562 & 3.287259 \\ \mathrm{H} & -3.749141 & 1.111193 & -0.248061 \\ \mathrm{O} & -1.822595 & 1.679505 & -0.483154 \\ \mathrm{O} & -1.860544 & 0.106850 & 4.107525 \\ \mathrm{H} & 0.099728 & 0.081671 & 3.606907\end{array}$

TS

$\begin{array}{lrrr}\mathrm{C} & 0.000000 & 0.000000 & 0.000000 \\ \mathrm{H} & 0.000000 & 0.000000 & 1.089830 \\ \mathrm{H} & 0.984842 & 0.000000 & -0.442048 \\ \mathrm{C} & -1.015314 & -0.682581 & -0.677066 \\ \mathrm{C} & -1.246344 & -0.460446 & -2.065226 \\ \mathrm{C} & -1.133107 & 0.819916 & -2.625623 \\ \mathrm{H} & -1.575021 & 0.911325 & -3.616083 \\ \mathrm{C} & -0.877087 & 1.983472 & -1.887533 \\ \mathrm{H} & -1.528271 & 2.829088 & -2.121829 \\ \mathrm{C} & -0.038333 & 2.106478 & -0.777820 \\ \mathrm{H} & 1.005843 & 1.849684 & -0.862829 \\ \mathrm{H} & -0.216239 & 2.938400 & -0.095500 \\ \mathrm{C} & -1.964155 & -1.470405 & 0.141517 \\ \mathrm{C} & -1.864896 & -1.511619 & -2.937177 \\ \mathrm{H} & -1.791160 & -1.367940 & 1.236344 \\ \mathrm{O} & -2.853697 & -2.190438 & -0.276936 \\ \mathrm{H} & -1.998264 & -2.501295 & -2.474016 \\ \mathrm{O} & -2.163327 & -1.315028 & -4.101385\end{array}$

\begin{tabular}{lrrr} 
\} $&{\multicolumn{3}{l}{\text { C }}\text { C }} \\
{\text { C }} &{ } &{ } &{ } \\
{\mathrm{C}} &{0.000000} &{0.000000} &{0.000000} \\
{\mathrm{H}} &{0.000000} &{0.000000} &{1.088024} \\
{\mathrm{H}} &{0.969782} &{0.000000} &{-0.487813} \\
{\mathrm{C}} &{-1.143489} &{0.000000} &{-0.709794} \\
{\mathrm{H}} &{-1.094785} &{0.000000} &{-1.791498} \\
{\mathrm{C}} &{-2.428481} &{0.000000} &{-0.044486} \\
{\mathrm{H}} &{-2.345574} &{0.000000} &{1.043658} \\
{\mathrm{C}} &{-3.713477} &{0.000000} &{-0.515669} \\
{\mathrm{C}} &{-4.152996} &{0.000000} &{-1.930193}$
\end{tabular}




$\begin{array}{lrrr}\mathrm{C} & -5.441654 & -0.000039 & -2.341729 \\ \mathrm{H} & -6.256921 & -0.000073 & -1.632503 \\ \mathrm{H} & -5.668962 & -0.000035 & -3.402658 \\ \mathrm{C} & -3.170726 & 0.000000 & -2.986539 \\ \mathrm{C} & -4.726284 & 0.000000 & 0.574891 \\ \mathrm{H} & -4.267093 & -0.000161 & 1.587173 \\ \mathrm{O} & -5.937966 & -0.000168 & 0.465131 \\ \mathrm{~N} & -2.414720 & 0.000000 & -3.870739\end{array}$

TS

$\begin{array}{lrrr}\mathrm{H} & 0.000000 & 0.000000 & 0.000000 \\ \mathrm{C} & 0.000000 & 0.000000 & 1.077975 \\ \mathrm{H} & 1.003427 & 0.000000 & 1.504504 \\ \mathrm{C} & -1.008352 & 0.655182 & 1.785405 \\ \mathrm{H} & -0.696741 & 1.313310 & 2.600052 \\ \mathrm{C} & -2.388205 & 0.424513 & 1.721207 \\ \mathrm{H} & -3.061209 & 1.174531 & 2.131976 \\ \mathrm{C} & -2.974965 & -0.839301 & 1.559333 \\ \mathrm{C} & -2.193897 & -2.003534 & 1.314366 \\ \mathrm{C} & -1.037849 & -1.962487 & 0.529380 \\ \mathrm{H} & -1.117716 & -1.457819 & -0.421049 \\ \mathrm{H} & -0.347193 & -2.801313 & 0.549899 \\ \mathrm{C} & -2.499670 & -3.207639 & 2.032519 \\ \mathrm{C} & -4.406320 & -0.974902 & 1.954505 \\ \mathrm{H} & -4.783810 & -2.014899 & 2.011492 \\ \mathrm{O} & -5.138818 & -0.030636 & 2.179408 \\ \mathrm{~N} & -2.743668 & -4.197203 & 2.596969\end{array}$

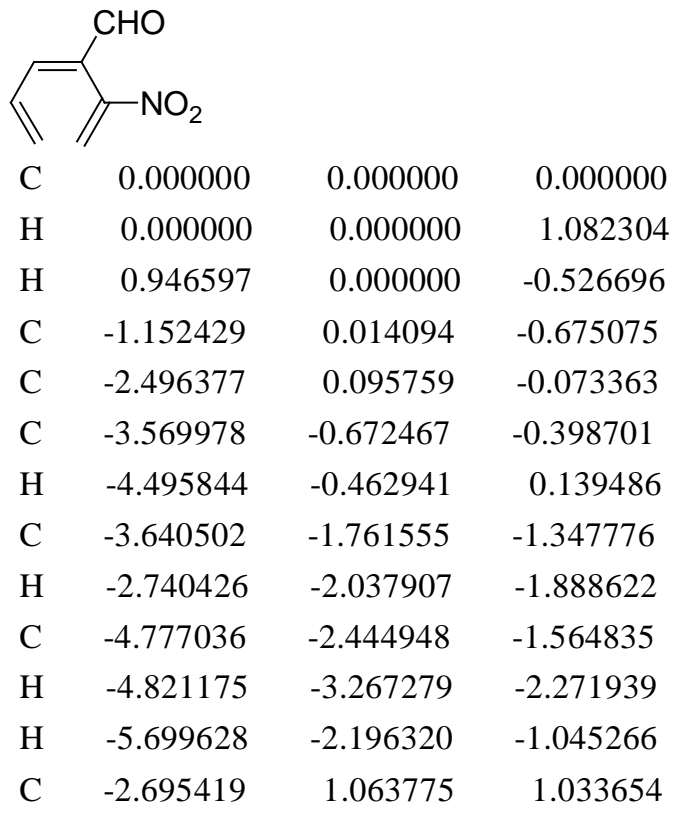




$\begin{array}{rrrr}\mathrm{N} & -1.054832 & 0.088592 & -2.163787 \\ \mathrm{O} & -2.073829 & 0.388375 & -2.783269 \\ \mathrm{O} & 0.035239 & -0.142443 & -2.681946 \\ \mathrm{O} & -1.826277 & 1.730683 & 1.559918 \\ \mathrm{H} & -3.749590 & 1.126889 & 1.381137\end{array}$

$\begin{array}{rrrr}\text { TS } & & & \\ \mathrm{C} & 0.000000 & 0.000000 & 0.000000 \\ \mathrm{H} & 0.000000 & 0.000000 & 1.078862 \\ \mathrm{H} & 1.002917 & 0.000000 & -0.426974 \\ \mathrm{C} & -1.005318 & -0.681138 & -0.688774 \\ \mathrm{H} & -0.703735 & -1.342352 & -1.504217 \\ \mathrm{C} & -2.385097 & -0.464155 & -0.583970 \\ \mathrm{H} & -3.074010 & -1.230482 & -0.935289 \\ \mathrm{C} & -2.974690 & 0.797154 & -0.424020 \\ \mathrm{C} & -2.165439 & 1.947548 & -0.246651 \\ \mathrm{C} & -0.980574 & 1.954486 & 0.479422 \\ \mathrm{H} & -1.024189 & 1.556337 & 1.481695 \\ \mathrm{H} & -0.285672 & 2.773443 & 0.325186 \\ \mathrm{~N} & -2.429607 & 3.102880 & -1.119731 \\ \mathrm{C} & -4.457274 & 0.871426 & -0.654834 \\ \mathrm{H} & -4.934097 & 1.831865 & -0.401953 \\ \mathrm{O} & -5.115207 & -0.081045 & -1.024008 \\ \mathrm{O} & -3.446623 & 3.072561 & -1.821509 \\ \mathrm{O} & -1.634124 & 4.041964 & -1.099510\end{array}$

6

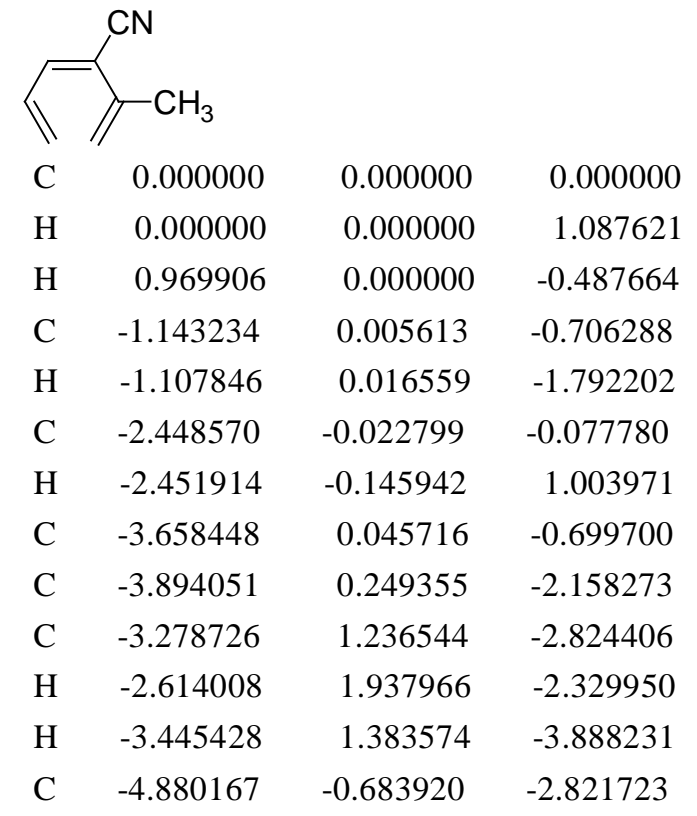




$\begin{array}{llll}\mathrm{C} & -4.832267 & -0.115373 & 0.113254 \\ \mathrm{~N} & -5.798291 & -0.244880 & 0.750944 \\ \mathrm{H} & -5.861715 & -0.629411 & -2.335423 \\ \mathrm{H} & -4.547674 & -1.727030 & -2.747278 \\ \mathrm{H} & -5.005466 & -0.434769 & -3.879135\end{array}$

TS

$\begin{array}{llll}\mathrm{H} & 0.000000 & 0.000000 & 0.000000 \\ \mathrm{H} & 0.000000 & 0.000000 & 1.802663 \\ \mathrm{C} & 0.603386 & 0.000000 & 0.909890 \\ \mathrm{C} & 1.824319 & 0.682944 & 0.867874 \\ \mathrm{H} & 1.977222 & 1.377802 & 0.038562 \\ \mathrm{C} & 2.970920 & 0.434108 & 1.626409 \\ \mathrm{H} & 3.754315 & 1.188507 & 1.604156 \\ \mathrm{C} & 3.378931 & -0.845865 & 2.071963 \\ \mathrm{C} & 2.599968 & -2.014942 & 1.883267 \\ \mathrm{C} & 1.211974 & -1.916059 & 1.968137 \\ \mathrm{H} & 0.597492 & -2.753911 & 1.644183 \\ \mathrm{H} & 0.811695 & -1.367555 & 2.807384 \\ \mathrm{C} & 3.274867 & -3.302767 & 1.462297 \\ \mathrm{C} & 4.770053 & -0.988951 & 2.400906 \\ \mathrm{~N} & 5.891166 & -1.110884 & 2.691650 \\ \mathrm{H} & 3.906996 & -3.171268 & 0.576121 \\ \mathrm{H} & 3.926648 & -3.672591 & 2.263758 \\ \mathrm{H} & 2.534163 & -4.079386 & 1.252411\end{array}$

\begin{tabular}{lrrr}
\multicolumn{3}{l}{ CN } \\
\hline
\end{tabular}




$\begin{array}{llll}\mathrm{H} & -2.703596 & -1.583056 & -2.472474 \\ \mathrm{H} & -3.426653 & -0.915656 & -3.814100\end{array}$

TS

$\begin{array}{lrrr}\mathrm{H} & 0.000000 & 0.000000 & 0.000000 \\ \mathrm{C} & 0.000000 & 0.000000 & 1.077186 \\ \mathrm{H} & 0.999348 & 0.000000 & 1.512503 \\ \mathrm{C} & -1.030729 & 0.625535 & 1.772760 \\ \mathrm{H} & -0.770859 & 1.250897 & 2.628474 \\ \mathrm{C} & -2.396730 & 0.385568 & 1.594427 \\ \mathrm{H} & -3.097081 & 1.116255 & 1.994531 \\ \mathrm{C} & -2.961285 & -0.852830 & 1.227388 \\ \mathrm{C} & -2.188897 & -2.056554 & 1.084656 \\ \mathrm{C} & -0.928564 & -2.068431 & 0.478172 \\ \mathrm{H} & -0.839912 & -1.617606 & -0.497389 \\ \mathrm{H} & -0.304335 & -2.948168 & 0.632934 \\ \mathrm{~N} & -2.636455 & -3.192470 & 1.738163 \\ \mathrm{C} & -4.374480 & -1.002900 & 1.415968 \\ \mathrm{H} & -3.520991 & -3.133881 & 2.226054 \\ \mathrm{H} & -2.483556 & -4.080472 & 1.281030 \\ \mathrm{~N} & -5.522915 & -1.151525 & 1.552221\end{array}$<smiles>N#Cc1ccccc1O</smiles>

$\begin{array}{lrrr}\mathrm{C} & 0.000000 & 0.000000 & 0.000000 \\ \mathrm{H} & 0.000000 & 0.000000 & 1.087849 \\ \mathrm{H} & 0.970195 & 0.000000 & -0.487278 \\ \mathrm{C} & -1.143306 & 0.000000 & -0.708962 \\ \mathrm{H} & -1.115085 & -0.000016 & -1.790986 \\ \mathrm{C} & -2.433466 & 0.000000 & -0.051253 \\ \mathrm{H} & -2.387508 & 0.000000 & 1.036635 \\ \mathrm{C} & -3.692539 & 0.000000 & -0.582614 \\ \mathrm{C} & -4.092322 & 0.000000 & -2.002797 \\ \mathrm{C} & -5.372995 & -0.000020 & -2.411782 \\ \mathrm{H} & -6.191176 & -0.000037 & -1.704301 \\ \mathrm{H} & -5.624433 & -0.000019 & -3.468845 \\ \mathrm{O} & -3.031717 & 0.000000 & -2.869811 \\ \mathrm{C} & -4.775945 & 0.000000 & 0.361304 \\ \mathrm{~N} & -5.647130 & -0.000002 & 1.132985 \\ \mathrm{H} & -3.377962 & 0.000000 & -3.776111\end{array}$

TS

$\begin{array}{llll}\mathrm{H} & 0.000000 & 0.000000 & 0.000000\end{array}$ 


\begin{tabular}{|c|c|c|c|}
\hline $\mathrm{C}$ & 0.000000 & 0.000000 & 1.076919 \\
\hline $\mathrm{H}$ & 1.001347 & 0.000000 & 1.507981 \\
\hline $\mathrm{C}$ & -1.020297 & 0.644631 & 1.776775 \\
\hline $\mathrm{H}$ & -0.737932 & 1.296944 & 2.605043 \\
\hline $\mathrm{C}$ & -2.388762 & 0.397212 & 1.658980 \\
\hline $\mathrm{H}$ & -3.074119 & 1.127623 & 2.083654 \\
\hline $\mathrm{C}$ & -2.962089 & -0.856916 & 1.351850 \\
\hline $\mathrm{C}$ & -2.189369 & -2.037758 & 1.142834 \\
\hline $\mathrm{C}$ & -0.958521 & -2.050269 & 0.492612 \\
\hline $\mathrm{H}$ & -0.902527 & -1.576847 & -0.473446 \\
\hline $\mathrm{H}$ & -0.353583 & -2.947364 & 0.604823 \\
\hline $\mathrm{O}$ & -2.591065 & -3.209529 & 1.712196 \\
\hline $\mathrm{C}$ & -4.344825 & -1.044163 & 1.680499 \\
\hline $\mathrm{N}$ & -5.461591 & -1.240184 & 1.951220 \\
\hline $\mathrm{H}$ & -3.318388 & -3.047306 & 2.337948 \\
\hline & & & \\
\hline & & & \\
\hline $\mathrm{C}$ & 0.000000 & 0.000000 & 0.000000 \\
\hline $\mathrm{H}$ & 0.000000 & 0.000000 & 1.087695 \\
\hline $\mathrm{H}$ & 0.969618 & 0.000000 & -0.488070 \\
\hline $\mathrm{C}$ & -1.143331 & 0.000000 & -0.708619 \\
\hline $\mathrm{H}$ & -1.108291 & 0.000000 & -1.790547 \\
\hline $\mathrm{C}$ & -2.433687 & 0.000000 & -0.052171 \\
\hline $\mathrm{H}$ & -2.394237 & 0.000000 & 1.035621 \\
\hline $\mathrm{C}$ & -3.687438 & 0.000018 & -0.595445 \\
\hline $\mathrm{C}$ & -4.056267 & 0.000043 & -2.016178 \\
\hline $\mathrm{C}$ & -5.290205 & 0.000005 & -2.526131 \\
\hline $\mathrm{H}$ & -6.158743 & -0.000050 & -1.880439 \\
\hline $\mathrm{H}$ & -5.434349 & -0.000003 & -3.599869 \\
\hline $\mathrm{F}$ & -3.000384 & 0.000027 & -2.866860 \\
\hline $\mathrm{C}$ & -4.794534 & 0.000013 & 0.319481 \\
\hline $\mathrm{N}$ & -5.694509 & 0.000008 & 1.057125 \\
\hline
\end{tabular}

$\begin{array}{crrr}\text { TS } & & & \\ \mathrm{H} & 0.000000 & 0.000000 & 0.000000 \\ \mathrm{C} & 0.000000 & 0.000000 & 1.077644 \\ \mathrm{H} & 1.001977 & 0.000000 & 1.506802 \\ \mathrm{C} & -1.011349 & 0.682626 & 1.761306 \\ \mathrm{H} & -0.719481 & 1.357589 & 2.567549 \\ \mathrm{C} & -2.379842 & 0.448458 & 1.648751 \\ \mathrm{H} & -3.062593 & 1.191874 & 2.053220 \\ \mathrm{C} & -2.955476 & -0.817554 & 1.387056\end{array}$




$\begin{array}{llll}\mathrm{C} & -2.175496 & -1.974570 & 1.188627 \\ \mathrm{C} & -0.927560 & -2.041611 & 0.585444 \\ \mathrm{H} & -0.824970 & -1.642331 & -0.409680 \\ \mathrm{H} & -0.341354 & -2.937350 & 0.780571 \\ \mathrm{~F} & -2.624986 & -3.087950 & 1.815319 \\ \mathrm{C} & -4.345557 & -0.988117 & 1.698028 \\ \mathrm{~N} & -5.480957 & -1.098124 & 1.927383\end{array}$

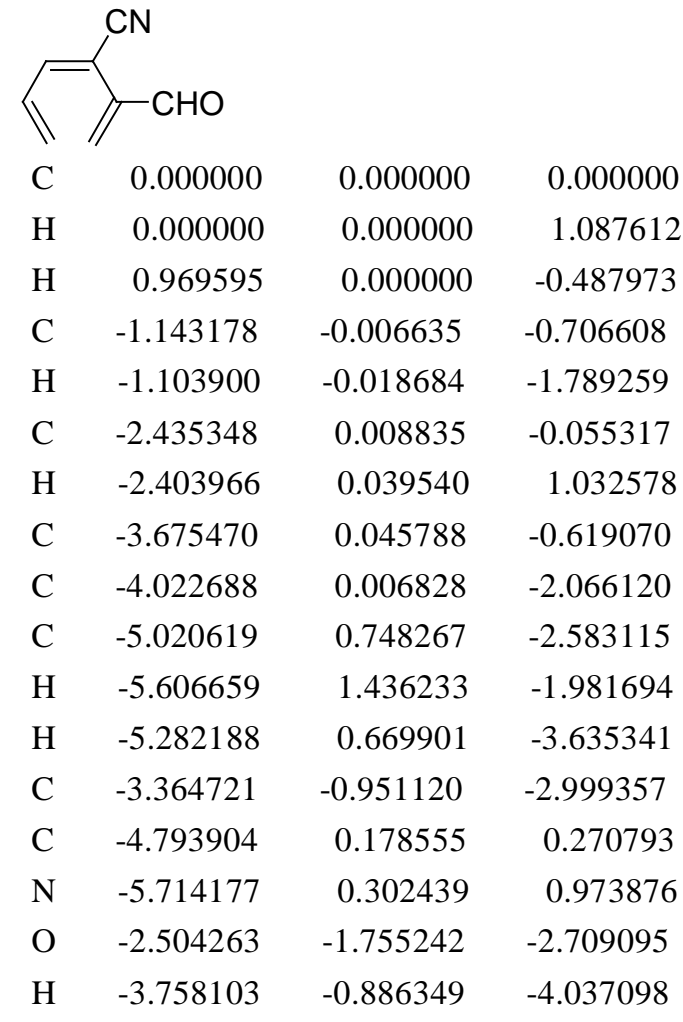

TS

$\begin{array}{lrrr}\mathrm{H} & 0.000000 & 0.000000 & 0.000000 \\ \mathrm{C} & 0.000000 & 0.000000 & 1.077577 \\ \mathrm{H} & 0.999834 & 0.000000 & 1.510472 \\ \mathrm{C} & -1.016258 & 0.647827 & 1.775128 \\ \mathrm{H} & -0.726443 & 1.289301 & 2.610404 \\ \mathrm{C} & -2.397042 & 0.430768 & 1.658844 \\ \mathrm{H} & -3.064188 & 1.186637 & 2.066563 \\ \mathrm{C} & -2.974395 & -0.832872 & 1.430896 \\ \mathrm{C} & -2.196477 & -2.010851 & 1.237823 \\ \mathrm{C} & -0.998049 & -1.990549 & 0.528811 \\ \mathrm{H} & -0.992620 & -1.527008 & -0.444913 \\ \mathrm{H} & -0.328880 & -2.838716 & 0.653185 \\ \mathrm{C} & -2.552753 & -3.239439 & 2.000546 \\ \mathrm{C} & -4.377017 & -0.953674 & 1.724886\end{array}$




$\begin{array}{llll}\mathrm{H} & -3.549014 & -3.226742 & 2.484969 \\ \mathrm{O} & -1.816784 & -4.203080 & 2.103654 \\ \mathrm{~N} & -5.519402 & -1.034927 & 1.933523\end{array}$

\begin{tabular}{lrrr} 
& \multicolumn{3}{l}{} \\
\hline & & & \\
$\mathrm{C}$ & $\mathrm{CN}$ & & \\
$\mathrm{H}$ & 0.000000 & 0.000000 & 0.000000 \\
$\mathrm{H}$ & 0.000000 & 0.000000 & 1.087701 \\
$\mathrm{C}$ & -1.143652 & 0.000000 & -0.708791 \\
$\mathrm{H}$ & -1.096984 & 0.000000 & -1.791107 \\
$\mathrm{C}$ & -2.428918 & 0.000000 & -0.044733 \\
$\mathrm{H}$ & -2.373271 & 0.000000 & 1.042484 \\
$\mathrm{C}$ & -3.695577 & 0.000000 & -0.558272 \\
$\mathrm{C}$ & -4.140646 & -0.000020 & -1.971828 \\
$\mathrm{C}$ & -5.442184 & 0.000101 & -2.332361 \\
$\mathrm{H}$ & -6.232612 & 0.000205 & -1.589528 \\
$\mathrm{H}$ & -5.729119 & 0.000147 & -3.377635 \\
$\mathrm{C}$ & -3.173995 & -0.000065 & -3.037223 \\
$\mathrm{C}$ & -4.763195 & 0.000000 & 0.403589 \\
$\mathrm{~N}$ & -5.635978 & 0.000000 & 1.173484 \\
$\mathrm{~N}$ & -2.426822 & -0.000102 & -3.928331
\end{tabular}

TA

$\begin{array}{lrrr}\mathrm{H} & 0.000000 & 0.000000 & 0.000000 \\ \mathrm{C} & 0.000000 & 0.000000 & 1.077767 \\ \mathrm{H} & 1.002414 & 0.000000 & 1.505932 \\ \mathrm{C} & -1.007742 & 0.656788 & 1.782081 \\ \mathrm{H} & -0.702877 & 1.315389 & 2.598342 \\ \mathrm{C} & -2.389255 & 0.430528 & 1.709096 \\ \mathrm{H} & -3.046527 & 1.191514 & 2.122748 \\ \mathrm{C} & -2.976580 & -0.836071 & 1.528237 \\ \mathrm{C} & -2.194815 & -2.006489 & 1.300548 \\ \mathrm{C} & -1.028460 & -1.958919 & 0.532061 \\ \mathrm{H} & -1.099107 & -1.467847 & -0.426015 \\ \mathrm{H} & -0.333754 & -2.793342 & 0.573132 \\ \mathrm{C} & -2.513228 & -3.205890 & 2.016702 \\ \mathrm{C} & -4.358419 & -0.967691 & 1.894230 \\ \mathrm{~N} & -5.487739 & -1.048040 & 2.162153 \\ \mathrm{~N} & -2.749376 & -4.197916 & 2.578786\end{array}$




\begin{tabular}{|c|c|c|c|}
\hline \multicolumn{4}{|c|}{$\mathrm{CN}$} \\
\hline & \multicolumn{3}{|l|}{$-\mathrm{NO}_{2}$} \\
\hline $\mathrm{C}$ & 0.000000 & 0.000000 & 0.000000 \\
\hline $\mathrm{H}$ & 0.000000 & 0.000000 & 1.087365 \\
\hline $\mathrm{H}$ & 0.969267 & 0.000000 & -0.488529 \\
\hline $\mathrm{C}$ & -1.143320 & -0.000639 & -0.705577 \\
\hline $\mathrm{H}$ & -1.106660 & 0.001736 & -1.791883 \\
\hline $\mathrm{C}$ & -2.444400 & -0.020174 & -0.069729 \\
\hline $\mathrm{H}$ & -2.446963 & -0.090007 & 1.016295 \\
\hline $\mathrm{C}$ & -3.655504 & 0.014719 & -0.687055 \\
\hline $\mathrm{C}$ & -3.833540 & 0.183336 & -2.148971 \\
\hline $\mathrm{C}$ & -3.415393 & 1.209910 & -2.888167 \\
\hline $\mathrm{H}$ & -2.902982 & 2.046663 & -2.426682 \\
\hline $\mathrm{H}$ & -3.591084 & 1.225696 & -3.957561 \\
\hline $\mathrm{N}$ & -4.553874 & -0.926668 & -2.840207 \\
\hline $\mathrm{C}$ & -4.845526 & -0.050672 & 0.110545 \\
\hline $\mathrm{N}$ & -5.809121 & -0.071322 & 0.762633 \\
\hline $\mathrm{O}$ & -4.835703 & -1.903883 & -2.151721 \\
\hline $\mathrm{O}$ & -4.804920 & -0.800676 & -4.034565 \\
\hline IN & & & \\
\hline $\mathrm{H}$ & 0.000000 & 0.000000 & 0.000000 \\
\hline $\mathrm{C}$ & 0.000000 & 0.000000 & 1.078151 \\
\hline $\mathrm{H}$ & 1.002634 & 0.000000 & 1.505707 \\
\hline $\mathrm{C}$ & -1.006701 & 0.663092 & 1.778536 \\
\hline $\mathrm{H}$ & -0.703231 & 1.330591 & 2.588086 \\
\hline $\mathrm{C}$ & -2.388571 & 0.435739 & 1.704108 \\
\hline $\mathrm{H}$ & -3.049628 & 1.209075 & 2.087350 \\
\hline $\mathrm{C}$ & -2.979495 & -0.834422 & 1.545881 \\
\hline $\mathrm{C}$ & -2.173193 & -1.978931 & 1.313034 \\
\hline $\mathrm{C}$ & -1.018485 & -1.933973 & 0.546063 \\
\hline $\mathrm{H}$ & -0.312589 & -2.753029 & 0.628540 \\
\hline $\mathrm{H}$ & -1.108629 & -1.475265 & -0.426587 \\
\hline $\mathrm{N}$ & -2.410833 & -3.189516 & 2.122868 \\
\hline $\mathrm{C}$ & -4.380906 & -0.910628 & 1.849918 \\
\hline $\mathrm{O}$ & -3.480739 & -3.278743 & 2.721060 \\
\hline $\mathrm{O}$ & -1.523594 & -4.043329 & 2.140203 \\
\hline $\mathrm{N}$ & -5.528088 & -0.842391 & 2.030843 \\
\hline
\end{tabular}




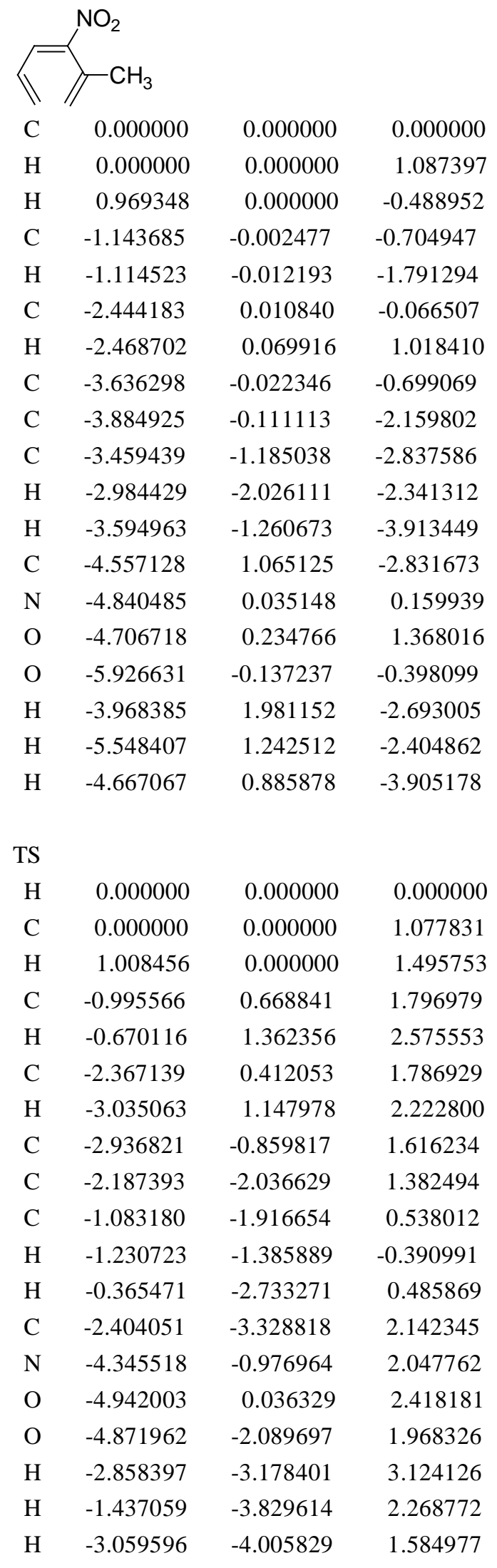




\begin{tabular}{lrrr} 
& \multicolumn{3}{l}{$\mathrm{NO}_{2}$} \\
$\mathrm{C}$ & & & \\
$\mathrm{C}$ & 0.000000 & 0.000000 & 0.000000 \\
$\mathrm{H}$ & 0.000000 & 0.000000 & 1.082568 \\
$\mathrm{H}$ & 0.964539 & 0.000000 & -0.500867 \\
$\mathrm{C}$ & -1.150865 & 0.002892 & -0.699794 \\
$\mathrm{C}$ & -2.445334 & -0.031994 & 0.030993 \\
$\mathrm{C}$ & -2.842051 & -0.954168 & 0.935295 \\
$\mathrm{H}$ & -3.806363 & -0.787156 & 1.407637 \\
$\mathrm{C}$ & -2.118080 & -2.150523 & 1.309320 \\
$\mathrm{H}$ & -1.184358 & -2.357598 & 0.794347 \\
$\mathrm{C}$ & -2.573443 & -2.989437 & 2.255479 \\
$\mathrm{H}$ & -3.498878 & -2.796588 & 2.793015 \\
$\mathrm{H}$ & -2.032297 & -3.891986 & 2.522399 \\
$\mathrm{~N}$ & -3.400085 & 1.058886 & -0.252722 \\
$\mathrm{~N}$ & -1.225005 & -0.082102 & -2.096933 \\
$\mathrm{H}$ & -1.960914 & 0.505777 & -2.475404 \\
$\mathrm{H}$ & -0.341215 & 0.133142 & -2.547546 \\
$\mathrm{O}$ & -3.103758 & 1.843381 & -1.162469 \\
$\mathrm{O}$ & -4.431477 & 1.142406 & 0.411766
\end{tabular}

$\begin{array}{crrr}\text { TS } & & & \\ \mathrm{C} & 0.000000 & 0.000000 & 0.000000 \\ \mathrm{H} & 0.000000 & 0.000000 & 1.078635 \\ \mathrm{H} & 0.983340 & 0.000000 & -0.467345 \\ \mathrm{C} & -1.035757 & 0.688468 & -0.635090 \\ \mathrm{C} & -2.378000 & 0.489700 & -0.151756 \\ \mathrm{C} & -2.889389 & -0.763161 & 0.208788 \\ \mathrm{H} & -3.971283 & -0.798329 & 0.297515 \\ \mathrm{C} & -2.193612 & -1.976565 & 0.146641 \\ \mathrm{H} & -2.767778 & -2.811299 & -0.260225 \\ \mathrm{C} & -0.838750 & -2.182903 & 0.380127 \\ \mathrm{H} & -0.368310 & -1.855149 & 1.291312 \\ \mathrm{H} & -0.368773 & -3.065359 & -0.055938 \\ \mathrm{~N} & -3.394526 & 1.520472 & -0.345419 \\ \mathrm{~N} & -0.794409 & 1.378933 & -1.799876 \\ \mathrm{O} & -3.176661 & 2.452758 & -1.140957 \\ \mathrm{O} & -4.430430 & 1.440101 & 0.317262 \\ \mathrm{H} & -1.569486 & 1.939693 & -2.133204 \\ \mathrm{H} & 0.110523 & 1.822726 & -1.876666\end{array}$




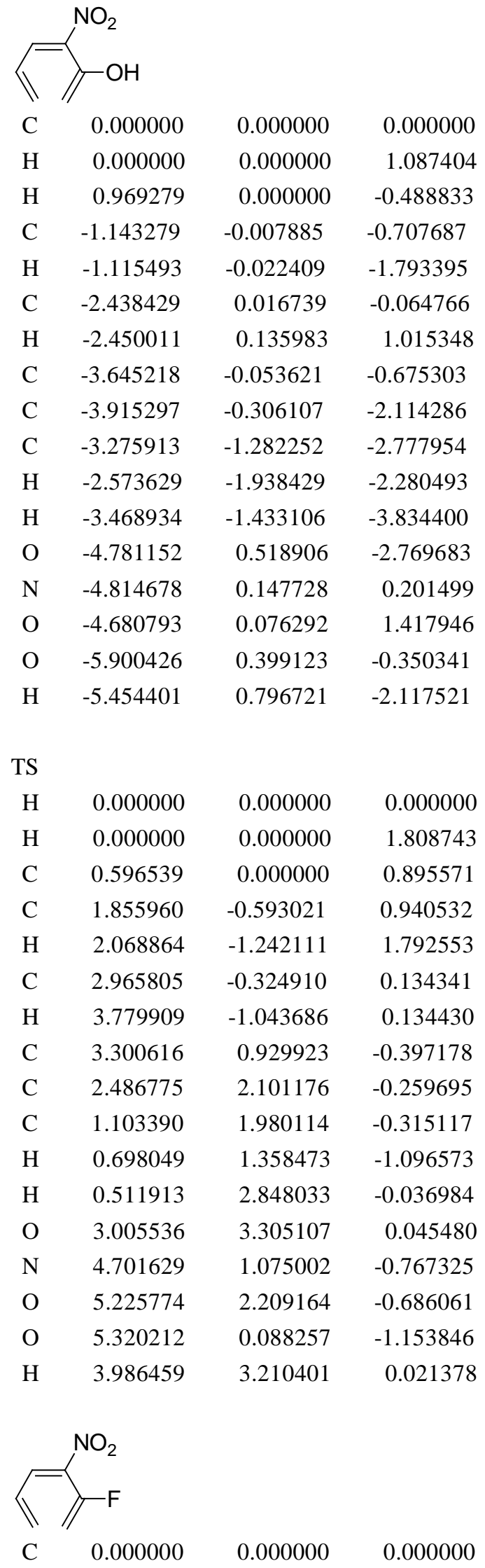




$\begin{array}{lrrr}\mathrm{H} & 0.000000 & 0.000000 & 1.087517 \\ \mathrm{H} & 0.969334 & 0.000000 & -0.488967 \\ \mathrm{C} & -1.144059 & 0.000000 & -0.709429 \\ \mathrm{H} & -1.109800 & 0.000000 & -1.789949 \\ \mathrm{C} & -2.421663 & -0.000022 & -0.031259 \\ \mathrm{H} & -2.367571 & -0.000089 & 1.053398 \\ \mathrm{C} & -3.692831 & -0.000008 & -0.516086 \\ \mathrm{C} & -4.107882 & -0.000007 & -1.924494 \\ \mathrm{C} & -5.298478 & -0.000317 & -2.528518 \\ \mathrm{H} & -6.223768 & -0.000445 & -1.978968 \\ \mathrm{H} & -5.314587 & -0.000441 & -3.612990 \\ \mathrm{~F} & -3.015516 & 0.000202 & -2.744308 \\ \mathrm{~N} & -4.746830 & -0.000041 & 0.532263 \\ \mathrm{O} & -4.400653 & -0.001399 & 1.714032 \\ \mathrm{O} & -5.923687 & 0.001445 & 0.173203\end{array}$

TS

$\begin{array}{lrrr}\mathrm{H} & 0.000000 & 0.000000 & 0.000000 \\ \mathrm{H} & 0.000000 & 0.000000 & 1.808787 \\ \mathrm{C} & 0.598060 & 0.000000 & 0.897183 \\ \mathrm{C} & 1.816311 & -0.689997 & 0.905895 \\ \mathrm{H} & 2.013491 & -1.377434 & 1.729441 \\ \mathrm{C} & 2.889821 & -0.450425 & 0.053277 \\ \mathrm{H} & 3.689816 & -1.180673 & -0.022043 \\ \mathrm{C} & 3.205498 & 0.809600 & -0.476414 \\ \mathrm{C} & 2.481431 & 1.977402 & -0.166462 \\ \mathrm{C} & 1.104801 & 2.020452 & 0.005493 \\ \mathrm{H} & 0.706406 & 2.882711 & 0.535811 \\ \mathrm{H} & 0.489578 & 1.655832 & -0.801192 \\ \mathrm{~F} & 3.188338 & 3.050884 & 0.240866 \\ \mathrm{~N} & 4.548379 & 0.959047 & -1.068482 \\ \mathrm{O} & 4.789474 & 2.018604 & -1.643956 \\ \mathrm{O} & 5.329168 & 0.007721 & -1.000066\end{array}$

$\begin{array}{lrrr}\text { N } & \mathrm{NO}_{2} \\ \mathrm{C} & 0.000000 & 0.000000 & 0.000000 \\ \mathrm{H} & 0.000000 & 0.000000 & 1.085372 \\ \mathrm{H} & 0.955748 & 0.000000 & -0.515845 \\ \mathrm{C} & -1.133954 & 0.021915 & -0.717568 \\ \mathrm{C} & -2.468605 & -0.024609 & -0.090345 \\ \mathrm{C} & -2.958784 & -0.938601 & 0.775404 \\ \mathrm{H} & -3.968589 & -0.767543 & 1.139347\end{array}$




$\begin{array}{lrrr}\mathrm{C} & -2.275959 & -2.129260 & 1.234944 \\ \mathrm{H} & -1.292152 & -2.341972 & 0.824646 \\ \mathrm{C} & -2.825105 & -2.966347 & 2.131406 \\ \mathrm{H} & -3.804187 & -2.776370 & 2.564458 \\ \mathrm{H} & -2.310385 & -3.863818 & 2.460076 \\ \mathrm{~N} & -3.369011 & 1.082263 & -0.474884 \\ \mathrm{C} & -1.038192 & 0.045386 & -2.220379 \\ \mathrm{H} & -1.996617 & -0.059282 & -2.762556 \\ \mathrm{O} & 0.013807 & 0.141194 & -2.815188 \\ \mathrm{O} & -2.922232 & 1.885256 & -1.299996 \\ \mathrm{O} & -4.488052 & 1.153045 & 0.028697\end{array}$

TS

$\begin{array}{lrrr}\mathrm{C} & 0.000000 & 0.000000 & 0.000000 \\ \mathrm{H} & 0.000000 & 0.000000 & 1.079124 \\ \mathrm{H} & 0.977501 & 0.000000 & -0.476083 \\ \mathrm{C} & -1.024731 & 0.692674 & -0.645361 \\ \mathrm{C} & -2.371141 & 0.426271 & -0.290295 \\ \mathrm{C} & -2.875335 & -0.848899 & -0.026466 \\ \mathrm{H} & -3.956689 & -0.935526 & -0.069788 \\ \mathrm{C} & -2.089489 & -2.005667 & -0.076080 \\ \mathrm{H} & -2.550934 & -2.858278 & -0.577971 \\ \mathrm{C} & -0.744222 & -2.108020 & 0.282150 \\ \mathrm{H} & -0.164643 & -2.931331 & -0.134885 \\ \mathrm{H} & -0.434495 & -1.832743 & 1.277585 \\ \mathrm{~N} & -3.382127 & 1.484439 & -0.521548 \\ \mathrm{C} & -0.681384 & 1.417150 & -1.911305 \\ \mathrm{O} & 0.459692 & 1.483858 & -2.327485 \\ \mathrm{H} & -1.519648 & 1.862679 & -2.470485 \\ \mathrm{O} & -2.954988 & 2.617040 & -0.757505 \\ \mathrm{O} & -4.574652 & 1.198337 & -0.427917\end{array}$

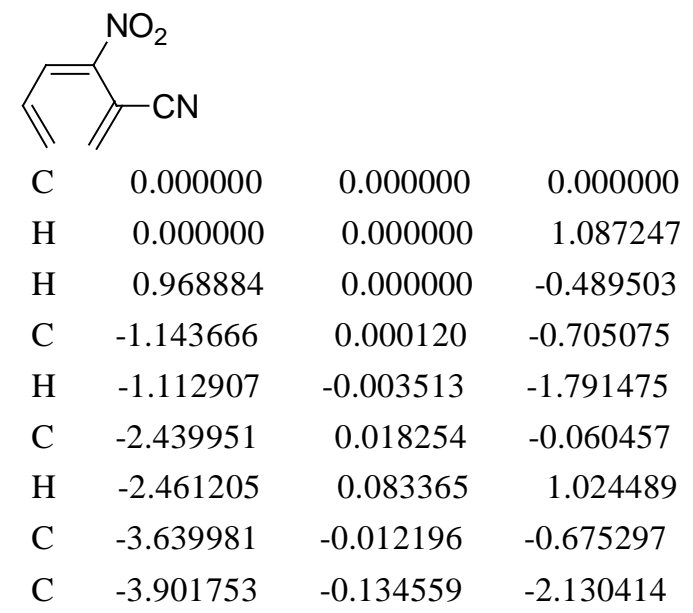




$\begin{array}{lrrc}\mathrm{C} & -3.472293 & -1.184313 & -2.851271 \\ \mathrm{H} & -2.960793 & -2.014070 & -2.374536 \\ \mathrm{H} & -3.636686 & -1.238048 & -3.922394 \\ \mathrm{C} & -4.586355 & 0.953161 & -2.781117 \\ \mathrm{~N} & -4.846900 & 0.066722 & 0.170309 \\ \mathrm{O} & -4.712779 & 0.206995 & 1.384689 \\ \mathrm{O} & -5.926669 & -0.024350 & -0.414858 \\ \mathrm{~N} & -5.101675 & 1.847217 & -3.316234\end{array}$

TS

$\begin{array}{lrrl}\mathrm{H} & 0.000000 & 0.000000 & 0.000000 \\ \mathrm{C} & 0.000000 & 0.000000 & 1.086328 \\ \mathrm{H} & 0.975827 & 0.000000 & 1.547662 \\ \mathrm{C} & -1.009818 & 0.693304 & 1.763913 \\ \mathrm{C} & -1.217252 & 0.433134 & 3.142501 \\ \mathrm{C} & -1.128135 & -0.839086 & 3.708258 \\ \mathrm{H} & -1.571084 & -0.926267 & 4.695612 \\ \mathrm{C} & -0.870596 & -2.000587 & 2.970684 \\ \mathrm{H} & -1.506355 & -2.850872 & 3.224860 \\ \mathrm{C} & -0.047118 & -2.119599 & 1.850498 \\ \mathrm{H} & -0.239844 & -2.943608 & 1.163255 \\ \mathrm{H} & 0.997734 & -1.861126 & 1.916212 \\ \mathrm{~N} & -1.873365 & 1.471818 & 3.971475 \\ \mathrm{C} & -1.958774 & 1.436435 & 0.987085 \\ \mathrm{~N} & -2.693122 & 1.983324 & 0.268413 \\ \mathrm{O} & -1.993093 & 2.586706 & 3.469155 \\ \mathrm{O} & -2.219074 & 1.175340 & 5.114625\end{array}$

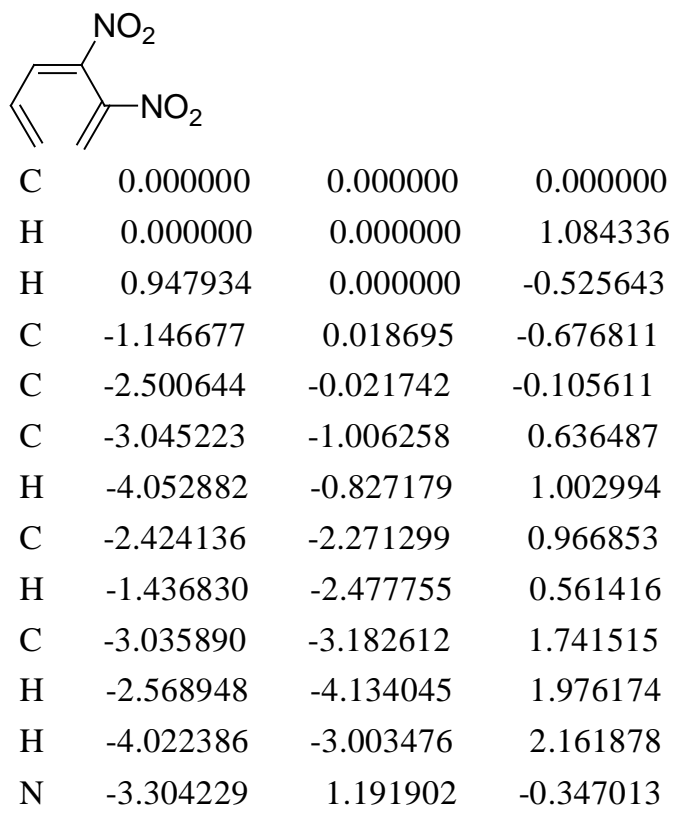




$\begin{array}{lrrr}\mathrm{N} & -1.084990 & 0.011286 & -2.168092 \\ \mathrm{O} & -2.147413 & -0.180439 & -2.752739 \\ \mathrm{O} & 0.006198 & 0.170268 & -2.703011 \\ \mathrm{O} & -2.730415 & 2.112068 & -0.929884 \\ \mathrm{O} & -4.465965 & 1.222106 & 0.051197\end{array}$

$\begin{array}{crrr}\text { TS } & & & \\ \mathrm{C} & 0.000000 & 0.000000 & 0.000000 \\ \mathrm{H} & 0.000000 & 0.000000 & 1.079331 \\ \mathrm{H} & 1.003200 & 0.000000 & -0.425771 \\ \mathrm{C} & -0.995909 & -0.718246 & -0.671024 \\ \mathrm{H} & -0.696705 & -1.406023 & -1.463346 \\ \mathrm{C} & -2.372646 & -0.500958 & -0.552358 \\ \mathrm{H} & -3.073406 & -1.262004 & -0.883317 \\ \mathrm{C} & -2.923346 & 0.766734 & -0.371916 \\ \mathrm{C} & -2.143973 & 1.935437 & -0.272077 \\ \mathrm{C} & -0.925852 & 1.949174 & 0.400955 \\ \mathrm{H} & -0.938563 & 1.633475 & 1.433054 \\ \mathrm{H} & -0.229609 & 2.747880 & 0.164184 \\ \mathrm{~N} & -2.408372 & 3.033162 & -1.226337 \\ \mathrm{~N} & -4.397722 & 0.874658 & -0.525112 \\ \mathrm{O} & -3.282192 & 2.862396 & -2.074191 \\ \mathrm{O} & -1.712946 & 4.044150 & -1.123102 \\ \mathrm{O} & -4.924653 & 1.843040 & 0.012045 \\ \mathrm{O} & -4.990886 & -0.031772 & -1.104031\end{array}$

\section{2, 4-substituted hexatriene}

1<smiles>Cc1cccc(C)c1</smiles>

$\begin{array}{llll}\mathrm{H} & 0.000000 & 0.000000 & 0.000000 \\ \mathrm{H} & 0.000000 & 0.000000 & 1.843924 \\ \mathrm{C} & 0.573635 & 0.000000 & 0.922233 \\ \mathrm{C} & 1.917349 & 0.000039 & 0.896057 \\ \mathrm{H} & 2.394318 & 0.000036 & -0.075883 \\ \mathrm{C} & 2.787908 & 0.000045 & 2.073539 \\ \mathrm{C} & 4.149332 & 0.000471 & 2.052342 \\ \mathrm{H} & 4.619092 & 0.000310 & 3.035668 \\ \mathrm{C} & 5.150537 & 0.001188 & 0.979395 \\ \mathrm{C} & 6.450569 & 0.001972 & 1.339584 \\ \mathrm{H} & 7.247322 & 0.002549 & 0.601317 \\ \mathrm{H} & 6.755403 & 0.002076 & 2.382846\end{array}$




$\begin{array}{rrrr}\text { C } & 4.796585 & 0.000979 & -0.491304 \\ \text { C } & 2.096586 & -0.000447 & 3.421193 \\ \text { H } & 1.453103 & -0.882111 & 3.538037 \\ \text { H } & 1.451804 & 0.880269 & 3.538015 \\ \text { H } & 2.819350 & 0.000140 & 4.241440 \\ \text { H } & 4.209141 & 0.884524 & -0.767679 \\ \text { H } & 4.209714 & -0.882983 & -0.767566 \\ \text { H } & 5.704794 & 0.001243 & -1.101204\end{array}$

TS

$\begin{array}{lrrr}\mathrm{H} & 0.000000 & 0.000000 & 0.000000 \\ \mathrm{C} & 0.000000 & 0.000000 & 1.078506 \\ \mathrm{H} & 1.000787 & 0.000000 & 1.512643 \\ \mathrm{C} & -1.016138 & -0.708288 & 1.729409 \\ \mathrm{C} & -2.369124 & -0.498637 & 1.418056 \\ \mathrm{H} & -3.078365 & -1.254538 & 1.757134 \\ \mathrm{C} & -2.945395 & 0.740432 & 1.076200 \\ \mathrm{C} & -2.194448 & 1.931358 & 1.055189 \\ \mathrm{H} & -2.679177 & 2.784749 & 1.536534 \\ \mathrm{C} & -0.854981 & 2.075931 & 0.691556 \\ \mathrm{H} & -0.528740 & 1.781939 & -0.292881 \\ \mathrm{H} & -0.321603 & 2.951409 & 1.066327 \\ \mathrm{C} & -4.456681 & 0.848519 & 1.073480 \\ \mathrm{C} & -0.682531 & -1.574007 & 2.931730 \\ \mathrm{H} & 0.163179 & -2.244835 & 2.743232 \\ \mathrm{H} & -0.415185 & -0.947460 & 3.794776 \\ \mathrm{H} & -1.541082 & -2.182997 & 3.233438 \\ \mathrm{H} & -4.933346 & 0.025396 & 1.616819 \\ \mathrm{H} & -4.788373 & 1.794020 & 1.519851 \\ \mathrm{H} & -4.846236 & 0.832253 & 0.046602\end{array}$

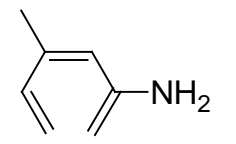

$\begin{array}{lrrr}\mathrm{H} & 0.000000 & 0.000000 & 0.000000 \\ \mathrm{H} & 0.000000 & 0.000000 & 1.844060 \\ \mathrm{C} & 0.574180 & 0.000000 & 0.921995 \\ \mathrm{C} & 1.917279 & 0.000081 & 0.948487 \\ \mathrm{H} & 2.417195 & 0.009267 & 1.912218 \\ \mathrm{C} & 2.786720 & 0.038026 & -0.232457 \\ \mathrm{C} & 4.142670 & -0.046719 & -0.188643 \\ \mathrm{H} & 4.664048 & 0.086353 & -1.134190 \\ \mathrm{C} & 5.068938 & -0.291153 & 0.925923 \\ \mathrm{C} & 6.371393 & 0.044811 & 0.794571\end{array}$




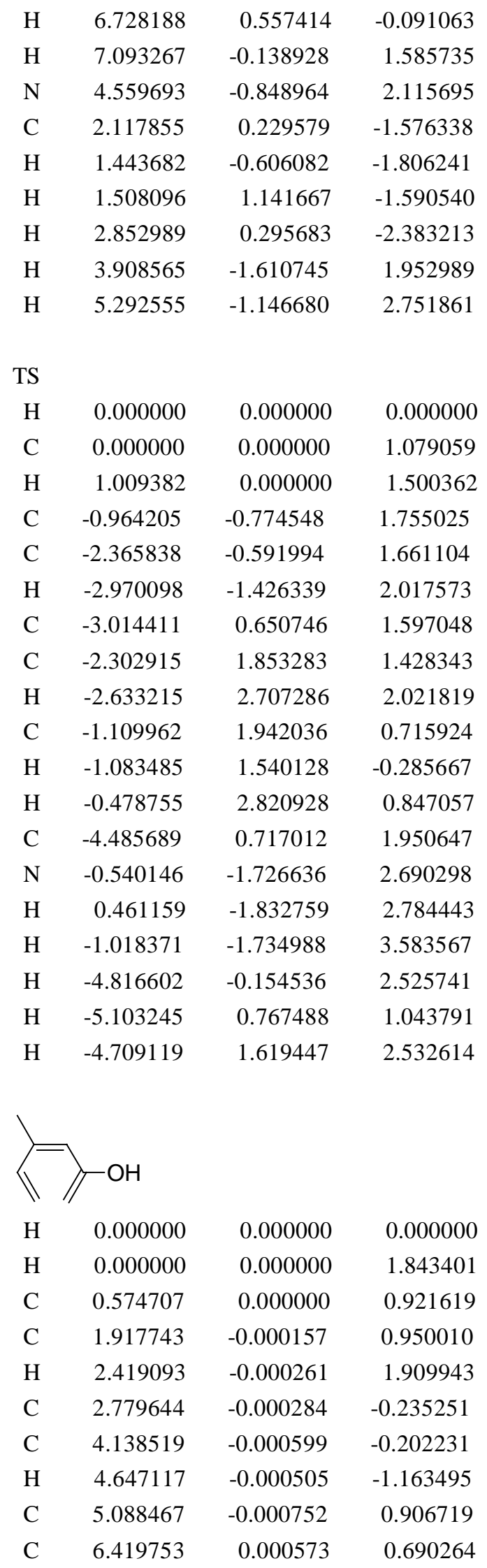




$\begin{array}{rrrr}\mathrm{H} & 6.819842 & 0.001724 & -0.315881 \\ \mathrm{H} & 7.135400 & 0.000730 & 1.508461 \\ \mathrm{O} & 4.554483 & -0.001900 & 2.170774 \\ \mathrm{C} & 2.099123 & -0.000022 & -1.587024 \\ \mathrm{H} & 1.456349 & 0.881403 & -1.706804 \\ \mathrm{H} & 1.455545 & -0.880876 & -1.706786 \\ \mathrm{H} & 2.826973 & -0.000355 & -2.403026 \\ \mathrm{H} & 5.284402 & -0.001332 & 2.810063\end{array}$

TS

$\begin{array}{lrrr}\mathrm{H} & 0.000000 & 0.000000 & 0.000000 \\ \mathrm{C} & 0.000000 & 0.000000 & 1.079091 \\ \mathrm{H} & 0.999444 & 0.000000 & 1.513268 \\ \mathrm{C} & -1.021869 & 0.681219 & 1.737216 \\ \mathrm{H} & -0.775905 & 1.293208 & 2.606353 \\ \mathrm{C} & -2.388301 & 0.473349 & 1.483835 \\ \mathrm{C} & -2.882035 & -0.781143 & 1.084131 \\ \mathrm{H} & -3.964308 & -0.907033 & 1.150367 \\ \mathrm{C} & -2.126486 & -1.969038 & 1.030867 \\ \mathrm{C} & -0.784593 & -2.095538 & 0.649305 \\ \mathrm{H} & -0.494233 & -1.795905 & -0.344299 \\ \mathrm{H} & -0.277946 & -2.993972 & 1.002778 \\ \mathrm{O} & -2.697003 & -3.113442 & 1.551100 \\ \mathrm{C} & -3.380763 & 1.543391 & 1.885212 \\ \mathrm{H} & -3.005924 & 2.128302 & 2.733308 \\ \mathrm{H} & -3.542878 & 2.251204 & 1.061304 \\ \mathrm{H} & -4.357225 & 1.127713 & 2.157058 \\ \mathrm{H} & -3.428372 & -2.860320 & 2.136622\end{array}$

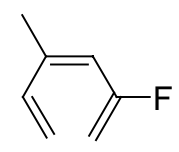

$\begin{array}{llll}\mathrm{H} & 0.000000 & 0.000000 & 0.000000 \\ \mathrm{H} & 0.000000 & 0.000000 & 1.843698 \\ \mathrm{C} & 0.574106 & 0.000000 & 0.921802 \\ \mathrm{C} & 1.916817 & 0.000137 & 0.949952 \\ \mathrm{H} & 2.413806 & 0.000160 & 1.911902 \\ \mathrm{C} & 2.778013 & 0.000324 & -0.235730 \\ \mathrm{C} & 4.137079 & 0.000404 & -0.196201 \\ \mathrm{H} & 4.660197 & 0.000645 & -1.150035 \\ \mathrm{C} & 5.064188 & 0.000525 & 0.920785 \\ \mathrm{C} & 6.399685 & 0.002027 & 0.815609 \\ \mathrm{H} & 6.874860 & 0.003173 & -0.157696 \\ \mathrm{H} & 7.027198 & 0.002319 & 1.698833\end{array}$




$\begin{array}{lrrr}\text { F } & 4.530106 & -0.000314 & 2.170566 \\ \mathrm{C} & 2.101623 & 0.000131 & -1.588573 \\ \mathrm{H} & 1.460385 & -0.882022 & -1.709999 \\ \mathrm{H} & 1.457839 & 0.880531 & -1.709008 \\ \mathrm{H} & 2.831828 & 0.001678 & -2.402287\end{array}$

TS

$\begin{array}{lrrc}\mathrm{H} & 0.000000 & 0.000000 & 0.000000 \\ \mathrm{C} & 0.000000 & 0.000000 & 1.079379 \\ \mathrm{H} & 0.998232 & 0.000000 & 1.515288 \\ \mathrm{C} & -1.022814 & 0.689196 & 1.729841 \\ \mathrm{H} & -0.777553 & 1.305920 & 2.595547 \\ \mathrm{C} & -2.386792 & 0.492323 & 1.468701 \\ \mathrm{C} & -2.881327 & -0.763821 & 1.067997 \\ \mathrm{H} & -3.955397 & -0.915087 & 1.155542 \\ \mathrm{C} & -2.121218 & -1.931485 & 0.985374 \\ \mathrm{C} & -0.772148 & -2.100828 & 0.670418 \\ \mathrm{H} & -0.409936 & -1.823211 & -0.305063 \\ \mathrm{H} & -0.312703 & -3.008919 & 1.062461 \\ \mathrm{~F} & -2.757247 & -3.038818 & 1.479078 \\ \mathrm{C} & -3.381973 & 1.556096 & 1.876226 \\ \mathrm{H} & -2.988645 & 2.169559 & 2.694673 \\ \mathrm{H} & -3.583944 & 2.236964 & 1.038446 \\ \mathrm{H} & -4.340438 & 1.130260 & 2.191353\end{array}$

\begin{tabular}{lrrr}
\hline$=$ & \multicolumn{3}{l}{} \\
\ & & & \\
$\mathrm{H}$ & 0.000000 & 0.000000 & 0.000000 \\
$\mathrm{H}$ & 0.000000 & 0.000000 & 1.845050 \\
$\mathrm{C}$ & 0.573131 & 0.000000 & 0.922602 \\
$\mathrm{C}$ & 1.915084 & 0.011274 & 0.942777 \\
$\mathrm{H}$ & 2.414568 & 0.019973 & 1.908838 \\
$\mathrm{C}$ & 2.786979 & -0.033759 & -0.234304 \\
$\mathrm{C}$ & 4.127976 & 0.163488 & -0.171864 \\
$\mathrm{H}$ & 4.707936 & -0.022370 & -1.073132 \\
$\mathrm{C}$ & 4.954283 & 0.552090 & 0.974878 \\
$\mathrm{C}$ & 4.622711 & 1.384385 & 1.984024 \\
$\mathrm{H}$ & 3.665994 & 1.891620 & 2.038106 \\
$\mathrm{H}$ & 5.335232 & 1.599206 & 2.777695 \\
$\mathrm{C}$ & 6.358210 & 0.045744 & 0.975471 \\
$\mathrm{C}$ & 2.152343 & -0.372861 & -1.563154 \\
$\mathrm{H}$ & 1.428521 & 0.397098 & -1.861491 \\
$\mathrm{H}$ & 1.604273 & -1.321583 & -1.511535
\end{tabular}




$\begin{array}{llrc}\mathrm{H} & 2.903143 & -0.448645 & -2.354218 \\ \mathrm{O} & 6.855908 & -0.615768 & 0.087070 \\ \mathrm{H} & 6.951166 & 0.340171 & 1.869863\end{array}$

TS

$\begin{array}{lrrr}\mathrm{H} & 0.000000 & 0.000000 & 0.000000 \\ \mathrm{C} & 0.000000 & 0.000000 & 1.078619 \\ \mathrm{H} & 1.000146 & 0.000000 & 1.511110 \\ \mathrm{C} & -1.008563 & 0.680899 & 1.753791 \\ \mathrm{H} & -0.722873 & 1.290003 & 2.614685 \\ \mathrm{C} & -2.400626 & 0.518920 & 1.573270 \\ \mathrm{C} & -2.948074 & -0.743095 & 1.311763 \\ \mathrm{H} & -4.009980 & -0.864761 & 1.517005 \\ \mathrm{C} & -2.204083 & -1.936132 & 1.214011 \\ \mathrm{C} & -0.923454 & -2.028591 & 0.653776 \\ \mathrm{H} & -0.771638 & -1.685712 & -0.356786 \\ \mathrm{H} & -0.311529 & -2.894793 & 0.912734 \\ \mathrm{C} & -2.753002 & -3.137517 & 1.883388 \\ \mathrm{C} & -3.322177 & 1.637656 & 2.009562 \\ \mathrm{H} & -2.899800 & 2.191858 & 2.856749 \\ \mathrm{H} & -3.461977 & 2.362916 & 1.197048 \\ \mathrm{H} & -4.311217 & 1.268359 & 2.297397 \\ \mathrm{O} & -3.914218 & -3.292885 & 2.217665 \\ \mathrm{H} & -2.003143 & -3.946212 & 2.043404\end{array}$

\begin{tabular}{lrrr}
\hline & \multicolumn{3}{l}{} \\
\} $&{ } &{ } &{ } \\
{\mathrm{H}} &{0.000000} &{0.000000} &{0.000000} \\
{\mathrm{H}} &{0.000000} &{0.000000} &{1.844475} \\
{\mathrm{C}} &{0.573130} &{0.000000} &{0.922279} \\
{\mathrm{C}} &{1.915731} &{0.000020} &{0.951621} \\
{\mathrm{H}} &{2.398509} &{0.000035} &{1.922002} \\
{\mathrm{C}} &{2.777609} &{0.000064} &{-0.231609} \\
{\mathrm{C}} &{4.137023} &{0.000240} &{-0.202516} \\
{\mathrm{H}} &{4.638843} &{0.000336} &{-1.168397} \\
{\mathrm{C}} &{5.106787} &{0.000388} &{0.896344} \\
{\mathrm{C}} &{6.437658} &{0.000939} &{0.649946} \\
{\mathrm{H}} &{6.812278} &{0.001283} &{-0.369341} \\
{\mathrm{H}} &{7.166734} &{0.001039} &{1.452089} \\
{\mathrm{C}} &{4.721239} &{-0.000118} &{2.285831} \\
{\mathrm{C}} &{2.098669} &{0.000090} &{-1.583764} \\
{\mathrm{H}} &{1.456419} &{0.881595} &{-1.701798} \\
{\mathrm{H}} &{1.456432} &{-0.881419} &{-1.701838}$
\end{tabular}




$\begin{array}{rrrr}\mathrm{H} & 2.827122 & 0.000105 & -2.398923 \\ \mathrm{~N} & 4.461828 & -0.000750 & 3.419658\end{array}$

$\begin{array}{rrrr}\text { TS } & & & \\ \mathrm{H} & 0.000000 & 0.000000 & 0.000000 \\ \mathrm{C} & 0.000000 & 0.000000 & 1.078213 \\ \mathrm{H} & 1.001273 & 0.000000 & 1.508683 \\ \mathrm{C} & -1.006507 & 0.671765 & 1.764887 \\ \mathrm{H} & -0.711515 & 1.292199 & 2.614307 \\ \mathrm{C} & -2.400349 & 0.491720 & 1.623472 \\ \mathrm{C} & -2.949524 & -0.778366 & 1.402078 \\ \mathrm{H} & -4.002698 & -0.892222 & 1.651160 \\ \mathrm{C} & -2.191720 & -1.963223 & 1.258067 \\ \mathrm{C} & -0.946965 & -2.015169 & 0.614263 \\ \mathrm{H} & -0.888655 & -1.617078 & -0.385781 \\ \mathrm{H} & -0.299671 & -2.870676 & 0.794678 \\ \mathrm{C} & -2.654756 & -3.120466 & 1.969868 \\ \mathrm{C} & -3.322085 & 1.608808 & 2.063524 \\ \mathrm{H} & -2.880484 & 2.184643 & 2.885606 \\ \mathrm{H} & -3.493929 & 2.314457 & 1.240236 \\ \mathrm{H} & -4.298347 & 1.236090 & 2.388879 \\ \mathrm{~N} & -3.040386 & -4.067051 & 2.529384\end{array}$

\begin{tabular}{lrrr}
\hline & & & \\
IV & & & \\
$\mathrm{H}$ & 0.000000 & 0.000000 & 0.000000 \\
$\mathrm{H}$ & 0.000000 & 0.000000 & 1.844892 \\
$\mathrm{C}$ & 0.573027 & 0.000000 & 0.922544 \\
$\mathrm{C}$ & 1.914604 & 0.009590 & 0.944203 \\
$\mathrm{H}$ & 2.414628 & 0.020384 & 1.909888 \\
$\mathrm{C}$ & 2.784008 & -0.031337 & -0.234814 \\
$\mathrm{C}$ & 4.126201 & 0.158578 & -0.173613 \\
$\mathrm{H}$ & 4.709810 & -0.007102 & -1.073317 \\
$\mathrm{C}$ & 4.906435 & 0.560460 & 0.993238 \\
$\mathrm{C}$ & 4.610952 & 1.452879 & 1.946124 \\
$\mathrm{H}$ & 3.680356 & 2.006368 & 1.909255 \\
$\mathrm{H}$ & 5.304158 & 1.646579 & 2.755155 \\
$\mathrm{~N}$ & 6.258417 & -0.083906 & 1.085935 \\
$\mathrm{C}$ & 2.148973 & -0.345970 & -1.568491 \\
$\mathrm{H}$ & 1.430420 & 0.433099 & -1.854458 \\
$\mathrm{H}$ & 1.595856 & -1.291945 & -1.529112 \\
$\mathrm{H}$ & 2.899310 & -0.415462 & -2.360325 \\
$\mathrm{O}$ & 6.561418 & -0.869649 & 0.189558
\end{tabular}




$\begin{array}{cccc}\mathrm{O} & 6.981865 & 0.203579 & 2.035753 \\ \mathrm{TS} & & & \\ \mathrm{H} & 0.000000 & 0.000000 & 0.000000 \\ \mathrm{H} & 0.000000 & 0.000000 & 1.812363 \\ \mathrm{C} & 0.594683 & 0.000000 & 0.899459 \\ \mathrm{C} & 1.815748 & -0.665506 & 0.911135 \\ \mathrm{H} & 2.048705 & -1.281840 & 1.782368 \\ \mathrm{C} & 2.890582 & -0.486188 & 0.011855 \\ \mathrm{C} & 3.212735 & 0.782369 & -0.488733 \\ \mathrm{H} & 4.218277 & 0.915929 & -0.873982 \\ \mathrm{C} & 2.483661 & 1.945549 & -0.196403 \\ \mathrm{C} & 1.107800 & 2.026018 & -0.004167 \\ \mathrm{H} & 0.475349 & 1.647534 & -0.790478 \\ \mathrm{H} & 0.716327 & 2.881220 & 0.537344 \\ \mathrm{~N} & 3.289073 & 3.138031 & 0.152999 \\ \mathrm{C} & 3.904038 & -1.598943 & -0.140556 \\ \mathrm{H} & 3.592576 & -2.298075 & -0.927675 \\ \mathrm{H} & 3.992167 & -2.182739 & 0.783309 \\ \mathrm{H} & 4.895148 & -1.219770 & -0.407634 \\ \mathrm{O} & 4.473488 & 3.153752 & -0.187905 \\ \mathrm{O} & 2.728314 & 4.060497 & 0.746615\end{array}$

\section{2}

$\mathrm{H}_{2} \mathrm{~N}$<smiles>C=CC(C)=CC(=C)C</smiles>

$\begin{array}{llll}\mathrm{H} & 0.000000 & 0.000000 & 0.000000\end{array}$

$\begin{array}{llll}\mathrm{H} & 0.000000 & 0.000000 & 1.848465\end{array}$

$\begin{array}{llll}\text { C } & 0.571130 & 0.000000 & 0.923546\end{array}$

$\begin{array}{llll}\text { C } & 1.921509 & 0.001984 & 0.921825\end{array}$

$\begin{array}{llll}\text { C } & 2.620426 & 0.021239 & 2.203915\end{array}$

$\begin{array}{llll}\mathrm{H} & 1.936534 & 0.084507 & 3.051990\end{array}$

$\begin{array}{llll}\text { C } & 3.944560 & 0.043759 & 2.535269\end{array}$

$\begin{array}{llll}\text { C } & 5.075349 & -0.122178 & 1.613029\end{array}$

$\begin{array}{llll}\mathrm{H} & 4.859100 & -0.581490 & 0.656893\end{array}$

$\begin{array}{llll}\text { C } & 6.344317 & 0.212794 & 1.893733\end{array}$

$\begin{array}{llll}\mathrm{H} & 6.620584 & 0.707428 & 2.819952\end{array}$

$\begin{array}{llll}\mathrm{H} & 7.141098 & 0.028395 & 1.179387\end{array}$

$\begin{array}{llll}\mathrm{N} & 4.324807 & 0.284950 & 3.868405\end{array}$

$\begin{array}{llll}\text { C } & 2.651692 & 0.001266 & -0.404140\end{array}$

$\begin{array}{llll}\mathrm{H} & 3.549374 & 0.287117 & 4.521754\end{array}$

$\begin{array}{llll}\mathrm{H} & 5.071232 & -0.320985 & 4.194484\end{array}$ 


$\begin{array}{rrrr}\mathrm{H} & 3.392879 & 0.806453 & -0.460559 \\ \mathrm{H} & 3.182094 & -0.943787 & -0.576874 \\ \mathrm{H} & 1.944528 & 0.132043 & -1.228504 \\ & & & \\ \mathrm{TS} & & & \\ \mathrm{H} & 0.000000 & 0.000000 & 0.000000 \\ \mathrm{C} & 0.000000 & 0.000000 & 1.078401 \\ \mathrm{H} & 1.005717 & 0.000000 & 1.500980 \\ \mathrm{C} & -0.993625 & 0.676941 & 1.780466 \\ \mathrm{H} & -0.703188 & 1.285038 & 2.640524 \\ \mathrm{C} & -2.382633 & 0.449974 & 1.658395 \\ \mathrm{C} & -2.916364 & -0.845130 & 1.478477 \\ \mathrm{H} & -3.958010 & -0.952694 & 1.788286 \\ \mathrm{C} & -2.168111 & -2.018856 & 1.328816 \\ \mathrm{C} & -0.941399 & -2.034553 & 0.648727 \\ \mathrm{H} & -0.911388 & -1.628790 & -0.350250 \\ \mathrm{H} & -0.286679 & -2.896837 & 0.785416 \\ \mathrm{C} & -2.634891 & -3.277313 & 2.039629 \\ \mathrm{~N} & -3.264057 & 1.477957 & 2.013691 \\ \mathrm{H} & -2.823224 & 2.283693 & 2.441550 \\ \mathrm{H} & -4.099096 & 1.191729 & 2.513365 \\ \mathrm{H} & -3.482335 & -3.089738 & 2.706737 \\ \mathrm{H} & -2.926814 & -4.064435 & 1.332055 \\ \mathrm{H} & -1.817593 & -3.688376 & 2.647073\end{array}$

$\begin{array}{lrrr}\mathrm{H}_{2} \mathrm{~N} & & & \\ & = & & \\ \mathrm{H} & 0.000000 & 0.000000 & 0.000000 \\ \mathrm{C} & 0.000000 & 0.000000 & 1.083825 \\ \mathrm{H} & 0.966539 & 0.000000 & 1.579919 \\ \mathrm{C} & -1.157599 & 0.053376 & 1.781993 \\ \mathrm{C} & -2.442285 & 0.002400 & 1.085157 \\ \mathrm{H} & -2.359000 & -0.355782 & 0.060207 \\ \mathrm{C} & -3.699907 & 0.295108 & 1.523792 \\ \mathrm{C} & -4.020014 & 0.926935 & 2.811953 \\ \mathrm{H} & -3.191610 & 1.420079 & 3.309325 \\ \mathrm{C} & -5.242851 & 0.952735 & 3.363918 \\ \mathrm{H} & -6.090229 & 0.435373 & 2.922921 \\ \mathrm{H} & -5.422414 & 1.475156 & 4.298959 \\ \mathrm{~N} & -4.816200 & -0.025719 & 0.732801 \\ \mathrm{~N} & -1.166848 & 0.193678 & 3.186728 \\ \mathrm{H} & -1.845190 & -0.410275 & 3.641535 \\ \mathrm{H} & -0.248038 & 0.054292 & 3.595148\end{array}$




\begin{tabular}{|c|c|c|c|}
\hline $\mathrm{H}$ & -5.520709 & 0.704673 & 0.709888 \\
\hline $\mathrm{H}$ & -4.581074 & -0.331631 & -0.205006 \\
\hline \multicolumn{4}{|l|}{ TS } \\
\hline $\mathrm{C}$ & 0.000000 & 0.000000 & 0.000000 \\
\hline $\mathrm{H}$ & 0.000000 & 0.000000 & 1.079966 \\
\hline $\mathrm{H}$ & 1.010447 & 0.000000 & -0.419306 \\
\hline $\mathrm{C}$ & -0.953255 & 0.799933 & -0.669044 \\
\hline $\mathrm{C}$ & -2.354389 & 0.628696 & -0.576665 \\
\hline $\mathrm{H}$ & -2.958127 & 1.471554 & -0.918369 \\
\hline $\mathrm{C}$ & -3.005982 & -0.619813 & -0.560510 \\
\hline $\mathrm{C}$ & -2.298614 & -1.833514 & -0.425429 \\
\hline $\mathrm{H}$ & -2.581545 & -2.640585 & -1.099825 \\
\hline $\mathrm{C}$ & -1.125964 & -1.910557 & 0.319966 \\
\hline $\mathrm{H}$ & -1.133213 & -1.539445 & 1.334661 \\
\hline $\mathrm{H}$ & -0.471245 & -2.770526 & 0.181834 \\
\hline $\mathrm{N}$ & -4.343495 & -0.682337 & -0.979529 \\
\hline $\mathrm{N}$ & -0.514354 & 1.777618 & -1.566269 \\
\hline $\mathrm{H}$ & 0.488294 & 1.882706 & -1.646177 \\
\hline $\mathrm{H}$ & -0.989071 & 1.827073 & -2.460309 \\
\hline $\mathrm{H}$ & -4.823114 & 0.208980 & -1.013194 \\
\hline $\mathrm{H}$ & -4.897932 & -1.384967 & -0.507931 \\
\hline \multicolumn{4}{|c|}{$\mathrm{H}_{2} \mathrm{~N}$} \\
\hline $\mathrm{H}$ & 0.000000 & 0.000000 & 0.000000 \\
\hline $\mathrm{H}$ & 0.000000 & 0.000000 & 1.847751 \\
\hline $\mathrm{C}$ & 0.571024 & 0.000000 & 0.924061 \\
\hline $\mathrm{C}$ & 1.911565 & -0.045037 & 0.945346 \\
\hline $\mathrm{H}$ & 2.434160 & -0.101827 & 1.891100 \\
\hline $\mathrm{C}$ & 2.761215 & -0.016709 & -0.252747 \\
\hline $\mathrm{C}$ & 4.085391 & -0.343779 & -0.288328 \\
\hline $\mathrm{H}$ & 4.589838 & -0.224415 & -1.245474 \\
\hline $\mathrm{C}$ & 4.960114 & -0.882345 & 0.738656 \\
\hline $\mathrm{C}$ & 6.262335 & -1.154297 & 0.507139 \\
\hline $\mathrm{H}$ & 6.703396 & -0.980021 & -0.466350 \\
\hline $\mathrm{H}$ & 6.909852 & -1.557667 & 1.281178 \\
\hline $\mathrm{O}$ & 4.384060 & -1.096783 & 1.969036 \\
\hline $\mathrm{N}$ & 2.101463 & 0.340270 & -1.437793 \\
\hline $\mathrm{H}$ & 1.447670 & 1.107856 & -1.328198 \\
\hline $\mathrm{H}$ & 2.717131 & 0.494626 & -2.227963 \\
\hline $\mathrm{H}$ & 5.065965 & -1.449207 & 2.562162 \\
\hline
\end{tabular}




\begin{tabular}{|c|c|c|c|}
\hline \multicolumn{4}{|l|}{ TS } \\
\hline $\mathrm{H}$ & 0.000000 & 0.000000 & 0.000000 \\
\hline $\mathrm{C}$ & 0.000000 & 0.000000 & 1.079592 \\
\hline $\mathrm{H}$ & 0.999608 & 0.000000 & 1.512492 \\
\hline $\mathrm{C}$ & -1.018177 & 0.672946 & 1.747446 \\
\hline $\mathrm{H}$ & -0.793914 & 1.244509 & 2.648744 \\
\hline $\mathrm{C}$ & -2.382432 & 0.432655 & 1.479125 \\
\hline $\mathrm{C}$ & -2.855268 & -0.846819 & 1.115138 \\
\hline $\mathrm{H}$ & -3.936909 & -0.986061 & 1.178826 \\
\hline $\mathrm{C}$ & -2.070952 & -2.012320 & 1.091560 \\
\hline $\mathrm{C}$ & -0.727670 & -2.110625 & 0.698762 \\
\hline $\mathrm{H}$ & -0.464754 & -1.834116 & -0.310067 \\
\hline $\mathrm{H}$ & -0.195710 & -2.988769 & 1.065630 \\
\hline $\mathrm{O}$ & -2.612572 & -3.165092 & 1.624126 \\
\hline $\mathrm{N}$ & -3.320610 & 1.421911 & 1.769746 \\
\hline $\mathrm{H}$ & -2.955957 & 2.233623 & 2.251788 \\
\hline $\mathrm{H}$ & -4.209983 & 1.111060 & 2.143302 \\
\hline $\mathrm{H}$ & -3.352753 & -2.922425 & 2.203564 \\
\hline \multicolumn{4}{|c|}{$\mathrm{H}_{2} \mathrm{~N}$} \\
\hline $\mathrm{H}$ & 0.000000 & 0.000000 & 0.000000 \\
\hline $\mathrm{H}$ & 0.000000 & 0.000000 & 1.847782 \\
\hline $\mathrm{C}$ & 0.570768 & 0.000000 & 0.924152 \\
\hline $\mathrm{C}$ & 1.910871 & -0.044690 & 0.945634 \\
\hline $\mathrm{H}$ & 2.429542 & -0.098514 & 1.893369 \\
\hline $\mathrm{C}$ & 2.761099 & -0.015248 & -0.252817 \\
\hline $\mathrm{C}$ & 4.082808 & -0.356643 & -0.282937 \\
\hline $\mathrm{H}$ & 4.604867 & -0.239673 & -1.231022 \\
\hline $\mathrm{C}$ & 4.926287 & -0.905344 & 0.753276 \\
\hline $\mathrm{C}$ & 6.225897 & -1.214917 & 0.637877 \\
\hline $\mathrm{H}$ & 6.747291 & -1.063786 & -0.299468 \\
\hline $\mathrm{H}$ & 6.774648 & -1.622355 & 1.478070 \\
\hline $\mathrm{F}$ & 4.336085 & -1.120404 & 1.962694 \\
\hline $\mathrm{N}$ & 2.109750 & 0.353774 & -1.434253 \\
\hline $\mathrm{H}$ & 1.447345 & 1.113414 & -1.325060 \\
\hline $\mathrm{H}$ & 2.722443 & 0.506241 & -2.226668 \\
\hline \multicolumn{4}{|l|}{ TS } \\
\hline $\mathrm{H}$ & 0.000000 & 0.000000 & 0.000000 \\
\hline $\mathrm{C}$ & 0.000000 & 0.000000 & 1.079908 \\
\hline $\mathrm{H}$ & 0.998750 & 0.000000 & 1.513825 \\
\hline $\mathrm{C}$ & -1.018201 & 0.680752 & 1.741663 \\
\hline
\end{tabular}




$\begin{array}{lrrr}\mathrm{H} & -0.794158 & 1.258275 & 2.638810 \\ \mathrm{C} & -2.380388 & 0.452544 & 1.464151 \\ \mathrm{C} & -2.853409 & -0.829242 & 1.096785 \\ \mathrm{H} & -3.925923 & -0.995145 & 1.188217 \\ \mathrm{C} & -2.066156 & -1.973734 & 1.042890 \\ \mathrm{C} & -0.713321 & -2.113051 & 0.718301 \\ \mathrm{H} & -0.379219 & -1.860410 & -0.275209 \\ \mathrm{H} & -0.227584 & -3.000153 & 1.126257 \\ \mathrm{~F} & -2.678031 & -3.092736 & 1.545804 \\ \mathrm{~N} & -3.320114 & 1.433302 & 1.759291 \\ \mathrm{H} & -2.966673 & 2.243348 & 2.251995 \\ \mathrm{H} & -4.216760 & 1.116789 & 2.109531\end{array}$

$\begin{array}{lrrr}\mathrm{H}_{2} \mathrm{~N} & & & \\ & = & & \\ \mathrm{C} & 0.000000 & 0.000000 & 0.000000 \\ \mathrm{H} & 0.000000 & 0.000000 & 1.083977 \\ \mathrm{H} & 0.971730 & 0.000000 & -0.488368 \\ \mathrm{C} & -1.134643 & 0.031234 & -0.737665 \\ \mathrm{C} & -2.514977 & -0.003031 & -0.282150 \\ \mathrm{H} & -3.220792 & 0.448257 & -0.976492 \\ \mathrm{C} & -3.009251 & -0.544644 & 0.867976 \\ \mathrm{C} & -2.222844 & -1.380692 & 1.784096 \\ \mathrm{H} & -1.343751 & -1.850724 & 1.350948 \\ \mathrm{C} & -2.539285 & -1.620198 & 3.064708 \\ \mathrm{H} & -3.380516 & -1.132612 & 3.549510 \\ \mathrm{H} & -1.942219 & -2.290626 & 3.675476 \\ \mathrm{~N} & -4.334257 & -0.329256 & 1.249785 \\ \mathrm{C} & -0.941710 & 0.183960 & -2.209854 \\ \mathrm{O} & -1.837757 & 0.329077 & -3.017602 \\ \mathrm{H} & 0.122205 & 0.177846 & -2.535378 \\ \mathrm{H} & -4.796380 & -1.138033 & 1.650592 \\ \mathrm{H} & -4.907767 & 0.127984 & 0.550207\end{array}$

$\begin{array}{cccc}\text { TS } & & & \\ \text { C } & 0.000000 & 0.000000 & 0.000000 \\ \text { H } & 0.000000 & 0.000000 & 1.079031 \\ \text { H } & 1.000867 & 0.000000 & -0.430524 \\ \text { C } & -1.002675 & -0.674202 & -0.687451 \\ \text { H } & -0.731333 & -1.261301 & -1.567374 \\ \text { C } & -2.396104 & -0.480631 & -0.506800 \\ \text { C } & -2.921667 & 0.809329 & -0.317587 \\ \text { H } & -3.969689 & 0.949856 & -0.581512\end{array}$




$\begin{array}{lrrr}\mathrm{C} & -2.150941 & 1.978743 & -0.221375 \\ \mathrm{C} & -0.888319 & 2.044182 & 0.388503 \\ \mathrm{H} & -0.790773 & 1.707725 & 1.408370 \\ \mathrm{H} & -0.248109 & 2.895089 & 0.149531 \\ \mathrm{C} & -2.652546 & 3.187389 & -0.912759 \\ \mathrm{~N} & -3.269450 & -1.519985 & -0.816756 \\ \mathrm{H} & -2.848609 & -2.331363 & -1.252367 \\ \mathrm{H} & -4.150112 & -1.257417 & -1.244296 \\ \mathrm{H} & -1.887167 & 3.988098 & -1.033734 \\ \mathrm{O} & -3.795033 & 3.357068 & -1.302928\end{array}$

$\begin{array}{lrrr}{ }^{\mathrm{H}_{2} \mathrm{~N}} & & & \\ & = & & \\ \mathrm{H} & 0.000000 & 0.000000 & 0.000000 \\ \mathrm{H} & 0.000000 & 0.000000 & 1.849321 \\ \mathrm{C} & 0.569370 & 0.000000 & 0.925063 \\ \mathrm{C} & 1.909154 & -0.043923 & 0.946077 \\ \mathrm{H} & 2.418174 & -0.092498 & 1.900827 \\ \mathrm{C} & 2.756197 & -0.015580 & -0.254654 \\ \mathrm{C} & 4.062301 & -0.412994 & -0.313419 \\ \mathrm{H} & 4.571001 & -0.260342 & -1.264619 \\ \mathrm{C} & 4.912690 & -1.066726 & 0.671462 \\ \mathrm{C} & 6.212339 & -1.361253 & 0.421742 \\ \mathrm{H} & 6.663196 & -1.114757 & -0.534883 \\ \mathrm{H} & 6.836428 & -1.851428 & 1.159419 \\ \mathrm{C} & 4.419191 & -1.453695 & 1.970986 \\ \mathrm{~N} & 2.117236 & 0.425674 & -1.413455 \\ \mathrm{H} & 1.478247 & 1.200306 & -1.277910 \\ \mathrm{H} & 2.720435 & 0.564262 & -2.215289 \\ \mathrm{~N} & 4.062185 & -1.777517 & 3.029646\end{array}$

$\begin{array}{cccc}\text { TS } & & & \\ \mathrm{H} & 0.000000 & 0.000000 & 0.000000 \\ \mathrm{C} & 0.000000 & 0.000000 & 1.078742 \\ \mathrm{H} & 1.001497 & 0.000000 & 1.508212 \\ \mathrm{C} & -1.001891 & 0.667017 & 1.773539 \\ \mathrm{H} & -0.726544 & 1.261918 & 2.646513 \\ \mathrm{C} & -2.395037 & 0.456897 & 1.616748 \\ \mathrm{C} & -2.918393 & -0.840589 & 1.455958 \\ \mathrm{H} & -3.961944 & -0.968455 & 1.741540 \\ \mathrm{C} & -2.133592 & -2.003282 & 1.333013 \\ \mathrm{C} & -0.896877 & -2.037674 & 0.666968 \\ \mathrm{H} & -0.871443 & -1.665969 & -0.345069\end{array}$




\begin{tabular}{|c|c|c|c|}
\hline $\mathrm{H}$ & -0.228601 & -2.875355 & 0.853300 \\
\hline $\mathrm{C}$ & -2.563941 & -3.162266 & 2.064571 \\
\hline $\mathrm{N}$ & -3.273100 & 1.491536 & 1.922432 \\
\hline $\mathrm{H}$ & -2.852232 & 2.321571 & 2.320431 \\
\hline $\mathrm{H}$ & -4.143547 & 1.235583 & 2.374096 \\
\hline $\mathrm{N}$ & -2.922665 & -4.109588 & 2.640539 \\
\hline \multicolumn{4}{|c|}{$\mathrm{H}_{2} \mathrm{~N}$} \\
\hline $\mathrm{C}$ & 0.000000 & 0.000000 & 0.000000 \\
\hline $\mathrm{H}$ & 0.000000 & 0.000000 & 1.083092 \\
\hline $\mathrm{H}$ & 0.947237 & 0.000000 & -0.523399 \\
\hline $\mathrm{C}$ & -1.158535 & 0.019077 & -0.678393 \\
\hline $\mathrm{C}$ & -2.522004 & -0.041740 & -0.196470 \\
\hline $\mathrm{H}$ & -3.255424 & 0.498597 & -0.785713 \\
\hline $\mathrm{C}$ & -2.944381 & -0.705444 & 0.919455 \\
\hline $\mathrm{C}$ & -2.107400 & -1.644041 & 1.679308 \\
\hline $\mathrm{H}$ & -1.268192 & -2.069952 & 1.135875 \\
\hline $\mathrm{C}$ & -2.333330 & -2.012410 & 2.948015 \\
\hline $\mathrm{H}$ & -3.127848 & -1.571315 & 3.543604 \\
\hline $\mathrm{H}$ & -1.704493 & -2.748843 & 3.438835 \\
\hline $\mathrm{N}$ & -4.230304 & -0.516643 & 1.414728 \\
\hline $\mathrm{N}$ & -1.039410 & 0.148125 & -2.172241 \\
\hline $\mathrm{O}$ & -2.085929 & 0.237232 & -2.813028 \\
\hline $\mathrm{O}$ & 0.082375 & 0.167368 & -2.672158 \\
\hline $\mathrm{H}$ & -4.849066 & 0.021426 & 0.819514 \\
\hline $\mathrm{H}$ & -4.675320 & -1.349294 & 1.783080 \\
\hline \multicolumn{4}{|l|}{ TS } \\
\hline $\mathrm{C}$ & 0.000000 & 0.000000 & 0.000000 \\
\hline $\mathrm{H}$ & 0.000000 & 0.000000 & 1.078530 \\
\hline $\mathrm{H}$ & 0.981010 & 0.000000 & -0.463596 \\
\hline $\mathrm{C}$ & -1.024586 & 0.699135 & -0.636762 \\
\hline $\mathrm{C}$ & -2.394995 & 0.504892 & -0.448586 \\
\hline $\mathrm{H}$ & -3.061402 & 1.282279 & -0.811341 \\
\hline $\mathrm{C}$ & -2.975988 & -0.749400 & -0.175785 \\
\hline $\mathrm{C}$ & -2.192745 & -1.931735 & -0.195853 \\
\hline $\mathrm{H}$ & -2.586348 & -2.731124 & -0.826149 \\
\hline $\mathrm{C}$ & -0.903617 & -2.049067 & 0.308304 \\
\hline $\mathrm{H}$ & -0.700085 & -1.768423 & 1.330026 \\
\hline $\mathrm{H}$ & -0.292710 & -2.880329 & -0.041604 \\
\hline $\mathrm{N}$ & -4.358553 & -0.850992 & -0.177728 \\
\hline $\mathrm{N}$ & -0.649294 & 1.587054 & -1.763934 \\
\hline
\end{tabular}




$\begin{array}{lrrr}\mathrm{O} & 0.518305 & 1.561427 & -2.156169 \\ \mathrm{O} & -1.520161 & 2.321295 & -2.237333 \\ \mathrm{H} & -4.873590 & -0.151820 & -0.699707 \\ \mathrm{H} & -4.739825 & -1.783724 & -0.268075\end{array}$

3

$\mathrm{HO}$<smiles>C=CC(C)=CC(=C)C</smiles>

$\begin{array}{llll}\mathrm{H} & 0.000000 & 0.000000 & 0.000000\end{array}$

$\begin{array}{llll}\mathrm{H} & 0.000000 & 0.000000 & 1.848215\end{array}$

$\begin{array}{llll}\text { C } & 0.571170 & 0.000000 & 0.923340\end{array}$

$\begin{array}{llll}\text { C } & 1.920652 & 0.000040 & 0.919878\end{array}$

$\begin{array}{llll}\text { C } & 2.619513 & 0.000152 & 2.203224\end{array}$

$\begin{array}{llll}\mathrm{H} & 1.931470 & 0.000156 & 3.051795\end{array}$

$\begin{array}{llll}\text { C } & 3.937658 & 0.000232 & 2.543384\end{array}$

$\begin{array}{llll}\text { C } & 5.104081 & 0.000310 & 1.664485\end{array}$

$\begin{array}{llll}\mathrm{H} & 4.911414 & -0.000219 & 0.600505\end{array}$

$\begin{array}{llll}\text { C } & 6.371581 & 0.000950 & 2.102802\end{array}$

$\begin{array}{llll}\mathrm{H} & 6.612553 & 0.001428 & 3.159943\end{array}$

$\begin{array}{llll}\mathrm{H} & 7.200634 & 0.001008 & 1.401602\end{array}$

$\begin{array}{llll}\text { O } & 4.302686 & 0.000331 & 3.868336\end{array}$

$\begin{array}{llll}\text { C } & 2.652137 & -0.000009 & -0.404003\end{array}$

$\begin{array}{llll}\mathrm{H} & 1.938768 & 0.000032 & -1.232933\end{array}$

$\mathrm{H} \quad 3.291584 \quad 0.884142 \quad-0.514103$

$\begin{array}{llll}\mathrm{H} & 3.291414 & -0.884294 & -0.514094\end{array}$

$\begin{array}{llll}\mathrm{H} & 3.500414 & 0.000089 & 4.413273\end{array}$

TS

$\begin{array}{llll}\mathrm{H} & 0.000000 & 0.000000 & 0.000000\end{array}$

$\begin{array}{llll}\text { C } & 0.000000 & 0.000000 & 1.077884\end{array}$

$\begin{array}{llll}\mathrm{H} & 1.005142 & 0.000000 & 1.501233\end{array}$

$\begin{array}{llll}\text { C } & -0.995219 & 0.676121 & 1.781942\end{array}$

$\begin{array}{llll}\mathrm{H} & -0.710766 & 1.284077 & 2.645054\end{array}$

$\begin{array}{llll}\text { C } & -2.374358 & 0.419765 & 1.676132\end{array}$

$\begin{array}{llll}\text { C } & -2.922214 & -0.861249 & 1.503976\end{array}$

$\begin{array}{llll}\mathrm{H} & -3.952569 & -0.943408 & 1.846908\end{array}$

$\begin{array}{llll}\text { C } & -2.172620 & -2.031049 & 1.328115\end{array}$

$\begin{array}{llll}\text { C } & -0.960186 & -2.031357 & 0.624770\end{array}$

H $\quad-0.949162 \quad-1.601139 \quad-0.364264$

$\begin{array}{llll}\mathrm{H} & -0.299342 & -2.892665 & 0.731666\end{array}$

$\begin{array}{llll}\text { C } & -2.625556 & -3.298367 & 2.030590\end{array}$

$\begin{array}{llll}\text { O } & -3.273225 & 1.360250 & 2.129135\end{array}$ 


\begin{tabular}{|c|c|c|c|}
\hline $\mathrm{H}$ & -2.806826 & 2.205742 & 2.212075 \\
\hline $\mathrm{H}$ & -1.780459 & -3.750448 & 2.565665 \\
\hline $\mathrm{H}$ & -2.985932 & -4.052114 & 1.318082 \\
\hline $\mathrm{H}$ & -3.421398 & -3.110869 & 2.757743 \\
\hline \multicolumn{4}{|c|}{$\mathrm{HO}$} \\
\hline $\mathrm{H}$ & 0.000000 & 0.000000 & 0.000000 \\
\hline $\mathrm{H}$ & 0.000000 & 0.000000 & 1.853809 \\
\hline $\mathrm{C}$ & 0.564705 & 0.000000 & 0.926200 \\
\hline $\mathrm{C}$ & 1.905316 & 0.006295 & 0.939879 \\
\hline $\mathrm{H}$ & 2.450032 & 0.016167 & 1.877363 \\
\hline $\mathrm{C}$ & 2.730034 & 0.044283 & -0.266867 \\
\hline $\mathrm{C}$ & 4.086892 & -0.011041 & -0.335750 \\
\hline $\mathrm{H}$ & 4.526856 & 0.133015 & -1.322489 \\
\hline $\mathrm{C}$ & 5.084113 & -0.223119 & 0.715575 \\
\hline $\mathrm{C}$ & 6.371925 & 0.135634 & 0.517942 \\
\hline $\mathrm{H}$ & 6.673833 & 0.666936 & -0.377206 \\
\hline $\mathrm{H}$ & 7.137785 & -0.052409 & 1.265127 \\
\hline $\mathrm{N}$ & 4.647630 & -0.787600 & 1.933368 \\
\hline $\mathrm{O}$ & 1.980718 & 0.188807 & -1.407547 \\
\hline $\mathrm{H}$ & 2.574513 & 0.182078 & -2.174585 \\
\hline $\mathrm{H}$ & 4.038947 & -1.590444 & 1.802316 \\
\hline $\mathrm{H}$ & 5.420004 & -1.033845 & 2.544385 \\
\hline \multicolumn{4}{|l|}{ TS } \\
\hline $\mathrm{H}$ & 0.000000 & 0.000000 & 0.000000 \\
\hline $\mathrm{C}$ & 0.000000 & 0.000000 & 1.079425 \\
\hline $\mathrm{H}$ & 0.998993 & 0.000000 & 1.513696 \\
\hline $\mathrm{C}$ & -1.023959 & 0.661770 & 1.749543 \\
\hline $\mathrm{H}$ & -0.816904 & 1.212385 & 2.668756 \\
\hline $\mathrm{C}$ & -2.377349 & 0.387319 & 1.473789 \\
\hline $\mathrm{C}$ & -2.862364 & -0.866439 & 1.092952 \\
\hline $\mathrm{H}$ & -3.942864 & -0.977929 & 1.160895 \\
\hline $\mathrm{C}$ & -2.082620 & -2.048702 & 1.090079 \\
\hline $\mathrm{C}$ & -0.732815 & -2.115536 & 0.686093 \\
\hline $\mathrm{H}$ & -0.489849 & -1.831149 & -0.326489 \\
\hline $\mathrm{H}$ & -0.161517 & -2.980461 & 1.033549 \\
\hline $\mathrm{N}$ & -2.697270 & -3.184795 & 1.612786 \\
\hline $\mathrm{O}$ & -3.347575 & 1.292536 & 1.839984 \\
\hline $\mathrm{H}$ & -2.914435 & 2.148586 & 1.978218 \\
\hline $\mathrm{H}$ & -3.281796 & -3.064831 & 2.431026 \\
\hline $\mathrm{H}$ & -2.138673 & -4.026792 & 1.636079 \\
\hline
\end{tabular}




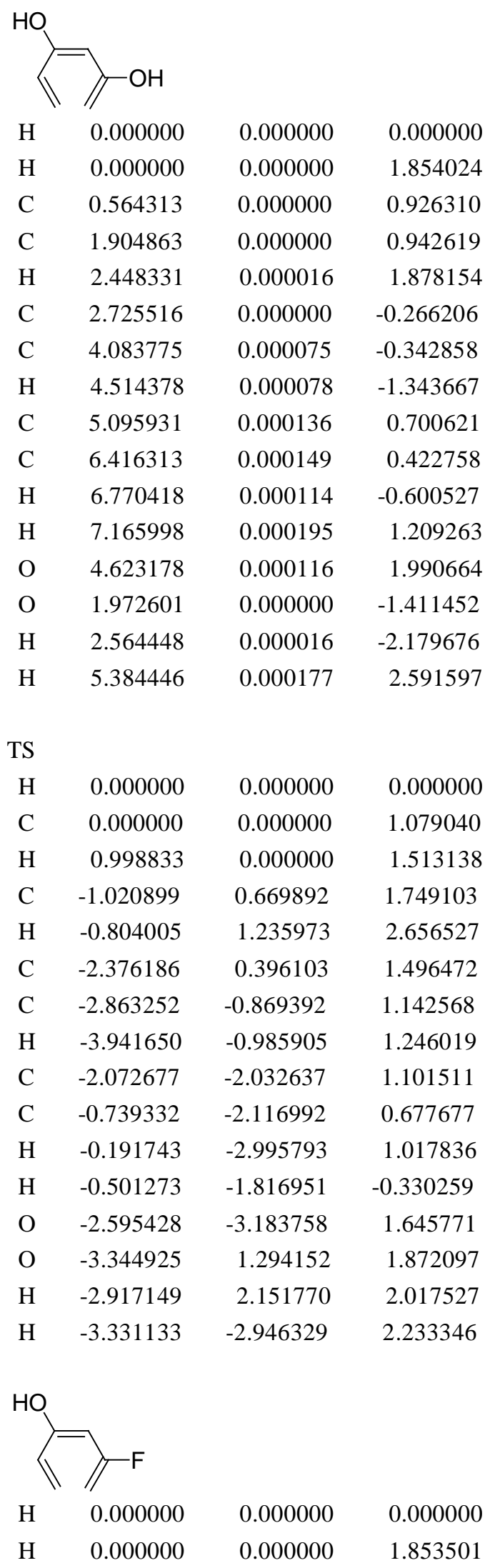




\begin{tabular}{|c|c|c|c|}
\hline $\mathrm{C}$ & 0.564580 & 0.000000 & 0.926093 \\
\hline $\mathrm{C}$ & 1.904840 & 0.000000 & 0.942445 \\
\hline $\mathrm{H}$ & 2.443857 & 0.000000 & 1.880089 \\
\hline $\mathrm{C}$ & 2.725459 & 0.000000 & -0.266193 \\
\hline $\mathrm{C}$ & 4.084854 & 0.000019 & -0.335985 \\
\hline $\mathrm{H}$ & 4.532308 & 0.000016 & -1.329388 \\
\hline $\mathrm{C}$ & 5.073181 & 0.000024 & 0.719869 \\
\hline $\mathrm{C}$ & 6.403134 & -0.000032 & 0.558408 \\
\hline $\mathrm{H}$ & 6.839000 & -0.000088 & -0.433205 \\
\hline $\mathrm{H}$ & 7.063825 & -0.000022 & 1.416613 \\
\hline $\mathrm{F}$ & 4.593155 & 0.000008 & 1.993985 \\
\hline $\mathrm{O}$ & 1.976229 & 0.000000 & -1.411310 \\
\hline $\mathrm{H}$ & 2.567436 & 0.000000 & -2.179979 \\
\hline \multicolumn{4}{|c|}{ TS } \\
\hline $\mathrm{H}$ & 0.000000 & 0.000000 & 0.000000 \\
\hline $\mathrm{C}$ & 0.000000 & 0.000000 & 1.079128 \\
\hline $\mathrm{H}$ & 0.998641 & 0.000000 & 1.513163 \\
\hline $\mathrm{C}$ & -1.017382 & 0.682674 & 1.744162 \\
\hline $\mathrm{H}$ & -0.792363 & 1.265521 & 2.638794 \\
\hline $\mathrm{C}$ & -2.374727 & 0.424176 & 1.498381 \\
\hline $\mathrm{C}$ & -2.871697 & -0.846956 & 1.165233 \\
\hline $\mathrm{H}$ & -3.938498 & -0.986881 & 1.317887 \\
\hline $\mathrm{C}$ & -2.080409 & -1.988965 & 1.080958 \\
\hline $\mathrm{C}$ & -0.744060 & -2.111539 & 0.693672 \\
\hline $\mathrm{H}$ & -0.231110 & -3.001137 & 1.059287 \\
\hline $\mathrm{H}$ & -0.458728 & -1.828648 & -0.306404 \\
\hline $\mathrm{F}$ & -2.657224 & -3.107758 & 1.611449 \\
\hline $\mathrm{O}$ & -3.335593 & 1.325199 & 1.872627 \\
\hline $\mathrm{H}$ & -2.904749 & 2.177076 & 2.041622 \\
\hline \multicolumn{4}{|l|}{$\mathrm{HO}$} \\
\hline $\mathrm{H}$ & 0.000000 & 0.000000 & 0.000000 \\
\hline $\mathrm{H}$ & 0.000000 & 0.000000 & 1.854208 \\
\hline $\mathrm{C}$ & 0.564248 & 0.000000 & 0.926603 \\
\hline $\mathrm{C}$ & 1.904014 & 0.000537 & 0.934554 \\
\hline $\mathrm{H}$ & 2.444110 & 0.003734 & 1.876086 \\
\hline $\mathrm{C}$ & 2.730128 & -0.049492 & -0.269217 \\
\hline $\mathrm{C}$ & 4.076876 & 0.118791 & -0.325069 \\
\hline $\mathrm{H}$ & 4.575696 & -0.106971 & -1.267075 \\
\hline $\mathrm{C}$ & 4.992780 & 0.507825 & 0.739454 \\
\hline $\mathrm{C}$ & 4.750805 & 1.278228 & 1.823467 \\
\hline
\end{tabular}




\begin{tabular}{|c|c|c|c|}
\hline $\mathrm{H}$ & 3.795288 & 1.754626 & 2.010957 \\
\hline $\mathrm{H}$ & 5.540037 & 1.468601 & 2.546890 \\
\hline $\mathrm{C}$ & 6.407045 & 0.063342 & 0.563438 \\
\hline $\mathrm{O}$ & 2.010500 & -0.356380 & -1.390171 \\
\hline $\mathrm{H}$ & 2.604018 & -0.332706 & -2.158065 \\
\hline $\mathrm{O}$ & 6.830002 & -0.530916 & -0.408004 \\
\hline $\mathrm{H}$ & 7.083259 & 0.342121 & 1.401043 \\
\hline \multicolumn{4}{|l|}{$\mathrm{TS}$} \\
\hline $\mathrm{H}$ & 0.000000 & 0.000000 & 0.000000 \\
\hline $\mathrm{C}$ & 0.000000 & 0.000000 & 1.078550 \\
\hline $\mathrm{H}$ & 1.001549 & 0.000000 & 1.507401 \\
\hline $\mathrm{C}$ & -0.996646 & 0.688465 & 1.764159 \\
\hline $\mathrm{H}$ & -0.733090 & 1.312300 & 2.618021 \\
\hline $\mathrm{C}$ & -2.381674 & 0.480891 & 1.604010 \\
\hline $\mathrm{C}$ & -2.931043 & -0.792202 & 1.410115 \\
\hline $\mathrm{H}$ & -3.974700 & -0.927182 & 1.697999 \\
\hline $\mathrm{C}$ & -2.171886 & -1.967520 & 1.286393 \\
\hline $\mathrm{C}$ & -0.915117 & -2.025694 & 0.665509 \\
\hline $\mathrm{H}$ & -0.275030 & -2.881615 & 0.885066 \\
\hline $\mathrm{H}$ & -0.828156 & -1.674639 & -0.350271 \\
\hline $\mathrm{C}$ & -2.676981 & -3.179987 & 1.968363 \\
\hline $\mathrm{O}$ & -3.177916 & 1.514648 & 2.017368 \\
\hline $\mathrm{H}$ & -4.082906 & 1.187442 & 2.149327 \\
\hline $\mathrm{O}$ & -3.819469 & -3.341768 & 2.360686 \\
\hline $\mathrm{H}$ & -1.917912 & -3.987379 & 2.077749 \\
\hline \multicolumn{4}{|l|}{$\mathrm{HO}$} \\
\hline $\mathrm{H}$ & 0.000000 & 0.000000 & 0.000000 \\
\hline $\mathrm{H}$ & 0.000000 & 0.000000 & 1.854117 \\
\hline $\mathrm{C}$ & 0.563872 & 0.000000 & 0.926459 \\
\hline $\mathrm{C}$ & 1.904179 & 0.000000 & 0.943934 \\
\hline $\mathrm{H}$ & 2.429743 & 0.000000 & 1.890401 \\
\hline $\mathrm{C}$ & 2.722616 & 0.000000 & -0.265438 \\
\hline $\mathrm{C}$ & 4.082150 & 0.000000 & -0.352021 \\
\hline $\mathrm{H}$ & 4.498783 & 0.000017 & -1.359752 \\
\hline $\mathrm{C}$ & 5.120629 & 0.000000 & 0.671415 \\
\hline $\mathrm{C}$ & 6.437951 & 0.000020 & 0.356005 \\
\hline $\mathrm{H}$ & 6.761691 & 0.000037 & -0.680473 \\
\hline $\mathrm{H}$ & 7.205151 & 0.000020 & 1.121066 \\
\hline $\mathrm{C}$ & 4.807887 & -0.000021 & 2.078965 \\
\hline $\mathrm{O}$ & 1.968858 & 0.000022 & -1.405322 \\
\hline
\end{tabular}




$\begin{array}{llrr}\mathrm{H} & 2.554251 & 0.000005 & -2.178845 \\ \mathrm{~N} & 4.595851 & -0.000040 & 3.222560\end{array}$

TS

$\begin{array}{lrrr}\mathrm{H} & 0.000000 & 0.000000 & 0.000000 \\ \mathrm{C} & 0.000000 & 0.000000 & 1.078057 \\ \mathrm{H} & 1.000937 & 0.000000 & 1.508445 \\ \mathrm{C} & -1.004434 & 0.662076 & 1.776624 \\ \mathrm{H} & -0.732753 & 1.263072 & 2.647803 \\ \mathrm{C} & -2.388319 & 0.422072 & 1.641919 \\ \mathrm{C} & -2.928324 & -0.859803 & 1.483968 \\ \mathrm{H} & -3.961422 & -0.961767 & 1.806622 \\ \mathrm{C} & -2.145474 & -2.018225 & 1.317605 \\ \mathrm{C} & -0.926898 & -2.033758 & 0.621282 \\ \mathrm{H} & -0.254167 & -2.876186 & 0.763424 \\ \mathrm{H} & -0.923101 & -1.622718 & -0.375644 \\ \mathrm{O} & -3.289780 & 1.369121 & 2.052741 \\ \mathrm{C} & -2.553681 & -3.195691 & 2.032046 \\ \mathrm{~N} & -2.887305 & -4.160644 & 2.592764 \\ \mathrm{H} & -2.832014 & 2.219619 & 2.135122\end{array}$

$\mathrm{HO}$<smiles>C=CC(C)=CC(=C)[N+](=O)[O-]</smiles>

$\begin{array}{llll}\mathrm{H} & 0.000000 & 0.000000 & 0.000000\end{array}$

$\begin{array}{llll}\mathrm{H} & 0.000000 & 0.000000 & 1.854191\end{array}$

$\begin{array}{llll}\text { C } & 0.564120 & 0.000000 & 0.926648\end{array}$

$\begin{array}{llll}\text { C } & 1.903561 & -0.000020 & 0.935976\end{array}$

$\begin{array}{llll}\mathrm{H} & 2.445261 & 0.007468 & 1.876472\end{array}$

$\begin{array}{llll}\text { C } & 2.726442 & -0.047080 & -0.270598\end{array}$

$\begin{array}{llll}\text { C } & 4.075099 & 0.115109 & -0.326745\end{array}$

$\begin{array}{llll}\mathrm{H} & 4.580567 & -0.093313 & -1.265482\end{array}$

$\begin{array}{llll}\text { C } & 4.936684 & 0.517163 & 0.769834\end{array}$

$\begin{array}{llll}\text { C } & 4.711192 & 1.381643 & 1.769112\end{array}$

$\begin{array}{llll}\mathrm{H} & 3.784021 & 1.940127 & 1.813447\end{array}$

$\begin{array}{llll}\mathrm{H} & 5.456563 & 1.543939 & 2.536980\end{array}$

$\begin{array}{llll}\mathrm{N} & 6.303613 & -0.108092 & 0.737313\end{array}$

$\begin{array}{llll}\text { O } & 2.006207 & -0.331531 & -1.392455\end{array}$

$\mathrm{H} \quad 2.596698 \quad-0.313350 \quad-2.162932$

$\begin{array}{llll}\text { O } & 6.568383 & -0.821156 & -0.229496\end{array}$

$\begin{array}{llll}\text { O } & 7.079141 & 0.130572 & 1.658595\end{array}$

TS

$\begin{array}{llll}\text { C } & 0.000000 & 0.000000 & 0.000000\end{array}$ 


$\begin{array}{rrrr}\mathrm{H} & 0.000000 & 0.000000 & 1.078377 \\ \mathrm{H} & 0.980499 & 0.000000 & -0.464737 \\ \mathrm{C} & -1.023429 & 0.702017 & -0.633526 \\ \mathrm{C} & -2.393413 & 0.502722 & -0.437531 \\ \mathrm{H} & -3.065920 & 1.270424 & -0.812861 \\ \mathrm{C} & -2.952434 & -0.750553 & -0.147979 \\ \mathrm{C} & -2.194162 & -1.938656 & -0.153715 \\ \mathrm{H} & -2.641740 & -2.750251 & -0.726570 \\ \mathrm{C} & -0.887018 & -2.057923 & 0.306695 \\ \mathrm{H} & -0.649157 & -1.775654 & 1.319925 \\ \mathrm{H} & -0.296700 & -2.898610 & -0.055837 \\ \mathrm{O} & -4.305318 & -0.918572 & -0.204532 \\ \mathrm{~N} & -0.657306 & 1.582852 & -1.766299 \\ \mathrm{O} & 0.502733 & 1.542501 & -2.176894 \\ \mathrm{O} & -1.529089 & 2.326226 & -2.222847 \\ \mathrm{H} & -4.723594 & -0.090330 & -0.492883\end{array}$

4<smiles>Cc1cccc(F)c1</smiles>

$\begin{array}{lrrr}\mathrm{H} & 0.000000 & 0.000000 & 0.000000 \\ \mathrm{H} & 0.000000 & 0.000000 & 1.849177 \\ \mathrm{C} & 0.570207 & 0.000000 & 0.924164 \\ \mathrm{C} & 1.918440 & 0.000000 & 0.921905 \\ \mathrm{C} & 2.610070 & 0.000000 & 2.209037 \\ \mathrm{H} & 1.954179 & 0.000000 & 3.076846 \\ \mathrm{C} & 3.923975 & 0.000000 & 2.525957 \\ \mathrm{C} & 5.124958 & 0.000000 & 1.710842 \\ \mathrm{H} & 4.984722 & 0.000000 & 0.637245 \\ \mathrm{C} & 6.365916 & 0.000000 & 2.221264 \\ \mathrm{H} & 6.543646 & 0.000000 & 3.291022 \\ \mathrm{H} & 7.235395 & 0.000000 & 1.571852 \\ \mathrm{~F} & 4.200348 & 0.000000 & 3.861100 \\ \mathrm{C} & 2.667132 & 0.000000 & -0.391223 \\ \mathrm{H} & 1.967164 & 0.000107 & -1.231379 \\ \mathrm{H} & 3.308607 & 0.884173 & -0.490614 \\ \mathrm{H} & 3.308451 & -0.884272 & -0.490723\end{array}$

TS

$\begin{array}{cccc}\mathrm{H} & 0.000000 & 0.000000 & 0.000000 \\ \mathrm{C} & 0.000000 & 0.000000 & 1.077165 \\ \mathrm{H} & 1.005734 & 0.000000 & 1.499420 \\ \mathrm{C} & -0.990620 & 0.681573 & 1.786136\end{array}$




\begin{tabular}{|c|c|c|c|}
\hline $\mathrm{H}$ & -0.709881 & 1.336483 & 2.611890 \\
\hline $\mathrm{C}$ & -2.356243 & 0.402221 & 1.714932 \\
\hline $\mathrm{C}$ & -2.940012 & -0.858358 & 1.556086 \\
\hline $\mathrm{H}$ & -3.953092 & -0.931238 & 1.946666 \\
\hline $\mathrm{C}$ & -2.207153 & -2.027162 & 1.316096 \\
\hline $\mathrm{C}$ & -1.023193 & -1.996231 & 0.568118 \\
\hline $\mathrm{H}$ & -1.053385 & -1.515521 & -0.397015 \\
\hline $\mathrm{H}$ & -0.356770 & -2.858207 & 0.608115 \\
\hline $\mathrm{C}$ & -2.632870 & -3.312847 & 2.000077 \\
\hline $\mathrm{F}$ & -3.181999 & 1.346720 & 2.244888 \\
\hline $\mathrm{H}$ & -1.773395 & -3.767795 & 2.508908 \\
\hline $\mathrm{H}$ & -3.001512 & -4.053849 & 1.278972 \\
\hline $\mathrm{H}$ & -3.414777 & -3.147915 & 2.747302 \\
\hline \multicolumn{4}{|c|}{\rangle$_{\|} \mathrm{NH}_{2}$} \\
\hline $\mathrm{H}$ & 0.000000 & 0.000000 & 0.000000 \\
\hline $\mathrm{H}$ & 0.000000 & 0.000000 & 1.852806 \\
\hline $\mathrm{C}$ & 0.565078 & 0.000000 & 0.926115 \\
\hline $\mathrm{C}$ & 1.906428 & 0.006729 & 0.937085 \\
\hline $\mathrm{H}$ & 2.459379 & 0.020388 & 1.870422 \\
\hline $\mathrm{C}$ & 2.715070 & 0.040207 & -0.269159 \\
\hline $\mathrm{C}$ & 4.054903 & 0.022361 & -0.434725 \\
\hline $\mathrm{H}$ & 4.396533 & 0.143211 & -1.458312 \\
\hline $\mathrm{C}$ & 5.114695 & -0.124873 & 0.564490 \\
\hline $\mathrm{C}$ & 6.375687 & 0.260877 & 0.273976 \\
\hline $\mathrm{H}$ & 6.607528 & 0.750276 & -0.664647 \\
\hline $\mathrm{H}$ & 7.188232 & 0.138431 & 0.984593 \\
\hline $\mathrm{N}$ & 4.755260 & -0.640883 & 1.827804 \\
\hline $\mathrm{F}$ & 1.977363 & 0.142455 & -1.408617 \\
\hline $\mathrm{H}$ & 4.177083 & -1.474143 & 1.764523 \\
\hline $\mathrm{H}$ & 5.562866 & -0.826745 & 2.414190 \\
\hline \multicolumn{4}{|c|}{ TS } \\
\hline $\mathrm{H}$ & 0.000000 & 0.000000 & 0.000000 \\
\hline $\mathrm{C}$ & 0.000000 & 0.000000 & 1.078560 \\
\hline $\mathrm{H}$ & 0.998886 & 0.000000 & 1.513137 \\
\hline $\mathrm{C}$ & -1.020660 & 0.669327 & 1.750257 \\
\hline $\mathrm{H}$ & -0.813997 & 1.274157 & 2.631742 \\
\hline $\mathrm{C}$ & -2.362078 & 0.376986 & 1.498280 \\
\hline $\mathrm{C}$ & -2.889400 & -0.857598 & 1.129421 \\
\hline $\mathrm{H}$ & -3.966997 & -0.952367 & 1.241905 \\
\hline $\mathrm{C}$ & -2.121297 & -2.045667 & 1.077441 \\
\hline
\end{tabular}




$\begin{array}{llll}\mathrm{C} & -0.783007 & -2.103795 & 0.638381 \\ \mathrm{H} & -0.565146 & -1.787283 & -0.369946 \\ \mathrm{H} & -0.203118 & -2.976993 & 0.947041 \\ \mathrm{~N} & -2.727135 & -3.187654 & 1.596163 \\ \mathrm{~F} & -3.277124 & 1.288651 & 1.925822 \\ \mathrm{H} & -3.280241 & -3.083731 & 2.437951 \\ \mathrm{H} & -2.176303 & -4.034789 & 1.579829\end{array}$

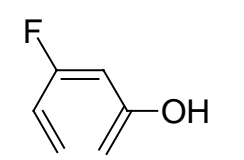

$\begin{array}{lccc}\mathrm{H} & 0.000000 & 0.000000 & 0.000000 \\ \mathrm{H} & 0.000000 & 0.000000 & 1.852536 \\ \mathrm{C} & 0.565225 & 0.000000 & 0.925950 \\ \mathrm{C} & 1.906613 & 0.000000 & 0.938616 \\ \mathrm{H} & 2.460516 & 0.000000 & 1.869163 \\ \mathrm{C} & 2.710077 & 0.000000 & -0.269642 \\ \mathrm{C} & 4.048933 & 0.000018 & -0.445240 \\ \mathrm{H} & 4.386607 & 0.000013 & -1.476587 \\ \mathrm{C} & 5.112850 & 0.000023 & 0.546220 \\ \mathrm{C} & 6.414403 & 0.000002 & 0.197080 \\ \mathrm{H} & 6.709279 & -0.000020 & -0.844712 \\ \mathrm{H} & 7.207816 & 0.000006 & 0.939543 \\ \mathrm{O} & 4.696231 & 0.000008 & 1.854419 \\ \mathrm{~F} & 1.970338 & 0.000000 & -1.411486 \\ \mathrm{H} & 5.480370 & 0.000013 & 2.425343\end{array}$

TS

$\begin{array}{lrrr}\mathrm{H} & 0.000000 & 0.000000 & 0.000000 \\ \mathrm{C} & 0.000000 & 0.000000 & 1.078084 \\ \mathrm{H} & 0.998974 & 0.000000 & 1.512119 \\ \mathrm{C} & -1.016878 & 0.676150 & 1.752170 \\ \mathrm{H} & -0.798219 & 1.297303 & 2.619409 \\ \mathrm{C} & -2.361536 & 0.382467 & 1.533933 \\ \mathrm{C} & -2.893576 & -0.864824 & 1.202180 \\ \mathrm{H} & -3.965578 & -0.965265 & 1.363506 \\ \mathrm{C} & -2.114654 & -2.032308 & 1.103653 \\ \mathrm{C} & -0.800260 & -2.102378 & 0.624911 \\ \mathrm{H} & -0.603620 & -1.761655 & -0.378494 \\ \mathrm{H} & -0.237451 & -2.989830 & 0.912164 \\ \mathrm{O} & -2.615330 & -3.192030 & 1.646914 \\ \mathrm{~F} & -3.268837 & 1.289383 & 1.979072 \\ \mathrm{H} & -3.321275 & -2.970132 & 2.275308\end{array}$




\begin{tabular}{|c|c|c|c|}
\hline $\mathrm{H}$ & 0.000000 & 0.000000 & 0.000000 \\
\hline$\Pi$ & 0.000000 & 0.000000 & 1.852562 \\
\hline C & 0.564983 & 0.000000 & 0.926026 \\
\hline $\mathrm{C}$ & 1.906124 & 0.000000 & 0.939018 \\
\hline $\mathrm{H}$ & 2.454873 & 0.000000 & 1.872359 \\
\hline $\mathrm{C}$ & 2.708877 & 0.000000 & -0.269680 \\
\hline $\mathrm{C}$ & 4.049504 & 0.000054 & -0.437876 \\
\hline $\mathrm{H}$ & 4.404096 & 0.000056 & -1.463844 \\
\hline $\mathrm{C}$ & 5.089596 & 0.000089 & 0.567230 \\
\hline $\mathrm{C}$ & 6.407636 & -0.000028 & 0.335417 \\
\hline $\mathrm{H}$ & 6.787453 & -0.000131 & -0.678882 \\
\hline $\mathrm{H}$ & 7.114672 & -0.000087 & 1.156088 \\
\hline $\mathrm{F}$ & 4.665868 & 0.000043 & 1.860186 \\
\hline $\mathrm{F}$ & 1.973818 & 0.000065 & -1.411012 \\
\hline \multicolumn{4}{|l|}{$\mathrm{TS}$} \\
\hline $\mathrm{H}$ & 0.000000 & 0.000000 & 0.000000 \\
\hline $\mathrm{C}$ & 0.000000 & 0.000000 & 1.078231 \\
\hline $\mathrm{H}$ & 0.998971 & 0.000000 & 1.511808 \\
\hline $\mathrm{C}$ & -1.012311 & 0.691847 & 1.746413 \\
\hline $\mathrm{H}$ & -0.785047 & 1.328889 & 2.599764 \\
\hline $\mathrm{C}$ & -2.359987 & 0.411965 & 1.540022 \\
\hline $\mathrm{C}$ & -2.901095 & -0.841145 & 1.235521 \\
\hline $\mathrm{H}$ & -3.959013 & -0.966197 & 1.447056 \\
\hline $\mathrm{C}$ & -2.122161 & -1.987631 & 1.098477 \\
\hline $\mathrm{C}$ & -0.805334 & -2.096503 & 0.649376 \\
\hline $\mathrm{H}$ & -0.568289 & -1.776356 & -0.351467 \\
\hline $\mathrm{H}$ & -0.272641 & -2.993608 & 0.963312 \\
\hline $\mathrm{F}$ & -2.672718 & -3.108499 & 1.644700 \\
\hline $\mathrm{F}$ & -3.254731 & 1.325194 & 1.987156 \\
\hline \multicolumn{4}{|c|}{$\sum_{\|}{ }_{\| \mathrm{CHO}}$} \\
\hline $\mathrm{H}$ & 0.000000 & 0.000000 & 0.000000 \\
\hline $\mathrm{H}$ & 0.000000 & 0.000000 & 1.853360 \\
\hline $\mathrm{C}$ & 0.564383 & 0.000000 & 0.926461 \\
\hline $\mathrm{C}$ & 1.904984 & -0.000498 & 0.932638 \\
\hline $\mathrm{H}$ & 2.451434 & -0.000113 & 1.870817 \\
\hline $\mathrm{C}$ & 2.714035 & -0.044197 & -0.272516 \\
\hline $\mathrm{C}$ & 4.051130 & 0.041749 & -0.417049 \\
\hline
\end{tabular}




$\begin{array}{lrrr}\mathrm{H} & 4.448710 & -0.160305 & -1.407401 \\ \mathrm{C} & 5.047682 & 0.314391 & 0.615954 \\ \mathrm{C} & 4.908198 & 1.079996 & 1.718495 \\ \mathrm{H} & 4.001136 & 1.628685 & 1.946767 \\ \mathrm{H} & 5.735021 & 1.194503 & 2.415501 \\ \mathrm{C} & 6.405782 & -0.257091 & 0.373900 \\ \mathrm{~F} & 1.991565 & -0.260595 & -1.399886 \\ \mathrm{O} & 6.726669 & -0.872771 & -0.621467 \\ \mathrm{H} & 7.137900 & -0.057823 & 1.186755\end{array}$

TS

$\begin{array}{lrrr}\mathrm{H} & 0.000000 & 0.000000 & 0.000000 \\ \mathrm{C} & 0.000000 & 0.000000 & 1.077755 \\ \mathrm{H} & 1.000319 & 0.000000 & 1.509016 \\ \mathrm{C} & -1.001366 & 0.681218 & 1.766266 \\ \mathrm{H} & -0.741449 & 1.316364 & 2.613654 \\ \mathrm{C} & -2.373777 & 0.438694 & 1.623939 \\ \mathrm{C} & -2.953413 & -0.811151 & 1.432781 \\ \mathrm{H} & -3.989083 & -0.912512 & 1.747159 \\ \mathrm{C} & -2.198112 & -1.981851 & 1.257222 \\ \mathrm{C} & -0.957028 & -2.019885 & 0.607547 \\ \mathrm{H} & -0.889834 & -1.621995 & -0.392243 \\ \mathrm{H} & -0.312429 & -2.883055 & 0.778441 \\ \mathrm{C} & -2.684822 & -3.217107 & 1.918488 \\ \mathrm{~F} & -3.200697 & 1.399938 & 2.105534 \\ \mathrm{H} & -1.914573 & -4.016687 & 2.003111 \\ \mathrm{O} & -3.819766 & -3.398100 & 2.317674\end{array}$

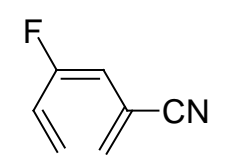

$\begin{array}{llll}\mathrm{H} & 0.000000 & 0.000000 & 0.000000 \\ \mathrm{H} & 0.000000 & 0.000000 & 1.853298 \\ \mathrm{C} & 0.564140 & 0.000000 & 0.926389 \\ \mathrm{C} & 1.905407 & 0.000000 & 0.940342 \\ \mathrm{H} & 2.442977 & 0.000000 & 1.881352 \\ \mathrm{C} & 2.703130 & 0.000000 & -0.271355 \\ \mathrm{C} & 4.041130 & 0.000000 & -0.463663 \\ \mathrm{H} & 4.356098 & 0.000000 & -1.503412 \\ \mathrm{C} & 5.140029 & 0.000000 & 0.496590 \\ \mathrm{C} & 6.432458 & 0.000000 & 0.096986 \\ \mathrm{H} & 6.686630 & 0.000000 & -0.958468 \\ \mathrm{H} & 7.247998 & 0.000000 & 0.810686 \\ \mathrm{C} & 4.896636 & 0.000000 & 1.917211\end{array}$




$\begin{array}{cccc}\mathrm{F} & 1.959445 & 0.000000 & -1.406441 \\ \mathrm{~N} & 4.722514 & 0.000001 & 3.067047\end{array}$

TS

$\begin{array}{lrrr}\mathrm{H} & 0.000000 & 0.000000 & 0.000000 \\ \mathrm{C} & 0.000000 & 0.000000 & 1.077403 \\ \mathrm{H} & 1.001069 & 0.000000 & 1.507434 \\ \mathrm{C} & -1.001942 & 0.666370 & 1.778051 \\ \mathrm{H} & -0.737108 & 1.308979 & 2.618278 \\ \mathrm{C} & -2.373138 & 0.402244 & 1.670080 \\ \mathrm{C} & -2.947682 & -0.856611 & 1.511428 \\ \mathrm{H} & -3.970744 & -0.949491 & 1.865103 \\ \mathrm{C} & -2.180391 & -2.016383 & 1.287320 \\ \mathrm{C} & -0.979764 & -2.006602 & 0.561884 \\ \mathrm{H} & -1.001635 & -1.550765 & -0.415154 \\ \mathrm{H} & -0.304724 & -2.854031 & 0.651399 \\ \mathrm{C} & -2.575573 & -3.211799 & 1.976732 \\ \mathrm{~F} & -3.204038 & 1.354666 & 2.158300 \\ \mathrm{~N} & -2.900446 & -4.190831 & 2.517383\end{array}$

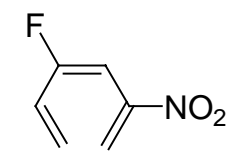

$\begin{array}{lrrc}\mathrm{H} & 0.000000 & 0.000000 & 0.000000 \\ \mathrm{H} & 0.000000 & 0.000000 & 1.853160 \\ \mathrm{C} & 0.564503 & 0.000000 & 0.926477 \\ \mathrm{C} & 1.904765 & -0.001592 & 0.934384 \\ \mathrm{H} & 2.452536 & 0.000914 & 1.871772 \\ \mathrm{C} & 2.711551 & -0.042636 & -0.272653 \\ \mathrm{C} & 4.049853 & 0.038543 & -0.415477 \\ \mathrm{H} & 4.456519 & -0.145574 & -1.403001 \\ \mathrm{C} & 4.995786 & 0.331370 & 0.650769 \\ \mathrm{C} & 4.883811 & 1.187133 & 1.673913 \\ \mathrm{H} & 4.003863 & 1.811097 & 1.776406 \\ \mathrm{H} & 5.679034 & 1.280938 & 2.402605 \\ \mathrm{~N} & 6.296833 & -0.407619 & 0.538380 \\ \mathrm{~F} & 1.993552 & -0.237843 & -1.401944 \\ \mathrm{O} & 6.436092 & -1.139206 & -0.439566 \\ \mathrm{O} & 7.140936 & -0.239033 & 1.413523\end{array}$

TS

$\begin{array}{llll}\mathrm{C} & 0.000000 & 0.000000 & 0.000000 \\ \mathrm{H} & 0.000000 & 0.000000 & 1.077491 \\ \mathrm{H} & 0.999644 & 0.000000 & -0.432502\end{array}$




$\begin{array}{lccc}\mathrm{C} & -1.005756 & -0.666073 & -0.695313 \\ \mathrm{H} & -0.747158 & -1.308387 & -1.537563 \\ \mathrm{C} & -2.376328 & -0.404513 & -0.573345 \\ \mathrm{C} & -2.947571 & 0.853383 & -0.397361 \\ \mathrm{C} & -2.169689 & 1.990721 & -0.162300 \\ \mathrm{C} & -0.953574 & 2.017503 & 0.514093 \\ \mathrm{H} & -0.935414 & 1.590356 & 1.503849 \\ \mathrm{H} & -0.300204 & 2.869600 & 0.358802 \\ \mathrm{H} & -3.971321 & 0.970243 & -0.734615 \\ \mathrm{~N} & -2.587970 & 3.217732 & -0.881510 \\ \mathrm{~F} & -3.211313 & -1.355112 & -1.051839 \\ \mathrm{O} & -3.744526 & 3.269752 & -1.299688 \\ \mathrm{O} & -1.760688 & 4.120747 & -1.001855\end{array}$

5

$\begin{array}{crrr}\mathrm{OHC} & & & \\ & = & & \\ \mathrm{H} & 0.000000 & 0.000000 & 0.000000 \\ \mathrm{H} & 0.000000 & 0.000000 & 1.847814 \\ \mathrm{C} & 0.571528 & 0.000000 & 0.923551 \\ \mathrm{C} & 1.921812 & -0.000040 & 0.921406 \\ \mathrm{C} & 2.587126 & -0.000084 & 2.223168 \\ \mathrm{H} & 1.867286 & 0.000064 & 3.042428 \\ \mathrm{C} & 3.895978 & -0.000302 & 2.622525 \\ \mathrm{C} & 5.074253 & -0.000635 & 1.749278 \\ \mathrm{H} & 4.873164 & -0.000730 & 0.684942 \\ \mathrm{C} & 6.361357 & -0.000850 & 2.133005 \\ \mathrm{H} & 6.659004 & -0.000794 & 3.172873 \\ \mathrm{H} & 7.145820 & -0.001107 & 1.380758 \\ \mathrm{C} & 4.044346 & -0.000190 & 4.105311 \\ \mathrm{C} & 2.666500 & -0.000038 & -0.394450 \\ \mathrm{H} & 1.960890 & -0.000038 & -1.230225 \\ \mathrm{H} & 3.306283 & -0.883788 & -0.497664 \\ \mathrm{H} & 3.306260 & 0.883723 & -0.497652 \\ \mathrm{H} & 3.070369 & 0.000069 & 4.644951 \\ \mathrm{O} & 5.082281 & -0.000316 & 4.739417 \\ & & & \\ \mathrm{TS} & & & \\ \mathrm{H} & 0.000000 & 0.000000 & 0.000000 \\ \mathrm{C} & 0.000000 & 0.000000 & 1.077624 \\ \mathrm{H} & 0.999571 & 0.000000 & 1.511607\end{array}$




$\begin{array}{lrrr}\mathrm{C} & -1.017921 & 0.661323 & 1.755290 \\ \mathrm{H} & -0.747160 & 1.280050 & 2.614623 \\ \mathrm{C} & -2.398761 & 0.459899 & 1.563534 \\ \mathrm{C} & -2.978142 & -0.785003 & 1.243942 \\ \mathrm{H} & -4.047416 & -0.835228 & 1.440926 \\ \mathrm{C} & -2.265028 & -1.981136 & 1.106371 \\ \mathrm{C} & -0.974678 & -2.037398 & 0.557493 \\ \mathrm{H} & -0.812939 & -1.617934 & -0.421925 \\ \mathrm{H} & -0.367119 & -2.922036 & 0.754597 \\ \mathrm{C} & -2.850573 & -3.243533 & 1.719741 \\ \mathrm{C} & -3.310383 & 1.503477 & 2.098329 \\ \mathrm{H} & -2.791683 & 2.352686 & 2.597356 \\ \mathrm{O} & -4.524073 & 1.489780 & 2.009158 \\ \mathrm{H} & -3.788517 & -3.046668 & 2.246134 \\ \mathrm{H} & -2.144135 & -3.669213 & 2.444686 \\ \mathrm{H} & -3.036921 & -4.016976 & 0.964463\end{array}$

$\begin{array}{cccc}\mathrm{OHC} & & & \\ & & & \\ \mathrm{C} & 0.000000 & 0.000000 & 0.000000 \\ \mathrm{H} & 0.000000 & 0.000000 & 1.083409 \\ \mathrm{H} & 0.966653 & 0.000000 & -0.495641 \\ \mathrm{C} & -1.159663 & -0.061405 & -0.696421 \\ \mathrm{C} & -2.421333 & -0.054701 & 0.042835 \\ \mathrm{H} & -2.293902 & 0.256571 & 1.078313 \\ \mathrm{C} & -3.699190 & -0.345442 & -0.341222 \\ \mathrm{C} & -4.094854 & -0.895541 & -1.645277 \\ \mathrm{H} & -3.281118 & -1.310239 & -2.233378 \\ \mathrm{C} & -5.341661 & -0.960608 & -2.138550 \\ \mathrm{H} & -6.201412 & -0.582416 & -1.601121 \\ \mathrm{H} & -5.514455 & -1.417242 & -3.109701 \\ \mathrm{C} & -4.706281 & -0.096310 & 0.726280 \\ \mathrm{~N} & -1.194060 & -0.174985 & -2.095261 \\ \mathrm{H} & -4.255301 & 0.222518 & 1.693324 \\ \mathrm{O} & -5.913902 & -0.198809 & 0.626384 \\ \mathrm{H} & -1.926351 & 0.371786 & -2.535068 \\ \mathrm{H} & -0.298193 & 0.013216 & -2.532374 \\ & & & \\ \mathrm{TS} & & & \\ \mathrm{H} & 0.000000 & 0.000000 & 0.000000 \\ \mathrm{C} & 0.000000 & 0.000000 & 1.079229 \\ \mathrm{H} & 0.993219 & 0.000000 & 1.525120 \\ \mathrm{C} & -1.046735 & 0.637777 & 1.728548\end{array}$




$\begin{array}{lrrr}\mathrm{H} & -0.847981 & 1.217153 & 2.631710 \\ \mathrm{C} & -2.397269 & 0.421763 & 1.387625 \\ \mathrm{C} & -2.896712 & -0.787766 & 0.883616 \\ \mathrm{H} & -3.982433 & -0.858901 & 0.859043 \\ \mathrm{C} & -2.169212 & -2.000102 & 0.856959 \\ \mathrm{C} & -0.789543 & -2.118751 & 0.581157 \\ \mathrm{H} & -0.419243 & -1.801781 & -0.380787 \\ \mathrm{H} & -0.291409 & -3.017832 & 0.952852 \\ \mathrm{~N} & -2.876299 & -3.137330 & 1.247266 \\ \mathrm{C} & -3.386900 & 1.432207 & 1.841439 \\ \mathrm{H} & -2.948423 & 2.261809 & 2.440298 \\ \mathrm{O} & -4.579092 & 1.411865 & 1.596851 \\ \mathrm{H} & -3.528476 & -3.044438 & 2.015666 \\ \mathrm{H} & -2.365875 & -4.009213 & 1.255772\end{array}$

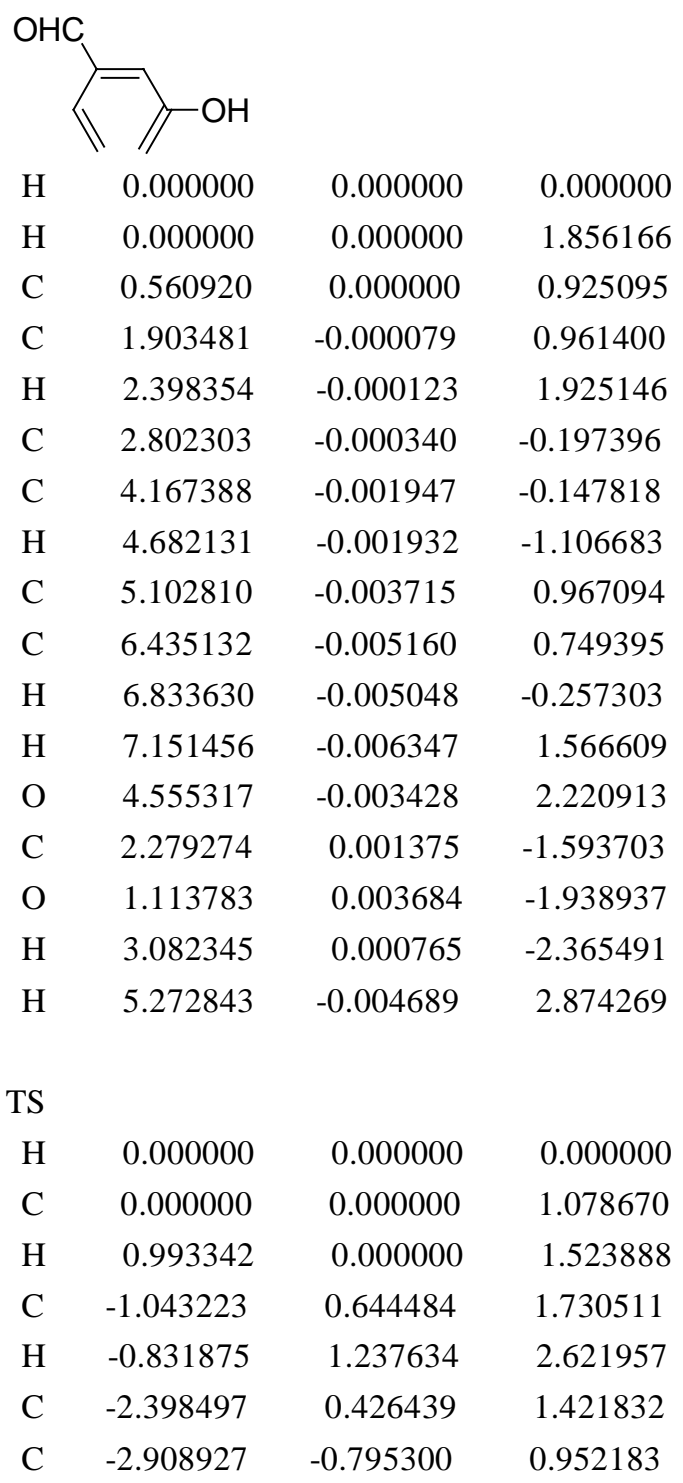




$\begin{array}{lrrr}\mathrm{H} & -3.995756 & -0.871627 & 0.978552 \\ \mathrm{C} & -2.168943 & -1.987705 & 0.883993 \\ \mathrm{C} & -0.808740 & -2.120972 & 0.565242 \\ \mathrm{H} & -0.455540 & -1.776264 & -0.391722 \\ \mathrm{H} & -0.330397 & -3.037492 & 0.909913 \\ \mathrm{O} & -2.777844 & -3.147480 & 1.307599 \\ \mathrm{C} & -3.383533 & 1.431139 & 1.899608 \\ \mathrm{H} & -2.937765 & 2.270646 & 2.477285 \\ \mathrm{O} & -4.581843 & 1.390211 & 1.691944 \\ \mathrm{H} & -3.558303 & -2.921241 & 1.838444\end{array}$

\begin{tabular}{|c|c|c|c|}
\hline $\mathrm{H}$ & 0.000000 & 0.000000 & 0.000000 \\
\hline $\mathrm{H}$ & 0.000000 & 0.000000 & 1.856661 \\
\hline $\mathrm{C}$ & 0.560248 & 0.000000 & 0.925481 \\
\hline $\mathrm{C}$ & 1.902717 & 0.000118 & 0.960846 \\
\hline $\mathrm{H}$ & 2.394969 & 0.000193 & 1.925738 \\
\hline $\mathrm{C}$ & 2.799800 & -0.000069 & -0.198380 \\
\hline $\mathrm{C}$ & 4.164658 & -0.002102 & -0.142107 \\
\hline $\mathrm{H}$ & 4.694453 & -0.001956 & -1.093258 \\
\hline $\mathrm{C}$ & 5.075111 & -0.004426 & 0.982170 \\
\hline $\mathrm{C}$ & 6.412106 & -0.006008 & 0.879950 \\
\hline $\mathrm{H}$ & 6.889712 & -0.005611 & -0.092169 \\
\hline $\mathrm{H}$ & 7.036906 & -0.007673 & 1.765170 \\
\hline $\mathrm{F}$ & 4.529205 & -0.004927 & 2.222540 \\
\hline $\mathrm{C}$ & 2.279501 & 0.002162 & -1.596455 \\
\hline $\mathrm{O}$ & 1.114471 & 0.004385 & -1.941043 \\
\hline $\mathrm{H}$ & 3.083933 & 0.001736 & -2.366369 \\
\hline \multicolumn{4}{|l|}{ TS } \\
\hline $\mathrm{H}$ & 0.000000 & 0.000000 & 0.000000 \\
\hline $\mathrm{C}$ & 0.000000 & 0.000000 & 1.078892 \\
\hline $\mathrm{H}$ & 0.993715 & 0.000000 & 1.523007 \\
\hline $\mathrm{C}$ & -1.037976 & 0.663150 & 1.725017 \\
\hline $\mathrm{H}$ & -0.816324 & 1.273716 & 2.602073 \\
\hline $\mathrm{C}$ & -2.395263 & 0.454508 & 1.430794 \\
\hline $\mathrm{C}$ & -2.917685 & -0.777123 & 0.996015 \\
\hline $\mathrm{H}$ & -3.997220 & -0.882369 & 1.065981 \\
\hline $\mathrm{C}$ & -2.166342 & -1.945462 & 0.898477 \\
\hline $\mathrm{C}$ & -0.811156 & -2.113560 & 0.600289 \\
\hline $\mathrm{H}$ & -0.359449 & -3.033944 & 0.970232 \\
\hline $\mathrm{H}$ & -0.425452 & -1.793435 & -0.352014 \\
\hline
\end{tabular}




$\begin{array}{lrrc}\mathrm{F} & -2.812743 & -3.064066 & 1.336403 \\ \mathrm{C} & -3.370487 & 1.472263 & 1.905606 \\ \mathrm{H} & -2.917483 & 2.303980 & 2.488724 \\ \mathrm{O} & -4.565446 & 1.446661 & 1.685310\end{array}$

\begin{tabular}{|c|c|c|c|}
\hline $\mathrm{C}$ & 0.000000 & 0.000000 & 0.000000 \\
\hline $\mathrm{H}$ & 0.000000 & 0.000000 & 1.083885 \\
\hline $\mathrm{H}$ & 0.971465 & 0.000000 & -0.489658 \\
\hline $\mathrm{C}$ & -1.131134 & 0.044734 & -0.735063 \\
\hline $\mathrm{C}$ & -2.505391 & 0.080216 & -0.238468 \\
\hline $\mathrm{H}$ & -3.195167 & 0.568275 & -0.925747 \\
\hline $\mathrm{C}$ & -3.024373 & -0.429445 & 0.912648 \\
\hline $\mathrm{C}$ & -2.282312 & -1.259601 & 1.865861 \\
\hline $\mathrm{H}$ & -1.373729 & -1.710397 & 1.470172 \\
\hline $\mathrm{C}$ & -2.630362 & -1.531764 & 3.132548 \\
\hline $\mathrm{H}$ & -2.012901 & -2.190693 & 3.737020 \\
\hline $\mathrm{H}$ & -3.521111 & -1.119761 & 3.588974 \\
\hline $\mathrm{C}$ & -4.469139 & -0.120649 & 1.114614 \\
\hline $\mathrm{C}$ & -0.965641 & 0.168742 & -2.214732 \\
\hline $\mathrm{O}$ & -1.879013 & 0.340049 & -2.995011 \\
\hline $\mathrm{H}$ & 0.087175 & 0.107126 & -2.566413 \\
\hline $\mathrm{H}$ & -4.928304 & 0.424253 & 0.260616 \\
\hline $\mathrm{O}$ & -5.132209 & -0.394257 & 2.095233 \\
\hline & & & \\
\hline $\mathrm{C}$ & 0.000000 & 0.000000 & 0.000000 \\
\hline $\mathrm{H}$ & 0.000000 & 0.000000 & 1.078966 \\
\hline $\mathrm{H}$ & 0.996156 & 0.000000 & -0.439565 \\
\hline $\mathrm{C}$ & -1.020827 & -0.669335 & -0.662259 \\
\hline $\mathrm{H}$ & -0.780659 & -1.284698 & -1.529362 \\
\hline $\mathrm{C}$ & -2.399993 & -0.496405 & -0.416777 \\
\hline $\mathrm{C}$ & -2.961911 & 0.744986 & -0.083028 \\
\hline $\mathrm{H}$ & -4.038892 & 0.847141 & -0.212331 \\
\hline $\mathrm{C}$ & -2.230026 & 1.941008 & -0.006818 \\
\hline $\mathrm{C}$ & -0.903282 & 2.044085 & 0.441844 \\
\hline $\mathrm{H}$ & -0.662485 & 1.719923 & 1.441013 \\
\hline $\mathrm{H}$ & -0.322596 & 2.910777 & 0.120240 \\
\hline $\mathrm{C}$ & -3.337046 & -1.531373 & -0.930016 \\
\hline $\mathrm{C}$ & -2.856715 & 3.153405 & -0.593818 \\
\hline $\mathrm{H}$ & -4.413991 & -1.287722 & -0.798962 \\
\hline $\mathrm{O}$ & -2.990890 & -2.572031 & -1.454195 \\
\hline
\end{tabular}




\begin{tabular}{|c|c|c|c|}
\hline $\mathrm{O}$ & -4.048308 & 3.298306 & -0.790584 \\
\hline $\mathrm{H}$ & -2.135268 & 3.968360 & -0.826898 \\
\hline \multicolumn{4}{|c|}{$\mathrm{OHC}$} \\
\hline & & & \\
\hline $\mathrm{H}$ & 0.000000 & 0.000000 & 0.000000 \\
\hline $\mathrm{H}$ & 0.000000 & 0.000000 & 1.857051 \\
\hline $\mathrm{C}$ & 0.559680 & 0.000000 & 0.925819 \\
\hline $\mathrm{C}$ & 1.902447 & 0.000039 & 0.961824 \\
\hline $\mathrm{H}$ & 2.381423 & 0.000070 & 1.934677 \\
\hline $\mathrm{C}$ & 2.799391 & 0.000046 & -0.194634 \\
\hline $\mathrm{C}$ & 4.163836 & -0.001033 & -0.150468 \\
\hline $\mathrm{H}$ & 4.671095 & -0.000619 & -1.114691 \\
\hline $\mathrm{C}$ & 5.120622 & -0.002736 & 0.953882 \\
\hline $\mathrm{C}$ & 6.452330 & -0.003209 & 0.708891 \\
\hline $\mathrm{H}$ & 6.829478 & -0.002299 & -0.309361 \\
\hline $\mathrm{H}$ & 7.179702 & -0.004434 & 1.512789 \\
\hline $\mathrm{C}$ & 4.718282 & -0.003969 & 2.338289 \\
\hline $\mathrm{C}$ & 2.273666 & 0.001407 & -1.594491 \\
\hline $\mathrm{O}$ & 1.108164 & 0.002253 & -1.933784 \\
\hline $\mathrm{H}$ & 3.075620 & 0.001598 & -2.366741 \\
\hline $\mathrm{N}$ & 4.447478 & -0.004930 & 3.468963 \\
\hline \multicolumn{4}{|l|}{$\mathrm{TS}$} \\
\hline $\mathrm{H}$ & 0.000000 & 0.000000 & 0.000000 \\
\hline $\mathrm{C}$ & 0.000000 & 0.000000 & 1.078328 \\
\hline $\mathrm{H}$ & 0.997780 & 0.000000 & 1.514890 \\
\hline $\mathrm{C}$ & -1.017223 & 0.657416 & 1.756923 \\
\hline $\mathrm{H}$ & -0.761594 & 1.285123 & 2.610821 \\
\hline $\mathrm{C}$ & -2.402375 & 0.465572 & 1.565068 \\
\hline $\mathrm{C}$ & -2.974029 & -0.785162 & 1.286828 \\
\hline $\mathrm{H}$ & -4.041391 & -0.879741 & 1.482021 \\
\hline $\mathrm{C}$ & -2.227067 & -1.972586 & 1.150597 \\
\hline $\mathrm{C}$ & -0.941890 & -2.028562 & 0.582558 \\
\hline $\mathrm{H}$ & -0.818080 & -1.630289 & -0.411060 \\
\hline $\mathrm{H}$ & -0.314630 & -2.890300 & 0.800414 \\
\hline $\mathrm{C}$ & -2.750598 & -3.147254 & 1.788193 \\
\hline $\mathrm{C}$ & -3.329332 & 1.505792 & 2.092300 \\
\hline $\mathrm{O}$ & -2.971322 & 2.559124 & 2.579227 \\
\hline $\mathrm{H}$ & -4.408744 & 1.251307 & 2.009477 \\
\hline $\mathrm{N}$ & -3.181045 & -4.107367 & 2.287783 \\
\hline
\end{tabular}




\begin{tabular}{|c|c|c|c|}
\hline $\mathrm{C}$ & 0.000000 & 0.000000 & 0.000000 \\
\hline $\mathrm{H}$ & 0.000000 & 0.000000 & 1.083149 \\
\hline $\mathrm{H}$ & 0.947659 & 0.000000 & -0.524280 \\
\hline $\mathrm{C}$ & -1.151769 & 0.033158 & -0.683101 \\
\hline $\mathrm{C}$ & -2.516481 & 0.074834 & -0.178866 \\
\hline $\mathrm{H}$ & -3.213281 & 0.619888 & -0.809023 \\
\hline $\mathrm{C}$ & -2.996197 & -0.484891 & 0.965976 \\
\hline $\mathrm{C}$ & -2.235898 & -1.381359 & 1.839890 \\
\hline $\mathrm{H}$ & -1.349461 & -1.822439 & 1.386966 \\
\hline $\mathrm{C}$ & -2.543944 & -1.718730 & 3.101398 \\
\hline $\mathrm{H}$ & -3.409445 & -1.318415 & 3.613304 \\
\hline $\mathrm{H}$ & -1.917684 & -2.421438 & 3.644094 \\
\hline $\mathrm{C}$ & -4.423711 & -0.147241 & 1.245905 \\
\hline $\mathrm{N}$ & -1.038395 & 0.109296 & -2.177395 \\
\hline $\mathrm{O}$ & -2.088063 & 0.210939 & -2.809505 \\
\hline $\mathrm{O}$ & 0.080298 & 0.064417 & -2.678041 \\
\hline $\mathrm{H}$ & -4.902817 & 0.452152 & 0.441101 \\
\hline $\mathrm{O}$ & -5.050176 & -0.455218 & 2.238872 \\
\hline \multicolumn{4}{|l|}{ TS } \\
\hline $\mathrm{C}$ & 0.000000 & 0.000000 & 0.000000 \\
\hline $\mathrm{H}$ & 0.000000 & 0.000000 & 1.077302 \\
\hline $\mathrm{H}$ & 0.981432 & 0.000000 & -0.463963 \\
\hline $\mathrm{C}$ & -1.025743 & 0.693619 & -0.642889 \\
\hline $\mathrm{C}$ & -2.399196 & 0.483109 & -0.482063 \\
\hline $\mathrm{H}$ & -3.069624 & 1.253176 & -0.853188 \\
\hline $\mathrm{C}$ & -2.955026 & -0.778912 & -0.224178 \\
\hline $\mathrm{C}$ & -2.186009 & -1.959136 & -0.139682 \\
\hline $\mathrm{H}$ & -2.609921 & -2.812946 & -0.668681 \\
\hline $\mathrm{C}$ & -0.909712 & -2.067256 & 0.396011 \\
\hline $\mathrm{H}$ & -0.308461 & -2.936097 & 0.132869 \\
\hline $\mathrm{H}$ & -0.724620 & -1.696766 & 1.391683 \\
\hline $\mathrm{C}$ & -4.417572 & -0.915137 & -0.477622 \\
\hline $\mathrm{N}$ & -0.641480 & 1.590220 & -1.760846 \\
\hline $\mathrm{O}$ & 0.503859 & 1.500404 & -2.200337 \\
\hline $\mathrm{O}$ & -1.486620 & 2.385773 & -2.170740 \\
\hline $\mathrm{H}$ & -4.951507 & 0.045356 & -0.643995 \\
\hline $\mathrm{O}$ & -5.009261 & -1.975075 & -0.507808 \\
\hline
\end{tabular}




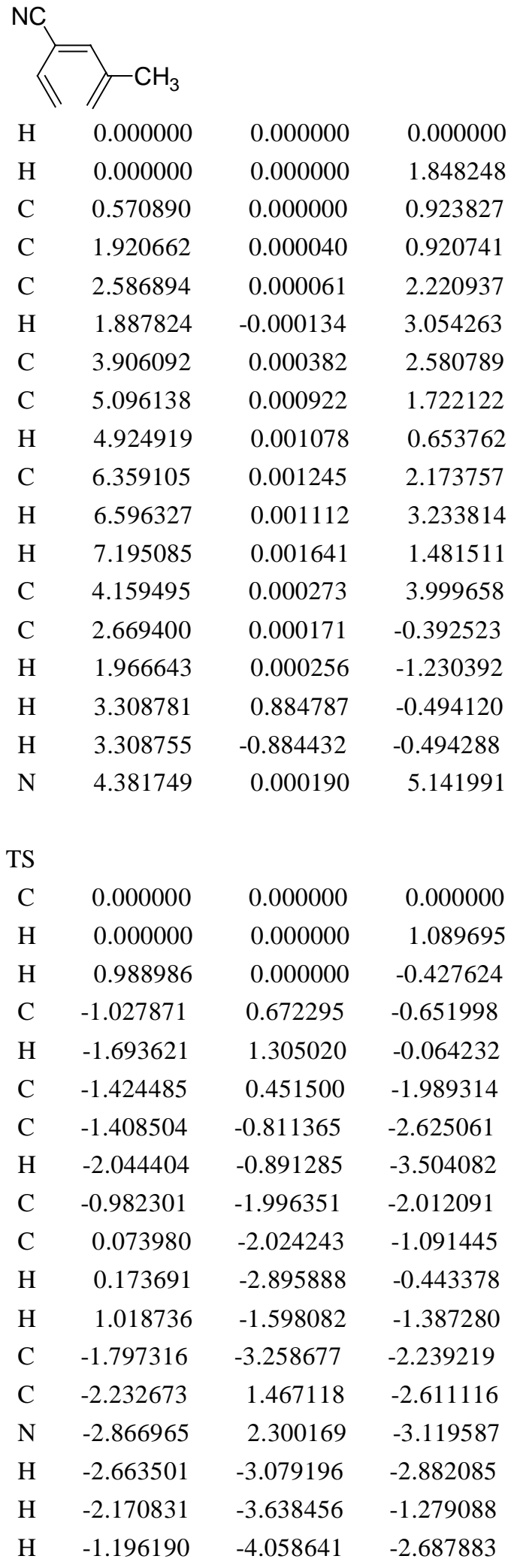




\begin{tabular}{|c|c|c|c|}
\hline $\mathrm{H}$ & 0.000000 & 0.000000 & 0.000000 \\
\hline $\mathrm{H}$ & 0.000000 & 0.000000 & 1.851693 \\
\hline $\mathrm{C}$ & 0.567788 & 0.000000 & 0.926490 \\
\hline $\mathrm{C}$ & 1.908519 & 0.006922 & 0.945926 \\
\hline $\mathrm{H}$ & 2.438367 & 0.022712 & 1.892214 \\
\hline $\mathrm{C}$ & 2.755615 & 0.042093 & -0.254388 \\
\hline $\mathrm{C}$ & 4.117845 & -0.027619 & -0.314368 \\
\hline $\mathrm{H}$ & 4.564273 & 0.104809 & -1.295374 \\
\hline $\mathrm{C}$ & 5.099422 & -0.257767 & 0.745676 \\
\hline $\mathrm{C}$ & 6.392310 & 0.065789 & 0.515821 \\
\hline $\mathrm{H}$ & 6.692381 & 0.540404 & -0.410814 \\
\hline $\mathrm{H}$ & 7.162802 & -0.089876 & 1.265558 \\
\hline $\mathrm{N}$ & 4.652082 & -0.765569 & 1.979459 \\
\hline $\mathrm{C}$ & 2.076065 & 0.212968 & -1.512931 \\
\hline $\mathrm{N}$ & 1.510234 & 0.345086 & -2.521203 \\
\hline $\mathrm{H}$ & 4.008279 & -1.545132 & 1.884994 \\
\hline $\mathrm{H}$ & 5.415502 & -1.020283 & 2.597969 \\
\hline \\
\hline $\mathrm{H}$ & 0.000000 & 0.000000 & 0.000000 \\
\hline $\mathrm{C}$ & 0.000000 & 0.000000 & 1.079178 \\
\hline $\mathrm{H}$ & 0.992679 & 0.000000 & 1.525576 \\
\hline $\mathrm{C}$ & -1.039427 & 0.649733 & 1.729187 \\
\hline $\mathrm{H}$ & -0.845720 & 1.238879 & 2.623958 \\
\hline $\mathrm{C}$ & -2.389056 & 0.418877 & 1.384690 \\
\hline $\mathrm{C}$ & -2.880912 & -0.807744 & 0.902981 \\
\hline $\mathrm{H}$ & -3.963038 & -0.910439 & 0.870911 \\
\hline $\mathrm{C}$ & -2.134801 & -2.012971 & 0.902524 \\
\hline $\mathrm{C}$ & -0.758391 & -2.118001 & 0.615589 \\
\hline $\mathrm{H}$ & -0.403650 & -1.814732 & -0.356665 \\
\hline $\mathrm{H}$ & -0.245216 & -3.002665 & 1.000176 \\
\hline $\mathrm{N}$ & -2.827444 & -3.143126 & 1.330287 \\
\hline $\mathrm{C}$ & -3.366387 & 1.394459 & 1.790373 \\
\hline $\mathrm{N}$ & -4.150853 & 2.192060 & 2.111706 \\
\hline $\mathrm{H}$ & -3.463270 & -3.042042 & 2.111398 \\
\hline 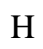 & -2.316236 & -4.014551 & 1.336782 \\
\hline & & & \\
\hline $\mathrm{H}$ & 0.000000 & 0.000000 & 0.000000 \\
\hline
\end{tabular}




\begin{tabular}{|c|c|c|c|}
\hline $\mathrm{H}$ & 0.000000 & 0.000000 & 55 \\
\hline $\mathrm{C}$ & 0.567611 & 0.000000 & 0.926511 \\
\hline $\mathrm{C}$ & 1.908615 & 0.000000 & 0.948069 \\
\hline $\mathrm{H}$ & 2.439984 & 0.000000 & 1.890982 \\
\hline $\mathrm{C}$ & 2.748634 & 0.000000 & -0.255264 \\
\hline $\mathrm{C}$ & 4.111487 & 0.000056 & -0.326020 \\
\hline $\mathrm{H}$ & 4.547309 & 0.000045 & -1.320170 \\
\hline $\mathrm{C}$ & 5.109052 & 0.000112 & 0.732792 \\
\hline $\mathrm{C}$ & 6.426220 & 0.000103 & 0.441049 \\
\hline $\mathrm{H}$ & 6.766598 & 0.000070 & -0.586653 \\
\hline $\mathrm{H}$ & 7.186157 & 0.000127 & 1.217613 \\
\hline $\mathrm{O}$ & 4.627743 & 0.000107 & 2.014516 \\
\hline $\mathrm{C}$ & 2.059238 & 0.000000 & -1.520703 \\
\hline $\mathrm{N}$ & 1.487365 & 0.000005 & -2.534043 \\
\hline $\mathrm{H}$ & 5.378070 & 0.000163 & 2.629811 \\
\hline \multicolumn{4}{|l|}{ is } \\
\hline $\mathrm{H}$ & 0.000000 & 0.000000 & 0.000000 \\
\hline $\mathrm{C}$ & 0.000000 & 0.000000 & 1.078611 \\
\hline $\mathrm{H}$ & 0.993186 & 0.000000 & 1.523696 \\
\hline $\mathrm{C}$ & -1.034488 & 0.658432 & 1.731555 \\
\hline $\mathrm{H}$ & -0.825270 & 1.264212 & 2.611827 \\
\hline $\mathrm{C}$ & -2.390487 & 0.425874 & 1.425124 \\
\hline $\mathrm{C}$ & -2.896240 & -0.815238 & 0.987288 \\
\hline $\mathrm{H}$ & -3.979161 & -0.923666 & 1.013554 \\
\hline $\mathrm{C}$ & -2.136402 & -1.999982 & 0.941608 \\
\hline $\mathrm{C}$ & -0.781785 & -2.117064 & 0.602649 \\
\hline $\mathrm{H}$ & -0.453923 & -1.787463 & -0.368731 \\
\hline $\mathrm{H}$ & -0.282572 & -3.017957 & 0.958220 \\
\hline $\mathrm{O}$ & -2.723441 & -3.153344 & 1.406088 \\
\hline $\mathrm{C}$ & -3.357458 & 1.403208 & 1.848884 \\
\hline $\mathrm{N}$ & -4.137130 & 2.200635 & 2.181507 \\
\hline $\mathrm{H}$ & -3.478716 & -2.927310 & 1.972443 \\
\hline \multicolumn{4}{|c|}{$\left.{ }_{\|}^{N C}\right\rangle_{F}$} \\
\hline $\mathrm{H}$ & 0.000000 & 0.000000 & 0.000000 \\
\hline $\mathrm{H}$ & 0.000000 & 0.000000 & 1.851557 \\
\hline $\mathrm{C}$ & 0.567598 & 0.000000 & 0.926453 \\
\hline $\mathrm{C}$ & 1.908384 & 0.000000 & 0.947914 \\
\hline $\mathrm{H}$ & 2.435609 & 0.000000 & 1.892897 \\
\hline $\mathrm{C}$ & 2.748034 & 0.000000 & -0.254936 \\
\hline $\mathrm{C}$ & 4.111390 & 0.000019 & -0.316893 \\
\hline
\end{tabular}




$\begin{array}{lrrr}\mathrm{H} & 4.564847 & -0.000001 & -1.303597 \\ \mathrm{C} & 5.082986 & 0.000044 & 0.754740 \\ \mathrm{C} & 6.411665 & -0.000054 & 0.581703 \\ \mathrm{H} & 6.836674 & -0.000145 & -0.414477 \\ \mathrm{H} & 7.081886 & -0.000093 & 1.432944 \\ \mathrm{~F} & 4.598247 & 0.000028 & 2.021558 \\ \mathrm{C} & 2.062612 & 0.000000 & -1.521806 \\ \mathrm{~N} & 1.494186 & 0.000006 & -2.536886\end{array}$

TS

$\begin{array}{lrrc}\mathrm{H} & 0.000000 & 0.000000 & 0.000000 \\ \mathrm{C} & 0.000000 & 0.000000 & 1.079010 \\ \mathrm{H} & 0.992708 & 0.000000 & 1.524547 \\ \mathrm{C} & -1.032198 & 0.673857 & 1.723334 \\ \mathrm{H} & -0.819034 & 1.292047 & 2.593900 \\ \mathrm{C} & -2.387705 & 0.451376 & 1.418149 \\ \mathrm{C} & -2.897755 & -0.795033 & 0.996440 \\ \mathrm{H} & -3.973548 & -0.931165 & 1.055616 \\ \mathrm{C} & -2.130140 & -1.957754 & 0.920850 \\ \mathrm{C} & -0.772338 & -2.115851 & 0.636797 \\ \mathrm{H} & -0.382964 & -1.812784 & -0.319664 \\ \mathrm{H} & -0.315307 & -3.024401 & 1.028868 \\ \mathrm{~F} & -2.774771 & -3.070576 & 1.370621 \\ \mathrm{C} & -3.348329 & 1.435914 & 1.838213 \\ \mathrm{~N} & -4.118009 & 2.245560 & 2.163451\end{array}$

$\begin{array}{lrrr}\mathrm{NC} & & & \\ & \end{array}$




$\begin{array}{rrrr}\mathrm{H} & 7.065260 & 0.193098 & 1.558636 \\ \mathrm{O} & 6.828951 & -0.690047 & -0.249890\end{array}$

TS

$\begin{array}{lrrr}\mathrm{H} & 0.000000 & 0.000000 & 0.000000 \\ \mathrm{C} & 0.000000 & 0.000000 & 1.078446 \\ \mathrm{H} & 0.994496 & 0.000000 & 1.520788 \\ \mathrm{C} & -1.019221 & 0.668816 & 1.742800 \\ \mathrm{H} & -0.772037 & 1.276330 & 2.613558 \\ \mathrm{C} & -2.401478 & 0.481562 & 1.497099 \\ \mathrm{C} & -2.963858 & -0.768049 & 1.181986 \\ \mathrm{H} & -4.036294 & -0.874654 & 1.322679 \\ \mathrm{C} & -2.215965 & -1.954115 & 1.105021 \\ \mathrm{C} & -0.899862 & -2.049639 & 0.632294 \\ \mathrm{H} & -0.677531 & -1.711932 & -0.366476 \\ \mathrm{H} & -0.308410 & -2.914430 & 0.938089 \\ \mathrm{C} & -2.825136 & -3.172568 & 1.704737 \\ \mathrm{C} & -3.301487 & 1.510924 & 1.944836 \\ \mathrm{~N} & -4.016681 & 2.362872 & 2.285847 \\ \mathrm{H} & -2.092312 & -3.978920 & 1.930945 \\ \mathrm{O} & -4.012013 & -3.326717 & 1.914245\end{array}$<smiles>C=CC(C#N)=CC(=C)C#N</smiles>

$\begin{array}{lrrr}\mathrm{H} & 0.000000 & 0.000000 & 0.000000 \\ \mathrm{H} & 0.000000 & 0.000000 & 1.851910 \\ \mathrm{C} & 0.567182 & 0.000000 & 0.926683 \\ \mathrm{C} & 1.908304 & 0.000000 & 0.949717 \\ \mathrm{H} & 2.421864 & 0.000000 & 1.903382 \\ \mathrm{C} & 2.745782 & 0.000000 & -0.253015 \\ \mathrm{C} & 4.107865 & 0.000000 & -0.335608 \\ \mathrm{H} & 4.529068 & 0.000000 & -1.337267 \\ \mathrm{C} & 5.135662 & 0.000000 & 0.702283 \\ \mathrm{C} & 6.447865 & 0.000000 & 0.369781 \\ \mathrm{H} & 6.756740 & 0.000000 & -0.671095 \\ \mathrm{H} & 7.225925 & 0.000000 & 1.124564 \\ \mathrm{C} & 4.816988 & 0.000000 & 2.107539 \\ \mathrm{C} & 2.053231 & 0.000000 & -1.517462 \\ \mathrm{~N} & 1.483193 & 0.000001 & -2.531350 \\ \mathrm{~N} & 4.598531 & -0.000002 & 3.249487\end{array}$

TS

$\begin{array}{llll}\text { H } & 0.000000 & 0.000000 & 0.000000\end{array}$ 


\begin{tabular}{|c|c|c|c|}
\hline $\mathrm{C}$ & 0.000000 & 0.000000 & 1.077901 \\
\hline $\mathrm{H}$ & 0.996294 & 0.000000 & 1.516987 \\
\hline $\mathrm{C}$ & -1.015982 & 0.655660 & 1.758792 \\
\hline $\mathrm{H}$ & -0.754101 & 1.275305 & 2.616798 \\
\hline $\mathrm{C}$ & -2.404234 & 0.447157 & 1.567384 \\
\hline $\mathrm{C}$ & -2.973256 & -0.813102 & 1.310791 \\
\hline $\mathrm{H}$ & -4.034554 & -0.913782 & 1.519748 \\
\hline $\mathrm{C}$ & -2.207665 & -1.988799 & 1.172473 \\
\hline $\mathrm{C}$ & -0.937646 & -2.031756 & 0.576102 \\
\hline $\mathrm{H}$ & -0.838689 & -1.620892 & -0.415191 \\
\hline $\mathrm{H}$ & -0.296087 & -2.886940 & 0.775960 \\
\hline $\mathrm{C}$ & -2.702765 & -3.165889 & 1.829136 \\
\hline $\mathrm{C}$ & -3.297658 & 1.478859 & 2.023348 \\
\hline $\mathrm{N}$ & -4.010638 & 2.330097 & 2.370072 \\
\hline $\mathrm{N}$ & -3.105345 & -4.129536 & 2.344148 \\
\hline \multicolumn{4}{|l|}{$\mathrm{NC}$} \\
\hline $\mathrm{H}$ & 0.000000 & 0.000000 & 0.000000 \\
\hline $\mathrm{H}$ & 0.000000 & 0.000000 & 1.851991 \\
\hline $\mathrm{C}$ & 0.567100 & 0.000000 & 0.926694 \\
\hline $\mathrm{C}$ & 1.907093 & -0.000653 & 0.943066 \\
\hline $\mathrm{H}$ & 2.433491 & 0.000082 & 1.893180 \\
\hline $\mathrm{C}$ & 2.754995 & -0.040814 & -0.253586 \\
\hline $\mathrm{C}$ & 4.107552 & 0.105930 & -0.289744 \\
\hline $\mathrm{H}$ & 4.618531 & -0.066990 & -1.229965 \\
\hline $\mathrm{C}$ & 4.964159 & 0.470065 & 0.832489 \\
\hline $\mathrm{C}$ & 4.732316 & 1.340272 & 1.822412 \\
\hline $\mathrm{H}$ & 3.800747 & 1.891585 & 1.868385 \\
\hline $\mathrm{H}$ & 5.487017 & 1.524140 & 2.577324 \\
\hline $\mathrm{N}$ & 6.317036 & -0.175810 & 0.808062 \\
\hline $\mathrm{C}$ & 2.095354 & -0.302697 & -1.506437 \\
\hline $\mathrm{N}$ & 1.536008 & -0.502457 & -2.506269 \\
\hline $\mathrm{O}$ & 6.553143 & -0.924486 & -0.137577 \\
\hline $\mathrm{O}$ & 7.097215 & 0.075439 & 1.720079 \\
\hline \multicolumn{4}{|c|}{ TS } \\
\hline $\mathrm{H}$ & 0.000000 & 0.000000 & 0.000000 \\
\hline $\mathrm{C}$ & 0.000000 & 0.000000 & 1.077970 \\
\hline $\mathrm{H}$ & 0.994759 & 0.000000 & 1.519763 \\
\hline $\mathrm{C}$ & -1.020152 & 0.654574 & 1.753862 \\
\hline $\mathrm{H}$ & -0.764823 & 1.274086 & 2.613870 \\
\hline $\mathrm{C}$ & -2.406287 & 0.448063 & 1.549765 \\
\hline
\end{tabular}




$\begin{array}{lrrr}\mathrm{C} & -2.971056 & -0.810049 & 1.272658 \\ \mathrm{H} & -4.030068 & -0.932705 & 1.469055 \\ \mathrm{C} & -2.195596 & -1.964056 & 1.120012 \\ \mathrm{C} & -0.915687 & -2.043434 & 0.577031 \\ \mathrm{H} & -0.772139 & -1.655357 & -0.417642 \\ \mathrm{H} & -0.306597 & -2.904115 & 0.834952 \\ \mathrm{~N} & -2.723990 & -3.179865 & 1.793457 \\ \mathrm{C} & -3.304212 & 1.476231 & 2.003881 \\ \mathrm{~N} & -4.018847 & 2.326203 & 2.350047 \\ \mathrm{O} & -3.929236 & -3.218448 & 2.033244 \\ \mathrm{O} & -1.927369 & -4.079710 & 2.052076\end{array}$

\begin{tabular}{|c|c|c|c|}
\hline \multicolumn{4}{|c|}{$\mathrm{O}_{2} \mathrm{~N}$} \\
\hline & $\geqslant \mathrm{CH}_{3}$ & & \\
\hline $\mathrm{H}$ & 0.000000 & 0.000000 & 0.000000 \\
\hline $\mathrm{H}$ & 0.000000 & 0.000000 & 1.848505 \\
\hline $\mathrm{C}$ & 0.570531 & 0.000000 & 0.924087 \\
\hline $\mathrm{C}$ & 1.920797 & 0.003085 & 0.921328 \\
\hline $\mathrm{C}$ & 2.570980 & 0.002716 & 2.229439 \\
\hline $\mathrm{H}$ & 1.871340 & 0.001561 & 3.058971 \\
\hline $\mathrm{C}$ & 3.875009 & -0.001708 & 2.613038 \\
\hline $\mathrm{C}$ & 5.067114 & 0.048812 & 1.772964 \\
\hline $\mathrm{H}$ & 4.831190 & 0.133344 & 0.719933 \\
\hline $\mathrm{C}$ & 6.368382 & 0.053003 & 2.103281 \\
\hline $\mathrm{H}$ & 6.728692 & -0.032520 & 3.118677 \\
\hline $\mathrm{H}$ & 7.109527 & 0.143830 & 1.314271 \\
\hline $\mathrm{N}$ & 4.050228 & -0.062642 & 4.092737 \\
\hline $\mathrm{C}$ & 2.668777 & 0.001527 & -0.392218 \\
\hline $\mathrm{H}$ & 3.280383 & 0.903426 & -0.512436 \\
\hline $\mathrm{H}$ & 1.964835 & -0.033893 & -1.228320 \\
\hline $\mathrm{H}$ & 3.334200 & -0.864941 & -0.479823 \\
\hline $\mathrm{O}$ & 3.147261 & 0.365871 & 4.812113 \\
\hline $\mathrm{O}$ & 5.092035 & -0.556252 & 4.523870 \\
\hline \multicolumn{4}{|l|}{$\mathrm{TS}$} \\
\hline $\mathrm{H}$ & 0.000000 & 0.000000 & 0.000000 \\
\hline $\mathrm{C}$ & 0.000000 & 0.000000 & 1.077476 \\
\hline $\mathrm{H}$ & 0.999266 & 0.000000 & 1.511202 \\
\hline $\mathrm{C}$ & -1.007023 & 0.668685 & 1.764709 \\
\hline $\mathrm{H}$ & -0.748834 & 1.307047 & 2.606323 \\
\hline
\end{tabular}




$\begin{array}{lrrr}\mathrm{C} & -2.375973 & 0.423801 & 1.583973 \\ \mathrm{C} & -2.962558 & -0.828839 & 1.352656 \\ \mathrm{H} & -4.016172 & -0.889405 & 1.606139 \\ \mathrm{C} & -2.228162 & -2.008312 & 1.192487 \\ \mathrm{C} & -0.970834 & -2.025531 & 0.571564 \\ \mathrm{H} & -0.882362 & -1.593416 & -0.412043 \\ \mathrm{H} & -0.326880 & -2.892021 & 0.726197 \\ \mathrm{C} & -2.747851 & -3.277238 & 1.846664 \\ \mathrm{~N} & -3.293597 & 1.467420 & 2.108260 \\ \mathrm{O} & -2.786335 & 2.485586 & 2.581416 \\ \mathrm{O} & -4.507066 & 1.278995 & 2.017024 \\ \mathrm{H} & -2.000758 & -3.666089 & 2.551030 \\ \mathrm{H} & -2.936877 & -4.068594 & 1.111385 \\ \mathrm{H} & -3.670708 & -3.102054 & 2.406127\end{array}$

$\begin{array}{lrrr}\mathrm{O}_{2} \mathrm{~N} & & & \\ & = & & \\ \mathrm{H} & 0.000000 & 0.000000 & 0.000000 \\ \mathrm{C} & 0.000000 & 0.000000 & 1.083190 \\ \mathrm{H} & 0.965908 & 0.000000 & 1.580196 \\ \mathrm{C} & -1.159109 & 0.062410 & 1.779560 \\ \mathrm{C} & -2.411650 & 0.063160 & 1.027775 \\ \mathrm{H} & -2.309084 & -0.239946 & -0.007580 \\ \mathrm{C} & -3.674388 & 0.367055 & 1.422797 \\ \mathrm{C} & -4.081676 & 0.942332 & 2.704252 \\ \mathrm{H} & -3.227754 & 1.318874 & 3.260014 \\ \mathrm{C} & -5.300278 & 1.106798 & 3.242639 \\ \mathrm{H} & -6.212295 & 0.767309 & 2.772949 \\ \mathrm{H} & -5.384312 & 1.610999 & 4.201464 \\ \mathrm{~N} & -4.702691 & 0.115518 & 0.375904 \\ \mathrm{~N} & -1.196139 & 0.177000 & 3.179617 \\ \mathrm{H} & -1.905764 & -0.399910 & 3.619856 \\ \mathrm{H} & -0.293136 & 0.012691 & 3.612263 \\ \mathrm{O} & -5.861367 & -0.065222 & 0.750101 \\ \mathrm{O} & -4.351312 & 0.082481 & -0.803694\end{array}$

$\begin{array}{rrrr}\text { TS } & & & \\ \mathrm{C} & 0.000000 & 0.000000 & 0.000000 \\ \mathrm{H} & 0.000000 & 0.000000 & 1.078405 \\ \mathrm{H} & 1.005540 & 0.000000 & -0.427075 \\ \mathrm{C} & -0.975593 & 0.757102 & -0.682350 \\ \mathrm{C} & -2.374983 & 0.557582 & -0.586869 \\ \mathrm{H} & -3.022824 & 1.353634 & -0.938562\end{array}$




$\begin{array}{lrrr}\mathrm{C} & -2.963203 & -0.702567 & -0.488701 \\ \mathrm{C} & -2.287317 & -1.916870 & -0.306896 \\ \mathrm{H} & -2.618677 & -2.771641 & -0.888509 \\ \mathrm{C} & -1.116536 & -1.965067 & 0.436313 \\ \mathrm{H} & -0.468207 & -2.835019 & 0.353727 \\ \mathrm{H} & -1.111537 & -1.497944 & 1.409164 \\ \mathrm{~N} & -4.382463 & -0.789861 & -0.920281 \\ \mathrm{~N} & -0.567855 & 1.712047 & -1.606904 \\ \mathrm{H} & 0.424667 & 1.879243 & -1.689811 \\ \mathrm{H} & -1.068387 & 1.774055 & -2.484106 \\ \mathrm{O} & -5.010024 & 0.255242 & -1.094275 \\ \mathrm{O} & -4.861055 & -1.915556 & -1.064046\end{array}$

$\begin{array}{lrrr}\mathrm{O}_{2} \mathrm{~N} & & & \\ & = & & \\ \mathrm{H} & 0.000000 & 0.000000 & 0.000000 \\ \mathrm{H} & 0.000000 & 0.000000 & 1.851843 \\ \mathrm{C} & 0.563245 & 0.000000 & 0.922925 \\ \mathrm{C} & 1.904459 & -0.004129 & 0.978651 \\ \mathrm{H} & 2.376737 & -0.010238 & 1.953462 \\ \mathrm{C} & 2.881866 & 0.049612 & -0.102948 \\ \mathrm{C} & 4.233748 & 0.102480 & 0.001037 \\ \mathrm{H} & 4.773202 & 0.170536 & -0.935975 \\ \mathrm{C} & 5.096599 & 0.087780 & 1.171147 \\ \mathrm{C} & 6.436914 & 0.143150 & 1.026132 \\ \mathrm{H} & 6.886102 & 0.196764 & 0.042655 \\ \mathrm{H} & 7.108299 & 0.138639 & 1.880444 \\ \mathrm{O} & 4.478379 & 0.016806 & 2.389517 \\ \mathrm{~N} & 2.404454 & 0.049690 & -1.516797 \\ \mathrm{O} & 1.312583 & -0.465804 & -1.750821 \\ \mathrm{O} & 3.127810 & 0.547911 & -2.380005 \\ \mathrm{H} & 5.154802 & 0.011187 & 3.085330\end{array}$

$\begin{array}{rrrr}\text { TS } & & & \\ \mathrm{H} & 0.000000 & 0.000000 & 0.000000 \\ \mathrm{C} & 0.000000 & 0.000000 & 1.078490 \\ \mathrm{H} & 0.992385 & 0.000000 & 1.524693 \\ \mathrm{C} & -1.035216 & 0.652314 & 1.736998 \\ \mathrm{H} & -0.839653 & 1.260877 & 2.615035 \\ \mathrm{C} & -2.374112 & 0.393501 & 1.424134 \\ \mathrm{C} & -2.897721 & -0.836460 & 1.016718 \\ \mathrm{H} & -3.979616 & -0.921572 & 1.064562 \\ \mathrm{C} & -2.135317 & -2.016764 & 0.956262\end{array}$




$\begin{array}{lrrc}\mathrm{C} & -0.783801 & -2.122695 & 0.597937 \\ \mathrm{H} & -0.471171 & -1.781172 & -0.374189 \\ \mathrm{H} & -0.272893 & -3.022379 & 0.939210 \\ \mathrm{O} & -2.710672 & -3.176769 & 1.414265 \\ \mathrm{~N} & -3.370858 & 1.394153 & 1.885802 \\ \mathrm{O} & -2.943665 & 2.391020 & 2.467718 \\ \mathrm{O} & -4.561065 & 1.186187 & 1.648263 \\ \mathrm{H} & -3.472087 & -2.961321 & 1.976565\end{array}$

$\begin{array}{llrr}\mathrm{O}_{2} \mathrm{~N} & & & \\ & & & \\ \mathrm{H} & 0.000000 & 0.000000 & 0.000000 \\ \mathrm{H} & 0.000000 & 0.000000 & 1.852317 \\ \mathrm{C} & 0.562507 & 0.000000 & 0.923164 \\ \mathrm{C} & 1.903671 & -0.003741 & 0.978746 \\ \mathrm{H} & 2.371692 & -0.008852 & 1.955255 \\ \mathrm{C} & 2.881841 & 0.047308 & -0.101660 \\ \mathrm{C} & 4.232999 & 0.100152 & 0.012897 \\ \mathrm{H} & 4.788817 & 0.164906 & -0.915437 \\ \mathrm{C} & 5.069044 & 0.089398 & 1.191827 \\ \mathrm{C} & 6.407987 & 0.141448 & 1.164010 \\ \mathrm{H} & 6.936386 & 0.193957 & 0.220161 \\ \mathrm{H} & 6.982541 & 0.133878 & 2.082636 \\ \mathrm{~F} & 4.453315 & 0.022708 & 2.397505 \\ \mathrm{~N} & 2.413056 & 0.043749 & -1.518165 \\ \mathrm{O} & 1.311205 & -0.448308 & -1.752979 \\ \mathrm{O} & 3.154431 & 0.516109 & -2.379953\end{array}$

$\begin{array}{cccc}\text { TS } & & & \\ \mathrm{H} & 0.000000 & 0.000000 & 0.000000 \\ \mathrm{C} & 0.000000 & 0.000000 & 1.078603 \\ \mathrm{H} & 0.993167 & 0.000000 & 1.523014 \\ \mathrm{C} & -1.027754 & 0.674107 & 1.731882 \\ \mathrm{H} & -0.819340 & 1.305206 & 2.591064 \\ \mathrm{C} & -2.371503 & 0.426658 & 1.442052 \\ \mathrm{C} & -2.912273 & -0.810930 & 1.077116 \\ \mathrm{H} & -3.985257 & -0.922107 & 1.186083 \\ \mathrm{C} & -2.144304 & -1.968906 & 0.975446 \\ \mathrm{C} & -0.799631 & -2.110117 & 0.625484 \\ \mathrm{H} & -0.463670 & -1.788785 & -0.345106 \\ \mathrm{H} & -0.312063 & -3.016354 & 0.983749 \\ \mathrm{~F} & -2.756262 & -3.085800 & 1.454067 \\ \mathrm{~N} & -3.353599 & 1.441395 & 1.906164\end{array}$




$\begin{array}{llll}\mathrm{O} & -2.913659 & 2.420332 & 2.508101 \\ \mathrm{O} & -4.540944 & 1.258942 & 1.643761\end{array}$

$\begin{array}{lrrr}\mathrm{O}_{2} \mathrm{~N} & & & \\ & = & & \\ \mathrm{C} & 0.000000 & 0.000000 & 0.000000 \\ \mathrm{H} & 0.000000 & 0.000000 & 1.084132 \\ \mathrm{H} & 0.971612 & 0.000000 & -0.489031 \\ \mathrm{C} & -1.130032 & 0.048034 & -0.734940 \\ \mathrm{C} & -2.503398 & 0.095866 & -0.235394 \\ \mathrm{H} & -3.203161 & 0.628855 & -0.870680 \\ \mathrm{C} & -2.995516 & -0.468175 & 0.890346 \\ \mathrm{C} & -2.275906 & -1.359611 & 1.795442 \\ \mathrm{H} & -1.379367 & -1.779585 & 1.344166 \\ \mathrm{C} & -2.561146 & -1.694282 & 3.061580 \\ \mathrm{H} & -1.905956 & -2.375666 & 3.596399 \\ \mathrm{H} & -3.428722 & -1.322560 & 3.590823 \\ \mathrm{~N} & -4.441844 & -0.187171 & 1.144317 \\ \mathrm{C} & -0.973370 & 0.172332 & -2.216386 \\ \mathrm{O} & -5.032505 & -0.902881 & 1.950523 \\ \mathrm{O} & -4.963621 & 0.743320 & 0.533184 \\ \mathrm{O} & -1.893559 & 0.335139 & -2.988730 \\ \mathrm{H} & 0.077791 & 0.116768 & -2.572955\end{array}$

$\begin{array}{cccc}\text { TS } & & & \\ \mathrm{C} & 0.000000 & 0.000000 & 0.000000 \\ \mathrm{H} & 0.000000 & 0.000000 & 1.078328 \\ \mathrm{H} & 0.994934 & 0.000000 & -0.441164 \\ \mathrm{C} & -1.016297 & -0.666897 & -0.671917 \\ \mathrm{H} & -0.777796 & -1.287785 & -1.532241 \\ \mathrm{C} & -2.385227 & -0.451203 & -0.434828 \\ \mathrm{C} & -2.967185 & 0.788166 & -0.172956 \\ \mathrm{H} & -4.032557 & 0.876100 & -0.361516 \\ \mathrm{C} & -2.214839 & 1.967121 & -0.064037 \\ \mathrm{C} & -0.918728 & 2.040462 & 0.465345 \\ \mathrm{H} & -0.745772 & 1.680639 & 1.466420 \\ \mathrm{H} & -0.303409 & 2.901724 & 0.200579 \\ \mathrm{~N} & -3.308982 & -1.509631 & -0.920661 \\ \mathrm{C} & -2.785607 & 3.193214 & -0.685411 \\ \mathrm{H} & -2.036158 & 3.993433 & -0.875545 \\ \mathrm{O} & -3.959641 & 3.356443 & -0.950073 \\ \mathrm{O} & -2.803447 & -2.497973 & -1.453168 \\ \mathrm{O} & -4.513477 & -1.354645 & -0.735187\end{array}$




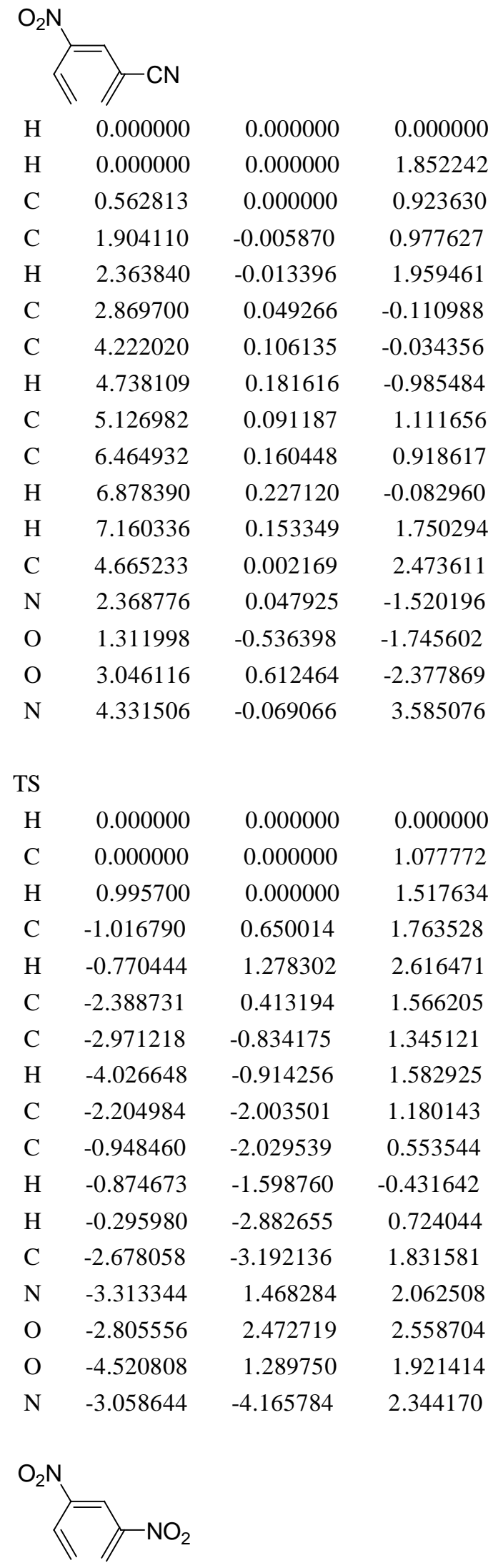




\begin{tabular}{|c|c|c|c|}
\hline $\mathrm{C}$ & 0.000000 & 0.000000 & 0.000000 \\
\hline $\mathrm{H}$ & 0.000000 & 0.000000 & 1.083391 \\
\hline $\mathrm{H}$ & 0.947845 & 0.000000 & -0.524296 \\
\hline $\mathrm{C}$ & -1.149772 & 0.035647 & -0.684508 \\
\hline $\mathrm{C}$ & -2.515382 & 0.092187 & -0.183424 \\
\hline $\mathrm{H}$ & -3.216467 & 0.681003 & -0.761693 \\
\hline $\mathrm{C}$ & -2.978535 & -0.517860 & 0.930611 \\
\hline $\mathrm{C}$ & -2.249969 & -1.468221 & 1.764071 \\
\hline $\mathrm{H}$ & -1.373978 & -1.879023 & 1.266499 \\
\hline $\mathrm{C}$ & -2.507171 & -1.861145 & 3.019620 \\
\hline $\mathrm{H}$ & -3.352197 & -1.500707 & 3.591488 \\
\hline $\mathrm{H}$ & -1.851004 & -2.580605 & 3.500356 \\
\hline $\mathrm{N}$ & -4.405808 & -0.206266 & 1.251798 \\
\hline $\mathrm{N}$ & -1.043486 & 0.104655 & -2.178486 \\
\hline $\mathrm{O}$ & -2.098951 & 0.170729 & -2.803769 \\
\hline $\mathrm{O}$ & 0.074939 & 0.085078 & -2.680562 \\
\hline $\mathrm{O}$ & -4.913362 & 0.775473 & 0.714192 \\
\hline $\mathrm{O}$ & -4.991892 & -0.952318 & 2.031975 \\
\hline \multicolumn{4}{|l|}{ 15 } \\
\hline $\mathrm{C}$ & 0.000000 & 0.000000 & 0.000000 \\
\hline $\mathrm{H}$ & 0.000000 & 0.000000 & 1.077451 \\
\hline $\mathrm{H}$ & 0.979013 & 0.000000 & -0.468581 \\
\hline $\mathrm{C}$ & -1.032632 & 0.680430 & -0.641294 \\
\hline $\mathrm{C}$ & -2.401931 & 0.481895 & -0.450687 \\
\hline $\mathrm{H}$ & -3.101202 & 1.225600 & -0.813046 \\
\hline $\mathrm{C}$ & -2.922135 & -0.771483 & -0.130767 \\
\hline $\mathrm{C}$ & -2.190717 & -1.970691 & -0.088244 \\
\hline $\mathrm{H}$ & -2.647525 & -2.812128 & -0.604297 \\
\hline $\mathrm{C}$ & -0.892890 & -2.085398 & 0.391393 \\
\hline $\mathrm{H}$ & -0.307439 & -2.950415 & 0.085811 \\
\hline $\mathrm{H}$ & -0.660223 & -1.727332 & 1.381010 \\
\hline $\mathrm{N}$ & -4.396831 & -0.906328 & -0.279969 \\
\hline $\mathrm{N}$ & -0.666162 & 1.560739 & -1.780679 \\
\hline $\mathrm{O}$ & 0.465778 & 1.445118 & -2.246246 \\
\hline $\mathrm{O}$ & -1.511762 & 2.360049 & -2.175639 \\
\hline $\mathrm{O}$ & -5.065913 & 0.122388 & -0.322227 \\
\hline $\mathrm{O}$ & -4.855323 & -2.047029 & -0.322383 \\
\hline
\end{tabular}

\section{2, 5-substituted hexatriene}

1<smiles>C=C(C)/C=C\C(=C)C</smiles> 


\begin{tabular}{|c|c|c|c|}
\hline $\mathrm{C}$ & 0.000000 & 0.000000 & 0.000000 \\
\hline $\mathrm{H}$ & 0.000000 & 0.000000 & 1.086451 \\
\hline $\mathrm{H}$ & 0.972706 & 0.000000 & -0.485673 \\
\hline $\mathrm{C}$ & -1.145780 & -0.006879 & -0.707688 \\
\hline $\mathrm{C}$ & -1.049756 & 0.030256 & -2.175073 \\
\hline $\mathrm{H}$ & -0.038296 & 0.236581 & -2.525879 \\
\hline $\mathrm{C}$ & -1.980251 & -0.091357 & -3.145355 \\
\hline $\mathrm{H}$ & -1.629262 & 0.109808 & -4.160671 \\
\hline $\mathrm{C}$ & -3.413290 & -0.451486 & -3.082623 \\
\hline $\mathrm{C}$ & -3.842170 & -1.597365 & -2.534196 \\
\hline $\mathrm{H}$ & -3.153125 & -2.305838 & -2.084378 \\
\hline $\mathrm{H}$ & -4.895257 & -1.868742 & -2.537541 \\
\hline $\mathrm{C}$ & -4.357389 & 0.498799 & -3.785800 \\
\hline $\mathrm{C}$ & -2.476450 & 0.024606 & 0.009065 \\
\hline $\mathrm{H}$ & -2.327566 & 0.216330 & 1.076227 \\
\hline $\mathrm{H}$ & -3.027158 & -0.914608 & -0.097783 \\
\hline $\mathrm{H}$ & -3.124134 & 0.811175 & -0.394778 \\
\hline $\mathrm{H}$ & -4.351339 & 1.487564 & -3.309202 \\
\hline $\mathrm{H}$ & -5.384882 & 0.121867 & -3.785312 \\
\hline $\mathrm{H}$ & -4.050611 & 0.653730 & -4.829311 \\
\hline \multicolumn{4}{|l|}{ TS } \\
\hline $\mathrm{C}$ & 0.000000 & 0.000000 & 0.000000 \\
\hline $\mathrm{H}$ & 0.000000 & 0.000000 & 1.091856 \\
\hline $\mathrm{H}$ & 0.990422 & 0.000000 & -0.424107 \\
\hline $\mathrm{C}$ & -1.018702 & -0.695950 & -0.660361 \\
\hline $\mathrm{C}$ & -1.338485 & -0.462341 & -2.009950 \\
\hline $\mathrm{H}$ & -1.954372 & -1.209837 & -2.510190 \\
\hline $\mathrm{C}$ & -1.294392 & 0.787889 & -2.644520 \\
\hline $\mathrm{H}$ & -1.881383 & 0.859074 & -3.560316 \\
\hline $\mathrm{C}$ & -0.923812 & 1.995810 & -2.026599 \\
\hline $\mathrm{C}$ & 0.072623 & 2.061540 & -1.046298 \\
\hline $\mathrm{H}$ & 1.049067 & 1.664132 & -1.268613 \\
\hline $\mathrm{H}$ & 0.103593 & 2.941978 & -0.401303 \\
\hline $\mathrm{C}$ & -1.759438 & 3.233768 & -2.309459 \\
\hline $\mathrm{C}$ & -1.930037 & -1.606040 & 0.147112 \\
\hline $\mathrm{H}$ & -2.818199 & -1.910002 & -0.415190 \\
\hline $\mathrm{H}$ & -1.412147 & -2.514847 & 0.480946 \\
\hline $\mathrm{H}$ & -2.271801 & -1.087615 & 1.052833 \\
\hline $\mathrm{H}$ & -2.645070 & 3.008915 & -2.911539 \\
\hline $\mathrm{H}$ & -2.103392 & 3.678382 & -1.366109 \\
\hline $\mathrm{H}$ & -1.182349 & 4.008799 & -2.830884 \\
\hline
\end{tabular}




\begin{tabular}{|c|c|c|c|}
\hline $\mathrm{C}$ & 0.000000 & 0.000000 & 0.000000 \\
\hline $\mathrm{H}$ & 0.000000 & 0.000000 & 1.087496 \\
\hline $\mathrm{H}$ & 0.968619 & 0.000000 & -0.492132 \\
\hline $\mathrm{C}$ & -1.145972 & -0.043172 & -0.700460 \\
\hline $\mathrm{C}$ & -1.169875 & -0.101959 & -2.179222 \\
\hline $\mathrm{H}$ & -1.732000 & -0.952865 & -2.570434 \\
\hline $\mathrm{C}$ & -0.625209 & 0.715486 & -3.103500 \\
\hline $\mathrm{H}$ & -0.736320 & 0.401991 & -4.139407 \\
\hline $\mathrm{C}$ & 0.073781 & 2.005574 & -2.978643 \\
\hline $\mathrm{C}$ & 0.679971 & 2.514940 & -4.076156 \\
\hline $\mathrm{H}$ & 1.216869 & 3.459127 & -4.047960 \\
\hline $\mathrm{H}$ & 0.625418 & 2.005841 & -5.030925 \\
\hline $\mathrm{N}$ & 0.002893 & 2.702067 & -1.770347 \\
\hline $\mathrm{C}$ & -2.493214 & -0.137167 & -0.020847 \\
\hline $\mathrm{H}$ & -0.025931 & 2.126639 & -0.937751 \\
\hline $\mathrm{H}$ & 0.670398 & 3.459622 & -1.690036 \\
\hline $\mathrm{H}$ & -3.122807 & 0.722969 & -0.280294 \\
\hline $\mathrm{H}$ & -3.033285 & -1.033706 & -0.353991 \\
\hline $\mathrm{H}$ & -2.396053 & -0.181773 & 1.068275 \\
\hline & & & \\
\hline $\mathrm{H}$ & 0.000000 & 0.000000 & 0.000000 \\
\hline $\mathrm{C}$ & 0.000000 & 0.000000 & 1.079454 \\
\hline $\mathrm{H}$ & 0.996721 & 0.000000 & 1.520744 \\
\hline $\mathrm{C}$ & -1.040224 & 0.678631 & 1.716422 \\
\hline $\mathrm{C}$ & -2.360983 & 0.442252 & 1.296584 \\
\hline $\mathrm{H}$ & -3.128366 & 1.165631 & 1.576208 \\
\hline $\mathrm{C}$ & -2.825647 & -0.776645 & 0.793708 \\
\hline $\mathrm{H}$ & -3.906138 & -0.877184 & 0.695608 \\
\hline $\mathrm{C}$ & -2.099438 & -1.994510 & 0.834171 \\
\hline $\mathrm{C}$ & -0.705823 & -2.122720 & 0.658601 \\
\hline $\mathrm{H}$ & -0.263419 & -1.862983 & -0.290308 \\
\hline $\mathrm{H}$ & -0.243499 & -3.009106 & 1.104265 \\
\hline $\mathrm{N}$ & -2.841654 & -3.121437 & 1.214521 \\
\hline $\mathrm{C}$ & -0.805527 & 1.495056 & 2.972457 \\
\hline $\mathrm{H}$ & -0.873387 & 0.876929 & 3.879892 \\
\hline $\mathrm{H}$ & -1.566733 & 2.278602 & 3.069358 \\
\hline $\mathrm{H}$ & 0.176487 & 1.979392 & 2.976935 \\
\hline $\mathrm{H}$ & -2.320714 & -3.985801 & 1.278998 \\
\hline $\mathrm{H}$ & -3.490873 & -3.002463 & 1.983651 \\
\hline
\end{tabular}




\begin{tabular}{|c|c|c|c|}
\hline $\mathrm{C}$ & 0.000000 & 0.000000 & 0.000000 \\
\hline $\mathrm{H}$ & 0.000000 & 0.000000 & 1.086206 \\
\hline $\mathrm{H}$ & 0.973270 & 0.000000 & -0.484131 \\
\hline $\mathrm{C}$ & -1.149325 & -0.000080 & -0.706876 \\
\hline $\mathrm{C}$ & -1.017099 & -0.000122 & -2.170548 \\
\hline $\mathrm{H}$ & 0.032862 & 0.000810 & -2.463466 \\
\hline $\mathrm{C}$ & -1.849879 & -0.001233 & -3.241420 \\
\hline $\mathrm{H}$ & -1.337079 & -0.000956 & -4.200843 \\
\hline $\mathrm{C}$ & -3.296951 & -0.002798 & -3.439516 \\
\hline $\mathrm{C}$ & -3.825723 & -0.003444 & -4.680524 \\
\hline $\mathrm{H}$ & -3.191196 & -0.002674 & -5.557797 \\
\hline $\mathrm{H}$ & -4.900054 & -0.004721 & -4.846187 \\
\hline $\mathrm{O}$ & -4.075842 & -0.003584 & -2.314402 \\
\hline $\mathrm{C}$ & -2.469514 & -0.000209 & 0.024947 \\
\hline $\mathrm{H}$ & -2.299267 & 0.000731 & 1.106070 \\
\hline $\mathrm{H}$ & -3.071764 & 0.874252 & -0.236447 \\
\hline $\mathrm{H}$ & -3.070701 & -0.875772 & -0.235203 \\
\hline $\mathrm{H}$ & -5.006851 & -0.003633 & -2.588235 \\
\hline & & & \\
\hline $\mathrm{H}$ & 0.000000 & 0.000000 & 0.000000 \\
\hline $\mathrm{C}$ & 0.000000 & 0.000000 & 1.079111 \\
\hline $\mathrm{H}$ & 0.995984 & 0.000000 & 1.521407 \\
\hline $\mathrm{C}$ & -1.040690 & 0.680749 & 1.715667 \\
\hline $\mathrm{C}$ & -2.362440 & 0.440455 & 1.308703 \\
\hline $\mathrm{H}$ & -3.131624 & 1.157216 & 1.597747 \\
\hline $\mathrm{C}$ & -2.825940 & -0.787647 & 0.820321 \\
\hline $\mathrm{H}$ & -3.908891 & -0.895257 & 0.743965 \\
\hline $\mathrm{C}$ & -2.083380 & -1.987821 & 0.830920 \\
\hline $\mathrm{C}$ & -0.702072 & -2.135049 & 0.643195 \\
\hline $\mathrm{H}$ & -0.269377 & -3.038286 & 1.076118 \\
\hline $\mathrm{H}$ & -0.253826 & -1.853381 & -0.294958 \\
\hline $\mathrm{O}$ & -2.739144 & -3.133968 & 1.238451 \\
\hline $\mathrm{C}$ & -0.797447 & 1.507098 & 2.964085 \\
\hline $\mathrm{H}$ & -0.784963 & 0.878658 & 3.866396 \\
\hline $\mathrm{H}$ & 0.153728 & 2.048132 & 2.927043 \\
\hline $\mathrm{H}$ & -1.598374 & 2.242534 & 3.102906 \\
\hline $\mathrm{H}$ & -3.533902 & -2.881861 & 1.734996 \\
\hline $\mathrm{C}$ & 0.000000 & 0.000000 & 0.000000 \\
\hline
\end{tabular}




\begin{tabular}{|c|c|c|c|}
\hline $\mathrm{H}$ & 0.000000 & 0.000000 & .086097 \\
\hline $\mathrm{H}$ & 0.972999 & 0.000000 & -0.484526 \\
\hline $\mathrm{C}$ & -1.149270 & 0.000020 & -0.706676 \\
\hline $\mathrm{C}$ & -1.019246 & -0.000004 & -2.169333 \\
\hline $\mathrm{H}$ & 0.027228 & -0.000053 & -2.472788 \\
\hline $\mathrm{C}$ & -1.869870 & -0.000013 & -3.226125 \\
\hline $\mathrm{H}$ & -1.386982 & -0.000084 & -4.201375 \\
\hline $\mathrm{C}$ & -3.313707 & 0.000033 & -3.372861 \\
\hline $\mathrm{C}$ & -3.962480 & -0.000141 & -4.545062 \\
\hline $\mathrm{H}$ & -3.414411 & -0.000349 & -5.479186 \\
\hline $\mathrm{H}$ & -5.045423 & -0.000077 & -4.579171 \\
\hline $\mathrm{F}$ & -4.058760 & 0.000291 & -2.240134 \\
\hline $\mathrm{C}$ & -2.472034 & 0.000043 & 0.021361 \\
\hline $\mathrm{H}$ & -2.305168 & -0.000014 & 1.102500 \\
\hline $\mathrm{H}$ & -3.072243 & 0.877428 & -0.236179 \\
\hline $\mathrm{H}$ & -3.072351 & -0.877230 & -0.236287 \\
\hline \multicolumn{4}{|c|}{$\mathrm{TS}$} \\
\hline $\mathrm{H}$ & 0.000000 & 0.000000 & 0.000000 \\
\hline $\mathrm{H}$ & 0.000000 & 0.000000 & 1.809848 \\
\hline $\mathrm{C}$ & 0.596706 & 0.000000 & 0.913810 \\
\hline $\mathrm{C}$ & 1.782972 & -0.732319 & 0.842609 \\
\hline $\mathrm{C}$ & 2.982246 & -0.579178 & 1.546149 \\
\hline $\mathrm{H}$ & 3.701536 & -1.385748 & 1.424353 \\
\hline $\mathrm{C}$ & 3.473711 & 0.645984 & 2.017750 \\
\hline $\mathrm{H}$ & 4.545194 & 0.681419 & 2.211792 \\
\hline $\mathrm{C}$ & 2.768089 & 1.854860 & 1.945377 \\
\hline $\mathrm{C}$ & 1.372474 & 1.838844 & 2.033937 \\
\hline $\mathrm{H}$ & 0.941037 & 1.276610 & 2.848076 \\
\hline $\mathrm{H}$ & 0.804090 & 2.726818 & 1.758608 \\
\hline $\mathrm{C}$ & 3.513426 & 3.122837 & 1.575541 \\
\hline $\mathrm{F}$ & 1.879973 & -1.593607 & -0.218133 \\
\hline $\mathrm{H}$ & 3.395371 & 3.348243 & 0.506261 \\
\hline $\mathrm{H}$ & 3.157430 & 3.994727 & 2.134849 \\
\hline $\mathrm{H}$ & 4.587324 & 3.014803 & 1.761264 \\
\hline $\mathrm{C}$ & 0.000000 & 0.000000 & 0.000000 \\
\hline $\mathrm{H}$ & 0.000000 & 0.000000 & 1.086414 \\
\hline $\mathrm{H}$ & 0.972441 & 0.000000 & -0.485944 \\
\hline $\mathrm{C}$ & -1.145720 & -0.006019 & -0.707732 \\
\hline $\mathrm{C}$ & -1.048437 & 0.037431 & -2.175009 \\
\hline $\mathrm{H}$ & -0.053360 & 0.313012 & -2.523732 \\
\hline
\end{tabular}




$\begin{array}{lrrr}\mathrm{C} & -1.964239 & -0.135829 & -3.151758 \\ \mathrm{H} & -1.645784 & 0.113649 & -4.162648 \\ \mathrm{C} & -3.353835 & -0.613673 & -3.087892 \\ \mathrm{C} & -3.781211 & -1.704554 & -2.423780 \\ \mathrm{H} & -3.108418 & -2.335405 & -1.852602 \\ \mathrm{H} & -4.825986 & -2.006293 & -2.460793 \\ \mathrm{C} & -4.347950 & 0.082898 & -3.950911 \\ \mathrm{C} & -2.476918 & 0.041172 & 0.008390 \\ \mathrm{H} & -2.328521 & 0.275087 & 1.066883 \\ \mathrm{H} & -3.022466 & -0.906191 & -0.051615 \\ \mathrm{H} & -3.129509 & 0.807359 & -0.424761 \\ \mathrm{O} & -4.085488 & 0.999370 & -4.703388 \\ \mathrm{H} & -5.384116 & -0.316149 & -3.872545\end{array}$

TS

$\begin{array}{llll}\mathrm{H} & 0.000000 & 0.000000 & 0.000000\end{array}$

$\begin{array}{llll}\mathrm{H} & 0.000000 & 0.000000 & 1.816381\end{array}$

$\begin{array}{llll}\text { C } & 0.591763 & 0.000000 & 0.914832\end{array}$

$\begin{array}{llll}\text { C } & 1.806293 & 0.686859 & 0.934369\end{array}$

$\begin{array}{llll}\text { C } & 2.747634 & 0.492902 & 1.971463\end{array}$

$\begin{array}{llll}\mathrm{H} & 3.515360 & 1.254504 & 2.105696\end{array}$

$\begin{array}{llll}\text { C } & 3.015538 & -0.745988 & 2.549146\end{array}$

$\begin{array}{llll}\mathrm{H} & 3.949117 & -0.844385 & 3.098603\end{array}$

$\begin{array}{llll}\text { C } & 2.404018 & -1.954152 & 2.144215\end{array}$

$\begin{array}{llll}\text { C } & 1.073451 & -2.075126 & 1.720938\end{array}$

$\begin{array}{llll}\mathrm{H} & 0.815492 & -2.934201 & 1.097424\end{array}$

$\begin{array}{llll}\mathrm{H} & 0.275216 & -1.780309 & 2.381919\end{array}$

$\begin{array}{llll}\text { C } & 3.281397 & -3.136365 & 1.995495\end{array}$

$\begin{array}{llll}\text { C } & 2.248821 & 1.511752 & -0.258664\end{array}$

$\begin{array}{llll}\mathrm{H} & 2.921249 & 0.937208 & -0.910748\end{array}$

$\mathrm{H} \quad 1.403336 \quad 1.854810 \quad-0.861511$

$\begin{array}{llll}\mathrm{H} & 2.810820 & 2.392670 & 0.073460\end{array}$

$\begin{array}{llll}\mathrm{H} & 2.838446 & -3.950162 & 1.376218\end{array}$

$\begin{array}{llll}\text { O } & 4.378917 & -3.273303 & 2.507357\end{array}$

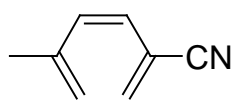

$\begin{array}{lrrr}\mathrm{C} & 0.000000 & 0.000000 & 0.000000 \\ \mathrm{H} & 0.000000 & 0.000000 & 1.086189 \\ \mathrm{H} & 0.961396 & 0.000000 & -0.501154 \\ \mathrm{C} & -1.161416 & 0.001663 & -0.689214 \\ \mathrm{C} & -2.462034 & -0.051960 & -0.004242 \\ \mathrm{H} & -2.379006 & -0.376994 & 1.031384 \\ \mathrm{C} & -3.701017 & 0.206121 & -0.468988\end{array}$




\begin{tabular}{|c|c|c|c|}
\hline $\mathrm{H}$ & -4.520802 & -0.0 & 055 \\
\hline $\mathrm{C}$ & -4.121683 & 0.750673 & -1.772559 \\
\hline $\mathrm{C}$ & -3.555816 & 1.849790 & -2.292259 \\
\hline $\mathrm{H}$ & -2.755168 & 2.377680 & -1.783540 \\
\hline $\mathrm{H}$ & -3.883433 & 2.258276 & -3.244411 \\
\hline $\mathrm{C}$ & -5.279612 & 0.045372 & -2.441021 \\
\hline $\mathrm{C}$ & -1.068790 & -0.046645 & -2.128515 \\
\hline $\mathrm{N}$ & -0.928159 & -0.124896 & -3.280017 \\
\hline $\mathrm{H}$ & -5.009959 & -0.983178 & -2.711980 \\
\hline $\mathrm{H}$ & -6.144195 & -0.019886 & -1.766364 \\
\hline $\mathrm{H}$ & -5.593756 & 0.566494 & -3.349930 \\
\hline \multicolumn{4}{|l|}{13} \\
\hline $\mathrm{H}$ & 0.000000 & 0.000000 & 0.000000 \\
\hline $\mathrm{C}$ & 0.000000 & 0.000000 & 1.089645 \\
\hline $\mathrm{H}$ & 0.986527 & 0.000000 & 1.524180 \\
\hline $\mathrm{C}$ & -1.014754 & -0.679825 & 1.763430 \\
\hline $\mathrm{C}$ & -1.262802 & -0.470002 & 3.138469 \\
\hline $\mathrm{H}$ & -1.830515 & -1.231704 & 3.671705 \\
\hline $\mathrm{C}$ & -1.203412 & 0.775466 & 3.761622 \\
\hline $\mathrm{H}$ & -1.711553 & 0.862213 & 4.719453 \\
\hline $\mathrm{C}$ & -0.880439 & 1.980563 & 3.087164 \\
\hline $\mathrm{C}$ & 0.055623 & 2.077514 & 2.045683 \\
\hline $\mathrm{H}$ & -0.010338 & 2.929708 & 1.371452 \\
\hline $\mathrm{H}$ & 1.062990 & 1.743131 & 2.229779 \\
\hline $\mathrm{C}$ & -1.716785 & 3.112201 & 3.366735 \\
\hline $\mathrm{C}$ & -2.022650 & -1.517927 & 0.999461 \\
\hline $\mathrm{H}$ & -1.619782 & -1.888632 & 0.052970 \\
\hline $\mathrm{H}$ & -2.931822 & -0.941347 & 0.780188 \\
\hline $\mathrm{H}$ & -2.334265 & -2.381414 & 1.598733 \\
\hline $\mathrm{N}$ & -2.381887 & 4.038721 & 3.606859 \\
\hline $\mathrm{C}$ & 0.000000 & 0.000000 & 0.000000 \\
\hline $\mathrm{H}$ & 0.000000 & 0.000000 & 1.086262 \\
\hline $\mathrm{H}$ & 0.972484 & 0.000000 & -0.485604 \\
\hline $\mathrm{C}$ & -1.146133 & -0.005121 & -0.707321 \\
\hline $\mathrm{C}$ & -1.041550 & 0.034281 & -2.172908 \\
\hline $\mathrm{H}$ & -0.038291 & 0.277382 & -2.520993 \\
\hline $\mathrm{C}$ & -1.956170 & -0.118820 & -3.154148 \\
\hline $\mathrm{H}$ & -1.632979 & 0.101248 & -4.168269 \\
\hline $\mathrm{C}$ & -3.340038 & -0.587789 & -3.054052 \\
\hline $\mathrm{C}$ & -3.800299 & -1.690858 & -2.457021 \\
\hline
\end{tabular}




$\begin{array}{cccc}\mathrm{H} & -3.117571 & -2.357427 & -1.943210 \\ \mathrm{H} & -4.853528 & -1.942152 & -2.490479 \\ \mathrm{~N} & -4.331024 & 0.221939 & -3.828347 \\ \mathrm{C} & -2.478885 & 0.037913 & 0.005635 \\ \mathrm{H} & -2.332513 & 0.265481 & 1.065560 \\ \mathrm{H} & -3.022925 & -0.910035 & -0.060571 \\ \mathrm{H} & -3.131669 & 0.806796 & -0.422464 \\ \mathrm{O} & -3.885619 & 1.183293 & -4.453490 \\ \mathrm{O} & -5.513616 & -0.109022 & -3.800730\end{array}$

$\begin{array}{crrr}\text { TS } & & & \\ \mathrm{H} & 0.000000 & 0.000000 & 0.000000 \\ \mathrm{H} & 0.000000 & 0.000000 & 1.824930 \\ \mathrm{C} & 0.580489 & 0.000000 & 0.907220 \\ \mathrm{C} & 1.796871 & 0.677850 & 0.910990 \\ \mathrm{C} & 2.889111 & 0.445804 & 0.051409 \\ \mathrm{H} & 3.645675 & 1.220349 & -0.009053 \\ \mathrm{C} & 3.229999 & -0.819177 & -0.422224 \\ \mathrm{H} & 4.242352 & -0.915556 & -0.812199 \\ \mathrm{C} & 2.543127 & -2.006244 & -0.077045 \\ \mathrm{C} & 1.154436 & -2.041457 & 0.041823 \\ \mathrm{H} & 0.688383 & -2.890710 & 0.540119 \\ \mathrm{H} & 0.565801 & -1.629829 & -0.762100 \\ \mathrm{C} & 3.386919 & -3.189262 & 0.358034 \\ \mathrm{~N} & 2.077094 & 1.554879 & 2.067621 \\ \mathrm{O} & 1.304652 & 1.513965 & 3.027233 \\ \mathrm{O} & 3.058410 & 2.298904 & 2.002417 \\ \mathrm{H} & 3.731200 & -3.066611 & 1.394094 \\ \mathrm{H} & 4.285113 & -3.268748 & -0.265037 \\ \mathrm{H} & 2.840464 & -4.133933 & 0.292065\end{array}$

2

$\begin{array}{lrrr}\mathrm{H}_{2} \mathrm{~N} & =\mathrm{NH}_{2} & & \\ \mathrm{C} & 0.000000 & 0.000000 & 0.000000 \\ \mathrm{H} & 0.000000 & 0.000000 & 1.086541 \\ \mathrm{H} & 0.964451 & 0.000000 & -0.493322 \\ \mathrm{C} & -1.150292 & 0.044040 & -0.711090 \\ \mathrm{C} & -1.063885 & 0.021454 & -2.178009 \\ \mathrm{H} & -0.044341 & -0.180670 & -2.498647 \\ \mathrm{C} & -1.934911 & 0.178753 & -3.205867 \\ \mathrm{H} & -1.479220 & 0.053401 & -4.185542 \\ \mathrm{C} & -3.364739 & 0.492556 & -3.336453\end{array}$




\begin{tabular}{|c|c|c|c|}
\hline $\mathrm{C}$ & -3.931159 & 0.405864 & -4.562174 \\
\hline $\mathrm{H}$ & -4.980064 & 0.638456 & -4.724259 \\
\hline $\mathrm{H}$ & -3.363235 & 0.064975 & -5.419373 \\
\hline $\mathrm{N}$ & -4.117245 & 0.826518 & -2.199812 \\
\hline $\mathrm{N}$ & -2.400236 & 0.155550 & -0.082629 \\
\hline $\mathrm{H}$ & -3.151570 & -0.347067 & -0.545018 \\
\hline $\mathrm{H}$ & -2.366755 & -0.057771 & 0.908281 \\
\hline $\mathrm{H}$ & -3.620051 & 1.386217 & -1.513892 \\
\hline $\mathrm{H}$ & -5.018789 & 1.232403 & -2.425724 \\
\hline \multicolumn{4}{|l|}{ TS } \\
\hline $\mathrm{C}$ & 0.000000 & 0.000000 & 0.000000 \\
\hline $\mathrm{H}$ & 0.000000 & 0.000000 & 1.079244 \\
\hline $\mathrm{H}$ & 1.008020 & 0.000000 & -0.424002 \\
\hline $\mathrm{C}$ & -0.977125 & 0.769530 & -0.660180 \\
\hline $\mathrm{C}$ & -2.360599 & 0.563354 & -0.484857 \\
\hline $\mathrm{H}$ & -3.017875 & 1.366077 & -0.818037 \\
\hline $\mathrm{C}$ & -2.958464 & -0.681722 & -0.262951 \\
\hline $\mathrm{H}$ & -4.031503 & -0.744832 & -0.441820 \\
\hline $\mathrm{C}$ & -2.261004 & -1.904227 & -0.183687 \\
\hline $\mathrm{C}$ & -0.965908 & -2.011498 & 0.358534 \\
\hline $\mathrm{H}$ & -0.819658 & -1.706784 & 1.383487 \\
\hline $\mathrm{H}$ & -0.383579 & -2.898086 & 0.092566 \\
\hline $\mathrm{N}$ & -2.873059 & -3.019931 & -0.790786 \\
\hline $\mathrm{N}$ & -0.599112 & 1.715767 & -1.634733 \\
\hline $\mathrm{H}$ & 0.400917 & 1.832407 & -1.739411 \\
\hline $\mathrm{H}$ & -1.053591 & 1.624363 & -2.537247 \\
\hline $\mathrm{H}$ & -3.214528 & -2.875502 & -1.735228 \\
\hline $\mathrm{H}$ & -2.341118 & -3.878366 & -0.721892 \\
\hline $\mathrm{C}$ & 0.000000 & 0.000000 & 0.000000 \\
\hline $\mathrm{H}$ & 0.000000 & 0.000000 & 1.086922 \\
\hline $\mathrm{H}$ & 0.963241 & 0.000000 & -0.494508 \\
\hline $\mathrm{C}$ & -1.141399 & 0.009005 & -0.714023 \\
\hline $\mathrm{C}$ & -1.141188 & -0.025601 & -2.175032 \\
\hline $\mathrm{H}$ & -0.162765 & -0.308019 & -2.556805 \\
\hline $\mathrm{C}$ & -2.075122 & 0.202528 & -3.129766 \\
\hline $\mathrm{H}$ & -1.716824 & 0.019182 & -4.140240 \\
\hline $\mathrm{C}$ & -3.472341 & 0.661173 & -3.139529 \\
\hline $\mathrm{C}$ & -4.147862 & 0.563421 & -4.311803 \\
\hline $\mathrm{H}$ & -5.183929 & 0.878082 & -4.400689 \\
\hline $\mathrm{H}$ & -3.668085 & 0.186228 & -5.206566 \\
\hline
\end{tabular}




$\begin{array}{rrrr}\mathrm{N} & -4.022602 & 1.255320 & -2.007122 \\ \mathrm{O} & -2.376848 & -0.002309 & -0.101840 \\ \mathrm{H} & -2.247686 & 0.079694 & 0.856454 \\ \mathrm{H} & -3.676147 & 0.898380 & -1.124377 \\ \mathrm{H} & -5.032573 & 1.325395 & -2.032411\end{array}$

TS

$\begin{array}{lrrr}\mathrm{H} & 0.000000 & 0.000000 & 0.000000 \\ \mathrm{C} & 0.000000 & 0.000000 & 1.078838 \\ \mathrm{H} & 1.006532 & 0.000000 & 1.504925 \\ \mathrm{C} & -0.980987 & 0.764063 & 1.740345 \\ \mathrm{C} & -2.363959 & 0.553860 & 1.569624 \\ \mathrm{H} & -3.023919 & 1.350398 & 1.910900 \\ \mathrm{C} & -2.955087 & -0.697404 & 1.355351 \\ \mathrm{H} & -4.026261 & -0.766731 & 1.551043 \\ \mathrm{C} & -2.247286 & -1.904680 & 1.239374 \\ \mathrm{C} & -0.963968 & -2.028080 & 0.693526 \\ \mathrm{H} & -0.807598 & -1.696704 & -0.320044 \\ \mathrm{H} & -0.414054 & -2.932834 & 0.953421 \\ \mathrm{O} & -2.765385 & -3.041804 & 1.836796 \\ \mathrm{~N} & -0.605857 & 1.709621 & 2.714341 \\ \mathrm{H} & 0.393082 & 1.835449 & 2.816753 \\ \mathrm{H} & -1.060570 & 1.621439 & 3.616739 \\ \mathrm{H} & -3.391988 & -2.773669 & 2.526597\end{array}$

$\begin{array}{lccc}\mathrm{H}_{2} \mathrm{~N} & =\mathrm{F} & & \\ \mathrm{C} & 0.000000 & 0.000000 & 0.000000 \\ \mathrm{H} & 0.000000 & 0.000000 & 1.086290 \\ \mathrm{H} & 0.963901 & 0.000000 & -0.493657 \\ \mathrm{C} & -1.154223 & -0.025891 & -0.711929 \\ \mathrm{C} & -1.037674 & -0.019080 & -2.176781 \\ \mathrm{H} & -0.009713 & -0.182109 & -2.491110 \\ \mathrm{C} & -1.904391 & 0.129710 & -3.208106 \\ \mathrm{H} & -1.467825 & 0.014630 & -4.197973 \\ \mathrm{C} & -3.321716 & 0.430658 & -3.301906 \\ \mathrm{C} & -4.068629 & 0.341491 & -4.408201 \\ \mathrm{H} & -5.117171 & 0.613564 & -4.395014 \\ \mathrm{H} & -3.635051 & -0.010130 & -5.336350 \\ \mathrm{~F} & -3.939256 & 0.895198 & -2.172229 \\ \mathrm{~N} & -2.400722 & -0.155492 & -0.105937 \\ \mathrm{H} & -2.405318 & 0.061298 & 0.882881 \\ \mathrm{H} & -3.174643 & 0.265524 & -0.598906\end{array}$




\begin{tabular}{|c|c|c|c|}
\hline \multicolumn{4}{|l|}{ ГS } \\
\hline $\mathrm{H}$ & 0.000000 & 0.000000 & 0.000000 \\
\hline $\mathrm{H}$ & 0.000000 & 0.000000 & 1.816019 \\
\hline $\mathrm{C}$ & 0.591570 & 0.000000 & 0.900295 \\
\hline $\mathrm{C}$ & 1.770408 & 0.743480 & 0.927476 \\
\hline $\mathrm{C}$ & 2.879870 & 0.596603 & 0.095669 \\
\hline $\mathrm{H}$ & 3.620594 & 1.392949 & 0.124322 \\
\hline $\mathrm{C}$ & 3.254137 & -0.608279 & -0.510577 \\
\hline $\mathrm{H}$ & 4.257240 & -0.646726 & -0.932072 \\
\hline $\mathrm{C}$ & 2.622890 & -1.845428 & -0.266542 \\
\hline $\mathrm{C}$ & 1.239171 & -1.974011 & -0.025544 \\
\hline $\mathrm{H}$ & 0.901441 & -2.888881 & 0.467342 \\
\hline $\mathrm{H}$ & 0.562395 & -1.636249 & -0.795087 \\
\hline $\mathrm{N}$ & 3.462935 & -2.962882 & -0.150849 \\
\hline $\mathrm{F}$ & 1.955752 & 1.569362 & 2.004206 \\
\hline $\mathrm{H}$ & 2.992946 & -3.838002 & 0.039776 \\
\hline $\mathrm{H}$ & 4.292955 & -2.846677 & 0.419247 \\
\hline $\mathrm{C}$ & 0.000000 & 0.000000 & 0.000000 \\
\hline $\mathrm{H}$ & 0.000000 & 0.000000 & 1.082837 \\
\hline $\mathrm{H}$ & 0.967734 & 0.000000 & -0.498501 \\
\hline $\mathrm{C}$ & -1.134688 & -0.009913 & -0.730111 \\
\hline $\mathrm{C}$ & -2.520642 & 0.046425 & -0.241423 \\
\hline $\mathrm{H}$ & -3.176488 & 0.559828 & -0.941694 \\
\hline $\mathrm{C}$ & -3.111443 & -0.411900 & 0.884477 \\
\hline $\mathrm{H}$ & -4.158920 & -0.143171 & 1.001210 \\
\hline $\mathrm{C}$ & -2.630021 & -1.257998 & 1.984822 \\
\hline $\mathrm{C}$ & -3.366234 & -1.343704 & 3.115070 \\
\hline $\mathrm{H}$ & -3.058116 & -1.960192 & 3.955028 \\
\hline $\mathrm{H}$ & -4.274297 & -0.764674 & 3.233226 \\
\hline $\mathrm{N}$ & -1.408615 & -1.933201 & 1.834660 \\
\hline $\mathrm{C}$ & -0.966330 & 0.063405 & -2.209296 \\
\hline $\mathrm{H}$ & -1.283692 & -2.684181 & 2.504690 \\
\hline $\mathrm{H}$ & -1.236492 & -2.262365 & 0.891698 \\
\hline $\mathrm{H}$ & 0.092502 & 0.028753 & -2.550163 \\
\hline $\mathrm{O}$ & -1.874959 & 0.166834 & -3.009176 \\
\hline & & & \\
\hline $\mathrm{C}$ & 0.000000 & 0.000000 & 0.000000 \\
\hline $\mathrm{H}$ & 0.000000 & 0.000000 & 1.091819 \\
\hline $\mathrm{H}$ & 0.988373 & 0.000000 & -0.432262 \\
\hline $\mathrm{C}$ & -1.003897 & 0.735666 & -0.645395 \\
\hline
\end{tabular}




$\begin{array}{lrrr}\mathrm{C} & -1.352919 & 0.582726 & -2.025796 \\ \mathrm{H} & -1.870321 & 1.421233 & -2.489415 \\ \mathrm{C} & -1.457415 & -0.644785 & -2.651931 \\ \mathrm{H} & -2.041614 & -0.692797 & -3.568450 \\ \mathrm{C} & -1.126657 & -1.882954 & -2.037522 \\ \mathrm{C} & -0.073248 & -2.012140 & -1.131676 \\ \mathrm{H} & -0.047964 & -2.886494 & -0.479235 \\ \mathrm{H} & 0.902680 & -1.643332 & -1.402822 \\ \mathrm{C} & -2.023639 & -3.026692 & -2.254126 \\ \mathrm{~N} & -1.811390 & 1.594006 & 0.082590 \\ \mathrm{H} & -2.795324 & 1.636510 & -0.150000 \\ \mathrm{H} & -1.618040 & 1.683283 & 1.070515 \\ \mathrm{O} & -2.880574 & -3.105671 & -3.121059 \\ \mathrm{H} & -1.844878 & -3.877996 & -1.556499 \\ & & & \\ \mathrm{H} & -1 & & \\ \mathrm{C} & -1 & & \\ \mathrm{H} & 0.000000 & 0.000000 & 0.000000 \\ \mathrm{H} & 0.963964 & 0.000000 & -0.494347 \\ \mathrm{C} & -1.148029 & -0.044143 & -0.712806 \\ \mathrm{C} & -1.063051 & -0.034399 & -2.179225 \\ \mathrm{H} & -0.069117 & 0.227121 & -2.534088 \\ \mathrm{C} & -1.981971 & -0.241901 & -3.147183 \\ \mathrm{H} & -1.648701 & -0.056814 & -4.167115 \\ \mathrm{C} & -3.386817 & -0.697959 & -3.067181 \\ \mathrm{C} & -3.868111 & -1.662780 & -2.257239 \\ \mathrm{H} & -3.224980 & -2.163693 & -1.544456 \\ \mathrm{H} & -4.913281 & -1.953216 & -2.292472 \\ \mathrm{C} & -4.279190 & -0.090655 & -4.025239 \\ \mathrm{~N} & -2.406459 & -0.128579 & -0.101661 \\ \mathrm{H} & -2.393403 & 0.132764 & 0.877972 \\ \mathrm{H} & -3.143688 & 0.360097 & -0.595325 \\ \mathrm{~N} & -4.977450 & 0.419535 & -4.803438 \\ & & & \end{array}$

$\begin{array}{rrrr}\text { TS } & & & \\ \mathrm{H} & 0.000000 & 0.000000 & 0.000000 \\ \mathrm{H} & 0.000000 & 0.000000 & 1.823803 \\ \mathrm{C} & 0.583833 & 0.000000 & 0.906131 \\ \mathrm{C} & 1.821116 & 0.648838 & 0.879937 \\ \mathrm{C} & 2.777859 & 0.393788 & -0.143235 \\ \mathrm{H} & 3.547061 & 1.142653 & -0.321295 \\ \mathrm{C} & 2.954749 & -0.842595 & -0.739785 \\ \mathrm{H} & 3.853525 & -0.969838 & -1.340564\end{array}$




$\begin{array}{lrrr}\mathrm{C} & 2.319380 & -2.042692 & -0.289080 \\ \mathrm{C} & 0.977533 & -2.130491 & 0.108103 \\ \mathrm{H} & 0.687335 & -2.999594 & 0.701807 \\ \mathrm{H} & 0.212992 & -1.803950 & -0.579097 \\ \mathrm{~N} & 3.156668 & -3.138475 & -0.145491 \\ \mathrm{C} & 2.239788 & 1.437311 & 1.997593 \\ \mathrm{~N} & 2.577912 & 2.096595 & 2.898143 \\ \mathrm{H} & 2.741776 & -3.991069 & 0.204273 \\ \mathrm{H} & 4.099434 & -2.977525 & 0.185477\end{array}$

$\begin{array}{lrrr} & = & \\ \mathrm{H} & \mathrm{N} & & \\ \mathrm{C} & 0.000000 & 0.000000 & 0.000000 \\ \mathrm{H} & 0.000000 & 0.000000 & 1.081887 \\ \mathrm{H} & 0.944923 & 0.000000 & -0.529638 \\ \mathrm{C} & -1.154730 & -0.018625 & -0.676603 \\ \mathrm{C} & -2.526825 & 0.027827 & -0.167347 \\ \mathrm{H} & -3.192265 & 0.643588 & -0.764330 \\ \mathrm{C} & -3.062833 & -0.546823 & 0.931735 \\ \mathrm{H} & -4.091129 & -0.266192 & 1.145531 \\ \mathrm{C} & -2.536703 & -1.524906 & 1.892864 \\ \mathrm{C} & -3.217588 & -1.735506 & 3.041449 \\ \mathrm{H} & -2.877545 & -2.449323 & 3.786491 \\ \mathrm{H} & -4.110493 & -1.167094 & 3.272420 \\ \mathrm{~N} & -1.331602 & -2.182138 & 1.603843 \\ \mathrm{~N} & -1.053429 & 0.018516 & -2.169504 \\ \mathrm{H} & -1.210125 & -2.411532 & 0.624275 \\ \mathrm{H} & -1.174724 & -2.999154 & 2.183861 \\ \mathrm{O} & 0.062530 & 0.031439 & -2.683459 \\ \mathrm{O} & -2.110853 & 0.026747 & -2.799450\end{array}$

$\begin{array}{rrrr}\text { TS } & & & \\ \mathrm{C} & 0.000000 & 0.000000 & 0.000000 \\ \mathrm{H} & 0.000000 & 0.000000 & 1.077470 \\ \mathrm{H} & 0.976255 & 0.000000 & -0.474023 \\ \mathrm{C} & -1.051375 & 0.630731 & -0.647164 \\ \mathrm{C} & -2.420764 & 0.391012 & -0.360893 \\ \mathrm{H} & -3.141397 & 1.151566 & -0.642539 \\ \mathrm{C} & -2.901796 & -0.844753 & 0.022039 \\ \mathrm{H} & -3.982525 & -0.973587 & 0.021529 \\ \mathrm{C} & -2.115334 & -2.045346 & 0.005509 \\ \mathrm{C} & -0.793059 & -2.129451 & 0.459436 \\ \mathrm{H} & -0.569774 & -1.783698 & 1.456407 \\ \mathrm{H} & -0.209245 & -2.999293 & 0.153736\end{array}$




$\begin{array}{lrrr}\mathrm{N} & -2.709417 & -3.131330 & -0.608153 \\ \mathrm{~N} & -0.763125 & 1.405153 & -1.849843 \\ \mathrm{O} & 0.398114 & 1.425083 & -2.271417 \\ \mathrm{O} & -1.697366 & 2.022433 & -2.376058 \\ \mathrm{H} & -2.171581 & -3.984344 & -0.672343 \\ \mathrm{H} & -3.323842 & -2.966309 & -1.394739\end{array}$

3

$\begin{array}{lrrr}\mathrm{HO} & =\mathrm{H} & \\ \mathrm{C} & 0.000000 & 0.000000 & 0.000000 \\ \mathrm{H} & 0.000000 & 0.000000 & 1.084044 \\ \mathrm{H} & 0.953027 & 0.000000 & -0.513753 \\ \mathrm{C} & -1.164960 & -0.005652 & -0.685209 \\ \mathrm{C} & -1.116749 & -0.010636 & -2.151025 \\ \mathrm{H} & -0.088510 & -0.042037 & -2.503339 \\ \mathrm{C} & -2.035593 & 0.003296 & -3.149386 \\ \mathrm{H} & -1.622359 & -0.037844 & -4.153962 \\ \mathrm{C} & -3.491386 & 0.056615 & -3.179166 \\ \mathrm{C} & -4.201470 & -0.067051 & -4.315141 \\ \mathrm{H} & -5.286645 & -0.013487 & -4.325248 \\ \mathrm{H} & -3.704354 & -0.227151 & -5.263444 \\ \mathrm{O} & -4.109038 & 0.264674 & -1.961528 \\ \mathrm{O} & -2.328536 & -0.030809 & 0.013140 \\ \mathrm{H} & -3.087546 & 0.040431 & -0.595596 \\ \mathrm{H} & -5.071561 & 0.200918 & -2.062096\end{array}$

TS

$\begin{array}{cccc}\mathrm{H} & 0.000000 & 0.000000 & 0.000000 \\ \mathrm{C} & 0.000000 & 0.000000 & 1.089801 \\ \mathrm{H} & 0.983564 & 0.000000 & 1.529359 \\ \mathrm{C} & -1.003591 & -0.755083 & 1.709489 \\ \mathrm{C} & -1.428342 & -0.591952 & 3.037354 \\ \mathrm{H} & -2.027257 & -1.393986 & 3.470348 \\ \mathrm{C} & -1.445669 & 0.632927 & 3.718946 \\ \mathrm{H} & -2.056546 & 0.674706 & 4.621457 \\ \mathrm{C} & -1.040426 & 1.856238 & 3.162589 \\ \mathrm{C} & -0.028165 & 2.006405 & 2.206488 \\ \mathrm{H} & 0.960266 & 1.653941 & 2.450191 \\ \mathrm{H} & -0.040959 & 2.932358 & 1.631939 \\ \mathrm{O} & -1.778001 & 2.991892 & 3.445996 \\ \mathrm{O} & -1.713143 & -1.610202 & 0.885206\end{array}$




\begin{tabular}{|c|c|c|c|}
\hline $\mathrm{H}$ & -2.603697 & -1.732115 & 1.249163 \\
\hline $\mathrm{H}$ & -2.666675 & 2.727772 & 3.730452 \\
\hline & & & \\
\hline $\mathrm{C}$ & 0.000000 & 0.000000 & 0.000000 \\
\hline $\mathrm{H}$ & 0.000000 & 0.000000 & 1.083521 \\
\hline $\mathrm{H}$ & 0.951095 & 0.000000 & -0.518403 \\
\hline $\mathrm{C}$ & -1.149449 & 0.004073 & -0.686655 \\
\hline $\mathrm{C}$ & -1.254044 & 0.018088 & -2.135250 \\
\hline $\mathrm{H}$ & -0.281149 & 0.100237 & -2.614571 \\
\hline $\mathrm{C}$ & -2.321548 & -0.017819 & -2.961218 \\
\hline $\mathrm{H}$ & -2.099550 & 0.099040 & -4.020749 \\
\hline $\mathrm{C}$ & -3.764765 & -0.177596 & -2.731160 \\
\hline $\mathrm{C}$ & -4.373543 & -0.905259 & -1.781433 \\
\hline $\mathrm{H}$ & -3.811200 & -1.412903 & -1.012743 \\
\hline $\mathrm{H}$ & -5.456933 & -0.997430 & -1.752470 \\
\hline $\mathrm{O}$ & -4.451999 & 0.463643 & -3.739583 \\
\hline $\mathrm{F}$ & -2.311875 & 0.020366 & 0.007025 \\
\hline $\mathrm{H}$ & -5.399973 & 0.293305 & -3.621436 \\
\hline \multicolumn{4}{|l|}{ TS } \\
\hline $\mathrm{H}$ & 0.000000 & 0.000000 & 0.000000 \\
\hline $\mathrm{C}$ & 0.000000 & 0.000000 & 1.090041 \\
\hline $\mathrm{H}$ & 0.984868 & 0.000000 & 1.525036 \\
\hline $\mathrm{C}$ & -1.010198 & 0.741392 & 1.703127 \\
\hline $\mathrm{C}$ & -1.516435 & 0.591013 & 2.993157 \\
\hline $\mathrm{H}$ & -2.166542 & 1.383626 & 3.356304 \\
\hline $\mathrm{C}$ & -1.528418 & -0.621382 & 3.695914 \\
\hline $\mathrm{H}$ & -2.180927 & -0.666605 & 4.567868 \\
\hline $\mathrm{C}$ & -1.077286 & -1.839667 & 3.164925 \\
\hline $\mathrm{C}$ & -0.020464 & -1.972812 & 2.253575 \\
\hline $\mathrm{H}$ & 0.017983 & -2.905150 & 1.691383 \\
\hline $\mathrm{H}$ & 0.945087 & -1.592803 & 2.544392 \\
\hline F & -1.735159 & 1.575120 & 0.896167 \\
\hline $\mathrm{O}$ & -1.799327 & -2.986108 & 3.423684 \\
\hline $\mathrm{H}$ & -2.701584 & -2.741601 & 3.682987 \\
\hline $\mathrm{C}$ & 0.000000 & 0.000000 & 0.000000 \\
\hline $\mathrm{H}$ & 0.000000 & 0.000000 & 1.086899 \\
\hline $\mathrm{H}$ & 0.963466 & 0.000000 & -0.494092 \\
\hline $\mathrm{C}$ & -1.143103 & 0.004050 & -0.713961 \\
\hline
\end{tabular}




$\begin{array}{cccc}\mathrm{C} & -1.123747 & 0.007609 & -2.174245 \\ \mathrm{H} & -0.110293 & 0.082914 & -2.561690 \\ \mathrm{C} & -2.097745 & -0.023279 & -3.110691 \\ \mathrm{H} & -1.751165 & 0.094004 & -4.135594 \\ \mathrm{C} & -3.559580 & -0.151299 & -3.056250 \\ \mathrm{C} & -4.323969 & -0.764961 & -2.131216 \\ \mathrm{H} & -3.914203 & -1.225012 & -1.242618 \\ \mathrm{H} & -5.403541 & -0.806975 & -2.263325 \\ \mathrm{C} & -4.283493 & 0.370476 & -4.251876 \\ \mathrm{O} & -2.381072 & 0.031691 & -0.126963 \\ \mathrm{H} & -2.262415 & 0.080969 & 0.835115 \\ \mathrm{O} & -3.755023 & 0.848406 & -5.235961 \\ \mathrm{H} & -5.390505 & 0.278643 & -4.188463\end{array}$

TS

$\begin{array}{llll}\mathrm{H} & 0.000000 & 0.000000 & 0.000000\end{array}$

$\begin{array}{llll}\mathrm{H} & 0.000000 & 0.000000 & 1.815904\end{array}$

$\begin{array}{llll}\text { C } & 0.592941 & 0.000000 & 0.915951\end{array}$

$\begin{array}{llll}\text { C } & 1.824207 & -0.661223 & 0.914468\end{array}$

$\begin{array}{llll}\text { C } & 2.835077 & -0.410122 & 1.873661\end{array}$

$\begin{array}{llll}\mathrm{H} & 3.608671 & -1.167365 & 1.979554\end{array}$

$\begin{array}{llll}\text { C } & 3.079924 & 0.835983 & 2.430652\end{array}$

$\begin{array}{llll}\mathrm{H} & 4.038421 & 0.972208 & 2.932003\end{array}$

$\begin{array}{llll}\text { C } & 2.385231 & 2.014089 & 2.043736\end{array}$

$\begin{array}{llll}\text { C } & 1.024754 & 2.105905 & 1.753495\end{array}$

$\begin{array}{llll}\mathrm{H} & 0.704487 & 2.992891 & 1.209320\end{array}$

$\begin{array}{llll}\mathrm{H} & 0.319810 & 1.747626 & 2.485416\end{array}$

$\begin{array}{llll}\text { O } & 3.130924 & 3.133085 & 1.781546\end{array}$

$\begin{array}{llll}\text { C } & 2.156115 & -1.543599 & -0.218591\end{array}$

$\mathrm{H} \quad 1.431933 \quad-1.484990 \quad-1.063352$

$\begin{array}{llll}\mathrm{O} & 3.104870 & -2.309149 & -0.265176\end{array}$

$\begin{array}{llll}\mathrm{H} & 4.061343 & 2.879826 & 1.667187\end{array}$

$\begin{array}{lrcc} & & & \\ \mathrm{C} & 0.000000 & 0.000000 & 0.000000 \\ \mathrm{H} & 0.000000 & 0.000000 & 1.086779 \\ \mathrm{H} & 0.963478 & 0.000000 & -0.494140 \\ \mathrm{C} & -1.143207 & 0.005746 & -0.713171 \\ \mathrm{C} & -1.118400 & 0.010577 & -2.174150 \\ \mathrm{H} & -0.106871 & 0.075790 & -2.567252 \\ \mathrm{C} & -2.101060 & -0.005269 & -3.099374 \\ \mathrm{H} & -1.772418 & 0.109886 & -4.131126 \\ \mathrm{C} & -3.566702 & -0.142691 & -2.972539\end{array}$




$\begin{array}{lrrr}\mathrm{C} & -4.233192 & -0.961704 & -2.137367 \\ \mathrm{H} & -3.705581 & -1.582971 & -1.425584 \\ \mathrm{H} & -5.316958 & -1.013081 & -2.152475 \\ \mathrm{O} & -2.383381 & 0.034229 & -0.136270 \\ \mathrm{C} & -4.324379 & 0.629520 & -3.927785 \\ \mathrm{~N} & -4.909135 & 1.259943 & -4.711343 \\ \mathrm{H} & -2.273626 & 0.095850 & 0.826296\end{array}$

$\begin{array}{crrr}\text { TS } & & & \\ \mathrm{H} & 0.000000 & 0.000000 & 0.000000 \\ \mathrm{C} & 0.000000 & 0.000000 & 1.077200 \\ \mathrm{H} & 0.990369 & 0.000000 & 1.529999 \\ \mathrm{C} & -0.985572 & 0.718744 & 1.752686 \\ \mathrm{C} & -2.390096 & 0.583110 & 1.588808 \\ \mathrm{H} & -3.005418 & 1.416784 & 1.926522 \\ \mathrm{C} & -3.035837 & -0.635286 & 1.426843 \\ \mathrm{H} & -4.100357 & -0.661211 & 1.649058 \\ \mathrm{C} & -2.354162 & -1.871583 & 1.281849 \\ \mathrm{C} & -1.122064 & -1.968782 & 0.624938 \\ \mathrm{H} & -1.047870 & -1.536182 & -0.359339 \\ \mathrm{H} & -0.503642 & -2.849450 & 0.781999 \\ \mathrm{C} & -2.867274 & -2.995158 & 2.005750 \\ \mathrm{O} & -0.543864 & 1.519823 & 2.773313 \\ \mathrm{H} & -1.265207 & 1.666372 & 3.406391 \\ \mathrm{~N} & -3.298619 & -3.913732 & 2.579248\end{array}$

$\begin{array}{cccc} & = & & \\ \mathrm{HO} & & & \\ \mathrm{C} & 0.000000 & 0.000000 & 0.000000 \\ \mathrm{H} & 0.000000 & 0.000000 & 1.086751 \\ \mathrm{H} & 0.963375 & 0.000000 & -0.494343 \\ \mathrm{C} & -1.142763 & 0.006781 & -0.713591 \\ \mathrm{C} & -1.119334 & 0.011543 & -2.174315 \\ \mathrm{H} & -0.108698 & 0.073155 & -2.569656 \\ \mathrm{C} & -2.104224 & -0.004586 & -3.097279 \\ \mathrm{H} & -1.790134 & 0.112990 & -4.129675 \\ \mathrm{C} & -3.551387 & -0.160736 & -2.944442 \\ \mathrm{C} & -4.243645 & -0.957075 & -2.124438 \\ \mathrm{H} & -3.731912 & -1.571669 & -1.396881 \\ \mathrm{H} & -5.325225 & -0.984515 & -2.173420 \\ \mathrm{~N} & -4.350616 & 0.624817 & -3.936523 \\ \mathrm{O} & -2.383823 & 0.035071 & -0.136709 \\ \mathrm{H} & -2.274901 & 0.099262 & 0.825711 \\ \mathrm{O} & -3.723001 & 1.339578 & -4.717898\end{array}$




$\begin{array}{crrr}\mathrm{O} & -5.574600 & 0.522511 & -3.919258 \\ \mathrm{TS} & & & \\ \mathrm{H} & 0.000000 & 0.000000 & 0.000000 \\ \mathrm{H} & 0.000000 & 0.000000 & 1.831609 \\ \mathrm{C} & 0.574877 & 0.000000 & 0.911113 \\ \mathrm{C} & 1.801991 & 0.650564 & 0.897120 \\ \mathrm{C} & 2.824130 & 0.414373 & -0.049028 \\ \mathrm{H} & 3.593267 & 1.170086 & -0.166240 \\ \mathrm{C} & 3.046019 & -0.828486 & -0.620601 \\ \mathrm{H} & 3.983344 & -0.956535 & -1.161330 \\ \mathrm{C} & 2.367947 & -2.014336 & -0.220672 \\ \mathrm{C} & 1.016375 & -2.122485 & 0.100077 \\ \mathrm{H} & 0.285723 & -1.770995 & -0.609120 \\ \mathrm{H} & 0.720604 & -3.008479 & 0.659095 \\ \mathrm{O} & 3.130145 & -3.124598 & 0.017333 \\ \mathrm{~N} & 2.170977 & 1.461400 & 2.061454 \\ \mathrm{O} & 1.416357 & 1.456387 & 3.037056 \\ \mathrm{O} & 3.210497 & 2.125370 & 1.993960 \\ \mathrm{H} & 4.060661 & -2.866208 & 0.119560\end{array}$

4

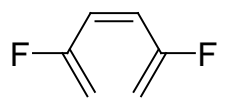

$\begin{array}{llll}\text { C } & 0.000000 & 0.000000 & 0.000000\end{array}$

$\begin{array}{llll}\mathrm{H} & 0.000000 & 0.000000 & 1.083488\end{array}$

$\begin{array}{llll}\mathrm{H} & 0.951242 & 0.000000 & -0.517859\end{array}$

$\begin{array}{llll}\text { C } & -1.152451 & -0.000161 & -0.685201\end{array}$

$\begin{array}{llll}\text { C } & -1.226956 & 0.000142 & -2.136403\end{array}$

$\begin{array}{llll}\mathrm{H} & -0.235555 & 0.003127 & -2.583685\end{array}$

$\begin{array}{llll}\text { C } & -2.255355 & -0.002307 & -3.017073\end{array}$

$\mathrm{H} \quad-1.965850 \quad-0.000121 \quad-4.065484$

$\begin{array}{llll}\text { C } & -3.700740 & -0.007479 & -2.867861\end{array}$

$\begin{array}{llll}\text { C } & -4.554798 & -0.006078 & -3.901434\end{array}$

$\mathrm{H} \quad-5.625405 \quad-0.010310 \quad-3.734836$

$\mathrm{H} \quad-4.189416 \quad-0.000544 \quad-4.920985$

F $\quad-4.205162 \quad-0.014515 \quad-1.619211$

$\begin{array}{llll}\text { F } & -2.308917 & -0.000172 & 0.004794\end{array}$

TS

$\begin{array}{llll}\mathrm{H} & 0.000000 & 0.000000 & 0.000000\end{array}$

$\begin{array}{llll}\mathrm{H} & 0.000000 & 0.000000 & 1.815933\end{array}$

$\begin{array}{llll}\text { C } & 0.590739 & 0.000000 & 0.915595\end{array}$ 


$\begin{array}{lccc}\text { C } & 1.765583 & -0.754434 & 0.888962 \\ \mathrm{C} & 2.884754 & -0.616488 & 1.706800 \\ \mathrm{H} & 3.625497 & -1.410649 & 1.659735 \\ \mathrm{C} & 3.283855 & 0.598920 & 2.279776 \\ \mathrm{H} & 4.307575 & 0.666537 & 2.638984 \\ \mathrm{C} & 2.604622 & 1.800749 & 2.093543 \\ \mathrm{C} & 1.241549 & 1.981943 & 1.849947 \\ \mathrm{H} & 0.959698 & 2.922664 & 1.377833 \\ \mathrm{H} & 0.537025 & 1.635393 & 2.586922 \\ \mathrm{~F} & 1.931813 & -1.591922 & -0.175291 \\ \mathrm{~F} & 3.405148 & 2.894916 & 1.939884\end{array}$

$\begin{array}{lrcc} & = & & \\ \mathrm{C} & & & \\ \mathrm{C} & 0.000000 & 0.000000 & 0.000000 \\ \mathrm{H} & 0.000000 & 0.000000 & 1.083521 \\ \mathrm{H} & 0.950845 & 0.000000 & -0.518719 \\ \mathrm{C} & -1.148385 & 0.004329 & -0.687680 \\ \mathrm{C} & -1.257659 & 0.016578 & -2.135049 \\ \mathrm{H} & -0.285702 & 0.107777 & -2.615210 \\ \mathrm{C} & -2.325988 & -0.026260 & -2.961786 \\ \mathrm{H} & -2.100109 & 0.098916 & -4.018553 \\ \mathrm{C} & -3.768618 & -0.178260 & -2.734215 \\ \mathrm{C} & -4.407874 & -0.793407 & -1.719385 \\ \mathrm{H} & -3.895201 & -1.245728 & -0.881809 \\ \mathrm{H} & -5.494300 & -0.854854 & -1.723211 \\ \mathrm{C} & -4.636374 & 0.320115 & -3.842054 \\ \mathrm{~F} & -2.312430 & 0.023964 & 0.010102 \\ \mathrm{O} & -4.230956 & 0.795076 & -4.883758 \\ \mathrm{H} & -5.726510 & 0.212562 & -3.650092\end{array}$

$\begin{array}{rrrr}\text { TS } & & & \\ \mathrm{H} & 0.000000 & 0.000000 & 0.000000 \\ \mathrm{C} & 0.000000 & 0.000000 & 1.091006 \\ \mathrm{H} & 0.987573 & 0.000000 & 1.523246 \\ \mathrm{C} & -1.021827 & 0.684822 & 1.758461 \\ \mathrm{C} & -1.362132 & 0.447977 & 3.106767 \\ \mathrm{H} & -1.964155 & 1.206918 & 3.600626 \\ \mathrm{C} & -1.288042 & -0.801536 & 3.713695 \\ \mathrm{H} & -1.858326 & -0.952887 & 4.626784 \\ \mathrm{C} & -0.884983 & -1.962949 & 3.031791 \\ \mathrm{C} & 0.052695 & -2.098220 & 2.014587 \\ \mathrm{H} & -0.027343 & -2.996961 & 1.404819 \\ \mathrm{H} & 1.063001 & -1.768817 & 2.188411\end{array}$




\begin{tabular}{|c|c|c|c|}
\hline $\mathrm{F}$ & -1.660468 & -3.049492 & 3.287941 \\
\hline $\mathrm{C}$ & -1.891304 & 1.592715 & 0.979308 \\
\hline $\mathrm{H}$ & -1.763136 & 1.504397 & -0.123482 \\
\hline $\mathrm{O}$ & -2.667520 & 2.402329 & 1.454127 \\
\hline & & & \\
\hline $\mathrm{C}$ & 0.000000 & 0.000000 & 0.000000 \\
\hline $\mathrm{H}$ & 0.000000 & 0.000000 & 1.083516 \\
\hline $\mathrm{H}$ & 0.951137 & 0.000000 & -0.518318 \\
\hline $\mathrm{C}$ & -1.148524 & 0.006415 & -0.686735 \\
\hline $\mathrm{C}$ & -1.252180 & 0.018943 & -2.135412 \\
\hline $\mathrm{H}$ & -0.281337 & 0.091393 & -2.620017 \\
\hline $\mathrm{C}$ & -2.326355 & -0.001880 & -2.953133 \\
\hline $\mathrm{H}$ & -2.111866 & 0.115748 & -4.013662 \\
\hline $\mathrm{C}$ & -3.767785 & -0.145028 & -2.662759 \\
\hline $\mathrm{C}$ & -4.335497 & -0.936899 & -1.732794 \\
\hline $\mathrm{H}$ & -3.735635 & -1.538340 & -1.062372 \\
\hline $\mathrm{H}$ & -5.414245 & -0.992144 & -1.631558 \\
\hline $\mathrm{C}$ & -4.630125 & 0.591110 & -3.556045 \\
\hline $\mathrm{F}$ & -2.314087 & 0.025684 & 0.002865 \\
\hline $\mathrm{N}$ & -5.300887 & 1.193086 & -4.291275 \\
\hline \multicolumn{4}{|l|}{$\mathrm{TS}$} \\
\hline $\mathrm{H}$ & 0.000000 & 0.000000 & 0.000000 \\
\hline $\mathrm{H}$ & 0.000000 & 0.000000 & 1.823591 \\
\hline $\mathrm{C}$ & 0.583898 & 0.000000 & 0.917173 \\
\hline $\mathrm{C}$ & 1.808555 & -0.680390 & 0.950944 \\
\hline $\mathrm{C}$ & 2.773654 & -0.442354 & 1.957162 \\
\hline $\mathrm{H}$ & 3.554873 & -1.183798 & 2.105319 \\
\hline $\mathrm{C}$ & 2.971658 & 0.802355 & 2.547597 \\
\hline $\mathrm{H}$ & 3.897294 & 0.948573 & 3.098188 \\
\hline $\mathrm{C}$ & 2.299469 & 1.971020 & 2.148125 \\
\hline $\mathrm{C}$ & 1.001132 & 2.128363 & 1.676893 \\
\hline $\mathrm{H}$ & 0.815932 & 3.024854 & 1.086304 \\
\hline $\mathrm{H}$ & 0.168374 & 1.823342 & 2.286901 \\
\hline $\mathrm{F}$ & 3.113959 & 3.053573 & 2.035593 \\
\hline $\mathrm{C}$ & 2.190900 & -1.511341 & -0.151898 \\
\hline $\mathrm{N}$ & 2.491035 & -2.204897 & -1.038616 \\
\hline $\mathrm{C}$ & 0.000000 & 0.000000 & 0.000000 \\
\hline $\mathrm{H}$ & 0.000000 & 0.000000 & 1.083514 \\
\hline
\end{tabular}




$\begin{array}{cccc}\mathrm{H} & 0.950991 & 0.000000 & -0.518562 \\ \mathrm{C} & -1.148310 & 0.006935 & -0.686881 \\ \mathrm{C} & -1.254565 & 0.019493 & -2.135030 \\ \mathrm{H} & -0.285234 & 0.091020 & -2.622329 \\ \mathrm{C} & -2.330601 & -0.004984 & -2.950386 \\ \mathrm{H} & -2.131421 & 0.115950 & -4.009990 \\ \mathrm{C} & -3.750486 & -0.169881 & -2.637123 \\ \mathrm{C} & -4.343964 & -0.938343 & -1.718318 \\ \mathrm{H} & -3.757689 & -1.526768 & -1.026184 \\ \mathrm{H} & -5.424410 & -0.975247 & -1.654041 \\ \mathrm{~F} & -2.314661 & 0.025676 & 0.003581 \\ \mathrm{~N} & -4.661282 & 0.571624 & -3.567788 \\ \mathrm{O} & -4.128881 & 1.261114 & -4.437114 \\ \mathrm{O} & -5.874004 & 0.458917 & -3.414628\end{array}$

TS

$\begin{array}{lrrr}\mathrm{H} & 0.000000 & 0.000000 & 0.000000 \\ \mathrm{H} & 0.000000 & 0.000000 & 1.831490 \\ \mathrm{C} & 0.575294 & 0.000000 & 0.920127 \\ \mathrm{C} & 1.790910 & -0.675389 & 0.941291 \\ \mathrm{C} & 2.815485 & -0.458352 & 1.879429 \\ \mathrm{H} & 3.587750 & -1.213750 & 1.973193 \\ \mathrm{C} & 3.054479 & 0.784570 & 2.455584 \\ \mathrm{H} & 4.007343 & 0.925550 & 2.958787 \\ \mathrm{C} & 2.365421 & 1.956056 & 2.090153 \\ \mathrm{C} & 1.042514 & 2.113537 & 1.694933 \\ \mathrm{H} & 0.817953 & 3.013313 & 1.124110 \\ \mathrm{H} & 0.250900 & 1.795706 & 2.351382 \\ \mathrm{~N} & 2.137336 & -1.515215 & -0.218037 \\ \mathrm{~F} & 3.174993 & 3.033325 & 1.929378 \\ \mathrm{O} & 1.413868 & -1.451807 & -1.212872 \\ \mathrm{O} & 3.120660 & -2.251907 & -0.117783\end{array}$

5<smiles>C=C(C=O)C=CC(=C)C=O</smiles>

$\begin{array}{llll}\text { C } & 0.000000 & 0.000000 & 0.000000\end{array}$

$\begin{array}{llll}\mathrm{H} & 0.000000 & 0.000000 & 1.087927\end{array}$

$\begin{array}{llll}\mathrm{H} & 0.967864 & 0.000000 & -0.489349\end{array}$

$\begin{array}{llll}\text { C } & -1.159928 & -0.009942 & -0.682487\end{array}$

$\begin{array}{llll}\text { C } & -1.329547 & -0.078193 & -2.145318\end{array}$

$\begin{array}{llll}\text { C } & -0.592240 & 0.439423 & -3.145443\end{array}$

$\begin{array}{llll}\text { C } & 0.569679 & 1.346444 & -3.121890\end{array}$ 


$\begin{array}{crcc}\mathrm{H} & -2.209228 & -0.647899 & -2.437626 \\ \mathrm{H} & -0.879318 & 0.154607 & -4.155548 \\ \mathrm{C} & 0.796587 & 2.351753 & -2.256328 \\ \mathrm{H} & 0.132014 & 2.573459 & -1.428433 \\ \mathrm{H} & 1.669480 & 2.991081 & -2.369913 \\ \mathrm{C} & -2.409815 & -0.087737 & 0.126849 \\ \mathrm{C} & 1.529289 & 1.212240 & -4.255215 \\ \mathrm{O} & 1.409034 & 0.424420 & -5.171227 \\ \mathrm{H} & 2.389259 & 1.916821 & -4.213392 \\ \mathrm{O} & -3.525752 & -0.204786 & -0.337026 \\ \mathrm{H} & -2.247172 & -0.043393 & 1.226537\end{array}$

TS

$\begin{array}{lrrr}\mathrm{C} & 0.000000 & 0.000000 & 0.000000 \\ \mathrm{H} & 0.000000 & 0.000000 & 1.077760 \\ \mathrm{H} & 0.999530 & 0.000000 & -0.437453 \\ \mathrm{C} & -1.017565 & 0.675454 & -0.678144 \\ \mathrm{C} & -2.401299 & 0.470347 & -0.472023 \\ \mathrm{H} & -3.066221 & 1.244123 & -0.848130 \\ \mathrm{C} & -2.956584 & -0.777193 & -0.213345 \\ \mathrm{H} & -4.020174 & -0.899045 & -0.403689 \\ \mathrm{C} & -2.192384 & -1.963995 & -0.130979 \\ \mathrm{C} & -0.914305 & -2.054315 & 0.425729 \\ \mathrm{H} & -0.753959 & -1.694437 & 1.428895 \\ \mathrm{H} & -0.298930 & -2.917331 & 0.166993 \\ \mathrm{C} & -2.731899 & -3.167491 & -0.818036 \\ \mathrm{C} & -0.623758 & 1.568772 & -1.799896 \\ \mathrm{H} & 0.433559 & 1.447770 & -2.126323 \\ \mathrm{O} & -1.347846 & 2.396362 & -2.318521 \\ \mathrm{O} & -3.895597 & -3.327548 & -1.131658 \\ \mathrm{H} & -1.973826 & -3.960810 & -1.005327\end{array}$

$\begin{array}{lrrr} & \mathrm{OHC} \\ \mathrm{C} & 0.000000 & 0.000000 & 0.000000 \\ \mathrm{H} & 0.000000 & 0.000000 & 1.086102 \\ \mathrm{H} & 0.961343 & 0.000000 & -0.501318 \\ \mathrm{C} & -1.161861 & -0.000902 & -0.689067 \\ \mathrm{C} & -2.459396 & -0.047633 & 0.000896 \\ \mathrm{H} & -2.361920 & -0.338305 & 1.045218 \\ \mathrm{C} & -3.714103 & 0.165350 & -0.445122 \\ \mathrm{H} & -4.517158 & -0.060210 & 0.253425 \\ \mathrm{C} & -4.194232 & 0.633103 & -1.751452 \\ \mathrm{C} & -3.632139 & 1.595016 & -2.506989\end{array}$




$\begin{array}{lrrr}\mathrm{H} & -2.737979 & 2.130819 & -2.209820 \\ \mathrm{H} & -4.075286 & 1.881111 & -3.458062 \\ \mathrm{C} & -5.491938 & 0.069929 & -2.216613 \\ \mathrm{C} & -1.069836 & -0.047173 & -2.127407 \\ \mathrm{~N} & -0.950197 & -0.095927 & -3.283022 \\ \mathrm{O} & -6.175239 & -0.700902 & -1.572970 \\ \mathrm{H} & -5.815956 & 0.428138 & -3.218009\end{array}$

$\begin{array}{cccc}\text { TS } & & & \\ \mathrm{H} & 0.000000 & 0.000000 & 0.000000 \\ \mathrm{C} & 0.000000 & 0.000000 & 1.077412 \\ \mathrm{H} & 1.000772 & 0.000000 & 1.512601 \\ \mathrm{C} & -1.015433 & 0.665570 & 1.767268 \\ \mathrm{C} & -2.401679 & 0.442127 & 1.600497 \\ \mathrm{H} & -3.063477 & 1.217194 & 1.979135 \\ \mathrm{C} & -2.959416 & -0.812462 & 1.386407 \\ \mathrm{H} & -4.014446 & -0.927434 & 1.622675 \\ \mathrm{C} & -2.180319 & -1.992381 & 1.255819 \\ \mathrm{C} & -0.941334 & -2.040165 & 0.608161 \\ \mathrm{H} & -0.881118 & -1.622368 & -0.383521 \\ \mathrm{H} & -0.288848 & -2.892161 & 0.783152 \\ \mathrm{C} & -2.625459 & -3.145429 & 1.986266 \\ \mathrm{C} & -0.613323 & 1.575741 & 2.874330 \\ \mathrm{H} & 0.448030 & 1.465533 & 3.189397 \\ \mathrm{O} & -1.338244 & 2.403986 & 3.389865 \\ \mathrm{~N} & -2.995250 & -4.088459 & 2.561033\end{array}$

$\begin{array}{lrrr}\mathrm{OHC} & =\mathrm{NO}_{2} & & \\ \mathrm{C} & 0.000000 & 0.000000 & 0.000000 \\ \mathrm{H} & 0.000000 & 0.000000 & 1.083257 \\ \mathrm{H} & 0.947746 & 0.000000 & -0.525928 \\ \mathrm{C} & -1.147960 & 0.005109 & -0.680451 \\ \mathrm{C} & -1.350712 & -0.050459 & -2.135189 \\ \mathrm{H} & -2.087807 & -0.779767 & -2.458994 \\ \mathrm{C} & -0.717463 & 0.655097 & -3.088555 \\ \mathrm{H} & -0.917655 & 0.370065 & -4.118930 \\ \mathrm{C} & 0.239267 & 1.770864 & -2.972661 \\ \mathrm{C} & 0.226046 & 2.750908 & -2.050828 \\ \mathrm{H} & -0.504602 & 2.796326 & -1.250664 \\ \mathrm{H} & 0.960148 & 3.552809 & -2.088910 \\ \mathrm{C} & 1.250616 & 1.881209 & -4.062197 \\ \mathrm{~N} & -2.409451 & -0.033784 & 0.119736 \\ \mathrm{O} & -2.331029 & -0.026255 & 1.345038\end{array}$




$\begin{array}{rrrr}\mathrm{O} & -3.461312 & -0.067020 & -0.517852 \\ \mathrm{H} & 1.950870 & 2.738233 & -3.953835 \\ \mathrm{O} & 1.324117 & 1.125617 & -5.009732\end{array}$

TS

$\begin{array}{lrrr}\mathrm{C} & 0.000000 & 0.000000 & 0.000000 \\ \mathrm{H} & 0.000000 & 0.000000 & 1.077487 \\ \mathrm{H} & 0.999577 & 0.000000 & -0.437245 \\ \mathrm{C} & -1.018718 & 0.665336 & -0.685150 \\ \mathrm{C} & -2.402792 & 0.444453 & -0.508102 \\ \mathrm{H} & -3.067845 & 1.216491 & -0.886818 \\ \mathrm{C} & -2.959263 & -0.808834 & -0.274036 \\ \mathrm{H} & -4.011931 & -0.942487 & -0.497233 \\ \mathrm{C} & -2.171411 & -1.966628 & -0.128364 \\ \mathrm{C} & -0.920205 & -2.049123 & 0.466773 \\ \mathrm{H} & -0.818667 & -1.656064 & 1.464918 \\ \mathrm{H} & -0.296066 & -2.905082 & 0.231312 \\ \mathrm{~N} & -2.645849 & -3.160204 & -0.877598 \\ \mathrm{C} & -0.621670 & 1.574307 & -1.797428 \\ \mathrm{O} & -1.823166 & -4.034380 & -1.144291 \\ \mathrm{O} & -3.840222 & -3.210136 & -1.167753 \\ \mathrm{H} & 0.435382 & 1.457527 & -2.123349 \\ \mathrm{O} & -1.347764 & 2.406016 & -2.304103\end{array}$

6

$\begin{array}{lrrr}\mathrm{NC} & -\mathbb{C} \text {. } & & \\ \mathrm{C} & 0.000000 & 0.000000 & 0.000000 \\ \mathrm{H} & 0.000000 & 0.000000 & 1.084177 \\ \mathrm{H} & 0.963102 & 0.000000 & -0.501957 \\ \mathrm{C} & -1.148878 & 0.000201 & -0.710226 \\ \mathrm{C} & -1.129512 & 0.039136 & -2.179402 \\ \mathrm{H} & -0.147932 & 0.292210 & -2.575019 \\ \mathrm{C} & -2.105057 & -0.147622 & -3.090490 \\ \mathrm{H} & -1.839311 & 0.047131 & -4.128122 \\ \mathrm{C} & -3.502647 & -0.582291 & -2.893300 \\ \mathrm{C} & -3.894598 & -1.592366 & -2.094745 \\ \mathrm{H} & -3.173988 & -2.166477 & -1.523635 \\ \mathrm{H} & -4.939641 & -1.867194 & -2.001862 \\ \mathrm{C} & -4.480680 & 0.103025 & -3.700386 \\ \mathrm{C} & -2.382470 & 0.040696 & 0.034674 \\ \mathrm{~N} & -3.348954 & 0.087357 & 0.679189\end{array}$




$\begin{array}{crrr}\mathrm{N} & -5.241431 & 0.668060 & -4.374701 \\ & & & \\ \mathrm{TS} & & & \\ \mathrm{H} & 0.000000 & 0.000000 & 0.000000 \\ \mathrm{C} & 0.000000 & 0.000000 & 1.077360 \\ \mathrm{H} & 0.988957 & 0.000000 & 1.529889 \\ \mathrm{C} & -1.040457 & 0.662436 & 1.736425 \\ \mathrm{C} & -2.420322 & 0.441697 & 1.481529 \\ \mathrm{H} & -3.120408 & 1.209135 & 1.801938 \\ \mathrm{C} & -2.957334 & -0.809831 & 1.207817 \\ \mathrm{H} & -4.031574 & -0.914371 & 1.337525 \\ \mathrm{C} & -2.182511 & -1.999167 & 1.154347 \\ \mathrm{C} & -0.891173 & -2.076953 & 0.623186 \\ \mathrm{H} & -0.726995 & -1.694458 & -0.370519 \\ \mathrm{H} & -0.266756 & -2.926516 & 0.889954 \\ \mathrm{C} & -2.711265 & -3.136719 & 1.852890 \\ \mathrm{C} & -0.725159 & 1.492017 & 2.865117 \\ \mathrm{~N} & -0.467336 & 2.183607 & 3.765911 \\ \mathrm{~N} & -3.149468 & -4.067298 & 2.398966\end{array}$<smiles>C=C(C#N)/C=C\C(=C)[N+](=O)[O-]</smiles>

$\begin{array}{llll}\text { C } & 0.000000 & 0.000000 & 0.000000\end{array}$

$\begin{array}{llll}\mathrm{H} & 0.000000 & 0.000000 & 1.084182\end{array}$

$\begin{array}{llll}\mathrm{H} & 0.962895 & 0.000000 & -0.502255\end{array}$

$\begin{array}{llll}\text { C } & -1.148845 & 0.000902 & -0.710511\end{array}$

$\begin{array}{llll}\text { C } & -1.130217 & 0.038876 & -2.179208\end{array}$

$\begin{array}{llll}\mathrm{H} & -0.147157 & 0.284115 & -2.575501\end{array}$

$\begin{array}{llll}\text { C } & -2.106229 & -0.142439 & -3.091429\end{array}$

$\begin{array}{llll}\mathrm{H} & -1.846367 & 0.050848 & -4.127698\end{array}$

$\begin{array}{llll}\text { C } & -3.485603 & -0.585013 & -2.883481\end{array}$

$\begin{array}{llll}\text { C } & -3.937653 & -1.565088 & -2.096578\end{array}$

$\begin{array}{llll}\mathrm{H} & -3.257250 & -2.145143 & -1.485577\end{array}$

$\begin{array}{llll}\mathrm{H} & -4.995110 & -1.796622 & -2.059026\end{array}$

$\begin{array}{llll}\mathrm{N} & -4.486930 & 0.111702 & -3.743761\end{array}$

$\begin{array}{llll}\text { C } & -2.383492 & 0.040370 & 0.032283\end{array}$

$\begin{array}{llll}\mathrm{N} & -3.356002 & 0.081796 & 0.668135\end{array}$

$\begin{array}{llll}\text { O } & -4.055042 & 0.999750 & -4.478989\end{array}$

$\begin{array}{llll}\text { O } & -5.662045 & -0.233211 & -3.675325\end{array}$

TS

$\begin{array}{llll}\mathrm{H} & 0.000000 & 0.000000 & 0.000000\end{array}$

$\begin{array}{llll}\mathrm{H} & 0.000000 & 0.000000 & 1.830353\end{array}$

$\begin{array}{llll}\text { C } & 0.575891 & 0.000000 & 0.910200\end{array}$ 


$\begin{array}{lrrc}\mathrm{C} & 1.797833 & 0.657538 & 0.906575 \\ \mathrm{C} & 2.867683 & 0.456008 & 0.013058 \\ \mathrm{H} & 3.623021 & 1.231535 & -0.046470 \\ \mathrm{C} & 3.196646 & -0.794527 & -0.497847 \\ \mathrm{H} & 4.189242 & -0.895746 & -0.929041 \\ \mathrm{C} & 2.501608 & -1.984936 & -0.158999 \\ \mathrm{C} & 1.119525 & -2.063295 & 0.041843 \\ \mathrm{H} & 0.716532 & -2.917328 & 0.581006 \\ \mathrm{H} & 0.474029 & -1.671107 & -0.726484 \\ \mathrm{C} & 3.311632 & -3.127106 & 0.159934 \\ \mathrm{~N} & 2.110980 & 1.510479 & 2.084896 \\ \mathrm{O} & 1.401695 & 1.392795 & 3.081556 \\ \mathrm{O} & 3.049585 & 2.298447 & 1.979843 \\ \mathrm{~N} & 3.965776 & -4.060951 & 0.396166\end{array}$

$\begin{array}{lrrr}\mathrm{O}_{2} \mathrm{~N} & =\mathrm{NO}_{2} & & \\ \mathrm{C} & 0.000000 & 0.000000 & 0.000000 \\ \mathrm{H} & 0.000000 & 0.000000 & 1.083434 \\ \mathrm{H} & 0.948783 & 0.000000 & -0.524102 \\ \mathrm{C} & -1.148639 & -0.004671 & -0.678957 \\ \mathrm{C} & -1.347116 & -0.058004 & -2.134560 \\ \mathrm{H} & -2.067837 & -0.796929 & -2.472260 \\ \mathrm{C} & -0.721078 & 0.673222 & -3.071415 \\ \mathrm{H} & -0.898634 & 0.415945 & -4.111502 \\ \mathrm{C} & 0.167273 & 1.825248 & -2.860024 \\ \mathrm{C} & -0.023140 & 2.883013 & -2.069300 \\ \mathrm{H} & -0.908544 & 2.953087 & -1.447995 \\ \mathrm{H} & 0.702114 & 3.687375 & -2.040371 \\ \mathrm{~N} & 1.388531 & 1.823037 & -3.717481 \\ \mathrm{~N} & -2.411927 & -0.048920 & 0.114016 \\ \mathrm{O} & -3.459691 & -0.065943 & -0.531944 \\ \mathrm{O} & -2.339664 & -0.060208 & 1.338857 \\ \mathrm{O} & 1.538514 & 0.843979 & -4.448445 \\ \mathrm{O} & 2.162033 & 2.772796 & -3.645136\end{array}$

TS

$\begin{array}{rrrr}\mathrm{C} & 0.000000 & 0.000000 & 0.000000 \\ \mathrm{H} & 0.000000 & 0.000000 & 1.077168 \\ \mathrm{H} & 0.978265 & 0.000000 & -0.470282 \\ \mathrm{C} & -1.035016 & 0.661361 & -0.646828 \\ \mathrm{C} & -2.415595 & 0.463049 & -0.464391\end{array}$




$\begin{array}{lrrr}\mathrm{H} & -3.084827 & 1.236110 & -0.824897 \\ \mathrm{C} & -2.968233 & -0.786138 & -0.195130 \\ \mathrm{H} & -4.032591 & -0.906163 & -0.363048 \\ \mathrm{C} & -2.191983 & -1.953845 & -0.083291 \\ \mathrm{C} & -0.912690 & -2.063292 & 0.444422 \\ \mathrm{H} & -0.314933 & -2.923233 & 0.159181 \\ \mathrm{H} & -0.747210 & -1.689798 & 1.441123 \\ \mathrm{~N} & -2.731550 & -3.141757 & -0.803069 \\ \mathrm{~N} & -0.668790 & 1.520666 & -1.807745 \\ \mathrm{O} & 0.453994 & 1.384740 & -2.286712 \\ \mathrm{O} & -1.508814 & 2.328239 & -2.199265 \\ \mathrm{O} & -1.939129 & -4.024269 & -1.122066 \\ \mathrm{O} & -3.942354 & -3.172415 & -1.013060\end{array}$

\section{3, 4-substituted hexatriene}

1<smiles>Cc1ccccc1C</smiles>

$\begin{array}{llll}\mathrm{H} & 0.000000 & 0.000000 & 0.000000\end{array}$

$\begin{array}{llll}\mathrm{H} & 0.000000 & 0.000000 & 1.842428\end{array}$

$\begin{array}{llll}\text { C } & 0.574449 & 0.000000 & 0.920740\end{array}$

$\begin{array}{llll}\mathrm{C} & 1.917746 & 0.000000 & 0.953335\end{array}$

$\begin{array}{llll}\mathrm{H} & 2.374556 & -0.000136 & 1.937089\end{array}$

$\begin{array}{llll}\text { C } & 2.810934 & -0.000041 & -0.212670\end{array}$

$\begin{array}{llll}\text { C } & 4.177995 & 0.001014 & -0.090831\end{array}$

$\begin{array}{llll}\mathrm{C} & 4.850840 & 0.002351 & 1.214794\end{array}$

$\begin{array}{llll}\mathrm{H} & 4.227245 & 0.003008 & 2.102243\end{array}$

$\begin{array}{llll}\text { C } & 6.178735 & 0.002921 & 1.420268\end{array}$

H $6.906928 \quad 0.002465 \quad 0.615606$

$\begin{array}{llll}\mathrm{H} & 6.581163 & 0.003920 & 2.429013\end{array}$

$\begin{array}{llll}\text { C } & 2.094224 & -0.001291 & -1.545751\end{array}$

$\begin{array}{llll}\text { C } & 5.119098 & 0.000951 & -1.276209\end{array}$

$\mathrm{H} \quad 1.445110 \quad-0.882150 \quad-1.631357$

$\mathrm{H} \quad 1.443292 \quad 0.878143 \quad-1.632109$

$\mathrm{H} \quad 2.760906 \quad-0.000900 \quad-2.406225$

$\mathrm{H} \quad 4.614922 \quad-0.000305 \quad-2.240936$

$\begin{array}{llll}\mathrm{H} & 5.773168 & 0.881793 & -1.246731\end{array}$

$\begin{array}{llll}\mathrm{H} & 5.774912 & -0.878537 & -1.245297\end{array}$

TS

$\begin{array}{llll}\text { C } & 0.000000 & 0.000000 & 0.000000\end{array}$

$\begin{array}{llll}\mathrm{H} & 0.000000 & 0.000000 & 1.078046\end{array}$ 


$\begin{array}{lrrr}\mathrm{H} & 1.004142 & 0.000000 & -0.425695 \\ \mathrm{C} & -0.995442 & -0.704551 & -0.676285 \\ \mathrm{H} & -0.682201 & -1.346786 & -1.503867 \\ \mathrm{C} & -2.386416 & -0.557721 & -0.534525 \\ \mathrm{C} & -2.994355 & 0.695776 & -0.265291 \\ \mathrm{C} & -2.248292 & 1.878715 & -0.121880 \\ \mathrm{H} & -2.671032 & 2.753260 & -0.623828 \\ \mathrm{C} & -0.974902 & 2.010860 & 0.431025 \\ \mathrm{H} & -0.799283 & 1.650217 & 1.431660 \\ \mathrm{H} & -0.408815 & 2.913637 & 0.198492 \\ \mathrm{C} & -4.491016 & 0.844149 & -0.475445 \\ \mathrm{C} & -3.255359 & -1.703283 & -1.023212 \\ \mathrm{H} & -2.639625 & -2.554943 & -1.327148 \\ \mathrm{H} & -3.924689 & -2.055678 & -0.227546 \\ \mathrm{H} & -3.894418 & -1.428773 & -1.872182 \\ \mathrm{H} & -5.051561 & 0.268579 & 0.272755 \\ \mathrm{H} & -4.826276 & 0.490580 & -1.458834 \\ \mathrm{H} & -4.795514 & 1.889806 & -0.372018\end{array}$

$\begin{array}{llrr} & \\ \end{array}$

TS

$\begin{array}{llll}\mathrm{H} & 0.000000 & 0.000000 & 0.000000\end{array}$ 


\begin{tabular}{|c|c|c|c|}
\hline $\mathrm{C}$ & 0.000000 & 0.000000 & 1.078766 \\
\hline $\mathrm{H}$ & 1.009841 & 0.000000 & 1.493365 \\
\hline $\mathrm{C}$ & -0.974585 & -0.707168 & 1.783299 \\
\hline $\mathrm{H}$ & -0.656666 & -1.351932 & 2.605627 \\
\hline $\mathrm{C}$ & -2.371673 & -0.512858 & 1.716547 \\
\hline $\mathrm{C}$ & -2.954261 & 0.782291 & 1.616566 \\
\hline $\mathrm{C}$ & -2.181426 & 1.928815 & 1.401101 \\
\hline $\mathrm{H}$ & -2.481278 & 2.818538 & 1.960873 \\
\hline $\mathrm{C}$ & -0.993696 & 1.974465 & 0.668059 \\
\hline $\mathrm{H}$ & -1.002650 & 1.575506 & -0.335039 \\
\hline $\mathrm{H}$ & -0.351620 & 2.848171 & 0.777433 \\
\hline $\mathrm{C}$ & -4.379870 & 0.953698 & 2.106180 \\
\hline $\mathrm{N}$ & -3.172124 & -1.567626 & 2.196361 \\
\hline $\mathrm{H}$ & -2.831003 & -2.484784 & 1.941542 \\
\hline $\mathrm{H}$ & -4.160647 & -1.482950 & 2.003062 \\
\hline $\mathrm{H}$ & -4.553981 & 0.461234 & 3.072189 \\
\hline $\mathrm{H}$ & -5.108783 & 0.539249 & 1.393470 \\
\hline $\mathrm{H}$ & -4.622100 & 2.014750 & 2.217127 \\
\hline \multicolumn{4}{|c|}{$\mathrm{HO}$} \\
\hline $\mathrm{H}$ & 0.000000 & 0.000000 & 0.000000 \\
\hline $\mathrm{H}$ & 0.000000 & 0.000000 & 1.845359 \\
\hline $\mathrm{C}$ & 0.572035 & 0.000000 & 0.922598 \\
\hline $\mathrm{C}$ & 1.916065 & 0.006115 & 0.945691 \\
\hline $\mathrm{H}$ & 2.394955 & 0.029513 & 1.921397 \\
\hline $\mathrm{C}$ & 2.786946 & 0.006279 & -0.224356 \\
\hline $\mathrm{C}$ & 4.147211 & 0.025820 & -0.103212 \\
\hline $\mathrm{C}$ & 4.898013 & -0.058874 & 1.148627 \\
\hline $\mathrm{H}$ & 4.362929 & -0.429517 & 2.016951 \\
\hline $\mathrm{C}$ & 6.198859 & 0.249727 & 1.280853 \\
\hline $\mathrm{H}$ & 6.788134 & 0.670573 & 0.469572 \\
\hline $\mathrm{H}$ & 6.711390 & 0.125797 & 2.229177 \\
\hline $\mathrm{O}$ & 4.899408 & 0.158977 & -1.249269 \\
\hline $\mathrm{C}$ & 2.146194 & 0.026799 & -1.590012 \\
\hline $\mathrm{H}$ & 1.592714 & 0.961144 & -1.752731 \\
\hline $\mathrm{H}$ & 1.423351 & -0.792854 & -1.688932 \\
\hline $\mathrm{H}$ & 2.891459 & -0.069125 & -2.379730 \\
\hline $\mathrm{H}$ & 5.766729 & -0.245423 & -1.085832 \\
\hline \multicolumn{4}{|l|}{ TS } \\
\hline $\mathrm{H}$ & 0.000000 & 0.000000 & 0.000000 \\
\hline $\mathrm{C}$ & 0.000000 & 0.000000 & 1.077935 \\
\hline
\end{tabular}




$\begin{array}{lrrr}\mathrm{H} & 1.006260 & 0.000000 & 1.499110 \\ \mathrm{C} & -0.986408 & -0.693105 & 1.779987 \\ \mathrm{H} & -0.682094 & -1.325822 & 2.619527 \\ \mathrm{C} & -2.372298 & -0.471526 & 1.697886 \\ \mathrm{C} & -2.970625 & 0.803822 & 1.575628 \\ \mathrm{C} & -2.198281 & 1.950206 & 1.353650 \\ \mathrm{H} & -2.515844 & 2.845253 & 1.893812 \\ \mathrm{C} & -1.000205 & 1.996561 & 0.639788 \\ \mathrm{H} & -0.982797 & 1.578817 & -0.355028 \\ \mathrm{H} & -0.363908 & 2.873923 & 0.751706 \\ \mathrm{C} & -4.409538 & 0.946281 & 2.026091 \\ \mathrm{O} & -3.225402 & -1.458233 & 2.149486 \\ \mathrm{H} & -2.715385 & -2.278357 & 2.231024 \\ \mathrm{H} & -4.603059 & 0.419006 & 2.966317 \\ \mathrm{H} & -5.095937 & 0.521609 & 1.282652 \\ \mathrm{H} & -4.668085 & 2.002587 & 2.149796\end{array}$

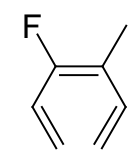

$\begin{array}{lrrr}\mathrm{H} & 0.000000 & 0.000000 & 0.000000 \\ \mathrm{H} & 0.000000 & 0.000000 & 1.845493 \\ \mathrm{C} & 0.571861 & 0.000000 & 0.922749 \\ \mathrm{C} & 1.914620 & 0.000039 & 0.945062 \\ \mathrm{H} & 2.399618 & 0.000120 & 1.917860 \\ \mathrm{C} & 2.778231 & 0.000191 & -0.232670 \\ \mathrm{C} & 4.131016 & -0.000124 & -0.114007 \\ \mathrm{C} & 4.954756 & -0.001022 & 1.080651 \\ \mathrm{H} & 4.433552 & -0.001781 & 2.032334 \\ \mathrm{C} & 6.296125 & -0.001092 & 1.061383 \\ \mathrm{H} & 6.853147 & -0.000404 & 0.130814 \\ \mathrm{H} & 6.867679 & -0.001846 & 1.983816 \\ \mathrm{~F} & 4.869411 & 0.000044 & -1.258456 \\ \mathrm{C} & 2.135684 & 0.000754 & -1.597657 \\ \mathrm{H} & 1.496163 & 0.883170 & -1.725502 \\ \mathrm{H} & 1.495501 & -0.881121 & -1.725898 \\ \mathrm{H} & 2.882954 & 0.000640 & -2.390946\end{array}$

$\begin{array}{cccc}\text { TS } & & & \\ \mathrm{H} & 0.000000 & 0.000000 & 0.000000 \\ \mathrm{C} & 0.000000 & 0.000000 & 1.077247 \\ \mathrm{H} & 1.006402 & 0.000000 & 1.498028 \\ \mathrm{C} & -0.983312 & -0.697977 & 1.782115 \\ \mathrm{H} & -0.688974 & -1.371685 & 2.588580\end{array}$




$\begin{array}{lrrc}\mathrm{C} & -2.355282 & -0.451950 & 1.723830 \\ \mathrm{C} & -2.986426 & 0.800414 & 1.608455 \\ \mathrm{C} & -2.232450 & 1.947453 & 1.334264 \\ \mathrm{H} & -2.535367 & 2.853857 & 1.861963 \\ \mathrm{C} & -1.054610 & 1.966723 & 0.587323 \\ \mathrm{H} & -0.412680 & 2.844480 & 0.646010 \\ \mathrm{H} & -1.067439 & 1.505073 & -0.388032 \\ \mathrm{C} & -4.409179 & 0.919123 & 2.110624 \\ \mathrm{~F} & -3.139195 & -1.436965 & 2.254805 \\ \mathrm{H} & -5.113582 & 0.480167 & 1.392981 \\ \mathrm{H} & -4.682105 & 1.970960 & 2.238124 \\ \mathrm{H} & -4.558698 & 0.396403 & 3.060857\end{array}$

$\begin{array}{lrrr}\mathrm{OHC} & & \\ \end{array}$

TS

$\begin{array}{cccc}\mathrm{H} & 0.000000 & 0.000000 & 0.000000 \\ \mathrm{C} & 0.000000 & 0.000000 & 1.078504 \\ \mathrm{H} & 0.997266 & 0.000000 & 1.516419 \\ \mathrm{C} & -1.018608 & -0.674279 & 1.738422 \\ \mathrm{H} & -0.751925 & -1.286787 & 2.602977 \\ \mathrm{C} & -2.403112 & -0.531687 & 1.497151 \\ \mathrm{C} & -3.015828 & 0.709299 & 1.156111\end{array}$




$\begin{array}{lrrr}\mathrm{C} & -2.251343 & 1.884699 & 1.080500 \\ \mathrm{H} & -2.718964 & 2.758261 & 1.541424 \\ \mathrm{C} & -0.931038 & 2.034388 & 0.650046 \\ \mathrm{H} & -0.655463 & 1.712748 & -0.341196 \\ \mathrm{H} & -0.389435 & 2.923844 & 0.974076 \\ \mathrm{C} & -4.522157 & 0.870406 & 1.210861 \\ \mathrm{C} & -3.208669 & -1.684731 & 1.964535 \\ \mathrm{H} & -2.616949 & -2.421078 & 2.555252 \\ \mathrm{O} & -4.385387 & -1.901099 & 1.734015 \\ \mathrm{H} & -4.972763 & 0.409235 & 2.093396 \\ \mathrm{H} & -4.989147 & 0.384803 & 0.347256 \\ \mathrm{H} & -4.786188 & 1.932445 & 1.182856\end{array}$

\begin{tabular}{|c|c|c|c|}
\hline $\mathrm{H}$ & 0.000000 & 0.000000 & 0.000000 \\
\hline $\mathrm{H}$ & 0.000000 & 0.000000 & 1.844442 \\
\hline $\mathrm{C}$ & 0.572472 & 0.000000 & 0.921973 \\
\hline $\mathrm{C}$ & 1.915890 & -0.000020 & 0.948096 \\
\hline $\mathrm{H}$ & 2.395198 & 0.000016 & 1.921793 \\
\hline $\mathrm{C}$ & 2.779706 & 0.000019 & -0.230302 \\
\hline $\mathrm{C}$ & 4.150120 & -0.000413 & -0.118163 \\
\hline $\mathrm{C}$ & 4.917695 & -0.001242 & 1.134307 \\
\hline $\mathrm{H}$ & 4.345507 & -0.001928 & 2.055929 \\
\hline $\mathrm{C}$ & 6.256027 & -0.001307 & 1.216725 \\
\hline $\mathrm{H}$ & 6.750915 & -0.001978 & 2.182657 \\
\hline $\mathrm{H}$ & 6.892182 & -0.000701 & 0.336329 \\
\hline $\mathrm{C}$ & 4.943919 & -0.000129 & -1.317554 \\
\hline $\mathrm{C}$ & 2.106820 & 0.000602 & -1.580671 \\
\hline $\mathrm{H}$ & 1.464070 & 0.882678 & -1.690118 \\
\hline $\mathrm{H}$ & 1.463588 & -0.881070 & -1.690601 \\
\hline $\mathrm{H}$ & 2.826294 & 0.000626 & -2.400496 \\
\hline $\mathrm{N}$ & 5.621621 & 0.000532 & -2.264197 \\
\hline \multicolumn{4}{|l|}{ TS } \\
\hline $\mathrm{H}$ & 0.000000 & 0.000000 & 0.000000 \\
\hline $\mathrm{H}$ & 0.000000 & 0.000000 & 1.808934 \\
\hline $\mathrm{C}$ & 0.597707 & 0.000000 & 0.897245 \\
\hline $\mathrm{C}$ & 1.805815 & -0.700417 & 0.908673 \\
\hline $\mathrm{H}$ & 1.995811 & -1.358489 & 1.759364 \\
\hline $\mathrm{C}$ & 2.895302 & -0.531469 & 0.042914 \\
\hline $\mathrm{C}$ & 3.222833 & 0.755235 & -0.471235 \\
\hline $\mathrm{C}$ & 2.517368 & 1.940142 & -0.167811 \\
\hline
\end{tabular}




$\begin{array}{lrrr}\mathrm{H} & 3.132878 & 2.810241 & 0.063143 \\ \mathrm{C} & 1.142288 & 2.024394 & 0.021460 \\ \mathrm{H} & 0.491107 & 1.640378 & -0.747316 \\ \mathrm{H} & 0.745601 & 2.901763 & 0.530533 \\ \mathrm{C} & 4.537385 & 0.933384 & -1.028026 \\ \mathrm{C} & 3.907227 & -1.651656 & -0.073169 \\ \mathrm{H} & 3.850976 & -2.112517 & -1.067852 \\ \mathrm{H} & 3.697434 & -2.437046 & 0.659286 \\ \mathrm{H} & 4.938298 & -1.310035 & 0.061586 \\ \mathrm{~N} & 5.587883 & 1.094813 & -1.503744\end{array}$<smiles>C=CC(C)=C(C=C)[N+](=O)[O-]</smiles>

$\begin{array}{lrrr}\mathrm{H} & 0.000000 & 0.000000 & 0.000000 \\ \mathrm{H} & 0.000000 & 0.000000 & 1.844211 \\ \mathrm{C} & 0.572598 & 0.000000 & 0.921888 \\ \mathrm{C} & 1.914667 & -0.003847 & 0.951035 \\ \mathrm{H} & 2.392447 & 0.001335 & 1.925608 \\ \mathrm{C} & 2.785602 & -0.005810 & -0.227510 \\ \mathrm{C} & 4.138911 & -0.052870 & -0.081829 \\ \mathrm{C} & 4.912809 & -0.160936 & 1.149901 \\ \mathrm{H} & 4.325605 & -0.355405 & 2.041284 \\ \mathrm{C} & 6.245795 & -0.085571 & 1.279354 \\ \mathrm{H} & 6.703857 & -0.217377 & 2.254320 \\ \mathrm{H} & 6.911208 & 0.116122 & 0.447172 \\ \mathrm{~N} & 4.968494 & -0.018184 & -1.311747 \\ \mathrm{C} & 2.110255 & 0.080095 & -1.575800 \\ \mathrm{H} & 1.443341 & 0.949060 & -1.605263 \\ \mathrm{H} & 1.492464 & -0.810094 & -1.745340 \\ \mathrm{H} & 2.809333 & 0.153874 & -2.406983 \\ \mathrm{O} & 4.895648 & -0.973841 & -2.080418 \\ \mathrm{O} & 5.693396 & 0.962199 & -1.470155\end{array}$

$\begin{array}{crrr}\text { TS } & & & \\ \mathrm{H} & 0.000000 & 0.000000 & 0.000000 \\ \mathrm{H} & 0.000000 & 0.000000 & 1.813867 \\ \mathrm{C} & 0.593707 & 0.000000 & 0.900790 \\ \mathrm{C} & 1.804188 & -0.692154 & 0.894030 \\ \mathrm{H} & 2.041147 & -1.318321 & 1.755395 \\ \mathrm{C} & 2.833614 & -0.544929 & -0.047550 \\ \mathrm{C} & 3.058694 & 0.730849 & -0.617800 \\ \mathrm{C} & 2.439459 & 1.931653 & -0.234653 \\ \mathrm{H} & 3.121026 & 2.767749 & -0.091714\end{array}$




$\begin{array}{lrrr}\mathrm{C} & 1.103630 & 2.069773 & 0.127518 \\ \mathrm{H} & 0.330684 & 1.755885 & -0.554386 \\ \mathrm{H} & 0.829509 & 2.946843 & 0.713274 \\ \mathrm{~N} & 4.316664 & 0.921068 & -1.382193 \\ \mathrm{C} & 3.848148 & -1.666480 & -0.159397 \\ \mathrm{H} & 3.684635 & -2.385380 & 0.649313 \\ \mathrm{H} & 3.729120 & -2.196965 & -1.109328 \\ \mathrm{H} & 4.885899 & -1.324317 & -0.117649 \\ \mathrm{O} & 4.605301 & 0.051975 & -2.204801 \\ \mathrm{O} & 4.977479 & 1.942225 & -1.191904\end{array}$

\section{2}

$\begin{array}{lrrr} & & \\ \mathrm{C} & 0.000000 & 0.000000 & 0.000000 \\ \mathrm{H} & 0.000000 & 0.000000 & 1.086289 \\ \mathrm{H} & 0.972403 & 0.000000 & -0.482472 \\ \mathrm{C} & -1.130591 & -0.038908 & -0.723833 \\ \mathrm{H} & -1.045511 & -0.093419 & -1.804284 \\ \mathrm{C} & -2.482778 & -0.004838 & -0.174460 \\ \mathrm{C} & -3.615827 & -0.291508 & -0.901445 \\ \mathrm{C} & -3.631149 & -0.601054 & -2.320727 \\ \mathrm{H} & -2.712480 & -0.413369 & -2.870269 \\ \mathrm{C} & -4.684209 & -1.061444 & -3.022110 \\ \mathrm{H} & -5.637325 & -1.309023 & -2.561789 \\ \mathrm{H} & -4.614128 & -1.225073 & -4.092425 \\ \mathrm{~N} & -2.607320 & 0.256832 & 1.193642 \\ \mathrm{~N} & -4.848135 & -0.265382 & -0.174950 \\ \mathrm{H} & -5.645360 & -0.167773 & -0.795806 \\ \mathrm{H} & -4.984787 & -1.101899 & 0.391052 \\ \mathrm{H} & -3.559253 & 0.536328 & 1.421573 \\ \mathrm{H} & -1.926407 & 0.926903 & 1.535921\end{array}$

TS

$\begin{array}{llll}\text { C } & 0.000000 & 0.000000 & 0.000000\end{array}$

$\begin{array}{llll}\mathrm{H} & 0.000000 & 0.000000 & 1.078562\end{array}$

$\begin{array}{llll}\mathrm{H} & 1.008581 & 0.000000 & -0.417170\end{array}$

$\begin{array}{llll}\text { C } & -0.977220 & 0.717026 & -0.696233\end{array}$

$\begin{array}{llll}\mathrm{H} & -0.660880 & 1.381317 & -1.505375\end{array}$

$\begin{array}{llll}\text { C } & -2.367755 & 0.530532 & -0.601896\end{array}$

$\begin{array}{llll}\text { C } & -2.955831 & -0.759307 & -0.468234\end{array}$

$\begin{array}{llll}\text { C } & -2.203699 & -1.924172 & -0.277530\end{array}$

$\begin{array}{llll}\mathrm{H} & -2.510676 & -2.789758 & -0.870303\end{array}$ 


\begin{tabular}{|c|c|c|c|}
\hline $\mathrm{C}$ & -1.008743 & -1.978826 & 0.440215 \\
\hline $\mathrm{H}$ & -0.989224 & -1.588191 & 1.446468 \\
\hline $\mathrm{H}$ & -0.366319 & -2.848036 & 0.303728 \\
\hline $\mathrm{N}$ & -4.321388 & -0.847991 & -0.838936 \\
\hline $\mathrm{N}$ & -3.227806 & 1.541209 & -1.097557 \\
\hline $\mathrm{H}$ & -4.151322 & 1.490623 & -0.677708 \\
\hline $\mathrm{H}$ & -2.844577 & 2.471002 & -0.976717 \\
\hline $\mathrm{H}$ & -4.524172 & -0.372391 & -1.717779 \\
\hline $\mathrm{H}$ & -4.645503 & -1.808622 & -0.879122 \\
\hline & $\mathrm{H}_{2}$ & & \\
\hline $\mathrm{H}$ & 0.000000 & 0.000000 & 0.000000 \\
\hline $\mathrm{H}$ & 0.000000 & 0.000000 & 1.846420 \\
\hline $\mathrm{C}$ & 0.571673 & 0.000000 & 0.924354 \\
\hline $\mathrm{C}$ & 1.917463 & -0.000020 & 0.938965 \\
\hline $\mathrm{H}$ & 2.413758 & -0.000102 & 1.906851 \\
\hline $\mathrm{C}$ & 2.770452 & 0.000039 & -0.232234 \\
\hline $\mathrm{C}$ & 4.143506 & 0.000155 & -0.226579 \\
\hline $\mathrm{C}$ & 4.997448 & 0.000405 & 0.954224 \\
\hline $\mathrm{H}$ & 4.499038 & -0.000150 & 1.918224 \\
\hline $\mathrm{C}$ & 6.336610 & 0.001290 & 0.899150 \\
\hline $\mathrm{H}$ & 6.863736 & 0.001796 & -0.048998 \\
\hline $\mathrm{H}$ & 6.934512 & 0.001461 & 1.805400 \\
\hline $\mathrm{O}$ & 4.801630 & 0.000198 & -1.408461 \\
\hline $\mathrm{N}$ & 2.198151 & 0.000237 & -1.562699 \\
\hline $\mathrm{H}$ & 1.601573 & 0.816659 & -1.699514 \\
\hline $\mathrm{H}$ & 1.602127 & -0.816533 & -1.699964 \\
\hline $\mathrm{H}$ & 4.071319 & 0.000363 & -2.073856 \\
\hline \multicolumn{4}{|l|}{ TS } \\
\hline $\mathrm{H}$ & 0.000000 & 0.000000 & 0.000000 \\
\hline $\mathrm{H}$ & 0.000000 & 0.000000 & 1.807167 \\
\hline $\mathrm{C}$ & 0.599533 & 0.000000 & 0.910796 \\
\hline $\mathrm{C}$ & 1.791159 & 0.734534 & 0.901553 \\
\hline $\mathrm{H}$ & 1.982481 & 1.404530 & 0.056616 \\
\hline $\mathrm{C}$ & 2.865434 & 0.554545 & 1.776437 \\
\hline $\mathrm{C}$ & 3.245835 & -0.714615 & 2.306017 \\
\hline $\mathrm{C}$ & 2.566831 & -1.901282 & 2.008527 \\
\hline $\mathrm{H}$ & 3.215123 & -2.742232 & 1.764368 \\
\hline $\mathrm{C}$ & 1.191076 & -1.996884 & 1.798495 \\
\hline $\mathrm{H}$ & 0.515144 & -1.656976 & 2.567716 \\
\hline $\mathrm{H}$ & 0.820028 & -2.871820 & 1.264622 \\
\hline
\end{tabular}




\begin{tabular}{|c|c|c|c|}
\hline $\mathrm{N}$ & 3.950322 & 1.520842 & 1.846030 \\
\hline $\mathrm{O}$ & 4.503324 & -0.818274 & 2.808291 \\
\hline $\mathrm{H}$ & 4.910964 & 0.059757 & 2.610756 \\
\hline $\mathrm{H}$ & 3.743151 & 2.242121 & 2.535797 \\
\hline $\mathrm{H}$ & 4.065573 & 1.998462 & 0.952839 \\
\hline \multicolumn{4}{|c|}{$\mathrm{NH}_{2}$} \\
\hline $\mathrm{H}$ & 0.000000 & 0.000000 & 0.000000 \\
\hline $\mathrm{H}$ & 0.000000 & 0.000000 & 1.849014 \\
\hline $\mathrm{C}$ & 0.569092 & 0.000000 & 0.924864 \\
\hline $\mathrm{C}$ & 1.910317 & -0.035594 & 0.939746 \\
\hline $\mathrm{H}$ & 2.423319 & -0.068438 & 1.896277 \\
\hline $\mathrm{C}$ & 2.755341 & -0.000455 & -0.251447 \\
\hline $\mathrm{C}$ & 4.086891 & -0.291869 & -0.210532 \\
\hline $\mathrm{C}$ & 4.896685 & -0.755821 & 0.885880 \\
\hline $\mathrm{H}$ & 4.381257 & -0.906217 & 1.830124 \\
\hline $\mathrm{C}$ & 6.213687 & -1.010736 & 0.802913 \\
\hline $\mathrm{H}$ & 6.760817 & -0.886313 & -0.125368 \\
\hline $\mathrm{H}$ & 6.770857 & -1.354567 & 1.667802 \\
\hline $\mathrm{F}$ & 4.760403 & -0.162183 & -1.397906 \\
\hline $\mathrm{N}$ & 2.137620 & 0.296398 & -1.469973 \\
\hline $\mathrm{H}$ & 1.469035 & 1.056908 & -1.411459 \\
\hline $\mathrm{H}$ & 2.798831 & 0.439850 & -2.223643 \\
\hline \multicolumn{4}{|c|}{ TS } \\
\hline $\mathrm{H}$ & 0.000000 & 0.000000 & 0.000000 \\
\hline $\mathrm{H}$ & 0.000000 & 0.000000 & 1.807392 \\
\hline $\mathrm{C}$ & 0.598722 & 0.000000 & 0.896444 \\
\hline $\mathrm{C}$ & 1.799085 & -0.719197 & 0.919013 \\
\hline $\mathrm{H}$ & 2.018876 & -1.394927 & 1.747628 \\
\hline $\mathrm{C}$ & 2.860982 & -0.500036 & 0.053183 \\
\hline $\mathrm{C}$ & 3.298450 & 0.742974 & -0.463708 \\
\hline $\mathrm{C}$ & 2.590742 & 1.921846 & -0.170170 \\
\hline $\mathrm{H}$ & 3.204038 & 2.776566 & 0.121993 \\
\hline $\mathrm{C}$ & 1.206429 & 1.993297 & -0.022789 \\
\hline $\mathrm{H}$ & 0.575869 & 1.622926 & -0.816392 \\
\hline $\mathrm{H}$ & 0.791211 & 2.862199 & 0.486433 \\
\hline $\mathrm{N}$ & 4.576338 & 0.788657 & -1.015794 \\
\hline $\mathrm{F}$ & 3.841279 & -1.463232 & 0.047627 \\
\hline $\mathrm{H}$ & 5.212847 & 0.072549 & -0.688006 \\
\hline $\mathrm{H}$ & 4.991812 & 1.711252 & -1.048724 \\
\hline
\end{tabular}




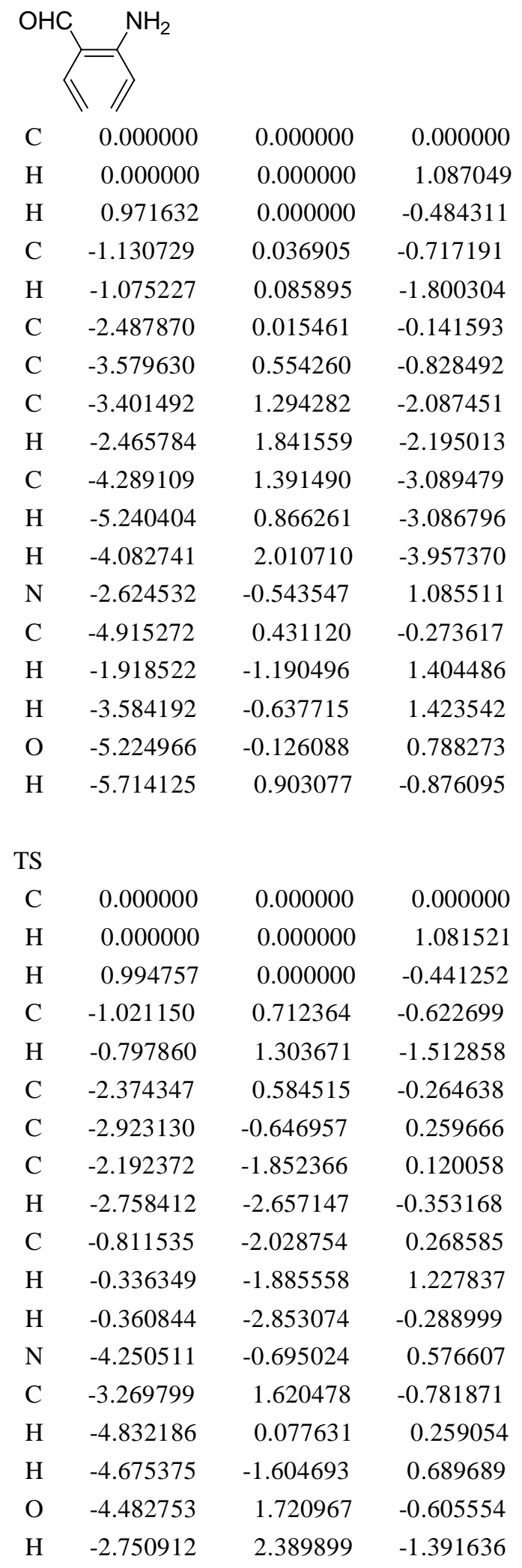




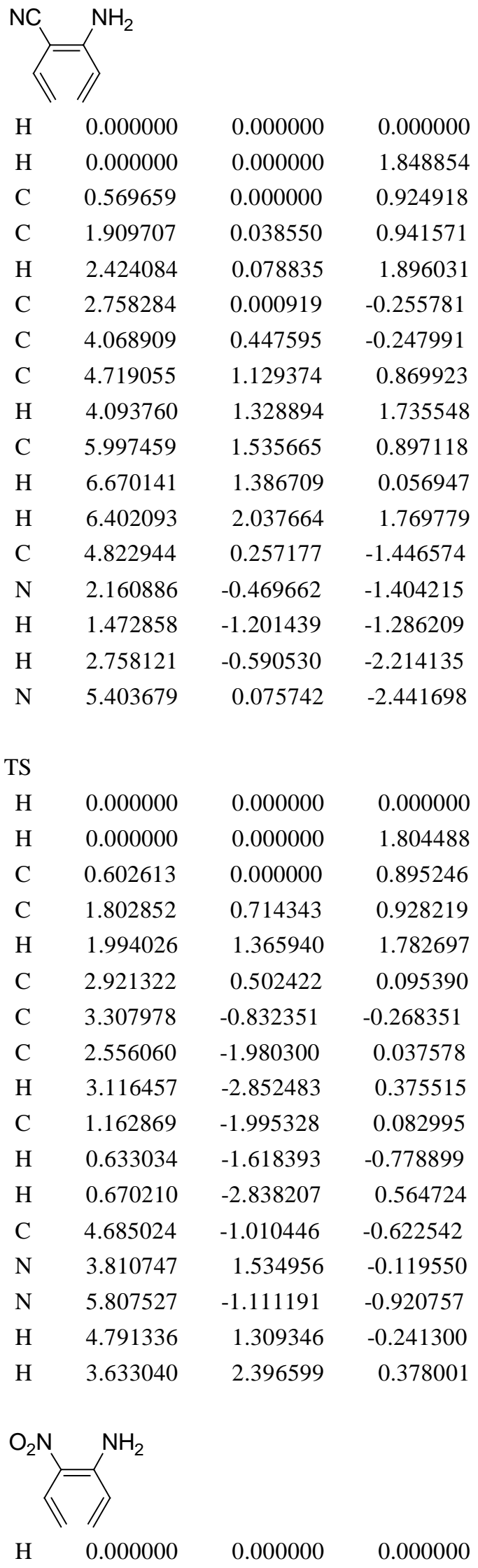




$\begin{array}{rrrr}\mathrm{C} & 0.000000 & 0.000000 & 1.086922 \\ \mathrm{H} & 0.969589 & 0.000000 & 1.575126 \\ \mathrm{C} & -1.131197 & -0.039027 & 1.801382 \\ \mathrm{H} & -1.081224 & -0.085019 & 2.884470 \\ \mathrm{C} & -2.484483 & 0.004234 & 1.201993 \\ \mathrm{C} & -3.539228 & -0.687904 & 1.807407 \\ \mathrm{C} & -3.284819 & -1.629888 & 2.898819 \\ \mathrm{H} & -2.231242 & -1.882454 & 2.984752 \\ \mathrm{C} & -4.111616 & -2.248171 & 3.757788 \\ \mathrm{H} & -3.687789 & -2.940613 & 4.480020 \\ \mathrm{H} & -5.181317 & -2.103249 & 3.767883 \\ \mathrm{~N} & -2.587658 & 0.742903 & 0.076568 \\ \mathrm{~N} & -4.879212 & -0.481909 & 1.339526 \\ \mathrm{H} & -1.870730 & 1.425920 & -0.118231 \\ \mathrm{H} & -3.517990 & 0.836607 & -0.323286 \\ \mathrm{O} & -5.080730 & 0.138693 & 0.265643 \\ \mathrm{O} & -5.816697 & -0.918024 & 2.013192\end{array}$

$\begin{array}{crrr}\text { TS } & & & \\ \mathrm{C} & 0.000000 & 0.000000 & 0.000000 \\ \mathrm{H} & 0.000000 & 0.000000 & 1.080120 \\ \mathrm{H} & 1.013437 & 0.000000 & -0.407009 \\ \mathrm{C} & -0.958496 & -0.729253 & -0.711047 \\ \mathrm{H} & -0.607491 & -1.417630 & -1.481122 \\ \mathrm{C} & -2.360982 & -0.519106 & -0.740393 \\ \mathrm{C} & -2.858320 & 0.839495 & -0.749590 \\ \mathrm{C} & -2.041202 & 1.957084 & -0.528664 \\ \mathrm{H} & -2.204909 & 2.820844 & -1.166862 \\ \mathrm{C} & -0.963708 & 1.915932 & 0.350554 \\ \mathrm{H} & -1.154508 & 1.514302 & 1.336099 \\ \mathrm{H} & -0.247637 & 2.734703 & 0.337149 \\ \mathrm{~N} & -4.133992 & 1.144510 & -1.392971 \\ \mathrm{~N} & -3.162743 & -1.586559 & -1.020491 \\ \mathrm{H} & -2.712597 & -2.479245 & -1.160933 \\ \mathrm{H} & -4.054624 & -1.407327 & -1.465871 \\ \mathrm{O} & -4.901194 & 0.224269 & -1.734767 \\ \mathrm{O} & -4.425745 & 2.330909 & -1.555082\end{array}$

3<smiles>Oc1ccccc1O</smiles>

$\begin{array}{llll}\mathrm{H} & 0.000000 & 0.000000 & 0.000000\end{array}$ 


\begin{tabular}{|c|c|c|c|}
\hline $\mathrm{H}$ & 0.000000 & 0.000000 & 1.846432 \\
\hline $\mathrm{C}$ & 0.571279 & 0.000000 & 0.924473 \\
\hline $\mathrm{C}$ & 1.916475 & -0.040589 & 0.938972 \\
\hline $\mathrm{H}$ & 2.428890 & -0.079805 & 1.896340 \\
\hline $\mathrm{C}$ & 2.754136 & 0.010844 & -0.237393 \\
\hline $\mathrm{C}$ & 4.106718 & -0.175448 & -0.301462 \\
\hline $\mathrm{C}$ & 4.959760 & -0.553414 & 0.811320 \\
\hline $\mathrm{H}$ & 4.467596 & -0.712599 & 1.765893 \\
\hline $\mathrm{C}$ & 6.287595 & -0.715668 & 0.713699 \\
\hline $\mathrm{H}$ & 6.808454 & -0.576278 & -0.227531 \\
\hline $\mathrm{H}$ & 6.881384 & -0.994522 & 1.578435 \\
\hline $\mathrm{O}$ & 4.743471 & -0.027854 & -1.496582 \\
\hline $\mathrm{O}$ & 2.148214 & 0.269392 & -1.466050 \\
\hline $\mathrm{H}$ & 1.474315 & 0.956430 & -1.330319 \\
\hline $\mathrm{H}$ & 4.056951 & 0.231805 & -2.138893 \\
\hline \multicolumn{4}{|l|}{ TS } \\
\hline $\mathrm{H}$ & 0.000000 & 0.000000 & 0.000000 \\
\hline $\mathrm{H}$ & 0.000000 & 0.000000 & 1.808772 \\
\hline $\mathrm{C}$ & 0.597922 & 0.000000 & 0.897657 \\
\hline $\mathrm{C}$ & 1.803594 & -0.702715 & 0.914496 \\
\hline $\mathrm{H}$ & 2.049183 & -1.349209 & 1.757235 \\
\hline $\mathrm{C}$ & 2.849358 & -0.506412 & 0.008485 \\
\hline $\mathrm{C}$ & 3.171355 & 0.765404 & -0.528261 \\
\hline $\mathrm{C}$ & 2.511927 & 1.952411 & -0.205938 \\
\hline $\mathrm{H}$ & 3.162772 & 2.799334 & 0.032384 \\
\hline $\mathrm{C}$ & 1.141067 & 2.047102 & 0.049122 \\
\hline $\mathrm{H}$ & 0.441584 & 1.715477 & -0.701604 \\
\hline $\mathrm{H}$ & 0.790731 & 2.914901 & 0.607248 \\
\hline $\mathrm{O}$ & 3.803324 & -1.483374 & -0.071702 \\
\hline $\mathrm{O}$ & 4.458481 & 0.794206 & -1.053566 \\
\hline $\mathrm{H}$ & 4.590272 & -1.090566 & -0.489548 \\
\hline $\mathrm{H}$ & 4.643843 & 1.686414 & -1.380105 \\
\hline & & & \\
\hline $\mathrm{H}$ & 0.000000 & 0.000000 & 0.000000 \\
\hline $\mathrm{H}$ & 0.000000 & 0.000000 & 1.853302 \\
\hline $\mathrm{C}$ & 0.563751 & 0.000000 & 0.926734 \\
\hline $\mathrm{C}$ & 1.906973 & -0.000020 & 0.936533 \\
\hline $\mathrm{H}$ & 2.437971 & -0.000019 & 1.884465 \\
\hline $\mathrm{C}$ & 2.728510 & -0.000043 & -0.244580 \\
\hline $\mathrm{C}$ & 4.082741 & -0.000046 & -0.377722 \\
\hline
\end{tabular}




$\begin{array}{llll}\mathrm{C} & 5.025117 & -0.000109 & 0.727962 \\ \mathrm{H} & 4.599097 & -0.000597 & 1.726656 \\ \mathrm{C} & 6.356657 & 0.000317 & 0.571424 \\ \mathrm{H} & 6.812758 & 0.000742 & -0.412691 \\ \mathrm{H} & 7.018917 & 0.000200 & 1.431169 \\ \mathrm{O} & 4.644187 & 0.000136 & -1.620434 \\ \mathrm{~F} & 2.037859 & 0.000065 & -1.434819 \\ \mathrm{H} & 3.916936 & 0.000492 & -2.265822\end{array}$

TS

$\begin{array}{lrrr}\mathrm{H} & 0.000000 & 0.000000 & 0.000000 \\ \mathrm{H} & 0.000000 & 0.000000 & 1.808027 \\ \mathrm{C} & 0.597698 & 0.000000 & 0.896836 \\ \mathrm{C} & 1.799167 & 0.717269 & 0.918410 \\ \mathrm{H} & 2.026049 & 1.390428 & 1.746657 \\ \mathrm{C} & 2.856576 & 0.477145 & 0.053958 \\ \mathrm{C} & 3.277475 & -0.775376 & -0.446568 \\ \mathrm{C} & 2.575594 & -1.950916 & -0.172052 \\ \mathrm{H} & 3.196688 & -2.808874 & 0.082761 \\ \mathrm{C} & 1.190107 & -2.006389 & -0.013921 \\ \mathrm{H} & 0.560699 & -1.627773 & -0.803907 \\ \mathrm{H} & 0.768916 & -2.872975 & 0.493852 \\ \mathrm{O} & 4.560253 & -0.882346 & -0.900623 \\ \mathrm{~F} & 3.866499 & 1.416068 & 0.037841 \\ \mathrm{H} & 4.992323 & -0.022427 & -0.757916\end{array}$

$\begin{array}{lrrr} & \mathrm{OHC} \\ & & & \\ \mathrm{H} & & & \\ \mathrm{H} & & & \\ \mathrm{H} & 0.000000 & 0.000000 & 0.000000 \\ \mathrm{C} & 0.572710 & 0.000000 & 0.923758 \\ \mathrm{C} & 1.913086 & 0.007339 & 0.946185 \\ \mathrm{H} & 2.399908 & 0.033011 & 1.919625 \\ \mathrm{C} & 2.794540 & -0.061606 & -0.229558 \\ \mathrm{C} & 4.068896 & 0.507393 & -0.256949 \\ \mathrm{C} & 4.637646 & 1.280444 & 0.847043 \\ \mathrm{H} & 3.999121 & 1.448802 & 1.707056 \\ \mathrm{C} & 5.878756 & 1.785753 & 0.819370 \\ \mathrm{H} & 6.268647 & 2.360248 & 1.653950 \\ \mathrm{H} & 6.529240 & 1.638176 & -0.036366 \\ \mathrm{O} & 4.857427 & 0.392510 & -1.319801 \\ \mathrm{C} & 2.359295 & -0.797956 & -1.396913 \\ \mathrm{H} & 1.377731 & -1.295300 & -1.325353\end{array}$




$\begin{array}{rrrr}\mathrm{O} & 3.016158 & -0.927651 & -2.446293 \\ \mathrm{H} & 4.344587 & -0.135571 & -2.009045 \\ \mathrm{TS} & & & \\ \mathrm{H} & 0.000000 & 0.000000 & 0.000000 \\ \mathrm{C} & 0.000000 & 0.000000 & 1.087986 \\ \mathrm{H} & 0.986998 & 0.000000 & 1.528789 \\ \mathrm{C} & -0.987203 & 0.703193 & 1.769911 \\ \mathrm{H} & -1.709131 & 1.300016 & 1.209217 \\ \mathrm{C} & -1.215865 & 0.557126 & 3.150570 \\ \mathrm{C} & -0.983397 & -0.680684 & 3.850173 \\ \mathrm{C} & -0.755607 & -1.878554 & 3.154438 \\ \mathrm{H} & -1.362740 & -2.707222 & 3.519665 \\ \mathrm{C} & -0.047542 & -2.046245 & 1.957344 \\ \mathrm{H} & 1.014874 & -1.862585 & 1.915677 \\ \mathrm{H} & -0.342263 & -2.888264 & 1.327582 \\ \mathrm{O} & -1.351006 & -0.795694 & 5.139242 \\ \mathrm{C} & -2.033394 & 1.577784 & 3.795627 \\ \mathrm{H} & -2.371052 & 2.397467 & 3.131772 \\ \mathrm{O} & -2.351419 & 1.604064 & 4.988007 \\ \mathrm{H} & -1.757721 & 0.064026 & 5.421900\end{array}$

$\begin{array}{lrrr}\text { NC } & \\ \end{array}$

TS

$\begin{array}{llll}\mathrm{H} & 0.000000 & 0.000000 & 0.000000\end{array}$ 


\begin{tabular}{|c|c|c|c|}
\hline $\mathrm{H}$ & 0.000000 & 0.000000 & 1.804770 \\
\hline $\mathrm{C}$ & 0.601463 & 0.000000 & 0.909643 \\
\hline $\mathrm{C}$ & 1.802510 & 0.715280 & 0.878362 \\
\hline $\mathrm{H}$ & 1.994539 & 1.398744 & 0.051742 \\
\hline $\mathrm{C}$ & 2.915509 & 0.488869 & 1.695961 \\
\hline $\mathrm{C}$ & 3.299700 & -0.827997 & 2.103168 \\
\hline $\mathrm{C}$ & 2.549936 & -1.984494 & 1.821421 \\
\hline $\mathrm{H}$ & 3.112398 & -2.863617 & 1.507120 \\
\hline $\mathrm{C}$ & 1.158294 & -1.995322 & 1.763126 \\
\hline $\mathrm{H}$ & 0.621181 & -1.595846 & 2.609791 \\
\hline $\mathrm{H}$ & 0.665920 & -2.843746 & 1.291551 \\
\hline $\mathrm{C}$ & 4.677209 & -0.971739 & 2.470526 \\
\hline $\mathrm{O}$ & 3.807630 & 1.511578 & 1.776592 \\
\hline $\mathrm{H}$ & 4.668209 & 1.186752 & 2.100061 \\
\hline $\mathrm{N}$ & 5.799319 & -1.016839 & 2.784295 \\
\hline & & & \\
\hline $\mathrm{H}$ & 0.000000 & 0.000000 & 0.000000 \\
\hline $\mathrm{H}$ & 0.000000 & 0.000000 & 1.852109 \\
\hline $\mathrm{C}$ & 0.561357 & 0.000000 & 0.922074 \\
\hline $\mathrm{C}$ & 1.902027 & -0.004140 & 0.982723 \\
\hline $\mathrm{H}$ & 2.333457 & -0.020450 & 1.978213 \\
\hline $\mathrm{C}$ & 2.917732 & -0.027189 & -0.071573 \\
\hline $\mathrm{C}$ & 4.271874 & 0.220289 & 0.188336 \\
\hline $\mathrm{C}$ & 4.757653 & 0.663836 & 1.498582 \\
\hline $\mathrm{H}$ & 4.024699 & 0.927549 & 2.249915 \\
\hline $\mathrm{C}$ & 6.064288 & 0.781726 & 1.772881 \\
\hline $\mathrm{H}$ & 6.824321 & 0.537625 & 1.038490 \\
\hline $\mathrm{H}$ & 6.398395 & 1.128624 & 2.745659 \\
\hline $\mathrm{O}$ & 5.222714 & 0.101752 & -0.722674 \\
\hline $\mathrm{N}$ & 2.534379 & -0.340068 & -1.417109 \\
\hline $\mathrm{O}$ & 1.346563 & -0.455318 & -1.701937 \\
\hline $\mathrm{O}$ & 3.428424 & -0.495382 & -2.305055 \\
\hline $\mathrm{H}$ & 4.757701 & -0.196874 & -1.567791 \\
\hline \multicolumn{4}{|c|}{ TS } \\
\hline $\mathrm{H}$ & 0.000000 & 0.000000 & 0.000000 \\
\hline $\mathrm{H}$ & 0.000000 & 0.000000 & 1.800380 \\
\hline $\mathrm{C}$ & 0.606365 & 0.000000 & 0.892547 \\
\hline $\mathrm{C}$ & 1.803449 & -0.724153 & 0.938166 \\
\hline $\mathrm{H}$ & 1.945115 & -1.447868 & 1.740436 \\
\hline $\mathrm{C}$ & 2.975253 & -0.500835 & 0.196988 \\
\hline
\end{tabular}




$\begin{array}{lrrr}\mathrm{C} & 3.375237 & 0.842113 & -0.145086 \\ \mathrm{C} & 2.580149 & 1.971424 & 0.101855 \\ \mathrm{H} & 3.081851 & 2.849279 & 0.500040 \\ \mathrm{C} & 1.194658 & 1.925412 & 0.004959 \\ \mathrm{H} & 0.781677 & 1.488857 & -0.893268 \\ \mathrm{H} & 0.613872 & 2.752525 & 0.406469 \\ \mathrm{O} & 3.824739 & -1.536648 & 0.121207 \\ \mathrm{~N} & 4.786372 & 1.124039 & -0.368885 \\ \mathrm{O} & 5.144221 & 2.294299 & -0.456623 \\ \mathrm{O} & 5.595847 & 0.174702 & -0.499142 \\ \mathrm{H} & 4.704952 & -1.177623 & -0.156838\end{array}$

4<smiles>Fc1ccccc1F</smiles>

$\begin{array}{llll}\mathrm{H} & 0.000000 & 0.000000 & 0.000000\end{array}$

$\begin{array}{llll}\mathrm{H} & 0.000000 & 0.000000 & 1.853799\end{array}$

$\begin{array}{llll}\text { C } & 0.563508 & 0.000000 & 0.926880\end{array}$

$\begin{array}{llll}\text { C } & 1.905001 & -0.000160 & 0.934045\end{array}$

$\begin{array}{llll}\mathrm{H} & 2.442643 & -0.000172 & 1.877703\end{array}$

$\begin{array}{llll}\text { C } & 2.709828 & -0.000448 & -0.267285\end{array}$

$\begin{array}{llll}\text { C } & 4.062426 & -0.000568 & -0.357646\end{array}$

$\begin{array}{llll}\text { C } & 5.020724 & -0.000297 & 0.725054\end{array}$

$\begin{array}{llll}\mathrm{H} & 4.614687 & -0.000177 & 1.732357\end{array}$

$\begin{array}{llll}\text { C } & 6.349269 & -0.000172 & 0.538221\end{array}$

$\mathrm{H} \quad 6.783372 \quad-0.000286 \quad-0.455828$

$\begin{array}{lllr}\mathrm{H} & 7.031888 & -0.000049 & 1.381275\end{array}$

F $\quad 4.593846 \quad-0.000003 \quad-1.600116$

$\begin{array}{llll}\text { F } & 2.017257 & -0.000209 & -1.427197\end{array}$

$\begin{array}{cccc}\text { TS } & & & \\ \text { H } & 0.000000 & 0.000000 & 0.000000 \\ \text { C } & 0.000000 & 0.000000 & 1.077735 \\ \text { H } & 0.999955 & 0.000000 & 1.509336 \\ \text { C } & -0.999993 & -0.699449 & 1.756546 \\ \text { H } & -0.747176 & -1.353006 & 2.591854 \\ \text { C } & -2.360999 & -0.467541 & 1.592291 \\ \text { C } & -2.933624 & 0.789521 & 1.325442 \\ \text { C } & -2.217595 & 1.973506 & 1.189131 \\ \text { C } & -0.935374 & 2.053390 & 0.641842 \\ \text { H } & -0.333374 & 2.926999 & 0.887996 \\ \text { H } & -0.768968 & 1.688080 & -0.358345 \\ \text { H } & -2.652873 & 2.830512 & 1.703783\end{array}$




$$
\begin{array}{rrrr}
\text { F } & -4.250270 & 0.884425 & 1.621653 \\
\text { F } & -3.218092 & -1.381408 & 2.102727
\end{array}
$$

$\begin{array}{lrrr} & \\ & & & \\ & & & \\ \mathrm{H} & 0.000000 & 0.000000 & 0.000000 \\ \mathrm{H} & 0.000000 & 0.000000 & 1.858667 \\ \mathrm{C} & 0.557923 & 0.000000 & 0.926342 \\ \mathrm{C} & 1.899358 & 0.000079 & 0.956947 \\ \mathrm{H} & 2.374541 & 0.000207 & 1.934853 \\ \mathrm{C} & 2.809469 & -0.000009 & -0.193223 \\ \mathrm{C} & 4.167376 & 0.000293 & -0.021149 \\ \mathrm{C} & 4.936815 & 0.000703 & 1.206774 \\ \mathrm{H} & 4.379105 & 0.000539 & 2.136318 \\ \mathrm{C} & 6.279109 & 0.001226 & 1.234587 \\ \mathrm{H} & 6.870922 & 0.001388 & 0.325674 \\ \mathrm{H} & 6.815838 & 0.001488 & 2.177560 \\ \mathrm{C} & 2.309678 & -0.000646 & -1.591852 \\ \mathrm{~F} & 4.952921 & 0.000170 & -1.125416 \\ \mathrm{H} & 3.095604 & -0.001766 & -2.366989 \\ \mathrm{O} & 1.134809 & -0.000118 & -1.918645\end{array}$

TS

$\begin{array}{llll}\mathrm{H} & 0.000000 & 0.000000 & 0.000000\end{array}$

$\begin{array}{llll}\text { C } & 0.000000 & 0.000000 & 1.079554\end{array}$

$\begin{array}{llll}\mathrm{H} & 0.994692 & 0.000000 & 1.521546\end{array}$

$\begin{array}{llll}\text { C } & -1.024066 & -0.684724 & 1.724256\end{array}$

$\begin{array}{llll}\mathrm{H} & -0.804014 & -1.312772 & 2.586536\end{array}$

$\begin{array}{llll}\text { C } & -2.387923 & -0.527320 & 1.429601\end{array}$

$\begin{array}{llll}\text { C } & -2.904866 & 0.713855 & 0.993849\end{array}$

$\begin{array}{llll}\text { C } & -2.214030 & 1.920823 & 0.959089\end{array}$

$\begin{array}{llll}\mathrm{H} & -2.770197 & 2.762130 & 1.374404\end{array}$

$\begin{array}{llll}\text { C } & -0.850867 & 2.073870 & 0.679903\end{array}$

$\mathrm{H} \quad-0.351184 \quad 2.944186 \quad 1.106554$

$\begin{array}{llll}\mathrm{H} & -0.460473 & 1.801187 & -0.286577\end{array}$

$\begin{array}{llll}\text { F } & -4.261445 & 0.823025 & 1.006764\end{array}$

$\begin{array}{llll}\text { C } & -3.334252 & -1.550333 & 1.949134\end{array}$

$\begin{array}{llll}\text { O } & -2.973407 & -2.554278 & 2.538128\end{array}$

$\begin{array}{llll}\mathrm{H} & -4.406007 & -1.357618 & 1.766223\end{array}$<smiles>N#Cc1ccccc1F</smiles> 


\begin{tabular}{|c|c|c|c|}
\hline $\mathrm{H}$ & 0.000000 & 0.000000 & 0.000000 \\
\hline $\mathrm{H}$ & 0.000000 & 0.000000 & 1.853207 \\
\hline $\mathrm{C}$ & 0.565529 & 0.000000 & 0.927285 \\
\hline $\mathrm{C}$ & 1.905511 & -0.000079 & 0.943891 \\
\hline $\mathrm{H}$ & 2.420230 & -0.000108 & 1.900307 \\
\hline $\mathrm{C}$ & 2.754463 & -0.000194 & -0.250039 \\
\hline $\mathrm{C}$ & 4.118872 & -0.000235 & -0.225055 \\
\hline $\mathrm{C}$ & 4.998283 & -0.000119 & 0.923296 \\
\hline $\mathrm{H}$ & 4.526631 & -0.000132 & 1.900347 \\
\hline $\mathrm{C}$ & 6.336697 & -0.000018 & 0.822026 \\
\hline $\mathrm{H}$ & 6.835644 & -0.000018 & -0.141075 \\
\hline $\mathrm{H}$ & 6.962123 & 0.000036 & 1.708426 \\
\hline $\mathrm{F}$ & 4.756830 & 0.000075 & -1.410765 \\
\hline $\mathrm{C}$ & 2.112325 & -0.000114 & -1.530627 \\
\hline $\mathrm{N}$ & 1.546807 & -0.000041 & -2.547288 \\
\hline \multicolumn{4}{|c|}{ TS } \\
\hline $\mathrm{H}$ & 0.000000 & 0.000000 & 0.000000 \\
\hline $\mathrm{H}$ & 0.000000 & 0.000000 & 1.818391 \\
\hline $\mathrm{C}$ & 0.589490 & 0.000000 & 0.903927 \\
\hline $\mathrm{C}$ & 1.797830 & 0.689030 & 0.886402 \\
\hline $\mathrm{H}$ & 2.080623 & 1.310980 & 1.734742 \\
\hline $\mathrm{C}$ & 2.777426 & 0.504731 & -0.106495 \\
\hline $\mathrm{C}$ & 2.987074 & -0.744582 & -0.735538 \\
\hline $\mathrm{C}$ & 2.383110 & -1.942886 & -0.368426 \\
\hline $\mathrm{H}$ & 3.074414 & -2.783431 & -0.299288 \\
\hline $\mathrm{C}$ & 1.080166 & -2.082521 & 0.119242 \\
\hline $\mathrm{H}$ & 0.239840 & -1.801463 & -0.493553 \\
\hline $\mathrm{H}$ & 0.875115 & -2.946552 & 0.751418 \\
\hline $\mathrm{F}$ & 4.133832 & -0.842496 & -1.443212 \\
\hline $\mathrm{C}$ & 3.818329 & 1.483082 & -0.238551 \\
\hline $\mathrm{N}$ & 4.630898 & 2.310309 & -0.332116 \\
\hline & & & \\
\hline $\mathrm{H}$ & 0.000000 & 0.000000 & 0.000000 \\
\hline $\mathrm{H}$ & 0.000000 & 0.000000 & 1.851792 \\
\hline $\mathrm{C}$ & 0.564458 & 0.000000 & 0.925058 \\
\hline $\mathrm{C}$ & 1.904760 & -0.014145 & 0.960624 \\
\hline $\mathrm{H}$ & 2.392473 & -0.030419 & 1.931186 \\
\hline $\mathrm{C}$ & 2.818935 & 0.024947 & -0.170758 \\
\hline $\mathrm{C}$ & 4.172213 & -0.069928 & -0.101551 \\
\hline $\mathrm{C}$ & 4.981127 & -0.261604 & 1.085784 \\
\hline
\end{tabular}




$\begin{array}{cccc}\mathrm{H} & 4.458208 & -0.321881 & 2.034074 \\ \mathrm{C} & 6.317948 & -0.363968 & 1.045841 \\ \mathrm{H} & 6.866266 & -0.310430 & 0.111634 \\ \mathrm{H} & 6.893051 & -0.503132 & 1.955095 \\ \mathrm{~F} & 4.875401 & -0.023979 & -1.245781 \\ \mathrm{~N} & 2.235163 & 0.200498 & -1.516705 \\ \mathrm{O} & 1.371540 & -0.606408 & -1.858575 \\ \mathrm{O} & 2.633292 & 1.142989 & -2.192353\end{array}$

TS

$\begin{array}{lrrr}\mathrm{H} & 0.000000 & 0.000000 & 0.000000 \\ \mathrm{H} & 0.000000 & 0.000000 & 1.813473 \\ \mathrm{C} & 0.592790 & 0.000000 & 0.900377 \\ \mathrm{C} & 1.798784 & 0.695258 & 0.907285 \\ \mathrm{H} & 2.056190 & 1.344420 & 1.741788 \\ \mathrm{C} & 2.822862 & 0.499154 & -0.024347 \\ \mathrm{C} & 3.161579 & -0.750779 & -0.581257 \\ \mathrm{C} & 2.503501 & -1.935977 & -0.268991 \\ \mathrm{H} & 3.161074 & -2.783356 & -0.076930 \\ \mathrm{C} & 1.139341 & -2.029064 & 0.013286 \\ \mathrm{H} & 0.426887 & -1.685373 & -0.718831 \\ \mathrm{H} & 0.795391 & -2.892507 & 0.580661 \\ \mathrm{~F} & 4.401538 & -0.871350 & -1.093026 \\ \mathrm{~N} & 3.842868 & 1.568251 & -0.110608 \\ \mathrm{O} & 4.093463 & 2.207676 & 0.910733 \\ \mathrm{O} & 4.338738 & 1.773593 & -1.213465\end{array}$

5

\begin{tabular}{|c|c|c|c|}
\hline $\mathrm{C}$ & 0.000000 & 0.000000 & 0.000000 \\
\hline $\mathrm{H}$ & 0.000000 & 0.000000 & 1.086680 \\
\hline $\mathrm{H}$ & 0.973814 & 0.000000 & -0.480317 \\
\hline $\mathrm{C}$ & -1.127169 & 0.020842 & -0.727940 \\
\hline $\mathrm{H}$ & -1.033852 & 0.046401 & -1.811216 \\
\hline $\mathrm{C}$ & -2.491096 & -0.015718 & -0.181702 \\
\hline $\mathrm{C}$ & -3.585428 & 0.509573 & -0.832641 \\
\hline $\mathrm{C}$ & -3.445647 & 1.300903 & -2.052949 \\
\hline $\mathrm{H}$ & -2.426830 & 1.542162 & -2.343776 \\
\hline $\mathrm{C}$ & -4.443882 & 1.772583 & -2.820908 \\
\hline $\mathrm{H}$ & -4.215916 & 2.365628 & -3.701976 \\
\hline
\end{tabular}




\begin{tabular}{|c|c|c|c|}
\hline $\mathrm{H}$ & -5.489120 & 1.604892 & -2.593247 \\
\hline $\mathrm{C}$ & -2.676832 & -0.687405 & 1.129340 \\
\hline $\mathrm{C}$ & -4.975644 & 0.231118 & -0.317635 \\
\hline $\mathrm{H}$ & -5.138998 & -0.787236 & 0.074818 \\
\hline $\mathrm{O}$ & -5.893518 & 1.023197 & -0.391571 \\
\hline $\mathrm{O}$ & -3.658069 & -0.556882 & 1.842928 \\
\hline $\mathrm{H}$ & -1.848630 & -1.355408 & 1.435709 \\
\hline \multicolumn{4}{|l|}{ TS } \\
\hline $\mathrm{C}$ & 0.000000 & 0.000000 & 0.000000 \\
\hline $\mathrm{H}$ & 0.000000 & 0.000000 & 1.077744 \\
\hline $\mathrm{H}$ & 0.998965 & 0.000000 & -0.434632 \\
\hline $\mathrm{C}$ & -1.015352 & 0.664200 & -0.679871 \\
\hline $\mathrm{H}$ & -0.721569 & 1.307081 & -1.512980 \\
\hline $\mathrm{C}$ & -2.407281 & 0.491801 & -0.522151 \\
\hline $\mathrm{C}$ & -2.997325 & -0.780634 & -0.260386 \\
\hline $\mathrm{C}$ & -2.217301 & -1.940197 & -0.107314 \\
\hline $\mathrm{H}$ & -2.618172 & -2.825845 & -0.599153 \\
\hline $\mathrm{C}$ & -0.966085 & -2.026030 & 0.493179 \\
\hline $\mathrm{H}$ & -0.355426 & -2.907535 & 0.302355 \\
\hline $\mathrm{H}$ & -0.831630 & -1.610210 & 1.479260 \\
\hline $\mathrm{C}$ & -4.446941 & -1.030003 & -0.563152 \\
\hline $\mathrm{C}$ & -3.192021 & 1.673484 & -0.976758 \\
\hline $\mathrm{O}$ & -4.356692 & 1.921852 & -0.727975 \\
\hline $\mathrm{H}$ & -2.581825 & 2.403097 & -1.554229 \\
\hline $\mathrm{H}$ & -5.070224 & -0.148124 & -0.764695 \\
\hline $\mathrm{O}$ & -4.919120 & -2.153957 & -0.577861 \\
\hline & & & \\
\hline $\mathrm{H}$ & 0.000000 & 0.000000 & 0.000000 \\
\hline $\mathrm{H}$ & 0.000000 & 0.000000 & 1.851943 \\
\hline $\mathrm{C}$ & 0.566654 & 0.000000 & 0.926400 \\
\hline $\mathrm{C}$ & 1.909233 & 0.000040 & 0.949413 \\
\hline $\mathrm{H}$ & 2.409329 & 0.000453 & 1.911113 \\
\hline $\mathrm{C}$ & 2.766172 & 0.000381 & -0.238763 \\
\hline $\mathrm{C}$ & 4.148545 & -0.001813 & -0.232082 \\
\hline $\mathrm{C}$ & 4.920458 & -0.007736 & 1.008096 \\
\hline $\mathrm{H}$ & 4.344592 & -0.016442 & 1.928531 \\
\hline $\mathrm{C}$ & 6.259761 & -0.004436 & 1.126594 \\
\hline $\mathrm{H}$ & 6.919647 & 0.003522 & 0.270056 \\
\hline $\mathrm{H}$ & 6.708594 & -0.010348 & 2.115939 \\
\hline $\mathrm{C}$ & 4.826994 & 0.001344 & -1.563369 \\
\hline
\end{tabular}




$\begin{array}{llll}\mathrm{C} & 2.046587 & 0.003757 & -1.487085 \\ \mathrm{~N} & 1.382607 & 0.006385 & -2.443140 \\ \mathrm{H} & 4.139118 & 0.003627 & -2.429856 \\ \mathrm{O} & 6.029608 & 0.001942 & -1.743118\end{array}$

$\begin{array}{crrr}\text { TS } & & & \\ \mathrm{H} & 0.000000 & 0.000000 & 0.000000 \\ \mathrm{H} & 0.000000 & 0.000000 & 1.812558 \\ \mathrm{C} & 0.594374 & 0.000000 & 0.912952 \\ \mathrm{C} & 1.809285 & 0.679442 & 0.908257 \\ \mathrm{H} & 2.033035 & 1.344652 & 0.074054 \\ \mathrm{C} & 2.872160 & 0.504353 & 1.807748 \\ \mathrm{C} & 3.198100 & -0.767222 & 2.363297 \\ \mathrm{C} & 2.493752 & -1.948395 & 2.060485 \\ \mathrm{H} & 3.107120 & -2.831439 & 1.880322 \\ \mathrm{C} & 1.124317 & -2.029841 & 1.816484 \\ \mathrm{H} & 0.443525 & -1.643607 & 2.557765 \\ \mathrm{H} & 0.747245 & -2.906137 & 1.291351 \\ \mathrm{C} & 4.491507 & -0.950868 & 2.970817 \\ \mathrm{C} & 3.894922 & 1.591727 & 1.851000 \\ \mathrm{O} & 3.799331 & 2.622879 & 1.213227 \\ \mathrm{H} & 4.761949 & 1.413522 & 2.515132 \\ \mathrm{~N} & 5.512353 & -1.148330 & 3.494095\end{array}$

\begin{tabular}{lrrr}
$\mathrm{O}_{2} \mathrm{~N}$ & \multicolumn{3}{c}{$\mathrm{CHO}$} \\
$\mathrm{C}$ & $\mathrm{C}$ & & \\
$\mathrm{C}$ & 0.000000 & 0.000000 & 0.000000 \\
$\mathrm{H}$ & 0.000000 & 0.000000 & 1.085386 \\
$\mathrm{H}$ & 0.968924 & 0.000000 & -0.488081 \\
$\mathrm{C}$ & -1.125850 & 0.015107 & -0.731998 \\
$\mathrm{H}$ & -1.045043 & 0.026238 & -1.813564 \\
$\mathrm{C}$ & -2.480296 & -0.010355 & -0.208323 \\
$\mathrm{C}$ & -3.656622 & 0.138791 & -0.888685 \\
$\mathrm{C}$ & -3.659813 & 0.437297 & -2.324843 \\
$\mathrm{H}$ & -2.692707 & 0.679552 & -2.756545 \\
$\mathrm{C}$ & -4.723178 & 0.457242 & -3.143732 \\
$\mathrm{H}$ & -5.726209 & 0.241743 & -2.802512 \\
$\mathrm{H}$ & -4.584718 & 0.701755 & -4.193194 \\
$\mathrm{~N}$ & -2.530742 & -0.270594 & 1.257990 \\
$\mathrm{O}$ & -2.293785 & 0.681870 & 1.995397 \\
$\mathrm{O}$ & -2.759161 & -1.420244 & 1.617188 \\
$\mathrm{C}$ & -4.934405 & 0.032637 & -0.119846 \\
$\mathrm{H}$ & -4.826249 & -0.090525 & 0.972636
\end{tabular}




$\begin{array}{rrrr}\mathrm{O} & -6.045683 & 0.080145 & -0.609284 \\ \mathrm{TS} & & & \\ \mathrm{C} & 0.000000 & 0.000000 & 0.000000 \\ \mathrm{H} & 0.000000 & 0.000000 & 1.090114 \\ \mathrm{H} & 0.991296 & 0.000000 & -0.421250 \\ \mathrm{C} & -1.042983 & 0.668750 & -0.637510 \\ \mathrm{H} & -1.652855 & 1.360386 & -0.058081 \\ \mathrm{C} & -1.530799 & 0.422133 & -1.927357 \\ \mathrm{C} & -1.660417 & -0.852334 & -2.532884 \\ \mathrm{C} & -1.069860 & -1.973520 & -1.929914 \\ \mathrm{H} & -1.650603 & -2.892904 & -1.966983 \\ \mathrm{C} & 0.120305 & -1.937822 & -1.214715 \\ \mathrm{H} & 0.953095 & -1.405800 & -1.650573 \\ \mathrm{H} & 0.403081 & -2.792974 & -0.604528 \\ \mathrm{C} & -2.738789 & -1.152670 & -3.535660 \\ \mathrm{~N} & -2.307712 & 1.549380 & -2.508471 \\ \mathrm{O} & -2.271889 & 1.665335 & -3.733555 \\ \mathrm{O} & -2.891782 & 2.319231 & -1.749069 \\ \mathrm{H} & -3.351116 & -0.312290 & -3.894864 \\ \mathrm{O} & -2.945306 & -2.285484 & -3.927179\end{array}$

6

\begin{tabular}{|c|c|c|c|}
\hline $\mathrm{H}$ & 0.000000 & 0.000000 & 0.000000 \\
\hline $\mathrm{H}$ & 0.000000 & 0.000000 & 1.852620 \\
\hline $\mathrm{C}$ & 0.566063 & 0.000000 & 0.926887 \\
\hline $\mathrm{C}$ & 1.908115 & -0.000020 & 0.946493 \\
\hline $\mathrm{H}$ & 2.417379 & -0.000019 & 1.904241 \\
\hline $\mathrm{C}$ & 2.751945 & -0.000043 & -0.247721 \\
\hline $\mathrm{C}$ & 4.130292 & -0.000004 & -0.237809 \\
\hline $\mathrm{C}$ & 4.957114 & 0.000087 & 0.968063 \\
\hline $\mathrm{H}$ & 4.434549 & 0.000093 & 1.918606 \\
\hline $\mathrm{C}$ & 6.299346 & 0.000163 & 0.967149 \\
\hline $\mathrm{H}$ & 6.878135 & 0.000161 & 0.048154 \\
\hline $\mathrm{H}$ & 6.852576 & 0.000212 & 1.900602 \\
\hline $\mathrm{C}$ & 4.830690 & 0.000185 & -1.491127 \\
\hline $\mathrm{C}$ & 2.068938 & 0.000000 & -1.510548 \\
\hline $\mathrm{N}$ & 1.464356 & 0.000038 & -2.504363 \\
\hline $\mathrm{N}$ & 5.448677 & 0.000342 & -2.476665 \\
\hline
\end{tabular}




\begin{tabular}{|c|c|c|c|}
\hline \multicolumn{4}{|l|}{ TS } \\
\hline $\mathrm{H}$ & 0.000000 & 0.000000 & 0.000000 \\
\hline $\mathrm{H}$ & 0.000000 & 0.000000 & 1.814388 \\
\hline $\mathrm{C}$ & 0.592239 & 0.000000 & 0.900711 \\
\hline $\mathrm{C}$ & 1.805524 & -0.682705 & 0.904617 \\
\hline $\mathrm{H}$ & 2.035024 & -1.333616 & 1.748358 \\
\hline $\mathrm{C}$ & 2.856849 & -0.487765 & -0.011226 \\
\hline $\mathrm{C}$ & 3.181337 & 0.788937 & -0.558494 \\
\hline $\mathrm{C}$ & 2.478055 & 1.963378 & -0.229646 \\
\hline $\mathrm{H}$ & 3.096125 & 2.841304 & -0.041259 \\
\hline $\mathrm{C}$ & 1.111659 & 2.043670 & 0.024677 \\
\hline $\mathrm{H}$ & 0.423483 & 1.666255 & -0.714249 \\
\hline $\mathrm{H}$ & 0.740194 & 2.912306 & 0.566007 \\
\hline $\mathrm{C}$ & 4.468643 & 0.956847 & -1.174503 \\
\hline $\mathrm{C}$ & 3.835007 & -1.536123 & -0.105946 \\
\hline $\mathrm{N}$ & 4.584316 & -2.423269 & -0.175319 \\
\hline $\mathrm{N}$ & 5.489559 & 1.138277 & -1.701871 \\
\hline \multicolumn{4}{|c|}{$\mathrm{O}_{2} \mathrm{~N} \quad \mathrm{CN}$} \\
\hline $\mathrm{H}$ & 0.000000 & 0.000000 & 0.000000 \\
\hline $\mathrm{H}$ & 0.000000 & 0.000000 & 1.851221 \\
\hline $\mathrm{C}$ & 0.564949 & 0.000000 & 0.924700 \\
\hline $\mathrm{C}$ & 1.907233 & -0.014774 & 0.962715 \\
\hline $\mathrm{H}$ & 2.391430 & -0.029352 & 1.933534 \\
\hline $\mathrm{C}$ & 2.817129 & 0.032529 & -0.168251 \\
\hline $\mathrm{C}$ & 4.179679 & -0.077153 & -0.121275 \\
\hline $\mathrm{C}$ & 4.930455 & -0.346038 & 1.109892 \\
\hline $\mathrm{H}$ & 4.349350 & -0.474360 & 2.016633 \\
\hline $\mathrm{C}$ & 6.265679 & -0.451424 & 1.176512 \\
\hline $\mathrm{H}$ & 6.901565 & -0.342147 & 0.303138 \\
\hline $\mathrm{H}$ & 6.755357 & -0.653241 & 2.123564 \\
\hline $\mathrm{C}$ & 4.964582 & 0.022001 & -1.319980 \\
\hline $\mathrm{N}$ & 2.196656 & 0.251944 & -1.497075 \\
\hline $\mathrm{O}$ & 1.346670 & -0.562484 & -1.850218 \\
\hline $\mathrm{O}$ & 2.560441 & 1.231740 & -2.136373 \\
\hline $\mathrm{N}$ & 5.683251 & 0.067302 & -2.232929 \\
\hline \multicolumn{4}{|c|}{$\mathrm{TS}$} \\
\hline $\mathrm{H}$ & 0.000000 & 0.000000 & 0.000000 \\
\hline $\mathrm{H}$ & 0.000000 & 0.000000 & 1.811151 \\
\hline $\mathrm{C}$ & 0.594493 & 0.000000 & 0.898469 \\
\hline
\end{tabular}




$\begin{array}{lrrc}\mathrm{C} & 1.813524 & 0.671357 & 0.923704 \\ \mathrm{H} & 2.034114 & 1.342390 & 1.751571 \\ \mathrm{C} & 2.890578 & 0.452118 & 0.058174 \\ \mathrm{C} & 3.293355 & -0.815621 & -0.430258 \\ \mathrm{C} & 2.528558 & -1.969717 & -0.170044 \\ \mathrm{H} & 3.090894 & -2.866178 & 0.087398 \\ \mathrm{C} & 1.140993 & -1.991783 & -0.087563 \\ \mathrm{H} & 0.576879 & -1.536077 & -0.886264 \\ \mathrm{H} & 0.657209 & -2.854998 & 0.364653 \\ \mathrm{C} & 4.639107 & -1.058402 & -0.876241 \\ \mathrm{~N} & 3.884642 & 1.553537 & -0.005621 \\ \mathrm{O} & 3.910190 & 2.366242 & 0.916860 \\ \mathrm{O} & 4.588861 & 1.601706 & -1.009347 \\ \mathrm{~N} & 5.690813 & -1.401724 & -1.235341\end{array}$

$\begin{array}{lrrr}\mathrm{O}_{2} \mathrm{~N} & \\ \mathrm{C} & 0.000000 & 0.000000 & 0.000000 \\ \mathrm{H} & 0.000000 & 0.000000 & 1.083663 \\ \mathrm{H} & 0.964822 & 0.000000 & -0.496596 \\ \mathrm{C} & -1.124941 & -0.014071 & -0.731221 \\ \mathrm{H} & -1.036926 & -0.022004 & -1.812942 \\ \mathrm{C} & -2.491867 & 0.026428 & -0.233192 \\ \mathrm{C} & -3.612649 & -0.081199 & -0.990960 \\ \mathrm{C} & -3.647748 & -0.383686 & -2.414152 \\ \mathrm{H} & -2.687047 & -0.646541 & -2.845287 \\ \mathrm{C} & -4.730325 & -0.407611 & -3.206530 \\ \mathrm{H} & -5.723547 & -0.151009 & -2.857273 \\ \mathrm{H} & -4.626873 & -0.682700 & -4.251092 \\ \mathrm{~N} & -2.648996 & 0.283453 & 1.220865 \\ \mathrm{~N} & -4.930479 & 0.040973 & -0.317524 \\ \mathrm{O} & -2.115181 & -0.515536 & 1.982378 \\ \mathrm{O} & -3.264891 & 1.291529 & 1.546637 \\ \mathrm{O} & -5.692573 & 0.898829 & -0.749268 \\ \mathrm{O} & -5.169506 & -0.747952 & 0.589282\end{array}$

TS

$\begin{array}{llll}\text { C } & 0.000000 & 0.000000 & 0.000000\end{array}$

$\begin{array}{llll}\mathrm{H} & 0.000000 & 0.000000 & 1.076759\end{array}$

$\begin{array}{llll}\mathrm{H} & 1.002535 & 0.000000 & -0.428072\end{array}$ 


$\begin{array}{lrrr}\mathrm{C} & -0.998959 & -0.651213 & -0.718581 \\ \mathrm{H} & -0.709619 & -1.359665 & -1.495320 \\ \mathrm{C} & -2.374950 & -0.393875 & -0.699673 \\ \mathrm{C} & -2.949902 & 0.881845 & -0.557458 \\ \mathrm{C} & -2.208025 & 2.003896 & -0.186928 \\ \mathrm{H} & -2.433286 & 2.938692 & -0.694059 \\ \mathrm{C} & -1.118067 & 1.910807 & 0.667334 \\ \mathrm{H} & -1.235061 & 1.320344 & 1.564102 \\ \mathrm{H} & -0.432579 & 2.749451 & 0.756035 \\ \mathrm{~N} & -4.235482 & 1.180222 & -1.232836 \\ \mathrm{~N} & -3.217051 & -1.535556 & -1.150573 \\ \mathrm{O} & -2.827616 & -2.203055 & -2.101492 \\ \mathrm{O} & -4.198602 & -1.767967 & -0.455583 \\ \mathrm{O} & -4.626321 & 0.427877 & -2.120756 \\ \mathrm{O} & -4.807826 & 2.206455 & -0.869304\end{array}$

\section{Table S6}

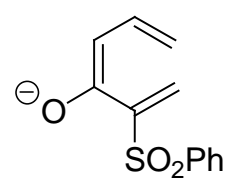

$\begin{array}{lrrr}\mathrm{C} & 0.000000 & 0.000000 & 0.000000 \\ \mathrm{H} & 0.000000 & 0.000000 & 1.087256 \\ \mathrm{H} & 0.973209 & 0.000000 & -0.489026 \\ \mathrm{C} & -1.157981 & -0.017019 & -0.709577 \\ \mathrm{H} & -2.084879 & -0.014202 & -0.133468 \\ \mathrm{C} & -1.299200 & -0.057566 & -2.133574 \\ \mathrm{H} & -0.388308 & -0.162292 & -2.724860 \\ \mathrm{C} & -2.468895 & -0.029479 & -2.896147 \\ \mathrm{C} & -3.789218 & 0.203114 & -2.184864 \\ \mathrm{C} & -4.099317 & 1.228127 & -1.383155 \\ \mathrm{H} & -5.085875 & 1.337219 & -0.942653 \\ \mathrm{H} & -3.342541 & 1.968625 & -1.141282 \\ \mathrm{O} & -2.529747 & -0.144205 & -4.160564 \\ \mathrm{~S} & -5.161018 & -0.956443 & -2.513978 \\ \mathrm{O} & -4.617130 & -2.298128 & -2.782534 \\ \mathrm{O} & -6.153489 & -0.799860 & -1.421883 \\ \mathrm{C} & -6.016791 & -0.373514 & -4.010717 \\ \mathrm{C} & -7.409568 & -0.290504 & -3.961674 \\ \mathrm{H} & -7.921600 & -0.511428 & -3.031041 \\ \mathrm{C} & -8.109351 & 0.082166 & -5.110406\end{array}$




\begin{tabular}{|c|c|c|c|}
\hline $\mathrm{H}$ & -9.194881 & 0.148298 & -5.0 \\
\hline $\mathrm{C}$ & -7.415433 & 0.375329 & -6.286917 \\
\hline $\mathrm{H}$ & -7.962349 & 0.670762 & -7.180166 \\
\hline $\mathrm{C}$ & -6.020514 & 0.298059 & -6.314016 \\
\hline $\mathrm{H}$ & -5.481316 & 0.537150 & -7.227968 \\
\hline $\mathrm{C}$ & -5.305375 & -0.081342 & -5.175919 \\
\hline $\mathrm{H}$ & -4.212920 & -0.121870 & -5.138418 \\
\hline \multicolumn{4}{|l|}{$\mathrm{TS}$} \\
\hline $\mathrm{C}$ & 0.000000 & 0.000000 & 0.000000 \\
\hline $\mathrm{H}$ & 0.000000 & 0.000000 & 1.080435 \\
\hline $\mathrm{H}$ & 1.003903 & 0.000000 & -0.429340 \\
\hline $\mathrm{C}$ & -1.012292 & 0.683314 & -0.690631 \\
\hline $\mathrm{H}$ & -0.712679 & 1.298864 & -1.548448 \\
\hline $\mathrm{C}$ & -2.381316 & 0.574071 & -0.477039 \\
\hline $\mathrm{H}$ & -3.030642 & 1.315408 & -0.944475 \\
\hline $\mathrm{C}$ & -3.141746 & -0.575965 & -0.021296 \\
\hline $\mathrm{C}$ & -2.371185 & -1.839167 & -0.064242 \\
\hline $\mathrm{C}$ & -1.103487 & -2.000493 & 0.471305 \\
\hline $\mathrm{H}$ & -0.920695 & -1.676267 & 1.487030 \\
\hline $\mathrm{H}$ & -0.497555 & -2.840180 & 0.141574 \\
\hline $\mathrm{O}$ & -4.388624 & -0.525081 & 0.115736 \\
\hline$S$ & -2.926333 & -3.074089 & -1.207406 \\
\hline $\mathrm{O}$ & -1.800910 & -4.001271 & -1.494929 \\
\hline $\mathrm{O}$ & -3.652028 & -2.486295 & -2.352313 \\
\hline $\mathrm{C}$ & -4.146789 & -4.082121 & -0.306956 \\
\hline $\mathrm{C}$ & -3.998763 & -5.469453 & -0.310393 \\
\hline $\mathrm{H}$ & -3.138563 & -5.904630 & -0.808759 \\
\hline $\mathrm{C}$ & -4.954422 & -6.262352 & 0.329449 \\
\hline $\mathrm{H}$ & -4.846505 & -7.345283 & 0.330212 \\
\hline $\mathrm{C}$ & -6.043072 & -5.666240 & 0.969376 \\
\hline $\mathrm{H}$ & -6.785105 & -6.284733 & 1.470853 \\
\hline $\mathrm{C}$ & -6.173783 & -4.273692 & 0.971677 \\
\hline $\mathrm{H}$ & -7.017671 & -3.809112 & 1.477706 \\
\hline $\mathrm{C}$ & -5.228609 & -3.469998 & 0.332779 \\
\hline $\mathrm{H}$ & -5.282663 & -2.379614 & 0.339529 \\
\hline $\mathrm{H}$ & 0.000000 & 0.000000 & 0.000000 \\
\hline $\mathrm{C}$ & 0.000000 & 0.000000 & 1.090853 \\
\hline $\mathrm{C}$ & 1.182426 & 0.000000 & 1.735961 \\
\hline
\end{tabular}




$\begin{array}{cccc}\mathrm{H} & 1.174427 & -0.004692 & 2.825281 \\ \mathrm{C} & 2.461837 & 0.054863 & 1.056089 \\ \mathrm{H} & 2.425629 & 0.212140 & -0.016218 \\ \mathrm{C} & 3.699810 & -0.026865 & 1.613856 \\ \mathrm{C} & 3.938723 & -0.329537 & 3.054434 \\ \mathrm{C} & 3.243830 & -1.308359 & 3.684995 \\ \mathrm{H} & 2.574407 & -1.926337 & 3.091089 \\ \mathrm{C} & -1.342871 & -0.000364 & 1.757314 \\ \mathrm{~N} & 4.888351 & 0.147172 & 0.869246 \\ \mathrm{C} & 5.009796 & 0.385024 & 3.813263 \\ \mathrm{C} & 3.320065 & -1.613101 & 5.153961 \\ \mathrm{H} & -1.937169 & -0.877541 & 1.464164 \\ \mathrm{H} & -1.933045 & 0.881757 & 1.470941 \\ \mathrm{H} & -1.252078 & -0.003765 & 2.849290 \\ \mathrm{H} & 2.325851 & -1.908526 & 5.513576 \\ \mathrm{H} & 3.960686 & -2.498029 & 5.305387 \\ \mathrm{C} & 3.863062 & -0.423116 & 5.950387 \\ \mathrm{H} & 3.111355 & 0.376926 & 5.956528 \\ \mathrm{HS} & 4.042383 & -0.702173 & 6.994721 \\ \mathrm{H} & 0.000000 & 0.000000 & 0.000000 \\ \mathrm{C} & 5.149578 & 0.089035 & 5.302631 \\ \mathrm{H} & 5.943044 & -0.669261 & 5.394899 \\ \mathrm{H} & 5.533352 & 0.995409 & 5.781504 \\ \mathrm{O} & 5.816192 & 1.131828 & 3.266364 \\ \mathrm{H} & 5.680244 & 0.480812 & 1.409695 \\ \mathrm{C} & 5.034911 & 0.173726 & -0.494985 \\ \mathrm{O} & 4.168368 & -0.065724 & -1.320537 \\ \mathrm{O} & 6.327102 & 0.494923 & -0.767056 \\ \mathrm{C} & 6.809540 & 0.592787 & -2.150918 \\ \mathrm{C} & 6.057277 & 1.700875 & -2.896642 \\ \mathrm{C} & 6.686378 & -0.765810 & -2.850461 \\ \mathrm{C} & 8.281201 & 0.971360 & -1.961231 \\ \mathrm{H} & 8.771166 & 1.078457 & -2.934811 \\ \mathrm{H} & 8.807737 & 0.199781 & -1.390165 \\ \mathrm{H} & 8.369213 & 1.919455 & -1.420976 \\ \mathrm{H} & 5.134946 & 2.647012 & -2.349688 \\ \mathrm{H} & 7.501577 & 1.844043 & -3.888269 \\ \mathrm{H} & & -1.539504 & -2.269519 \\ \mathrm{H} & -0.714700 & -3.839246 \\ \mathrm{H} & & -1.052495 & -2.972439 \\ \mathrm{H} & & \\ \mathrm{H} & & & \\ \mathrm{H} & & & \\ \mathrm{H} & & & \\ \mathrm{H} & & & \\ \mathrm{H} & & & \\ \mathrm{H} & & & \end{array}$




\begin{tabular}{|c|c|c|c|}
\hline $\mathrm{C}$ & 1.247523 & 0.000000 & -0.636550 \\
\hline $\mathrm{H}$ & 1.387494 & -0.722138 & -1.447189 \\
\hline$C$ & 2.306322 & 0.896707 & -0.474473 \\
\hline $\mathrm{H}$ & 3.278944 & 0.591242 & -0.841616 \\
\hline $\mathrm{C}$ & 2.168131 & 2.279377 & -0.236934 \\
\hline $\mathrm{C}$ & 0.871454 & 2.884460 & -0.133821 \\
\hline $\mathrm{C}$ & -0.175599 & 2.198840 & 0.526599 \\
\hline $\mathrm{H}$ & 0.079775 & 1.825840 & 1.509956 \\
\hline $\mathrm{C}$ & -1.165361 & -0.744101 & -0.608117 \\
\hline $\mathrm{C}$ & -1.613061 & 2.642898 & 0.378873 \\
\hline $\mathrm{C}$ & 0.594603 & 4.183480 & -0.766757 \\
\hline $\mathrm{N}$ & 3.256664 & 3.138614 & -0.397923 \\
\hline $\mathrm{H}$ & -1.319928 & -1.719651 & -0.125778 \\
\hline $\mathrm{H}$ & -1.024074 & -0.910431 & -1.681409 \\
\hline $\mathrm{H}$ & -2.102556 & -0.185595 & -0.483619 \\
\hline $\mathrm{H}$ & -2.286618 & 1.781793 & 0.472521 \\
\hline $\mathrm{H}$ & -1.872108 & 3.311830 & 1.216376 \\
\hline $\mathrm{C}$ & -1.828082 & 3.375930 & -0.945481 \\
\hline $\mathrm{H}$ & -2.864801 & 3.718833 & -1.039150 \\
\hline $\mathrm{H}$ & -1.640238 & 2.685488 & -1.778415 \\
\hline $\mathrm{C}$ & -0.862752 & 4.558792 & -1.022703 \\
\hline $\mathrm{H}$ & -1.135065 & 5.316535 & -0.272221 \\
\hline $\mathrm{H}$ & -0.896204 & 5.066847 & -1.992599 \\
\hline $\mathrm{O}$ & 1.475041 & 5.004044 & -1.057837 \\
\hline $\mathrm{H}$ & 2.992810 & 4.114533 & -0.554799 \\
\hline $\mathrm{C}$ & 4.593556 & 2.823300 & -0.502218 \\
\hline $\mathrm{O}$ & 5.083308 & 1.709851 & -0.406998 \\
\hline $\mathrm{O}$ & 5.271559 & 3.977074 & -0.708833 \\
\hline $\mathrm{C}$ & 6.736278 & 3.988770 & -0.843195 \\
\hline $\mathrm{C}$ & 7.385951 & 3.492547 & 0.453141 \\
\hline $\mathrm{C}$ & 7.161037 & 3.161832 & -2.061623 \\
\hline $\mathrm{C}$ & 7.033964 & 5.474152 & -1.065592 \\
\hline $\mathrm{H}$ & 7.161586 & 2.439133 & 0.627710 \\
\hline $\mathrm{H}$ & 7.026590 & 4.078571 & 1.306202 \\
\hline $\mathrm{H}$ & 8.473091 & 3.615759 & 0.389971 \\
\hline $\mathrm{H}$ & 6.640682 & 3.513570 & -2.959311 \\
\hline $\mathrm{H}$ & 6.939609 & 2.103495 & -1.915970 \\
\hline $\mathrm{H}$ & 8.238337 & 3.278174 & -2.225838 \\
\hline $\mathrm{H}$ & 8.112184 & 5.628388 & -1.179675 \\
\hline $\mathrm{H}$ & 6.532639 & 5.838046 & -1.968146 \\
\hline & 6.685665 & 6.069346 & -0.215371 \\
\hline
\end{tabular}

